Supporting Information

\title{
Enantioselective Copper-Catalyzed Electrophilic Dearomative Azidation of $\beta$-Naphthols
}

\author{
Chong-Ji Wang, $\$$ Jian Sun, $\$$ Wei Zhou, Jing Xue, Bing-Tao Ren, Guang-Yi Zhang, Yan-Le Mei \\ and Qing-Hai Deng*
}

The Education Ministry Key Lab of Resource Chemistry and Shanghai Key Laboratory of Rare

Earth Functional Materials, Shanghai Normal University, Shanghai 200234, P. R. China

E-mail: qinghaideng@shnu.edu.cn

Table of Contents:

I. General Information

II. Substrate Preparation

III. Catalytic Enantioselective Reaction

IV. Further Transformations

V. Control Experiments

VI. Crystallographic Data

VII. Reference

VIII. Chromatographic Data for Chiral Products

IX. NMR Spectra of New Compounds $\left({ }^{1} \mathrm{H}\right.$ NMR, ${ }^{13} \mathrm{C}$ NMR, ${ }^{19}$ F NMR) 


\section{General Information}

All manipulations were maintained under an atmosphere of argon unless otherwise stated. Commercially available reagents were used without further purification. Solvents were pre-dried over activated $4 \AA$ molecular sieves and were refluxed over sodium-benzophenone (toluene, tetrahydrofurane), phosphorus pentoxide (chloroform) or calcium hydride (dichloromethane, dichloroethane, acetonitrile) under an argon atmosphere and collected by distillation. Column chromatography was performed on silica gel (200-300 mesh). ${ }^{1} \mathrm{H}$ NMR spectra were recorded on a 400 or $600 \mathrm{MHz}$ NMR spectrometer and ${ }^{13} \mathrm{C}$ NMR spectra were recorded on a 101 or $150 \mathrm{MHz}$ NMR spectrometer. Infrared spectra were prepared as $\mathrm{KBr}$ pellets and were recorded on a Varian Excalibur 3100 series FT-IR spectrometer. Mass spectra were recorded by the mass spectrometry service of Shanghai Institute of Organic Chemistry. HPLC analyses on a Waters 1596 or Shimadzu SPD-15C. Optical rotations were measured with Rudolph Research Analytical in a 1 dm cuvette.I. L2 ${ }^{[1]}, \mathbf{L 3}^{[2]}, \mathbf{L 4}^{-\mathbf{L}} 7^{[3]}, \mathbf{2 a}^{[4]}$, $\mathbf{2} \mathbf{b}^{[5]}$, and $\mathbf{4}^{[6]}$ were synthesized according to the literature procedures. 
II. Substrate Preparation
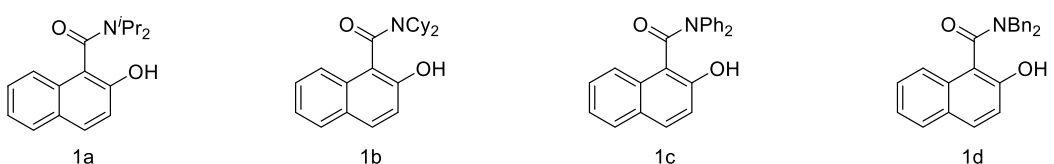

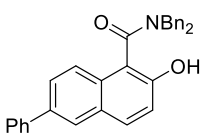

$1 \mathrm{e}$<smiles>CCCCC(=O)c1c(O)ccc2ccc(-c3ccccc3)cc12</smiles>

$1 \mathrm{i}$
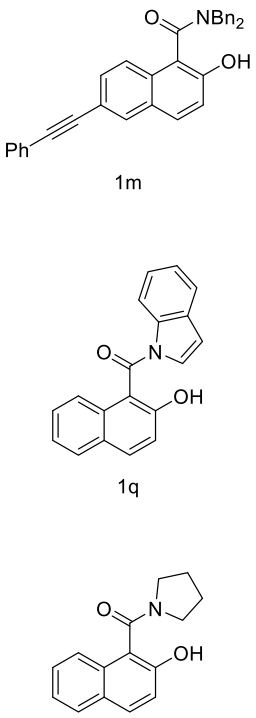

14
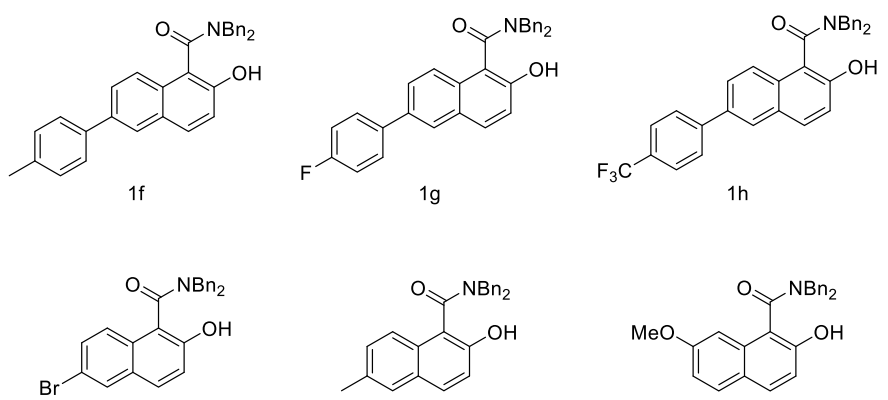

11
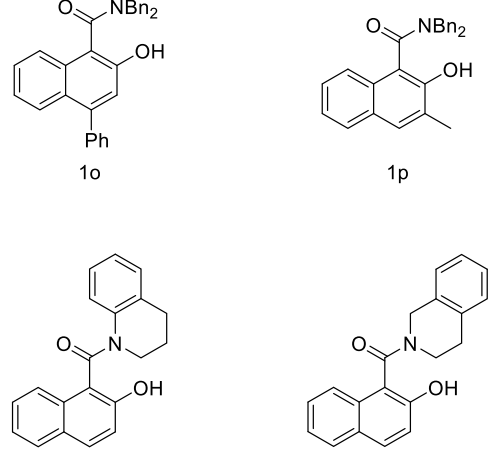

1t

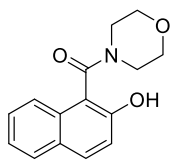

$1 \mathrm{w}$

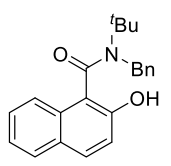

$1 \mathrm{x}$

\section{Synthetic Procedure for 1}<smiles></smiles><smiles></smiles><smiles>[R]CC1=CC=[R17]C=C2C1=CC=CC(C(=O)O)=C2O</smiles><smiles>[R]c1cc2c(c(C(=O)O)c1O)C=C[R17]C=C2</smiles>

1. $\mathrm{SOCl}_{2}$, toluene, $80^{\circ} \mathrm{C}, 6 \mathrm{~h}$

2. $\mathrm{HNBn}_{2}, \mathrm{Et}_{3} \mathrm{~N}$, toluene, $80^{\circ} \mathrm{C}$

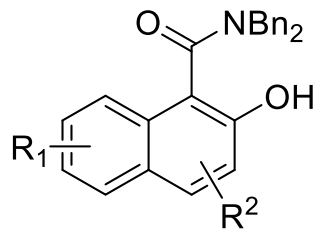

General Procedure for Step $1^{[7]}$ 
Sodium hydroxide ( 0.144 mol, 20 equiv) was added portionwise over a five minute period to a solution of methyl 2-hydroxy-1-naphthoate (7.2 mmol, 1 equiv) in methanol $(60 \mathrm{~mL})$ and water $(30 \mathrm{~mL})$ at $0{ }^{\circ} \mathrm{C}$. The resultant solution was warmed to $60{ }^{\circ} \mathrm{C}$ for about $12 \mathrm{~h}$ and then cooled to $25^{\circ} \mathrm{C}$. The reaction mixture was poured into $500 \mathrm{~mL}$ cold $\left(0{ }^{\circ} \mathrm{C}\right) 6 \mathrm{~N}$ hydrochloric acid, and the precipitate was collected by filtration and dried. Then the residue was purified by silica gel column chromatography (ethyl acetate/petroleum ether $=1 / 10-\mathrm{MeOH})$, then recrystallized in the mixture of dichloromethane/petroleum ether to afford the product.

\section{General Procedure for Step $2^{[8]}$}

In a round bottom flask 2-hydroxy-1-naphthoic acid ( 2 mmol, 1 equiv) was added, then $20 \mathrm{~mL}$ of toluene was added to it. The round bottom was connected with reflux condenser and $\mathrm{SOCl}_{2}(8 \mathrm{mmol}, 4$ equiv $)$ was added to the suspension of the acid in toluene in Ar atmosphere. Then the mixture was stirred for $6 \mathrm{~h}$ at $80^{\circ} \mathrm{C}$. After that the solvent was pumped out in high vacuum to get a sticky solid. Another portion of toluene (20 mL), $\mathrm{Et}_{3} \mathrm{~N}$ ( $2 \mathrm{mmol}, 1$ equiv), $\mathrm{HNBn}_{2}(2 \mathrm{mmol}, 1$ equiv) were added to it and the mixture was stirred for $10 \mathrm{~h}$ at $80{ }^{\circ} \mathrm{C}$. After that the volatile materials was evaporated in high vacuum. Water and DCM was added, organic portion was washed with saturated $\mathrm{NaHCO}_{3}$. The organic layer was dried over $\mathrm{Mg}_{2} \mathrm{SO}_{4}$, filtered and then concentrated. The residue was purified by silica gel column chromatography (ethyl acetate/petroleum ether $=1 / 10-1 / 2)$, then recrystallized in the mixture of dichloromethane/petroleum ether to afford the product.<smiles>CCCCNC(=O)c1c(O)ccc2ccccc12</smiles>

$1 \mathrm{a}$

2-Hydroxy-N,N-diisopropyl-1-naphthamide ${ }^{[9]}$; white solid; ${ }^{1} \mathrm{H}$ NMR (400 MHz, DMSO-d6) $9.80(\mathrm{~s}, 1 \mathrm{H}), 7.80(\mathrm{~d}, J=8.4 \mathrm{~Hz}, 1 \mathrm{H}), 7.75(\mathrm{~d}, J=8.8 \mathrm{~Hz}, 1 \mathrm{H}), 7.50$ (d, $J$ $=8.4 \mathrm{~Hz}, 1 \mathrm{H}), 7.44(\mathrm{ddd}, J=8.4,6.8,1.2 \mathrm{~Hz}, 1 \mathrm{H}), 7.29$ (ddd, $J=8.4,6.8,1.2 \mathrm{~Hz}$, 
1H), $7.17(\mathrm{~d}, J=8.8 \mathrm{~Hz}, 1 \mathrm{H}), 3.60(\mathrm{~m}, 2 \mathrm{H}), 1.60(\mathrm{~d}, J=6.8 \mathrm{~Hz}, 3 \mathrm{H}), 1.52(\mathrm{~d}, J=6.8$ $\mathrm{Hz}, 3 \mathrm{H}), 1.13(\mathrm{~d}, J=6.8 \mathrm{~Hz}, 3 \mathrm{H}), \delta 1.02(\mathrm{~d}, J=6.8 \mathrm{~Hz}, 3 \mathrm{H})$.<smiles>CCCCNC(=O)c1c(O)ccc2ccccc12</smiles>

1b

N,N-Dicyclohexyl-2-hydroxy-1-naphthamide; white solid (mp: 225 - $230{ }^{\circ} \mathrm{C}$ ); ${ }^{1} \mathbf{H}$ NMR (400 MHz, DMSO-d $) \delta 9.80(\mathrm{~s}, 1 \mathrm{H}), 7.79$ (d, $J=8.0 \mathrm{~Hz}, 1 \mathrm{H}), 7.75$ (d, $J=8.8$ $\mathrm{Hz}, 1 \mathrm{H}), 7.50$ (d, $J=8.4 \mathrm{~Hz}, 1 \mathrm{H}), 7.41$ (ddd, $J=8.4,6.8,1.2 \mathrm{~Hz}, 1 \mathrm{H}), 7.28$ (ddd, $J=$ 8.4, 6.8, $1.2 \mathrm{~Hz}, 1 \mathrm{H}), 7.17(\mathrm{~d}, J=8.8 \mathrm{~Hz}, 1 \mathrm{H}), 3.20-3.11(\mathrm{~m}, 2 \mathrm{H}), 2.78-2.60(\mathrm{~m}$, $2 \mathrm{H}), 1.87-1.77(\mathrm{~m}, 3 \mathrm{H}), 1.71-1.58(\mathrm{~m}, 4 \mathrm{H}), 1.51-1.42(\mathrm{~m}, 3 \mathrm{H}), 1.39-1.29(\mathrm{~m}$, 4H), $1.22-1.10(\mathrm{~m}, 1 \mathrm{H}), 0.87-0.78(\mathrm{~m}, 2 \mathrm{H}), 0.58-0.48(\mathrm{~m}, 1 \mathrm{H}) ;{ }^{13} \mathbf{C}\left\{{ }^{1} \mathbf{H}\right\} \mathbf{N M R}$ (101 MHz, DMSO-d $) \delta 167.2,150.1,131.0,129.0,128.0,127.5,126.6,123.1,122.9$, $118.8,117.9,59.5,54.9,30.9,30.8,29.83,29.80,26.0,25.5,25.34,25.30,24.5$; IR v (neat, $\mathbf{c m}^{-1}$ ): 3005, 2989, 1599, 1455, 1275, 1261, 750; HRMS (ESI, m/z): calcd for $\mathrm{C}_{23} \mathrm{H}_{30} \mathrm{NO}_{2}{ }^{+}[\mathrm{M}+\mathrm{H}]^{+}:$352.2271, found: 352.2278.<smiles>CCCCCC(C)C(=O)c1c(O)ccc2ccccc12</smiles>

2-Hydroxy-N,N-diphenyl-1-naphthamide; yellow solid (mp: $198-203{ }^{\circ} \mathrm{C}$ ); ${ }^{\mathbf{1}} \mathbf{H}$ NMR (400 MHz, DMSO-d6) $\delta 10.85$ (s, 1H), 8.13 (d, $J=8.4 \mathrm{~Hz}, 1 \mathrm{H}), 8.05$ (t, $J=$ $8.8 \mathrm{~Hz}, 2 \mathrm{H}), 7.99-7.94(\mathrm{~m}, 2 \mathrm{H}), 7.70(\mathrm{t}, J=7.4 \mathrm{~Hz}, 1 \mathrm{H}), 7.57$ (t, $J=7.4 \mathrm{~Hz}, 1 \mathrm{H})$, $7.49(\mathrm{~d}, J=8.8 \mathrm{~Hz}, 1 \mathrm{H}), 7.45-7.42(\mathrm{~m}, 1 \mathrm{H}), 7.37$ (d, $J=8.8 \mathrm{~Hz}, 1 \mathrm{H}), 7.31-7.28(\mathrm{~m}$, 3H), 7.10 - $7.08(\mathrm{~m}, 1 \mathrm{H}), 7.00-6.96(\mathrm{~m}, 2 \mathrm{H}) ;{ }^{13} \mathbf{C}\left\{{ }^{1} \mathbf{H}\right\}$ NMR (101 MHz, DMSO-d6) $\delta 167.7,150.9,142.9,142.3,131.0,130.1,129.0,128.3,127.9,127.2,127.04,127.00$, 126.9, 126.3, 123.2, 122.9, 117.8, 117.7; IR v (neat, $\mathbf{c m}^{-1}$ ): 3005, 2989, 1627, 1492 , 1275, 1261, 750; HRMS (ESI, m/z): calcd for $\mathrm{C}_{23} \mathrm{H}_{18} \mathrm{NO}_{2}{ }^{+}[\mathrm{M}+\mathrm{H}]^{+}: 340.1332$, found: 340.1339 . 
<smiles>CCCCCCCNC(=O)c1c(O)ccc2ccccc12</smiles>

N,N-Dibenzyl-2-hydroxy-1-naphthamide; white solid; ${ }^{1} \mathrm{H}$ NMR (400 MHz, DMSO-d6) $\delta 10.39$ (s, 1H), $7.84-7.82(\mathrm{~m}, 2 \mathrm{H}), 7.59$ (d, J=8.4 Hz, 1H), $7.47-7.37$ (m, 5H), $7.31(\mathrm{t}, J=7.2 \mathrm{~Hz}, 2 \mathrm{H}), 7.23-7.18(\mathrm{~m}, 4 \mathrm{H}), 7.09-7.04(\mathrm{~m}, 2 \mathrm{H}), 4.92(\mathrm{~d}, J$ $=15.2 \mathrm{~Hz}, 1 \mathrm{H}), 4.43(\mathrm{~d}, J=15.2 \mathrm{~Hz}, 1 \mathrm{H}), 4.35(\mathrm{~d}, J=15.6 \mathrm{~Hz}, 1 \mathrm{H}), 4.18(\mathrm{~d}, J=15.6$ $\mathrm{Hz}, 1 \mathrm{H})$.<smiles>CCCCC(=O)c1c(O)ccc2cc(-c3ccccc3)ccc12</smiles>

N,N-Dibenzyl-2-hydroxy-6-phenyl-1-naphthamide; white solid (mp: 250 - $255^{\circ} \mathrm{C}$ ); ${ }^{1}$ H NMR (400 MHz, DMSO-d6) $\delta 10.46$ (s, 1H), 8.15 (s, 1H), 7.93 (d, $J=9.2 \mathrm{~Hz}$, 1H), $7.82-7.77(\mathrm{~m}, 3 \mathrm{H}), 7.69(\mathrm{~d}, J=8.8 \mathrm{~Hz}, 1 \mathrm{H}), 7.51-7.44(\mathrm{~m}, 4 \mathrm{H}), 7.42-7.30$ (m, 4H), $7.27(\mathrm{~d}, J=8.8 \mathrm{~Hz}, 1 \mathrm{H}), 7.24-7.16(\mathrm{~m}, 3 \mathrm{H}), 7.10(\mathrm{~d}, J=7.2 \mathrm{~Hz}, 2 \mathrm{H}), 4.94$ $(\mathrm{d}, J=15.2 \mathrm{~Hz}, 1 \mathrm{H}), 4.46(\mathrm{~d}, J=15.2 \mathrm{~Hz}, 1 \mathrm{H}), 4.39$ (d, $J=16.0 \mathrm{~Hz}, 1 \mathrm{H}), 4.23(\mathrm{~d}, J=$ $16.0 \mathrm{~Hz}, 1 \mathrm{H}) ;{ }^{13} \mathbf{C}\left\{{ }^{1} \mathbf{H}\right\}$ NMR (101 MHz, DMSO-d6) $\delta$ 168.3, 151.1, 139.8, 137.1, $136.3,134.7,130.6,130.4,129.9,128.9,128.6,128.40,128.37,127.9,127.8,127.7$, 127.3, 127.2, 127.1, 126.6, 126.0, 125.6, 124.0, 118.4, 116.1, 50.9, 46.1; IR v (neat, $\mathbf{c m}^{-1}$ ): 3005, 2990, 1463, 1275, 1261, 753; HRMS (ESI, m/z): calcd for $\mathrm{C}_{31} \mathrm{H}_{26} \mathrm{NO}_{2}{ }^{+}$ [M+H $]^{+}$Calcd.: 444.1958, found: 444.1955.<smiles>CCCNC(=O)c1c(O)ccc2cc(-c3ccc(C)cc3)ccc12</smiles>

N,N-Dibenzyl-2-hydroxy-6-(p-tolyl)-1-naphthamide; white solid (mp: 259 - 262 $\left.{ }^{\circ} \mathrm{C}\right) ;{ }^{1}$ H NMR (400 MHz, DMSO-d6) $\delta 10.43$ (s, 1H), $8.12(\mathrm{~s}, 1 \mathrm{H}), 7.91$ (d, $J=8.8$ 
Hz, 1H), $7.79(\mathrm{~d}, J=8.4 \mathrm{~Hz}, 1 \mathrm{H}), 7.72-7.63(\mathrm{~m}, 3 \mathrm{H}), 7.46-7.38(\mathrm{~m}, 4 \mathrm{H}), 7.34-$ $7.18(\mathrm{~m}, 7 \mathrm{H}), 7.14-7.06(\mathrm{~m}, 2 \mathrm{H}), 4.93(\mathrm{~d}, J=14.8 \mathrm{~Hz}, 1 \mathrm{H}), 4.46(\mathrm{~d}, J=14.8 \mathrm{~Hz}$, 1H), $4.38(\mathrm{~d}, J=15.6 \mathrm{~Hz}, 1 \mathrm{H}), 4.22(\mathrm{~d}, J=15.6 \mathrm{~Hz}, 1 \mathrm{H}), 2.36(\mathrm{~s}, 3 \mathrm{H}) ;{ }^{13} \mathbf{C}\left\{{ }^{1} \mathbf{H}\right\} \mathbf{N M R}$ (101 MHz, DMSO-d6) $\delta 168.3,151.0,137.1,136.9,136.5,136.3,134.6,130.5$, $130.3,129.9,129.5,128.6,128.41,128.38,128.0,127.8,127.7,127.3,127.1,126.4$, 125.9, 125.1, 124.0, 118.3, 116.0, 50.9, 46.1, 20.6; IR v (neat, $\left.\mathbf{c m}^{-1}\right)$ : 3005, 2996, 1600, 1453, 1275, 1260, 750; HRMS (ESI, m/z): calcd for $\mathrm{C}_{32} \mathrm{H}_{28} \mathrm{NO}_{2}{ }^{+}[\mathrm{M}+\mathrm{H}]^{+}$: 458.2115, found: 458.2116 .<smiles>CCCCC(=O)c1c(O)ccc2cc(-c3ccc(F)cc3)ccc12</smiles>

N,N-Dibenzyl-6-(4-fluorophenyl)-2-hydroxy-1-naphthamide; yellow solid (mp: 241 - $\left.244{ }^{\circ} \mathrm{C}\right) ;{ }^{1} \mathbf{H}$ NMR (400 MHz, DMSO-d6) $\delta 10.45$ (s, 1H), 8.13 (d, $J=2.0 \mathrm{~Hz}$, 1H), $7.91(\mathrm{~d}, J=8.8 \mathrm{~Hz}, 1 \mathrm{H}), 7.83-7.77(\mathrm{~m}, 3 \mathrm{H}), 7.67(\mathrm{~d}, J=8.4 \mathrm{~Hz}, 1 \mathrm{H}), 7.45-$ $7.38(\mathrm{~m}, 4 \mathrm{H}), 7.35-7.29(\mathrm{~m}, 3 \mathrm{H}), 7.27-7.18(\mathrm{~m}, 4 \mathrm{H}), 7.11-7.09(\mathrm{~m}, 2 \mathrm{H}), 4.93$ (d, $J=15.2 \mathrm{~Hz}, 1 \mathrm{H}), 4.46(\mathrm{~d}, J=15.2 \mathrm{~Hz}, 1 \mathrm{H}), 4.38(\mathrm{~d}, J=15.6 \mathrm{~Hz}, 1 \mathrm{H}), 4.22(\mathrm{~d}, J=$ $15.6 \mathrm{~Hz}, 1 \mathrm{H}) ;{ }^{13} \mathbf{C}\left\{{ }^{1} \mathbf{H}\right\}$ NMR (101 MHz, DMSO-d6) $\delta 168.3,161.8(\mathrm{~d}, J=245.1 \mathrm{~Hz})$, $151.2,137.1,136.30,136.27,133.7,130.6,130.4,128.6(\mathrm{~d}, J=8.2 \mathrm{~Hz}), 128.4(\mathrm{~d}, J=$ $3.4 \mathrm{~Hz}), 127.9,127.8,127.7,127.3,127.1,125.9,125.5,124.1,118.5,116.1,115.70(\mathrm{~d}$, $J=21.4 \mathrm{~Hz}), 50.9,46.1 ;{ }^{19}$ F NMR (376 MHz, DMSO-d6) $\delta-115.67--115.74(\mathrm{~m}, 1$ F); IR $v$ (neat, $\left.\mathbf{c m}^{-1}\right):$ 3366, 2922, 2851, 2106, 1470, 1275, 1262, 822, 739; HRMS (ESI, m/z): calcd for $\mathrm{C}_{31} \mathrm{H}_{25} \mathrm{FNO}_{2}{ }^{+}[\mathrm{M}+\mathrm{H}]^{+}:$462.1864, found: 462.1869 .<smiles>CCCCC(=O)c1c(O)ccc2cc(-c3ccc(C(F)(F)F)cc3)ccc12</smiles>

N,N-Dibenzyl-2-hydroxy-6-(4-(trifluoromethyl)phenyl)-1-naphthamide; yellow 
solid (mp: 251 - $\left.252{ }^{\circ} \mathrm{C}\right)$; ${ }^{1} \mathbf{H}$ NMR (400 MHz, DMSO-d6) $\delta 10.58$ (s, 1H), 8.26 (s, 1H), $8.00(\mathrm{~d}, J=8.0 \mathrm{~Hz}, 2 \mathrm{H}), 7.96(\mathrm{~d}, J=9.2 \mathrm{~Hz}, 1 \mathrm{H}), 7.87-7.82(\mathrm{~m}, 3 \mathrm{H}), 7.72(\mathrm{~d}, J$ $=8.8 \mathrm{~Hz}, 1 \mathrm{H}), 7.46-7.44(\mathrm{~m}, 2 \mathrm{H}), 7.40(\mathrm{t}, J=7.4 \mathrm{~Hz}, 2 \mathrm{H}), 7.34-7.28(\mathrm{~m}, 2 \mathrm{H}), 7.23$ $-7.15(\mathrm{~m}, 3 \mathrm{H}), 7.10(\mathrm{~d}, J=7.2 \mathrm{~Hz}, 2 \mathrm{H}), 4.95(\mathrm{~d}, J=15.2 \mathrm{~Hz}, 1 \mathrm{H}), 4.47(\mathrm{~d}, J=15.2$ $\mathrm{Hz}, 1 \mathrm{H}), 4.39(\mathrm{~d}, J=15.6 \mathrm{~Hz}, 1 \mathrm{H}), 4.23(\mathrm{~d}, J=15.6 \mathrm{~Hz}, 1 \mathrm{H}) ;{ }^{13} \mathbf{C}\left\{{ }^{1} \mathbf{H}\right\}$ NMR $(\mathbf{1 0 1}$ MHz, DMSO-d6) $\delta$ 168.3, 151.7, 143.8, 137.1, 136.3, 133.0, 131.0, 130.9, 128.5, $128.4,127.9,127.7,127.44,127.38,127.2,126.5,125.84,125.80,124.4(q, J=269.5$ $\mathrm{Hz}$ ), 124.3, 118.7, 116.1, 51.0, 46.2; ${ }^{19}$ F NMR (376 MHz, DMSO-d6) $\delta$-60.80 (s, 3F); IR v (neat, $\mathbf{c m}^{-1}$ ): 3005, 2989, 1563, 1474, 1275, 1261, 757; HRMS (ESI, m/z): calcd for $\mathrm{C}_{32} \mathrm{H}_{25} \mathrm{~F}_{3} \mathrm{NO}_{2}{ }^{+}[\mathrm{M}+\mathrm{H}]^{+}:$512.1832, found: 512.1829 .<smiles>CCCCC(=O)c1c(O)ccc2ccc(-c3ccccc3)cc12</smiles>

1i

N,N-Dibenzyl-2-hydroxy-7-phenyl-1-naphthamide; white solid (mp: 229 - $231{ }^{\circ} \mathrm{C}$ ); ${ }^{1}$ H NMR (400 MHz, DMSO-d6) $\delta 10.42$ (s, 1H), 7.92 (d, $J=8.4$ Hz, 1H), 7.86 (d, $J$ $=8.8 \mathrm{~Hz}, 1 \mathrm{H}), 7.67(\mathrm{~d}, J=1.2 \mathrm{~Hz}, 1 \mathrm{H}), 7.63(\mathrm{dd}, J=8.4,1.6 \mathrm{~Hz}, 1 \mathrm{H}), 7.58-7.56(\mathrm{~m}$, 2H), $7.53-7.46(\mathrm{~m}, 4 \mathrm{H}), 7.44-7.35(\mathrm{~m}, 4 \mathrm{H}), 7.25-7.18(\mathrm{~m}, 6 \mathrm{H}), 4.92(\mathrm{~d}, J=14.8$ $\mathrm{Hz}, 1 \mathrm{H}), 4.45(\mathrm{~d}, J=14.8 \mathrm{~Hz}, 1 \mathrm{H}), 4.29(\mathrm{~s}, 2 \mathrm{H}) ;{ }^{13} \mathbf{C}\left\{{ }^{1} \mathbf{H}\right\}$ NMR (101 MHz, DMSO-d6) $\delta 168.3,151.4,139.9,138.5,137.4,136.3,131.4,129.8,129.0,128.9$, $128.5,128.3,128.2,127.8,127.6,127.3,127.2,126.8,122.3,120.4,118.2,116.5$, 51.0, 46.2; IR $v$ (neat, $\mathbf{c m}^{-1}$ ): 3006, 2990, 1601, 1494, 1453, 1275, 1261, 769, 742; HRMS (ESI, m/z): calcd for $\mathrm{C}_{31} \mathrm{H}_{26} \mathrm{NO}_{2}{ }^{+}[\mathrm{M}+\mathrm{H}]^{+}$: 444.1958, found: 444.1966.<smiles>CCCCC(=O)c1c(O)ccc2cc(Br)ccc12</smiles>

1j

N,N-Dibenzyl-6-bromo-2-hydroxy-1-naphthamide; white solid (mp: 198 - $203{ }^{\circ} \mathrm{C}$ ); ${ }^{1}$ H NMR (400 MHz, DMSO-d6) $\delta 10.60$ (s, 1H), 8.10 (d, $\left.J=2.0 \mathrm{~Hz}, 1 \mathrm{H}\right), 7.84$ (d, $J$ $=8.8 \mathrm{~Hz}, 1 \mathrm{H}), 7.58-7.52(\mathrm{~m}, 2 \mathrm{H}), 7.43-7.37(\mathrm{~m}, 4 \mathrm{H}), 7.31(\mathrm{t}, J=7.0 \mathrm{~Hz}, 1 \mathrm{H}), 7.26$ 
(d, $J=9.2 \mathrm{~Hz}, 1 \mathrm{H}), 7.22-7.16(\mathrm{~m}, 3 \mathrm{H}), 7.04-7.02(\mathrm{~m}, 2 \mathrm{H}), 5.01(\mathrm{~d}, J=15.2 \mathrm{~Hz}$, 1H), $4.39-4.34(\mathrm{~m}, 2 \mathrm{H}), 4.17(\mathrm{~d}, J=16.0 \mathrm{~Hz}, 1 \mathrm{H}) ;{ }^{13} \mathbf{C}\left\{{ }^{1} \mathbf{H}\right\}$ NMR (101 MHz, DMSO-d6) $\delta 168.0,151.4,137.0,136.3,129.93,129.85,129.8,129.6,128.9,128.5$, 128.4, 127.8, 127.6, 127.4, 127.1, 125.7, 119.1, 116.4, 115.9, 50.9, 46.3; IR v (neat, $\left.\mathbf{c m}^{-1}\right)$ : 3005, 2989, 1601, 1499, 1275, 1261, 757; HRMS (ESI, m/z): calcd for $\mathrm{C}_{25} \mathrm{H}_{21} \mathrm{BrNO}_{2}{ }^{+}[\mathrm{M}+\mathrm{H}]^{+}:$446.0750, found: 446.0755.<smiles>CCCCC(=O)c1c(O)ccc2cc(C)ccc12</smiles>

$1 k$

N,N-Dibenzyl-2-hydroxy-6-methyl-1-naphthamide; yellow solid (mp: 110 - 112 $\left.{ }^{\circ} \mathrm{C}\right) ;{ }^{1}$ H NMR (400 MHz, DMSO-d6) $\delta 10.23$ (s, 1H), 7.73 (d, $\left.J=8.8 \mathrm{~Hz}, 1 \mathrm{H}\right), 7.59$ (s, 1H), $7.51(\mathrm{~d}, J=8.4 \mathrm{~Hz}, 1 \mathrm{H}), 7.44-7.37(\mathrm{~m}, 4 \mathrm{H}), 7.33-7.29(\mathrm{~m}, 2 \mathrm{H}), 7.24-$ $7.17(\mathrm{~m}, 4 \mathrm{H}), 7.12-7.04(\mathrm{~m}, 2 \mathrm{H}), 4.91$ (d, $J=15.2 \mathrm{~Hz}, 1 \mathrm{H}), 4.42$ (d, $J=15.2 \mathrm{~Hz}$, 1H), $4.34(\mathrm{~d}, J=16.0 \mathrm{~Hz}, 1 \mathrm{H}), 4.17(\mathrm{~d}, J=16.0 \mathrm{~Hz}, 1 \mathrm{H}), 2.40(\mathrm{~s}, 3 \mathrm{H}) ;{ }^{13} \mathbf{C}\left\{{ }^{1} \mathbf{H}\right\} \mathbf{~ N M R}$ (101 MHz, DMSO-d6) $\delta$ 168.5, 150.2, 137.1, 136.3, 132.1, 129.44, 129.36, 129.1, $128.4,127.8,127.7,127.4,127.1,127.0,123.2,117.9,116.04,116.00,50.9$, 45.9, 20.9; IR v (neat, $\mathbf{c m}^{-\mathbf{1}}$ ): 3005, 2990, 1602, 1453, 1275, 1261, 897, 750; HRMS (ESI, $\mathbf{m} / \mathbf{z}$ ): calcd for $\mathrm{C}_{26} \mathrm{H}_{24} \mathrm{NO}_{2}{ }^{+}[\mathrm{M}+\mathrm{H}]^{+}:$382.1802, found: 382.1801 .<smiles>CCCCC(=O)c1c(O)ccc2ccc(OC)cc12</smiles>

11

N,N-Dibenzyl-2-hydroxy-7-methoxy-1-naphthamide; yellow solid (mp: 225 - 230 $\left.{ }^{\circ} \mathrm{C}\right) ;{ }^{1}$ H NMR (400 MHz, DMSO-d6) $\delta 7.68(\mathrm{dd}, J=8.8,2.8 \mathrm{~Hz}, 2 \mathrm{H}), 7.48(\mathrm{~d}, J=$ $7.2 \mathrm{~Hz}, 2 \mathrm{H}), 7.39-7.36(\mathrm{~m}, 2 \mathrm{H}), 7.30(\mathrm{t}, J=7.2 \mathrm{~Hz}, 1 \mathrm{H}), 7.26-7.17(\mathrm{~m}, 5 \mathrm{H}), 7.12$ $(\mathrm{d}, J=8.8 \mathrm{~Hz}, 1 \mathrm{H}), 6.88(\mathrm{dd}, J=8.8,2.4 \mathrm{~Hz}, 1 \mathrm{H}), 6.72(\mathrm{~d}, J=2.4 \mathrm{~Hz}, 1 \mathrm{H}), 4.78(\mathrm{~d}, J$ $=14.8 \mathrm{~Hz}, 1 \mathrm{H}), 4.51(\mathrm{~d}, J=14.8 \mathrm{~Hz}, 1 \mathrm{H}), 4.32(\mathrm{~d}, J=15.6 \mathrm{~Hz}, 1 \mathrm{H}), 4.25(\mathrm{~d}, J=15.6$ $\mathrm{Hz}, 1 \mathrm{H}), 3.63$ (s, 3H); ${ }^{13} \mathbf{C}\left\{{ }^{1} \mathbf{H}\right\}$ NMR (101 MHz, DMSO-d6) $\delta 169.0,158.0,153.3$, $137.4,136.5,132.7,129.7,129.5,128.4,128.3,128.1,127.8,127.3,127.1,122.4$, 
116.1, 115.3, 114.8, 101.3, 54.8, 50.9, 46.0; IR v (neat, $\mathbf{c m}^{-1}$ ): 3005, 2990, 1470, 1275, 1261, 897, 750; HRMS (ESI, m/z): calcd for $\mathrm{C}_{26} \mathrm{H}_{24} \mathrm{NO}_{3}{ }^{+}[\mathrm{M}+\mathrm{H}]^{+}:$: 398.1751, found: 398.1759 .

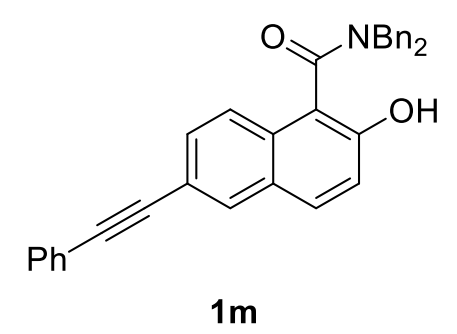

N,N-Dibenzyl-2-hydroxy-6-(phenylethynyl)-1-naphthamide; white solid (mp: 240 - $\left.242{ }^{\circ} \mathrm{C}\right) ;{ }^{1}$ H NMR (400 MHz, DMSO-d6) $\delta 10.65$ (s, 1H), 8.10 (d, $\left.J=1.2 \mathrm{~Hz}, 1 \mathrm{H}\right)$, $7.89(\mathrm{~d}, J=8.8 \mathrm{~Hz}, 1 \mathrm{H}), 7.63-7.54(\mathrm{~m}, 4 \mathrm{H}), 7.47-7.38(\mathrm{~m}, 7 \mathrm{H}), 7.32(\mathrm{t}, J=7.0 \mathrm{~Hz}$, 1H), $7.27(\mathrm{~d}, J=9.2 \mathrm{~Hz}, 1 \mathrm{H}), 7.23-7.17(\mathrm{~m}, 3 \mathrm{H}), 7.06-7.04(\mathrm{~m}, 2 \mathrm{H}), 5.04(\mathrm{~d}, J=$ 15.2 Hz, 1H), $4.41-4.35(\mathrm{~m}, 2 \mathrm{H}), 4.19(\mathrm{~d}, J=15.6 \mathrm{~Hz}, 1 \mathrm{H}) ;{ }^{13} \mathbf{C}\left\{{ }^{1} \mathbf{H}\right\}$ NMR $(\mathbf{1 0 1}$ MHz, DMSO-d6) $\delta$ 168.1, 152.0, 137.0, 136.3, 131.6, 131.3, 130.8, 130.4, 129.1, $128.8,128.7,128.45,128.41,127.8,127.7,127.4,127.3,127.1,123.9,122.4,118.8$, 116.6, 116.5, 89.7, 89.2, 50.9, 46.3; IR $v$ (neat, $\left.\mathbf{c m}^{-1}\right):$ 3005, 2990, 1474, 1275, 1261 , 897, 750; HRMS (ESI, m/z): calcd for $\mathrm{C}_{33} \mathrm{H}_{26} \mathrm{NO}_{2}{ }^{+}[\mathrm{M}+\mathrm{H}]^{+}$: 468.1958, found: 468.1949 .<smiles>CCCCC(=O)c1c(O)cc(Br)c2ccccc12</smiles>

N,N-Dibenzyl-4-bromo-2-hydroxy-1-naphthamide; white solid (mp: 211 - $215^{\circ} \mathrm{C}$ ); ${ }^{1}$ H NMR (400 MHz, DMSO-d6) $\delta 7.96$ (d, $\left.J=8.0 \mathrm{~Hz}, 1 \mathrm{H}\right), 7.67$ (s, 1H), 7.60 (d, $J=$ $8.4 \mathrm{~Hz}, 1 \mathrm{H}), 7.51-7.45(\mathrm{~m}, 3 \mathrm{H}), 7.39-7.32(\mathrm{~m}, 3 \mathrm{H}), 7.31-7.26(\mathrm{~m}, 1 \mathrm{H}), 7.21-$ $7.13(\mathrm{~m}, 3 \mathrm{H}), 7.08-7.06(\mathrm{~m}, 2 \mathrm{H}), 5.06$ (d, $J=15.2 \mathrm{~Hz}, 1 \mathrm{H}), 4.49$ (d, $J=16.0 \mathrm{~Hz}$, 1H), $4.33(\mathrm{~d}, J=15.2 \mathrm{~Hz}, 1 \mathrm{H}), 4.23(\mathrm{~d}, J=16.0 \mathrm{~Hz}, 1 \mathrm{H}) ;{ }^{13} \mathbf{C}\left\{{ }^{1} \mathbf{H}\right\} \mathbf{N M R}(\mathbf{1 0 1} \mathbf{M H z}$, DMSO-d6) $\delta 168.8,155.0,137.1,136.5,132.5,128.4,128.3,127.69,127.67,127.3$, $127.2,126.9,126.5,124.5,123.6,123.2,122.8,116.3,51.0,46.1 ;$ IR $v$ (neat, $\mathbf{~ c m}^{-1}$ ): 
3005, 2989, 1597, 1509, 1470, 1275, 1261, 750; HRMS (ESI, m/z): calcd for $\mathrm{C}_{25} \mathrm{H}_{21} \mathrm{BrNO}_{2}{ }^{+}[\mathrm{M}+\mathrm{H}]^{+}:$446.0750, found: 446.0747.<smiles>CCCCCC(=O)c1c(O)cc(-c2ccccc2)c2ccccc12</smiles>

N,N-Dibenzyl-2-hydroxy-4-phenyl-1-naphthamide; white solid (mp: 215 - $219{ }^{\circ} \mathrm{C}$ ); ${ }^{1}$ H NMR (400 MHz, DMSO-d6) $\delta 10.50(\mathrm{~s}, 1 \mathrm{H}), 7.69(\mathrm{~d}, J=8.4 \mathrm{~Hz}, 1 \mathrm{H}), 7.66(\mathrm{~d}, J$ $=8.4 \mathrm{~Hz}, 1 \mathrm{H}), 7.55-7.38(\mathrm{~m}, 10 \mathrm{H}), 7.34-7.27(\mathrm{~m}, 2 \mathrm{H}), 7.26-7.18(\mathrm{~m}, 3 \mathrm{H}), 7.14-$ $7.09(\mathrm{~m}, 3 \mathrm{H}), 4.96(\mathrm{~d}, J=15.2 \mathrm{~Hz}, 1 \mathrm{H}), 4.50-4.40(\mathrm{~m}, 2 \mathrm{H}), 4.26(\mathrm{~d}, J=15.6 \mathrm{~Hz}$, $1 \mathrm{H}) ;{ }^{13} \mathbf{C}\left\{{ }^{1} \mathbf{H}\right\}$ NMR (101 MHz, DMSO-d6) $\delta$ 168.4, 150.2, 141.9, 139.3, 137.1, 136.3, 131.8, 129.5, 128.5, 128.40, 128.38, 128.2, 127.8, 127.74, 127.69, 127.3, 127.1, 126.9, 125.7, 123.9, 123.4, 118.6, 115.9, 51.0, 46.1; IR v (neat, $\left.\mathbf{c m}^{-1}\right): 3005,2989$, 1454, 1275, 1261, 761; HRMS (ESI, m/z): calcd for $\mathrm{C}_{31} \mathrm{H}_{26} \mathrm{NO}_{2}{ }^{+}[\mathrm{M}+\mathrm{H}]^{+}: 444.1958$, found: 444.1959 .<smiles>CCCCC(=O)c1c(O)c(C)cc2ccccc12</smiles>

N,N-Dibenzyl-2-hydroxy-3-methyl-1-naphthamide; white solid (mp: 167 - $170{ }^{\circ} \mathrm{C}$ ); ${ }^{1}$ H NMR (400 MHz, DMSO-d6) $\delta 9.38$ (s, 1H), 7.75 (d, J=8.0 Hz, 1H), 7.71 (s, 1H), $7.55(\mathrm{~d}, J=8.0 \mathrm{~Hz}, 1 \mathrm{H}), 7.48-7.44(\mathrm{~m}, 2 \mathrm{H}), 7.41-7.35(\mathrm{~m}, 3 \mathrm{H}), 7.33-7.29(\mathrm{~m}$, 2H), $7.22-7.16(\mathrm{~m}, 3 \mathrm{H}), 7.08-7.02(\mathrm{~m}, 2 \mathrm{H}), 4.95(\mathrm{~d}, J=15.2 \mathrm{~Hz}, 1 \mathrm{H}), 4.43(\mathrm{~d}, J=$ $15.2 \mathrm{~Hz}, 1 \mathrm{H}), 4.33(\mathrm{~d}, J=16.0 \mathrm{~Hz}, 1 \mathrm{H}), 4.16(\mathrm{~d}, J=16.0 \mathrm{~Hz}, 1 \mathrm{H}), 2.41(\mathrm{~s}, 3 \mathrm{H})$; ${ }^{13} \mathbf{C}\left\{{ }^{1} \mathbf{H}\right\}$ NMR (101 MHz, DMSO-d6) $\delta$ 168.7, 149.7, 137.1, 136.2, 129.7, 129.5, $128.4,128.3,128.2$, 128.0, 127.8, 127.6, 127.3, 127.2, 127.1, 125.9, 123.4, 123.2, 118.1, 51.0, 46.1, 17.2; IR $v$ (neat, $\mathbf{c m}^{-1}$ ): 3005, 2989, 1595, 1454, 1275, 1261, 750; HRMS (ESI, m/z): calcd for $\mathrm{C}_{26} \mathrm{H}_{24} \mathrm{NO}_{2}{ }^{+}[\mathrm{M}+\mathrm{H}]^{+}: 382.1802$, found: 382.1796 . 


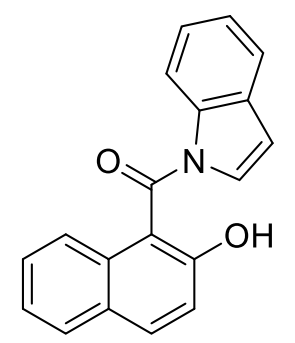

$1 q$

(2-Hydroxynaphthalen-1-yl)(1H-indol-1-yl)methanone; white solid (mp: 124 - 127 $\left.{ }^{\circ} \mathrm{C}\right) ;{ }^{1}$ H NMR (400 MHz, DMSO-d6) $\delta 10.54(\mathrm{~s}, 1 \mathrm{H}), 8.75-8.44(\mathrm{~m}, 1 \mathrm{H}), 8.02(\mathrm{~d}, J$ $=8.8 \mathrm{~Hz}, 1 \mathrm{H}), 7.94(\mathrm{~d}, J=8.0 \mathrm{~Hz}, 1 \mathrm{H}), 7.65(\mathrm{~d}, J=7.6 \mathrm{~Hz}, 1 \mathrm{H}), 7.45-7.30(\mathrm{~m}, 6 \mathrm{H})$, $7.09-6.84(\mathrm{~m}, 1 \mathrm{H}), 6.73-6.50(\mathrm{~m}, 1 \mathrm{H}) ;{ }^{13} \mathrm{C}\left\{{ }^{1} \mathbf{H}\right\}$ NMR (101 MHz, DMSO-d6) $\delta$ $167.0,152.4,134.7,131.8,131.0,130.9,128.3,127.7,127.6,127.4,124.7,123.9$, 123.5, 122.5, 121.0, 118.1, 115.9, 115.1, 108.8; IR v (neat, $\left.\mathbf{c m}^{-1}\right): 3006,1688,1515$, 1451, 1276, 1261, 750; HRMS (ESI, m/z): calcd for $\mathrm{C}_{19} \mathrm{H}_{13} \mathrm{NNaO}_{2}{ }^{+}[\mathrm{M}+\mathrm{Na}]^{+}$: 310.0838, found: 310.0873 .<smiles>O=C(c1c(O)ccc2ccccc12)N1Cc2ccccc2C1</smiles>

(2-Hydroxynaphthalen-1-yl)(isoindolin-2-yl)methanone ${ }^{[10]}$; white solid; ${ }^{1} \mathbf{H}$ NMR (400 MHz, DMSO-d6) $\delta 10.13(\mathrm{~s}, 1 \mathrm{H}), 7.86(\mathrm{~d}, J=8.8 \mathrm{~Hz}, 2 \mathrm{H}), 7.57$ (d, $J=8.4 \mathrm{~Hz}$, 1H), $7.45-7.41(\mathrm{~m}, 2 \mathrm{H}), 7.34(\mathrm{~d}, J=7.6 \mathrm{~Hz}, 1 \mathrm{H}), 7.30$ (d, $J=7.6 \mathrm{~Hz}, 1 \mathrm{H}), 7.26-$ $7.18(\mathrm{~m}, 3 \mathrm{H}), 5.01(\mathrm{~d}, J=16.0 \mathrm{~Hz}, 1 \mathrm{H}), 4.90(\mathrm{~d}, J=16.0 \mathrm{~Hz}, 1 \mathrm{H}), 4.61(\mathrm{~d}, J=14.4$ $\mathrm{Hz}, 1 \mathrm{H}), 4.24(\mathrm{~d}, J=14.4 \mathrm{~Hz}, 1 \mathrm{H})$.<smiles>O=C(c1c(O)ccc2ccccc12)N1CCCc2ccccc21</smiles>

$1 \mathrm{~s}$

(3,4-Dihydroquinolin-1(2H)-yl)(2-hydroxynaphthalen-1-yl)methanone; white 
solid (mp: 188 - $\left.191{ }^{\circ} \mathrm{C}\right)$; ${ }^{1} \mathbf{H}$ NMR (400 MHz, $\left.\mathbf{C D C l}_{3}\right) \delta 8.56(\mathrm{~s}, 1 \mathrm{H}), 7.78(\mathrm{~d}, J=8.8$ $\mathrm{Hz}, 1 \mathrm{H}), 7.70(\mathrm{~d}, J=8.0 \mathrm{~Hz}, 1 \mathrm{H}), 7.65(\mathrm{~d}, J=7.6 \mathrm{~Hz}, 1 \mathrm{H}), 7.30$ (t, $J=7.4 \mathrm{~Hz}, 1 \mathrm{H})$, $7.26-7.23(\mathrm{~m}, 2 \mathrm{H}), 7.19(\mathrm{~d}, J=8.8 \mathrm{~Hz}, 1 \mathrm{H}), 7.12(\mathrm{~d}, J=7.6 \mathrm{~Hz}, 1 \mathrm{H}), 6.97(\mathrm{t}, J=7.4$ $\mathrm{Hz}, 1 \mathrm{H}), 6.91-6.87(\mathrm{~m}, 1 \mathrm{H}), 3.96-3.83(\mathrm{~m}, 1 \mathrm{H}), 3.71(\mathrm{dt}, J=12.8,5.2 \mathrm{~Hz}, 1 \mathrm{H})$, $2.95-2.82(\mathrm{~m}, 2 \mathrm{H}), 2.14-2.05(\mathrm{~m}, 1 \mathrm{H}), 1.96-1.85(\mathrm{~m}, 1 \mathrm{H}) ;{ }^{13} \mathbf{C}\left\{{ }^{1} \mathbf{H}\right\}$ NMR $(\mathbf{1 0 1}$ MHz, $\left.\mathbf{C D C l}_{3}\right) \delta 170.2,156.4,137.9,132.9,131.1,130.4,128.9,128.5,128.3,127.5$, $125.9,125.3,124.2,123.7,123.5,119.0,113.7,46.2,27.0,24.1$; IR $v$ (neat, $\mathbf{c m}^{-1}$ ): 3005, 2989, 1600, 1577, 1440, 1275, 1261, 750; HRMS (ESI, m/z): calcd for $\mathrm{C}_{20} \mathrm{H}_{18} \mathrm{NO}_{2}^{+}[\mathrm{M}+\mathrm{H}]^{+}:$304.1332, found: 304.1326.<smiles>O=C(c1c(O)ccc2ccccc12)N1CCc2ccccc2C1</smiles>

(3,4-Dihydroisoquinolin-2(1H)-yl)(2-hydroxynaphthalen-1-yl)methanone ${ }^{[8]}$; white solid; ${ }^{1} \mathbf{H}$ NMR (400 MHz, CDCl $) \delta 8.08-7.92(\mathrm{~m}, 1 \mathrm{H}), 7.80(\mathrm{~d}, J=8.8 \mathrm{~Hz}, 2 \mathrm{H})$, $7.68(\mathrm{~d}, J=8.4 \mathrm{~Hz}, 1 \mathrm{H}), 7.46(\mathrm{t}, J=7.6 \mathrm{~Hz}, 1 \mathrm{H}), 7.36(\mathrm{t}, J=7.6 \mathrm{~Hz}, 1 \mathrm{H}), 7.20-7.16$ (m, 3H), $7.03-6.92(\mathrm{~m}, 1 \mathrm{H}), 4.92-4.62(\mathrm{~m}, 2 \mathrm{H}), 3.76-3.60(\mathrm{~m}, 2 \mathrm{H}), 3.00-2.85$ $(\mathrm{m}, 2 \mathrm{H})$.<smiles>O=C(c1c(O)ccc2ccccc12)N1CCCC1</smiles>

(2-Hydroxynaphthalen-1-yl)(pyrrolidin-1-yl)methanone ${ }^{[8]}$; white solid; ${ }^{1}$ H NMR (400 MHz, DMSO-d6) $\delta 9.98$ (s, 1H), $7.82-7.78$ (m, 2H), 7.50 (d, $J=8.0 \mathrm{~Hz}, 1 \mathrm{H})$, $7.43(\mathrm{t}, J=7.6 \mathrm{~Hz}, 1 \mathrm{H}), 7.30(\mathrm{t}, J=7.6 \mathrm{~Hz}, 1 \mathrm{H}), 7.20(\mathrm{~d}, J=8.8 \mathrm{~Hz}, 1 \mathrm{H}), 3.64-3.52$ (m, 2H), $3.24-3.18(\mathrm{~m}, 1 \mathrm{H}), 2.87-2.81(\mathrm{~m}, 1 \mathrm{H}), 1.91-1.84(\mathrm{~m}, 2 \mathrm{H}), 1.81-1.69$ $(\mathrm{m}, 2 \mathrm{H})$. 
<smiles>O=C(c1c(O)ccc2ccccc12)N1CCCCC1</smiles>

(2-Hydroxynaphthalen-1-yl)(piperidin-1-yl)methanone ${ }^{[12]}$; white solid; ${ }^{1} \mathbf{H}$ NMR (400 MHz, DMSO-d6) $\delta 9.92(\mathrm{~s}, 1 \mathrm{H}), 7.81$ (d, $J=8.0 \mathrm{~Hz}, 1 \mathrm{H}), 7.78$ (d, $J=8.8 \mathrm{~Hz}$, $1 \mathrm{H}), 7.48(\mathrm{~d}, J=8.0 \mathrm{~Hz}, 1 \mathrm{H}), 7.43(\mathrm{t}, J=8.0 \mathrm{~Hz}, 1 \mathrm{H}), 7.30(\mathrm{td}, J=8.0,1.2 \mathrm{~Hz} 1 \mathrm{H})$, $7.17(\mathrm{~d}, J=8.8 \mathrm{~Hz}, 1 \mathrm{H}), 3.98-3.85(\mathrm{~m}, 1 \mathrm{H}), 3.61-3.47(\mathrm{~m}, 1 \mathrm{H}), 3.15-3.02(\mathrm{~m}$, $2 \mathrm{H}), 1.70-1.52(\mathrm{~m}, 4 \mathrm{H}), 1.49-1.39(\mathrm{~m}, 1 \mathrm{H}), 1.30-1.16(\mathrm{~m}, 1 \mathrm{H})$.<smiles>N#Cc1cccc2c(C(=O)N3CCOCC3)c(O)ccc12</smiles>

(2-Hydroxynaphthalen-1-yl)(morpholino)methanone; white solid; ${ }^{1}$ H NMR (400 MHz, DMSO-d6) $\delta 10.06$ (s, 1H), $7.83-7.80$ (m, 2H), 7.52 (d, J=8.4 Hz, 1H), 7.45 (ddd, $J=8.4,8.0,1.2 \mathrm{~Hz} 1 \mathrm{H}), 7.32(\mathrm{ddd}, J=8.4,8.0,1.6 \mathrm{~Hz}, 1 \mathrm{H}), 7.18$ (d, $J=8.8 \mathrm{~Hz}$, $1 \mathrm{H}), 3.87-3.83(\mathrm{~m}, 1 \mathrm{H}), 3.73-3.66(\mathrm{~m}, 3 \mathrm{H}), 3.54-3.51(\mathrm{~m}, 1 \mathrm{H}), 3.38-3.34(\mathrm{~m}$, $1 \mathrm{H}), 3.19-3.05(\mathrm{~m}, 2 \mathrm{H})$.<smiles>[Y2]c1ccc(O)c(C(=O)N(Cc2ccccc2)Cc2ccccc2)c1</smiles>

N-Benzyl-N-(tert-butyl)-2-hydroxy-1-naphthamide; white solid (mp: 188 - $191{ }^{\circ} \mathrm{C}$ ); ${ }^{1}$ H NMR (400 MHz, DMSO-d6) $\delta 10.05$ (s, 1H), 7.76 (d, $J=8.4$ Hz, 1H), 7.71 (d, $J$ $=9.2 \mathrm{~Hz}, 1 \mathrm{H}), 7.67(\mathrm{~d}, J=8.8 \mathrm{~Hz}, 1 \mathrm{H}), 7.50(\mathrm{ddd}, J=8.4,7.2,1.2 \mathrm{~Hz}, 1 \mathrm{H}), 7.31-$ $7.27(\mathrm{~m}, 3 \mathrm{H}), 7.19$ (t, $J=7.6 \mathrm{~Hz}, 2 \mathrm{H}), 7.14(\mathrm{~d}, J=8.8 \mathrm{~Hz}, 1 \mathrm{H}), 7.10(\mathrm{t}, J=7.2 \mathrm{~Hz}$, 1H), $4.56(\mathrm{~d}, J=17.6 \mathrm{~Hz}, 1 \mathrm{H}), 4.50(\mathrm{~d}, J=17.6 \mathrm{~Hz}, 1 \mathrm{H}), 1.51(\mathrm{~s}, 9 \mathrm{H}) ;{ }^{13} \mathbf{C}\left\{{ }^{1} \mathbf{H}\right\} \mathbf{N M R}$ (101 MHz, DMSO-d6) $\delta 169.5,150.5,140.5,131.0,129.3,128.1,127.5,126.9$, 
126.4, 126.3, 123.1, 123.0, 119.1, 118.3, 57.8, 50.5, 28.5; IR v (neat, $\mathbf{c m}^{-1}$ ): 3005, 2989, 1600, 1515, 1275, 1261, 1195, 750; HRMS (ESI, m/z): calcd for $\mathrm{C}_{22} \mathrm{H}_{24} \mathrm{NO}_{2}{ }^{+}$ $[\mathrm{M}+\mathrm{H}]^{+}:$334.1802, found: 334.1811.

\section{Catalytic Enantioselective Reaction}

General Procedure for Catalytic Asymmetric Azidation Dearomatization of Naphthols:<smiles>[R]CC(=O)c1c([OH2+])ccc2cc[R1]cc12</smiles>

After stirring a mixture of $\mathrm{Cu}\left(\mathrm{CH}_{3} \mathrm{CN}\right)_{4} \cdot \mathrm{PF}_{6}(0.01 \mathrm{mmol}, 10 \mathrm{~mol} \%)$ and $\mathbf{L 4}(0.012$ mmol, $12 \mathrm{~mol} \%)$ in dry dichloromethane $(1 \mathrm{~mL})$ at room temperature for $2 \mathrm{~h}$, substrates 1 or $4(0.10 \mathrm{mmol})$ was added. After stirring $30 \mathrm{~min}, \mathrm{~N}_{3}$-transfer reagent $\mathbf{2 a}$ $(0.2 \mathrm{mmol})$ was added and the reaction mixture was stirred at room temperature under argon atmosphere. After the disappearance of substrate $\mathbf{1}$ or $\mathbf{4}$ (monitored by TLC) and then the crude product was purified by silica gel flash chromatography to afford the desired product $\mathbf{3}$ or $\mathbf{5}$.

\section{General Procedure for $1 \mathrm{mmol}$ Scale:}<smiles>O=C(NCCc1ccccc1)c1c(O)ccc2ccccc12</smiles>

$1 \mathrm{~d}$<smiles>CC1(C)OI(N)c2ccccc21</smiles>

2a (2 equiv)
$\mathrm{Cu}\left(\mathrm{CH}_{3} \mathrm{CN}\right)_{4} \cdot \mathrm{PF}_{6}(10 \mathrm{~mol} \%)$

$\underset{\mathrm{CH}_{2} \mathrm{Cl}_{2}, \mathrm{rt}}{\mathrm{L}}$

$\mathrm{CH}_{2} \mathrm{Cl}_{2}$, $\mathrm{rt}$<smiles>NC(=O)C(=O)C1(N)C(=O)C=Cc2ccccc21</smiles>

$3 d$

After stirring a mixture of $\mathrm{Cu}\left(\mathrm{CH}_{3} \mathrm{CN}\right)_{4} \cdot \mathrm{PF}_{6}(0.14 \mathrm{mmol}, 10 \mathrm{~mol} \%)$ and $\mathbf{L 4}(0.16$ mmol, $12 \mathrm{~mol} \%)$ in dry dichloromethane $(5 \mathrm{~mL})$ at room temperature for $2 \mathrm{~h}$, substrates $\mathbf{1 d}(1.36 \mathrm{mmol})$ was added. After stirring $30 \mathrm{~min}, \mathrm{~N}_{3}$-transfer reagent $\mathbf{2 a}$ (2.72 mmol) was added and the reaction mixture was stirred at room temperature under argon atmosphere. After the disappearance of substrate 1d (monitored by TLC) 
and then the crude product was purified by silica gel flash chromatography to afford the desired product 3d (488.3mg, $88 \%$ yield, $94 \%$ ee).<smiles></smiles>

3a

(S)-1-Azido-N,N-diisopropyl-2-oxo-1,2-dihydronaphthalene-1-carboxamide;

reaction temperature: $\mathrm{rt}$; reaction time: $13 \mathrm{~h}$; petroleum ether/ethylacetate $=30: 1$; TLC: $R_{f}=0.4(\mathrm{PE} / \mathrm{EA}=10: 1, \mathrm{UV})$; white solid (mp: $\left.155-158^{\circ} \mathrm{C}\right) ; 90 \%$ yield $(28.1$ $\mathrm{mg}, 0.090 \mathrm{mmol}) ;[\alpha]_{\mathbf{D}}{ }^{24}=-53.57\left(c=0.28, \mathrm{CH}_{2} \mathrm{Cl}_{2}\right) ;{ }^{1} \mathbf{H}$ NMR (400 MHz, DMSO-d6) $\delta 7.93(\mathrm{~d}, J=10.0 \mathrm{~Hz}, 1 \mathrm{H}), 7.70(\mathrm{~d}, J=7.6 \mathrm{~Hz}, 1 \mathrm{H}), 7.62-7.54(\mathrm{~m}, 2 \mathrm{H})$, $7.20(\mathrm{~d}, J=7.6 \mathrm{~Hz}, 1 \mathrm{H}), 6.40(\mathrm{~d}, J=10.0 \mathrm{~Hz}, 1 \mathrm{H}), 3.40-3.33(\mathrm{~m}, 1 \mathrm{H}), 2.70-2.63$ (m, 1H), $1.40-1.29(\mathrm{~m}, 6 \mathrm{H}), 0.82(\mathrm{~d}, J=6.8 \mathrm{~Hz}, 3 \mathrm{H}), 0.27$ (d, $J=6.4 \mathrm{~Hz}, 3 \mathrm{H})$; ${ }^{13} \mathbf{C}\left\{{ }^{1} \mathbf{H}\right\}$ NMR (101 MHz, DMSO-d6) $\delta$ 189.3, 164.1, 146.8, 135.3, 131.2, 131.0, 130.1, 128.1, 127.5, 122.9, 71.0, 47.9, 45.7, 19.8, 19.2, 18.0, 17.6; IR v (neat, $\mathbf{c m}^{-\mathbf{1}}$ ): 3005, 2100, 1650, 1275, 1261, 752, 750; HRMS (ESI, m/z): calcd for $\mathrm{C}_{17} \mathrm{H}_{21} \mathrm{~N}_{4} \mathrm{O}_{2}{ }^{+}$ $[\mathrm{M}+\mathrm{H}]^{+}:$313.1659, found: 313.1648.<smiles></smiles>

3b

(S)-1-Azido-N,N-dicyclohexyl-2-oxo-1,2-dihydronaphthalene-1-carboxamide;

reaction temperature: $\mathrm{rt}$; reaction time: $11 \mathrm{~h}$; petroleum ether/ethylacetate $=30: 1$; TLC: $R_{f}=0.5(\mathrm{PE} / \mathrm{EA}=10: 1, \mathrm{UV})$; white solid $\left(\mathrm{mp}: 172-176^{\circ} \mathrm{C}\right) ; 75 \%$ yield $(29.4$ $\mathrm{mg}, 0.075 \mathrm{mmol}) ;[\alpha]_{\mathrm{D}}{ }^{24}=-13.50\left(c=0.24, \mathrm{CH}_{2} \mathrm{Cl}_{2}\right) ;{ }^{1} \mathrm{H}$ NMR (400 MHz, DMSO-d6) $\delta 7.97(\mathrm{~d}, J=10.0 \mathrm{~Hz}, 1 \mathrm{H}), 7.70(\mathrm{dd}, J=7.2,2.0 \mathrm{~Hz}, 1 \mathrm{H}), 7.62-7.54(\mathrm{~m}$, 
2H), $7.19(\mathrm{dd}, J=7.2,2.0 \mathrm{~Hz}, 1 \mathrm{H}), 6.46(\mathrm{~d}, J=10.0 \mathrm{~Hz}, 1 \mathrm{H}), 2.90-2.85(\mathrm{~m}, 1 \mathrm{H})$, $2.45-2.40(\mathrm{~m}, 2 \mathrm{H}), 2.29-2.23(\mathrm{~m}, 1 \mathrm{H}), 1.76-1.67(\mathrm{~m}, 2 \mathrm{H}), 1.64-1.53(\mathrm{~m}, 2 \mathrm{H})$, $1.42-1.09(\mathrm{~m}, 10 \mathrm{H}), 0.91-0.77(\mathrm{~m}, 2 \mathrm{H}), 0.24-0.14(\mathrm{~m}, 2 \mathrm{H}) ;{ }^{13} \mathbf{C}\left\{{ }^{1} \mathbf{H}\right\}$ NMR $(\mathbf{1 0 1}$ MHz, DMSO-d6) $\delta$ 189.1, 164.4, 146.6, 135.5, 131.1, 130.7, 130.0, 128.2, 127.5, 123.0, 71.1, 55.8, 55.3, 28.9, 28.6, 28.23, 28.17, 25.54, 25.46, 25.4, 25.1, 25.0, 24.1; IR $v$ (neat, $\mathbf{c m}^{-1}$ ): 3006, 2989, 2105, 1660, 1470, 1275, 1261, 750; HRMS (ESI, m/z): calcd for $\mathrm{C}_{23} \mathrm{H}_{29} \mathrm{~N}_{4} \mathrm{O}_{2}{ }^{+}[\mathrm{M}+\mathrm{H}]^{+}:$393.2285, found: 393.2287.<smiles></smiles>

$3 c$

(S)-1-Azido-2-oxo-N,N-diphenyl-1,2-dihydronaphthalene-1-carboxamide;

reaction temperature: $\mathrm{rt}$; reaction time: $15 \mathrm{~h}$; petroleum ether/ethylacetate $=20: 1$; TLC: $R_{f}=0.5(\mathrm{PE} / \mathrm{EA}=5: 1, \mathrm{UV})$; white solid $\left(\mathrm{mp}: 154-157{ }^{\circ} \mathrm{C}\right) ; 94 \%$ yield $(35.8$ $\mathrm{mg}, 0.094 \mathrm{mmol}) ;[\boldsymbol{\alpha}]_{\mathbf{D}}{ }^{21}=-71.54\left(c=1.72, \mathrm{CH}_{2} \mathrm{Cl}_{2}\right) ;{ }^{1} \mathbf{H}$ NMR (400 MHz, DMSO-d6) $\delta 7.64(\mathrm{t}, J=7.5 \mathrm{~Hz}, 1 \mathrm{H}), 7.57-7.50(\mathrm{~m}, 2 \mathrm{H}), 7.46-7.13(\mathrm{~m}, 8 \mathrm{H}), 7.11$ $-6.85(\mathrm{~m}, 2 \mathrm{H}), 6.73-6.12(\mathrm{~m}, 2 \mathrm{H}), 5.82(\mathrm{~d}, J=10.0 \mathrm{~Hz}, 1 \mathrm{H}) ;{ }^{13} \mathbf{C}\left\{{ }^{1} \mathbf{H}\right\} \mathbf{N M R}(\mathbf{1 0 1}$ MHz, DMSO-d6) $\delta$ 190.2, 166.5, 145.1, 135.4, 131.8, 131.0, 130.3, 129.9, 129.1, 128.7, 127.9, 126.7, 123.2, 70.8; IR $v$ (neat, $\mathbf{c m}^{-1}$ ): 3006, 2106, 1674, 1490, 1275, 1261, 751, 703; HRMS (ESI, m/z): calcd for $\mathrm{C}_{23} \mathrm{H}_{17} \mathrm{~N}_{4} \mathrm{O}_{2}{ }^{+}[\mathrm{M}+\mathrm{H}]^{+}$: 381.1346, found: 381.1324 .<smiles></smiles>

3d

(S)-1-Azido-N,N-dibenzyl-2-oxo-1,2-dihydronaphthalene-1-carboxamide; reaction temperature: rt; reaction time: $19 \mathrm{~h}$; petroleum ether/ethylacetate $=20: 1$; TLC: $R_{f}=$ $0.4(\mathrm{PE} / \mathrm{EA}=5: 1, \mathrm{UV})$; white solid (mp: $\left.145-147{ }^{\circ} \mathrm{C}\right) ; 86 \%$ yield $(35.1 \mathrm{mg}, 0.086$ 
mmol); $[\boldsymbol{\alpha}]_{\mathbf{D}^{21}}=-5.96\left(c=1.91, \mathrm{CH}_{2} \mathrm{Cl}_{2}\right) ;{ }^{\mathbf{1}} \mathbf{H} \mathbf{~ N M R}\left(400 \mathbf{M H z}, \mathbf{C D C l}_{3}\right) \delta 7.54-7.47$ (m, 3H), $7.45-7.41(\mathrm{~m}, 1 \mathrm{H}), 7.36(\mathrm{~d}, J=7.6 \mathrm{~Hz}, 1 \mathrm{H}), 7.33-7.25(\mathrm{~m}, 3 \mathrm{H}), 7.20-7.14$ (m, 5H), $6.70-6.54(\mathrm{~m}, 2 \mathrm{H}), 6.28(\mathrm{~d}, J=10.0 \mathrm{~Hz}, 1 \mathrm{H}), 4.70(\mathrm{~d}, J=14.8 \mathrm{~Hz}, 1 \mathrm{H})$, $4.33(\mathrm{~d}, J=14.8 \mathrm{~Hz}, 1 \mathrm{H}), 3.70(\mathrm{~d}, J=15.2 \mathrm{~Hz}, 1 \mathrm{H}), 3.61(\mathrm{~d}, J=15.2 \mathrm{~Hz}, 1 \mathrm{H}) ;{ }^{13} \mathbf{C}\left\{{ }^{1} \mathbf{H}\right\}$ NMR (100 MHz, CDCl $) \delta 189.7,167.3,146.0,136.0,135.7,134.3,131.4,130.7$, $130.2,128.9,128.7,128.5,128.4,128.1,127.8,127.6,123.9,71.6,49.2,47.8$; IR v (neat, $\mathbf{c m}^{-1}$ ): 3006, 2104, 1662, 1418, 1275, 1261, 836, 750; HRMS (ESI, m/z): calcd for $\mathrm{C}_{25} \mathrm{H}_{20} \mathrm{~N}_{4} \mathrm{NaO}_{2}^{+}[\mathrm{M}+\mathrm{Na}]^{+}$: 431.1478, found: 431.1487 .<smiles>CCCCC(=O)C1(N)C(=O)C=Cc2cc(-c3ccccc3)ccc21</smiles>

$3 e$

\section{(S)-1-Azido-N,N-dibenzyl-2-oxo-6-phenyl-1,2-dihydronaphthalene-1-carboxamid}

e; reaction temperature: $\mathrm{rt}$; reaction time: $15 \mathrm{~h}$; petroleum ether/ethylacetate $=20: 1$; TLC: $R_{f}=0.5(\mathrm{PE} / \mathrm{EA}=5: 1, \mathrm{UV})$; white solid (mp: $\left.136-139{ }^{\circ} \mathrm{C}\right) ; 89 \%$ yield $(43.1$ $\mathrm{mg}, 0.089 \mathrm{mmol}) ;[\boldsymbol{\alpha}]_{\mathbf{D}}{ }^{\mathbf{2 4}}=+19.88\left(c=0.33, \mathrm{CH}_{2} \mathrm{Cl}_{2}\right) ;{ }^{\mathbf{1}} \mathbf{H} \mathbf{N M R}$ (400 MHz, DMSO-d6) $\delta 7.98(\mathrm{~d}, J=10.0 \mathrm{~Hz}, 1 \mathrm{H}), 7.94(\mathrm{~d}, J=1.6 \mathrm{~Hz}, 1 \mathrm{H}), 7.86(\mathrm{dd}, J=8.0,1.6$ $\mathrm{Hz}, 1 \mathrm{H}), 7.71-7.64(\mathrm{~m}, 2 \mathrm{H}), 7.53-7.49(\mathrm{~m}, 2 \mathrm{H}), 7.45-7.41(\mathrm{~m}, 2 \mathrm{H}), 7.33(\mathrm{t}, J=$ $7.2 \mathrm{~Hz}, 2 \mathrm{H}), 7.26(\mathrm{t}, J=7.2 \mathrm{~Hz}, 1 \mathrm{H}), 7.17-7.08(\mathrm{~m}, 5 \mathrm{H}), 6.73-6.64(\mathrm{~m}, 2 \mathrm{H}), 6.47$ $(\mathrm{d}, J=10.0 \mathrm{~Hz}, 1 \mathrm{H}), 4.63(\mathrm{~d}, J=15.2 \mathrm{~Hz}, 1 \mathrm{H}), 4.25(\mathrm{~d}, J=15.2 \mathrm{~Hz}, 1 \mathrm{H}), 3.79$ (d, $J=$ $16.0 \mathrm{~Hz}, 1 \mathrm{H}), 3.74(\mathrm{~d}, J=16.0 \mathrm{~Hz}, 1 \mathrm{H}) ;{ }^{13} \mathbf{C}\left\{{ }^{1} \mathrm{H}\right\}$ NMR (101 MHz, DMSO-d6) $\delta$ $189.6,166.5,147.2,142.0,138.3,135.9,134.0,133.2$, 129.4, 129.2, 129.0, 128.9, 128.6, 128.4, 128.3, 128.2, 127.6, 127.5, 127.2, 127.1, 126.8, 123.3, 70.7, 48.6, 47.8; IR $v$ (neat, $\left.\mathbf{c m}^{-1}\right):$ 3006, 2989, 2101, 1662, 1453, 1275, 1261, 1220, 764, 750; HRMS (ESI, m/z): calcd for $\mathrm{C}_{31} \mathrm{H}_{25} \mathrm{~N}_{4} \mathrm{O}_{2}{ }^{+}[\mathrm{M}+\mathrm{H}]^{+}:$485.1972, found: 485.1975 . 
<smiles>Cc1ccc(-c2ccc3c(c2)C=CC(=O)C3(N)C(=O)NCCc2ccccc2)cc1</smiles>

$3 f$

(S)-1-Azido-N,N-dibenzyl-2-oxo-6-(p-tolyl)-1,2-dihydronaphthalene-1-carboxami de; reaction temperature: rt; reaction time: $24 \mathrm{~h}$; petroleum ether/ethylacetate $=20: 1$; TLC: $R_{f}=0.55(\mathrm{PE} / \mathrm{EA}=5: 1, \mathrm{UV})$; white solid (mp: $\left.164-167^{\circ} \mathrm{C}\right) ; 99 \%$ yield $(49.8$ mg, $0.099 \mathrm{mmol}) ;[\boldsymbol{\alpha}]_{\mathbf{D}}{ }^{23}=+25.95\left(c=0.39, \mathrm{CH}_{2} \mathrm{Cl}_{2}\right) ;{ }^{1} \mathbf{H}$ NMR (400 MHz, DMSO-d6) $\delta 7.97(\mathrm{~d}, J=10.0 \mathrm{~Hz}, 1 \mathrm{H}), 7.92(\mathrm{~s}, 1 \mathrm{H}), 7.84(\mathrm{dd}, J=8.0,1.2 \mathrm{~Hz}, 1 \mathrm{H})$, $7.58(\mathrm{~d}, J=8.0 \mathrm{~Hz}, 2 \mathrm{H}), 7.41(\mathrm{~d}, J=8.0 \mathrm{~Hz}, 1 \mathrm{H}), 7.35-7.24(\mathrm{~m}, 5 \mathrm{H}), 7.17-7.08(\mathrm{~m}$, $5 \mathrm{H}), 6.72-6.64(\mathrm{~m}, 2 \mathrm{H}), 6.45(\mathrm{~d}, J=10.0 \mathrm{~Hz}, 1 \mathrm{H}), 4.61(\mathrm{~d}, J=15.2 \mathrm{~Hz}, 1 \mathrm{H}), 4.25(\mathrm{~d}$, $J=15.2 \mathrm{~Hz}, 1 \mathrm{H}), 3.78(\mathrm{~d}, J=16.0 \mathrm{~Hz}, 1 \mathrm{H}), 3.73(\mathrm{~d}, J=16.0 \mathrm{~Hz}, 1 \mathrm{H}), 2.36(\mathrm{~s}, 3 \mathrm{H})$; ${ }^{13} \mathbf{C}\left\{{ }^{1} \mathrm{H}\right\}$ NMR (101 MHz, DMSO-d6) $\delta$ 189.6, 166.6, 147.4, 142.0, 137.9, 136.0, $135.4,134.0,132.9,129.7,129.1,128.93,128.87,128.6,128.4,128.3,127.7,127.5$, 127.3, 127.1, 126.6, 123.3, 70.8, 48.6, 47.8, 20.7; IR $v$ (neat, $\mathbf{c m}^{-1}$ ): 3005, 2989, 2100, 1660, 1460, 1275, 1261, 897, 752, 750; HRMS (ESI, m/z): calcd for $\mathrm{C}_{32} \mathrm{H}_{27} \mathrm{~N}_{4} \mathrm{O}_{2}{ }^{+}$ $[\mathrm{M}+\mathrm{H}]^{+}:$499.2129, found: 499.2131 .<smiles>CCCNC(=O)C1(NC(=O)CCC)C(=O)C=Cc2cc(-c3ccc(F)cc3)ccc21</smiles>

$3 g$

(S)-1-Azido-N,N-dibenzyl-6-(4-fluorophenyl)-2-oxo-1,2-dihydronaphthalene-1-ca rboxamide; reaction temperature: $\mathrm{rt}$; reaction time: $15 \mathrm{~h}$; petroleum ether/ ethylacetate $=20: 1 ;$ TLC: $R_{f}=0.5(\mathrm{PE} / \mathrm{EA}=5: 1, \mathrm{UV})$; white solid (mp: $141-142$ $\left.{ }^{\circ} \mathrm{C}\right) ; 92 \%$ yield $(46.1 \mathrm{mg}, 0.092 \mathrm{mmol}) ;[\boldsymbol{\alpha}]_{\mathbf{D}}{ }^{23}=+17.46\left(c=0.32, \mathrm{CH}_{2} \mathrm{Cl}_{2}\right) ;{ }^{1} \mathbf{H} \mathbf{~ N M R}$ (400 MHz, DMSO-d6) $\delta 7.96$ (d, $J=10.0$ Hz, 1H), 7.92 (d, $J=2.0 \mathrm{~Hz}, 1 \mathrm{H}), 7.84$ (dd, 
$J=8.0,2.0 \mathrm{~Hz}, 1 \mathrm{H}), 7.74-7.71(\mathrm{~m}, 2 \mathrm{H}), 7.42(\mathrm{~d}, J=8.0 \mathrm{~Hz}, 1 \mathrm{H}), 7.37-7.32(\mathrm{~m}$, 4H), $7.27(\mathrm{~d}, J=7.2 \mathrm{~Hz}, 1 \mathrm{H}), 7.17-7.09(\mathrm{~m}, 5 \mathrm{H}), 6.72-6.65(\mathrm{~m}, 2 \mathrm{H}), 6.47(\mathrm{~d}, J=$ $10.0 \mathrm{~Hz}, 1 \mathrm{H}), 4.62$ (d, $J=15.2 \mathrm{~Hz}, 1 \mathrm{H}), 4.25$ (d, $J=15.2 \mathrm{~Hz}, 1 \mathrm{H}), 3.79$ (d, $J=16.0$ $\mathrm{Hz}, 1 \mathrm{H}), 3.73(\mathrm{~d}, J=16.0 \mathrm{~Hz}, 1 \mathrm{H}) ;{ }^{13} \mathbf{C}\left\{{ }^{1} \mathbf{H}\right\}$ NMR (101 MHz, DMSO-d6) $\delta$ 189.6, $166.5,162.4(\mathrm{~d}, J=246.5 \mathrm{~Hz}), 147.3,147.2,141.0,136.0,134.8(\mathrm{~d}, J=2.9 \mathrm{~Hz}), 134.0$, 133.2, 129.4, 129.2, 129.0(d, $J=8.4 \mathrm{~Hz}), 128.9,128.6,128.5,128.3,127.7,127.5$, 127.3, 127.1, 123.5, 116.0(d, $J=21.5 \mathrm{~Hz}), 70.7,48.6,47.8$; IR $\boldsymbol{v}$ (neat, $\left.\mathbf{c m}^{-1}\right)$ : 3006, 2989, 2101, 1660, 1458, 1275, 1261, 897, 750; ${ }^{19}$ F NMR (376 MHz, DMSO-d6) $\delta$ $-114.01--114.08(\mathrm{~m}, 1 \mathrm{~F})$; HRMS (ESI, m/z): calcd for $\mathrm{C}_{31} \mathrm{H}_{24} \mathrm{FN}_{4} \mathrm{O}_{2}{ }^{+}[\mathrm{M}+\mathrm{H}]^{+}$: 503.1878, found: 503.1895 .

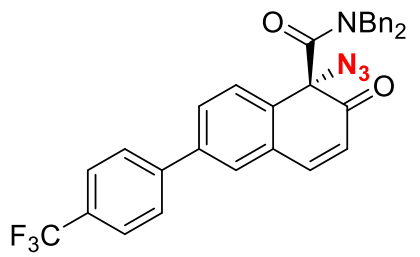

$3 \mathrm{~h}$

(S)-1-Azido-N,N-dibenzyl-2-oxo-6-(4-(trifluoromethyl)phenyl)-1,2-dihydronapht halene-1-carboxamide; reaction temperature: $\mathrm{rt}$; reaction time: $15 \mathrm{~h}$; petroleum ether/ ethylacetate $=20: 1 ;$ TLC: $R_{f}=0.5(\mathrm{PE} / \mathrm{EA}=5: 1, \mathrm{UV})$; white solid (mp: $165-167$ $\left.{ }^{\circ} \mathrm{C}\right) ; 92 \%$ yield $(50.8 \mathrm{mg}, 0.092 \mathrm{mmol}) ;[\boldsymbol{\alpha}]_{\mathbf{D}}{ }^{\mathbf{2 0}}=+10.60\left(c=0.61, \mathrm{CH}_{2} \mathrm{Cl}_{2}\right) ;{ }^{\mathbf{1}} \mathbf{H} \mathbf{~ N M R}$ (400 MHz, DMSO-d6) $\delta 8.01(\mathrm{~d}, J=1.6 \mathrm{~Hz}, 1 \mathrm{H}), 7.98$ (d, $J=10.0 \mathrm{~Hz}, 1 \mathrm{H}), 7.93-$ $7.86(\mathrm{~m}, 5 \mathrm{H}), 7.47$ (d, $J=8.0 \mathrm{~Hz}, 1 \mathrm{H}), 7.34(\mathrm{t}, J=7.2 \mathrm{~Hz}, 2 \mathrm{H}), 7.27$ (t, $J=7.2 \mathrm{~Hz}$, 1H), $7.17-7.10(\mathrm{~m}, 5 \mathrm{H}), 6.72-6.65(\mathrm{~m}, 2 \mathrm{H}), 6.50(\mathrm{~d}, J=10.0 \mathrm{~Hz}, 1 \mathrm{H}), 4.66(\mathrm{~d}, J=$ $15.2 \mathrm{~Hz}, 1 \mathrm{H}), 4.25(\mathrm{~d}, J=15.2 \mathrm{~Hz}, 1 \mathrm{H}), 3.80(\mathrm{~d}, J=16.0 \mathrm{~Hz}, 1 \mathrm{H}), 3.74(\mathrm{~d}, J=16.0$ $\mathrm{Hz}, 1 \mathrm{H}) ;{ }^{13} \mathbf{C}\left\{{ }^{1} \mathbf{H}\right\}$ NMR (101 MHz, DMSO-d6) $\delta$ 189.5, 166.4, 147.0, 142.3, 140.4, $135.9,134.1,133.9,129.7,129.5,129.1,128.8,128.7,128.4,128.2,128.1,127.7$, 127.5, 127.4(q, $J=32.7 \mathrm{~Hz}), 127.0,126.9(\mathrm{q}, J=273.2 \mathrm{~Hz}), 125.9(\mathrm{q}, J=3.8 \mathrm{~Hz})$, 123.6, 70.6, 48.6, 47.9; ${ }^{19}$ F NMR (376 MHz, DMSO-d6) $\delta$-60.97 (s, 3F); IR v (neat, $\mathbf{c m}^{-1}$ ): 3006, 2989, 1660, 1459, 1325, 1275, 1261, 750; HRMS (ESI, m/z): calcd for $\mathrm{C}_{32} \mathrm{H}_{24} \mathrm{~F}_{3} \mathrm{~N}_{4} \mathrm{O}_{2}{ }^{+}[\mathrm{M}+\mathrm{H}]^{+}:$553.1846, found: 553.1830. 
<smiles></smiles>

$3 \mathbf{i}$

(S)-1-Azido-N,N-dibenzyl-2-oxo-7-phenyl-1,2-dihydronaphthalene-1-carboxamid e; reaction temperature: $\mathrm{rt}$; reaction time: $16 \mathrm{~h}$; petroleum ether/ethylacetate $=20: 1$; TLC: $R_{f}=0.5(\mathrm{PE} / \mathrm{EA}=5: 1, \mathrm{UV})$; white solid (mp: $\left.67-69{ }^{\circ} \mathrm{C}\right) ; 94 \%$ yield $(45.7 \mathrm{mg}$, $0.094 \mathrm{mmol}) ;[\boldsymbol{\alpha}]_{\mathbf{D}}{ }^{\mathbf{2 4}}=+284.06\left(c=0.32, \mathrm{CH}_{2} \mathrm{Cl}_{2}\right) ;{ }^{1} \mathbf{H}$ NMR (400 MHz, DMSO-d6) $\delta 7.96(\mathrm{~d}, J=10.0 \mathrm{~Hz}, 1 \mathrm{H}), 7.80(\mathrm{~d}, J=8.0 \mathrm{~Hz}, 1 \mathrm{H}), 7.71(\mathrm{~d}, J=8.0 \mathrm{~Hz}, 1 \mathrm{H}), 7.56-$ 7.48 (m, 5H), 7.40 (s, 1H), $7.19-7.10(\mathrm{~m}, 8 \mathrm{H}), 6.80-6.73(\mathrm{~m}, 2 \mathrm{H}), 6.40(\mathrm{~d}, J=10.0$ $\mathrm{Hz}, 1 \mathrm{H}), 4.51(\mathrm{~d}, J=15.2 \mathrm{~Hz}, 1 \mathrm{H}), 4.31(\mathrm{~d}, J=15.2 \mathrm{~Hz}, 1 \mathrm{H}), 3.81(\mathrm{~d}, J=16.0 \mathrm{~Hz}$, 1H), $3.72(\mathrm{~d}, J=16.0 \mathrm{~Hz}, 1 \mathrm{H}) ;{ }^{13} \mathbf{C}\left\{{ }^{1} \mathbf{H}\right\}$ NMR (101 MHz, DMSO-d6) $\delta$ 189.4, 166.4, $146.8,143.0,138.1,136.1,135.2,134.0,131.7,129.2,128.6,128.4,128.31,128.28$, 127.7, 127.2, 127.0, 126.7, 126.1, 122.8, 70.9, 48.7, 47.8; IR v (neat, $\mathbf{c m}^{-1}$ ): 3006, 2989, 2102, 1662, 1459, 1275, 1261, 750; HRMS (ESI, m/z): calcd for $\mathrm{C}_{31} \mathrm{H}_{25} \mathrm{~N}_{4} \mathrm{O}_{2}{ }^{+}$ $[\mathrm{M}+\mathrm{H}]^{+}:$485.1972, found: 485.1966 .<smiles></smiles>

3j

(S)-1-Azido-N,N-dibenzyl-6-bromo-2-oxo-1,2-dihydronaphthalene-1-carboxamid e; reaction temperature: $\mathrm{rt}$; reaction time: $22 \mathrm{~h}$; petroleum ether/ ethylacetate $=20: 1$; TLC: $R_{f}=0.5(\mathrm{PE} / \mathrm{EA}=5: 1, \mathrm{UV}) ;$ white solid (mp: $\left.158-161{ }^{\circ} \mathrm{C}\right) ; 99 \%$ yield $(48.7$ $\mathrm{mg}, 0.099 \mathrm{mmol}) ;[\boldsymbol{\alpha}]_{\mathbf{D}}{ }^{21}=-3.30\left(c=0.93, \mathrm{CH}_{2} \mathrm{Cl}_{2}\right) ;{ }^{1} \mathbf{H}$ NMR (400 $\left.\mathbf{M H z}, \mathbf{C D C l}_{3}\right) \delta$ $7.56(\mathrm{dd}, J=8.4,2.0 \mathrm{~Hz}, 1 \mathrm{H}), 7.47(\mathrm{~d}, J=2.0 \mathrm{~Hz}, 1 \mathrm{H}), 7.41(\mathrm{~d}, J=10.0 \mathrm{~Hz}, 1 \mathrm{H})$, $7.33-7.26(\mathrm{~m}, 4 \mathrm{H}), 7.25-7.19(\mathrm{~m}, 3 \mathrm{H}), 7.15-7.11(\mathrm{~m}, 2 \mathrm{H}), 6.71-6.65(\mathrm{~m}, 2 \mathrm{H})$, $6.31(\mathrm{~d}, J=10.0 \mathrm{~Hz}, 1 \mathrm{H}), 4.62(\mathrm{~d}, J=14.8 \mathrm{~Hz}, 1 \mathrm{H}), 4.42(\mathrm{~d}, J=14.8 \mathrm{~Hz}, 1 \mathrm{H}), 3.71(\mathrm{~d}$, $J=15.6 \mathrm{~Hz}, 1 \mathrm{H}), 3.60(\mathrm{~d}, J=15.6 \mathrm{~Hz}, 1 \mathrm{H}) ;{ }^{13} \mathbf{C}\left\{{ }^{1} \mathbf{H}\right\}$ NMR $(\mathbf{1 0 1} \mathbf{M H z}$, DMSO-d6) $\delta$ 
189.2, 166.2, 145.8, 135.9, 133.9, 133.4, 133.3, 130.2, 130.1, 128.5, 128.4, 127.7, 127.6, 127.4, 126.9, 124.2, 123.4, 70.4, 48.5, 48.0; IR v (neat, $\mathbf{c m}^{-1}$ ): 3006, 2989, 2103, 1663, 1420, 1275, 1261, 750; HRMS (ESI, m/z): calcd for $\mathrm{C}_{25} \mathrm{H}_{20} \mathrm{BrN}_{4} \mathrm{O}_{2}{ }^{+}$ $[\mathrm{M}+\mathrm{H}]^{+}:$487.0764, found: 487.0774 .<smiles>CCCCNC(=O)[C@@]1(N)C(=O)C=Cc2cc(C)ccc21</smiles>

3k

(S)-1-Azido-N,N-dibenzyl-6-methyl-2-oxo-1,2-dihydronaphthalene-1-carboxamid e; reaction temperature: $\mathrm{rt}$; reaction time: $11 \mathrm{~h}$; petroleum ether/ethylacetate $=20: 1$; TLC: $R_{f}=0.5(\mathrm{PE} / \mathrm{EA}=5: 1, \mathrm{UV})$; white solid (mp: $\left.152-154{ }^{\circ} \mathrm{C}\right) ; 82 \%$ yield $(34.6$ $\mathrm{mg}, 0.082 \mathrm{mmol}) ;[\boldsymbol{\alpha}]_{\mathbf{D}}{ }^{21}=+6.06\left(c=1.12, \mathrm{CH}_{2} \mathrm{Cl}_{2}\right) ;{ }^{1} \mathbf{H}$ NMR (400 MHz, DMSO-d6) $\delta 7.84(\mathrm{~d}, J=10.0 \mathrm{~Hz}, 1 \mathrm{H}), 7.43(\mathrm{~s}, 1 \mathrm{H}), 7.39(\mathrm{~d}, J=8.0 \mathrm{~Hz}, 1 \mathrm{H}), 7.34-7.30(\mathrm{~m}, 2 \mathrm{H})$, $7.27(\mathrm{~d}, J=6.8 \mathrm{~Hz}, 1 \mathrm{H}), 7.23(\mathrm{~d}, J=7.6 \mathrm{~Hz}, 1 \mathrm{H}), 7.20-7.14(\mathrm{~m}, 3 \mathrm{H}), 7.13-7.07(\mathrm{~m}$, 2H), $6.70-6.64(\mathrm{~m}, 2 \mathrm{H}), 6.37(\mathrm{~d}, J=10.0 \mathrm{~Hz}, 1 \mathrm{H}), 4.48(\mathrm{~d}, J=15.2 \mathrm{~Hz}, 1 \mathrm{H}), 4.31(\mathrm{~d}$, $J=15.2 \mathrm{~Hz}, 1 \mathrm{H}), 3.72(\mathrm{~d}, J=16.0 \mathrm{~Hz}, 1 \mathrm{H}), 3.65(\mathrm{~d}, J=16.0 \mathrm{~Hz}, 1 \mathrm{H}), 2.32(\mathrm{~s}, 3 \mathrm{H})$; ${ }^{13} \mathbf{C}\left\{{ }^{1} \mathbf{H}\right\}$ NMR (101 MHz, DMSO-d6) $\delta$ 189.7, 166.6, 147.3, 140.1, 136.0, 134.0, 131.9, 131.7, 131.4, 128.4, 128.3, 128.1, 127.9, 127.7, 127.6, 127.22, 127.19, 123.0, 70.8, 48.6, 47.6, 20.5; IR v (neat, $\mathbf{c m}^{-1}$ ): 3006, 2989, 2102, 1660, 1459, 1275, 1261, 750; HRMS (ESI, m/z): calcd for $\mathrm{C}_{26} \mathrm{H}_{23} \mathrm{~N}_{4} \mathrm{O}_{2}{ }^{+}[\mathrm{M}+\mathrm{H}]^{+}$: 423.1816 , found: 423.1814 .<smiles>CCCCNC(=O)[C@@]1(N)C(=O)C=Cc2ccc(OC)cc21</smiles>

31

\section{(S)-1-Azido-N,N-dibenzyl-7-methoxy-2-oxo-1,2-dihydronaphthalene-1-carboxam}

ide; reaction temperature: $\mathrm{rt}$; reaction time: $15 \mathrm{~h}$; petroleum ether/ethylacetate $=20: 1$; TLC: $R_{f}=0.5(\mathrm{PE} / \mathrm{EA}=5: 1, \mathrm{UV})$; white solid (mp: $\left.109-111^{\circ} \mathrm{C}\right) ; 85 \%$ yield $(37.3$ mg, $0.085 \mathrm{mmol}) ;[\boldsymbol{\alpha}]_{\mathbf{D}}{ }^{\mathbf{2 0}}=+172.19\left(c=0.98, \mathrm{CH}_{2} \mathrm{Cl}_{2}\right) ;{ }^{\mathbf{1}} \mathbf{H}$ NMR (400 MHz, 
DMSO-d6) $\delta 7.84(\mathrm{~d}, J=10.0 \mathrm{~Hz}, 1 \mathrm{H}), 7.55(\mathrm{~d}, J=8.4 \mathrm{~Hz}, 1 \mathrm{H}), 7.33-7.24(\mathrm{~m}, 3 \mathrm{H})$, $7.20-7.17(\mathrm{~m}, 3 \mathrm{H}), 7.15-7.10(\mathrm{~m}, 2 \mathrm{H}), 7.03(\mathrm{dd}, J=8.4,2.4 \mathrm{~Hz}, 1 \mathrm{H}), 6.77-6.75$ $(\mathrm{m}, 2 \mathrm{H}), 6.72(\mathrm{~d}, J=2.4 \mathrm{~Hz}, 1 \mathrm{H}), 6.22(\mathrm{~d}, J=10.0 \mathrm{~Hz}, 1 \mathrm{H}), 4.45-4.38(\mathrm{~m}, 2 \mathrm{H}), 3.81$ - $3.77(\mathrm{~m}, 4 \mathrm{H}), 3.70(\mathrm{~d}, J=16.0 \mathrm{~Hz}, 1 \mathrm{H}) ;{ }^{13} \mathbf{C}\left\{{ }^{1} \mathbf{H}\right\}$ NMR (101 MHz, DMSO-d6) $\delta$ $189.5,166.5,161.7,147.3,136.6,136.1,134.1,133.0,128.4,128.3,127.7,127.3$, 127.0, 121.0, 120.1, 115.6, 113.9, 70.8, 55.7, 48.4, 47.8; IR v (neat, $\left.\mathbf{c m}^{-1}\right): 3006$, 2103, 1660, 1601, 1420, 1275, 1261, 750, 700; HRMS (ESI, m/z): calcd for $\mathrm{C}_{26} \mathrm{H}_{23} \mathrm{~N}_{4} \mathrm{O}_{3}{ }^{+}[\mathrm{M}+\mathrm{H}]^{+}:$439.1765, found: 439.1773 .

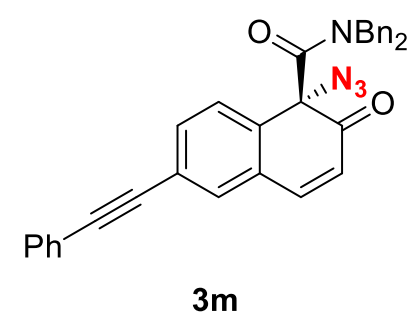

(S)-1-Azido-N,N-dibenzyl-2-oxo-6-(phenylethynyl)-1,2-dihydronaphthalene-1-car boxamide; reaction temperature: rt; reaction time: $16 \mathrm{~h}$; petroleum ether/ethylacetate $=20: 1 ;$ TLC: $R_{f}=0.5(\mathrm{PE} / \mathrm{EA}=5: 1, \mathrm{UV})$; white solid (mp: $\left.162-166{ }^{\circ} \mathrm{C}\right) ; 99 \%$ yield $(50.8 \mathrm{mg}, 0.099 \mathrm{mmol}) ;[\boldsymbol{\alpha}]_{\mathbf{D}}{ }^{23}=+23.08\left(c=0.40, \mathrm{CH}_{2} \mathrm{Cl}_{2}\right) ;{ }^{1} \mathbf{H}$ NMR (400 MHz, DMSO-d6) $\delta 7.92(\mathrm{~d}, J=10.0 \mathrm{~Hz}, 1 \mathrm{H}), 7.85(\mathrm{~d}, J=1.6 \mathrm{~Hz}, 1 \mathrm{H}), 7.72(\mathrm{dd}, J=$ 8.0, 1.6 Hz, 1H), $7.60-7.57(\mathrm{~m}, 2 \mathrm{H}), 7.47-7.45(\mathrm{~m}, 3 \mathrm{H}), 7.39$ (d, J=8.0 Hz, 1H), $7.35-7.31(\mathrm{~m}, 2 \mathrm{H}), 7.29-7.25(\mathrm{~m}, 1 \mathrm{H}), 7.23-7.17(\mathrm{~m}, 3 \mathrm{H}), 7.11(\mathrm{~d}, J=7.2 \mathrm{~Hz}$, 2H), $6.77-6.70(\mathrm{~m}, 2 \mathrm{H}), 6.47(\mathrm{~d}, J=10.0 \mathrm{~Hz}, 1 \mathrm{H}), 4.51(\mathrm{~d}, J=15.2 \mathrm{~Hz}, 1 \mathrm{H}), 4.33(\mathrm{~d}$, $J=15.2 \mathrm{~Hz}, 1 \mathrm{H}), 3.75(\mathrm{~d}, J=16.0 \mathrm{~Hz}, 1 \mathrm{H}), 3.67(\mathrm{~d}, J=16.0 \mathrm{~Hz}, 1 \mathrm{H}) ;{ }^{13} \mathbf{C}\left\{{ }^{1} \mathbf{H}\right\} \mathbf{~ N M R}$ (101 MHz, DMSO-d6) $\delta$ 189.2, 166.2, 146.3, 135.9, 134.2, 133.9, 133.7, 133.5, $131.5,129.3,128.8,128.6,128.5,128.41,128.38,127.8,127.6,127.3,127.1,124.2$, 123.8, 121.6, 91.3, 87.6, 70.6, 48.6, 47.8; IR v (neat, $\mathbf{c m}^{-1}$ ): 3005, 2989, 2102, 1662 , 1457, 1275, 1261, 750; HRMS (ESI, m/z): calcd for $\mathrm{C}_{33} \mathrm{H}_{25} \mathrm{~N}_{4} \mathrm{O}_{2}{ }^{+}[\mathrm{M}+\mathrm{H}]^{+}:$509.1972, found: 509.1977 . 
<smiles></smiles>

(S)-1-Azido-N,N-dibenzyl-4-bromo-2-oxo-1,2-dihydronaphthalene-1-carboxamid e; reaction temperature: $\mathrm{rt}$; reaction time: $15 \mathrm{~h}$; petroleum ether/ethylacetate $=20: 1$; TLC: $R_{f}=0.5(\mathrm{PE} / \mathrm{EA}=5: 1, \mathrm{UV})$; white solid $\left(\mathrm{mp}: 62-65^{\circ} \mathrm{C}\right) ; 67 \%$ yield $(32.6 \mathrm{mg}$, $0.067 \mathrm{mmol}) ;[\boldsymbol{\alpha}]_{\mathrm{D}}{ }^{21}=-41.15\left(c=0.53, \mathrm{CH}_{2} \mathrm{Cl}_{2}\right) ;{ }^{1} \mathrm{H}$ NMR (400 MHz, DMSO-d6) $\delta$ $7.91(\mathrm{dd}, J=8.0,1.2 \mathrm{~Hz}, 1 \mathrm{H}), 7.69-7.61(\mathrm{~m}, 2 \mathrm{H}), 7.39$ (dd, $J=8.0,1.2 \mathrm{~Hz}, 1 \mathrm{H})$, $7.34-7.25(\mathrm{~m}, 3 \mathrm{H}), 7.17-7.10(\mathrm{~m}, 6 \mathrm{H}), 6.71-6.63(\mathrm{~m}, 2 \mathrm{H}), 4.60(\mathrm{~d}, J=15.2 \mathrm{~Hz}$, 1H), $4.24(\mathrm{~d}, J=15.2 \mathrm{~Hz}, 1 \mathrm{H}), 3.73(\mathrm{~d}, J=16.0 \mathrm{~Hz}, 1 \mathrm{H}), 3.65(\mathrm{~d}, J=16.0 \mathrm{~Hz}, 1 \mathrm{H})$; ${ }^{13} \mathbf{C}\left\{{ }^{1} \mathbf{H}\right\}$ NMR (101 MHz, DMSO-d6) $\delta$ 186.4, 165.9, 146.0, 135.8, 133.8, 133.2, $132.7,130.9,130.6,128.4,128.3,127.7,127.5,127.3,127.2,127.13,127.09,71.2$, 48.8, 47.9; IR $v$ (neat, $\mathbf{c m}^{-1}$ ): 3005, 2105, 1662, 1557, 1417, 1275, 1261, 750; HRMS (ESI, m/z): calcd for $\mathrm{C}_{25} \mathrm{H}_{20} \mathrm{BrN}_{4} \mathrm{O}_{2}{ }^{+}[\mathrm{M}+\mathrm{H}]^{+}:$487.0764, found: 487.0781 .<smiles></smiles>

30

(S)-1-Azido-N,N-dibenzyl-2-oxo-4-phenyl-1,2-dihydronaphthalene-1-carboxamid e; reaction temperature: $\mathrm{rt}$; reaction time: $15 \mathrm{~h}$; petroleum ether/ethylacetate $=20: 1$; TLC: $R_{f}=0.5(\mathrm{PE} / \mathrm{EA}=5: 1, \mathrm{UV})$; white solid $\left(\mathrm{mp}: 66-70{ }^{\circ} \mathrm{C}\right) ; 99 \%$ yield $(48.4 \mathrm{mg}$, 0.099 mmol); $[\alpha]_{\mathbf{D}}{ }^{21}=-89.22\left(c=1.45, \mathrm{CH}_{2} \mathrm{Cl}_{2}\right) ;{ }^{1} \mathbf{H}$ NMR (400 MHz, DMSO-d6) $\delta$ $7.60(\mathrm{t}, J=7.0 \mathrm{~Hz}, 1 \mathrm{H}), 7.56-7.52(\mathrm{~m}, 3 \mathrm{H}), 7.46-7.40(\mathrm{~m}, 4 \mathrm{H}), 7.37-7.32(\mathrm{~m}, 2 \mathrm{H})$, $7.29-7.22(\mathrm{~m}, 2 \mathrm{H}), 7.20-7.09(\mathrm{~m}, 5 \mathrm{H}), 6.74-6.64(\mathrm{~m}, 2 \mathrm{H}), 6.32(\mathrm{~d}, J=2.0 \mathrm{~Hz}$, 1H), $4.68(\mathrm{~d}, J=15.2 \mathrm{~Hz}, 1 \mathrm{H}), 4.26(\mathrm{~d}, J=15.2 \mathrm{~Hz}, 1 \mathrm{H}), 3.86(\mathrm{~s}, 2 \mathrm{H}) ;{ }^{13} \mathbf{C}\left\{{ }^{1} \mathbf{H}\right\} \mathbf{N M R}$ 
(101 MHz, DMSO-d6) $\delta$ 188.6, 166.6, 157.4, 136.0, 135.9, 134.9, 134.0, 131.5, $130.0,129.6,129.4,128.9,128.7,128.4,128.2$, 128.1, 127.5, 127.2, 126.8, 122.7, 70.9, 48.7, 47.9; IR $v$ (neat, $\mathbf{c m}^{-1}$ ): 3006, 2104, 1660, 1605, 1275, 1261, 951, 750; HRMS (ESI, m/z): calcd for $\mathrm{C}_{31} \mathrm{H}_{25} \mathrm{~N}_{4} \mathrm{O}_{2}{ }^{+}[\mathrm{M}+\mathrm{H}]^{+}: 485.1972$, found: 485.1957 .

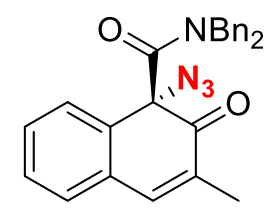

$3 p$

(S)-1-Azido-N,N-dibenzyl-3-methyl-2-oxo-1,2-dihydronaphthalene-1-carboxamid e; reaction temperature: $\mathrm{rt}$; reaction time: $6 \mathrm{~h}$; petroleum ether/ethylacetate $=20: 1$; TLC: $R_{f}=0.5(\mathrm{PE} / \mathrm{EA}=5: 1, \mathrm{UV})$; white solid (mp: $\left.128-130{ }^{\circ} \mathrm{C}\right) ; 99 \%$ yield $(41.9$ $\mathrm{mg}, 0.099 \mathrm{mmol}) ;[\boldsymbol{\alpha}]_{\mathbf{D}}{ }^{23}=+8.80\left(c=0.62, \mathrm{CH}_{2} \mathrm{Cl}_{2}\right) ;{ }^{1} \mathbf{H}$ NMR $\left(400 \mathrm{MHz}, \mathbf{C D}_{3} \mathbf{C N}\right) \delta$ $7.49-7.42(\mathrm{~m}, 3 \mathrm{H}), 7.40-7.37(\mathrm{~m}, 2 \mathrm{H}), 7.33-7.25(\mathrm{~m}, 3 \mathrm{H}), 7.21-7.10(\mathrm{~m}, 5 \mathrm{H})$, $6.71-6.63(\mathrm{~m}, 2 \mathrm{H}), 4.56(\mathrm{~d}, J=14.8 \mathrm{~Hz}, 1 \mathrm{H}), 4.37(\mathrm{~d}, J=14.8 \mathrm{~Hz}, 1 \mathrm{H}), 3.75(\mathrm{~d}, J=$ 16.0 Hz, 1H), $3.67(\mathrm{~d}, J=16.0 \mathrm{~Hz}, 1 \mathrm{H}), 1.89(\mathrm{~d}, J=1.2 \mathrm{~Hz}, 3 \mathrm{H}) ;{ }^{13} \mathbf{C}\left\{{ }^{1} \mathbf{H}\right\}$ NMR $(\mathbf{1 0 1}$ MHz, CD $_{3}$ CN) $\delta 191.4,168.3,143.8,137.5,135.7,135.5,132.7,131.3,131.2,131.0$, $130.2,129.5,129.4,129.3,129.0,128.7,128.4,72.4,50.0,48.9,15.5$; IR v (neat, $\left.\mathbf{c m}^{\mathbf{- 1}}\right)$ : 3006, 2102, 1658, 1456, 1275, 1261, 750; HRMS (ESI, m/z): calcd for $\mathrm{C}_{26} \mathrm{H}_{23} \mathrm{~N}_{4} \mathrm{O}_{2}{ }^{+}[\mathrm{M}+\mathrm{H}]^{+}$: 423.1816, found: 423.1818 .

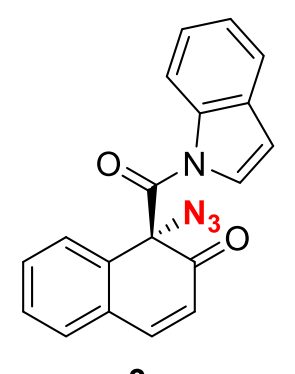

$3 q$

(S)-1-Azido-1-(1H-indole-1-carbonyl)naphthalen-2(1H)-one; reaction temperature: $\mathrm{rt}$; reaction time: $15 \mathrm{~h}$; petroleum ether/ethylacetate $=20: 1$; TLC: $R_{f}=0.3(\mathrm{PE} / \mathrm{EA}=$ 5:1, UV); white solid (mp: $\left.125-128{ }^{\circ} \mathrm{C}\right) ; 99 \%$ yield $(32.7 \mathrm{mg}, 0.099 \mathrm{mmol}) ;[\boldsymbol{\alpha}]_{\mathbf{D}}{ }^{\mathbf{2 4}}=$ 
-98.72 ( $\left.c=0.63, \mathrm{CH}_{2} \mathrm{Cl}_{2}\right)$; ${ }^{1} \mathrm{H}$ NMR (400 MHz, DMSO-d6) $\delta 8.53(\mathrm{~d}, J=8.4 \mathrm{~Hz}$, $1 \mathrm{H}), 8.21(\mathrm{~d}, J=10.0 \mathrm{~Hz}, 1 \mathrm{H}), 7.90(\mathrm{~d}, J=7.6 \mathrm{~Hz}, 1 \mathrm{H}), 7.70-7.63(\mathrm{~m}, 2 \mathrm{H}), 7.58$ (t, $J=7.6 \mathrm{~Hz}, 1 \mathrm{H}), 7.51(\mathrm{t}, J=7.6 \mathrm{~Hz}, 1 \mathrm{H}), 7.41-7.35(\mathrm{~m}, 2 \mathrm{H}), 6.71(\mathrm{~d}, J=3.6 \mathrm{~Hz}, 1 \mathrm{H})$, $6.62(\mathrm{~d}, J=10.0 \mathrm{~Hz}, 1 \mathrm{H}), 6.54(\mathrm{~d}, J=3.6 \mathrm{~Hz}, 1 \mathrm{H}) ;{ }^{13} \mathbf{C}\left\{{ }^{1} \mathbf{H}\right\}$ NMR (101 MHz, DMSO-d6) $\delta 189.8,164.6,148.4,135.6,134.3,132.0,131.8,131.0,129.6,128.1$, 128.0, 125.8, 124.8, 122.7, 122.4, 121.4, 116.2, 111.8, 72.1; IR v (neat, $\mathbf{c m}^{-1}$ ): 3006, 2989, 2109, 1664, 1452, 1275, 1261, 750; HRMS (ESI, m/z): calcd for $\mathrm{C}_{19} \mathrm{H}_{13} \mathrm{~N}_{4} \mathrm{O}_{2}{ }^{+}$ $[\mathrm{M}+\mathrm{H}]^{+}:$329.1033, found: 329.1035 .

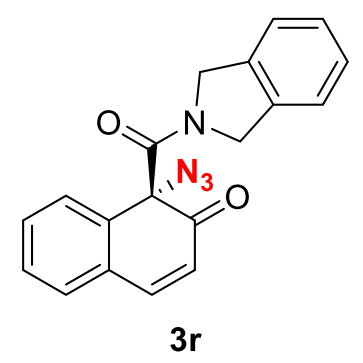

(S)-1-Azido-1-(isoindoline-2-carbonyl)naphthalen-2(1H)-one; reaction temperature: $\mathrm{rt}$; reaction time: $12.5 \mathrm{~h}$; petroleum ether/ethylacetate $=15: 1 ; \mathbf{T L C}: R_{f}=0.5(\mathrm{PE} / \mathrm{EA}$ = 3:1, UV); white solid (mp: $\left.163-166{ }^{\circ} \mathrm{C}\right) ; 94 \%$ yield (31.1 mg, $\left.0.094 \mathrm{mmol}\right) ;[\boldsymbol{\alpha}]_{\mathbf{D}}{ }^{25}$ $=-50.57\left(c=0.41, \mathrm{CH}_{2} \mathrm{Cl}_{2}\right) ;{ }^{1} \mathbf{H}$ NMR (400 MHz, DMSO-d6) $\delta 8.02(\mathrm{~d}, J=10.0 \mathrm{~Hz}$, 1H), $7.74(\mathrm{dd}, J=7.2,1.6 \mathrm{~Hz}, 1 \mathrm{H}), 7.60-7.52(\mathrm{~m}, 2 \mathrm{H}), 7.34-7.29(\mathrm{~m}, 2 \mathrm{H}), 7.25(\mathrm{t}$, $J=7.4 \mathrm{~Hz}, 1 \mathrm{H}), 7.18(\mathrm{t}, J=7.4 \mathrm{~Hz}, 1 \mathrm{H}), 7.11(\mathrm{~d}, J=7.6 \mathrm{~Hz}, 1 \mathrm{H}), 6.48(\mathrm{~d}, J=10.0$ $\mathrm{Hz}, 1 \mathrm{H}), 4.80(\mathrm{~s}, 2 \mathrm{H}), 4.17(\mathrm{~d}, J=13.6 \mathrm{~Hz}, 1 \mathrm{H}), 3.61(\mathrm{~d}, J=13.6 \mathrm{~Hz}, 1 \mathrm{H}) ;{ }^{13} \mathbf{C}\left\{{ }^{1} \mathbf{H}\right\}$ NMR (101 MHz, DMSO-d6) $\delta$ 190.7, 164.9, 147.6, 134.93, 134.86, 133.8, 131.6, $130.8,130.4,129.3,128.0,127.7,127.4,123.6,122.8,122.7,70.8,53.5,49.4$; IR v (neat, $\mathbf{c m}^{-1}$ ): 3006, 2989, 2104, 1660, 1464, 1275, 1261, 897, 756; HRMS (ESI, m/z): calcd for $\mathrm{C}_{19} \mathrm{H}_{15} \mathrm{~N}_{4} \mathrm{O}_{2}{ }^{+}[\mathrm{M}+\mathrm{H}]^{+}:$331.1190, found: 331.1103 . 
<smiles></smiles>

3s

(S)-1-Azido-1-(1,2,3,4-tetrahydroquinoline-1-carbonyl)naphthalen-2(1H)-one;

reaction temperature: $\mathrm{rt}$; reaction time: $9.5 \mathrm{~h}$; petroleum ether/ethylacetate $=20: 1$; TLC: $R_{f}=0.4(\mathrm{PE} / \mathrm{EA}=5: 1, \mathrm{UV})$; white solid (mp: $\left.149-152{ }^{\circ} \mathrm{C}\right) ; 96 \%$ yield $(33.1$ $\mathrm{mg}, 0.096 \mathrm{mmol}) ;[\boldsymbol{\alpha}]_{\mathbf{D}}{ }^{24}=-41.69\left(c=0.30, \mathrm{CH}_{2} \mathrm{Cl}_{2}\right) ;{ }^{1} \mathbf{H}$ NMR (400 MHz, DMSO-d6) $\delta 7.98-7.79(\mathrm{~m}, 1 \mathrm{H}), 7.73-7.65(\mathrm{~m}, 1 \mathrm{H}), 7.62-7.56(\mathrm{~m}, 2 \mathrm{H}), 7.41-$ $7.36(\mathrm{~m}, 1 \mathrm{H}), 7.16-7.01(\mathrm{~m}, 3 \mathrm{H}), 6.54-6.12(\mathrm{~m}, 1 \mathrm{H}), 3.20-2.74(\mathrm{~m}, 2 \mathrm{H}), 2.65-$ $2.54(\mathrm{~m}, 2 \mathrm{H}), 1.61-1.26(\mathrm{~m}, 2 \mathrm{H}) ;{ }^{13} \mathbf{C}\left\{{ }^{1} \mathbf{H}\right\}$ NMR (101 MHz, DMSO-d6) $\delta$ 189.2, $165.1,146.9,137.5,135.0,131.4,131.0,130.3,128.7,128.2,127.6,125.7,125.5$, 124.5, 122.8, 71.5, 43.6, 25.0, 21.6; IR v (neat, $\left.\mathbf{c m}^{-1}\right)$ : 3006, 2989, 2102, 1663, 1458, 1275, 1261, 750; HRMS (ESI, m/z): calcd for $\mathrm{C}_{20} \mathrm{H}_{17} \mathrm{~N}_{4} \mathrm{O}_{2}{ }^{+}[\mathrm{M}+\mathrm{H}]^{+}:$345.1346, found: 345.1379 .<smiles>NC(=O)[C@]12CC=Cc3ccccc3[C@]1(N)C(=O)N2CCc1ccccc1</smiles>

(S)-1-Azido-1-(1,2,3,4-tetrahydroisoquinoline-2-carbonyl)naphthalen-2(1H)-one; reaction temperature: $\mathrm{rt}$; reaction time: $9.5 \mathrm{~h}$; petroleum ether/ethylacetate $=5: 1$; TLC: $R_{f}=0.6(\mathrm{PE} / \mathrm{EA}=1: 1, \mathrm{UV}) ;$ white solid; $91 \%$ yield $(31.4 \mathrm{mg}, 0.091 \mathrm{mmol}) ;[\boldsymbol{\alpha}]_{\mathrm{D}}{ }^{21}=$ $+2.89\left(c=2.18, \mathrm{CH}_{2} \mathrm{Cl}_{2}\right)$; ${ }^{1} \mathbf{H}$ NMR (400 MHz, DMSO-d6) $\delta 8.01(\mathrm{~d}, J=10.0 \mathrm{~Hz}$, 1H), $7.80-7.68(\mathrm{~m}, 1 \mathrm{H}), 7.63-7.51(\mathrm{~m}, 1 \mathrm{H}), 7.50-7.30(\mathrm{~m}, 1 \mathrm{H}), 7.23-6.89(\mathrm{~m}$, $5 \mathrm{H}), 6.47(\mathrm{~d}, J=9.2 \mathrm{~Hz}, 1 \mathrm{H}), 4.73(\mathrm{dd}, J=50.8,16.8 \mathrm{~Hz}, 1 \mathrm{H}), 3.85-3.59(\mathrm{~m}, 1 \mathrm{H})$, 
$3.39-3.29(\mathrm{~m}, 1 \mathrm{H}), 2.97-2.73(\mathrm{~m}, 2 \mathrm{H}), 2.34-1.91(\mathrm{~m}, 1 \mathrm{H}) ;{ }^{13} \mathbf{C}\left\{{ }^{1} \mathbf{H}\right\}$ NMR (101 MHz, DMSO-d6) $\delta 189.7,164.7,147.2,134.7,133.1,132.2,131.5,131.0,130.3$, $128.4,128.2,127.9,126.4,126.2,125.9,122.9,70.8,45.0,41.7,26.7$; IR $v$ (neat, $\left.\mathbf{c m}^{-\mathbf{1}}\right)$ : 3004, 2990, 2095, 1650, 1273, 1260, 752, 750; HRMS (ESI, m/z): calcd for $\mathrm{C}_{20} \mathrm{H}_{17} \mathrm{~N}_{4} \mathrm{O}_{2}{ }^{+}[\mathrm{M}+\mathrm{H}]^{+}:$345.1346, found: 345.1347.<smiles>N[C@]1(C(=O)N2CCCC2)C(=O)C=Cc2ccccc21</smiles>

$3 u$

(S)-1-Azido-1-(pyrrolidine-1-carbonyl)naphthalen-2(1H)-one;

reaction temperature: $\mathrm{rt}$; reaction time: $9.5 \mathrm{~h}$; petroleum ether/ethylacetate $=5: 1$; TLC: $R_{f}=$ $0.6(\mathrm{PE} / \mathrm{EA}=1: 1, \mathrm{UV})$; white solid (mp: $\left.150-152{ }^{\circ} \mathrm{C}\right) ; 89 \%$ yield $(25.0 \mathrm{mg}, 0.089$ mmol); $[\boldsymbol{\alpha}]_{\mathbf{D}}{ }^{24}=-154.33\left(c=0.34, \mathrm{CH}_{2} \mathrm{Cl}_{2}\right) ;{ }^{1} \mathbf{H}$ NMR (400 MHz, DMSO-d6) $\delta 7.93$ $(\mathrm{d}, J=10.0 \mathrm{~Hz}, 1 \mathrm{H}), 7.69-7.66(\mathrm{~m}, 1 \mathrm{H}), 7.58-7.54(\mathrm{~m}, 2 \mathrm{H}), 7.26-7.21(\mathrm{~m}, 1 \mathrm{H})$, $6.41(\mathrm{~d}, J=10.0 \mathrm{~Hz}, 1 \mathrm{H}), 3.46-3.33(\mathrm{~m}, 2 \mathrm{H}), 2.72-2.67(\mathrm{~m}, 1 \mathrm{H}), 2.26-2.21(\mathrm{~m}$, 1H), $1.72-1.58(\mathrm{~m}, 3 \mathrm{H}), 1.50-1.39(\mathrm{~m}, 1 \mathrm{H}) ;{ }^{13} \mathbf{C}\left\{{ }^{1} \mathbf{H}\right\}$ NMR (101 MHz, DMSO-d6) $\delta 190.8,164.2,147.3,134.5,131.5,130.6,130.2$, 129.1, 127.9, 123.5, 70.6, 47.1, 44.1, 25.4, 23.0; IR $v$ (neat, $\mathbf{c m}^{-\mathbf{1}}$ ): 3005, 2989, 2108, 1659, 1411, 1275, 1261, 750; HRMS (ESI, m/z): calcd for $\mathrm{C}_{15} \mathrm{H}_{15} \mathrm{~N}_{4} \mathrm{O}_{2}{ }^{+}[\mathrm{M}+\mathrm{H}]^{+}:$283.1190, found: 283.1208 .<smiles></smiles>

(S)-1-Azido-1-(piperidine-1-carbonyl)naphthalen-2(1H)-one; reaction temperature: $\mathrm{rt}$; reaction time: $15 \mathrm{~h}$; petroleum ether/ethylacetate $=20: 1 ;$ TLC: $R_{f}=0.3(\mathrm{PE} / \mathrm{EA}=$ 5:1, UV); white solid (mp: $\left.135-139{ }^{\circ} \mathrm{C}\right) ; 95 \%$ yield $(28.1 \mathrm{mg}, 0.095 \mathrm{mmol}) ;[\boldsymbol{\alpha}]_{\mathbf{D}}{ }^{\mathbf{2 4}}=$ 
-63.74 (c = 0.55, $\left.\mathrm{CH}_{2} \mathrm{Cl}_{2}\right) ;{ }^{1} \mathrm{H}$ NMR (400 MHz, DMSO-d6) $\delta 7.93(\mathrm{~d}, J=10.0 \mathrm{~Hz}$, 1H), $7.70(\mathrm{dd}, J=6.4,2.0 \mathrm{~Hz} 1 \mathrm{H}), 7.61-7.54(\mathrm{~m}, 2 \mathrm{H}), 7.23(\mathrm{dd}, J=6.4,2.0 \mathrm{~Hz} 1 \mathrm{H})$, $6.41(\mathrm{~d}, J=10.0 \mathrm{~Hz}, 1 \mathrm{H}), 3.81-3.59(\mathrm{~m}, 1 \mathrm{H}), 3.39-3.24(\mathrm{~m}, 1 \mathrm{H}), 2.69-2.53(\mathrm{~m}$, 1H), $2.47-2.35(\mathrm{~m}, 1 \mathrm{H}), 1.53-1.28(\mathrm{~m}, 4 \mathrm{H}), 1.09-0.90(\mathrm{~m}, 1 \mathrm{H}), 0.67-0.44(\mathrm{~m}$, 1H); ${ }^{13} \mathbf{C}\left\{{ }^{1} \mathbf{H}\right\}$ NMR (101 MHz, DMSO-d6) $\delta$ 189.6, 163.9, 146.8, 135.0, 131.3, 130.9, 130.1, 128.1, 127.8, 122.9, 70.8, 45.1, 43.6, 24.8, 23.8, 23.4; IR v (neat, $\mathbf{~ c m}^{-1}$ ): 3005, 2107, 1659, 1439, 1276, 1261, 764, 750; HRMS (ESI, m/z): calcd for $\mathrm{C}_{16} \mathrm{H}_{16} \mathrm{~N}_{4} \mathrm{NaO}_{2}{ }^{+}[\mathrm{M}+\mathrm{Na}]^{+}:$319.1165, found: 319.1174 .<smiles>O=C1C=Cc2ccccc2C12C(=O)N1CCOCC12</smiles>

(S)-1-Azido-1-(morpholine-4-carbonyl)naphthalen-2(1H)-one;

reaction temperature: rt; reaction time: $8 \mathrm{~h}$; petroleum ether/ethylacetate $=20: 1 ;$ TLC: $R_{f}=0.2$ $(\mathrm{PE} / \mathrm{EA}=5: 1, \mathrm{UV})$; white solid (mp: $\left.142-144{ }^{\circ} \mathrm{C}\right) ; 74 \%$ yield $(22.0 \mathrm{mg}, 0.074$ mmol); $[\alpha]_{\mathbf{D}}{ }^{21}=-74.58\left(c=2.10, \mathrm{CH}_{2} \mathrm{Cl}_{2}\right) ;{ }^{1} \mathbf{H}$ NMR (400 MHz, DMSO-d6) $\delta 7.94$ $(\mathrm{d}, J=10.0 \mathrm{~Hz}, 1 \mathrm{H}), 7.70(\mathrm{dd}, J=6.4,2.4 \mathrm{~Hz} 1 \mathrm{H}), 7.62-7.56(\mathrm{~m}, 2 \mathrm{H}), 7.26(\mathrm{dd}, J=$ 6.4, 2.4 Hz 1H), 6.41 (d, $J=10.0 \mathrm{~Hz}, 1 \mathrm{H}), 3.84-3.35(\mathrm{~m}, 4 \mathrm{H}), 3.24-3.08(\mathrm{~m}, 1 \mathrm{H})$, $2.87-2.56(\mathrm{~m}, 2 \mathrm{H}), 2.47-2.35(\mathrm{~m}, 1 \mathrm{H}) ;{ }^{13} \mathbf{C}\left\{{ }^{1} \mathbf{H}\right\}$ NMR (101 MHz, DMSO-d6) $\delta$ 189.8, 164.6, 147.2, 134.6, 131.5, 131.1, 130.4, 128.2, 127.9, 122.8, 70.7, 65.7, 64.4, 45.1, 43.2; IR $v$ (neat, $\mathbf{c m}^{-1}$ ): 3005, 2989, 2110, 1663, 1434, 1275, 1261, 745; HRMS (ESI, m/z): calcd for $\mathrm{C}_{15} \mathrm{H}_{15} \mathrm{~N}_{4} \mathrm{O}_{3}{ }^{+}[\mathrm{M}+\mathrm{H}]^{+}:$299.1139, found: 299.1120 .<smiles>CC(C)(C)CN(Cc1ccccc1)C(=O)[C@@]1(N)C(=O)C=Cc2ccccc21</smiles>

$3 x$ 


\section{(S)-1-Azido-N-benzyl-N-(tert-butyl)-2-oxo-1,2-dihydronaphthalene-1-carboxami}

de; reaction temperature: $\mathrm{rt}$; reaction time: $13.5 \mathrm{~h}$; petroleum ether/ethylacetate $=20: 1$; TLC: $R_{f}=0.4(\mathrm{PE} / \mathrm{EA}=5: 1, \mathrm{UV})$; white solid (mp: $\left.141-144{ }^{\circ} \mathrm{C}\right) ; 73 \%$ yield $(27.3$ $\mathrm{mg}, 0.073 \mathrm{mmol}) ;[\boldsymbol{\alpha}]_{\mathbf{D}}{ }^{21}=+16.89\left(c=1.13, \mathrm{CH}_{2} \mathrm{Cl}_{2}\right) ;{ }^{1} \mathbf{H}$ NMR (400 MHz, DMSO-d6) $\delta 7.78(\mathrm{~d}, J=10.0 \mathrm{~Hz}, 1 \mathrm{H}), 7.59-7.44(\mathrm{~m}, 3 \mathrm{H}), 7.33(\mathrm{~d}, J=7.2 \mathrm{~Hz}, 1 \mathrm{H})$, $7.19-7.08(\mathrm{~m}, 3 \mathrm{H}), 6.93-6.82(\mathrm{~m}, 2 \mathrm{H}), 6.26-6.07(\mathrm{~m}, 1 \mathrm{H}), 3.88(\mathrm{~s}, 2 \mathrm{H}), 1.31$ (s, 9H); ${ }^{13} \mathbf{C}\left\{{ }^{1} \mathbf{H}\right\}$ NMR (101 MHz, DMSO-d6) $\delta$ 188.8, 166.8, 146.5, 137.8, 135.0, $131.4,130.7,129.8,127.82,127.76,127.7,126.6,125.5,123.0,72.0,59.5,47.3,27.6$; IR $v$ (neat, $\mathbf{c m}^{-1}$ ): 3005, 2105, 1663, 1275, 1261, 750; HRMS (ESI, m/z): calcd for $\mathrm{C}_{22} \mathrm{H}_{23} \mathrm{~N}_{4} \mathrm{O}_{2}{ }^{+}[\mathrm{M}+\mathrm{H}]^{+}:$375.1816, found: 375.1811 .<smiles>CC(=O)C1(C#N)C(=O)C=Cc2ccccc21</smiles>

$5 a$

(S)-Methyl-1-azido-2-oxo-1,2-dihydronaphthalene-1-carboxylate ${ }^{[12]} ; \quad$ reaction temperature: rt; reaction time: $3 \mathrm{~h}$; petroleum ether/ethylacetate $=25: 1$; TLC: $R_{f}=0.2$ $(\mathrm{PE} / \mathrm{EA}=10: 1, \mathrm{UV})$; brown solid $\left(\mathrm{mp}: 78-80{ }^{\circ} \mathrm{C}\right) ; 98 \%$ yield $(23.8 \mathrm{mg}, 0.098$ mmol $) ;[\boldsymbol{\alpha}]_{\mathbf{D}}{ }^{25}=-254.4\left(c=1.10, \mathrm{CHCl}_{3}\right) ;{ }^{1} \mathbf{H}$ NMR (400 MHz, $\left.\mathbf{C D C l}_{3}\right) \delta 7.53-7.39$ (m, 4H), $7.38-7.33(\mathrm{~m}, 1 \mathrm{H}), 6.24(\mathrm{~d}, J=10.0 \mathrm{~Hz}, 1 \mathrm{H}), 3.71(\mathrm{~s}, 3 \mathrm{H})$.<smiles>COC(=O)C1(C#N)C(=O)C=Cc2cc(-c3ccccc3)ccc21</smiles>

5b

(S)-Methyl-1-azido-2-oxo-6-phenyl-1,2-dihydronaphthalene-1-carboxylate;

reaction temperature: $\mathrm{rt}$; reaction time: $15 \mathrm{~h}$; petroleum ether/ethylacetate $=30: 1$; TLC: $R_{f}=0.4(\mathrm{PE} / \mathrm{EA}=10: 1, \mathrm{UV})$; yellow solid $\left(\mathrm{mp}: 142-146{ }^{\circ} \mathrm{C}\right) ; 99 \%$ yield (31.9 mg, $0.099 \mathrm{mmol}) ;[\boldsymbol{\alpha}]_{\mathrm{D}}{ }^{\mathbf{3 0}}=-217.8\left(\mathrm{c}=1.33, \mathrm{CHCl}_{3}\right) ;{ }^{1} \mathbf{H}$ NMR (400 MHz, $\left.\mathbf{C D C l}_{3}\right) \delta 7.65(\mathrm{dd}, J=8.2,1.8 \mathrm{~Hz}, 1 \mathrm{H}), 7.60-7.55(\mathrm{~m}, 5 \mathrm{H}), 7.49-7.39(\mathrm{~m}, 3 \mathrm{H})$, 
$6.29(\mathrm{~d}, J=9.6 \mathrm{~Hz}, 1 \mathrm{H}), 3.74(\mathrm{~s}, 3 \mathrm{H}) ;{ }^{13} \mathbf{C}\left\{{ }^{1} \mathbf{H}\right\}$ NMR (101 MHz, $\left.\mathbf{C D C l}_{3}\right) \delta$ 193.2, $167.5,146.8,143.1,139.4,133.9,129.7,129.6,129.2,128.9,128.8,128.4,127.2$, 123.9, 70.5, 53.9; IR $v$ (neat, $\mathbf{c m}^{-1}$ ): 2949, 2924, 2843, 2113, 1748, 1669, 1222; HRMS (ESI, m/z): calcd for $\mathrm{C}_{18} \mathrm{H}_{17} \mathrm{~N}_{4} \mathrm{O}_{3}{ }^{+}\left[\mathrm{M}+\mathrm{NH}_{4}\right]^{+}$Calcd.: 337.1295, found: 337.1292.<smiles>COC1(C#N)C(=O)C=Cc2ccc(-c3ccccc3)cc21</smiles>

(S)-Methyl-1-azido-2-oxo-7-phenyl-1,2-dihydronaphthalene-1-carboxylate, reaction temperature: $\mathrm{rt}$; reaction time: $12 \mathrm{~h}$; petroleum ether/ethylacetate $=30: 1$; TLC: $R_{f}=0.3(\mathrm{PE} / \mathrm{EA}=10: 1, \mathrm{UV})$; yellow solid (mp: $\left.149-155^{\circ} \mathrm{C}\right) ; 97 \%$ yield $(31.0 \mathrm{mg}, 0.097 \mathrm{mmol}) ;[\boldsymbol{\alpha}]_{\mathbf{D}}{ }^{20}=-187.3\left(\mathrm{c}=1.50, \mathrm{CHCl}_{3}\right) ;{ }^{\mathbf{1}} \mathbf{H}$ NMR (400 MHz, $\left.\mathbf{C D C l}_{3}\right) \delta 7.73-7.39(\mathrm{~m}, 9 \mathrm{H}), 6.25(\mathrm{~d}, J=10 \mathrm{~Hz}, 1 \mathrm{H}), 3.72(\mathrm{~s}, 3 \mathrm{H}) ;{ }^{13} \mathbf{C}\left\{{ }^{1} \mathbf{H}\right\} \mathbf{N M R}$ (101 MHz, CDCl $) \delta$ 193.2, 167.5, 146.5, 144.0, 139.2, 135.9, 130.6, 129.2, 128.6, 128.3, 128.2, 127.3, 127.1, 123.3, 70.7, 53.9; IR $v$ (neat, $\mathbf{c m}^{-1}$ ): 2914, 2950, 2104, 1745, 1672, 1599, 1220; HRMS (ESI, m/z): calcd for $\mathrm{C}_{18} \mathrm{H}_{17} \mathrm{~N}_{4} \mathrm{O}_{3}{ }^{+}\left[\mathrm{M}+\mathrm{NH}_{4}\right]^{+}$: 337.1293, found: 337.1295 ;<smiles>CC(=O)C1(C#N)C(=O)C=Cc2cc(-c3cc(C)cc(C)c3)ccc21</smiles>

5d

(S)-Methyl-1-azido-6-(3,5-dimethylphenyl)-2-oxo-1,2-dihydronaphthalene-1-carb oxylate; reaction temperature: rt; reaction time: $12 \mathrm{~h}$; petroleum ether/ethylacetate $=$ 30:1; TLC: $R_{f}=0.2(\mathrm{PE} / \mathrm{EA}=10: 1, \mathrm{UV})$; yellow solid $\left(\mathrm{mp}: 114-118{ }^{\circ} \mathrm{C}\right) ; 97 \%$ yield (33.7 mg, $0.097 \mathrm{mmol}) ;[\boldsymbol{\alpha}]_{\mathbf{D}}{ }^{21}=-154.5\left(\mathrm{c}=1.50, \mathrm{CHCl}_{3}\right) ;{ }^{1} \mathbf{H} \mathbf{~ N M R}(400 \mathrm{MHz}$, $\left.\mathbf{C D C l}_{3}\right) \delta 7.63(\mathrm{dd}, J=8.0,2.0 \mathrm{~Hz}, 1 \mathrm{H}), 7.58-7.52(\mathrm{~m}, 3 \mathrm{H}), 7.19(\mathrm{~s}, 2 \mathrm{H}), 7.06$ (s, 
1H), $6.28(\mathrm{~d}, J=10.0 \mathrm{~Hz}, 1 \mathrm{H}), 3.73(\mathrm{~s}, 3 \mathrm{H}), 2.40(\mathrm{~s}, 6 \mathrm{H}) ;{ }^{13} \mathbf{C}\left\{{ }^{1} \mathbf{H}\right\}$ NMR (101 MHz, $\left.\mathbf{C D C l}_{3}\right) \delta 193.3,167.5,146.9,143.4,139.4,138.7,133.7,130.0,129.59,129.56$, 128.81, 128.76, 125.1, 123.8, 70.5, 53.9, 21.5; IR $v$ (neat, $\left.\mathbf{c m}^{-1}\right): 2952,2918,2112$, 1759, 1669, 1219, 828; HRMS (ESI, m/z): calcd for $\mathrm{C}_{20} \mathrm{H}_{21} \mathrm{~N}_{4} \mathrm{O}_{3}{ }^{+}\left[\mathrm{M}+\mathrm{NH}_{4}\right]^{+}$: 365.1608, found: 365.1602 .<smiles>COC(=O)C1(C#N)C(=O)C=Cc2cc(Br)ccc21</smiles>

$5 e$

(S)-Methyl-1-azido-6-bromo-2-oxo-1,2-dihydronaphthalene-1-carboxylate;

reaction temperature: $\mathrm{rt}$; reaction time: $12 \mathrm{~h}$; petroleum ether/ethylacetate $=30: 1$; TLC: $R_{f}=0.4(\mathrm{PE} / \mathrm{EA}=10: 1, \mathrm{UV})$; yellow solid $\left(\mathrm{mp}: 115-118{ }^{\circ} \mathrm{C}\right) ; 99 \%$ yield (32.2 $\mathrm{mg}, 0.099 \mathrm{mmol}) ;[\boldsymbol{\alpha}]_{\mathbf{D}}{ }^{\mathbf{3 0}}=-273.5\left(\mathrm{c}=1.80, \mathrm{CHCl}_{3}\right) ;{ }^{1} \mathbf{H} \mathbf{~ N M R}(\mathbf{4 0 0} \mathbf{~ M H z}$, $\left.\mathbf{C D C l}_{3}\right) \delta 7.56(\mathrm{dd}, J=8.2,2.2 \mathrm{~Hz}, 1 \mathrm{H}), 7.50(\mathrm{~d}, J=2.0 \mathrm{~Hz}, 1 \mathrm{H}), 7.42(\mathrm{~d}, J=10.0 \mathrm{~Hz}$, 1H), $7.36(\mathrm{~d}, J=8.4 \mathrm{~Hz}, 1 \mathrm{H}), 6.27(\mathrm{~d}, J=10.0 \mathrm{~Hz}, 1 \mathrm{H}), 3.71(\mathrm{~s}, 3 \mathrm{H}) ;{ }^{13} \mathbf{C}\left\{{ }^{1} \mathbf{H}\right\} \mathbf{N M R}$ (101 MHz, CDCl $) \delta$ 192.8, 167.0, 145.2, 134.1, 133.7, 132.6, 131.1, 130.0, 124.7, 124.0, 70.3, 54.1; IR $v$ (neat, $\mathbf{c m}^{-1}$ ): 2955, 2924, 2851, 2111, 1752, 1673, 1224; HRMS (ESI, m/z): calcd for $\mathrm{C}_{12} \mathrm{H}_{12} \mathrm{BrN}_{4} \mathrm{O}_{3}{ }^{+}\left[\mathrm{M}+\mathrm{NH}_{4}\right]^{+}: 339.0087$, found: 339.0086 .<smiles>COc1ccc2c(c1)C(C#N)(C(C)O)C(=O)C=C2</smiles>

(S)-Methyl-1-azido-7-methoxy-2-oxo-1,2-dihydronaphthalene-1-carboxylate;

reaction temperature: $\mathrm{rt}$; reaction time: $10 \mathrm{~h}$; petroleum ether/ethylacetate $=30: 1$; TLC: $R_{f}=0.5(\mathrm{PE} / \mathrm{EA}=10: 1, \mathrm{UV})$; yellow solid $\left(\mathrm{mp}: 119-125^{\circ} \mathrm{C}\right) ; 99 \%$ yield $(27.3 \mathrm{mg}, 0.099 \mathrm{mmol}) ;[\boldsymbol{\alpha}]_{\mathbf{D}}{ }^{23}=-266.0\left(\mathrm{c}=1.50, \mathrm{CHCl}_{3}\right) ;{ }^{1} \mathbf{H} \mathbf{~ N M R}$ (400 MHz, $\left.\mathbf{C D C l}_{3}\right) \delta 7.43(\mathrm{~d}, J=10.0 \mathrm{~Hz}, 1 \mathrm{H}), 7.26(\mathrm{~d}, J=8.4 \mathrm{~Hz}, 1 \mathrm{H}), 6.98(\mathrm{~s}, 1 \mathrm{H}), 6.88(\mathrm{dd}, J$ $=8.4,2.4 \mathrm{~Hz}, 1 \mathrm{H}), 6.06(\mathrm{~d}, J=10.0 \mathrm{~Hz}, 1 \mathrm{H}), 3.82(\mathrm{~s}, 3 \mathrm{H}), 3.68(\mathrm{~s}, 3 \mathrm{H}) ;{ }^{13} \mathbf{C}\left\{{ }^{1} \mathbf{H}\right\}$ 
NMR (101 MHz, $\left.\mathbf{C D C l}_{3}\right) \delta$ 193.2, 167.5, 162.0, 147.1, 137.3, 131.8, 122.2, 120.7, 115.1, 114.4, 70.5, 55.7, 53.9; IR $v$ (neat, $\mathbf{c m}^{-1}$ ): 2960, 2926, 2845, 2118, 1745, 1598, 1552, 1222; HRMS (ESI, m/z): calcd for $\mathrm{C}_{13} \mathrm{H}_{15} \mathrm{~N}_{4} \mathrm{O}_{4}{ }^{+}\left[\mathrm{M}+\mathrm{NH}_{4}\right]^{+}:$291.1088, found: 291.1084 .<smiles>COC1(C#N)C(=O)C=Cc2cc(C#Cc3ccccc3)ccc21</smiles>

$5 g$

(S)-Methyl-1-azido-2-oxo-6-(phenylethynyl)-1,2-dihydronaphthalene-1-carboxyla te; reaction temperature: $\mathrm{rt}$; reaction time: $18 \mathrm{~h}$; petroleum ether/ethylacetate $=30: 1$; TLC: $R_{f}=0.5(\mathrm{PE} / \mathrm{EA}=10: 1, \mathrm{UV})$; yellow solid $\left(\mathrm{mp}: 118-123{ }^{\circ} \mathrm{C}\right) ; 95 \%$ yield (32.6 mg, $0.095 \mathrm{mmol}) ;[\boldsymbol{\alpha}]_{\mathrm{D}}{ }^{30}=-134.8\left(\mathrm{c}=2.00, \mathrm{CHCl}_{3}\right) ;{ }^{1} \mathbf{H}$ NMR (400 MHz, $\left.\mathbf{C D C l}_{3}\right) \delta 7.59-7.47(\mathrm{~m}, 6 \mathrm{H}), 7.38-7.36(\mathrm{~m}, 3 \mathrm{H}), 6.28(\mathrm{~d}, J=10.0 \mathrm{~Hz}, 1 \mathrm{H}), 3.71(\mathrm{~s}$, $3 \mathrm{H}) ;{ }^{13} \mathbf{C}\left\{{ }^{1} \mathbf{H}\right\}$ NMR (101 MHz, $\left.\mathbf{C D C l}_{3}\right) \delta$ 193.0, 167.2, 146.1, 134.6, 133.7, 132.8, $131.8,129.5,129.0,128.6,128.5,125.4,124.3,122.6,91.8,87.7,70.5,54.0 ;$ IR $v$ (neat, $\mathbf{c m}^{-1}$ ): 2955, 2924, 2846, 2114, 1753, 1674, 1223; HRMS (ESI, m/z): calcd for $\mathrm{C}_{20} \mathrm{H}_{17} \mathrm{~N}_{4} \mathrm{O}_{3}{ }^{+}\left[\mathrm{M}+\mathrm{NH}_{4}{ }^{++}:\right.$361.1295, found: 361.1292.

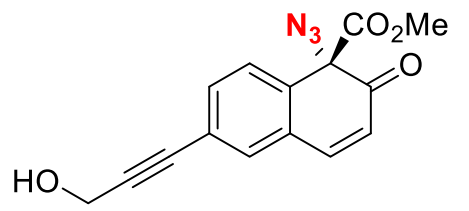

$5 h$

(S)-Methyl-1-azido-6-(3-hydroxyprop-1-yn-1-yl)-2-oxo-1,2-dihydronaphthalene-1 -carboxylate; reaction temperature: $\mathrm{rt}$; reaction time: $18 \mathrm{~h}$; petroleum ether/ ethylacetate $=30: 1 ;$ TLC: $R_{f}=0.5(\mathrm{PE} / \mathrm{EA}=10: 1, \mathrm{UV})$; yellow solid (mp: $110-$ $\left.117{ }^{\circ} \mathrm{C}\right) ; 83 \%$ yield $(24.7 \mathrm{mg}, 0.083 \mathrm{mmol}) ;[\boldsymbol{\alpha}]_{\mathbf{D}}{ }^{25}=-233.7\left(\mathrm{c}=0.80, \mathrm{CHCl}_{3}\right) ;{ }^{1} \mathbf{H}$ NMR (400 MHz, CDCl $) \delta 7.47-7.39(\mathrm{~m}, 4 \mathrm{H}), 6.25$ (d, J = 10.0 Hz, 1H), 4.49 (s, 2H), 3.69 (s, 3H); ${ }^{13} \mathbf{C}\left\{{ }^{1} \mathbf{H}\right\}$ NMR (101 MHz, $\left.\mathbf{C D C l}_{3}\right) \delta$ 193.0, 167.1, 146.0, 134.9, 
$133.8,132.9,129.4,128.5,124.7,124.3,89.8,84.0,70.5,54.0,51.6$; IR $v$ (neat, $\mathbf{c m}^{\mathbf{- 1}}$ ): 2958, 2912, 2842, 2113, 1748, 1672, 1219, 1030; HRMS (ESI, m/z): calcd for $\mathrm{C}_{15} \mathrm{H}_{15} \mathrm{~N}_{4} \mathrm{O}_{4}{ }^{+}\left[\mathrm{M}+\mathrm{NH}_{4}\right]^{+}: 315.1088$, found: 315.1085 .<smiles>COC(=O)C1(C#N)C(=O)C=C(c2ccccc2)c2ccccc21</smiles>

$5 i$

(S)-Methyl-1-azido-2-oxo-4-phenyl-1,2-dihydronaphthalene-1-carboxylate;

reaction temperature: $\mathrm{rt}$; reaction time: $16 \mathrm{~h}$; petroleum ether/ethylacetate $=30: 1$; TLC: $R_{f}=0.3(\mathrm{PE} / \mathrm{EA}=10: 1, \mathrm{UV})$; yellow oil; $84 \%$ yield $(26.8 \mathrm{mg}, 0.084 \mathrm{mmol})$; $[\alpha]_{\mathbf{D}}{ }^{\mathbf{2 4}}=-246.8\left(\mathrm{c}=1.40, \mathrm{CHCl}_{3}\right) ;{ }^{1} \mathbf{H}$ NMR (400 MHz, $\left.\mathbf{C D C l}_{3}\right) \delta 7.57-7.41(\mathrm{~m}, 7 \mathrm{H})$, $7.37-7.32(\mathrm{~m}, 2 \mathrm{H}), 6.22(\mathrm{~s}, 1 \mathrm{H}), 3.76(\mathrm{~s}, 3 \mathrm{H}) ;{ }^{13} \mathbf{C}\left\{{ }^{1} \mathbf{H}\right\}$ NMR (101 MHz, CDCl $) \delta$ 192.4, 167.7, 158.4, 137.0, 135.7, 130.9, 130.1, 129.6, 129.5, 129.4, 128.8, 128.7, 128.4, 123.1, 71.0, 54.0; IR $v$ (neat, $\mathbf{c m}^{-\mathbf{1}}$ ): 2950, 2920, 2842, 2107, 1758, 1662, 1343, 1226; HRMS (ESI, m/z): calcd for $\mathrm{C}_{18} \mathrm{H}_{17} \mathrm{~N}_{4} \mathrm{O}_{3}{ }^{+}\left[\mathrm{M}+\mathrm{NH}_{4}\right]^{+}$: 337.1295, found: 337.1291 .<smiles>CC(=O)C1(C#N)C(=O)C=C(Br)c2ccccc21</smiles>

(S)-Methyl-1-azido-4-bromo-2-oxo-1,2-dihydronaphthalene-1-carboxylate;

reaction temperature: $\mathrm{rt}$; reaction time: $21 \mathrm{~h}$; petroleum ether/ethylacetate $=30: 1$; TLC: $R_{f}=0.6(\mathrm{PE} / \mathrm{EA}=10: 1, \mathrm{UV})$; yellow solid $\left(\mathrm{mp}: 138-142^{\circ} \mathrm{C}\right) ; 97 \%$ yield $(36.0 \mathrm{mg}$, $0.097 \mathrm{mmol}) ;[\boldsymbol{\alpha}]_{\mathbf{D}}{ }^{\mathbf{3 0}}=-286.9\left(\mathrm{c}=1.20, \mathrm{CHCl}_{3}\right) ;{ }^{1} \mathbf{H} \mathbf{N M R}\left(\mathbf{4 0 0} \mathbf{M H z}, \mathbf{C D C l}_{3}\right) \delta 7.94$ - 7.92 (m, 1H), $7.51-7.49$ (m, 3H), 6.80 (s, 1H), 3.70 (s, 3H) ${ }^{13} \mathbf{C}\left\{{ }^{1} \mathbf{H}\right\}$ NMR (101 MHz, $\left.\mathbf{C D C l}_{3}\right) \delta 190.0,166.9,147.0,134.1,132.0,130.4,130.1,128.7,128.1,127.5$, 
70.9, 54.1; IR $v$ (neat, $\mathbf{c m}^{-1}$ ): 2960, 2921, 2101, 1739, 1666, 1556, 1217; HRMS (ESI, m/z): calcd for $\mathrm{C}_{12} \mathrm{H}_{12} \mathrm{BrN}_{4} \mathrm{O}_{3}{ }^{+}\left[\mathrm{M}+\mathrm{NH}_{4}\right]^{+}: 339.0087$, found: 339.0085 .<smiles>COC1(C#N)C(=O)C(Br)=Cc2ccccc21</smiles>

$5 \mathbf{k}$

(S)-Methyl-1-azido-3-bromo-2-oxo-1,2-dihydronaphthalene-1-carboxylate;

reaction temperature: $\mathrm{rt}$; reaction time: $32 \mathrm{~h}$; petroleum ether/ethylacetate $=80: 1$; TLC: $R_{f}=0.3(\mathrm{PE} / \mathrm{EA}=30: 1, \mathrm{UV})$; white solid $\left(\mathrm{mp}: 99-105{ }^{\circ} \mathrm{C}\right) ; 93 \%$ yield $(30.0 \mathrm{mg}$, $0.093 \mathrm{mmol}) ;[\boldsymbol{\alpha}]_{\mathbf{D}}{ }^{28}=-428.0\left(\mathrm{c}=0.80, \mathrm{CHCl}_{3}\right) ;{ }^{1} \mathbf{H} \mathbf{~ N M R}\left(400 \mathbf{~ M H z}, \mathbf{C D C l}_{3}\right) \delta 7.93$ (s, 1H), $7.50-7.41(\mathrm{~m}, 3 \mathrm{H}), 7.32(\mathrm{~d}, J=6.8 \mathrm{~Hz}, 1 \mathrm{H}), 3.70(\mathrm{~s}, 3 \mathrm{H}) ;{ }^{13} \mathbf{C}\left\{{ }^{1} \mathbf{H}\right\} \mathbf{N M R}$ (101 MHz, CDCl $) \delta 187.3,166.7,148.2,134.6,131.3,130.3,129.7,129.5,128.5$, 119.1, 71.6, 54.2; IR $v$ (neat, $\mathbf{c m}^{-1}$ ): 2963, 2924, 2847, 2107, 1751, 1683, $1228 \mathrm{~cm}^{-1}$; HRMS (ESI, m/z): calcd for $\mathrm{C}_{12} \mathrm{H}_{12} \mathrm{BrN}_{4} \mathrm{O}_{3}{ }^{+}\left[\mathrm{M}+\mathrm{NH}_{4}\right]^{+}:$339.0087, found: 339.0086 .<smiles>C=CCOC(=O)[C@@]1(N)C(=O)C=Cc2ccccc21</smiles>

5

(S)-Allyl-1-azido-2-oxo-1,2-dihydronaphthalene-1-carboxylate ${ }^{[12]}$;

reaction temperature: $\mathrm{rt}$; reaction time: $24 \mathrm{~h}$; petroleum ether/ethylacetate $=30: 1$; TLC: $R_{f}=$ $0.3(\mathrm{PE} / \mathrm{EA}=10: 1, \mathrm{UV})$; black oil; $89 \%$ yield $(23.9 \mathrm{mg}, 0.089 \mathrm{mmol}) ;[\boldsymbol{\alpha}]_{\mathbf{D}}{ }^{\mathbf{2 8}}=$ -145.1 (c = 1.00, $\left.\mathrm{CHCl}_{3}\right) ;{ }^{1} \mathbf{H}$ NMR (400 MHz, $\left.\mathbf{C D C l}_{3}\right) \delta 7.52-7.50$ (m, 2H), 7.47 $7.40(\mathrm{~m}, 2 \mathrm{H}), 7.37-7.35(\mathrm{~m}, 1 \mathrm{H}), 6.25(\mathrm{~d}, J=10.0 \mathrm{~Hz}, 1 \mathrm{H}), 5.77-5.67(\mathrm{~m}, 1 \mathrm{H}), 5.16$ $-5.06(\mathrm{~m}, 2 \mathrm{H}), 4.60(\mathrm{dt}, J=5.6,1.2 \mathrm{~Hz}, 2 \mathrm{H})$.<smiles>CC(C)=CCOC(=O)[C@@]1(N)C(=O)C=Cc2ccccc21</smiles> 


\section{(S)-3-Methylbut-2-en-1-yl-1-azido-2-oxo-1,2-dihydronaphthalene-1-carboxylate ${ }^{[1}$}

2]; reaction temperature: $\mathrm{rt}$; reaction time: $24 \mathrm{~h}$; petroleum ether/ethylacetate $=30: 1$;

TLC: $R_{f}=0.3(\mathrm{PE} / \mathrm{EA}=10: 1, \mathrm{UV})$; black oil; $94 \%$ yield $(27.9 \mathrm{mg}, 0.094 \mathrm{mmol})$; $[\boldsymbol{\alpha}]_{\mathbf{D}}{ }^{25}=-259.3\left(\mathrm{c}=0.70, \mathrm{CHCl}_{3}\right) ;{ }^{1} \mathbf{H}$ NMR (400 MHz, DMSO-d6) $\delta 7.50-7.47(\mathrm{~m}$, 2H), $7.45-7.38(\mathrm{~m}, 2 \mathrm{H}), 7.35-7.33(\mathrm{~m}, 1 \mathrm{H}), 6.23(\mathrm{~d}, J=10.0 \mathrm{~Hz}, 1 \mathrm{H}), 5.15(\mathrm{t}, J=$ $7.2 \mathrm{~Hz}, 1 \mathrm{H}), 4.58(\mathrm{~d}, J=6.8 \mathrm{~Hz}, 2 \mathrm{H}), 1.67$ (s, 3H), $1.55(\mathrm{~s}, 3 \mathrm{H})$.<smiles></smiles>

(S)-Prop-2-yn-1-yl-1-azido-2-oxo-1,2-dihydronaphthalene-1-carboxylate ${ }^{[12]}$; reaction temperature: $\mathrm{rt}$; reaction time: $24 \mathrm{~h}$; petroleum ether/ethylacetate $=30: 1$; TLC: $R_{f}=0.3(\mathrm{PE} / \mathrm{EA}=10: 1, \mathrm{UV})$; black solid $\left(\mathrm{mp}: 92-93{ }^{\circ} \mathrm{C}\right) ; 96 \%$ yield $(25.6 \mathrm{mg}$, 0.096 mmol $) ;[\alpha]_{\mathbf{D}}{ }^{25}=-156.2\left(\mathrm{c}=1.00, \mathrm{CHCl}_{3}\right) ;{ }^{1} \mathbf{H} \mathbf{~ N M R}\left(400 \mathbf{~ M H z}, \mathbf{C D C l}_{3}\right) \delta 7.52$ $(\mathrm{d}, J=10.0 \mathrm{~Hz}, 1 \mathrm{H}), 7.49-7.42(\mathrm{~m}, 3 \mathrm{H}), 7.36-7.38(\mathrm{~m}, 1 \mathrm{H}), 6.25(\mathrm{~d}, J=10.0 \mathrm{~Hz}$, $1 \mathrm{H}), 4.68-4.70(\mathrm{~m}, 2 \mathrm{H}), 2.42(\mathrm{t}, J=2.4 \mathrm{~Hz}, 1 \mathrm{H})$.<smiles>CCOC(=O)C1(C#N)C(=O)C=Cc2ccccc21</smiles>

50

(S)-Isopropyl-1-azido-2-oxo-1,2-dihydronaphthalene-1-carboxylate; reaction temperature: $\mathrm{rt}$; reaction time: $24 \mathrm{~h}$; petroleum ether/ethylacetate $=30: 1$; TLC: $R_{f}=$ $0.3(\mathrm{PE} / \mathrm{EA}=10: 1, \mathrm{UV})$; yellow oil; 87\% yield $(23.6 \mathrm{mg}, 0.087 \mathrm{mmol}) ;[\boldsymbol{\alpha}]_{\mathrm{D}}{ }^{25}=$ -324.2 ( $\left.c=0.70, \mathrm{CHCl}_{3}\right) ;{ }^{1} \mathbf{H}$ NMR (400 MHz, $\left.\mathbf{C D C l}_{3}\right) \delta 7.50-7.45(\mathrm{~m}, 2 \mathrm{H}), 7.44-$ $7.37(\mathrm{~m}, 2 \mathrm{H}), 7.35-7.33(\mathrm{~m}, 1 \mathrm{H}), 6.21(\mathrm{~d}, J=10.0 \mathrm{~Hz}, 1 \mathrm{H}), 5.01$ (hept, $J=6.3 \mathrm{~Hz}$, $1 \mathrm{H}), 1.16(\mathrm{~d}, J=6.0 \mathrm{~Hz}, 3 \mathrm{H}), 1.03(\mathrm{~d}, J=6.4 \mathrm{~Hz}, 3 \mathrm{H}) ;{ }^{13} \mathbf{C}\left\{{ }^{1} \mathbf{H}\right\}$ NMR (101 MHz, CDCl$\left._{3}\right) \delta 193.6,166.3,146.7,135.6,130.8,130.0,129.7,129.3,128.1,123.6,71.5$, 
70.7, 21.3, 21.2; IR $v$ (neat, $\mathbf{c m}^{-1}$ ): 2988, 2923, 2106, 1739, 1674, 1214, 1101; HRMS (ESI, m/z): calcd for $\mathrm{C}_{14} \mathrm{H}_{17} \mathrm{~N}_{4} \mathrm{O}_{3}{ }^{+}\left[\mathrm{M}+\mathrm{NH}_{4}\right]^{+}:$289.1295, found: 289.1291.<smiles>NC1(C(=O)Cc2ccccc2)C(=O)C=Cc2ccccc21</smiles>

$5 p$

(S)-Benzyl-1-azido-2-oxo-1,2-dihydronaphthalene-1-carboxylate ${ }^{[12]}$; reaction temperature: $\mathrm{rt}$; reaction time: $20 \mathrm{~h}$; petroleum ether/ethylacetate $=30: 1$; TLC: $R_{f}=$ $0.2(\mathrm{PE} / \mathrm{EA}=10: 1, \mathrm{UV})$; white solid (mp: $\left.70-72{ }^{\circ} \mathrm{C}\right) ; 90 \%$ yield $(28.7 \mathrm{mg}, 0.090$ mmol); $[\boldsymbol{\alpha}]_{\mathbf{D}}{ }^{24}=-190.8\left(\mathrm{c}=0.80, \mathrm{CHCl}_{3}\right) ;{ }^{1} \mathbf{H}$ NMR (400 $\left.\mathbf{~ M H z}, \mathbf{C D C l} 3\right) \delta 7.50-7.33$ (m, 5H), $7.26-7.25(\mathrm{~m}, 3 \mathrm{H}), 7.06-7.03(\mathrm{~m}, 2 \mathrm{H}), 6.23(\mathrm{~d}, J=10.0 \mathrm{~Hz}, 1 \mathrm{H}), 5.18-$ $5.11(\mathrm{~m}, 2 \mathrm{H})$.

Table S1. Optimization of the Reaction Conditions for the Synthesis of Product 3.
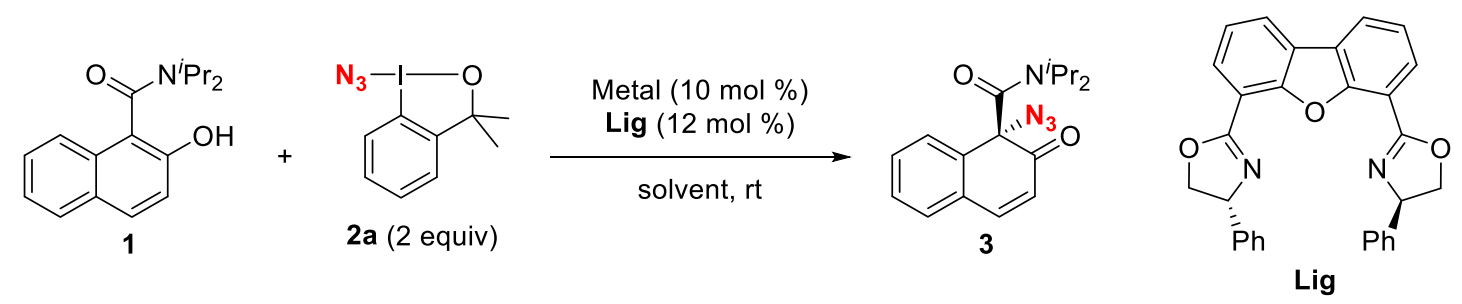

\begin{tabular}{cccccc}
\hline entry & metal & solvent & $\mathrm{t}[\mathrm{h}]$ & $\begin{array}{c}\text { yield }^{b} \\
(\%)\end{array}$ & $\begin{array}{c}\mathrm{ee}^{c} \\
(\%)\end{array}$ \\
\hline 1 & $\mathrm{Zn}(\mathrm{OTf})_{2}$ & $\mathrm{DCM}$ & 30 & $\mathrm{NR}^{d}$ & \\
2 & $\mathrm{Mg}\left(\mathrm{ClO}_{4}\right)_{2}$ & $\mathrm{DCM}$ & 30 & $\mathrm{NR}^{d}$ & \\
3 & $\mathrm{Ni}\left(\mathrm{ClO}_{4}\right)_{2}$ & $\mathrm{DCM}$ & 30 & $\mathrm{NR}^{d}$ & \\
4 & $\mathrm{Fe}(\mathrm{OTf})_{2}$ & $\mathrm{DCM}$ & 30 & 15 & 9 \\
5 & $\mathrm{FeCl}$ & $\mathrm{DCM}$ & 30 & 10 & 21 \\
6 & $\mathrm{Sc}(\mathrm{OTf})_{3}$ & $\mathrm{DCM}$ & 30 & $\mathrm{NR}^{d}$ & \\
7 & $\mathrm{Cu}(\mathrm{OTf})_{2}$ & $\mathrm{DCE}$ & 20 & 82 & 60 \\
8 & $\mathrm{Cu}(\mathrm{OTf})_{2}$ & $\mathrm{THF}$ & 30 & $\mathrm{NR}^{d}$ & \\
9 & $\mathrm{Cu}(\mathrm{OTf})_{2}$ & toluene & 30 & $\mathrm{NR}^{d}$ & \\
10 & $\mathrm{Cu}(\mathrm{OTf})_{2}$ & $\mathrm{CHCl}$ & 20 & 37 & 58
\end{tabular}


${ }^{a}$ Reaction conditions: $1(0.10 \mathrm{mmol}), 2 \mathrm{a}(2$ equiv), metal salt $(10 \mathrm{~mol} \%)$, ligand (12 mol \%), solvent $(1.0 \mathrm{~mL}), \mathrm{rt}$, argon. ${ }^{b}$ The yields of isolated products. ${ }^{c}$ Determined by HPLC analysis. ${ }^{d}$ No Reaction. DCE $=1$,2-dichloroethane, $\mathrm{DCM}=$ dichloromethane.

\section{Table S2. Optimization of the Reaction Conditions for the Synthesis of Product}

5.<smiles>CC(=O)c1c(O)cc(C)c2ccccc12</smiles><smiles>CC1(C)OI(N)c2ccccc21</smiles>

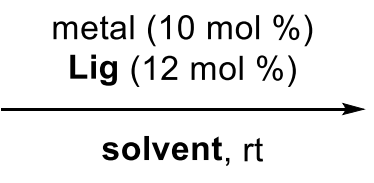<smiles>COC1(C#N)C(=O)C=Cc2ccccc21</smiles><smiles>COI(C#N)OC(=O)c1ccccc1</smiles><smiles>CC(C)[C@H]1COC(c2cccc(C3=N[C@H](C(C)C)CO3)n2)=N1</smiles>

L1<smiles>CC(C1=NC(c2ccccc2)CO1)(C1=NC(c2ccccc2)CO1)C1=NC(c2ccccc2)CO1</smiles><smiles>c1ccc(-c2ccccc2Oc2ccccc2C2=NC(c3ccccc3)CO2)cc1</smiles><smiles>[R]C1N=C(c2cccc3c2oc2c(C4=NC([R])C([R])O4)cccc23)OC1[R]</smiles>

L4: $\mathrm{R}^{1}=\mathrm{Ph}, \mathrm{R}^{2}=\mathrm{H}$

L5: $\mathrm{R}^{1}={ }^{i} \mathrm{Pr}, \mathrm{R}^{2}=\mathrm{H}$

L6: $R^{1}=B n, R^{2}=H$

L3

L7: $\mathrm{R}^{1}=\mathrm{Ph}, \mathrm{R}^{2}=\mathrm{Ph}$

\begin{tabular}{ccccccc}
\hline entry & metal & Lig & solvent & time $(\mathrm{h})$ & $\begin{array}{c}\text { yield }^{b} \\
(\%)\end{array}$ & $\begin{array}{c}\mathrm{ee}^{c} \\
(\%)\end{array}$ \\
\hline 1 & $\mathrm{Cu}(\mathrm{OTf})_{2}$ & $\mathbf{L 1}$ & $\mathrm{DCM}$ & 49 & 37 & 9 \\
2 & $\mathrm{Cu}(\mathrm{OTf})_{2}$ & $\mathbf{L 2}$ & $\mathrm{DCM}$ & 24 & 92 & -12 \\
3 & $\mathrm{Cu}(\mathrm{OTf})_{2}$ & $\mathbf{L 3}$ & $\mathrm{DCM}$ & 8 & 82 & 76 \\
4 & $\mathrm{Cu}(\mathrm{OTf})_{2}$ & $\mathbf{L 4}$ & $\mathrm{DCM}$ & 20 & 97 & 86 \\
5 & $\mathrm{Cu}(\mathrm{OTf})_{2}$ & $\mathbf{L 5}$ & $\mathrm{DCM}$ & 10 & 89 & 72 \\
6 & $\mathrm{Cu}(\mathrm{OTf})_{2}$ & $\mathbf{L 6}$ & $\mathrm{DCM}$ & 12 & 95 & 86 \\
7 & $\mathrm{Cu}(\mathrm{OTf})_{2}$ & $\mathbf{L 7}$ & $\mathrm{DCM}$ & 12 & 94 & 76 \\
8 & $\mathrm{Zn}(\mathrm{OTf})_{2}$ & $\mathbf{L 4}$ & $\mathrm{DCM}$ & 30 & $\mathrm{NR}^{d}$ & \\
9 & $\mathrm{Mg}(\mathrm{ClO})_{2}$ & $\mathbf{L 4}$ & $\mathrm{DCM}$ & 30 & $\mathrm{NR}^{d}$ & \\
10 & $\mathrm{Ni}(\mathrm{ClO})_{2}$ & $\mathbf{L 4}$ & $\mathrm{DCM}$ & 30 & $\mathrm{NR}^{d}$ & \\
11 & $\mathrm{Fe}(\mathrm{OTf})_{2}$ & $\mathbf{L 4}$ & $\mathrm{DCM}$ & 30 & $\mathrm{NR}^{d}$ & \\
12 & $\mathrm{FeCl}$ & $\mathbf{L 4}$ & $\mathrm{DCM}$ & 30 & $\mathrm{NR}^{d}$ & \\
13 & $\mathrm{Sc}(\mathrm{OTf})_{3}$ & $\mathbf{L 4}$ & $\mathrm{DCM}$ & 30 & $\mathrm{NR}^{d}$ & \\
14 & $\mathrm{Cu}(\mathrm{OTf})_{2}$ & $\mathbf{L 4}$ & $\mathrm{DCE}$ & 19 & 97 & 80 \\
\hline
\end{tabular}




\begin{tabular}{|c|c|c|c|c|c|c|}
\hline 15 & $\mathrm{Cu}(\mathrm{OTf})_{2}$ & L4 & THF & 30 & $\mathrm{NR}^{d}$ & \\
\hline 16 & $\mathrm{Cu}(\mathrm{OTf})_{2}$ & L4 & toluene & 30 & $\mathrm{NR}^{d}$ & \\
\hline 17 & $\mathrm{Cu}(\mathrm{OTf})_{2}$ & L4 & $\mathrm{CHCl}_{3}$ & 20 & 36 & 78 \\
\hline 18 & $\mathrm{Cu}(\mathrm{OTf})_{2}$ & L4 & $\mathrm{MeCN}$ & 24 & 92 & 85 \\
\hline 19 & $\mathrm{Cu}(\mathrm{OAc})_{2} \cdot \mathrm{H}_{2} \mathrm{O}$ & L4 & DCM & 23 & 63 & 26 \\
\hline 20 & (CuOTf) $\cdot 0.5$ tol. & L4 & DCM & 25 & 95 & 83 \\
\hline 21 & $\mathrm{Cu}\left(\mathrm{ClO}_{4}\right)_{2} \cdot 6 \mathrm{H}_{2} \mathrm{O}$ & L4 & DCM & 4 & 97 & 82 \\
\hline 22 & $\mathrm{Cu}\left(\mathrm{CH}_{3} \mathrm{CN}\right)_{4} \cdot \mathrm{PF}_{6}$ & L4 & DCM & 3 & 98 & 88 \\
\hline 23 & $\mathrm{Cu}\left(\mathrm{CH}_{3} \mathrm{CN}\right)_{4} \cdot \mathrm{BF}_{4}$ & L4 & DCM & 3 & 96 & 84 \\
\hline $24^{e}$ & $\mathrm{Cu}\left(\mathrm{CH}_{3} \mathrm{CN}\right)_{4} \cdot \mathrm{PF}_{6}$ & L4 & DCM & 22 & 45 & 80 \\
\hline $25^{f}$ & $\mathrm{Cu}\left(\mathrm{CH}_{3} \mathrm{CN}\right)_{4} \cdot \mathrm{PF}_{6}$ & L4 & DCM & 5 & 93 & 86 \\
\hline $26^{g}$ & $\mathrm{Cu}\left(\mathrm{CH}_{3} \mathrm{CN}\right)_{4} \cdot \mathrm{PF}_{6}$ & L4 & DCM & 45 & 72 & 89 \\
\hline
\end{tabular}

${ }^{a}$ Reaction conditions: 4 (0.10 mmol), 2a (2 equiv), metal salt (10 mol \%), Ligand (12 mol \%), solvent $(1.0 \mathrm{~mL}), \mathrm{rt}$, argon. ${ }^{b}$ The yields of isolated products. ${ }^{c}$ Determined by HPLC analysis. ${ }^{d}$ No Reaction. ${ }^{e} \mathbf{2 a}$ was replaced by $\mathbf{2 b} .{ }^{f} 50 \mathrm{mg}$ of $4 \AA$ molecular sieve was added. ${ }^{g}$ The reaction was carried out under $0^{\circ} \mathrm{C}$. DCE $=1,2$-dichloroethane, $\mathrm{DCM}=$ dichloromethane.

\section{Further Transformations}

\section{Procedure for Formation of 6-9 ${ }^{[13]}$ :}

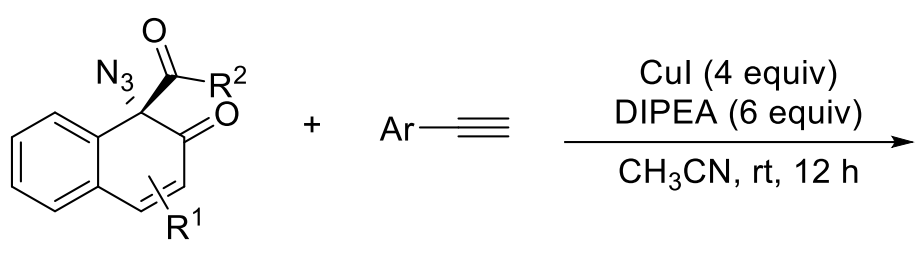<smiles>[R]CC(=O)C1(C([R])=O)c2ccccc2CC1[R]</smiles>

To a solution of phenylacetylene $(0.40 \mathrm{mmol})$ and azide $(0.10 \mathrm{mmol})$ in $1 \mathrm{~mL}$ of anhydrous acetonitrile were added $\mathrm{CuI}(0.40 \mathrm{mmol}$,$) and DIPEA$ (N,N-diisopropylethylamine) $(0.60 \mathrm{mmol})$ at room temperature, and the mixture was stirred until the disappearance of azide (monitored by TLC). The reaction was quenched with $\mathrm{NH}_{4} \mathrm{Cl}$ (aq.), extracted with ethyl acetate, washed with brine, dried over $\mathrm{Mg}_{2} \mathrm{SO}_{4}$, concentrated in vacuo and purified by column chromatography to afford the product. 


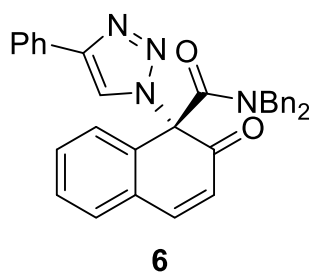

(S)-N,N-Dibenzyl-2-oxo-1-(4-phenyl-1H-1,2,3-triazol-1-yl)-1,2-dihydronaphthale ne-1-carboxamide; reaction temperature: rt; reaction time: $24 \mathrm{~h}$; petroleum ether/ ethylacetate $=5: 1 ;$ TLC: $R_{f}=0.2(\mathrm{PE} / \mathrm{EA}=3: 1, \mathrm{UV}) ;$ white solid $\left(\mathrm{mp}: 112-115^{\circ} \mathrm{C}\right)$; 96\% yield (49.0 mg, $0.096 \mathrm{mmol}) ;[\boldsymbol{\alpha}]_{\mathbf{D}}{ }^{\mathbf{2 0}}=-84.15\left(c=2.27, \mathrm{CH}_{2} \mathrm{Cl}_{2}\right) ;{ }^{1} \mathbf{H}$ NMR (400 MHz, DMSO-d6) $\delta 8.68(\mathrm{~s}, 1 \mathrm{H}), 7.92-7.88(\mathrm{~m}, 3 \mathrm{H}), 7.68-7.58(\mathrm{~m}, 4 \mathrm{H}), 7.43(\mathrm{t}, J$ $=7.6 \mathrm{~Hz}, 2 \mathrm{H}), 7.35-7.28(\mathrm{~m}, 4 \mathrm{H}), 7.22-7.18(\mathrm{~m}, 5 \mathrm{H}), 6.83-6.72(\mathrm{~m}, 2 \mathrm{H}), 6.47(\mathrm{~d}$, $J=10.0 \mathrm{~Hz}, 1 \mathrm{H}), 4.64(\mathrm{~d}, J=15.2 \mathrm{~Hz}, 1 \mathrm{H}), 4.30(\mathrm{~d}, J=15.2 \mathrm{~Hz}, 1 \mathrm{H}), 3.83(\mathrm{~d}, J=$ 15.6 Hz, 1H), $3.77(\mathrm{~d}, J=15.6 \mathrm{~Hz}, 1 \mathrm{H}) ;{ }^{13} \mathbf{C}\left\{{ }^{1} \mathbf{H}\right\}$ NMR (101 MHz, DMSO-d6) $\delta$ $189.3,165.9,146.8,145.6,135.8,133.8,131.2,131.1,130.7,130.1,129.8,128.8$, $128.7,128.4,128.0,127.9,127.8,127.5,127.3,125.3,123.3,122.8,73.3,49.5,47.7$; IR $v$ (neat, $\mathbf{c m}^{-1}$ ): 3006, 1670, 1418, 1275, 1261, 1073, 1025, 750; HRMS (ESI, m/z): calcd for $\mathrm{C}_{33} \mathrm{H}_{27} \mathrm{~N}_{4} \mathrm{O}_{2}{ }^{+}[\mathrm{M}+\mathrm{H}]^{+}$: 511.2129, found: 511.2125.

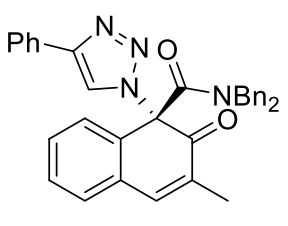

7

(S)-N,N-Dibenzyl-3-methyl-2-oxo-1-(4-phenyl-1H-1,2,3-triazol-1-yl)-1,2-dihydron aphthalene-1-carboxamide; reaction temperature: $\mathrm{rt}$; reaction time: $24 \mathrm{~h}$; petroleum ether/ethylacetate $=5: 1 ;$ TLC: $R_{f}=0.2(\mathrm{PE} / \mathrm{EA}=5: 1, \mathrm{UV})$; white solid (mp: 110 $\left.111{ }^{\circ} \mathrm{C}\right) ; 98 \%$ yield $(51.4 \mathrm{mg}, 0.098 \mathrm{mmol}) ;[\boldsymbol{\alpha}]_{\mathbf{D}}{ }^{23}=+53.81\left(c=1.12, \mathrm{CH}_{2} \mathrm{Cl}_{2}\right) ;{ }^{1} \mathbf{H}$ NMR (400 MHz, CD 3 CN) $\delta 8.31(\mathrm{~s}, 1 \mathrm{H}), 7.83(\mathrm{~d}, J=7.6 \mathrm{~Hz}, 2 \mathrm{H}), 7.59-7.56(\mathrm{~m}$, 1H), $7.52-7.29(\mathrm{~m}, 10 \mathrm{H}), 7.24-7.17(\mathrm{~m}, 5 \mathrm{H}), 6.83-6.74(\mathrm{~m}, 2 \mathrm{H}), 4.73(\mathrm{~d}, J=15.2$ $\mathrm{Hz}, 1 \mathrm{H}), 4.32(\mathrm{~d}, J=15.2 \mathrm{~Hz}, 1 \mathrm{H}), 3.86-3.78(\mathrm{~m}, 2 \mathrm{H}), 1.92(\mathrm{~s}, 3 \mathrm{H}) ;{ }^{13} \mathbf{C}\left\{{ }^{1} \mathbf{H}\right\} \mathbf{N M R}$ (101 MHz, CD $\mathbf{3}$ CN) $\delta 191.4,167.7,147.2,143.5,137.3,135.2,134.3,132.9,131.7$, 
131.6, 131.1, 131.0, 130.89, 130.86, 129.8, 129.44, 129.40, 129.2, 129.1, 128.9, 128.6, 128.4, 126.5, 123.4, 74.7, 50.9, 48.9, 15.7; IR v (neat, $\mathbf{c m}^{-1}$ ): 3006, 1672, 1425, 1261, 1073, 1027, 750; HRMS (ESI, m/z): calcd for $\mathrm{C}_{34} \mathrm{H}_{29} \mathrm{~N}_{4} \mathrm{O}_{2}{ }^{+}[\mathrm{M}+\mathrm{H}]^{+}:$525.2285, found: 525.2289 .

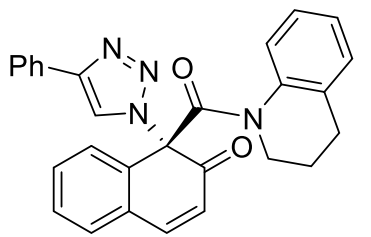

8

(S)-1-(4-Phenyl-1H-1,2,3-triazol-1-yl)-1-(1,2,3,4-tetrahydroquinoline-1-carbonyl) naphthalen-2(1H)-one; reaction temperature: rt; reaction time: $24 \mathrm{~h}$; petroleum ether/ ethylacetate $=5: 1 ;$ TLC: $R_{f}=0.2(\mathrm{PE} / \mathrm{EA}=5: 1, \mathrm{UV})$; white solid $\left(\mathrm{mp}: 117-120{ }^{\circ} \mathrm{C}\right)$; 97\% yield (43.3 mg, $0.097 \mathrm{mmol}) ;[\boldsymbol{\alpha}]_{\mathbf{D}}{ }^{\mathbf{2 4}}=-78.39\left(c=0.96, \mathrm{CH}_{2} \mathrm{Cl}_{2}\right) ;{ }^{\mathbf{1}} \mathbf{H}$ NMR $(400$ MHz, CD $\left.\mathbf{C}_{3} \mathrm{CN}\right) \delta 8.21(\mathrm{~s}, 1 \mathrm{H}), 7.81(\mathrm{~d}, J=7.6 \mathrm{~Hz}, 2 \mathrm{H}), 7.71-7.58(\mathrm{~m}, 6 \mathrm{H}), 7.42(\mathrm{t}, J$ $=7.4 \mathrm{~Hz}, 2 \mathrm{H}), 7.37-7.32(\mathrm{~m}, 1 \mathrm{H}), 7.19-7.05(\mathrm{~m}, 3 \mathrm{H}), 6.38-6.20(\mathrm{~m}, 1 \mathrm{H}), 3.19-$ $2.98(\mathrm{~m}, 2 \mathrm{H}), 2.75-2.62(\mathrm{~m}, 2 \mathrm{H}), 1.66-1.51(\mathrm{~m}, 2 \mathrm{H}) ;{ }^{13} \mathbf{C}\left\{{ }^{1} \mathbf{H}\right\}$ NMR (101 MHz, CD ${ }_{3}$ CN) $\delta 190.7,165.9,147.5,147.2,139.1,132.1,131.7,131.5,130.7,130.3,130.0$, $129.8,129.1,126.8,126.6,126.4,125.8,124.2,123.4,75.1,45.8,26.3,22.6$; IR v (neat, $\mathbf{c m}^{-1}$ ): 3005, 1670, 1420, 1274, 1261, 1060, 1019, 750; HRMS (ESI, m/z): calcd for $\mathrm{C}_{28} \mathrm{H}_{23} \mathrm{~N}_{4} \mathrm{O}_{2}{ }^{+}[\mathrm{M}+\mathrm{H}]^{+}$: 447.1816, found: 447.1811.<smiles>COC(=O)C1(c2ccccc2)C(=O)C=Cc2ccccc21</smiles>

(S)-Methyl-2-oxo-1-(4-(p-tolyl)-1H-1,2,3-triazol-1-yl)-1,2-dihydronaphthalene-1-c arboxylate; reaction temperature: $\mathrm{rt}$; reaction time: $12 \mathrm{~h}$; petroleum ether/ ethylacetate $=10: 1 ;$ TLC: $R_{f}=0.1(\mathrm{PE} / \mathrm{EA}=10: 1, \mathrm{UV})$; white solid $\left(\mathrm{mp}: 60-65^{\circ} \mathrm{C}\right)$; $73 \%$ yield $(26.2 \mathrm{mg}, 0.073 \mathrm{mmol}) ;[\boldsymbol{\alpha}]_{\mathbf{D}}{ }^{28}=-158.4\left(\mathrm{c}=1.00, \mathrm{CHCl}_{3}\right) ;{ }^{1} \mathbf{H}$ NMR $(400$ 
MHz, $\left.\mathbf{C D C l}_{3}\right) \delta 8.35(\mathrm{~s}, 1 \mathrm{H}), 7.76(\mathrm{~d}, J=8.0 \mathrm{~Hz}, 2 \mathrm{H}), 7.59(\mathrm{~d}, J=9.6 \mathrm{~Hz}, 1 \mathrm{H}), 7.49$ $-7.43(\mathrm{~m}, 3 \mathrm{H}), 7.33(\mathrm{~d}, J=7.2 \mathrm{~Hz}, 1 \mathrm{H}), 7.23(\mathrm{~d}, J=7.6 \mathrm{~Hz}, 2 \mathrm{H}), 6.25$ (d, $J=10.0 \mathrm{~Hz}$, 1H), 3.80 (s, 3H), 2.38 (s, 3H); ${ }^{13} \mathbf{C}\left\{{ }^{1} \mathbf{H}\right\}$ NMR (101 MHz, CDCl $) \delta 189.7,166.8$, 147.3 , 147.1, 138.1, 134.5, 131.1, 130.6, 130.4, 129.7, 129.6, 129.5, 127.8, 125.9, 123.1, 121.5, 73.1, 54.3, 21.4; IR v (neat, $\mathbf{c m}^{-1}$ ): 3158, 2956, 2921, 2854, 1755, 1678, 1262, 1223; HRMS (ESI, m/z): calcd for $\mathrm{C}_{21} \mathrm{H}_{18} \mathrm{~N}_{3} \mathrm{O}_{3}{ }^{+}[\mathrm{M}+\mathrm{H}]^{+}:$: 360.1343, found: 360.1349 .

\section{Procedure for Formation of $\mathbf{5} \mathbf{k}^{[6]}$}

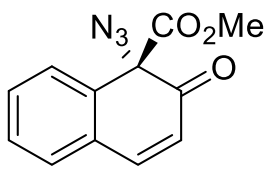

(5a, $88 \%$ ee)

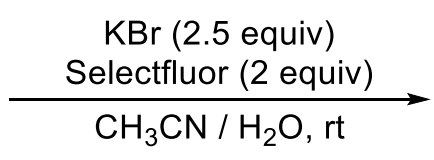

$\mathrm{CH}_{3} \mathrm{CN} / \mathrm{H}_{2} \mathrm{O}, \mathrm{rt}$

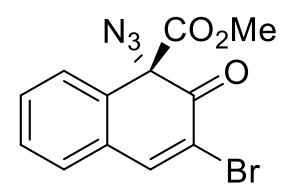

5k, Y: $72 \%$, ee: $86 \%$

To a stirred mixture of $\mathbf{5 a}(20.0 \mathrm{mg}, 0.08 \mathrm{mmol})$ and $\mathrm{KBr}(23.8 \mathrm{mg}, 0.20 \mathrm{mmol})$ in $\mathrm{CH}_{3} \mathrm{CN}(1.5 \mathrm{~mL})$ and water $(40 \mu \mathrm{L})$, selectfluor $(56.6 \mathrm{mg}, 0.16 \mathrm{mmol})$ was added. Then the reaction mixture was stirred at room temperature until the reaction was completed (monitored by TLC). The reaction was quenched by the addition of saturated $\mathrm{Mg}_{2} \mathrm{SO}_{4}$ aqueous solution $(3.0 \mathrm{~mL}$ ). The aqueous layer was extracted with ethyl acetate. The organic layer was washed with brine, dried over $\mathrm{Mg}_{2} \mathrm{SO}_{4}$, filtered and then concentrated. The residue was purified by silica gel column chromatography (ethyl acetate/petroleum ether $=1 / 25)$ to afford $\mathbf{5 k}(23.2 \mathrm{mg}, 72 \%$ yield, $86 \%$ ee $)$.

\section{Control Experiments}

After stirring a mixture of $\mathrm{Cu}\left(\mathrm{CH}_{3} \mathrm{CN}\right)_{4} \cdot \mathrm{PF}_{6}(0.01 \mathrm{mmol}, 10 \mathrm{~mol} \%)$ and $\mathbf{L 4}(0.012$ mmol, $12 \mathrm{~mol} \%)$ in dry dichloromethane $(1 \mathrm{~mL})$ at room temperature for $2 \mathrm{~h}$, substrate $1 \mathbf{d}(0.10 \mathrm{mmol})$ and the radical inhibitor $(0.20 \mathrm{mmol})$ were added. After stirring $30 \mathrm{~min}, \mathrm{~N}_{3}$-transfer reagent $\mathbf{2 a}(0.2 \mathrm{mmol})$ was added and the reaction mixture was stirred at room temperature under argon atmosphere. After the disappearance of substrate 1d (monitored by TLC) and then the crude product was purified by silica gel flash chromatography to afford the product $\mathbf{3 d}$. 


\section{Crystallographic Data}

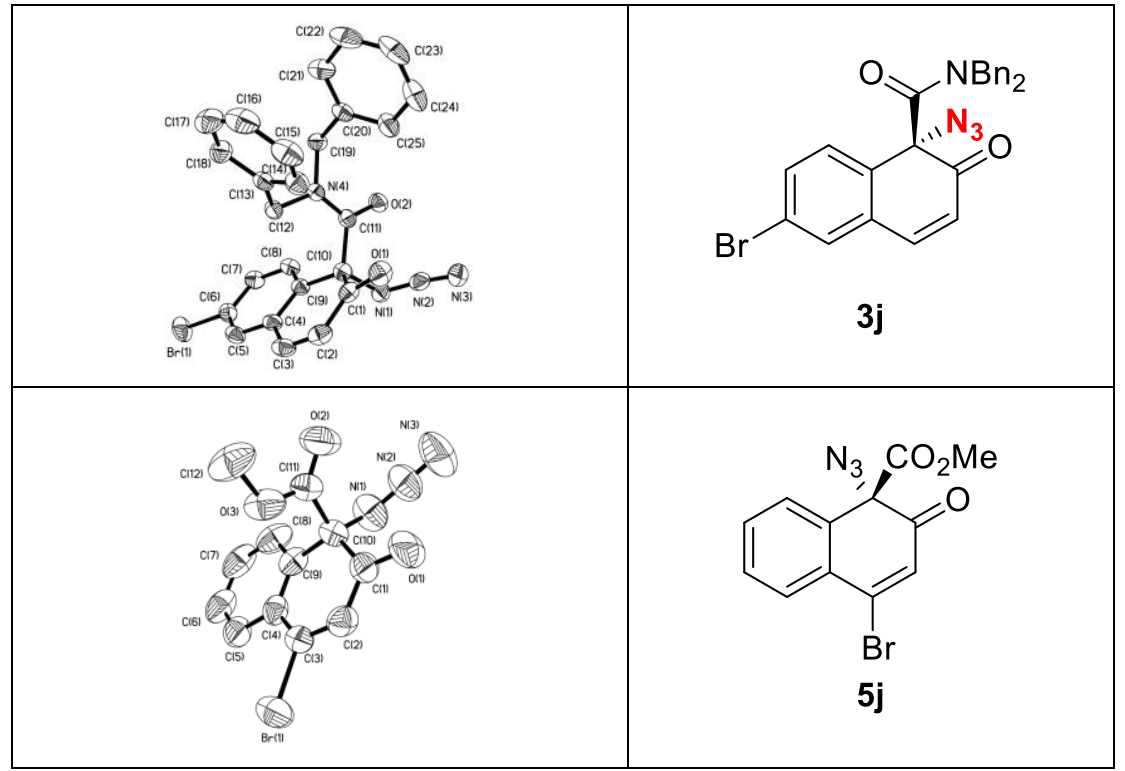

Table S3. Crystal data and structure refinement for $\mathbf{3 j}$ and $\mathbf{5 j}$.

\begin{tabular}{|c|c|c|c|c|}
\hline & \multicolumn{2}{|l|}{$\mathbf{3 j}$} & \multicolumn{2}{|c|}{$5 \mathbf{j}$} \\
\hline Identification code & \multicolumn{2}{|c|}{ mjr18224_0m } & \multicolumn{2}{|c|}{ mo_d8v17519_0m } \\
\hline Empirical formula & \multicolumn{2}{|c|}{$\mathrm{C}_{25} \mathrm{H}_{19} \mathrm{BrN}_{4} \mathrm{O}_{2}$} & \multicolumn{2}{|c|}{$\mathrm{C}_{12} \mathrm{H}_{8} \mathrm{BrN}_{3} \mathrm{O}_{3}$} \\
\hline Formula weight & \multicolumn{2}{|l|}{487.35} & \multicolumn{2}{|c|}{322.12} \\
\hline Temperature & \multicolumn{2}{|c|}{$173(2) \mathrm{K}$} & \multicolumn{2}{|c|}{ 299(2) K } \\
\hline Wavelength & \multicolumn{2}{|c|}{$1.34139 \AA$} & \multicolumn{2}{|c|}{$0.71073 \AA$} \\
\hline Crystal system & \multicolumn{2}{|c|}{ Orthorhombic } & \multicolumn{2}{|c|}{ Monoclinic } \\
\hline Space group & \multicolumn{2}{|c|}{ P 212121} & \multicolumn{2}{|c|}{ P 21} \\
\hline & $a=6.6491(4) \AA$ & $\alpha=90^{\circ}$. & $a=6.0629(5) \AA$ & $\alpha=90^{\circ}$. \\
\hline Unit cell dimensions & $\mathrm{b}=13.5692(7) \AA$ & $\beta=90^{\circ}$. & $\mathrm{b}=9.7025(8) \AA$ & $\beta=104.637(3)^{\circ}$ \\
\hline & $c=23.9241(13) \AA$ & $\gamma=90^{\circ}$. & $c=11.3609(10) \AA$ & $\gamma=90^{\circ}$. \\
\hline Volume & \multicolumn{2}{|c|}{$2158.5(2) \AA^{3}$} & \multicolumn{2}{|c|}{$646.62(9) \AA^{3}$} \\
\hline $\mathrm{Z}$ & \multicolumn{2}{|l|}{4} & \multicolumn{2}{|c|}{2} \\
\hline Density (calculated) & \multicolumn{2}{|c|}{$1.500 \mathrm{Mg} / \mathrm{m}^{3}$} & \multicolumn{2}{|c|}{$1.654 \mathrm{Mg} / \mathrm{m}^{3}$} \\
\hline bsorption coefficient & \multicolumn{2}{|c|}{$1.873 \mathrm{~mm}^{-1}$} & \multicolumn{2}{|c|}{$3.186 \mathrm{~mm}^{-1}$} \\
\hline $\mathrm{F}(000)$ & \multicolumn{2}{|l|}{992} & \multicolumn{2}{|c|}{320} \\
\hline Crystal size & \multicolumn{2}{|c|}{$0.170 \times 0.140 \times 0.100 \mathrm{~mm}^{3}$} & \multicolumn{2}{|c|}{$0.200 \times 0.150 \times 0.110 \mathrm{~mm}^{3}$} \\
\hline range for data collection & \multicolumn{2}{|c|}{5.673 to $54.994^{\circ}$. } & \multicolumn{2}{|c|}{2.800 to $25.494^{\circ}$. } \\
\hline Index ranges & \multicolumn{2}{|c|}{$\begin{array}{c}-7<=\mathrm{h}<=8,-16<=\mathrm{k}<=14, \\
-27<=1<=29\end{array}$} & \multicolumn{2}{|c|}{$-7<=\mathrm{h}<=7,-11<=\mathrm{k}<=11,-13<=\mathrm{l}<=13$} \\
\hline Reflections collected & \multicolumn{2}{|l|}{13564} & \multicolumn{2}{|c|}{6104} \\
\hline
\end{tabular}




\begin{tabular}{c|c|c} 
Independent reflections & $3900[\mathrm{R}(\mathrm{int})=0.0462]$ & 2347 [R(int) $=0.0285]$ \\
Completeness to theta $=53.594^{\circ}$ & $94.7 \%$ & $99.3 \%$ \\
Absorption correction & Semi-empirical from equivalents & Semi-empirical from equivalents \\
Max. and min. transmission & 0.7456 and 0.4677 & 0.7456 and 0.4240 \\
Refinement method & Full-matrix least-squares on $\mathrm{F}^{2}$ & Full-matrix least-squares on $\mathrm{F}^{2}$ \\
Data / restraints / parameters & $3900 / 0 / 290$ & $2347 / 1 / 174$ \\
Goodness-of-fit on $\mathrm{F}^{2}$ & 1.045 & 1.069 \\
Final R indices [I>2sigma(I)] & $\mathrm{R} 1=0.0269, \mathrm{wR} 2=0.0663$ & $\mathrm{R} 1=0.0372, \mathrm{wR} 2=0.0994$ \\
R indices (all data) & $\mathrm{R} 1=0.0274, \mathrm{wR} 2=0.0667$ & $\mathrm{R} 1=0.0457, \mathrm{wR} 2=0.1041$ \\
Absolute structure parameter & $0.097(7)$ & $0.047(8)$ \\
Extinction coefficient & $0.0147(16)$ & 0.601 and -0.339 e. $\AA^{-3}$
\end{tabular}

\section{Reference}

[1]. A. Cornejo, J. M. Fraile, J. I. García, M. J. Gil, V. Martínez-Merino, J. A. Mayoral, E. Pires, I. Villalba, Synlett. 2005, 15, 2321-2324

[2]. John C. K. Chu, Derek M. Dalton, and Tomislav Rovis, J. Am. Chem. Soc. 2015, $137,4445-4452$

[3]. Ulrich Iserloh, Yoji Oderaotoshi, Shuji Kanemasa, and Dennis P. Curran, Org. Synth. 2003, 80, 46

[4]. Maria Victoria Vita, Paola Caramenti, and Jerome Waser, Org. Lett. 2015, 17, $5832-5835$

[5]. Sebastien Alazet, Johannes Preindl, Raphael Simonet-Davin, Stefano Nicolai, Annik Nanchen, Thierry Meyer, and Jerome Waser, J. Org. Chem. 2018, 83, $12334-12356$

[6]. Qin Yin, Shou-Guo Wang, Xiao-Wei Liang, De-Wei Gao, Jun Zhenga and Shu-Li You, Chem. Sci. 2015, 6, 4179

[7]. Andrew G. Myers, Ralf Glatthar, Marlys Hammond, Philip M. Harrington, Elaine Y. Kuo, Jun Liang, Scott E. Schaus, Yusheng Wu, and Jia-Ning Xiang, J. Am. Chem. Soc. 2002, 124, 5380-5401

[8]. Atanu Modak, Uttam Dutta, Rajesh Kancherla, Soham Maity, Mohitosh Bhadra, 
Shaikh M. Mobin, and Debabrata Maiti, Org. Lett. 2014, 16, 2602-2605

[9]. Noriyuki Tezuka, Kohei Shimojo, Keiichi Hirano, Shinsuke Komagawa, Kengo Yoshida, Chao Wang, Kazunori Miyamoto, Tatsuo Saito, Ryo Takita, and Masanobu Uchiyama, J. Am. Chem. Soc. 2016, 138, 9166-9171

[10]. Funk, Lee, Andrew, Johnson, Mary, Catherine, Kung, Pei-Pei, Meng, Jerry, Jialun, Zhou, Joe, Zhongxiang, PCT Int. Appl., 2006117669, 09 Nov 2006

[11]. Alan R. Katritzky, Sanjay K. Singh, Chunming Cai, and Sergey Bobrov, J. Org. Chem. 2006, 71, 3364-3374

[12]. Jayaraman Dhineshkumar, Prasanjit Samaddara and Kandikere Ramaiah Prabhu, Chem. Commun., 2016, 52, 11084

[13]. Liping Zhu, Hongmei Yu, Zhaoqing Xu, Xianxing Jiang, Li Lin, and Rui Wang, Org. Lett. 2014, 16, 1562-1565 


\section{Chromatographic Data for Chiral Products}

\section{Compound 3a}

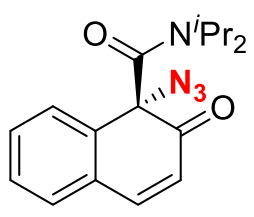

$3 a$

\section{HPLC Conditions}

Column: Chiralcel OD-H, Daicel Chemical Industries, Ltd.

Eluent: Hexanes / isopropanol (80:20)

Flow rate: $1.0 \mathrm{~mL} / \mathrm{min}$

Detection: UV $254 \mathrm{~nm}$

\section{Racemic}

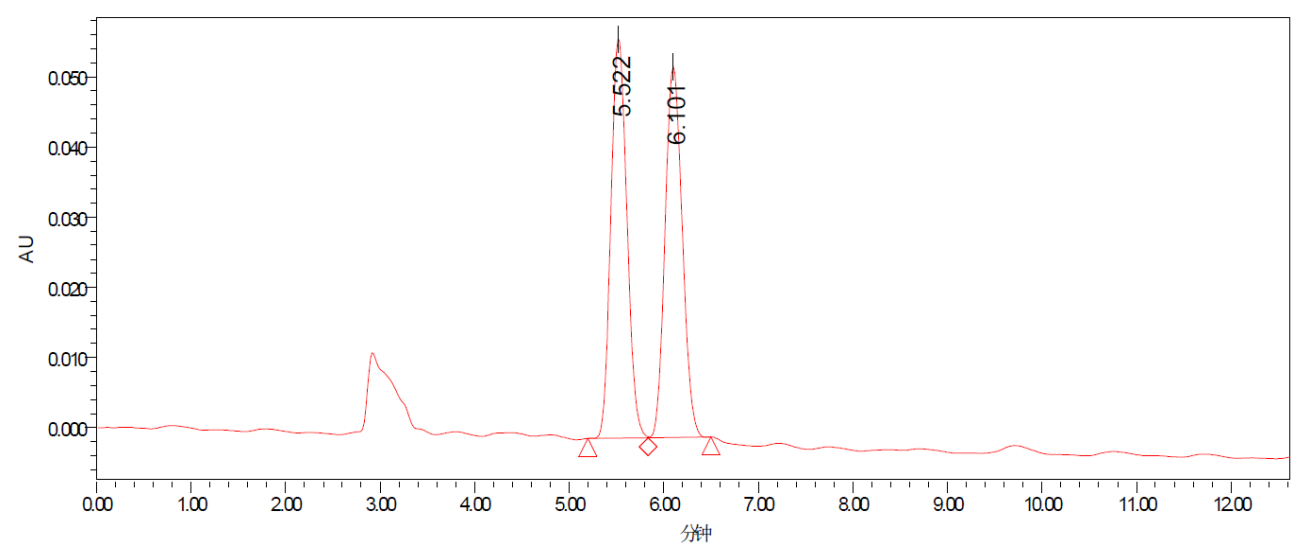

\begin{tabular}{|c|r|r|r|}
\hline & Ret. Tine & Area & Area $\%$ \\
\hline 1 & 5.522 & 670376 & 50.093 \\
\hline 2 & 6.101 & 667880 & 49.907 \\
\hline Sum & & 1338255 & 100.000 \\
\hline
\end{tabular}

\section{Chiral}

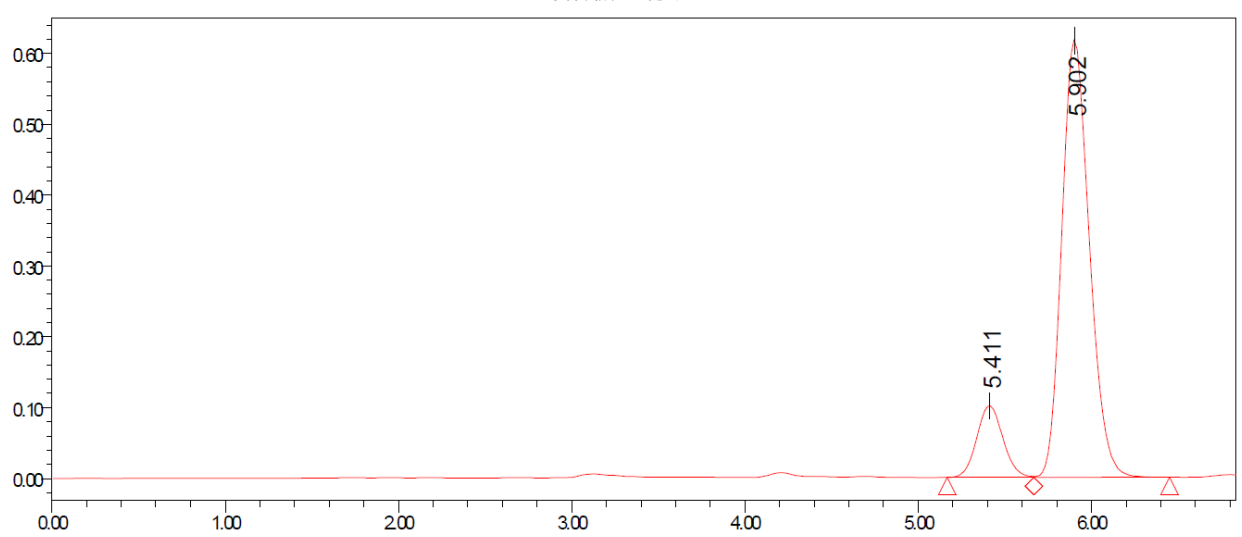

\begin{tabular}{|c|r|c|r|}
\hline & Ret. Time & Area & Area $\%$ \\
\hline 1 & 5.411 & 1064312 & 13.469 \\
\hline 2 & 5.902 & 6837688 & 86.531 \\
\hline Sum & & 7902000 & 100.000 \\
\hline
\end{tabular}




\section{Compound 3b}<smiles>NC1(C(=O)O)C(=O)C=Cc2ccccc21</smiles>

3b

\section{HPLC Conditions}

Column: Chiralcel OD-H, Daicel Chemical Industries, Ltd. Eluent: Hexanes / isopropanol (95:05)

Flow rate: $1.0 \mathrm{~mL} / \mathrm{min}$

Detection: UV $254 \mathrm{~nm}$

\section{Racemic}

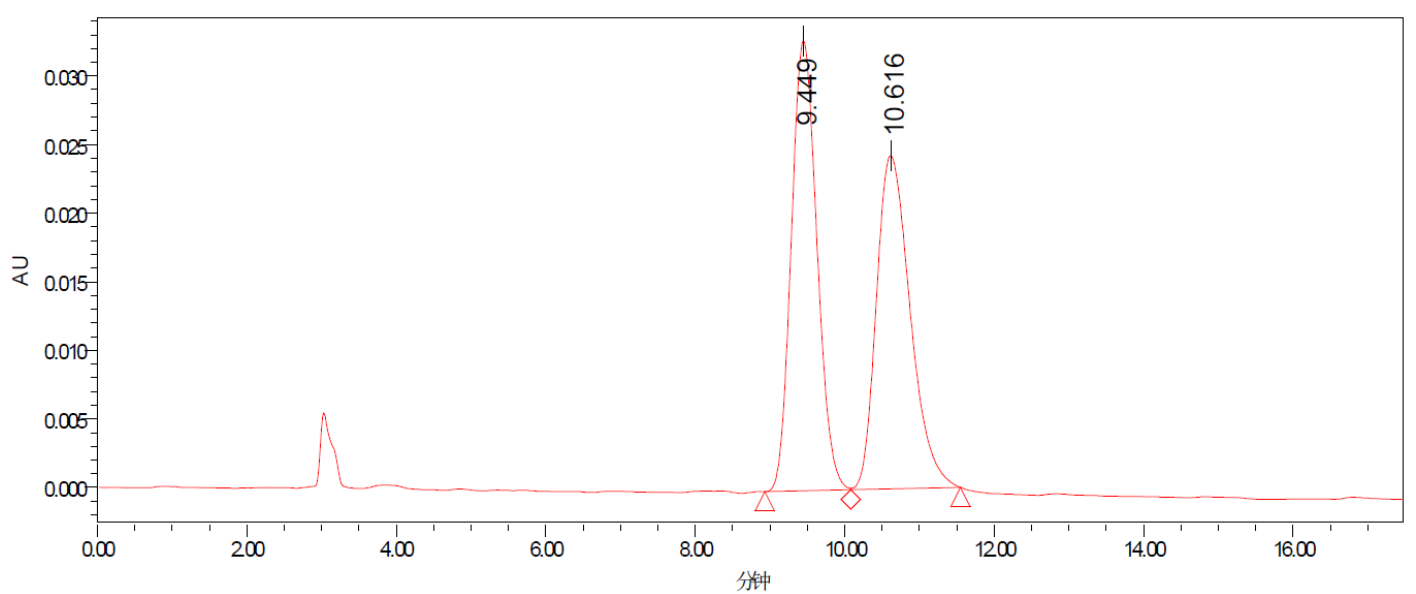

\begin{tabular}{|c|r|c|r|}
\hline & Ret. Time & Area & Area $\%$ \\
\hline 1 & 9.449 & 781845 & 50.584 \\
\hline 2 & 10.616 & 763779 & 49.416 \\
\hline Sum & & 1545624 & 100.000 \\
\hline
\end{tabular}

\section{Chiral}

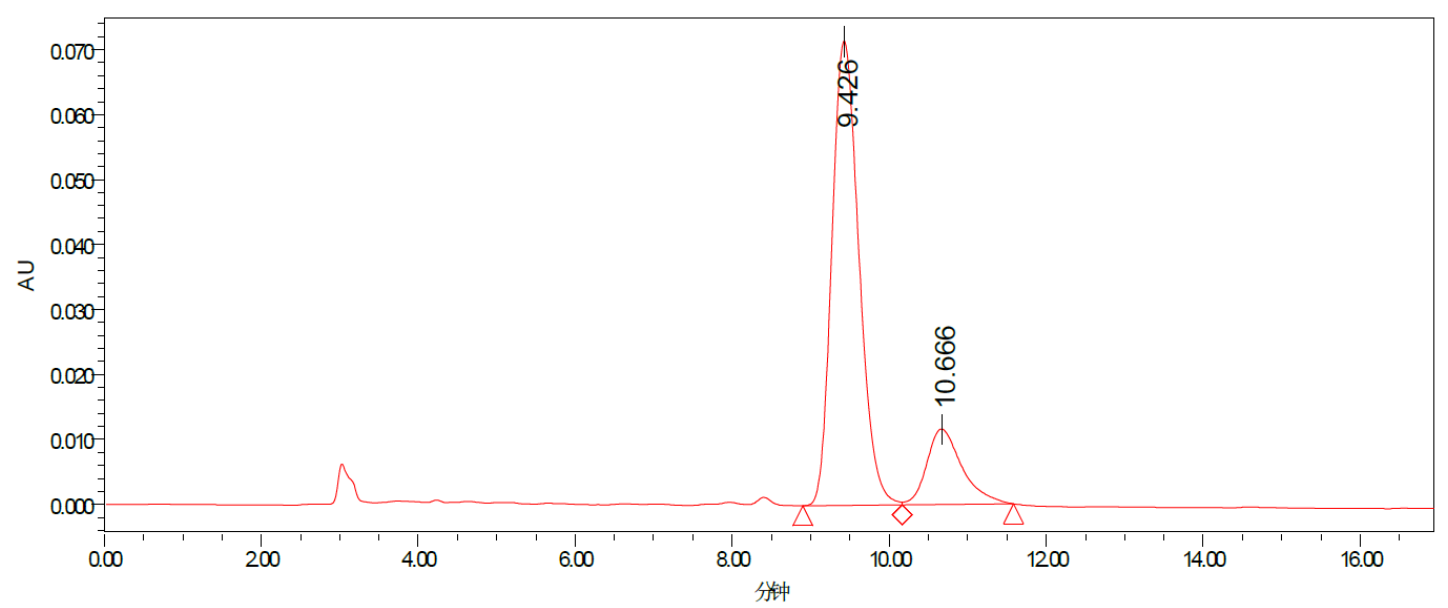

\begin{tabular}{|c|r|c|c|}
\hline & Pet. Time & Area & Area $\%$ \\
\hline 1 & 9.426 & 1709487 & 82484 \\
\hline 2 & 10.666 & 363024 & 17.516 \\
\hline Sum & & 2072511 & 100.000 \\
\hline
\end{tabular}




\section{Compound 3c}

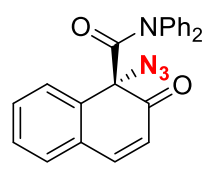

3c

\section{HPLC Conditions}

Column: Chiralcel OD-H, Daicel Chemical Industries, Ltd. Eluent: Hexanes / isopropanol (95:05)

Flow rate: $1.0 \mathrm{~mL} / \mathrm{min}$

Detection: UV $254 \mathrm{~nm}$

\section{Racemic}

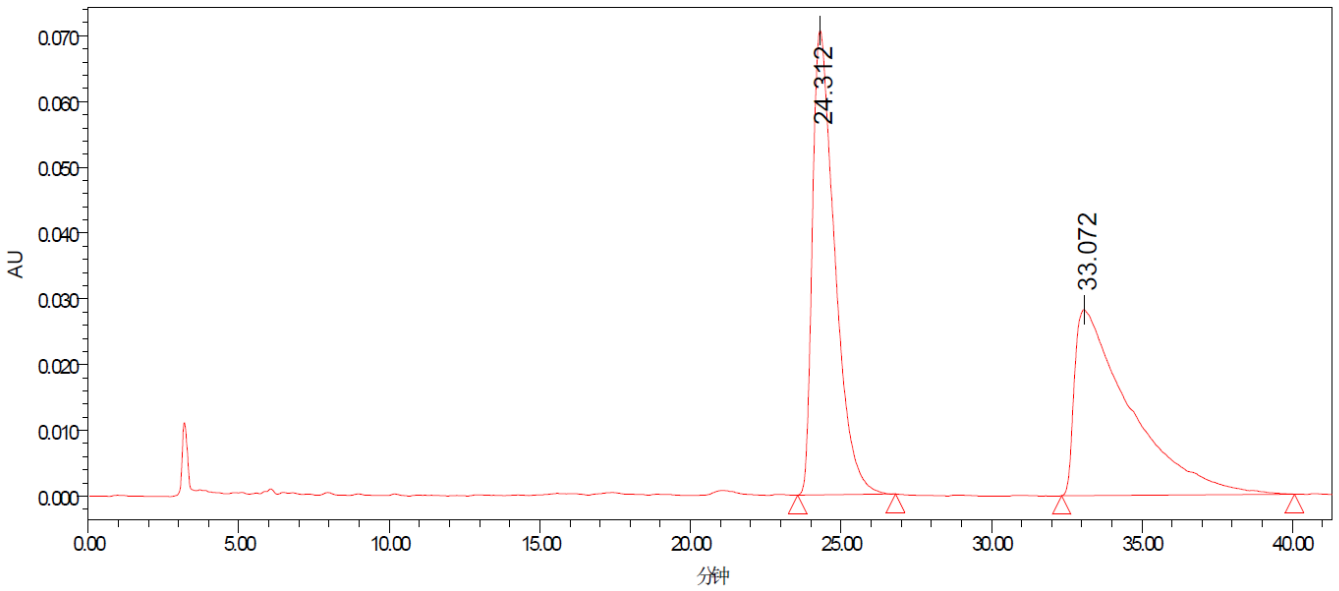

\begin{tabular}{|c|r|c|r|}
\hline & Ret. Time & Area & Area $\%$ \\
\hline 1 & 24.312 & 3641321 & 50.060 \\
\hline 2 & 33.072 & 3632576 & 49.940 \\
\hline Sum & & 7273897 & 100.000 \\
\hline
\end{tabular}

\section{Chiral}

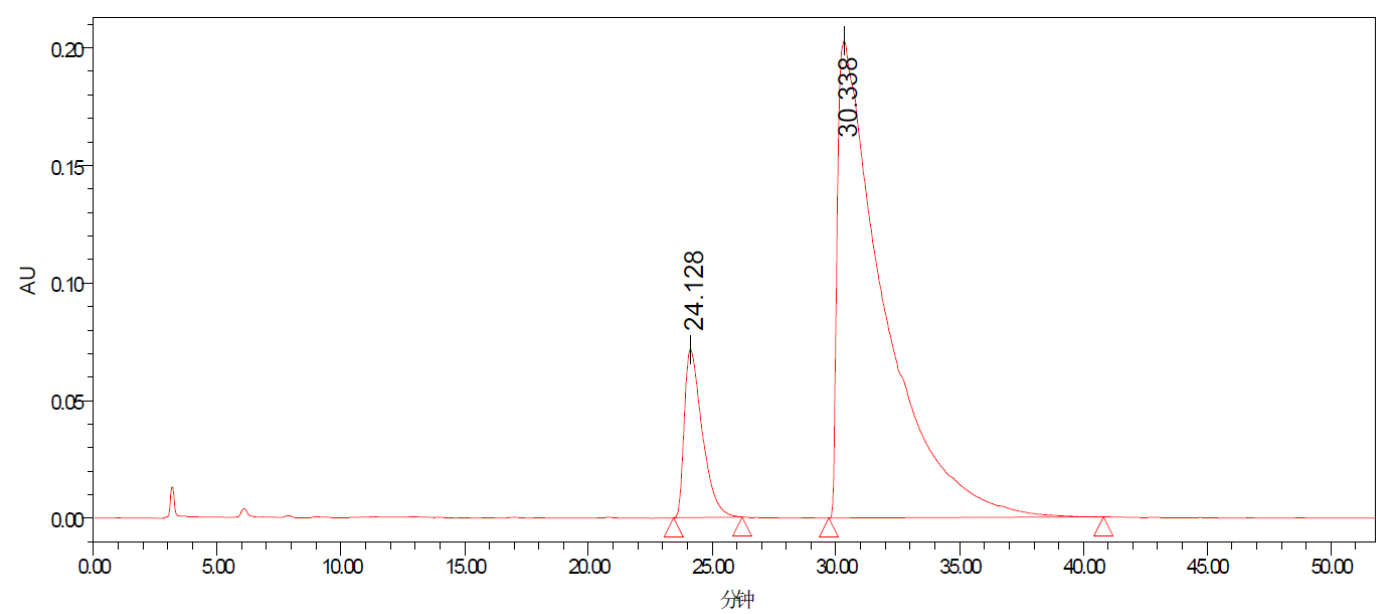

\begin{tabular}{|c|r|c|r|}
\hline & Ret. Time & Area & Area $\%$ \\
\hline 1 & 24.128 & 3606840 & $11.96 C$ \\
\hline 2 & 30.338 & 2655165 & 88.040 \\
\hline Sum & & 30158497 & 100.000 \\
\hline
\end{tabular}




\section{Compound 3d}<smiles></smiles>

3d

\section{HPLC Conditions}

Column: Chiralcel OD-H, Daicel Chemical Industries, Ltd.

Eluent: Hexanes / isopropanol (95:05)

Flow rate: $1.0 \mathrm{~mL} / \mathrm{min}$

Detection: UV $254 \mathrm{~nm}$

\section{Racemic}

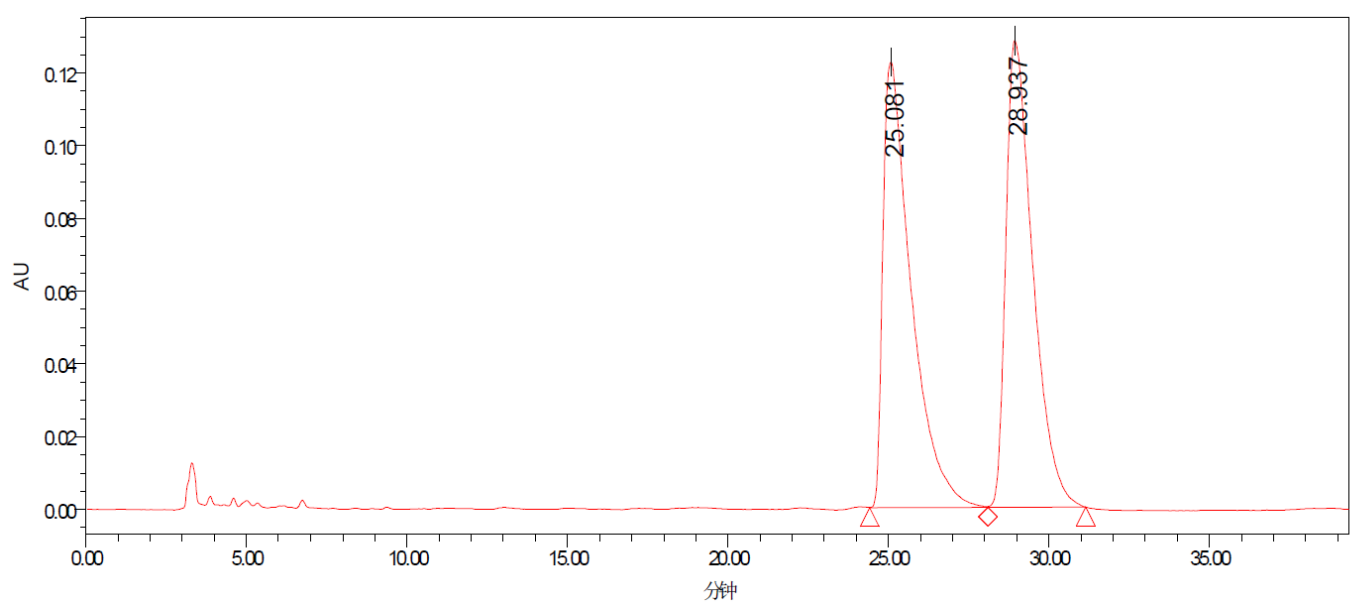

\begin{tabular}{|c|r|c|c|}
\hline & Ret. Time & Area & Area $\%$ \\
\hline 1 & 25.081 & 7480364 & 50.038 \\
\hline 2 & 28.937 & 7469046 & 49.962 \\
\hline Sum & & 14949411 & 100.000 \\
\hline
\end{tabular}

\section{Chiral}

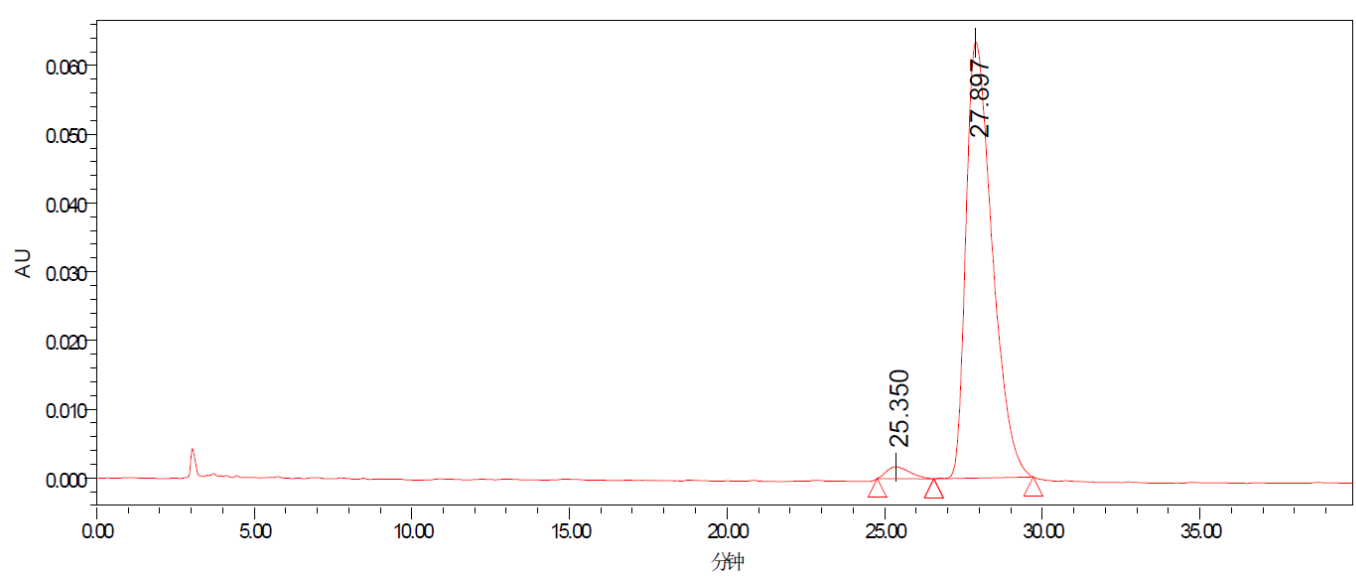

\begin{tabular}{|c|r|l|r|}
\hline & Ret. Time & Area & Area $\%$ \\
\hline 1 & 25.350 & 89621 & 2354 \\
\hline 2 & 27.897 & 3675913 & 97.646 \\
\hline Sum & & 3764534 & 100.000 \\
\hline
\end{tabular}




\section{Compound 3e}<smiles>CCCCC(=O)C1(N)C(=O)C=Cc2cc(-c3ccccc3)ccc21</smiles>

\section{HPLC Conditions}

Column: Chiralcel OJ-H, Daicel Chemical Industries, Ltd.

Eluent: Hexanes / isopropanol (75:25)

Flow rate: $1.0 \mathrm{~mL} / \mathrm{min}$

Detection: UV $254 \mathrm{~nm}$

\section{Racemic}

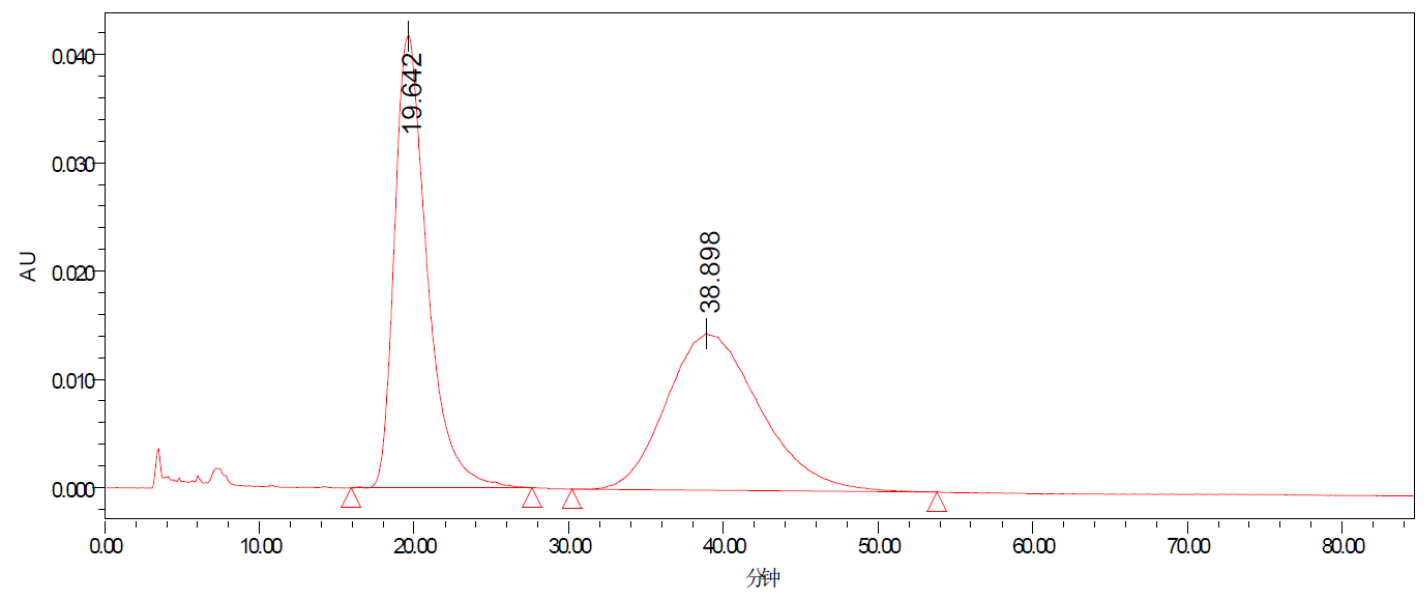

\begin{tabular}{|c|r|c|r|}
\hline & Ret. Time & Area & Area \% \\
\hline 1 & 19.642 & 6112361 & 50.182 \\
\hline 2 & 38.898 & 6067909 & 49.818 \\
\hline Sum & & 12180271 & 100.000 \\
\hline
\end{tabular}

\section{Chiral}

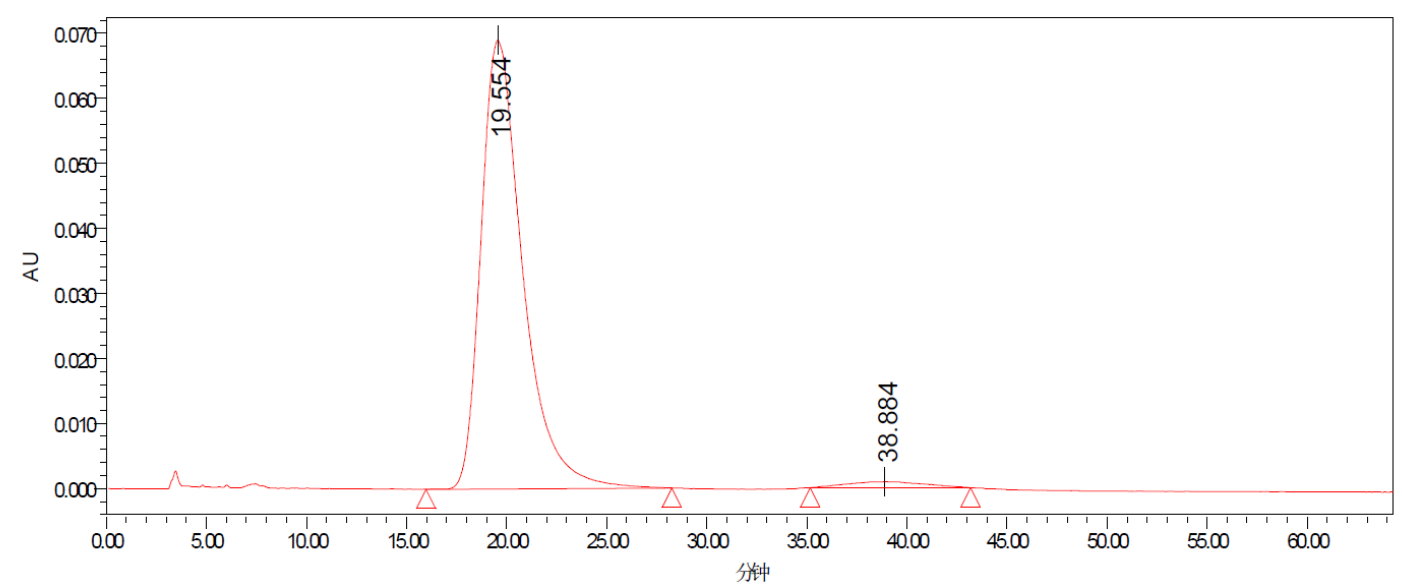

\begin{tabular}{|c|r|r|r|}
\hline & Ret. Time & Area & Area $\%$ \\
\hline 1 & 19.554 & 9992309 & $97.60 \mathrm{c}$ \\
\hline 2 & 38.884 & 244728 & 2391 \\
\hline Sum & & 10237037 & 100.000 \\
\hline
\end{tabular}




\section{Compound 3f}<smiles>CCCCNC(=O)[C@]1(N)C(=O)C=Cc2cc(-c3ccc(C)cc3)ccc21</smiles>

\section{HPLC Conditions}

Column: Chiralcel AS-H, Daicel Chemical Industries, Ltd.

Eluent: Hexanes / isopropanol (90:10)

Flow rate: $1.0 \mathrm{~mL} / \mathrm{min}$

Detection: UV $254 \mathrm{~nm}$

\section{Racemic}

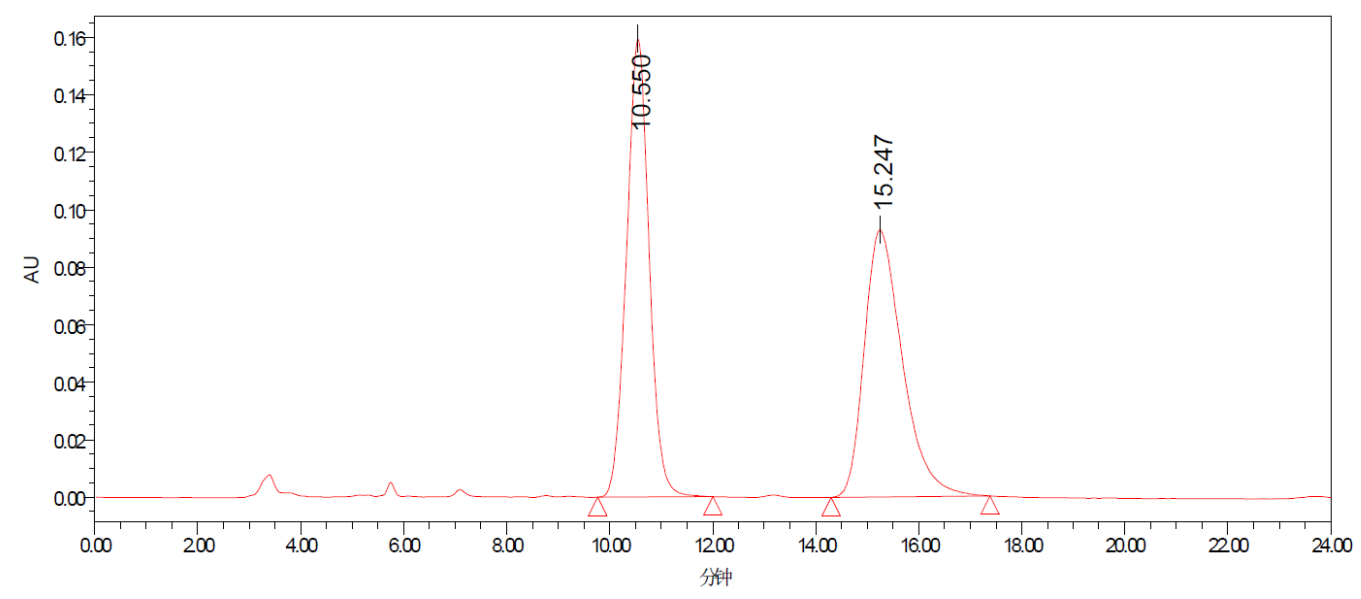

\begin{tabular}{|c|r|c|r|}
\hline & Ret. Time & Area & Area $\%$ \\
\hline 1 & 10.550 & 4857665 & 50.419 \\
\hline 2 & 15.247 & 4776838 & 49.581 \\
\hline Sum & & 9634503 & 100.000 \\
\hline
\end{tabular}

\section{Chiral}

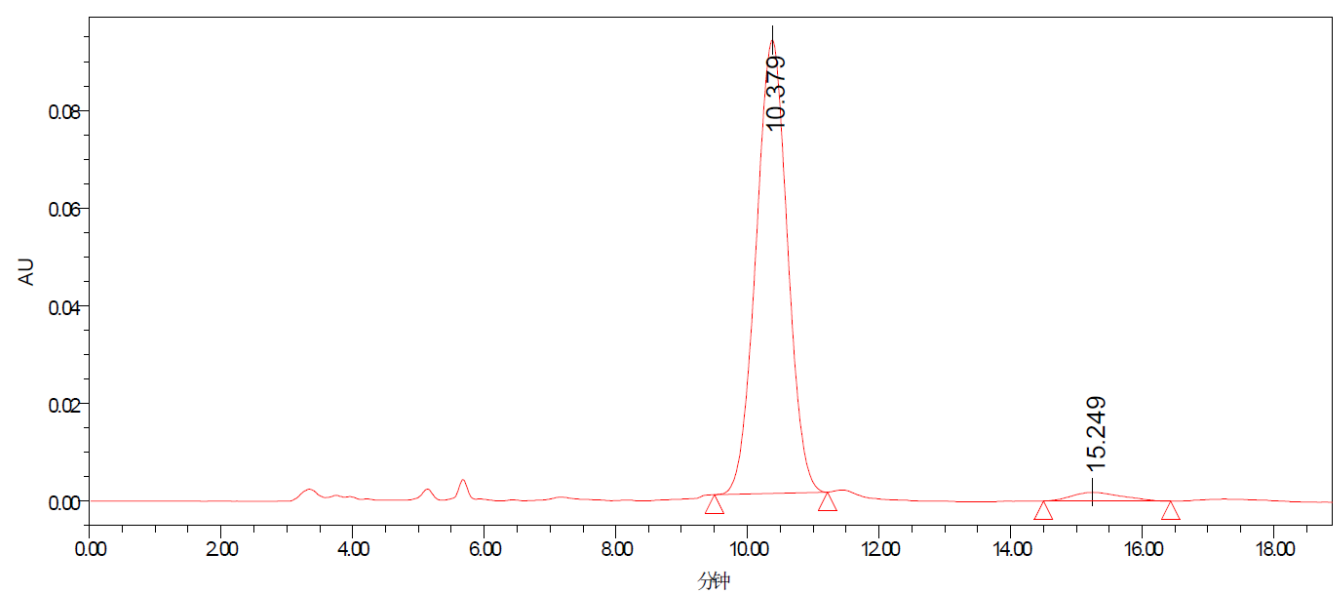

\begin{tabular}{|c|r|r|r|}
\hline & Ret. Time & Area & Area $\%$ \\
\hline 1 & 10.379 & 3079584 & 97.010 \\
\hline 2 & 15.249 & 94903 & 2990 \\
\hline Sum & & 3174487 & 100.000 \\
\hline
\end{tabular}




\section{Compound 3g}<smiles>CCCCC(=O)N[C@]1(C(N)=O)C(=O)C=Cc2cc(-c3ccc(F)cc3)ccc21</smiles>

\section{HPLC Conditions}

Column: Chiralcel OJ-H, Daicel Chemical Industries, Ltd.

Eluent: Hexanes / isopropanol (65:35)

Flow rate: $1.0 \mathrm{~mL} / \mathrm{min}$

Detection: UV $254 \mathrm{~nm}$

\section{Racemic}

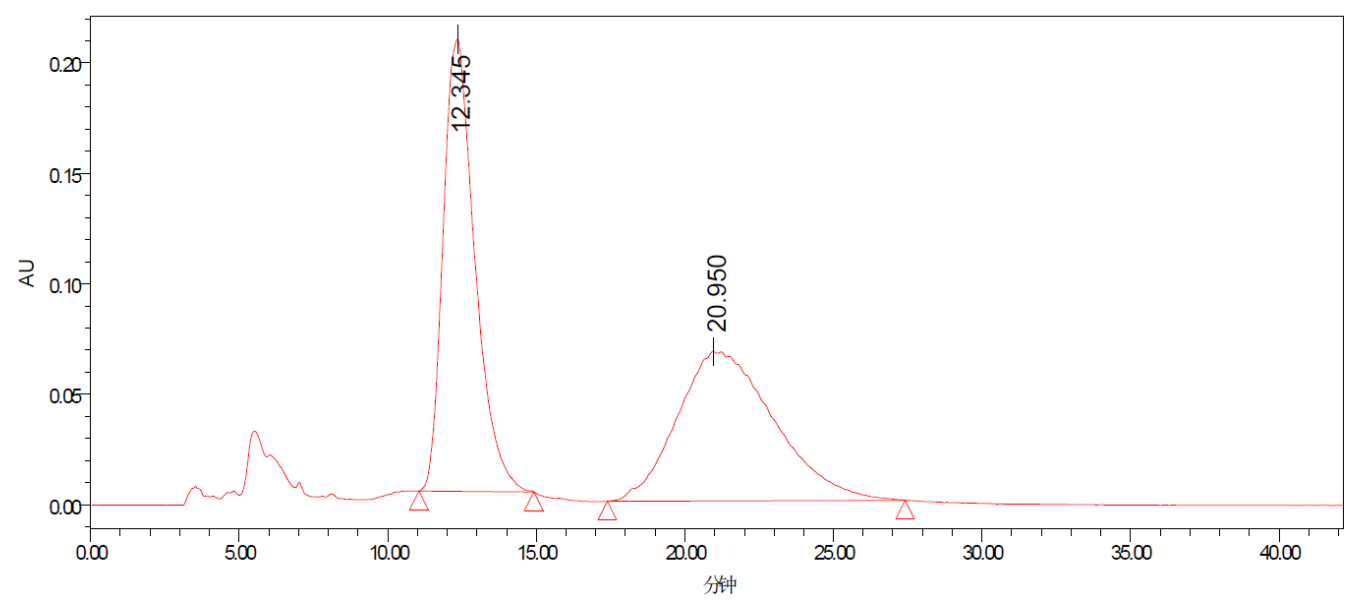

\begin{tabular}{|c|r|c|r|}
\hline & Pet. Time & Area & Area $\%$ \\
\hline 1 & 12345 & 15224881 & 49.845 \\
\hline 2 & 20.950 & 15319378 & 50.155 \\
\hline Sum & & 30544259 & 100.000 \\
\hline
\end{tabular}

\section{Chiral}

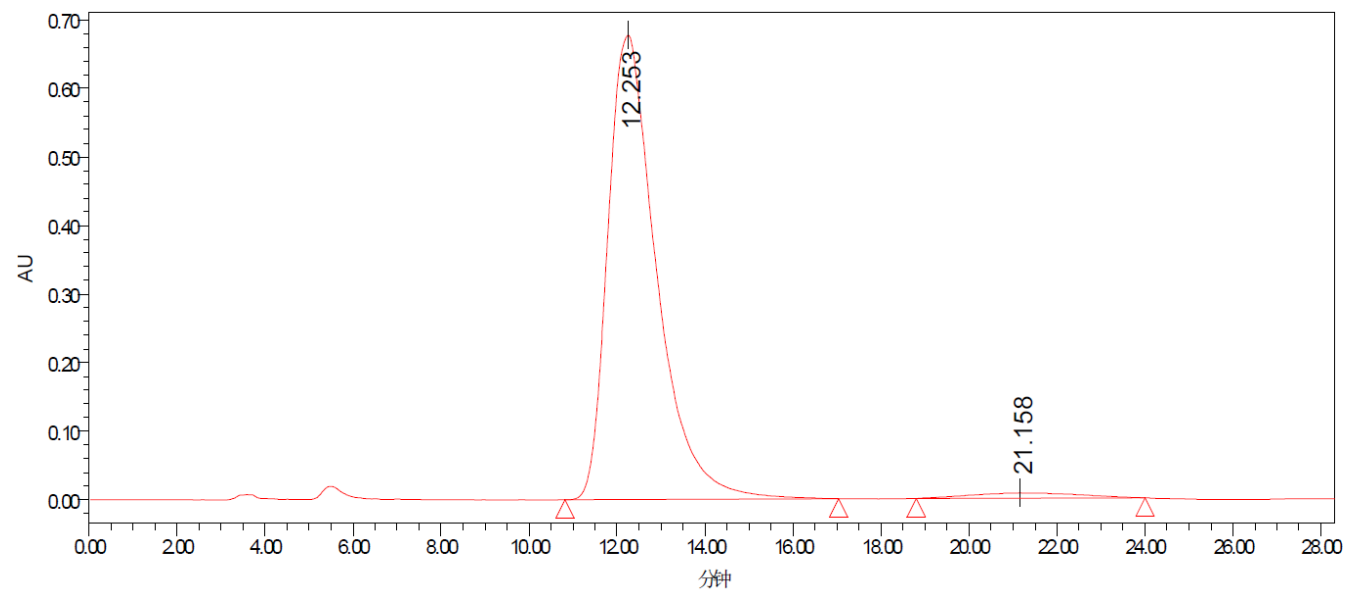

\begin{tabular}{|c|r|c|r|}
\hline & Pet. Time & Area & Area $\%$ \\
\hline 1 & 12253 & 52078303 & 97.566 \\
\hline 2 & 21.158 & 1299213 & 2434 \\
\hline Sum & & 53377516 & 100.000 \\
\hline
\end{tabular}




\section{Compound 3h}<smiles>CCCCC(=O)C1(N)C(=O)C=Cc2cc(-c3ccc(C(F)(F)F)cc3)ccc21</smiles>

\section{HPLC Conditions}

Column: Chiralcel AD-H, Daicel Chemical Industries, Ltd.

Eluent: Hexanes / isopropanol (90:10)

Flow rate: $1.0 \mathrm{~mL} / \mathrm{min}$

Detection: UV $254 \mathrm{~nm}$

\section{Racemic}

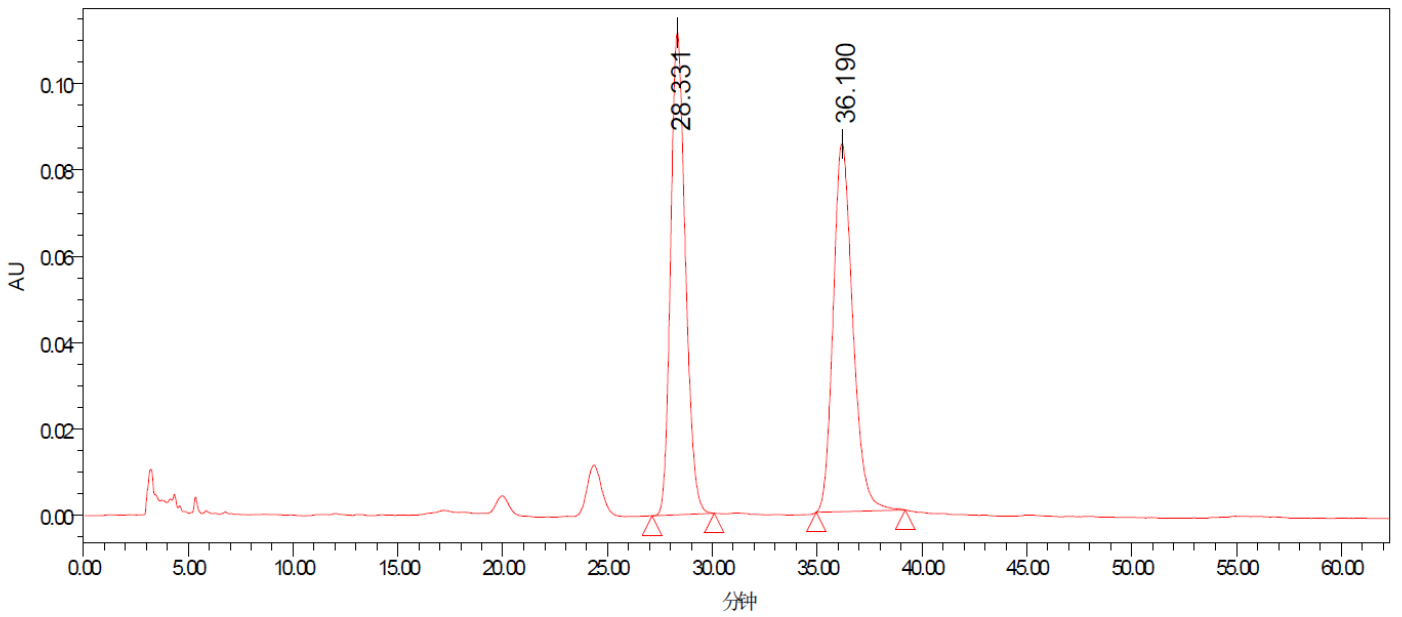

\begin{tabular}{|c|r|c|c|}
\hline & Ret. Time & Area & Area $\%$ \\
\hline 1 & 28.331 & 5470002 & 49.651 \\
\hline 2 & 36.190 & 5546840 & 50.349 \\
\hline Sum & & 11016842 & 100.000 \\
\hline
\end{tabular}

\section{Chiral}

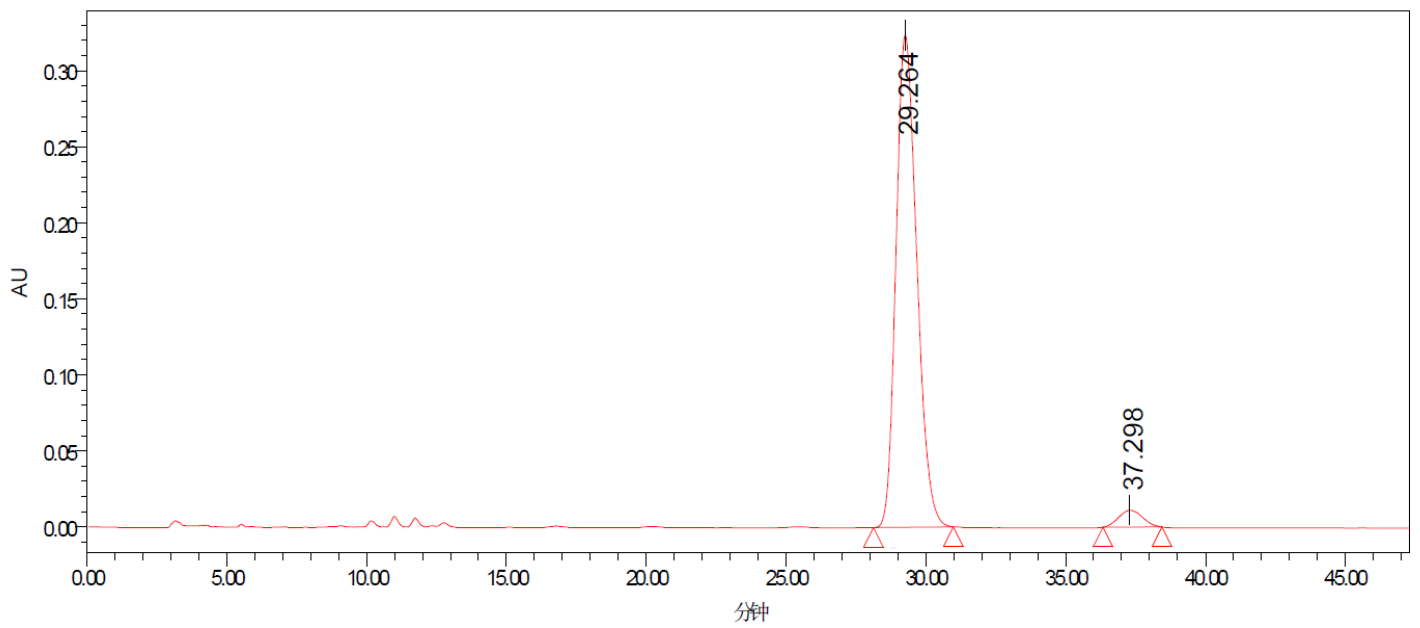

\begin{tabular}{|c|r|c|r|}
\hline & Ret. Time & Area & Area $\%$ \\
\hline 1 & 29.264 & 16686214 & 96.204 \\
\hline 2 & 37.298 & 658356 & 3.796 \\
\hline Sum & & 17344570 & 100.000 \\
\hline
\end{tabular}




\section{Compound 3i}<smiles>CCCCNC(=O)C1(N)C(=O)C=Cc2ccc(-c3ccccc3)cc21</smiles>

$3 \mathbf{i}$

\section{HPLC Conditions}

Column: Chiralcel AD-H, Daicel Chemical Industries, Ltd.

Eluent: Hexanes / isopropanol (90:10)

Flow rate: $1.0 \mathrm{~mL} / \mathrm{min}$

Detection: UV $254 \mathrm{~nm}$

\section{Racemic}

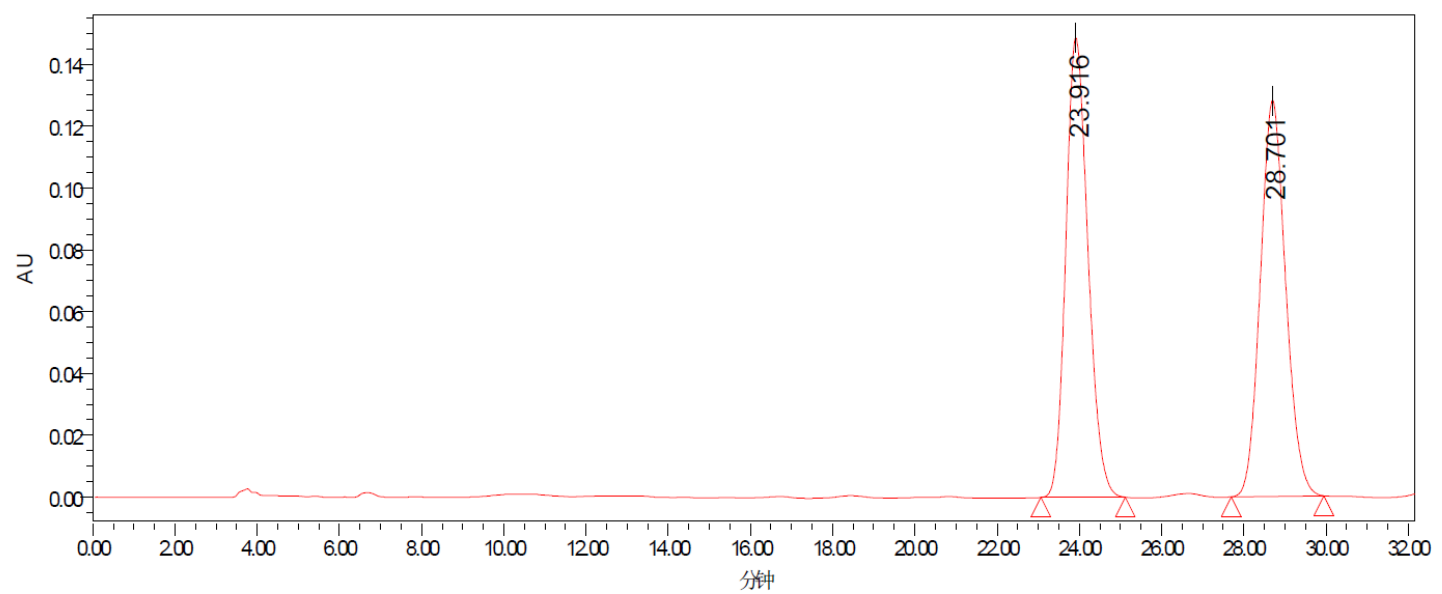

\begin{tabular}{|c|r|c|c|}
\hline & Ret. Time & Area & Area $\%$ \\
\hline 1 & 23.916 & 5567132 & 50.438 \\
\hline 2 & 28.701 & 5470425 & 49.562 \\
\hline Sum & & 11037551 & 100.000 \\
\hline
\end{tabular}

\section{Chiral}

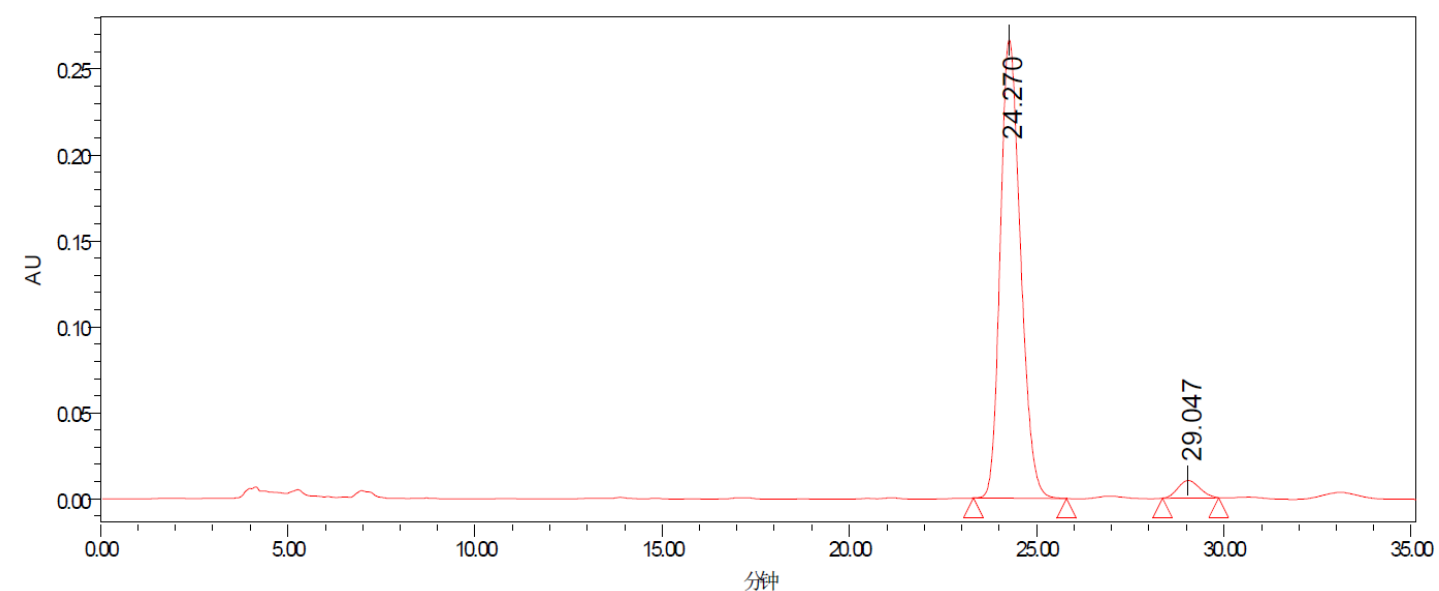

\begin{tabular}{|c|r|c|r|}
\hline & Ret. Tire & Area & Area $\%$ \\
\hline 1 & 24.270 & 10022297 & 96.073 \\
\hline 2 & 29.047 & 409669 & 3.927 \\
\hline Sum & & 10431966 & 100.000 \\
\hline
\end{tabular}




\section{Compound 3j}<smiles>CCCCC(=O)C1(N)C(=O)C=Cc2cc(Br)ccc21</smiles>

\section{HPLC Conditions}

Column: Chiralcel AD-H, Daicel Chemical Industries, Ltd.

Eluent: Hexanes / isopropanol (90:10)

Flow rate: $1.0 \mathrm{~mL} / \mathrm{min}$

Detection: UV $254 \mathrm{~nm}$

\section{Racemic}

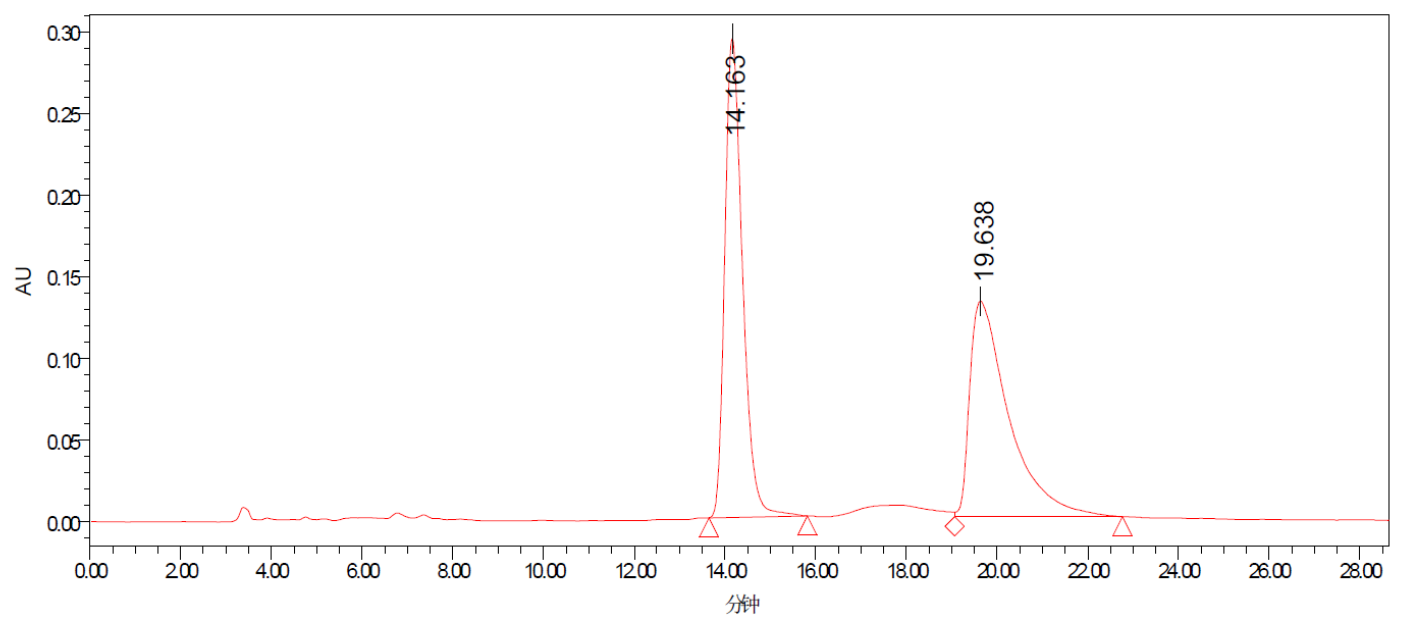

\begin{tabular}{|c|r|c|c|}
\hline & Ret. Time & Area & Area $\%$ \\
\hline 1 & $14.1 \widetilde{1}$ & 7966761 & 50.200 \\
\hline 2 & 19.638 & 7903256 & 49.800 \\
\hline Sum & & 15870017 & 100.000 \\
\hline
\end{tabular}

\section{Chiral}

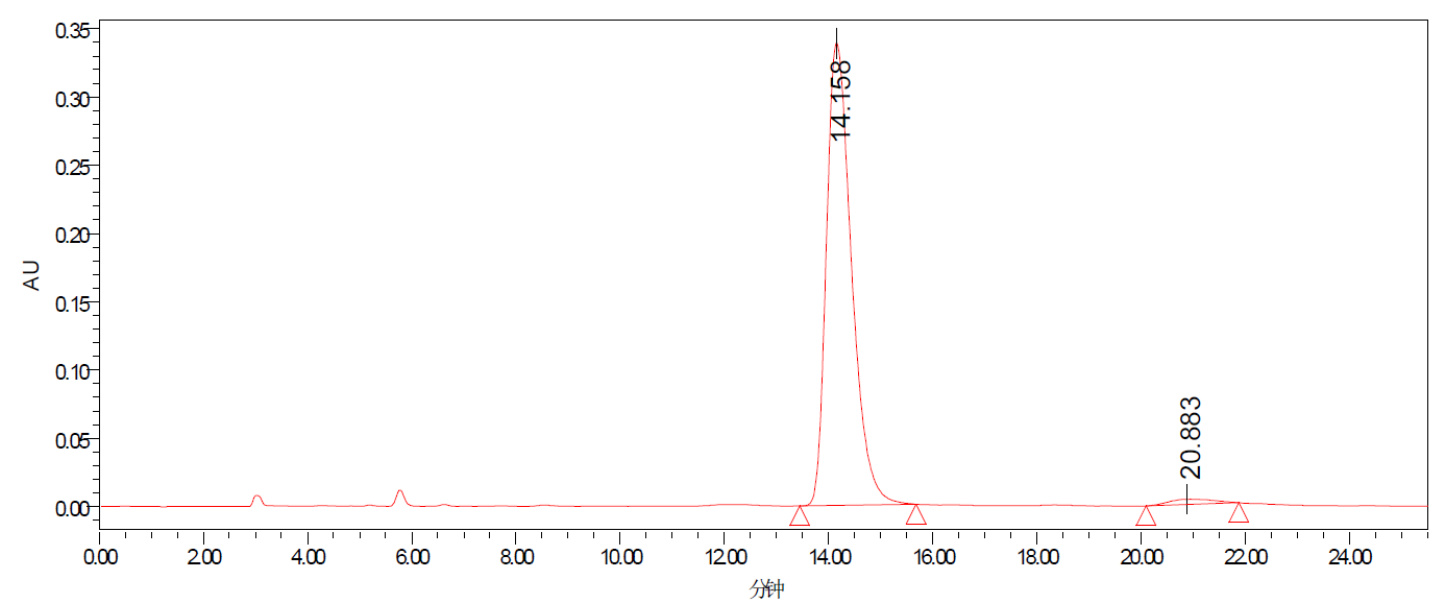

\begin{tabular}{|c|r|l|r|}
\hline & Ret. Time & Area & Area $\%$ \\
\hline 1 & 14.158 & 11345233 & 98.004 \\
\hline 2 & 20.883 & 231020 & 1.996 \\
\hline Sum & & 11576253 & 100.000 \\
\hline
\end{tabular}




\section{Compound 3k}<smiles>Cc1ccc2c(c1)C=CC(=O)[C@]2(N)C(=O)NC(=O)c1ccccc1</smiles>

\section{HPLC Conditions}

Column: Chiralcel AD-H, Daicel Chemical Industries, Ltd.

Eluent: Hexanes / isopropanol (90:10)

Flow rate: $1.0 \mathrm{~mL} / \mathrm{min}$

Detection: UV $254 \mathrm{~nm}$

\section{Racemic}

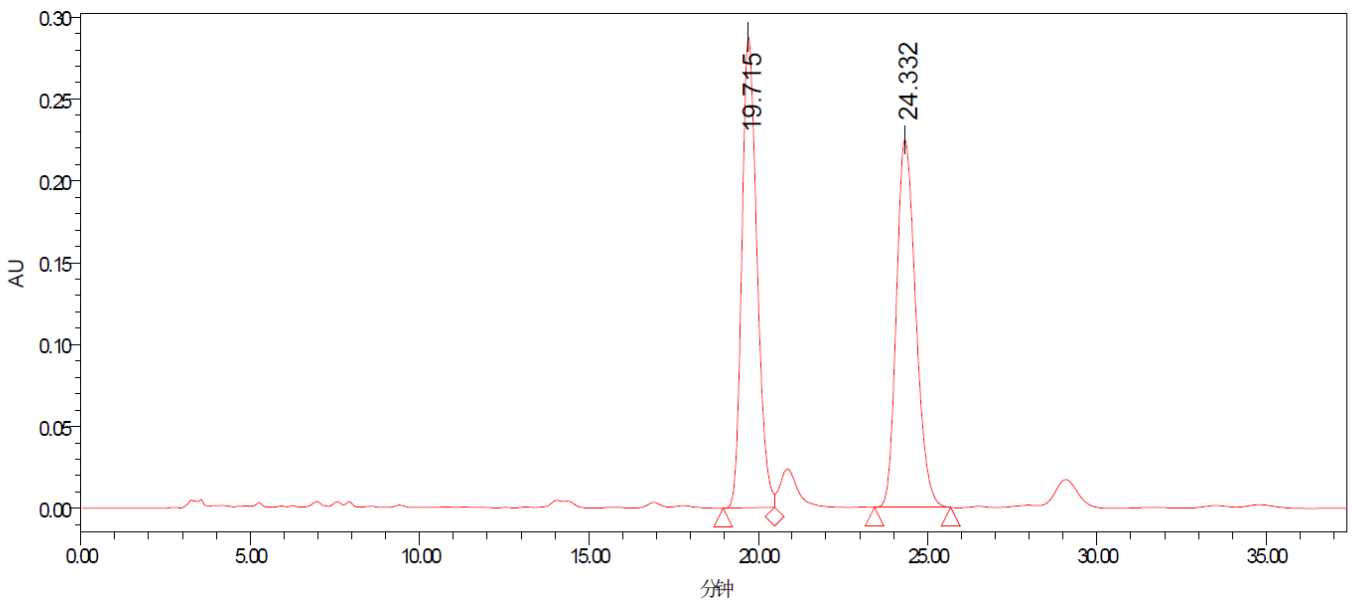

\begin{tabular}{|c|r|c|r|}
\hline & Pet. Time & Area & Area \% \\
\hline 1 & 19.715 & 8905459 & 50.049 \\
\hline 2 & 24.332 & 8888029 & 49.951 \\
\hline Sum & & 17793488 & 100.000 \\
\hline
\end{tabular}

\section{Chiral}

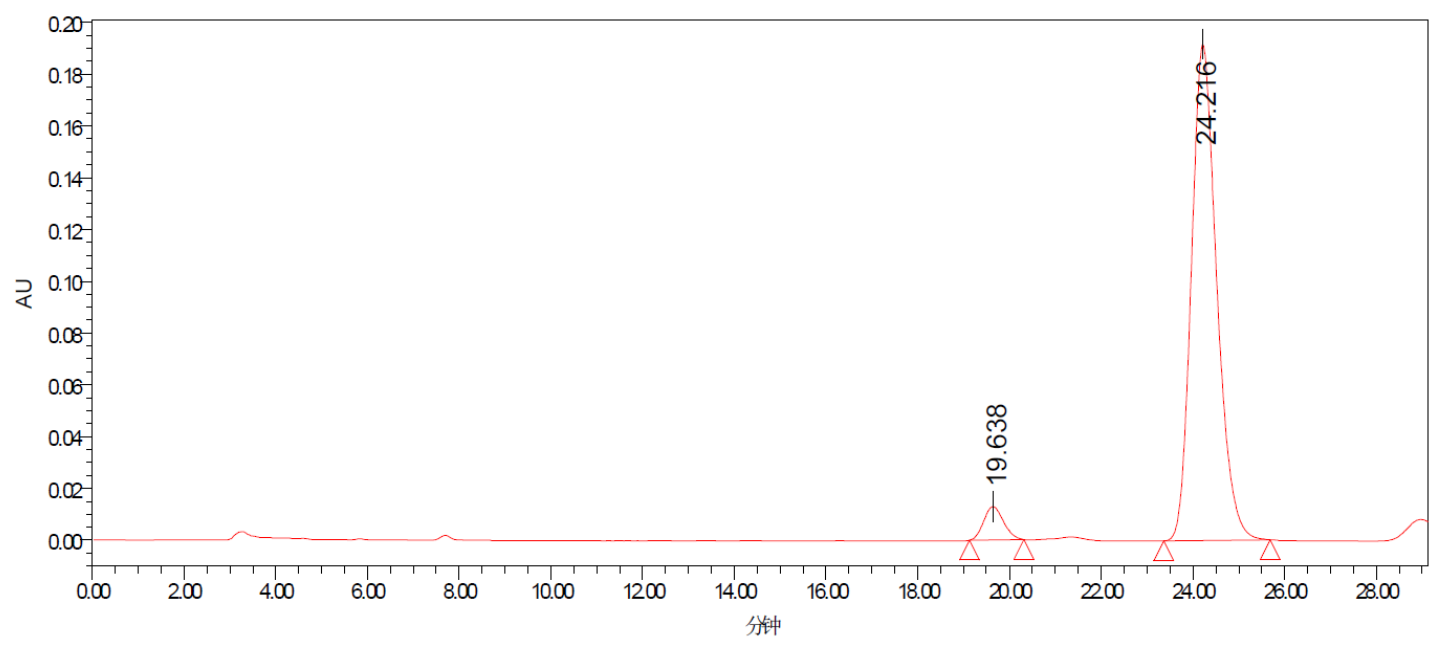

\begin{tabular}{|c|r|c|r|}
\hline & Ret. Time & Area & Area $\%$ \\
\hline 1 & 19.638 & 384298 & 4.983 \\
\hline 2 & 24.216 & 7327869 & 95.017 \\
\hline Sum & & 7712167 & 100.000 \\
\hline
\end{tabular}




\section{Compound 31}<smiles>CCCCNC(=O)C1(C(=O)CCCC)C(=O)C=Cc2ccc(OC)cc21</smiles>

31

\section{HPLC Conditions}

Column: Chiralcel AD-H, Daicel Chemical Industries, Ltd.

Eluent: Hexanes / isopropanol (90:10)

Flow rate: $1.0 \mathrm{~mL} / \mathrm{min}$

Detection: UV $254 \mathrm{~nm}$

\section{Racemic}

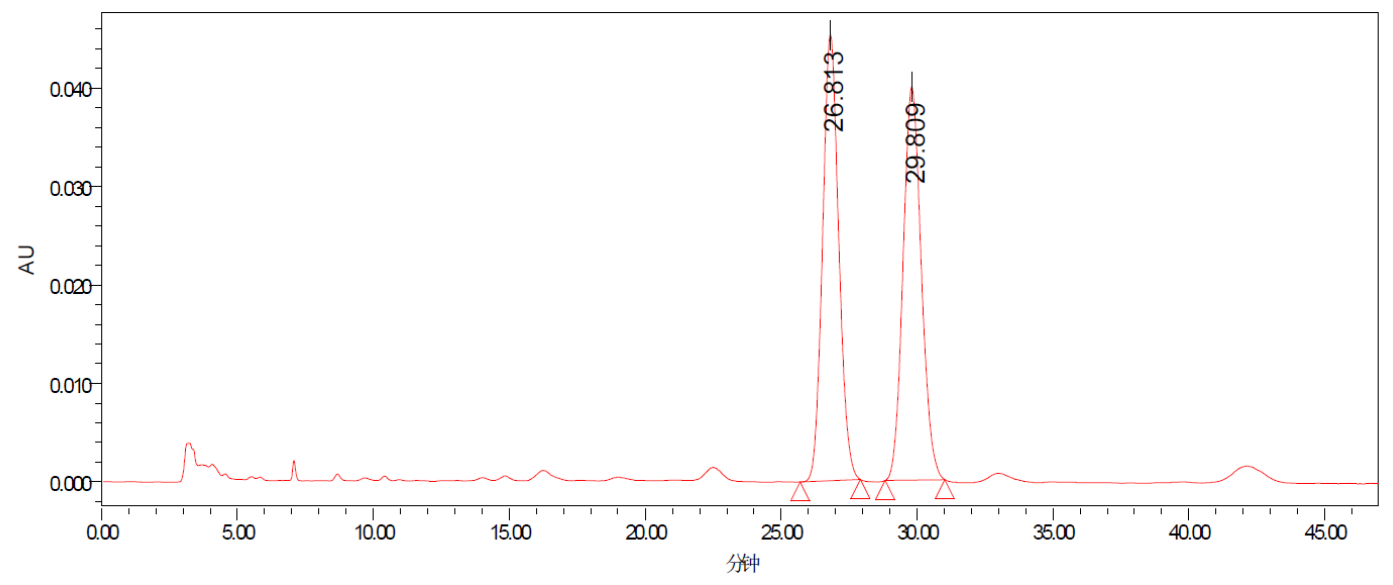

\begin{tabular}{|c|r|c|r|}
\hline & Ret. Time & Area & Area $\%$ \\
\hline 1 & 26.813 & 1870170 & 50.139 \\
\hline 2 & 29.809 & 1859824 & 49.861 \\
\hline Sum & & 3729994 & 100.000 \\
\hline
\end{tabular}

\section{Chiral}

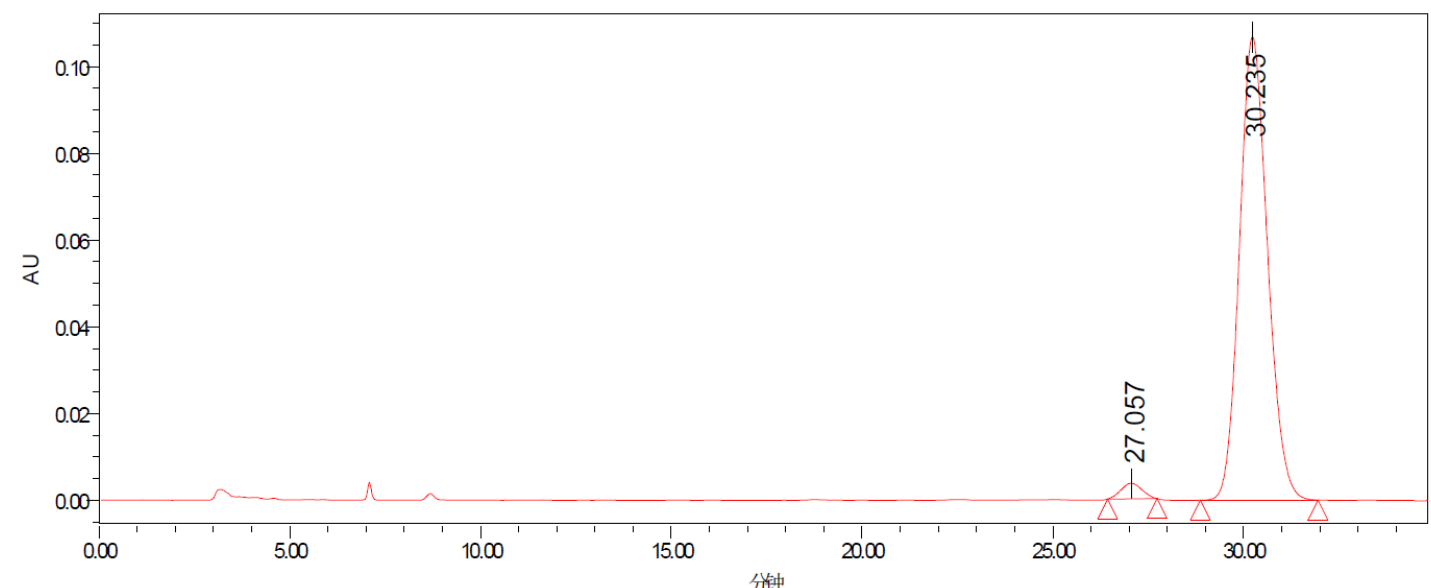

\begin{tabular}{|c|r|r|r|}
\hline & Ret. Tine & Area & Area $\%$ \\
\hline 1 & 27.057 & 139241 & 2488 \\
\hline 2 & 30.235 & 5458229 & 97.512 \\
\hline Sum & & 5597470 & 100.000 \\
\hline
\end{tabular}




\section{Compound 3m}<smiles>CCCCC(=O)C1(N)C(=O)C=Cc2cc(C#Cc3ccccc3)ccc21</smiles>

\section{HPLC Conditions}

Column: Chiralcel AD-H, Daicel Chemical Industries, Ltd.

Eluent: Hexanes / isopropanol (80:20)

Flow rate: $1.0 \mathrm{~mL} / \mathrm{min}$

Detection: UV $254 \mathrm{~nm}$

\section{Racemic}

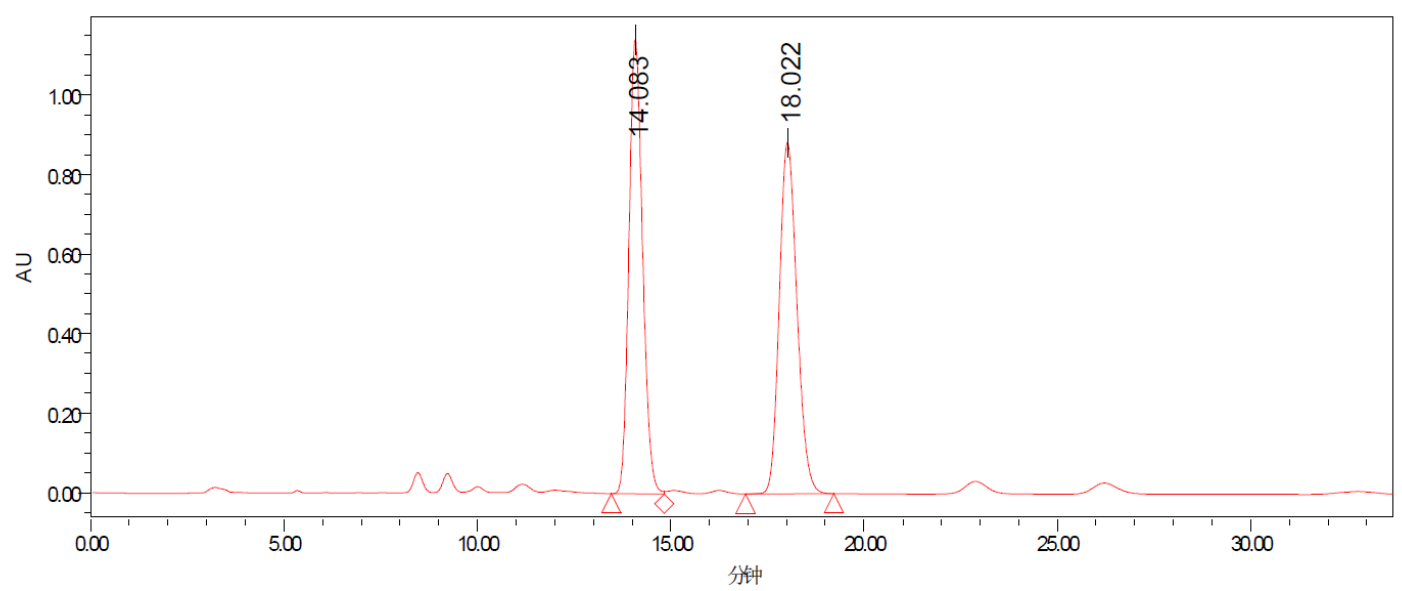

\begin{tabular}{|c|r|c|r|}
\hline & Ret. Time & Area & Area $\%$ \\
\hline 1 & 14.083 & 27975231 & $49.95 \mathrm{C}$ \\
\hline 2 & 18.022 & 28031450 & $50.05 \mathrm{C}$ \\
\hline Sum & & 56006681 & $100.00 \mathrm{C}$ \\
\hline
\end{tabular}

\section{Chiral}

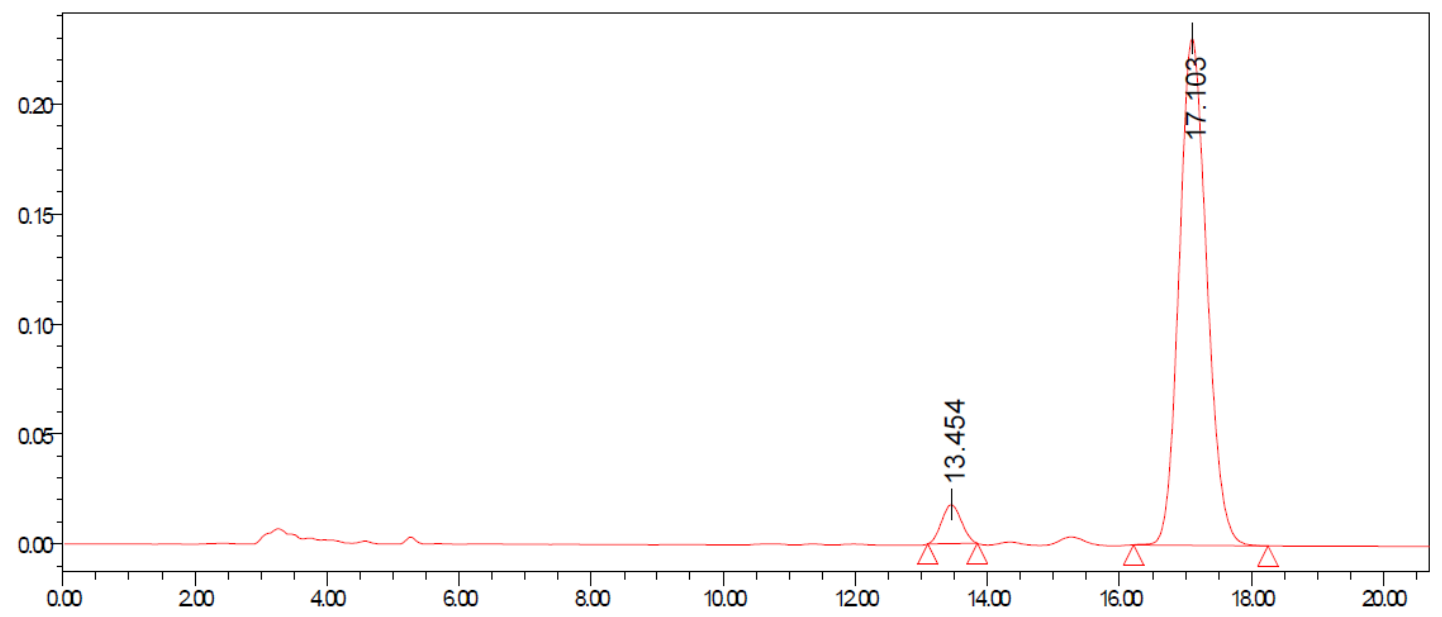

\begin{tabular}{|c|r|r|r|}
\hline & Pet. Time & Area & Area $\%$ \\
\hline 1 & 13.454 & 368737 & 5.212 \\
\hline 2 & 17.103 & 6706279 & 94.788 \\
\hline Sum & & 7075017 & 100.000 \\
\hline
\end{tabular}




\section{Compound 3n}<smiles>CCCCC(=O)C1(C(=O)Cc2ccccc2)C(=O)C=C(Br)c2ccccc21</smiles>

\section{HPLC Conditions}

Column: Chiralcel AD-H, Daicel Chemical Industries, Ltd.

Eluent: Hexanes / isopropanol (94:06)

Flow rate: $1.0 \mathrm{~mL} / \mathrm{min}$

Detection: UV $254 \mathrm{~nm}$

\section{Racemic}

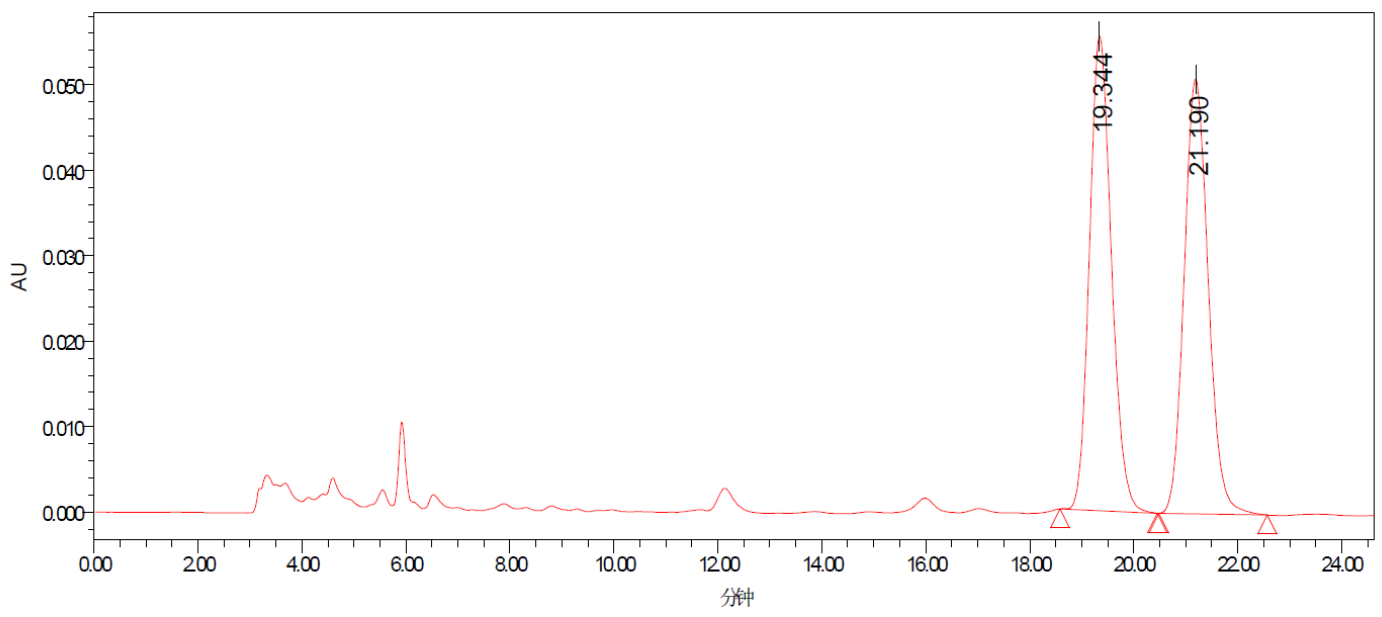

\begin{tabular}{|c|r|c|r|}
\hline & Ret. Time & Area & Area $\%$ \\
\hline 1 & 19.344 & 1627566 & 49.923 \\
\hline 2 & 21.190 & 1632579 & 50.077 \\
\hline Sum & & 3260145 & 100.000 \\
\hline
\end{tabular}

\section{Chiral}

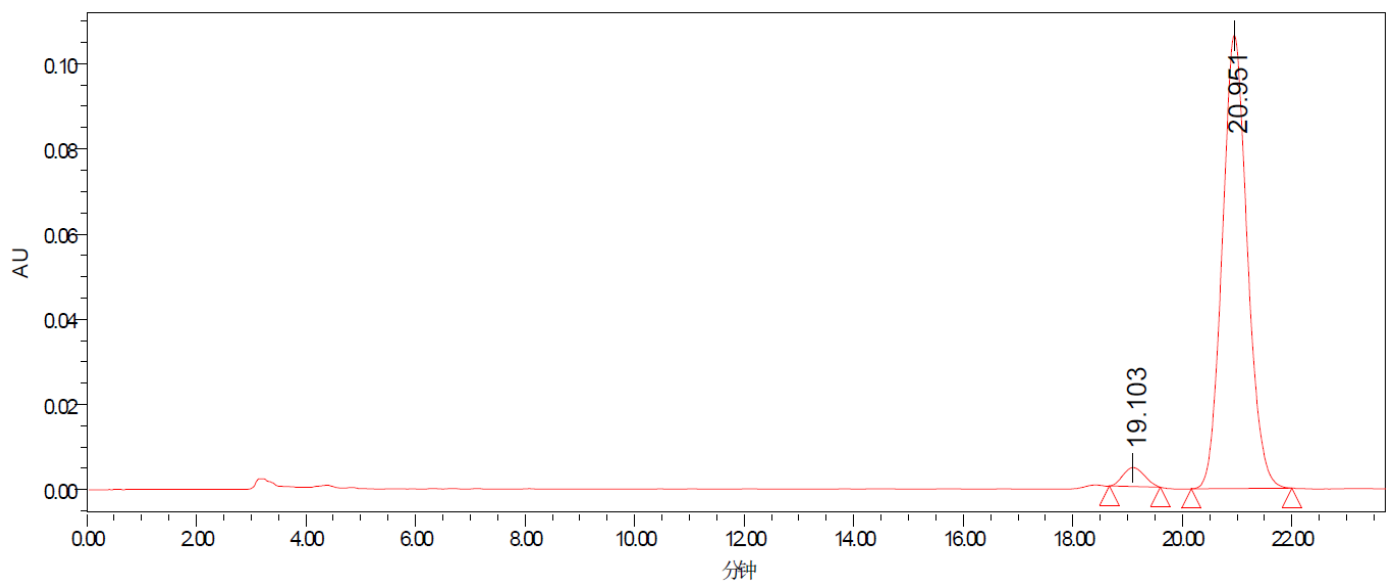

\begin{tabular}{|c|r|r|r|}
\hline & Ret. Time & Area & Area $\%$ \\
\hline 1 & 19.103 & 120826 & 3.451 \\
\hline 2 & 20.951 & 3380659 & 96.549 \\
\hline Sum & & 3501485 & 100.000 \\
\hline
\end{tabular}




\section{Compound 30}<smiles>CCCCC(=O)C1(C(=O)Cc2ccccc2)C(=O)C=C(c2ccccc2)c2ccccc21</smiles>

\section{HPLC Conditions}

Column: Chiralcel AD-H, Daicel Chemical Industries, Ltd.

Eluent: Hexanes / isopropanol (94:06)

Flow rate: $1.0 \mathrm{~mL} / \mathrm{min}$

Detection: UV $254 \mathrm{~nm}$

\section{Racemic}

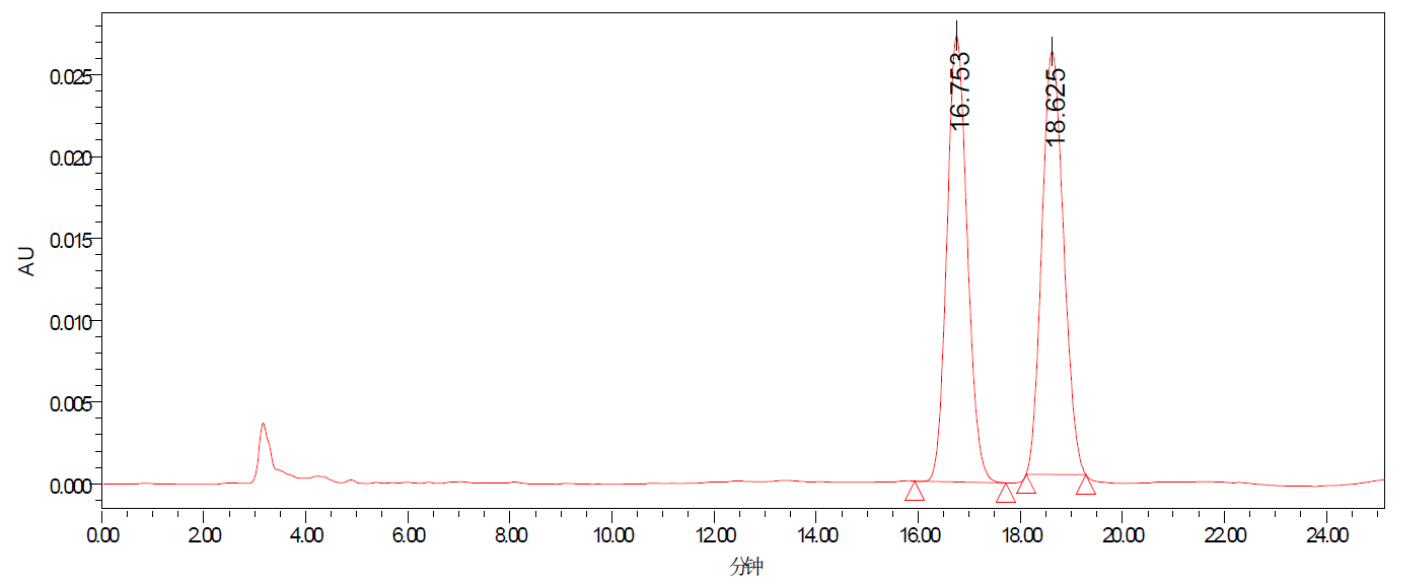

\begin{tabular}{|c|r|r|r|}
\hline & Ret. Time & Area & Area $\%$ \\
\hline 1 & 16.753 & 755178 & 49.728 \\
\hline 2 & 18.625 & 763439 & 50.272 \\
\hline Sum & & 1518617 & 100.000 \\
\hline
\end{tabular}

\section{Chiral}

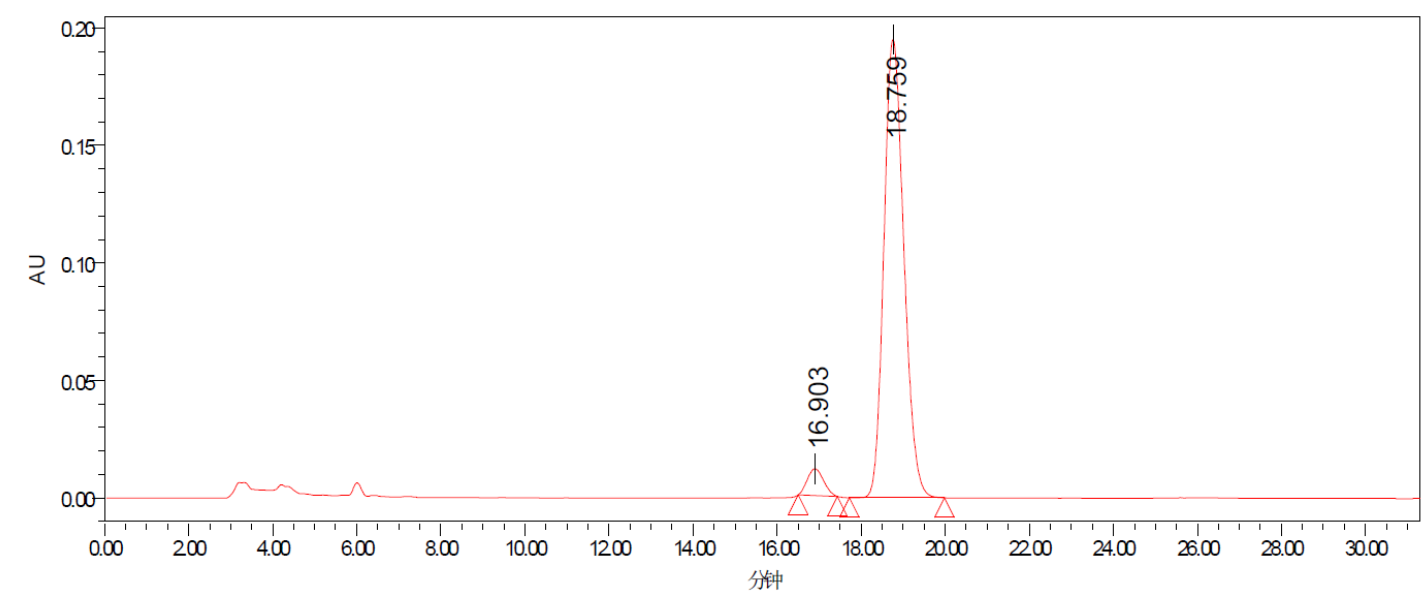

\begin{tabular}{|c|r|c|r|}
\hline & Ret. Time & Area & Area $\%$ \\
\hline 1 & 16.903 & 306264 & 4.55 \\
\hline 2 & 18.759 & 6414319 & 95.443 \\
\hline Sum & & 6720583 & 100.000 \\
\hline
\end{tabular}




\section{Compound 3p}<smiles></smiles>

$3 p$

\section{HPLC Conditions}

Column: Chiralcel OD-H, Daicel Chemical Industries, Ltd.

Eluent: Hexanes / isopropanol (97:03)

Flow rate: $1.0 \mathrm{~mL} / \mathrm{min}$

Detection: UV $254 \mathrm{~nm}$

\section{Racemic}

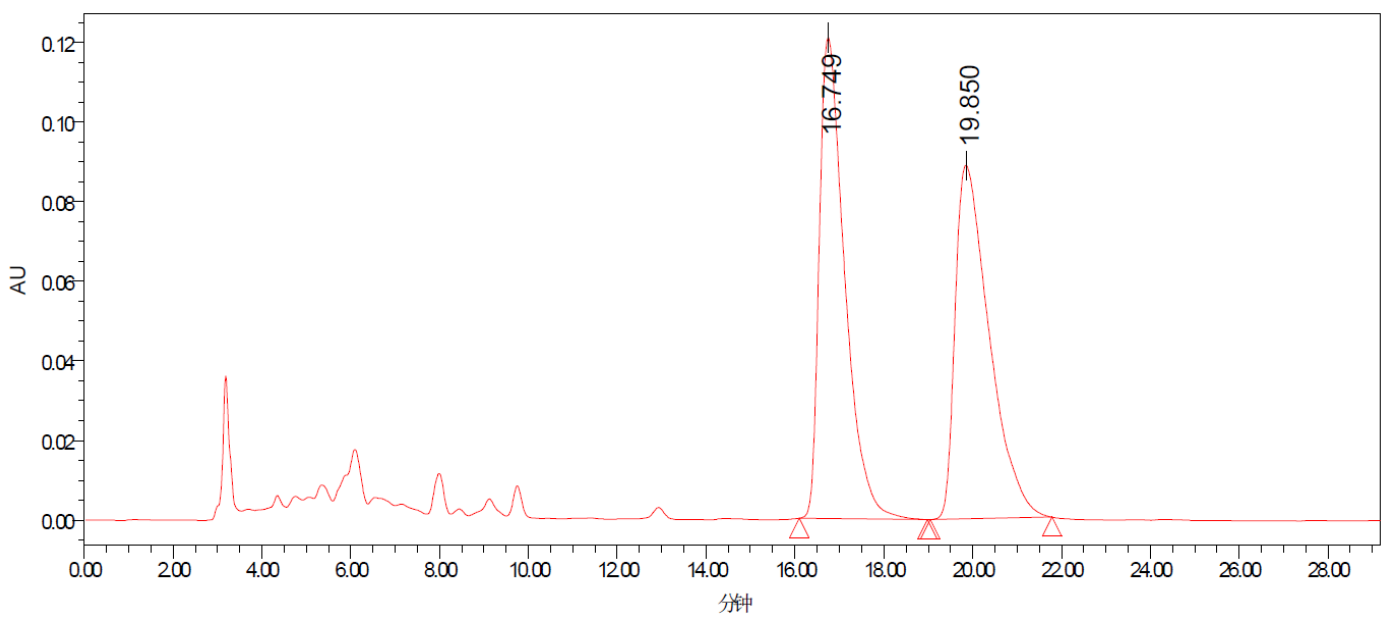

\begin{tabular}{|c|r|c|c|}
\hline & Ret. Time & Area & Area $\%$ \\
\hline 1 & 16.749 & 4796569 & 50.345 \\
\hline 2 & 19.850 & 4730908 & 49.655 \\
\hline Sum & & 9627477 & 100.000 \\
\hline
\end{tabular}

\section{Chiral}

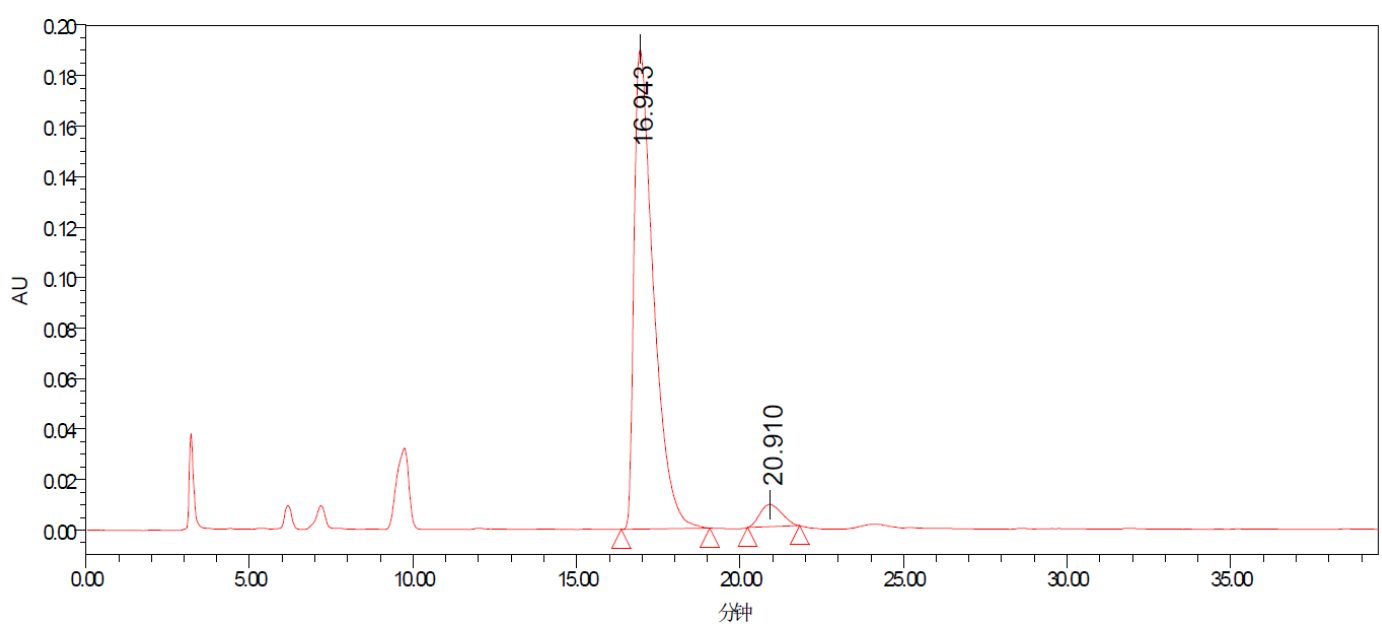

\begin{tabular}{|c|r|c|r|}
\hline & Ret. Time & Area & Area $\%$ \\
\hline 1 & 16.943 & 7841526 & 95.042 \\
\hline 2 & 20.910 & 409041 & 4.958 \\
\hline Sum & & 8250567 & 100.000 \\
\hline
\end{tabular}




\section{Compound 3q}<smiles>N[C@@]1(C(=O)n2ccc3ccccc32)C(=O)C=Cc2ccccc21</smiles>

\section{HPLC Conditions}

Column: Chiralcel AD-H, Daicel Chemical Industries, Ltd.

Eluent: Hexanes / isopropanol (80:20)

Flow rate: $1.0 \mathrm{~mL} / \mathrm{min}$

Detection: UV $254 \mathrm{~nm}$

\section{Racemic}

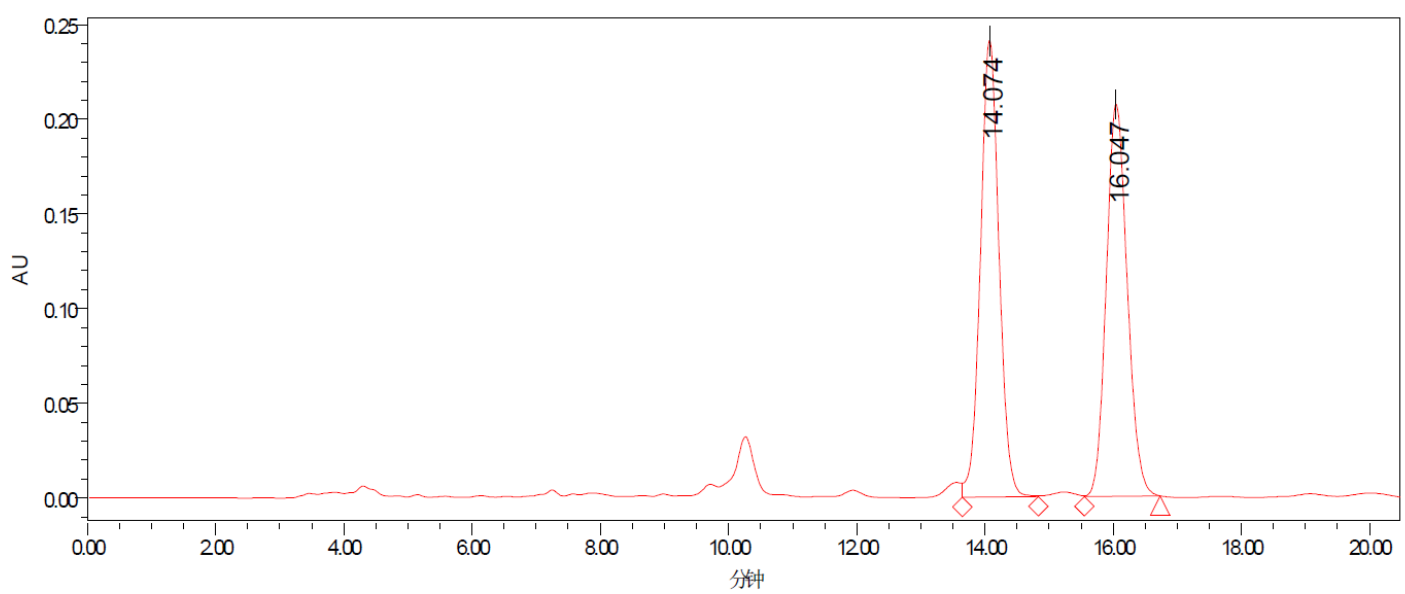

\begin{tabular}{|c|r|c|c|}
\hline & Ret. Tire & Area & Area $\%$ \\
\hline 1 & 14.074 & 4864310 & 50.833 \\
\hline 2 & 16.047 & 4704919 & 49.167 \\
\hline Sum & & 9569229 & 100.000 \\
\hline
\end{tabular}

\section{Chiral}

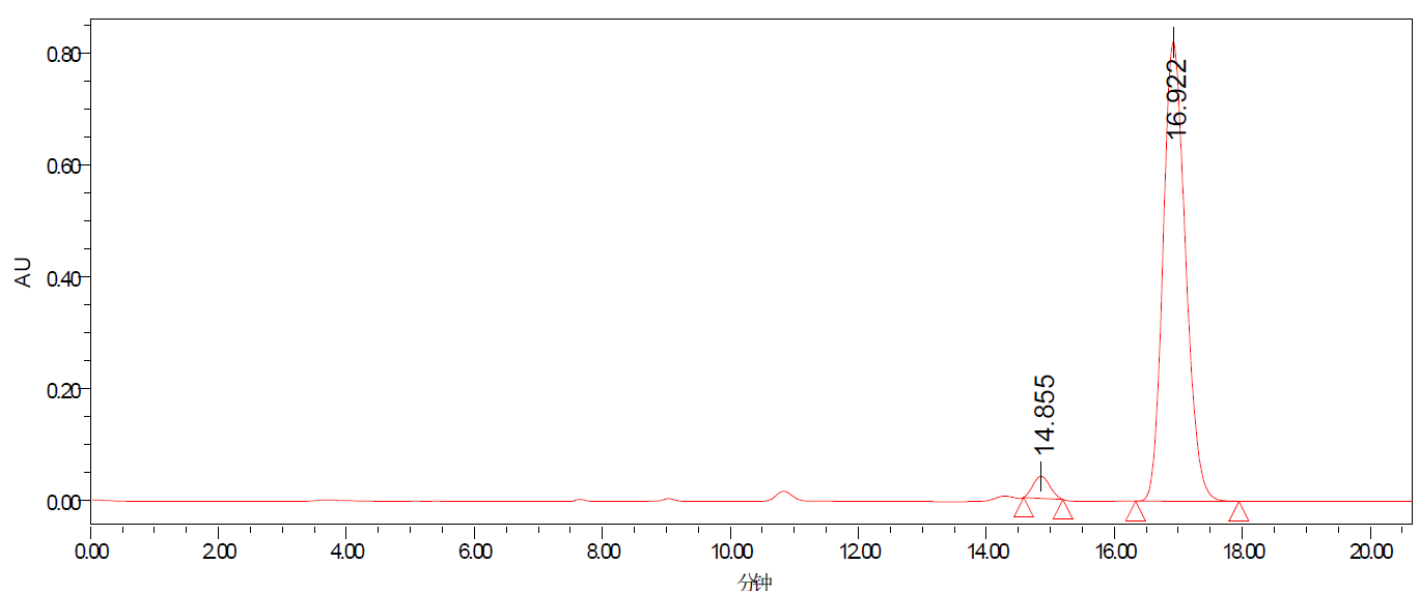

\begin{tabular}{|c|r|c|r|}
\hline & Ret. Time & Area & Area $\%$ \\
\hline 1 & 14.855 & 719765 & 3.467 \\
\hline 2 & 16.922 & 20040535 & 96.533 \\
\hline Sum & & 20760301 & 100.000 \\
\hline
\end{tabular}




\section{Compound 3r}

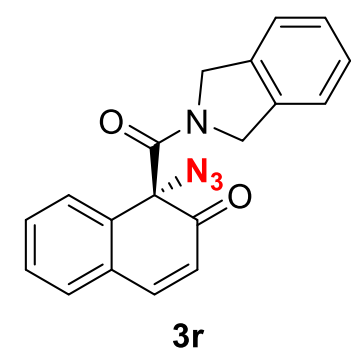

\section{HPLC Conditions}

Column: Chiralcel OD-H, Daicel Chemical Industries, Ltd.

Eluent: Hexanes / isopropanol (94:06)

Flow rate: $1.0 \mathrm{~mL} / \mathrm{min}$

Detection: UV $254 \mathrm{~nm}$

\section{Racemic}

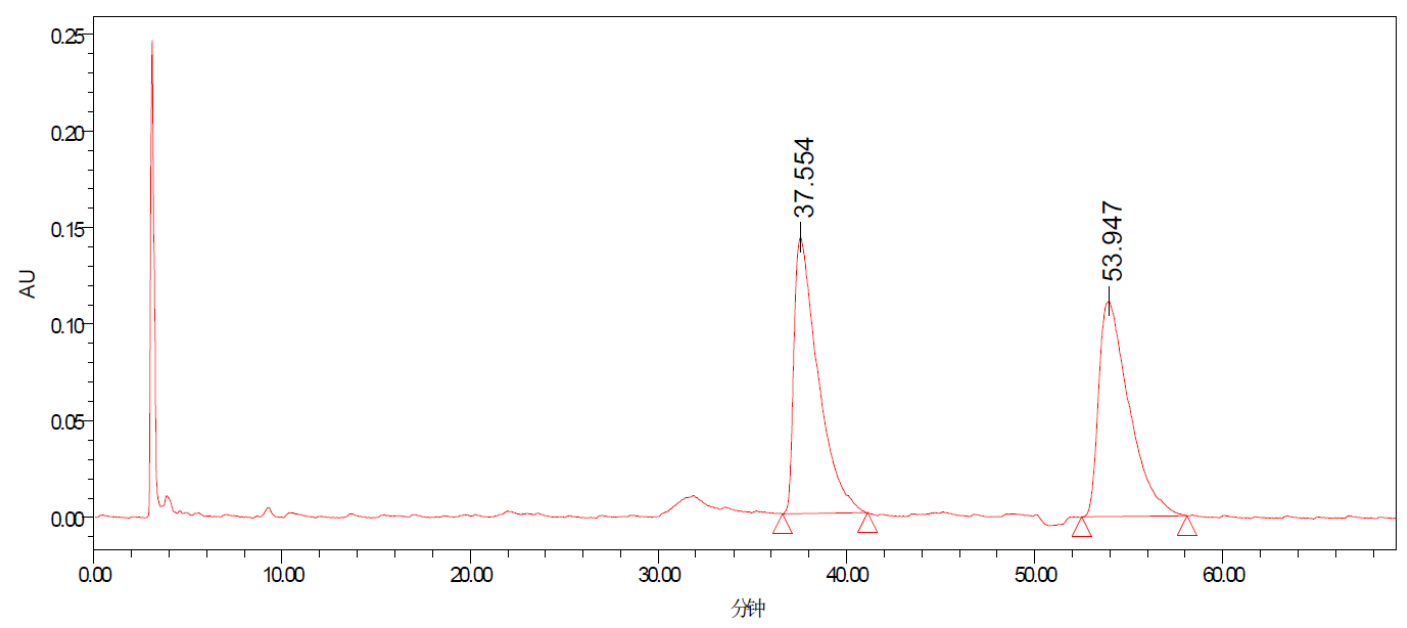

\begin{tabular}{|c|r|c|r|}
\hline & Ret. Tire & Area & Area $\%$ \\
\hline 1 & 37.554 & 12695875 & 50.297 \\
\hline 2 & 53.947 & 12546075 & 49.703 \\
\hline Sum & & 25241950 & 100.000 \\
\hline
\end{tabular}

\section{Chiral}

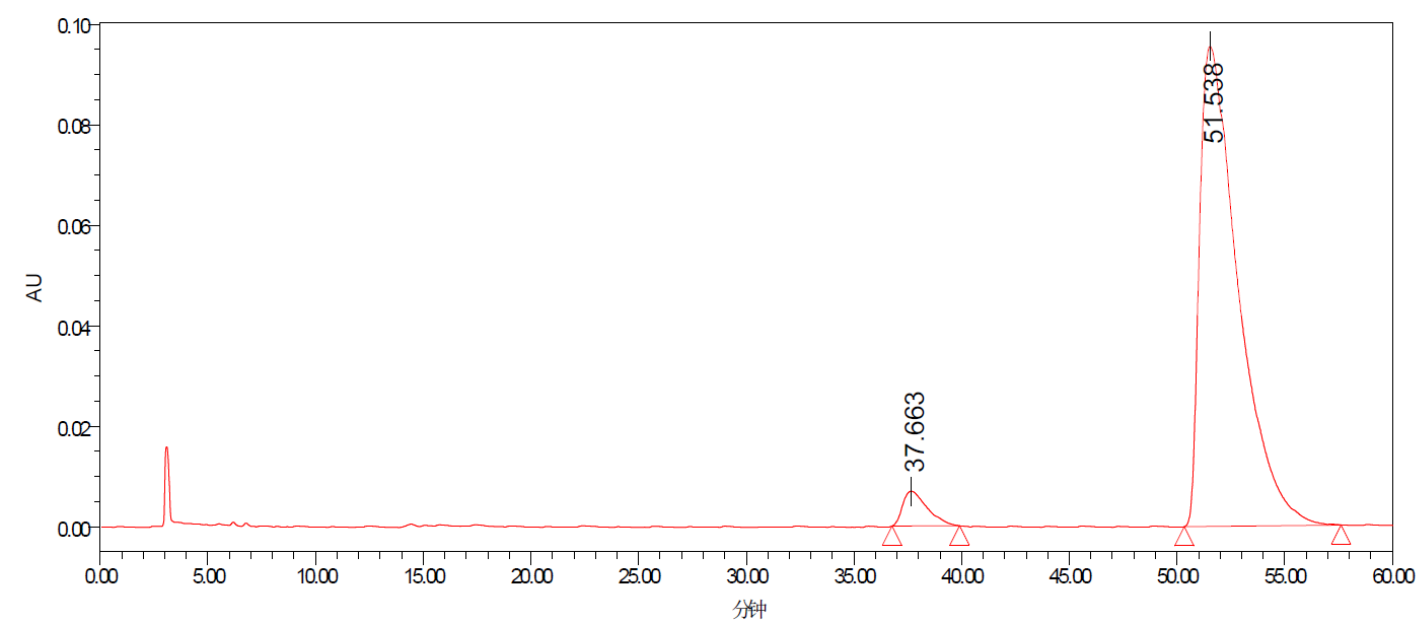

\begin{tabular}{|c|r|r|r|}
\hline & Ret. Time & Area & Area $\%$ \\
\hline 1 & 37.663 & 54835 & 4.408 \\
\hline 2 & 51.538 & $1189228<$ & 95.592 \\
\hline Sum & & 12440638 & 100.000 \\
\hline
\end{tabular}




\section{Compound 3s}<smiles></smiles>

3s

\section{HPLC Conditions}

Column: Chiralcel OD-H, Daicel Chemical Industries, Ltd.

Eluent: Hexanes / isopropanol (80:20)

Flow rate: $1.0 \mathrm{~mL} / \mathrm{min}$

Detection: UV $254 \mathrm{~nm}$

\section{Racemic}

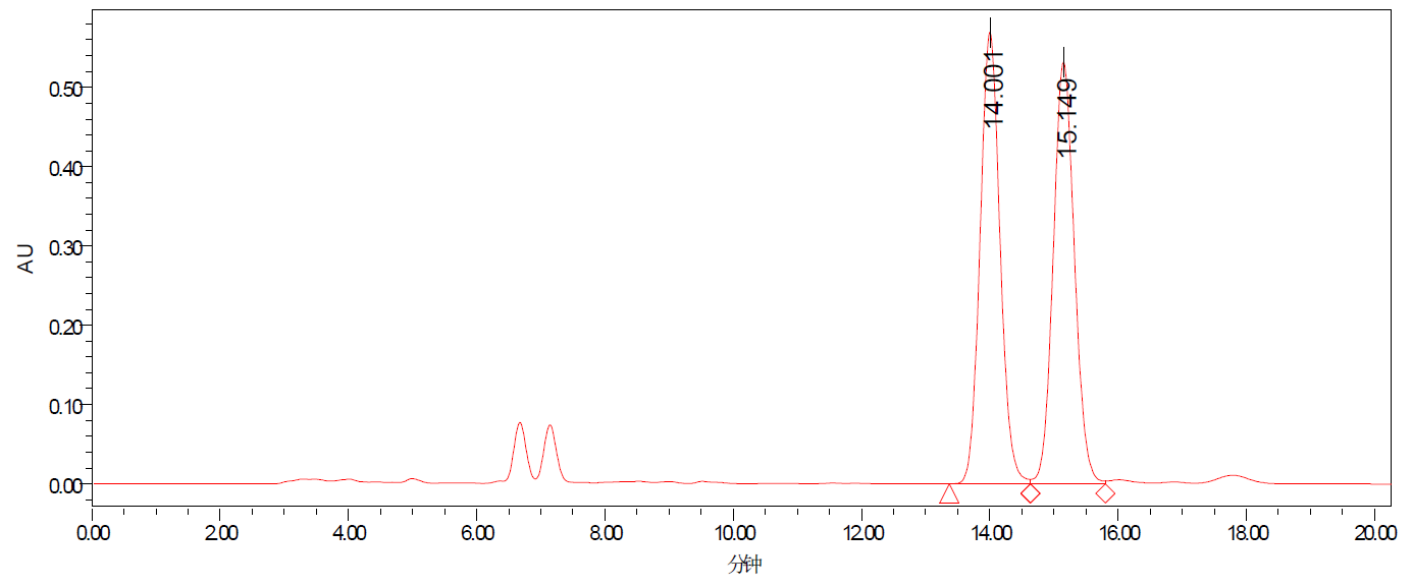

\begin{tabular}{|c|r|c|r|}
\hline & Ret. Tire & Area & Area $\%$ \\
\hline 1 & 14.001 & 12313505 & 50.181 \\
\hline 2 & 15.149 & 12224685 & 49.818 \\
\hline Sum & & 24538190 & 100.000 \\
\hline
\end{tabular}

Chiral

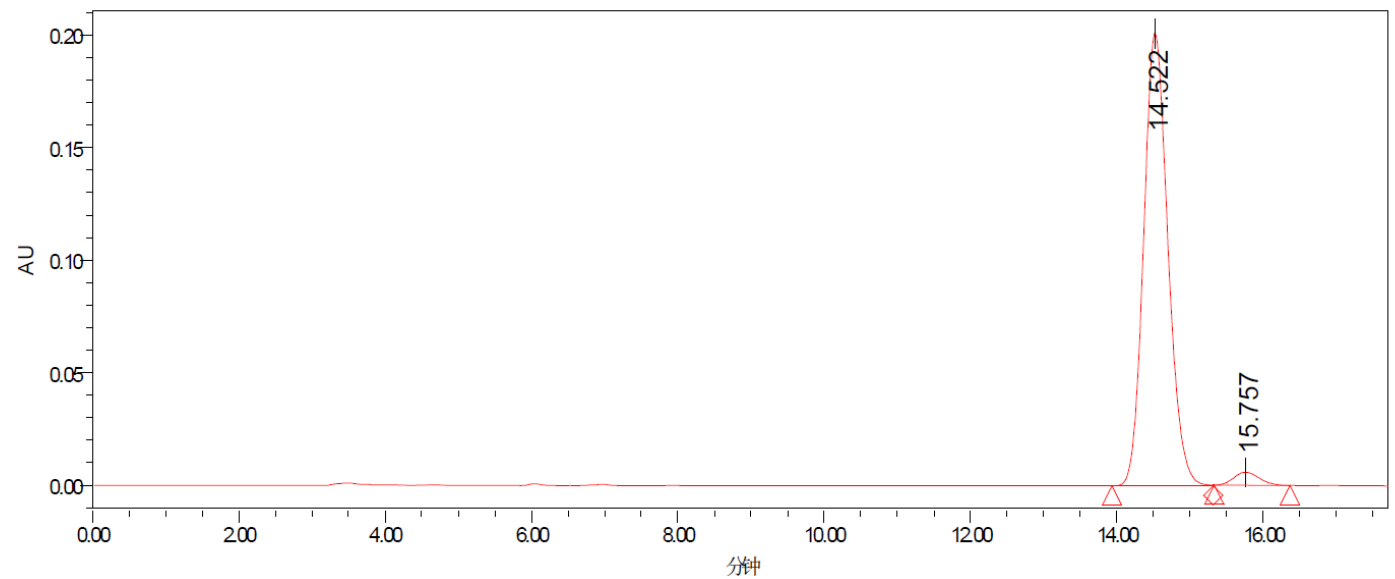

\begin{tabular}{|c|r|c|r|}
\hline & Pet. Time & Area & Area \% \\
\hline 1 & 14.522 & 4616828 & 97.022 \\
\hline 2 & 15.75 & 141692 & 2978 \\
\hline Sum & & 4758520 & 100.000 \\
\hline
\end{tabular}




\section{Compound 3t}<smiles>NC(=O)[C@@]12C(=O)C=Cc3ccccc3[C@]1(N)C(=O)N2CCc1ccccc1</smiles>

\section{HPLC Conditions}

Column: Chiralcel AD-H, Daicel Chemical Industries, Ltd.

Eluent: Hexanes / isopropanol (80:20)

Flow rate: $1.0 \mathrm{~mL} / \mathrm{min}$

Detection: UV $254 \mathrm{~nm}$

3t

\section{Racemic}

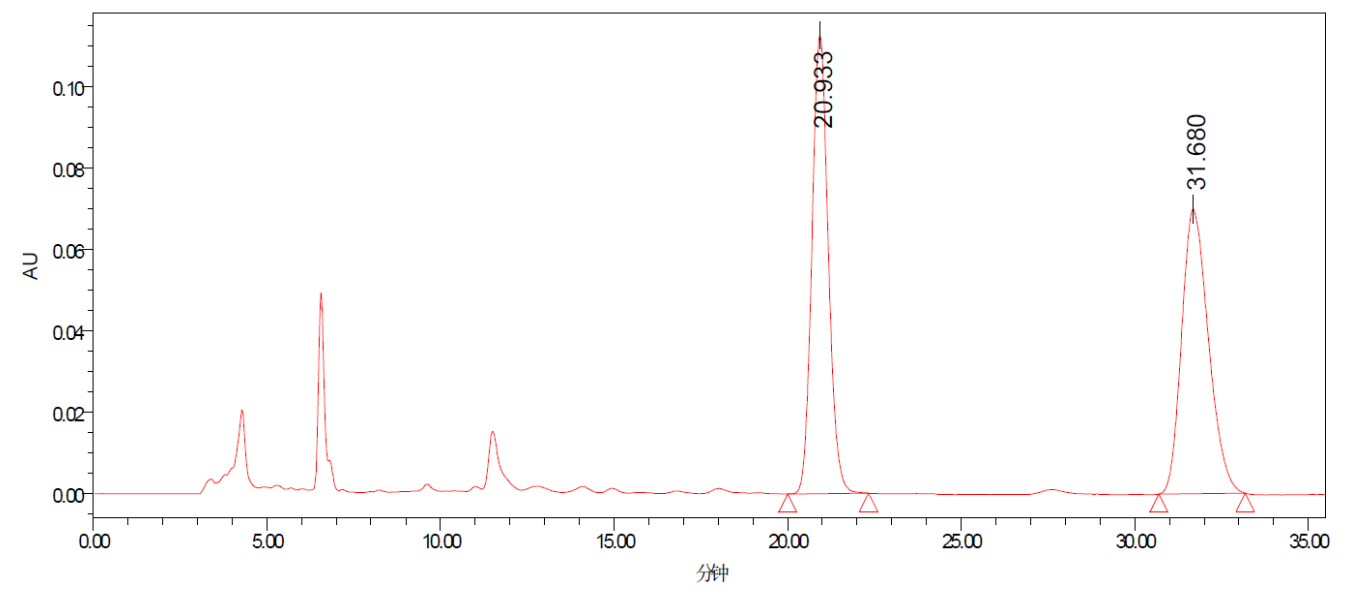

\begin{tabular}{|c|r|c|r|}
\hline & Ret. Time & Area & Area $\%$ \\
\hline 1 & 20.933 & 3689017 & 49.556 \\
\hline 2 & 31.680 & 3755121 & 50.444 \\
\hline Sum & & 7444138 & 100.000 \\
\hline
\end{tabular}

\section{Chiral}

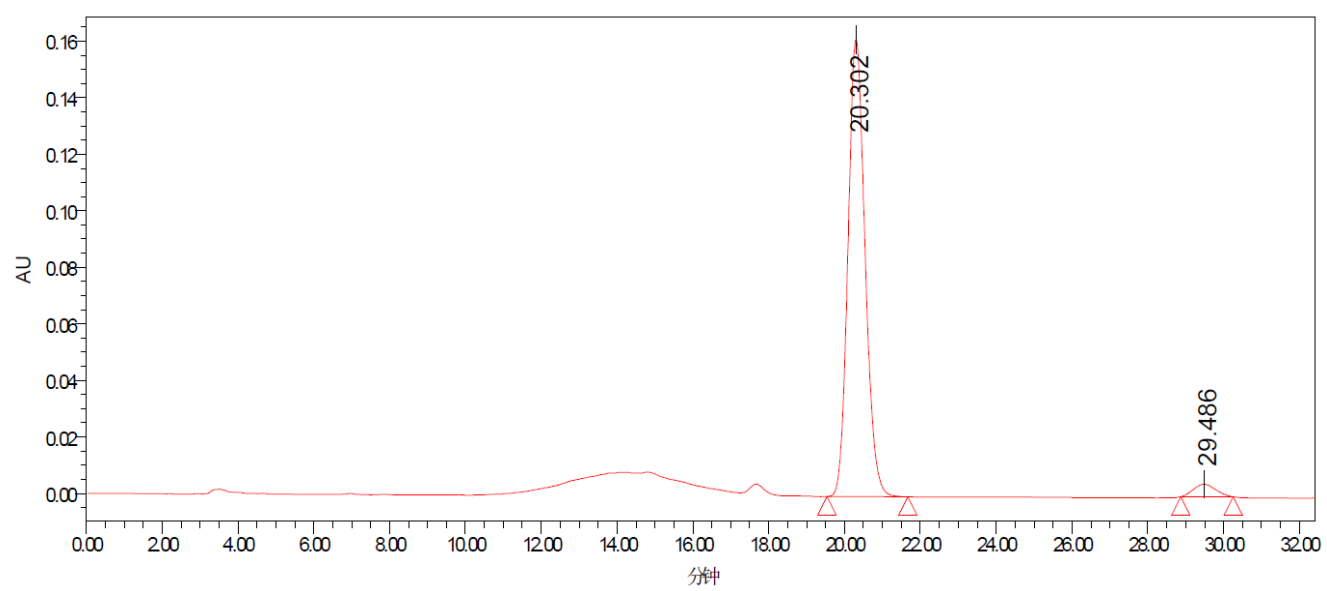

\begin{tabular}{|c|r|r|r|}
\hline & Ret. Time & Area & Area $\%$ \\
\hline 1 & 20.302 & 5058830 & 96.543 \\
\hline 2 & 29.486 & 18116 & 3.45 \\
\hline Sum & & 5239998 & 100.000 \\
\hline
\end{tabular}




\section{Compound 3u}<smiles></smiles>

3u

\section{HPLC Conditions}

Column: Chiralcel OD-H, Daicel Chemical Industries, Ltd.

Eluent: Hexanes / isopropanol (80:20)

Flow rate: $1.0 \mathrm{~mL} / \mathrm{min}$

Detection: UV $254 \mathrm{~nm}$

\section{Racemic}

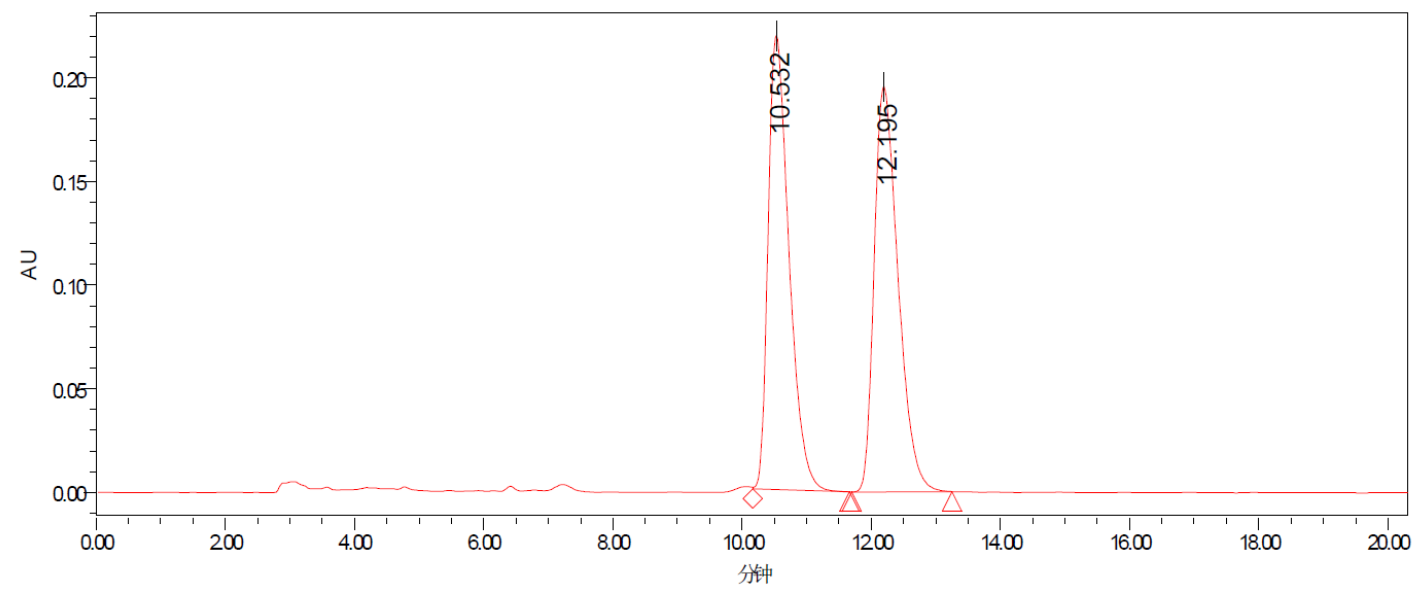

\begin{tabular}{|c|r|c|c|}
\hline & Ret. Time & Area & Area $\%$ \\
\hline 1 & 10.532 & 4921550 & 49.468 \\
\hline 2 & 12195 & 5027389 & 50.532 \\
\hline Sum & & 9948939 & 100.000 \\
\hline
\end{tabular}

\section{Chiral}

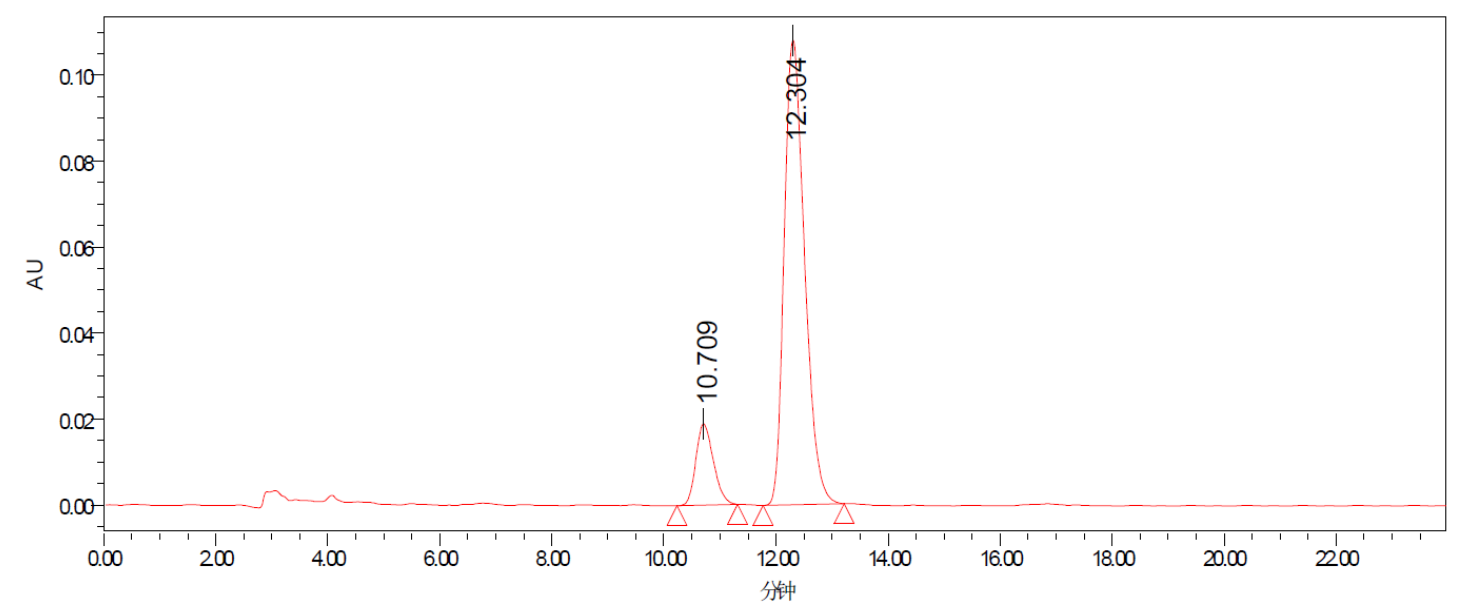

\begin{tabular}{|c|r|r|r|}
\hline & Ret. Time & Area & Area $\%$ \\
\hline 1 & 10.709 & 412145 & 13.139 \\
\hline 2 & 12304 & 2724570 & 86.861 \\
\hline Sum & & 3136715 & 100.000 \\
\hline
\end{tabular}




\section{Compound 3v}<smiles>NC(=O)[C@]1(C(=O)N2CCCCC2)C(=O)C=Cc2ccccc21</smiles>

\section{HPLC Conditions}

Column: Chiralcel AS-H, Daicel Chemical Industries, Ltd.

Eluent: Hexanes / isopropanol (90:10)

Flow rate: $1.0 \mathrm{~mL} / \mathrm{min}$

Detection: UV $254 \mathrm{~nm}$

\section{Racemic}

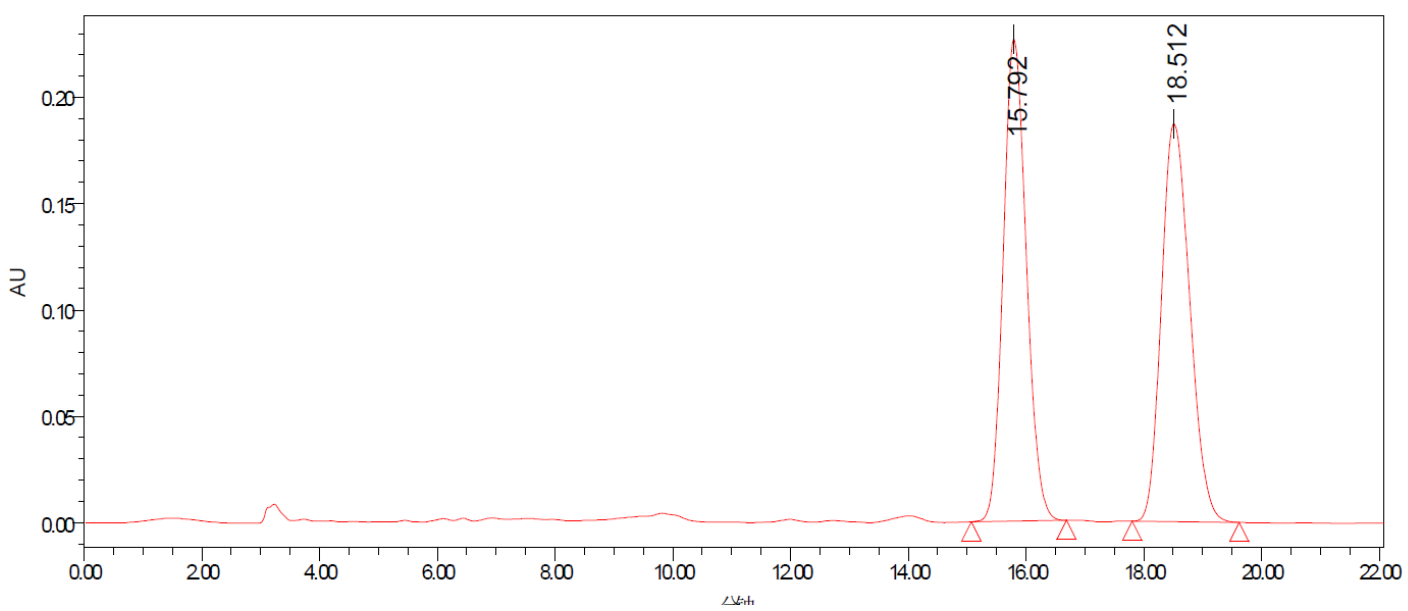

\begin{tabular}{|c|r|c|r|}
\hline & Ret. Time & Area & Area $\%$ \\
\hline 1 & 15.792 & 6196647 & 49.765 \\
\hline 2 & 18.512 & 6255080 & 50.235 \\
\hline Sum & & 12451727 & 100.000 \\
\hline
\end{tabular}

\section{Chiral}

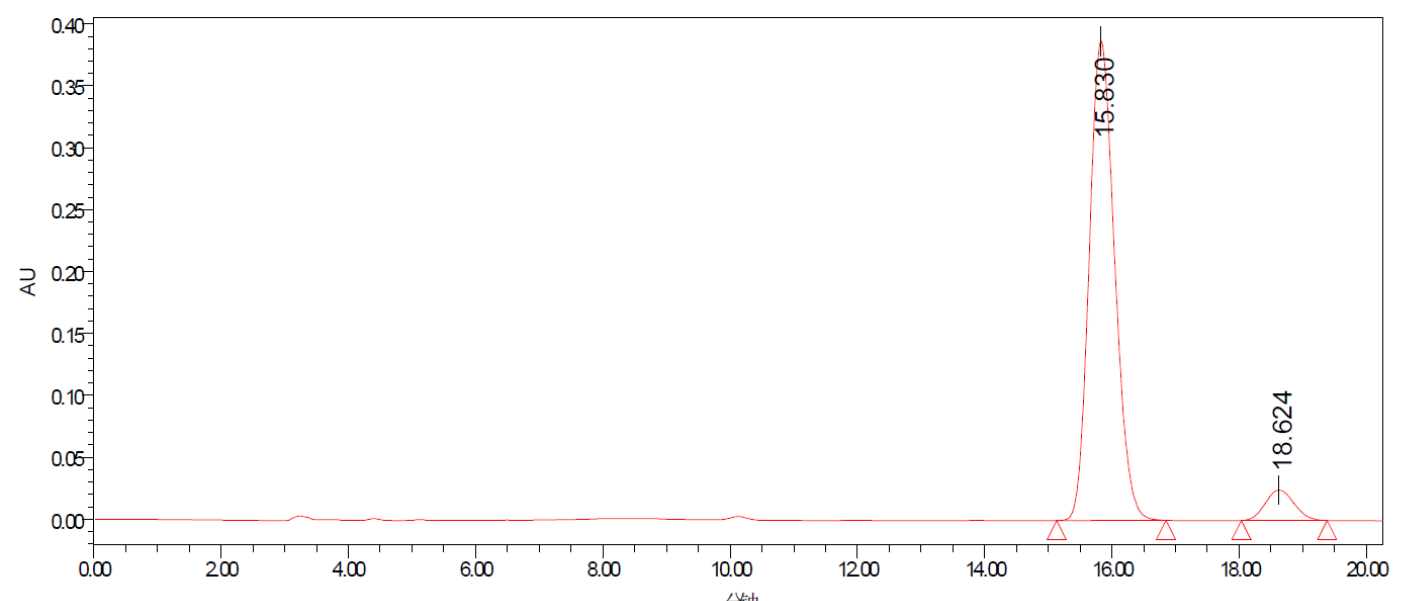

\begin{tabular}{|c|r|r|r|}
\hline & Ret. Time & Area & Area $\%$ \\
\hline 1 & 15.830 & 10715854 & 93.250 \\
\hline 2 & 18.624 & 775620 & 6.750 \\
\hline Sum & & 11491474 & 100.000 \\
\hline
\end{tabular}




\section{Compound 3w}<smiles>N[C@]1(C(=O)N2CCOCC2)C(=O)C=Cc2ccccc21</smiles>

\section{HPLC Conditions}

Column: Chiralcel AD-H, Daicel Chemical Industries, Ltd.

Eluent: Hexanes / isopropanol (80:20)

Flow rate: $1.0 \mathrm{~mL} / \mathrm{min}$

Detection: UV $254 \mathrm{~nm}$

\section{Racemic}

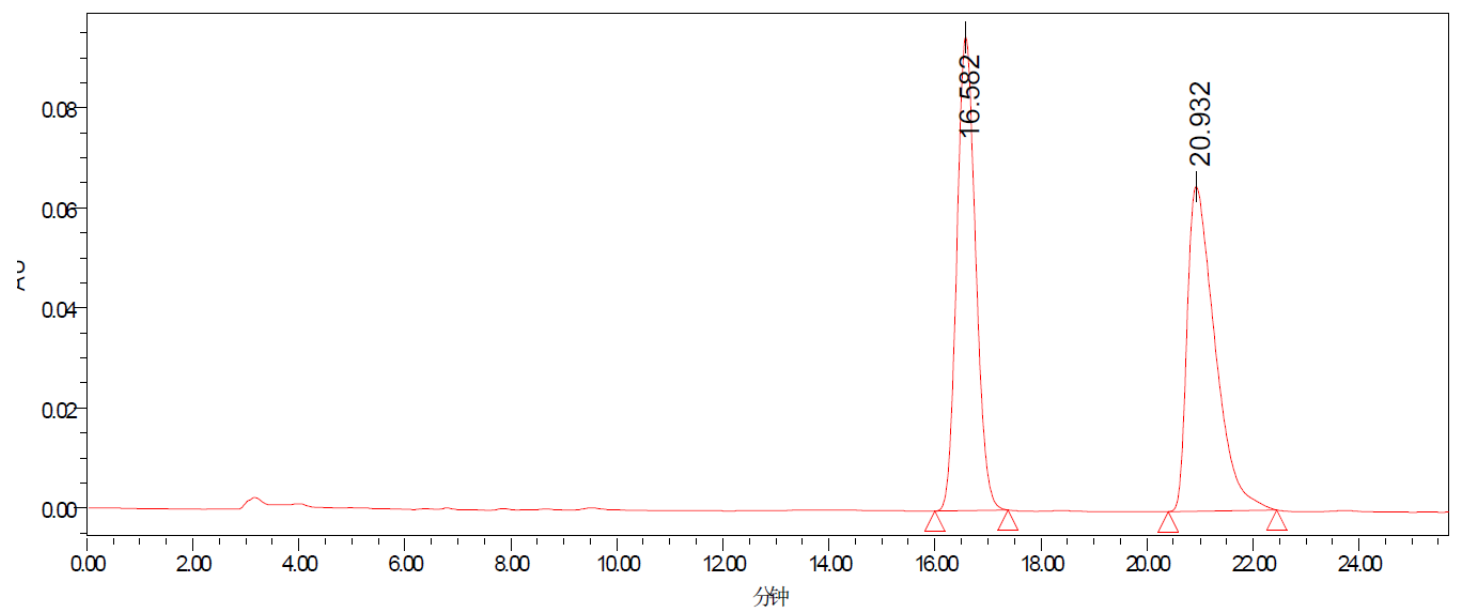

\begin{tabular}{|c|r|c|c|}
\hline & Ret. Tire & Area & Area $\%$ \\
\hline 1 & 16.582 & 2333254 & 49.672 \\
\hline 2 & 20.932 & 2364028 & 50.328 \\
\hline Sum & & 4697282 & 100.000 \\
\hline
\end{tabular}

\section{Chiral}

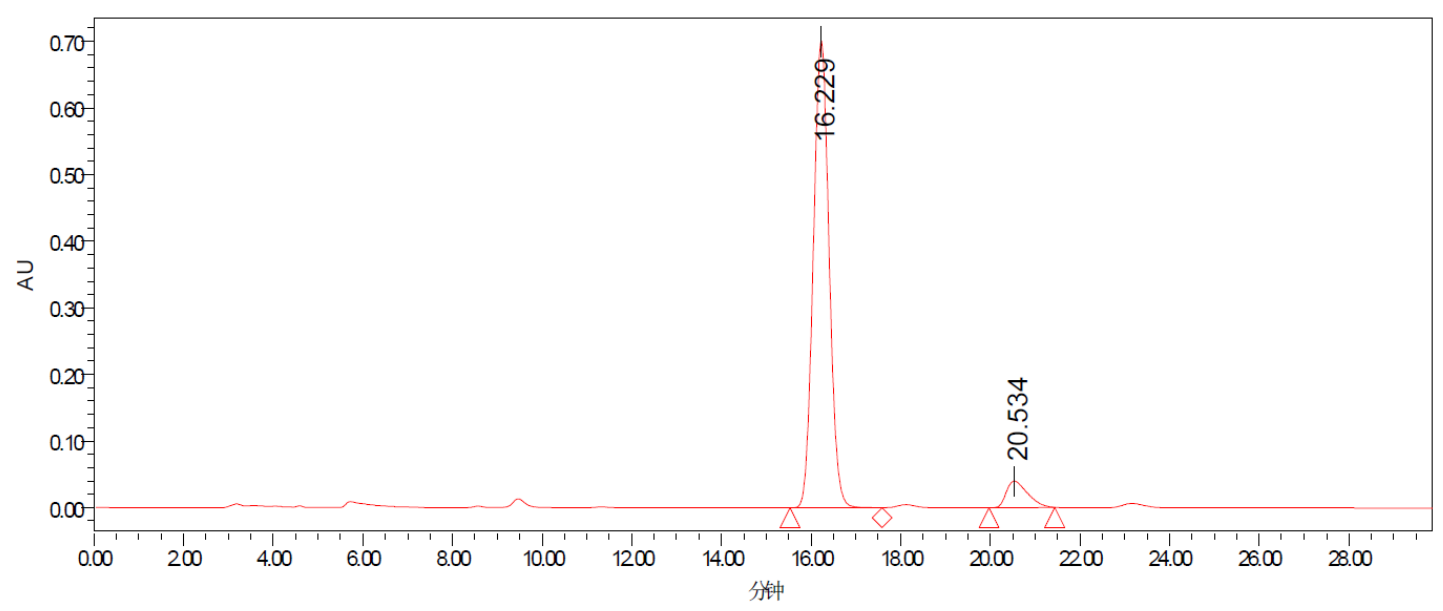

\begin{tabular}{|c|r|c|r|}
\hline & Ret. Time & Area & Area $\%$ \\
\hline 1 & 16.229 & 17111917 & 92869 \\
\hline 2 & 20.534 & 1314034 & 7.131 \\
\hline Sum & & 18425950 & 100.000 \\
\hline
\end{tabular}




\section{Compound 3x}<smiles>CC(C)(C)CN(Cc1ccccc1)C(=O)[C@]1(N)C(=O)C=Cc2ccccc21</smiles>

\section{HPLC Conditions}

Column: Chiralcel OD-H, Daicel Chemical Industries, Ltd.

Eluent: Hexanes / isopropanol (95:05)

Flow rate: $1.0 \mathrm{~mL} / \mathrm{min}$

Detection: UV $254 \mathrm{~nm}$

\section{Racemic}

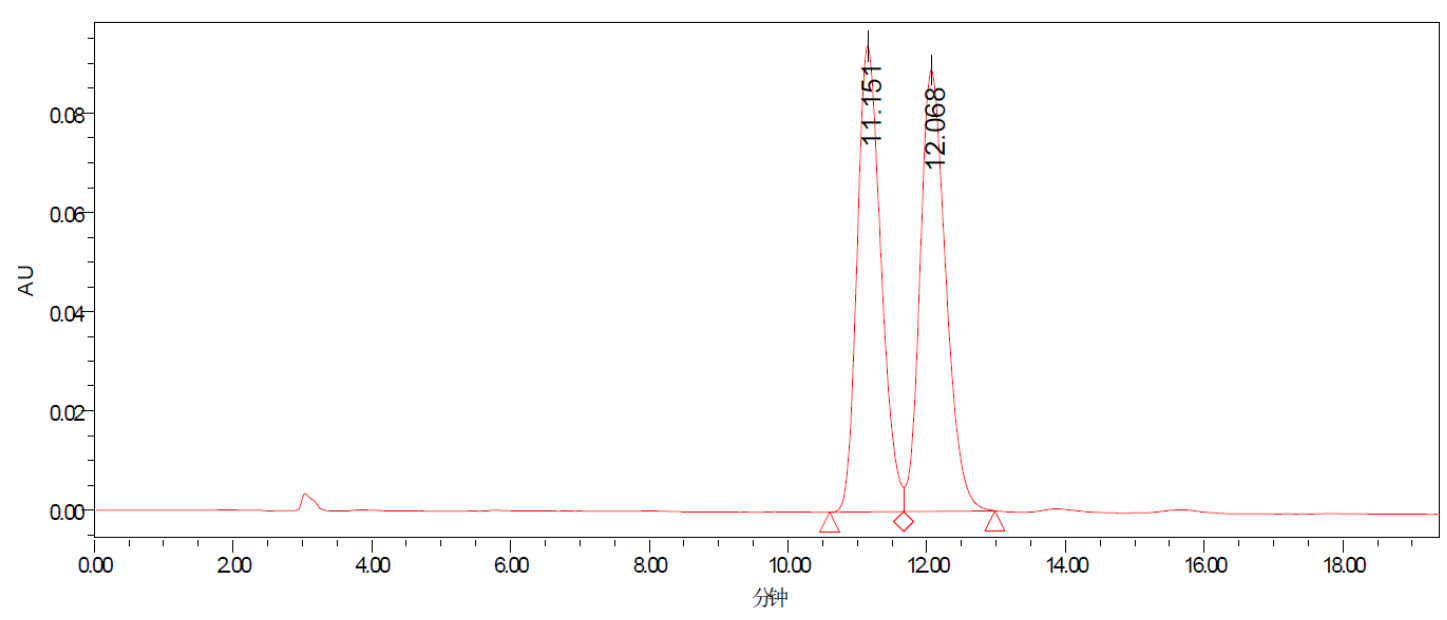

\begin{tabular}{|c|r|c|r|}
\hline & Ret. Time & Area & Area $\%$ \\
\hline 1 & 11.151 & 2246280 & 49.475 \\
\hline 2 & 12068 & 2293997 & 50.525 \\
\hline Sum & & 4540277 & 100.000 \\
\hline
\end{tabular}

Chiral

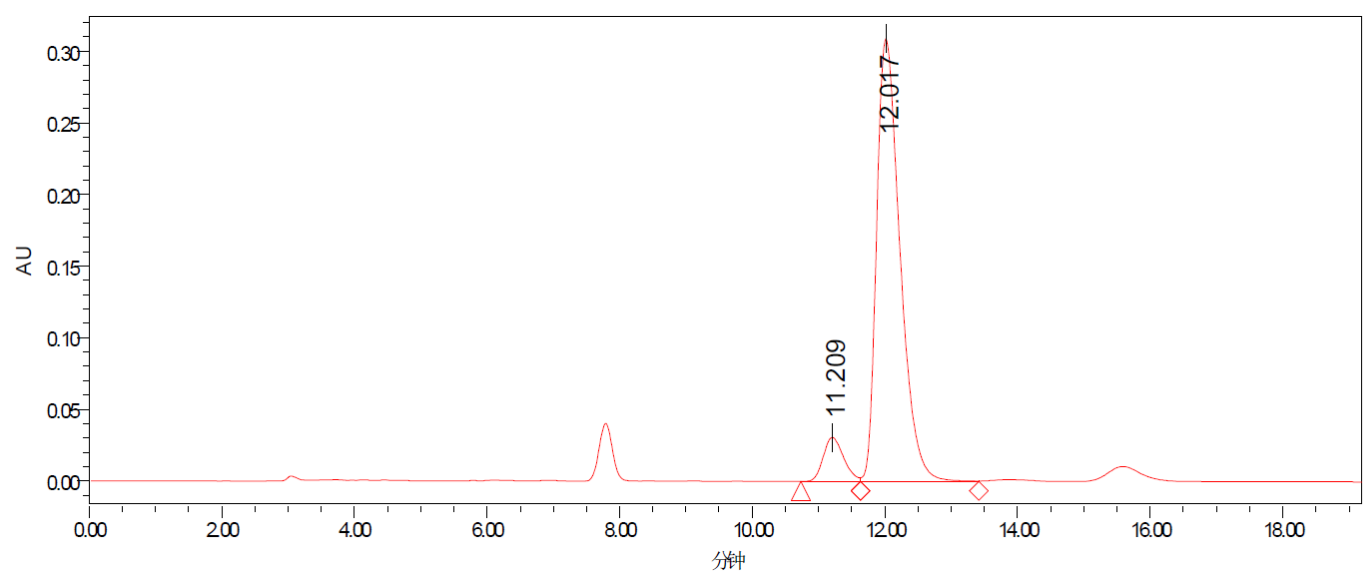

\begin{tabular}{|c|r|c|r|}
\hline & Ret. Time & Area & Area $\%$ \\
\hline 1 & 11.20 & 659124 & 8.006 \\
\hline 2 & 12017 & 7573537 & 91.994 \\
\hline Sum & & 8232661 & 100.000 \\
\hline
\end{tabular}




\section{Compound 5a}<smiles>COC(=O)[C@]1(C#N)C(=O)C=Cc2ccccc21</smiles>

$5 a$

\section{HPLC Conditions}

Column: Chiralcel OD-H, Daicel Chemical Industries, Ltd.

Eluent: Hexanes / isopropanol (96:04)

Flow rate: $1.0 \mathrm{~mL} / \mathrm{min}$

Detection: UV $254 \mathrm{~nm}$

\section{Racemic}

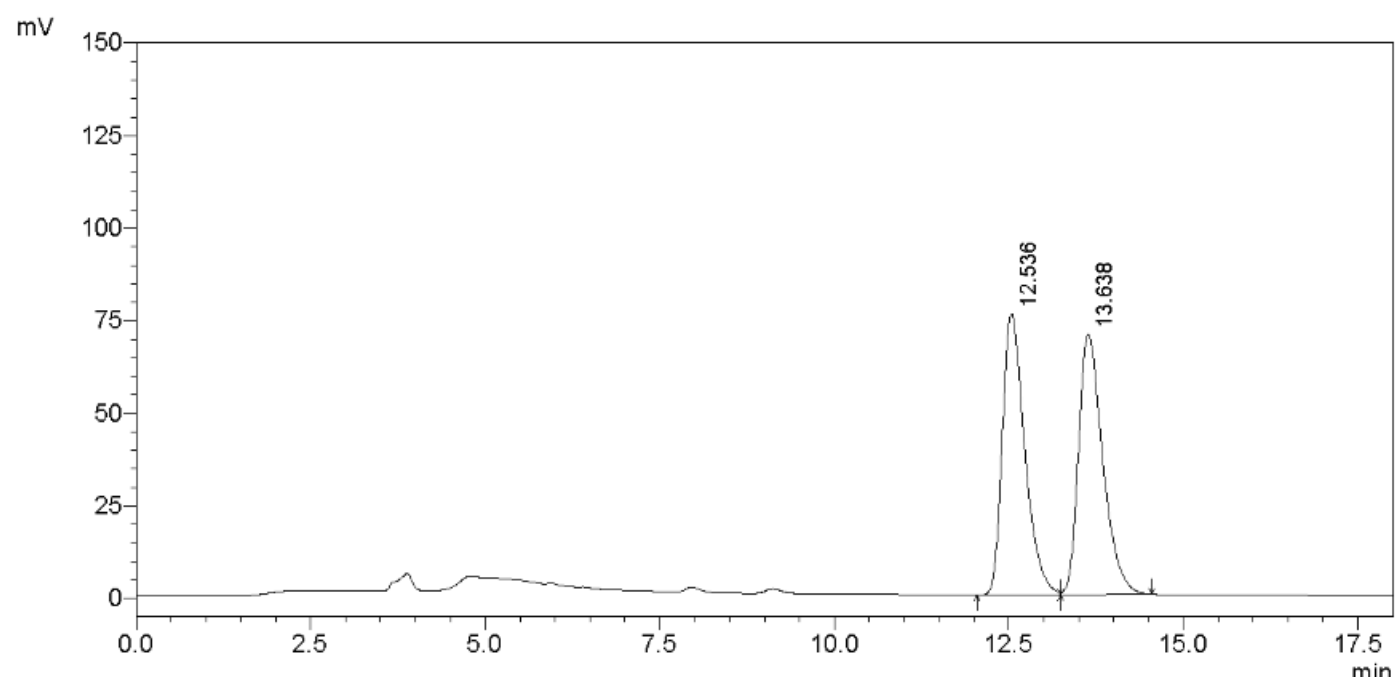

Detector A Ch1 254nm

\begin{tabular}{|r|r|l|r|}
\hline Peak\# & Ret. time & Area & Area $\not$ \\
\hline 1 & 12.536 & 1693993 & 49.940 \\
\hline 2 & 13.638 & 1698060 & 50.060 \\
\hline & & 3392053 & 100.000 \\
\hline
\end{tabular}

\section{Chiral}

$\mathrm{mV}$

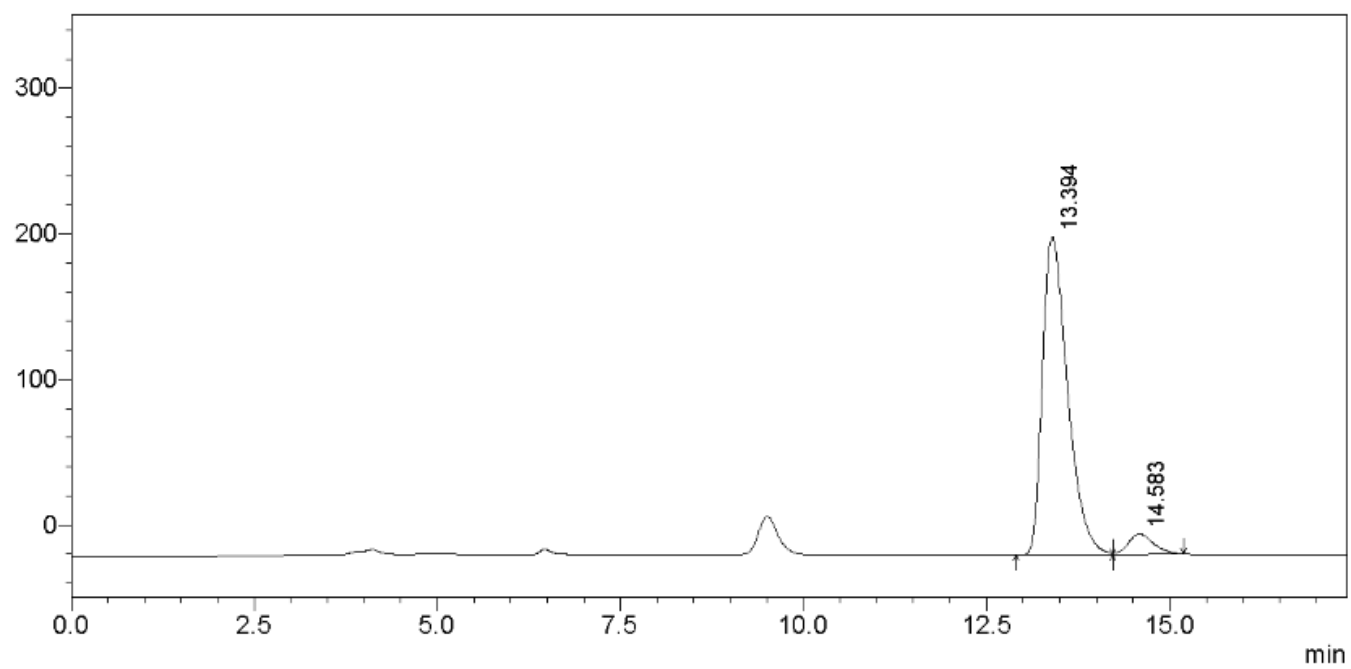

Detector A Ch1 254nm

\begin{tabular}{|r|r|r|r|}
\hline Peak\# & Ret. time & \multicolumn{1}{|c|}{ Area } & Area $\not$ \\
\hline 1 & 13.394 & 5191864 & 93.984 \\
\hline 2 & 14.583 & 332332 & 6.016 \\
\hline & & 5524196 & 100.000 \\
\hline
\end{tabular}




\section{Compound 5b}

\section{HPLC Conditions}<smiles>COC1(C#N)C(=O)C=Cc2cc(-c3ccccc3)ccc21</smiles>

$5 b$

Column: Chiralcel OD-H, Daicel Chemical Industries, Ltd.

Eluent: Hexanes / isopropanol (98:02)

Flow rate: $1.0 \mathrm{~mL} / \mathrm{min}$

Detection: UV $254 \mathrm{~nm}$

\section{Racemic}

$\mathrm{mV}$

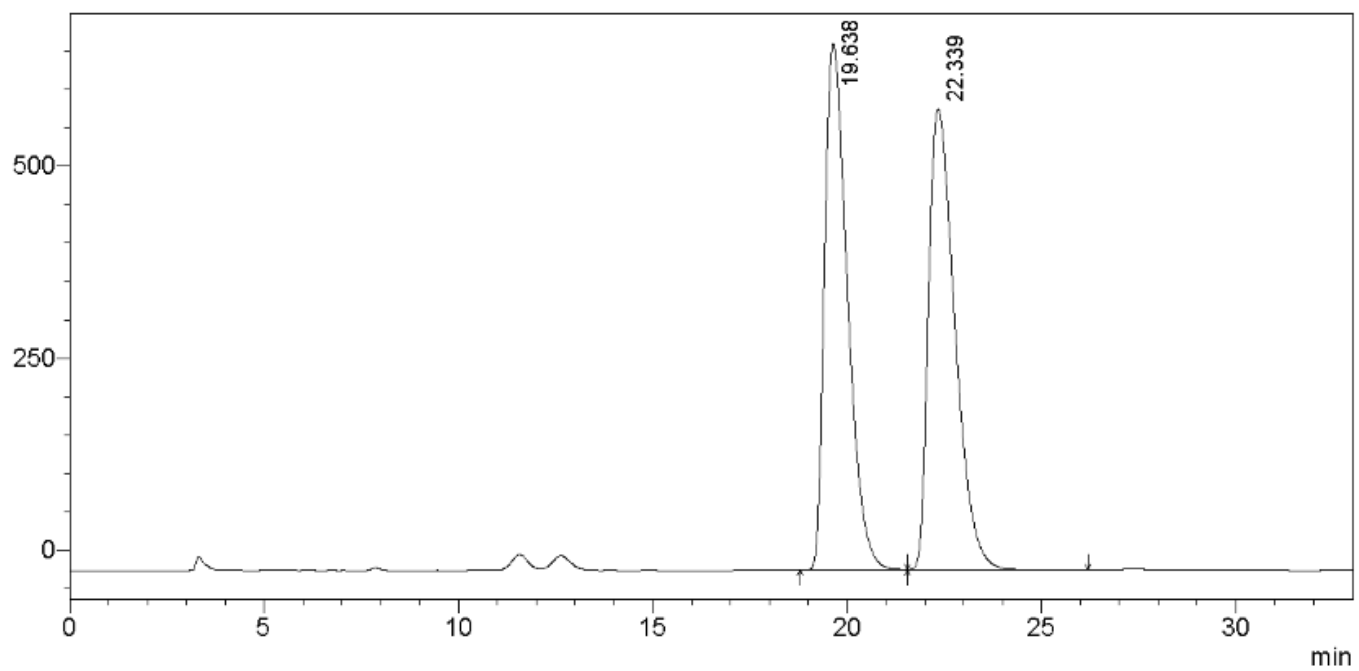

\begin{tabular}{|c|c|c|c|}
\hline \multicolumn{4}{|c|}{ etector A Ch1 $254 \mathrm{~nm}$} \\
\hline $\begin{array}{l}\text { Peak\# } \\
1\end{array}$ & $\begin{array}{l}\text { Ret. time } \\
19.638\end{array}$ & $\begin{array}{l}\text { Area } \\
28131807\end{array}$ & $\begin{array}{l}\text { Area } \% \\
49.749\end{array}$ \\
\hline 2 & 22.339 & 28415405 & 50. \\
\hline
\end{tabular}

\section{Chiral}

$\mathrm{mV}$

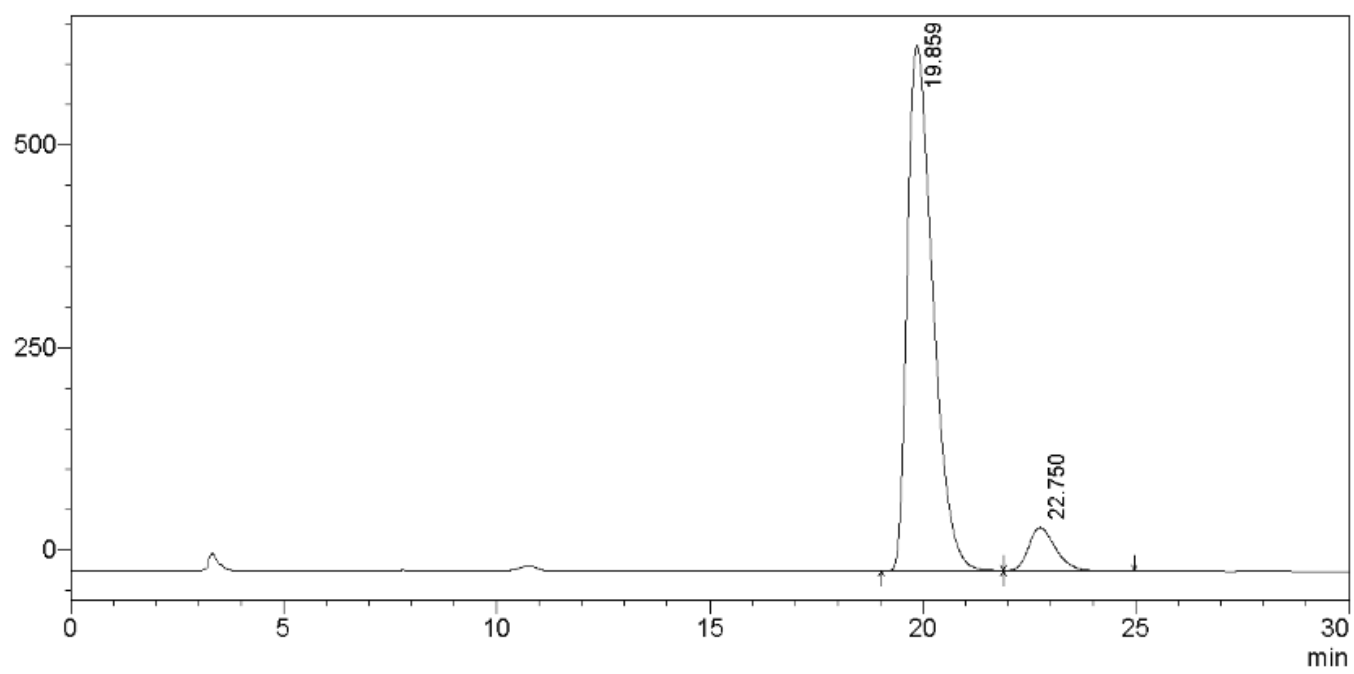

Detector A Ch1 254nm

\begin{tabular}{|r|r|r|r|}
\hline Peak\#\# & Ret. time & \multicolumn{1}{|c|}{ Area } & Area $\not$ \\
\hline 1 & 19.859 & 27024543 & 91.851 \\
\hline 2 & 22.750 & 2397636 & 8.149 \\
\hline & & 29422179 & 100.000 \\
\hline
\end{tabular}




\section{Compound 5c}<smiles>COC1(C#N)C(=O)C=Cc2ccc(-c3ccccc3)cc21</smiles>

\section{HPLC Conditions}

Column: Chiralcel AD-H, Daicel Chemical Industries, Ltd.

Eluent: Hexanes / isopropanol (95:05)

Flow rate: $1.0 \mathrm{~mL} / \mathrm{min}$

Detection: UV $254 \mathrm{~nm}$

\section{Racemic}

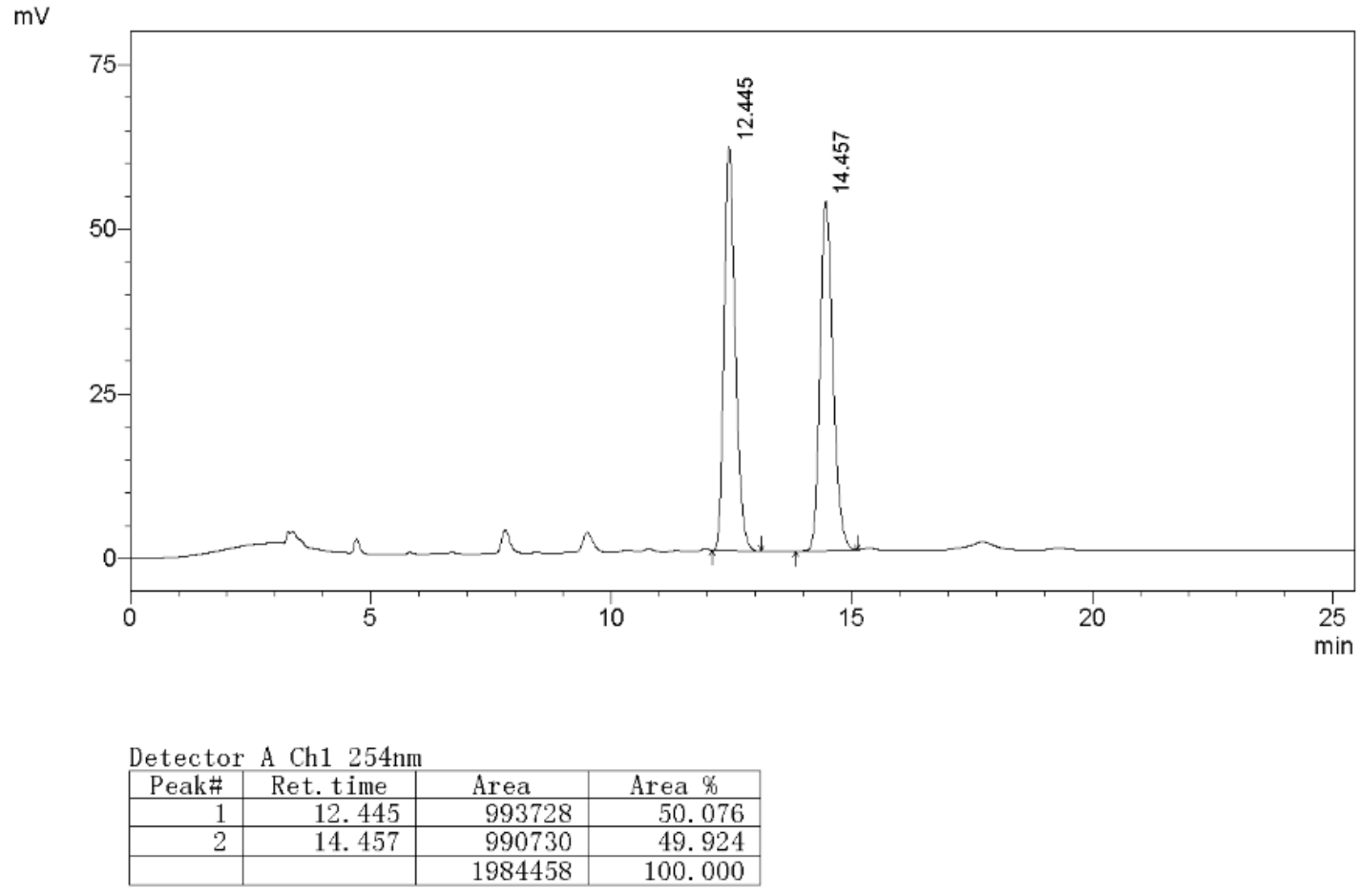

\section{Chiral}

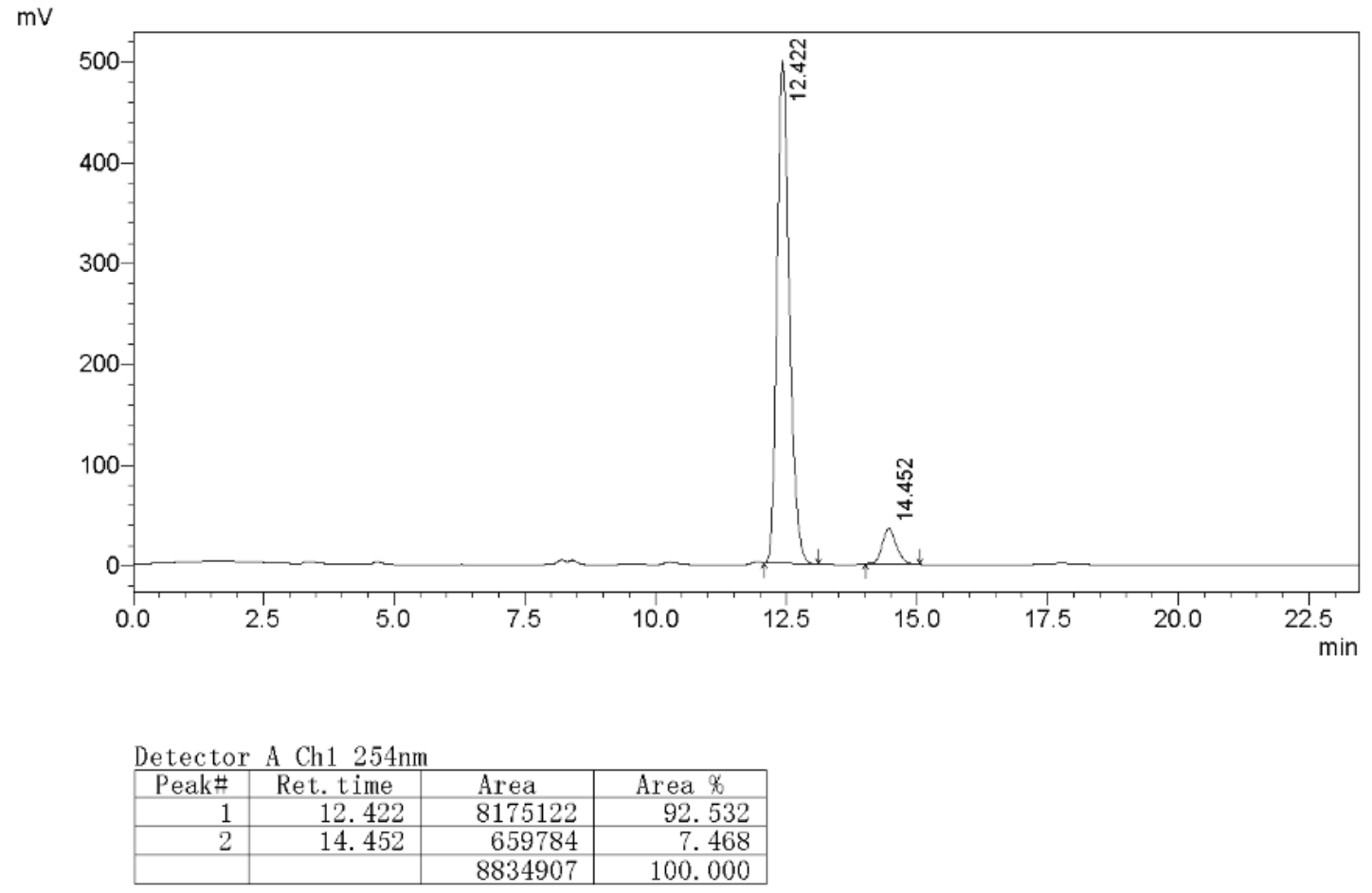




\section{Compound 5d}<smiles>COC(=O)C1(C#N)C(=O)C=Cc2cc(-c3cc(C)cc(C)c3)ccc21</smiles>

$5 d$

\section{HPLC Conditions}

Column: Chiralcel OD-H, Daicel Chemical Industries, Ltd.

Eluent: Hexanes / isopropanol (98:02)

Flow rate: $1.0 \mathrm{~mL} / \mathrm{min}$

Detection: UV $254 \mathrm{~nm}$

\section{Racemic}

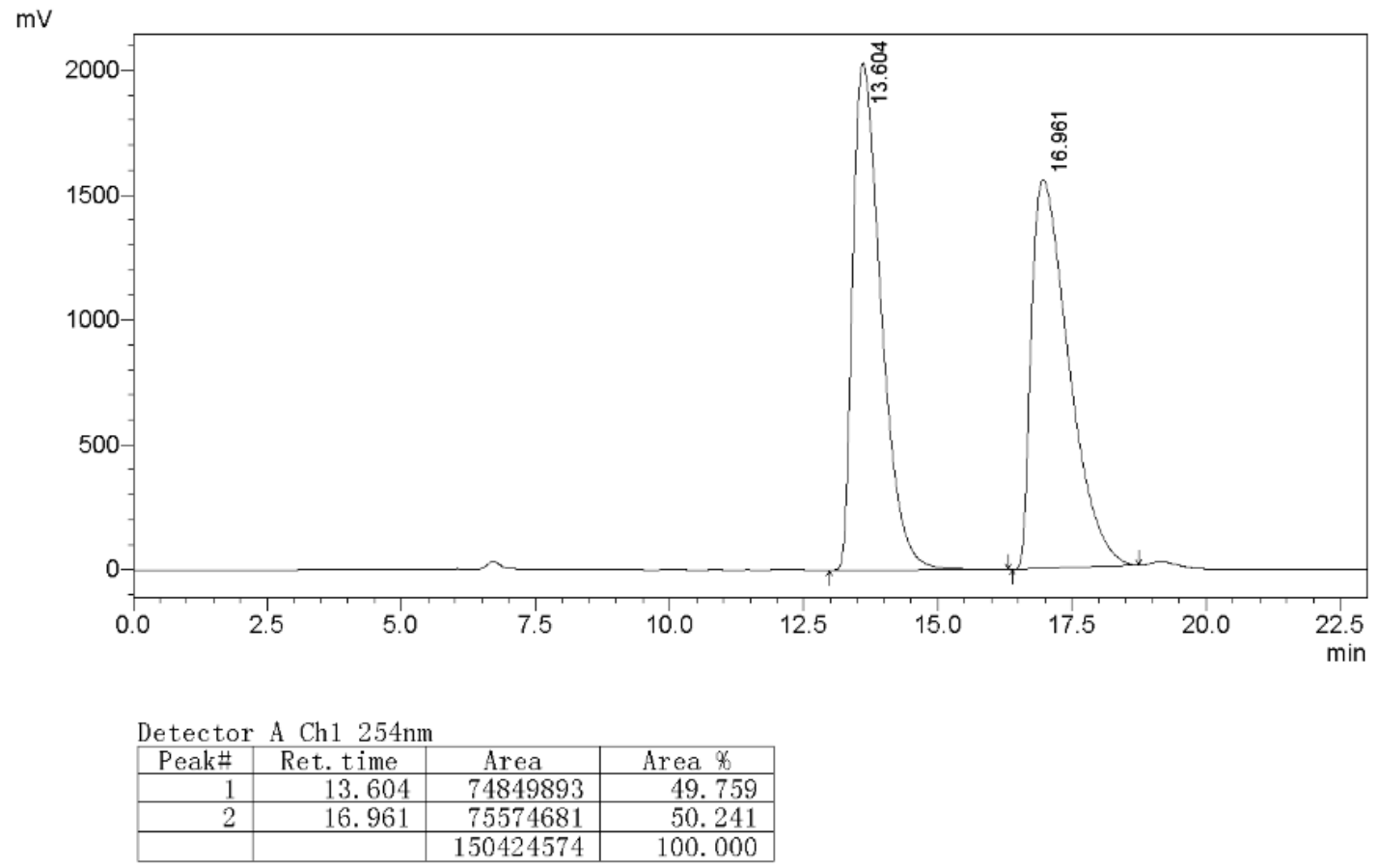

\section{Chiral}

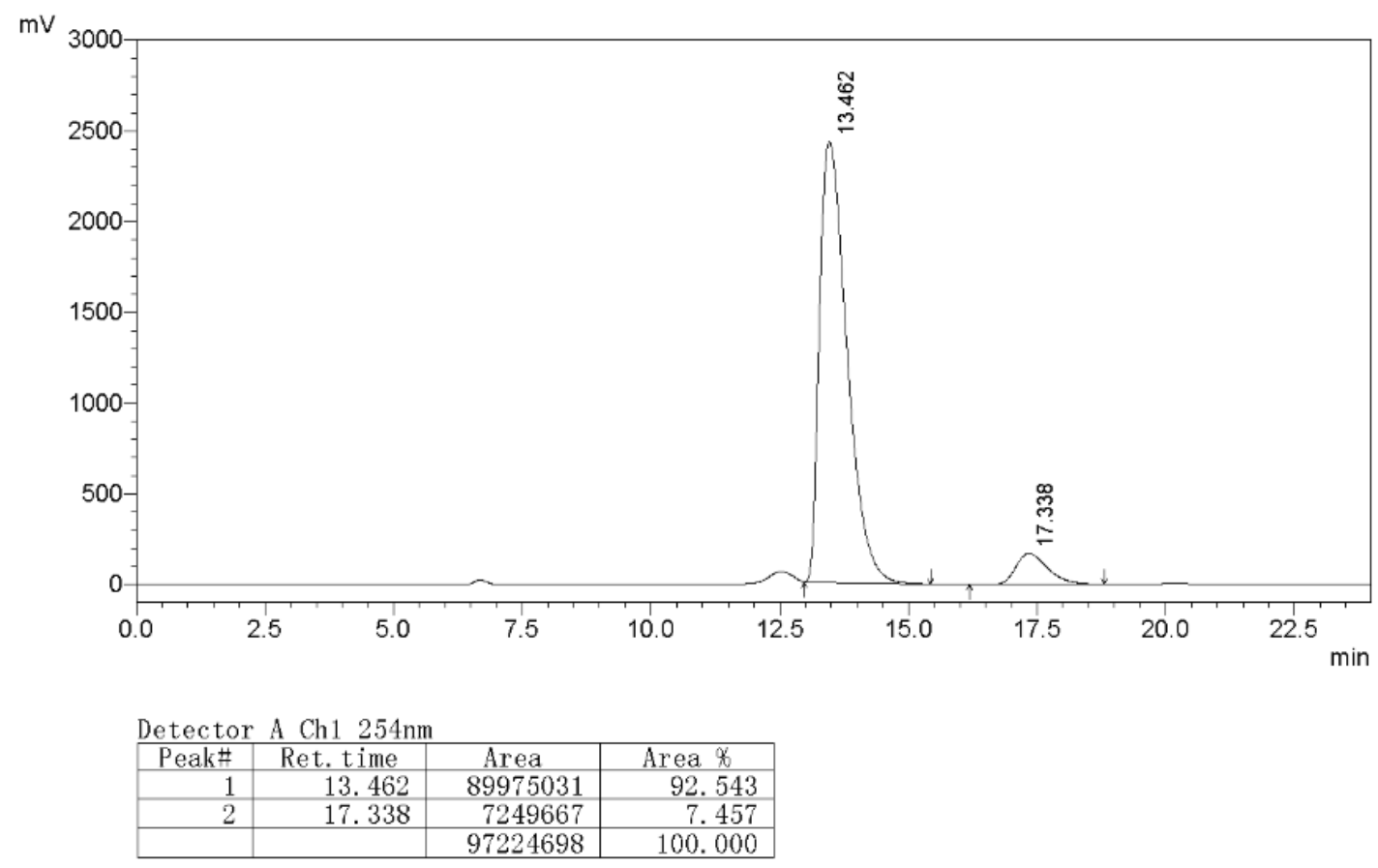




\section{Compound 5e}<smiles>COC1(C#N)C(=O)C=Cc2cc(Br)ccc21</smiles>

$5 e$

\section{HPLC Conditions}

Column: Chiralcel OD-H, Daicel Chemical Industries, Ltd.

Eluent: Hexanes / isopropanol (95:05)

Flow rate: $1.0 \mathrm{~mL} / \mathrm{min}$

Detection: UV $254 \mathrm{~nm}$

\section{Racemic}

$\mathrm{mV}$

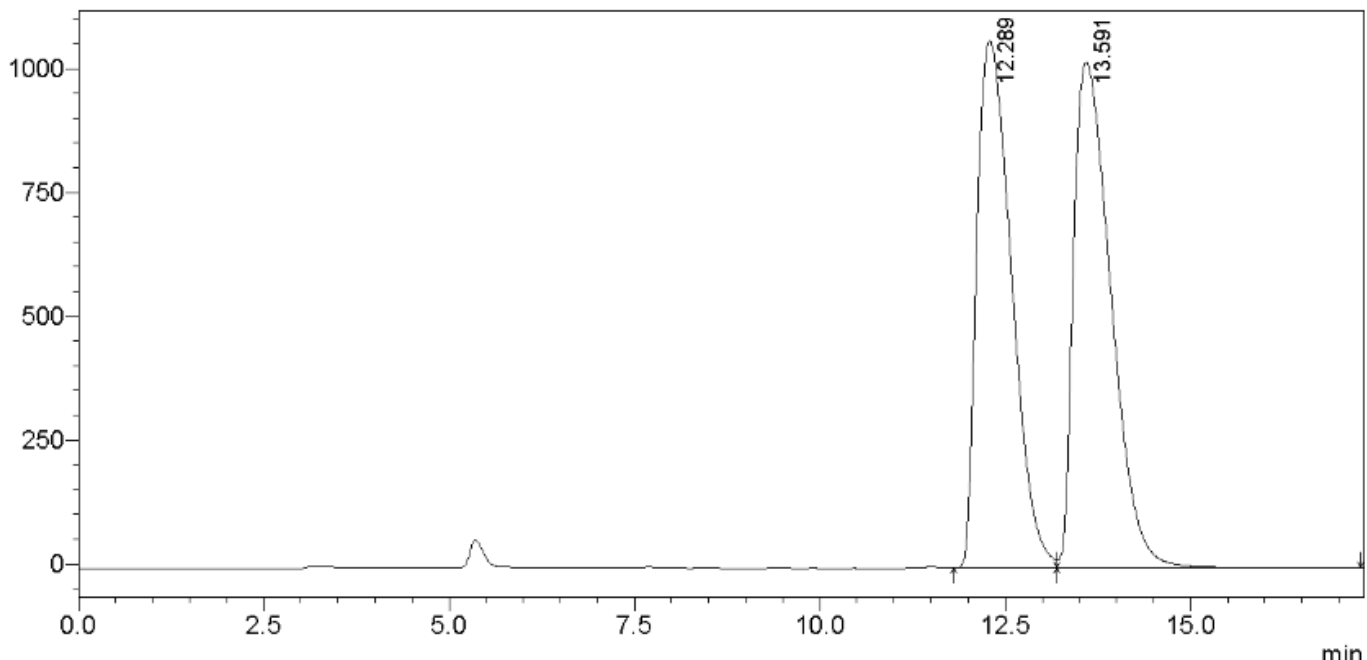

Detector A Ch1 254nm

\begin{tabular}{|r|r|r|r|}
\hline Peak\# & Ret. time & Area & Area $\not$ \\
\hline 1 & 12.289 & 34663411 & 49.103 \\
\hline 2 & 13.591 & 35929476 & 50.897 \\
\hline & & 70592887 & 100.000 \\
\hline
\end{tabular}

\section{Chiral}

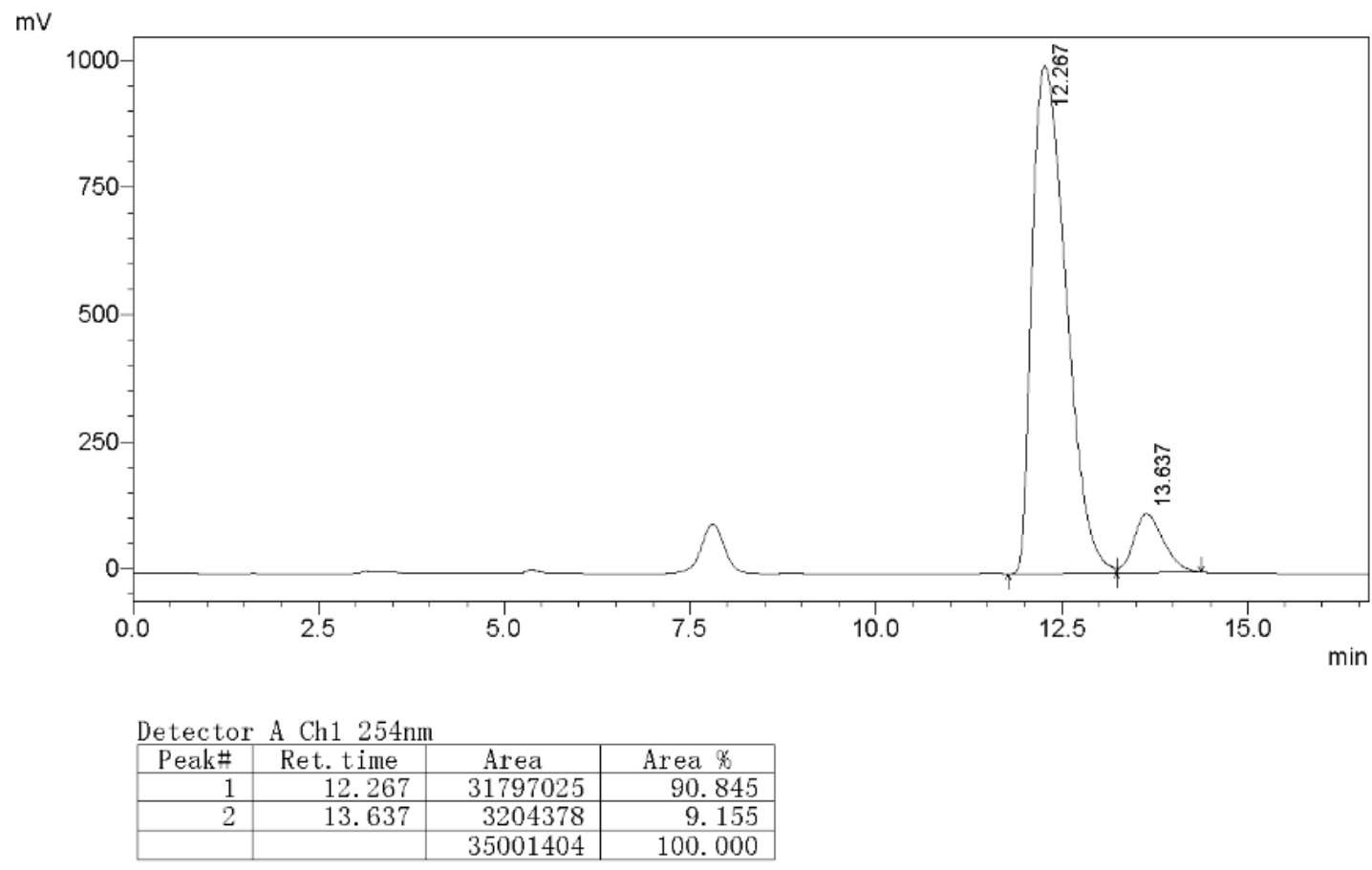




\section{Compound $5 f$}<smiles>COc1ccc2c(c1)C(C#N)(C(C)=O)C(=O)C=C2</smiles>

$5 f$

\section{HPLC Conditions}

Column: Chiralcel OD-3, Daicel Chemical Industries, Ltd.

Eluent: Hexanes / isopropanol (85:15)

Flow rate: $0.24 \mathrm{~mL} / \mathrm{min}$

Detection: UV $254 \mathrm{~nm}$

\section{Racemic}

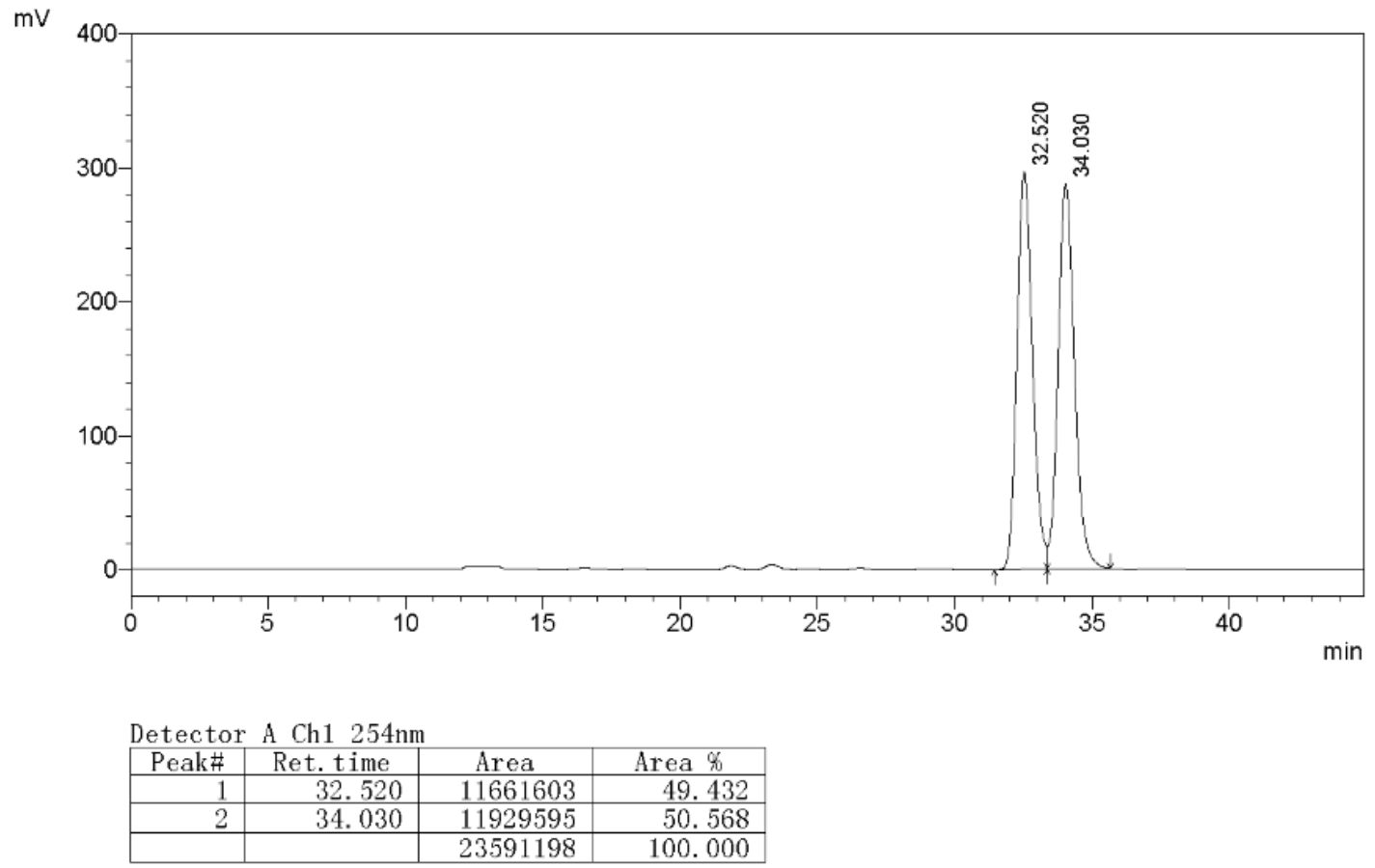

\section{Chiral}

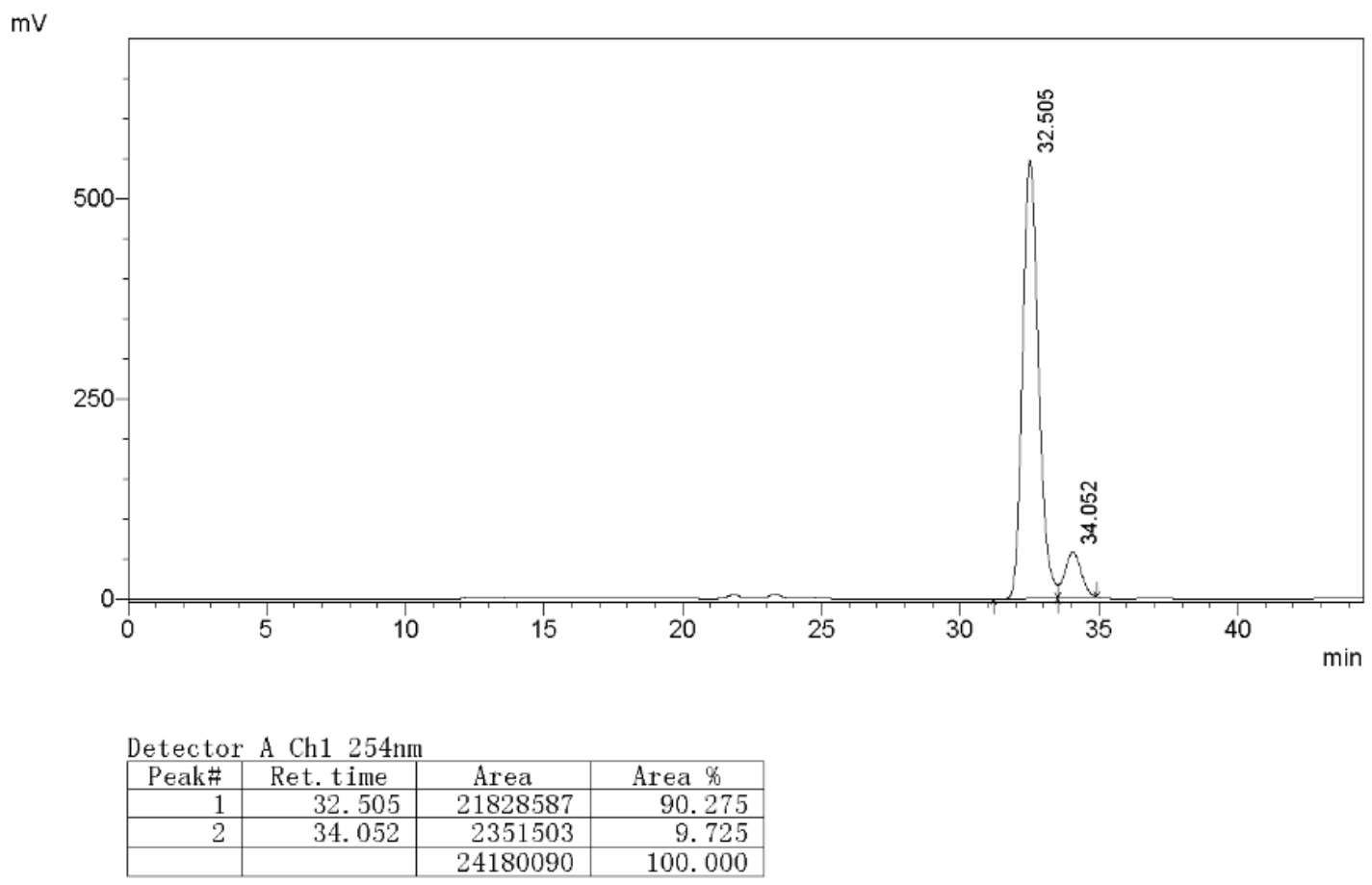




\section{Compound 5g}

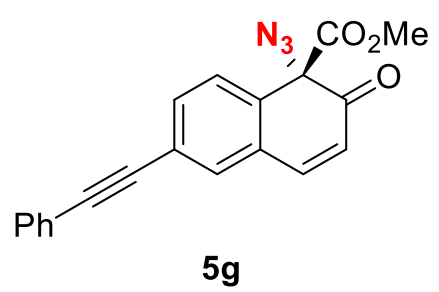

\section{HPLC Conditions}

Column: Chiralcel AS-H, Daicel Chemical Industries, Ltd.

Eluent: Hexanes / isopropanol (90:10)

Flow rate: $1.0 \mathrm{~mL} / \mathrm{min}$

Detection: UV $254 \mathrm{~nm}$

\section{Racemic}

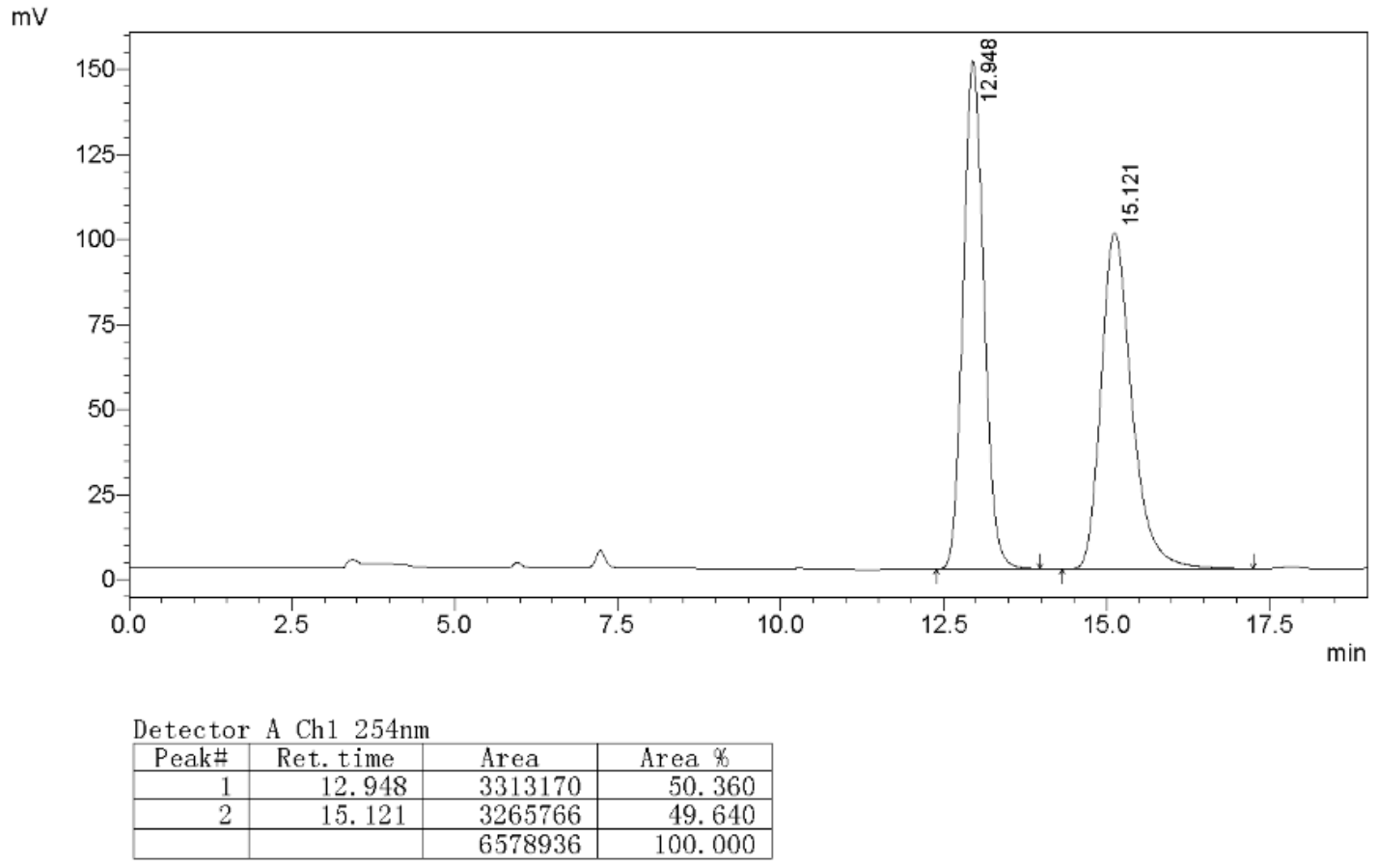

\section{Chiral}

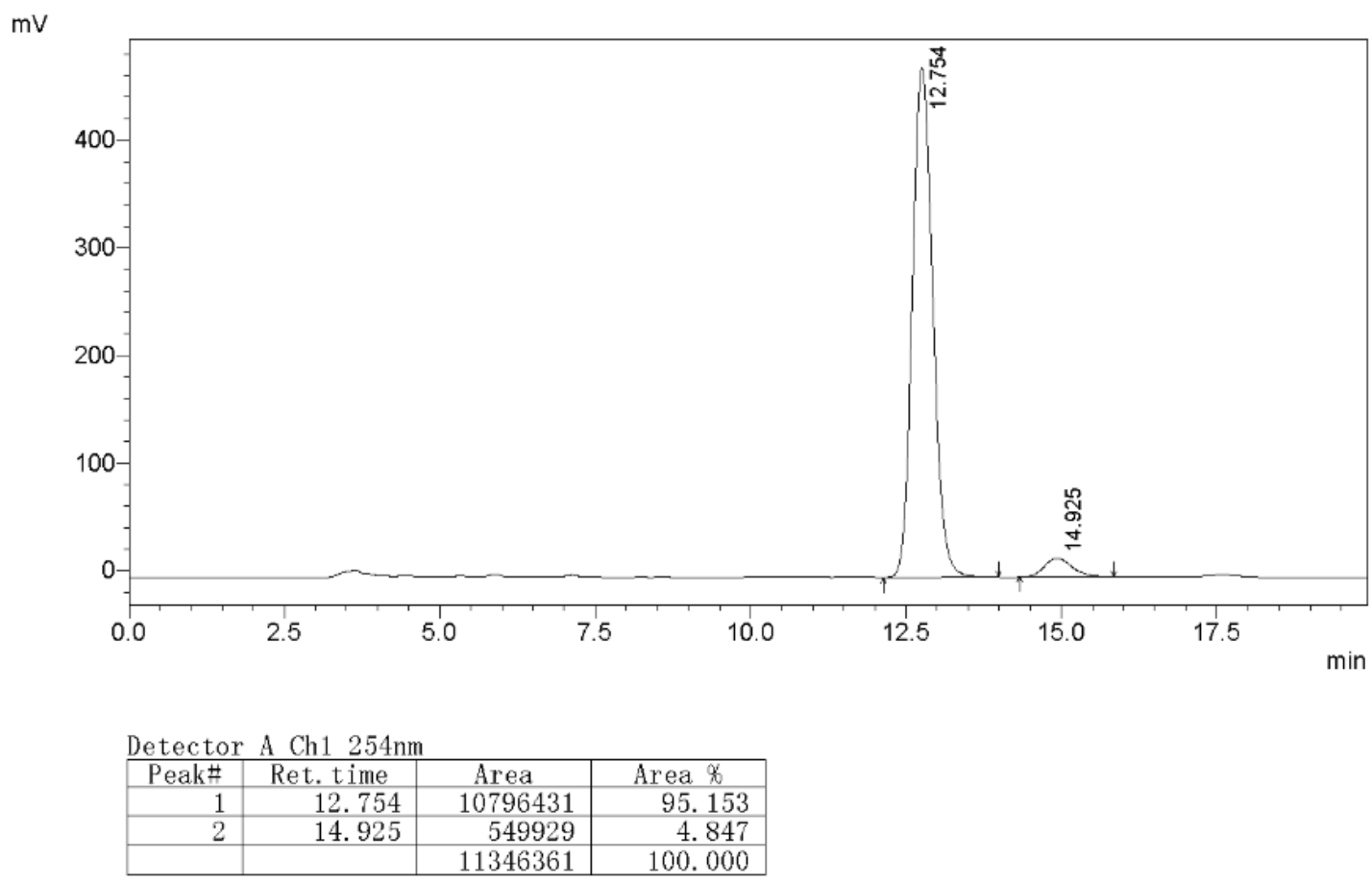




\section{Compound 5h}<smiles>COC1(C#N)C(=O)C=Cc2cc(C#CCO)ccc21</smiles>

$5 \mathrm{~h}$

\section{HPLC Conditions}

Column: Chiralcel OJ-H, Daicel Chemical Industries, Ltd.

Eluent: Hexanes / isopropanol (85:15)

Flow rate: $1.0 \mathrm{~mL} / \mathrm{min}$

Detection: UV $254 \mathrm{~nm}$

\section{Racemic}

$\mathrm{mV}$

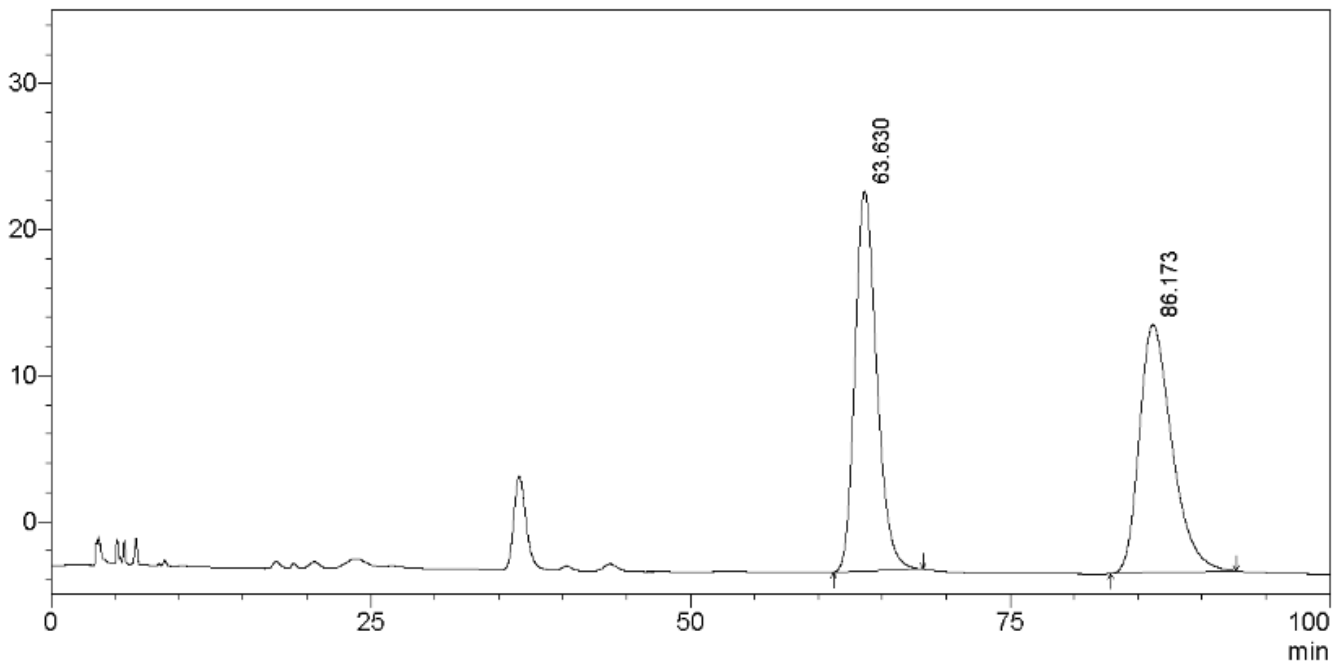

Detector A Ch1 254nm

Detector A Ch1 254nm
\begin{tabular}{|r|r|r|r|}
\hline Peak\# & Ret. time & Area & Area \% \\
\hline 1 & 63.630 & 3075125 & 50.437 \\
\hline 2 & 86.173 & 3021800 & 49.563 \\
\hline & & 6096925 & 100.000 \\
\hline
\end{tabular}

\section{Chiral}

$\mathrm{mV}$

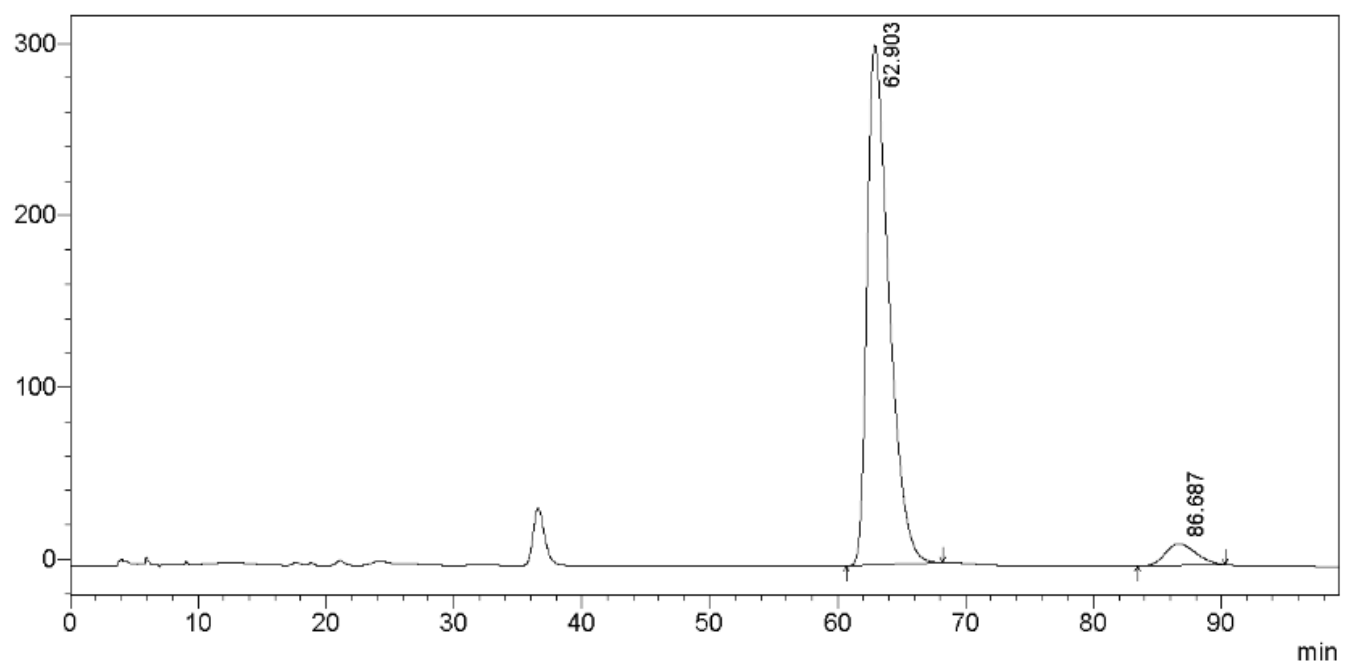

Detector A Ch1 254nm

\begin{tabular}{|c|c|c|c|}
\hline Peak\# & Ret. time & Area & Area $\phi$ \\
\hline 1 & 62.903 & 35908613 & 94.473 \\
\hline 2 & 86.687 & 2100765 & 5.527 \\
\hline & & 38009377 & 100.000 \\
\hline
\end{tabular}




\section{Compound 5i}<smiles>COC(=O)C1(C#N)C(=O)C=C(c2ccccc2)c2ccccc21</smiles>

$5 i$

\section{HPLC Conditions}

Column: Chiralcel OJ-H, Daicel Chemical Industries, Ltd.

Eluent: Hexanes / isopropanol (90:10)

Flow rate: $1.0 \mathrm{~mL} / \mathrm{min}$

Detection: UV $254 \mathrm{~nm}$

\section{Racemic}

$\mathrm{mV}$

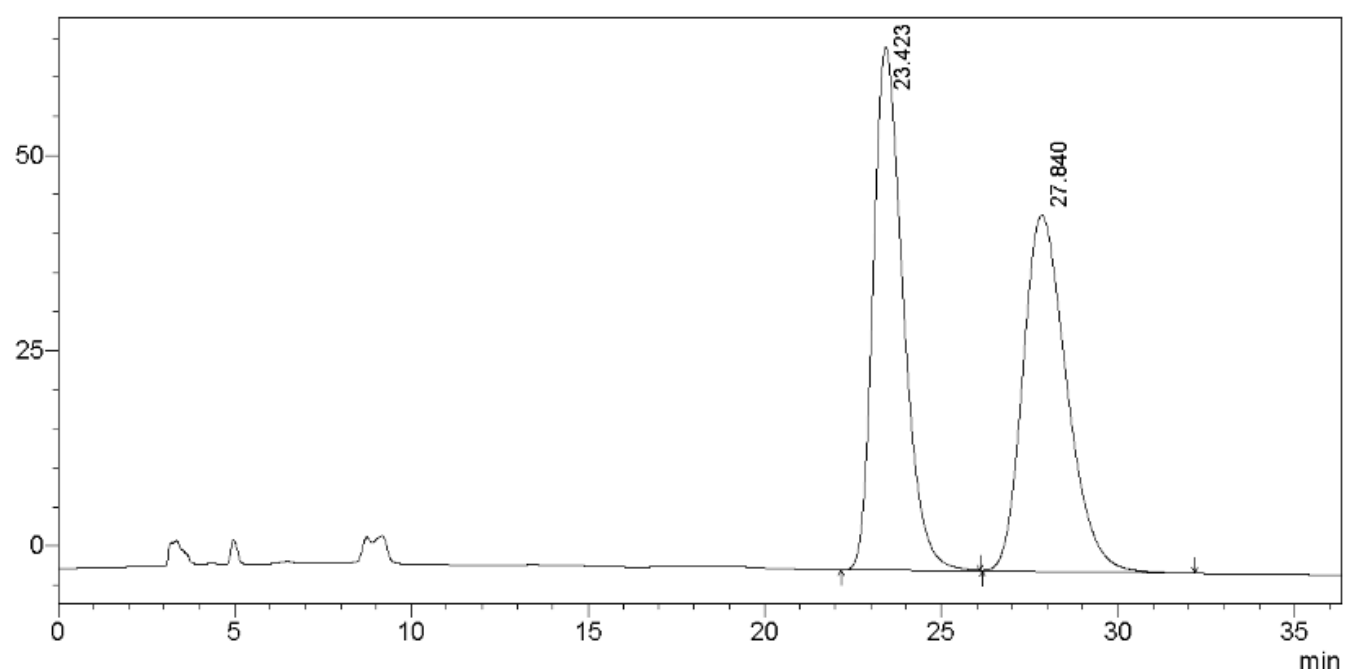

Detector A Ch1 254nm

\begin{tabular}{|r|r|r|r|}
\hline Peak\# & Ret. time & Area & Area \% \\
\hline 1 & 23.423 & 3936840 & 50.071 \\
\hline 2 & 27.840 & 3925603 & 49.929 \\
\hline & & 7862443 & 100.000 \\
\hline
\end{tabular}

\section{Chiral}

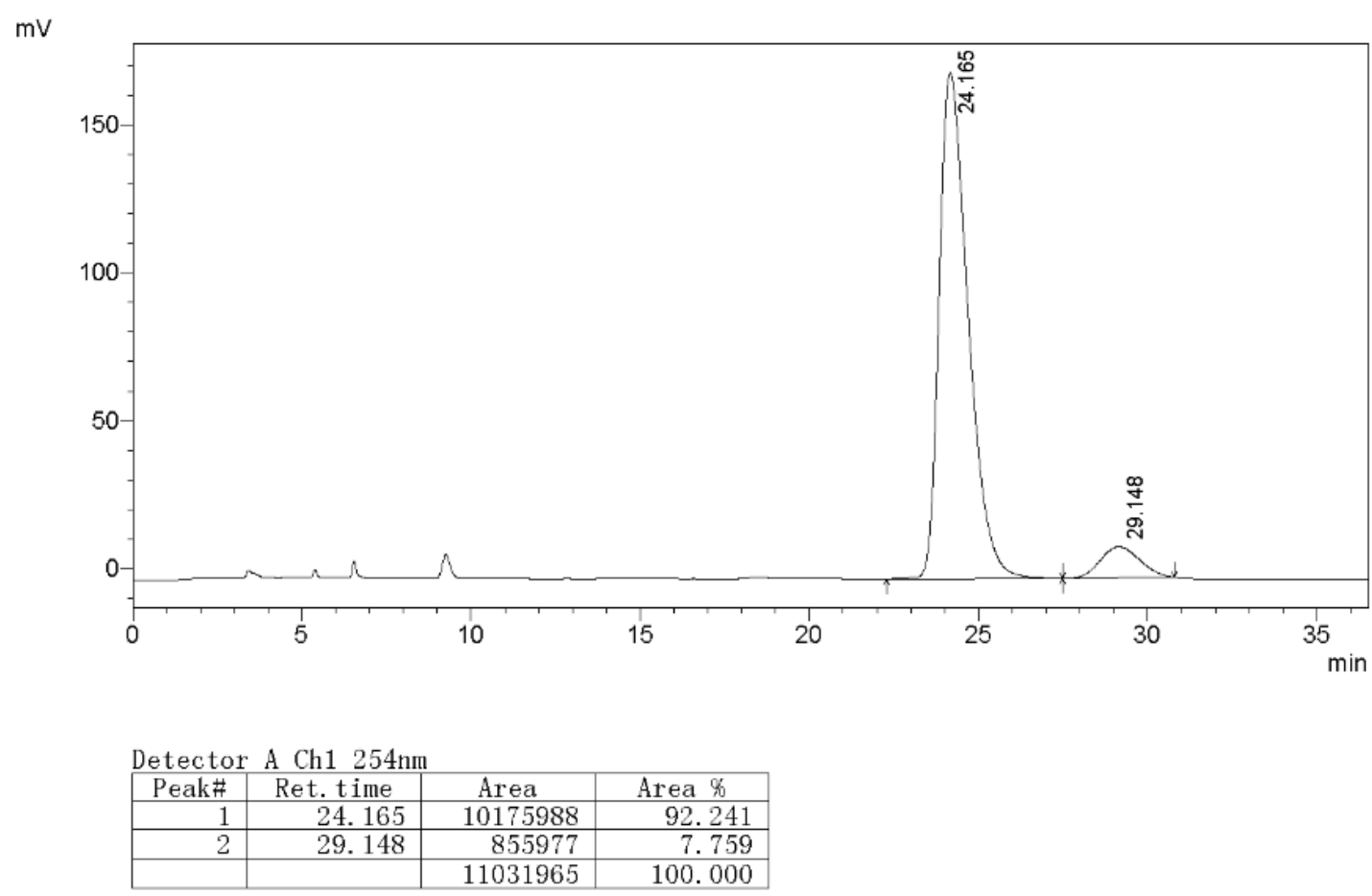




\section{Compound 5j}<smiles>COC(=O)C1(C#N)C(=O)C=C(Br)c2ccccc21</smiles>

5j

\section{HPLC Conditions}

Column: Chiralcel OD-H, Daicel Chemical Industries, Ltd.

Eluent: Hexanes / isopropanol (98:02)

Flow rate: $1.0 \mathrm{~mL} / \mathrm{min}$

Detection: UV $254 \mathrm{~nm}$

\section{Racemic}

mV

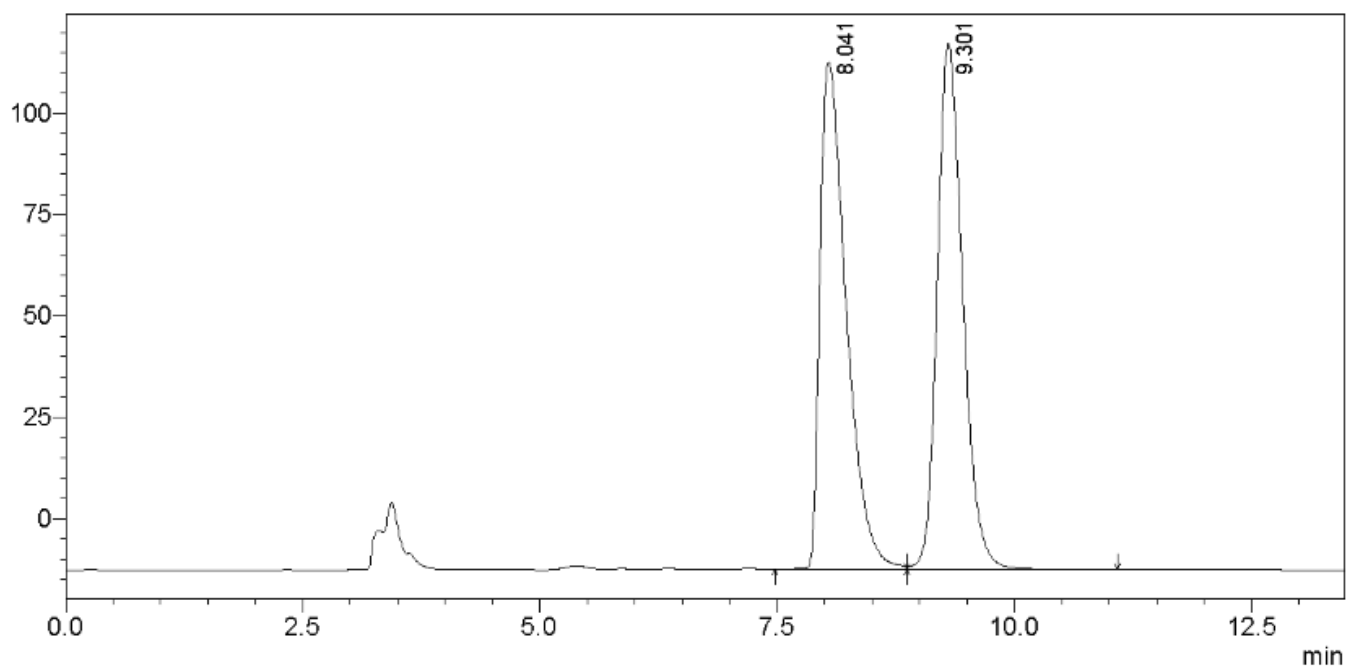

Detector A Ch1 $254 \mathrm{~nm}$
\begin{tabular}{|r|r|r|r|}
\hline Peak\# & Ret. time & \multicolumn{1}{|c|}{ Area } & \multicolumn{1}{|c|}{ Area $\%$} \\
\hline 1 & 8.041 & 2428598 & 49.881 \\
\hline 2 & 9.301 & 2440181 & 50.119 \\
\hline & & 4868779 & 100.000 \\
\hline
\end{tabular}

\section{Chiral sample a}

$\mathrm{mV}$

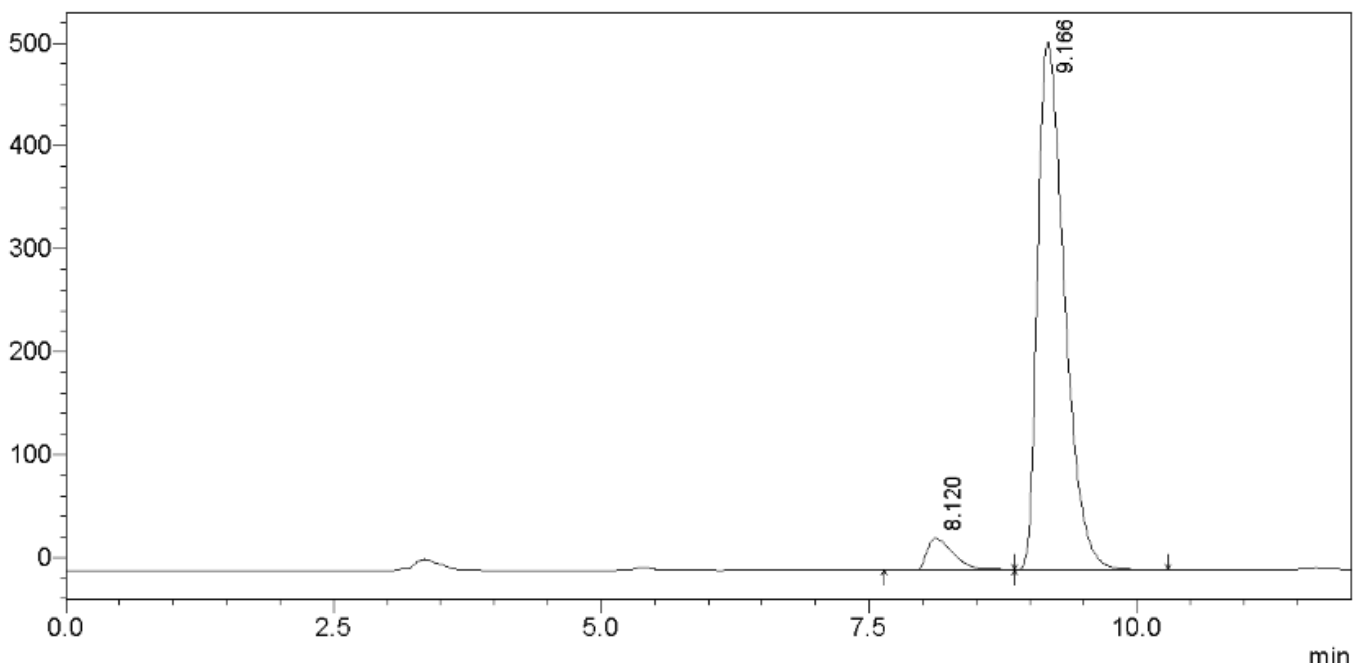

Detector A Ch1 $254 \mathrm{~nm}$
\begin{tabular}{|r|r|r|r|}
\hline Peak\# & Ret. time & \multicolumn{1}{|c|}{ Area } & Area $\not$ \\
\hline 1 & 8.120 & 534381 & 5.628 \\
\hline 2 & 9.166 & 8960738 & 94.372 \\
\hline & & 9495119 & 100.000 \\
\hline
\end{tabular}


Chiral sample b

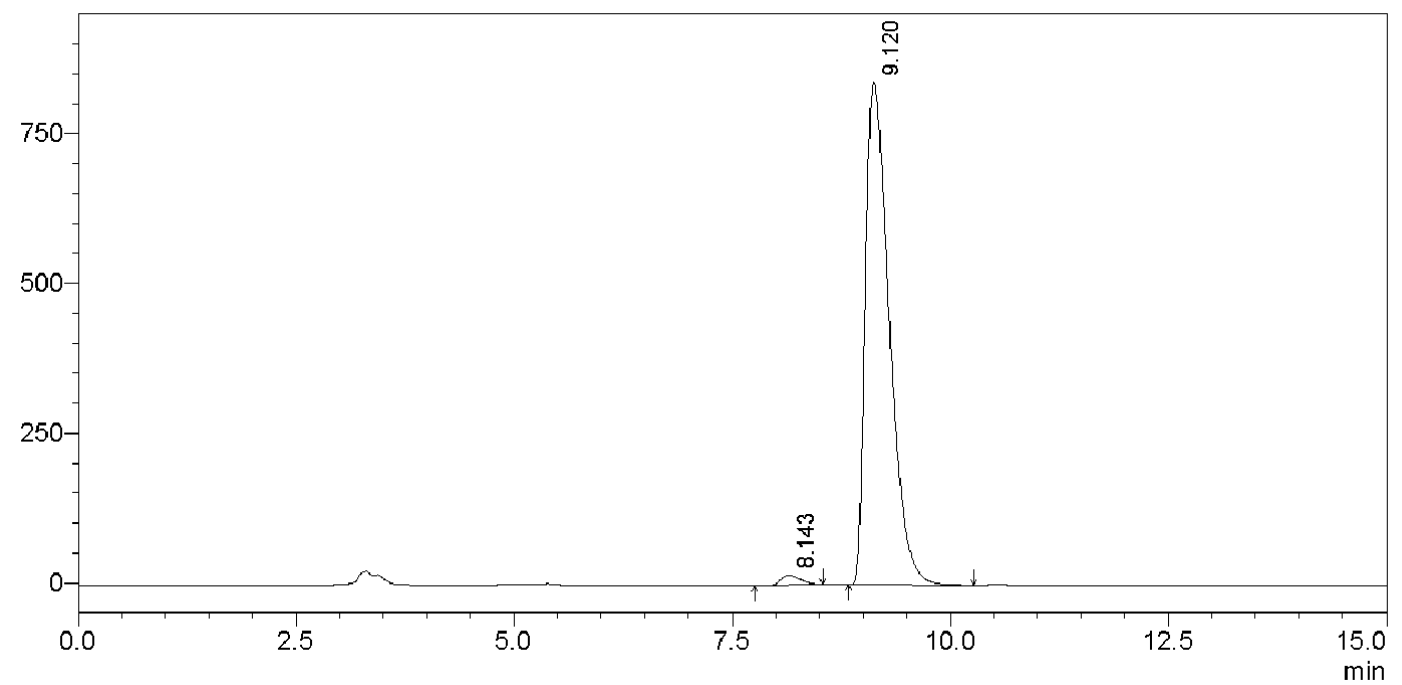

Detector A Ch1 254nm

\begin{tabular}{|c|c|c|r|}
\hline Peak\# & Ret. time & Area & Area \% \\
\hline 1 & 8.143 & 246608 & 1.514 \\
\hline 2 & 9.120 & 16046563 & 98.486 \\
\hline 总计 & & 16293171 & 100.000 \\
\hline
\end{tabular}




\section{Compound 5k}<smiles>COC1(C#N)C(=O)C(Br)=Cc2ccccc21</smiles>

$5 k$

\section{HPLC Conditions}

Column: Chiralcel OD-H, Daicel Chemical Industries, Ltd.

Eluent: Hexanes / isopropanol (98:02)

Flow rate: $1.0 \mathrm{~mL} / \mathrm{min}$

Detection: UV $254 \mathrm{~nm}$

\section{Racemic}

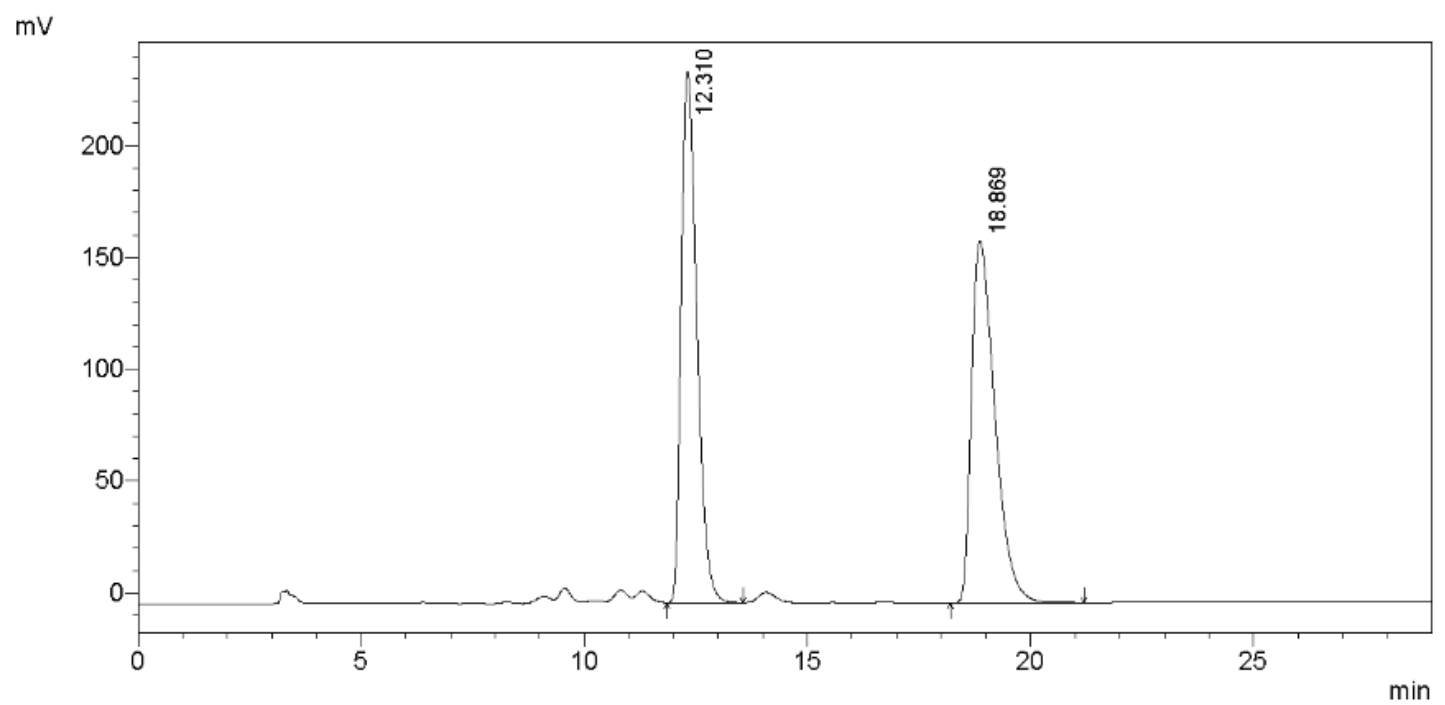

Detector A Ch1 254nm

\begin{tabular}{|r|r|r|r|}
\hline Peak\# & Ret. time & \multicolumn{1}{|c|}{ Area } & \multicolumn{1}{|c|}{ Area $\not$} \\
\hline 1 & 12.310 & 5776000 & 49.519 \\
\hline 2 & 18.869 & 5888241 & 50.481 \\
\hline & & 11664241 & 100.000 \\
\hline
\end{tabular}

\section{Chiral}

$\mathrm{mV}$

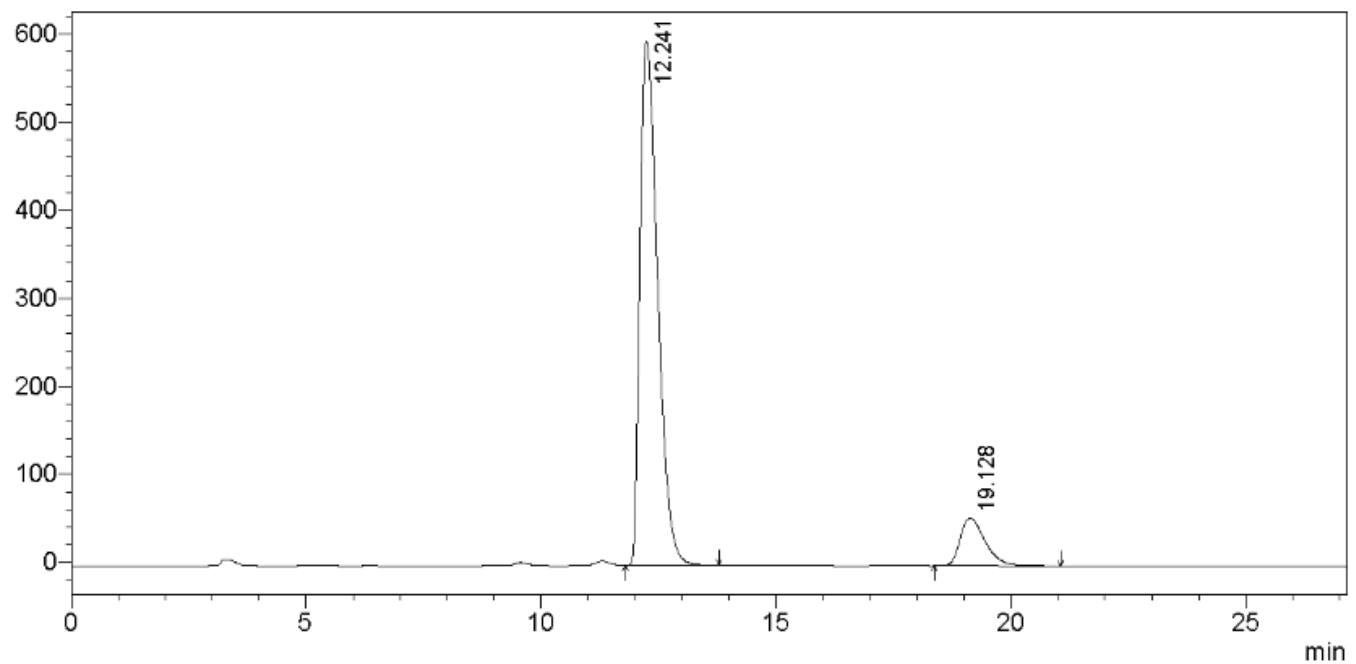

Detector A Ch1 254nm

\begin{tabular}{|r|r|r|r|}
\hline Peak\# & Ret. time & \multicolumn{1}{|c|}{ Area } & Area \% \\
\hline 1 & 12.241 & 15660503 & 88.726 \\
\hline 2 & 19.128 & 1989905 & 11.274 \\
\hline & & 17650408 & 100.000 \\
\hline
\end{tabular}




\section{Compound 5k}

\section{HPLC Conditions}<smiles>COC1(C#N)C(=O)C(Br)=Cc2ccccc21</smiles>

$5 k$

Column: Chiralcel OD-H, Daicel Chemical Industries, Ltd.

Eluent: Hexanes / isopropanol (98:02)

Flow rate: $1.0 \mathrm{~mL} / \mathrm{min}$

Detection: UV $254 \mathrm{~nm}$

\section{Racemic}

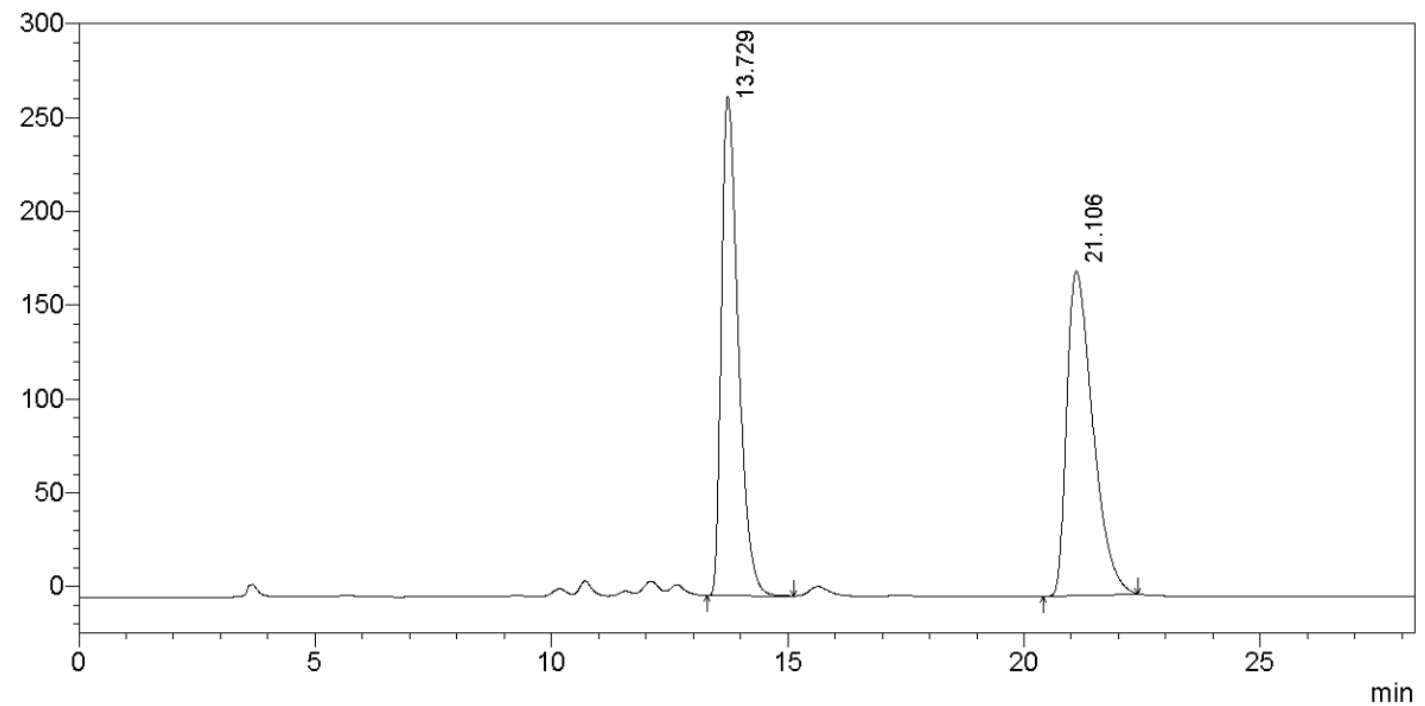

Detector A Ch1 254nm

\begin{tabular}{|c|c|c|c|}
\hline Peak\# & Ret.time & Area & Area \% \\
\hline 1 & 13.729 & 6619361 & 49.904 \\
\hline 2 & 21.106 & 6644919 & 50.096 \\
\hline 总计 & & 13264280 & 100.000 \\
\hline
\end{tabular}

\section{Chiral}

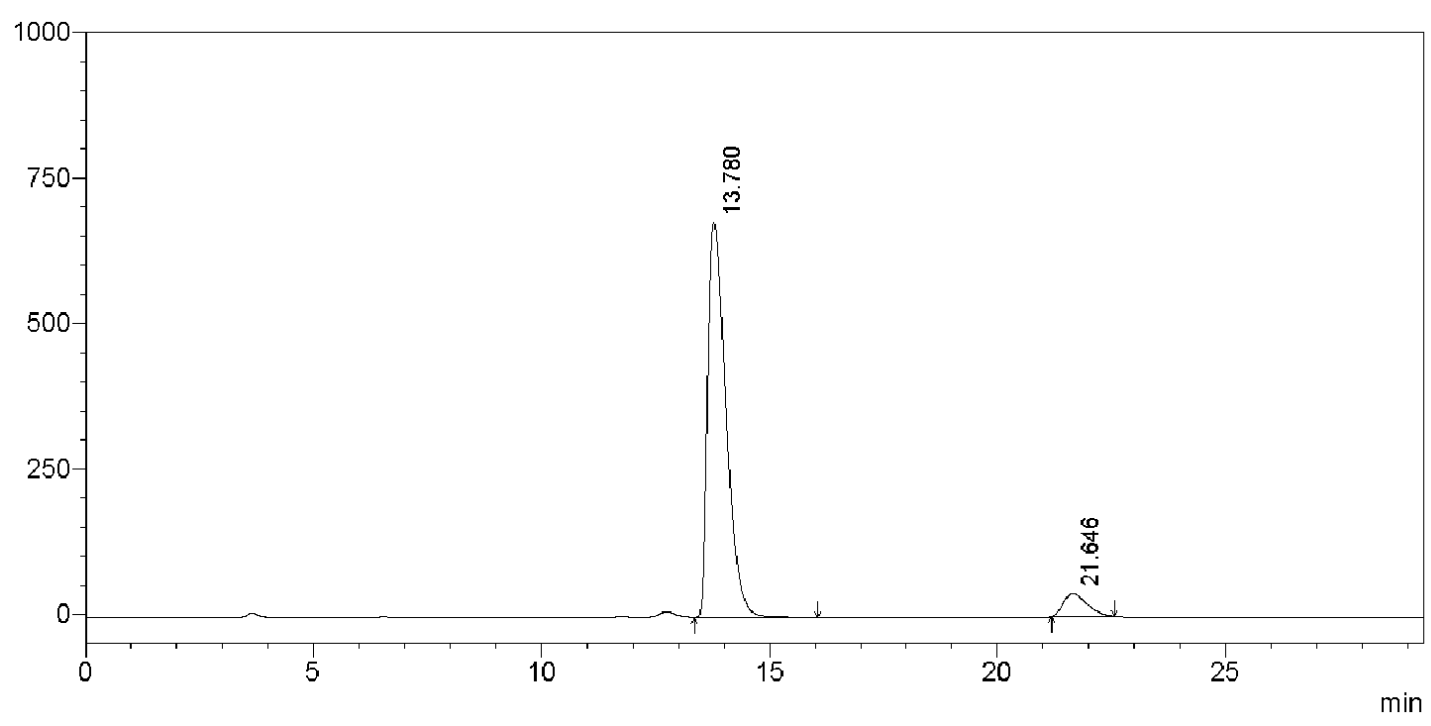

Detector A Ch1 254nm

\begin{tabular}{|r|r|r|r|}
\hline Peak\# & Ret. time & \multicolumn{1}{|c|}{ Area } & \multicolumn{1}{|c|}{ Area \% } \\
\hline 1 & 13.780 & 18920449 & 92.940 \\
\hline 2 & 21.646 & 1437314 & 7.060 \\
\hline 总计 & & 20357763 & 100.000 \\
\hline
\end{tabular}




\section{Compound 5l}

\section{HPLC Conditions}<smiles></smiles>

Column: Chiralcel OD-H, Daicel Chemical Industries, Ltd.

Eluent: Hexanes / isopropanol (97:03)

Flow rate: $1.0 \mathrm{~mL} / \mathrm{min}$

Detection: UV $254 \mathrm{~nm}$

\section{Racemic}

$\mathrm{mV}$

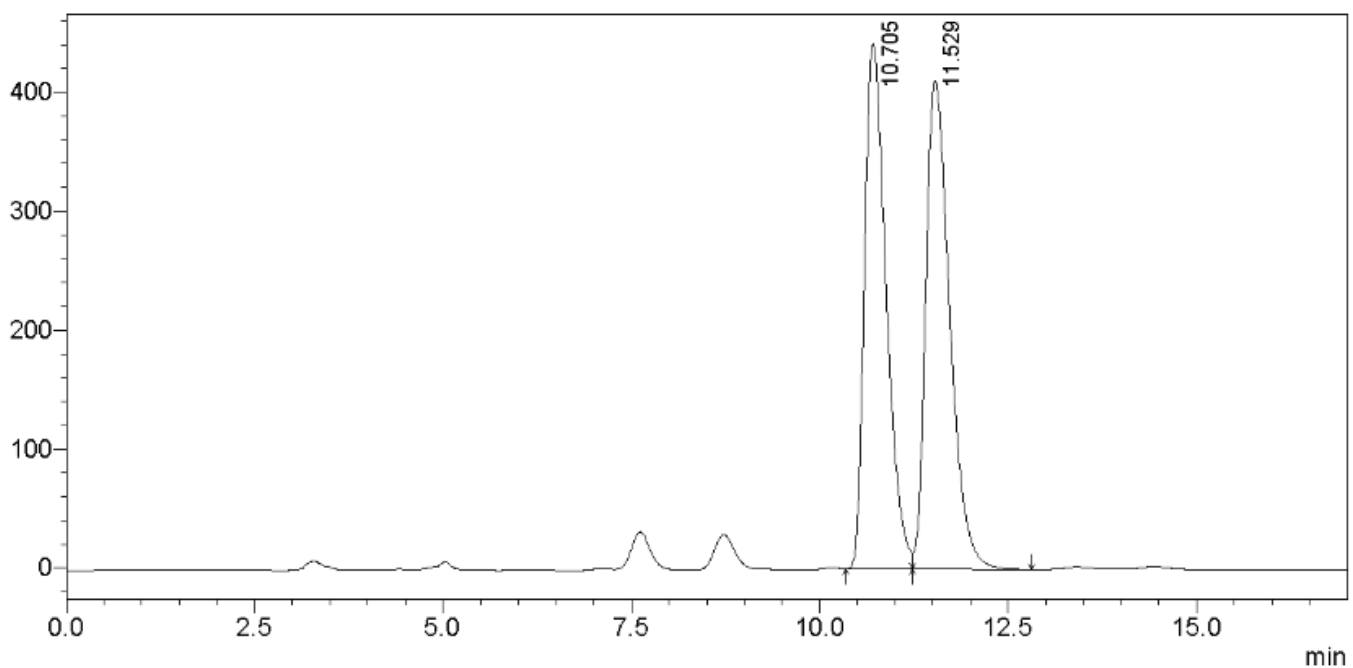

Detector A Ch1 254nm

\begin{tabular}{|r|r|r|r|}
\hline Peak\# & Ret. time & \multicolumn{1}{|c|}{ Area } & Area $\not 6$ \\
\hline 1 & 10.705 & 8838589 & 49.328 \\
\hline 2 & 11.529 & 9079303 & 50.672 \\
\hline & & 17917892 & 100.000 \\
\hline
\end{tabular}

\section{Chiral}

$\mathrm{mV}$

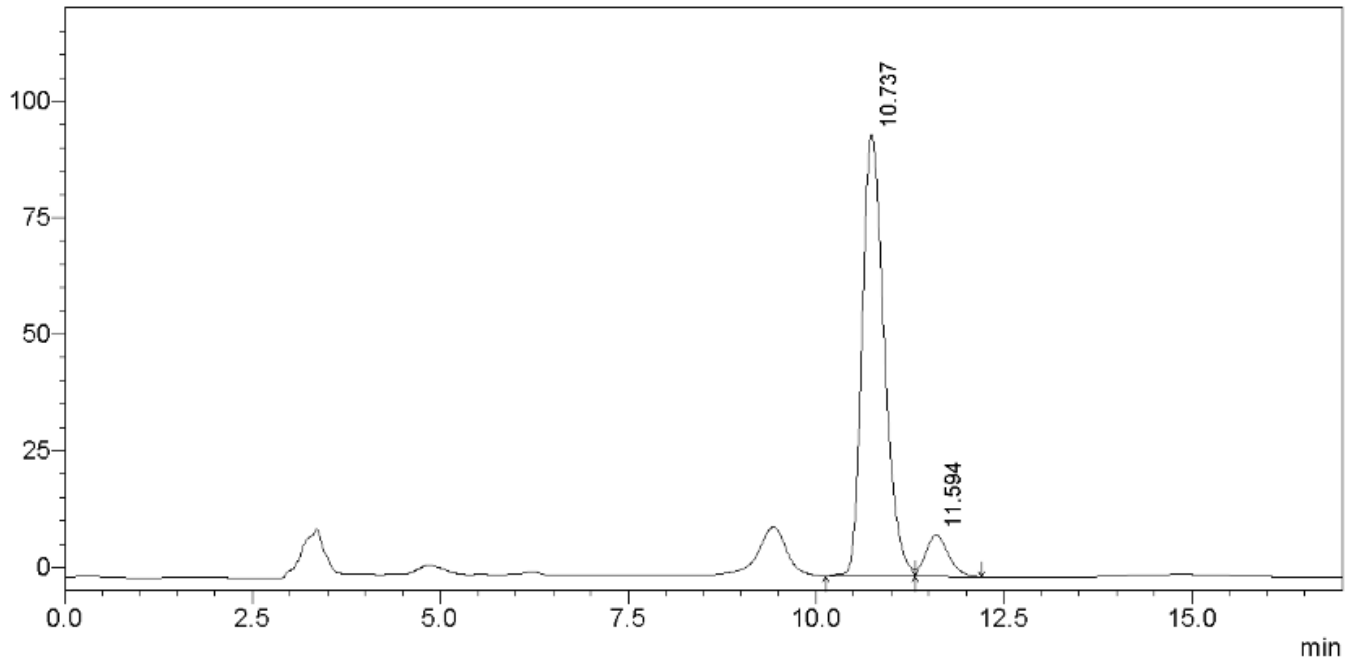

Detector A Ch1 254nm
\begin{tabular}{|r|r|r|r|}
\hline Peak\# & Ret. time & Area & Area $\%$ \\
\hline 1 & 10.737 & 1942703 & 91.075 \\
\hline 2 & 11.594 & 190376 & 8.925 \\
\hline & & 2133079 & 100.000 \\
\hline
\end{tabular}




\section{Compound 5m}

\section{HPLC Conditions}<smiles></smiles>

$5 \mathrm{~m}$
Column: Chiralcel OD-H, Daicel Chemical Industries, Ltd.

Eluent: Hexanes / isopropanol (95:05)

Flow rate: $1.0 \mathrm{~mL} / \mathrm{min}$

Detection: UV $254 \mathrm{~nm}$

\section{Racemic}

$\mathrm{mV}$

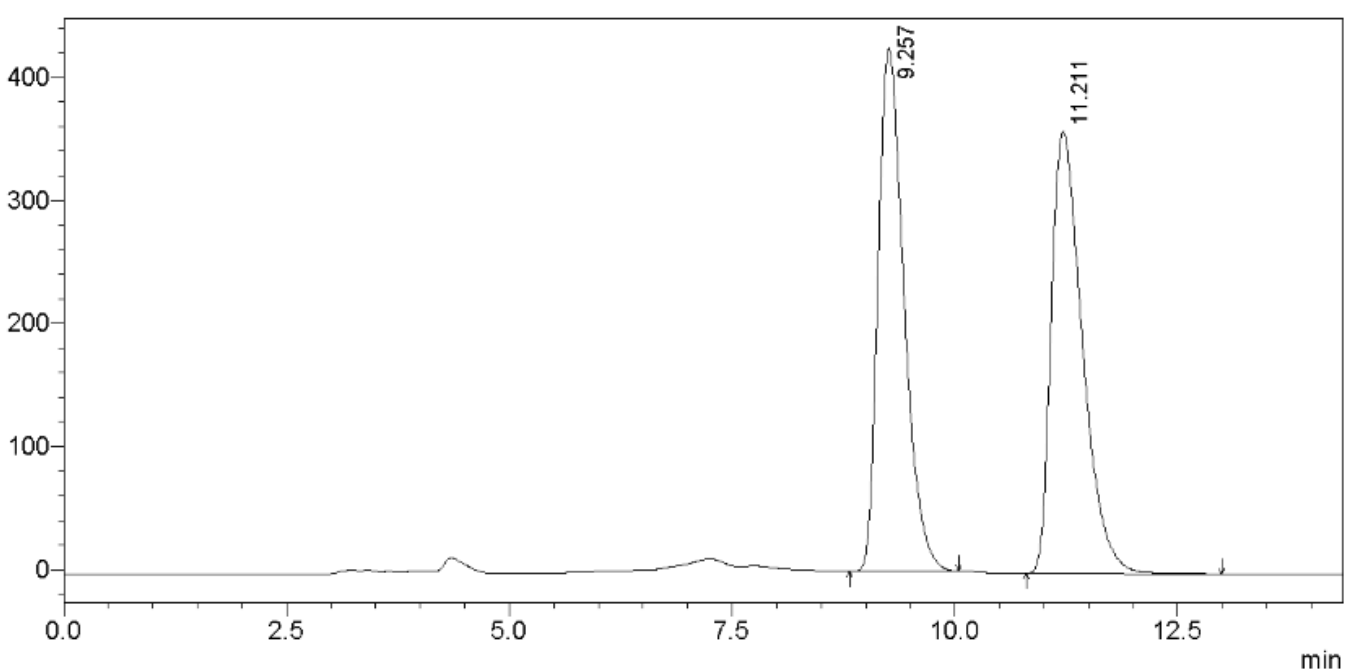

Detector A Ch1 254nm

\begin{tabular}{|r|r|r|r|}
\hline Peak\# & Ret. time & Area & Area 96 \\
\hline 1 & 9.257 & 8616767 & 49.622 \\
\hline 2 & 11.211 & 8747879 & 50.378 \\
\hline & & 17364646 & 100.000 \\
\hline
\end{tabular}

\section{Chiral}

$\mathrm{mV}$

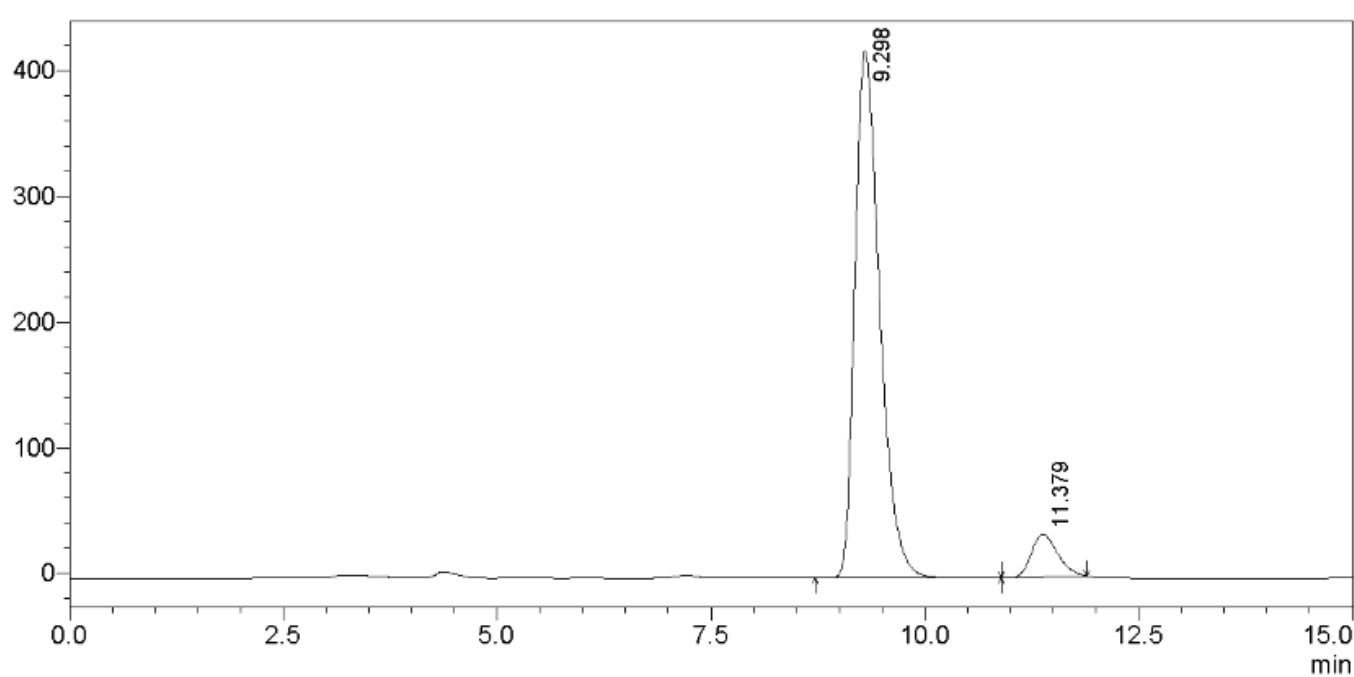

Detector A Ch1 $254 \mathrm{~nm}$
\begin{tabular}{|r|r|r|r|}
\hline Peak\# & Ret. time & \multicolumn{1}{|c|}{ Area } & \multicolumn{1}{c|}{ Area $\%$} \\
\hline 1 & 9.298 & 8489468 & 92.067 \\
\hline 2 & 11.379 & 731454 & 7.933 \\
\hline & & 9220921 & 100.000 \\
\hline
\end{tabular}




\section{Compound 5n}<smiles></smiles>

\section{HPLC Conditions}

Column: Chiralcel OD-H, Daicel Chemical Industries, Ltd.

Eluent: Hexanes / isopropanol (95:05)

Flow rate: $1.0 \mathrm{~mL} / \mathrm{min}$

Detection: UV $254 \mathrm{~nm}$

\section{Racemic}

$\mathrm{mV}$

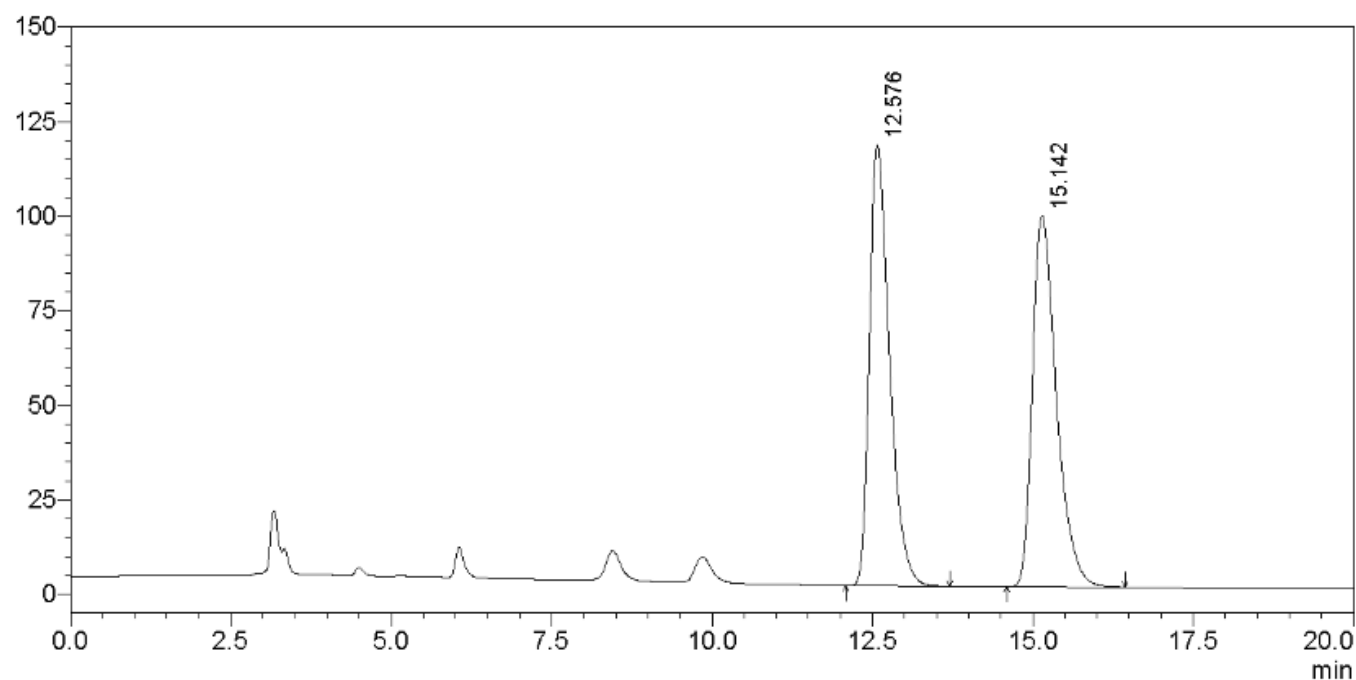

Detector A Ch1 254nm

\begin{tabular}{|r|r|r|r|}
\hline Peak\# & Ret. time & Area & Area \% \\
\hline 1 & 12.576 & 2550668 & 49.901 \\
\hline 2 & 15.142 & 2560795 & 50.099 \\
\hline & & 5111463 & 100.000 \\
\hline
\end{tabular}

\section{Chiral}

$\mathrm{mv}$

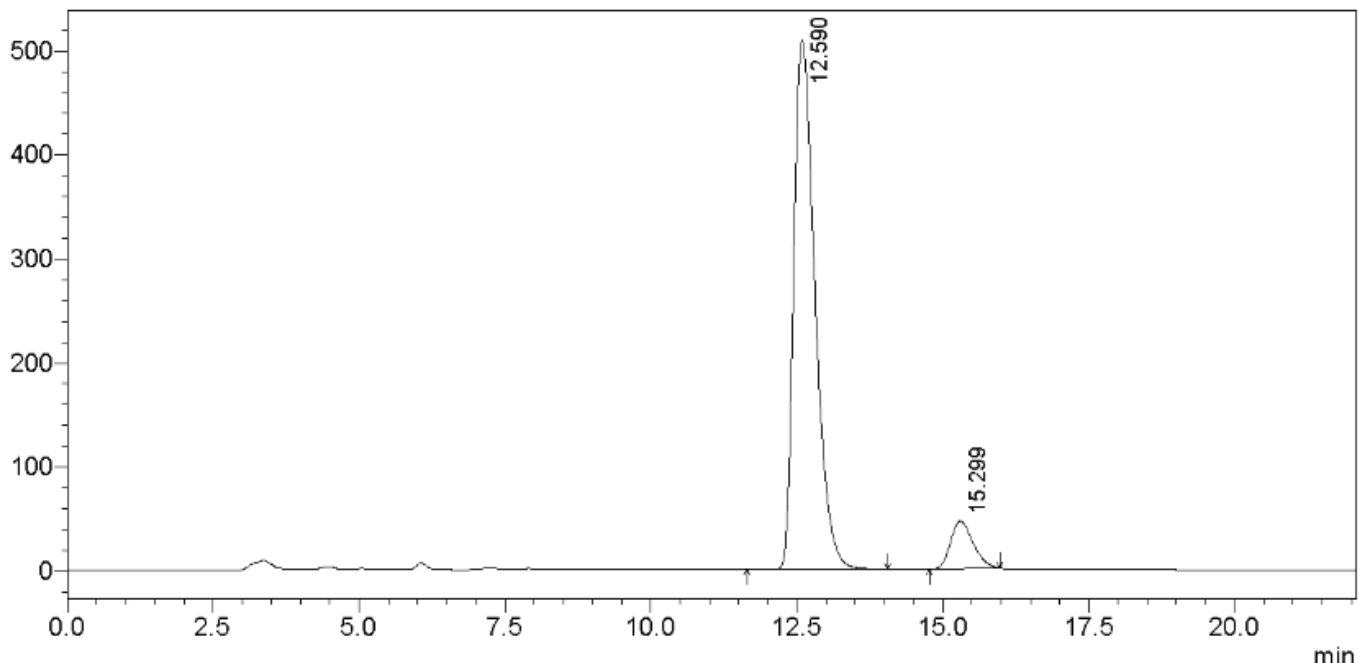

Detector A Ch1 254nm

\begin{tabular}{|r|r|r|r|}
\hline Detector A Ch1 $254 \mathrm{~nm}$ & \multicolumn{1}{|c|}{ Area } & Area $\%$ \\
\hline Peak\# & Ret. time & \multicolumn{1}{|c|}{ Area } & 91.208 \\
\hline 1 & 12.590 & 12831696 & 8.792 \\
\hline 2 & 15.299 & 1236959 & 100.000 \\
\hline & & 14068656 & \\
\hline
\end{tabular}




\section{Compound 50}<smiles>CCCOC(=O)C1(C#N)C(=O)C=Cc2ccccc21</smiles>

50

\section{HPLC Conditions}

Column: Chiralcel AD-H, Daicel Chemical Industries, Ltd.

Eluent: Hexanes / isopropanol (98:02)

Flow rate: $1.0 \mathrm{~mL} / \mathrm{min}$

Detection: UV $254 \mathrm{~nm}$

\section{Racemic}

$\mathrm{mV}$

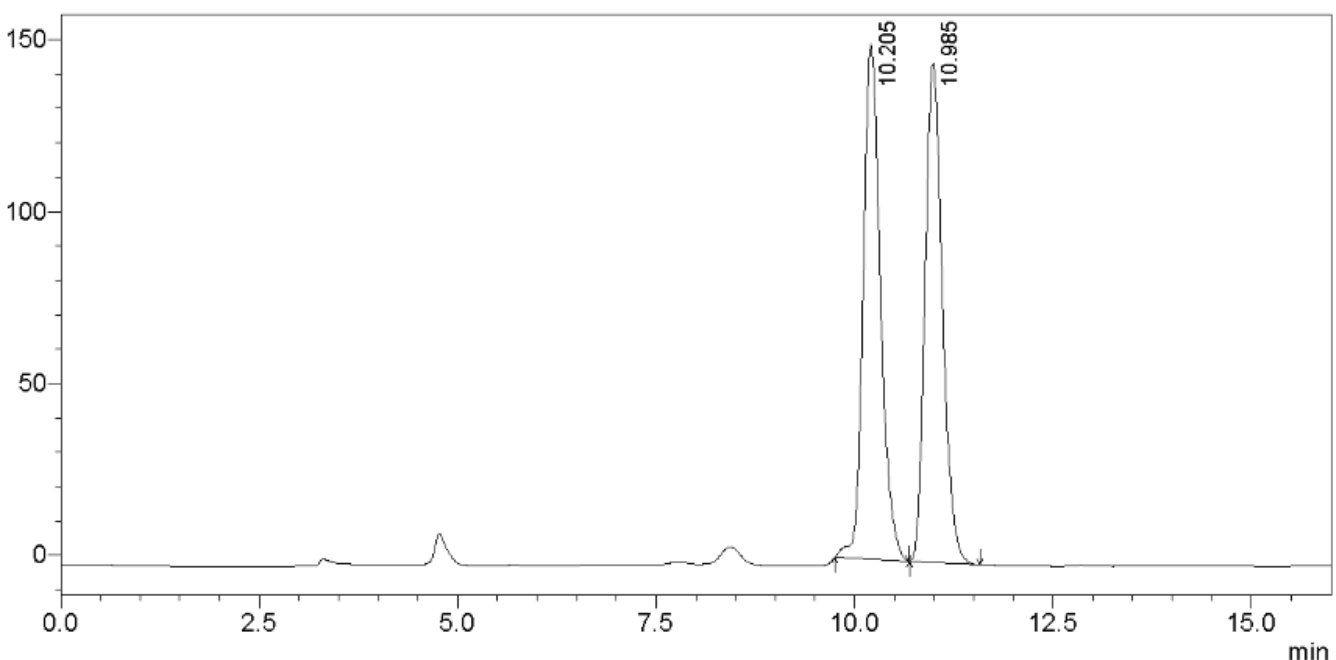

Detector A Ch1 254nm

\begin{tabular}{|r|r|r|r|}
\hline Peak\# & Ret. time & Area & Area $\varnothing$ \\
\hline 1 & 10.205 & 2272048 & 50.666 \\
\hline 2 & 10.985 & 2212355 & 49.334 \\
\hline & & 4484404 & 100.000 \\
\hline
\end{tabular}

\section{Chiral}

$\mathrm{mV}$

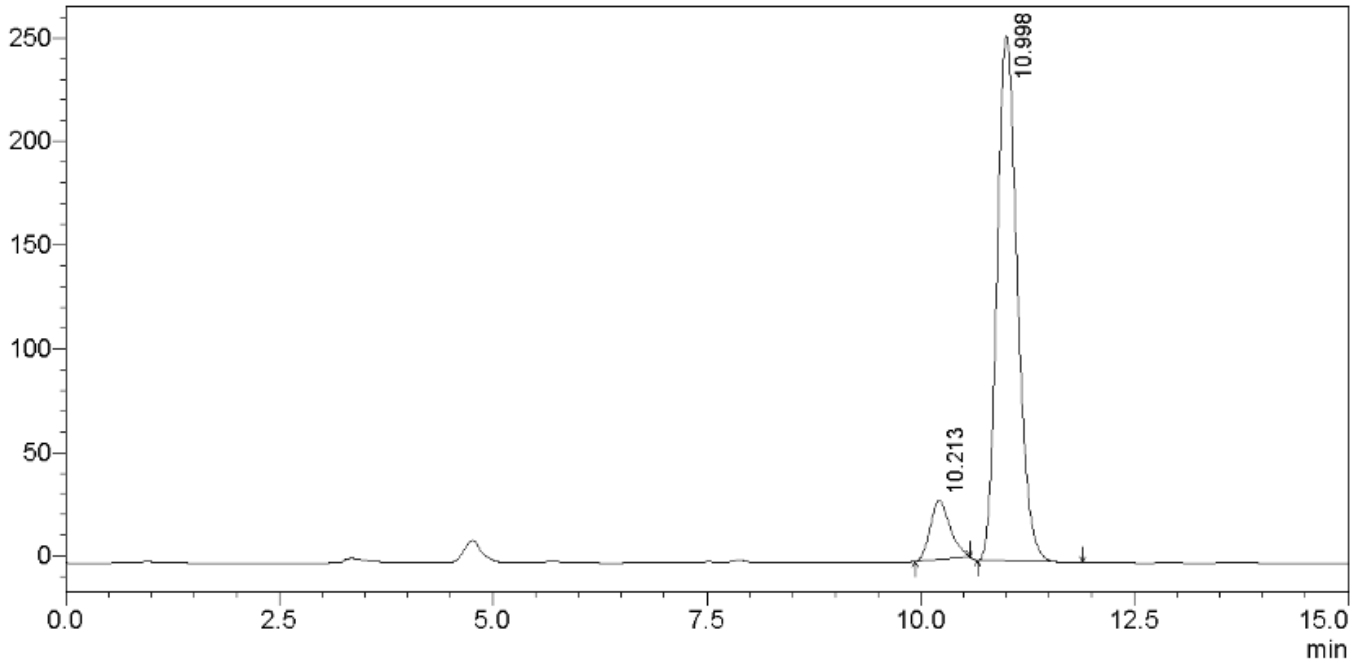

Detector A Ch1 254nm

\begin{tabular}{|r|r|r|r|}
\hline Peak\# & Ret. time & \multicolumn{1}{|c|}{ Area } & Area $\%$ \\
\hline 1 & 10.213 & 460595 & 9.870 \\
\hline 2 & 10.998 & 4205899 & 90.130 \\
\hline & & 4666495 & 100.000 \\
\hline
\end{tabular}




\section{Compound 5p}<smiles>N#CC1(C(=O)Br)C(=O)C=Cc2ccccc21</smiles>

\section{HPLC Conditions}

Column: Chiralcel AD-H, Daicel Chemical Industries, Ltd.

Eluent: Hexanes / isopropanol (95:05)

Flow rate: $1.0 \mathrm{~mL} / \mathrm{min}$

Detection: UV 254 nm

\section{Racemic}

$\mathrm{mV}$

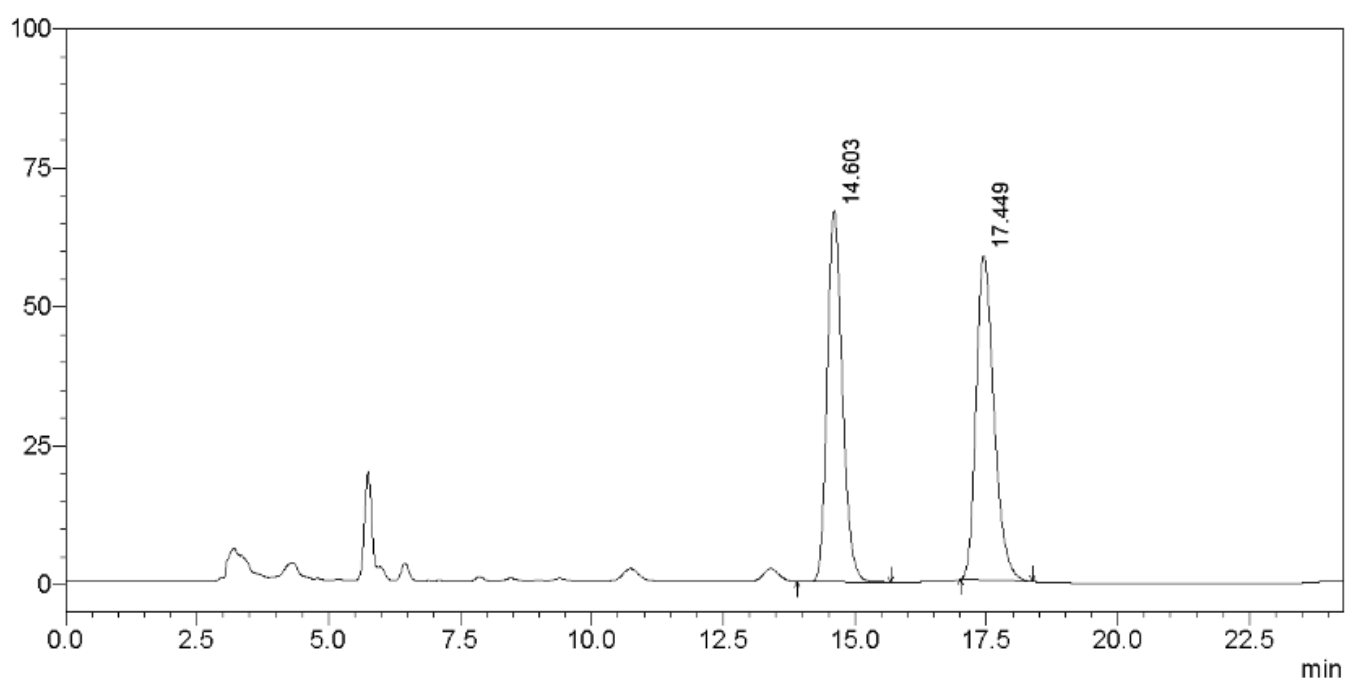

Detector A Ch1 254nm

Detector A Ch1 $254 \mathrm{~nm}$
\begin{tabular}{|r|r|r|r|}
\hline Peak\# & Ret. time & Area & Area \% \\
\hline 1 & 14.603 & 1344297 & 49.166 \\
\hline 2 & 17.449 & 1389931 & 50.834 \\
\hline & & 2734228 & 100.000 \\
\hline
\end{tabular}

\section{Chiral}

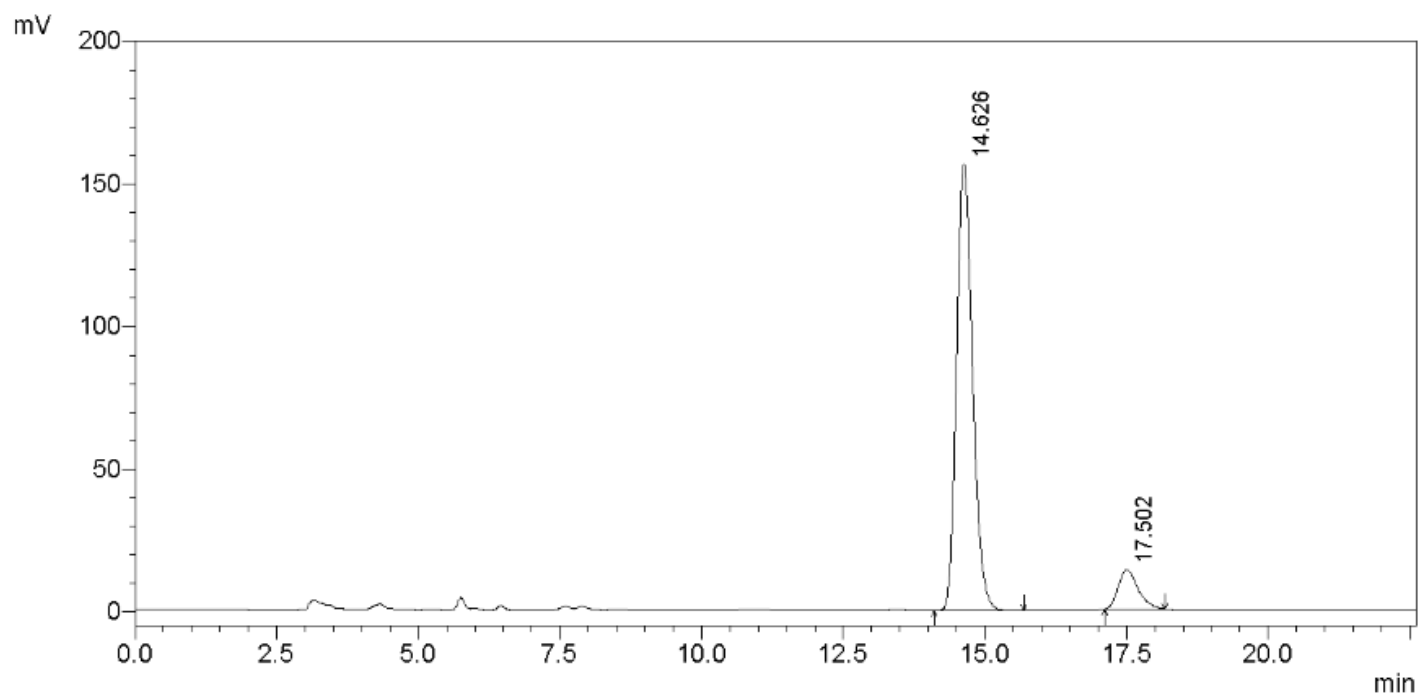

Detector A Ch1 254nm

\begin{tabular}{|r|r|r|r|}
\hline Peak\# & Ret. time & \multicolumn{1}{|c|}{ Area } & Area $\%$ \\
\hline 1 & 14.626 & 3082245 & 90.207 \\
\hline 2 & 17.502 & 334615 & 9.793 \\
\hline & & 3416860 & 100.000 \\
\hline
\end{tabular}




\section{Compound 6}

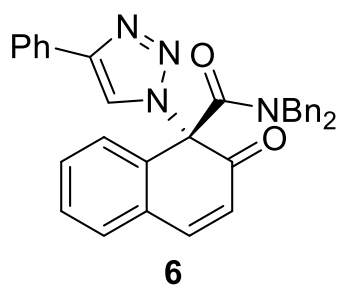

\section{HPLC Conditions}

Column: Chiralcel OD-H, Daicel Chemical Industries, Ltd.

Eluent: Hexanes / isopropanol (90:10)

Flow rate: $1.0 \mathrm{~mL} / \mathrm{min}$

Detection: UV $254 \mathrm{~nm}$

\section{Racemic}

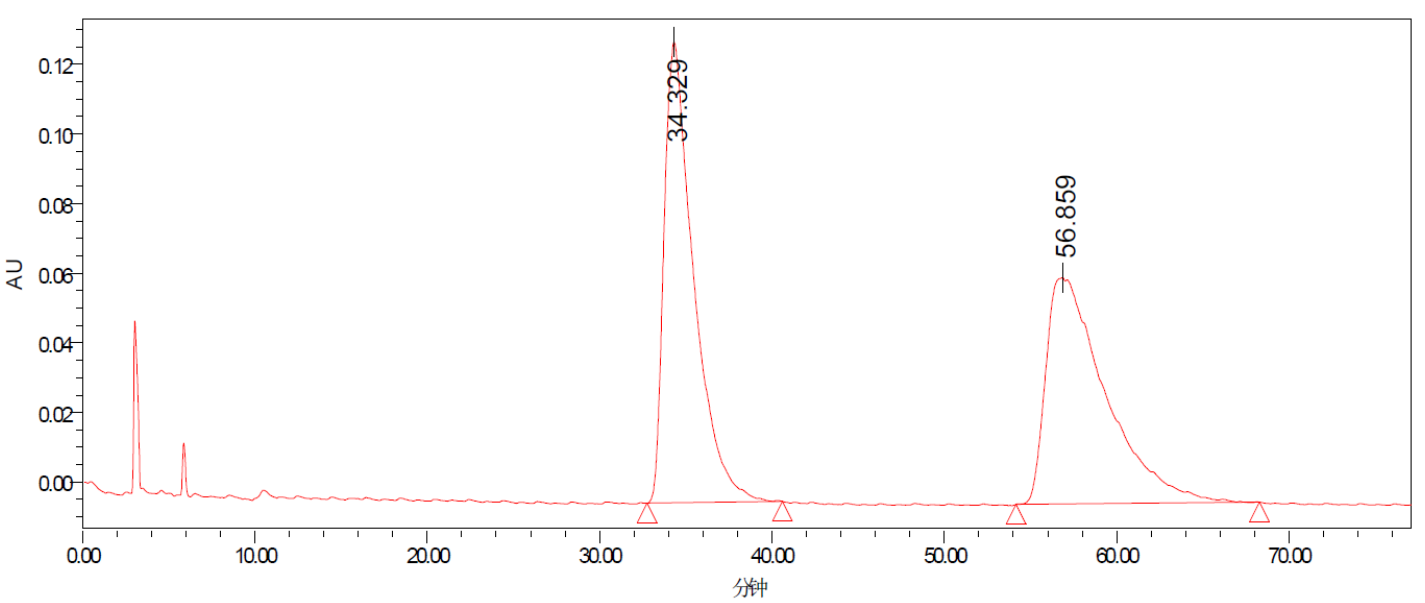

\begin{tabular}{|c|r|c|r|}
\hline & Ret. Time & Area & Area $\%$ \\
\hline 1 & 34.329 & 15806992 & 50.308 \\
\hline 2 & 56.859 & 15613596 & 49.692 \\
\hline Sum & & 31420588 & 100.000 \\
\hline
\end{tabular}

\section{Chiral}

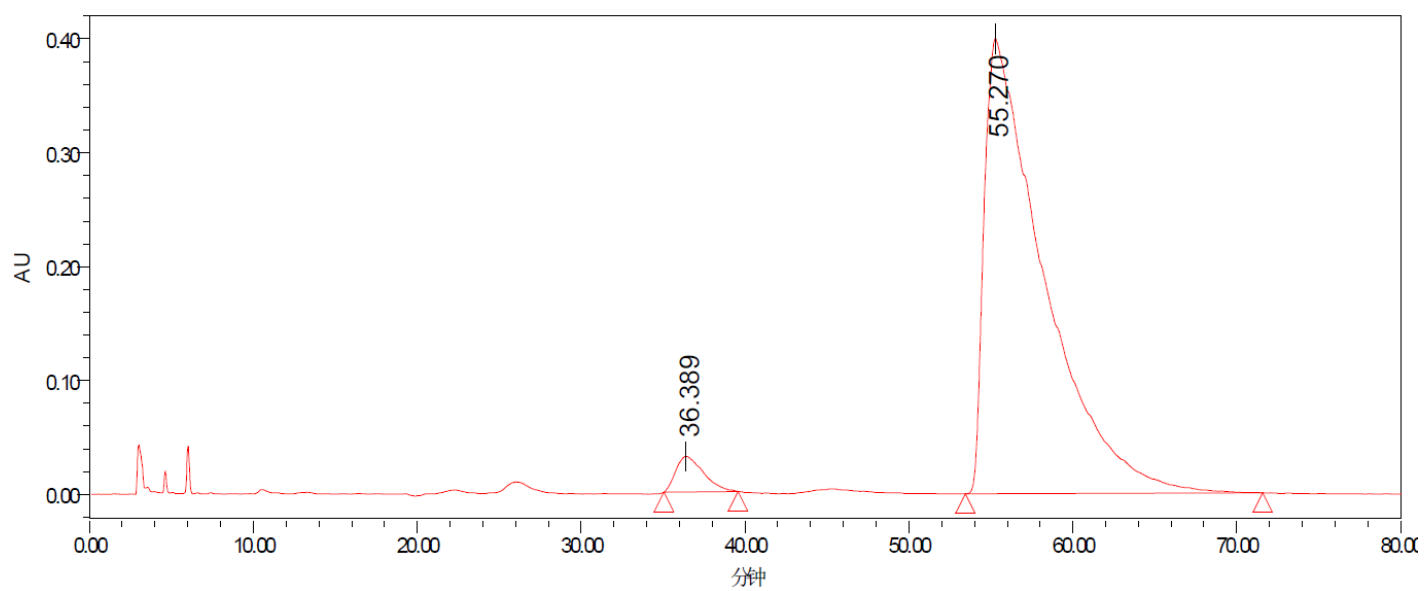

\begin{tabular}{|c|r|c|r|}
\hline & Pet. Time & Area & Area $\%$ \\
\hline 1 & 36.389 & 3656010 & 3.490 \\
\hline 2 & 55.270 & 101095393 & 96.510 \\
\hline Sum & & 104751403 & 100.000 \\
\hline
\end{tabular}




\section{Compound 7}

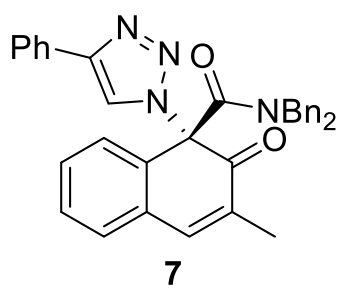

\section{HPLC Conditions}

Column: Chiralcel OD-H, Daicel Chemical Industries, Ltd.

Eluent: Hexanes / isopropanol (80:20)

Flow rate: $1.0 \mathrm{~mL} / \mathrm{min}$

Detection: UV $254 \mathrm{~nm}$

\section{Racemic}

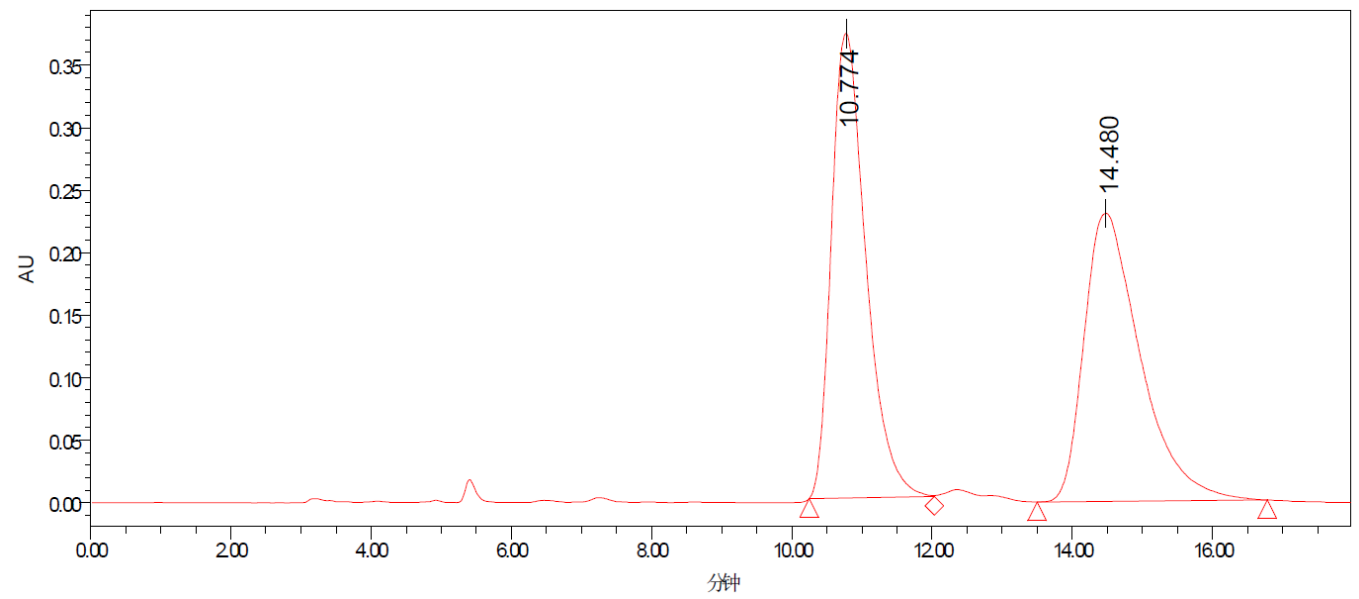

\begin{tabular}{|c|r|c|c|}
\hline & Ret. Time & Area & Area $\%$ \\
\hline 1 & 10.774 & 12705808 & 49.737 \\
\hline 2 & 14.480 & 12839971 & 50.263 \\
\hline Sum & & 25545779 & 100.000 \\
\hline
\end{tabular}

\section{Chiral}

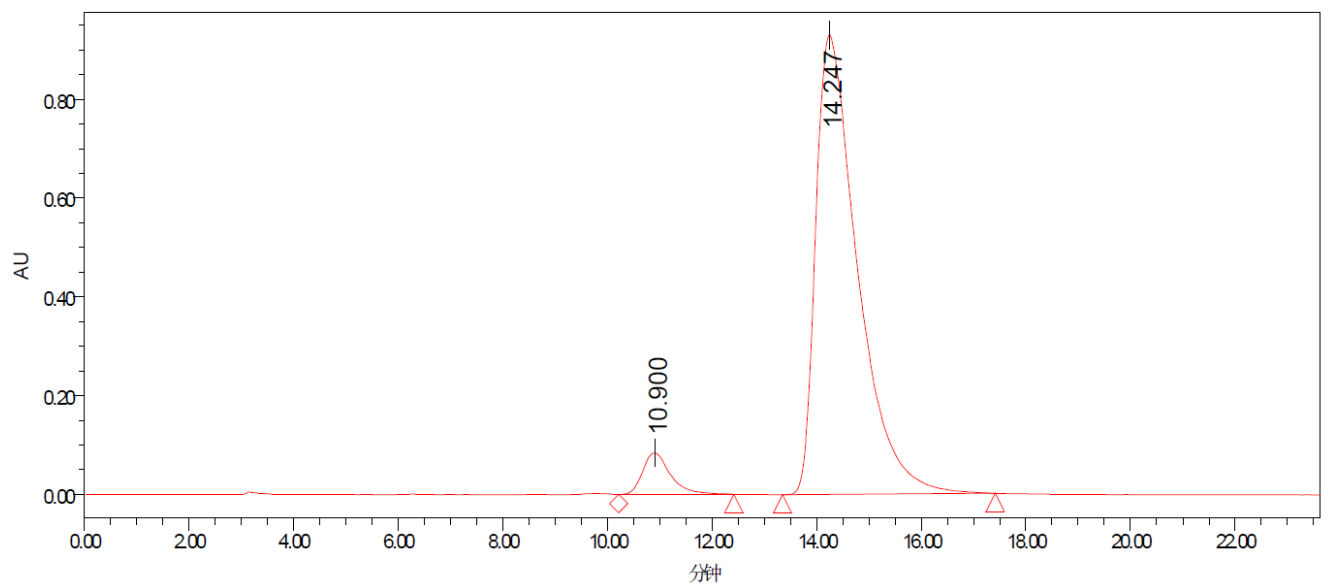

\begin{tabular}{|c|r|c|r|}
\hline & Ret. Time & Area & Area $\%$ \\
\hline 1 & 10.900 & 3078694 & 5.625 \\
\hline 2 & 14.247 & 51651770 & 94.375 \\
\hline Sum & & 54730464 & 100.000 \\
\hline
\end{tabular}




\section{Compound 8}

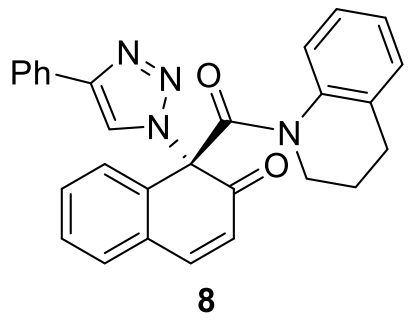

\section{HPLC Conditions}

Column: Chiralcel AS-H, Daicel Chemical Industries, Ltd. Eluent: Hexanes / isopropanol (80:20)

Flow rate: $1.0 \mathrm{~mL} / \mathrm{min}$

Detection: UV $254 \mathrm{~nm}$

\section{Racemic}

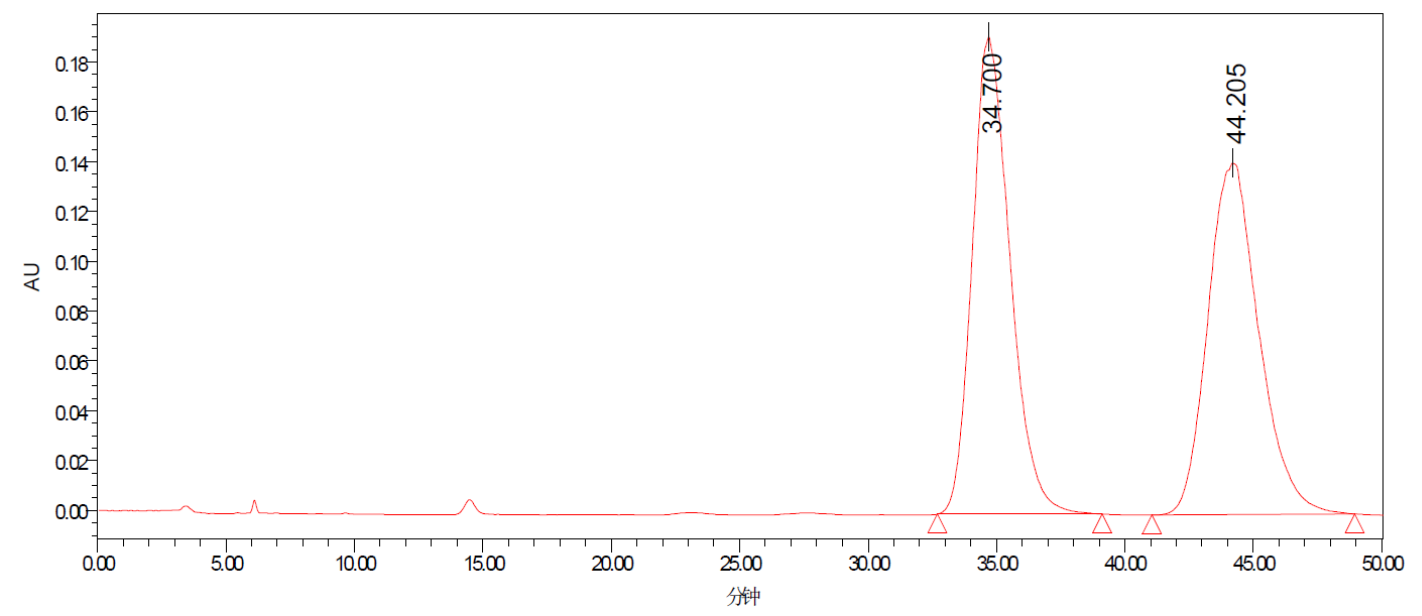

\begin{tabular}{|c|r|c|c|}
\hline & Ret. Time & Area & Area $\%$ \\
\hline 1 & 34.700 & 19604178 & 50.145 \\
\hline 2 & 44.205 & 19490960 & 49.855 \\
\hline S.m & & 39095138 & 100.000 \\
\hline
\end{tabular}

\section{Chiral}

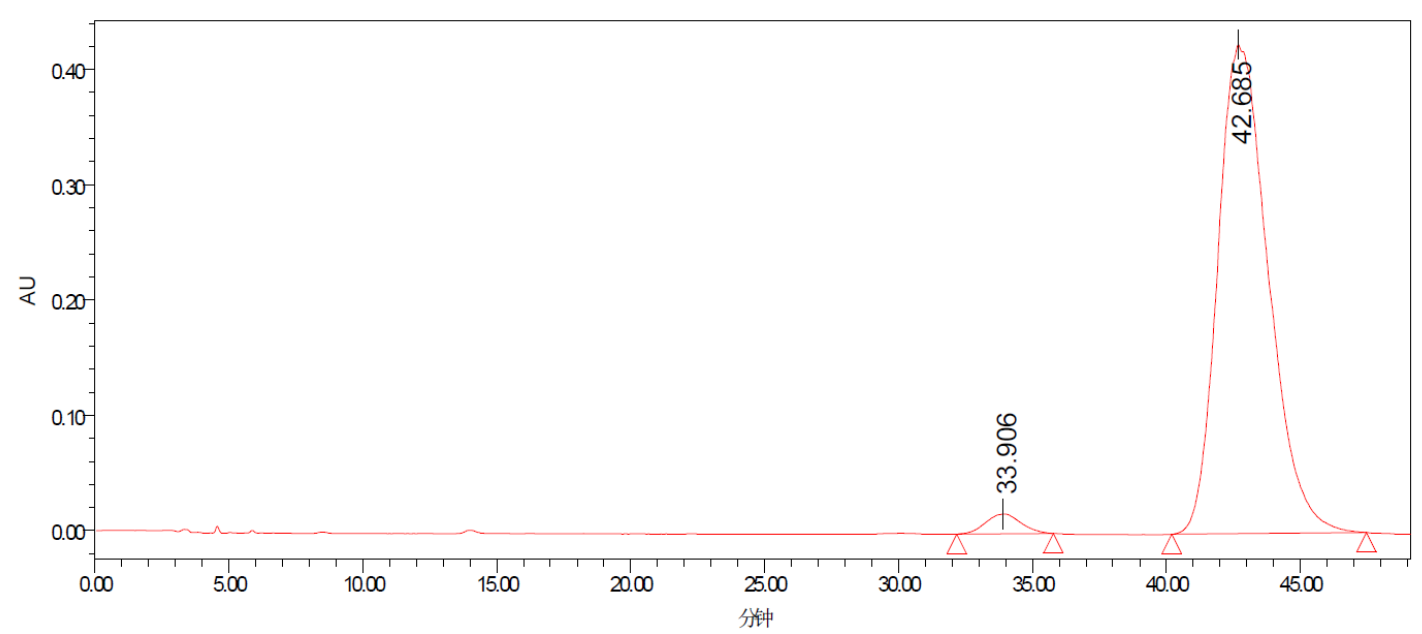

\begin{tabular}{|c|r|r|r|}
\hline & Ret. Time & Area & Area \% \\
\hline 1 & 33.906 & 1563936 & 2736 \\
\hline 2 & 42685 & 55594040 & 97.264 \\
\hline Sum & & 57157977 & 100.000 \\
\hline
\end{tabular}




\section{Compound 9}<smiles>COC(=O)C1(C(=O)C2(C)CCCCC2)C(=O)C=Cc2ccccc21</smiles>

\section{HPLC Conditions}

Column: Chiralcel OJ-H, Daicel Chemical Industries, Ltd.

Eluent: Hexanes / isopropanol (75:25)

Flow rate: $1.5 \mathrm{~mL} / \mathrm{min}$

Detection: UV $254 \mathrm{~nm}$

\section{Racemic}

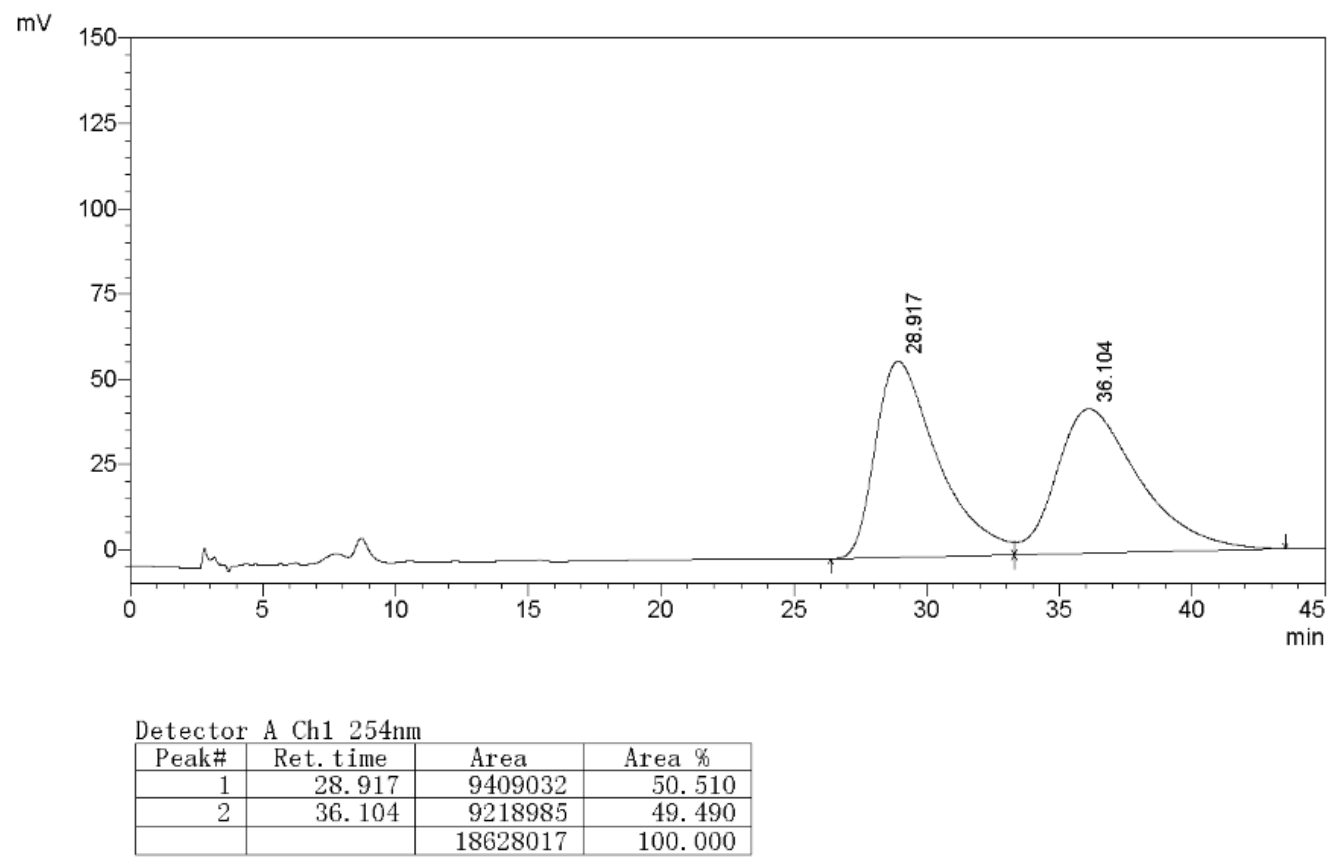

\section{Chiral}

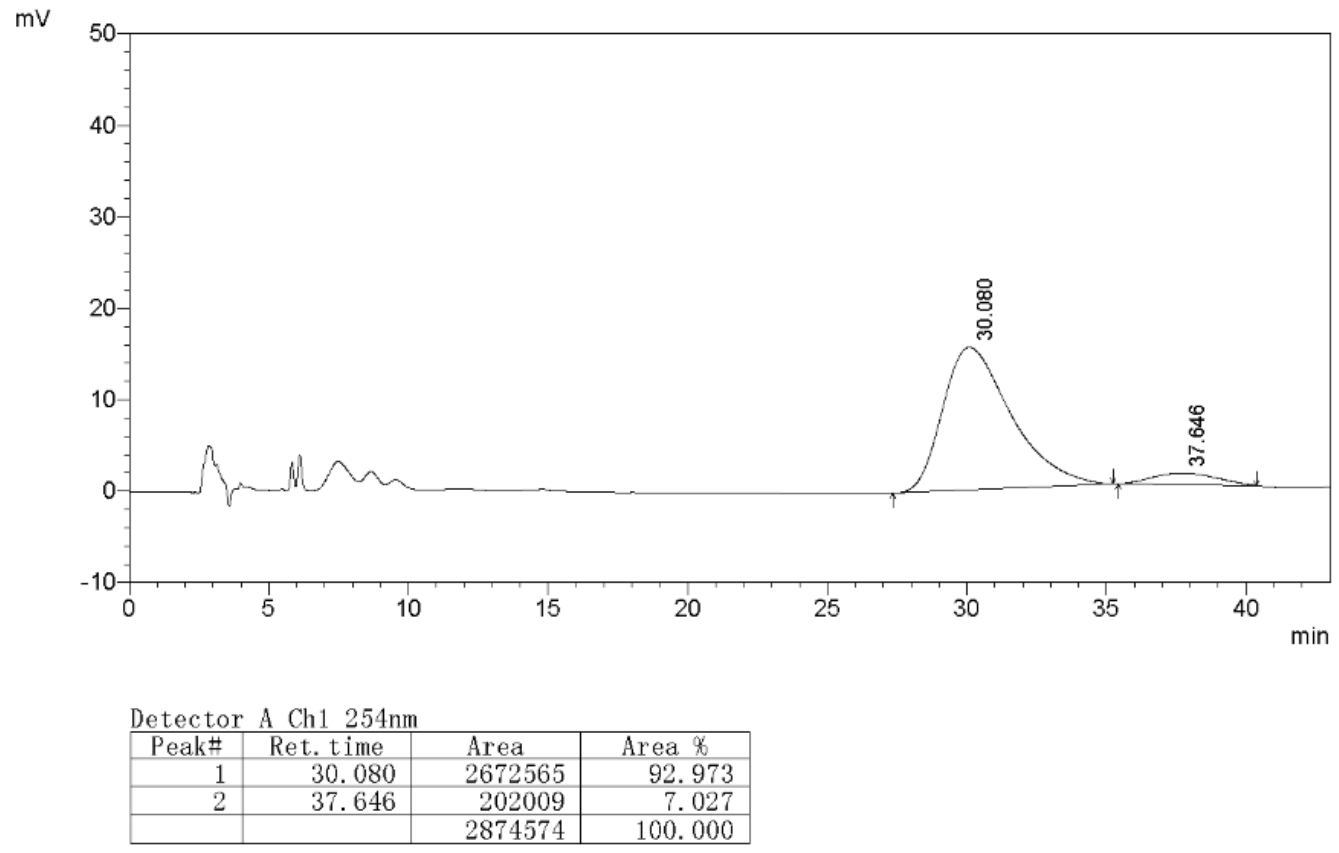


<smiles>CCCCN(C(=O)O)C(=O)c1c(O)ccc2ccccc12</smiles>

$1 \mathrm{~b}$

IX. NMR Spectra of New Compounds $\left({ }^{1} \mathrm{H}\right.$ NMR, ${ }^{13} \mathrm{C}$ NMR, ${ }^{19}$ F NMR)

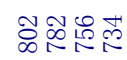

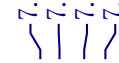

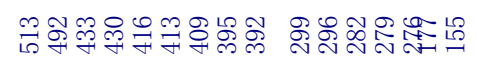

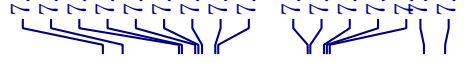

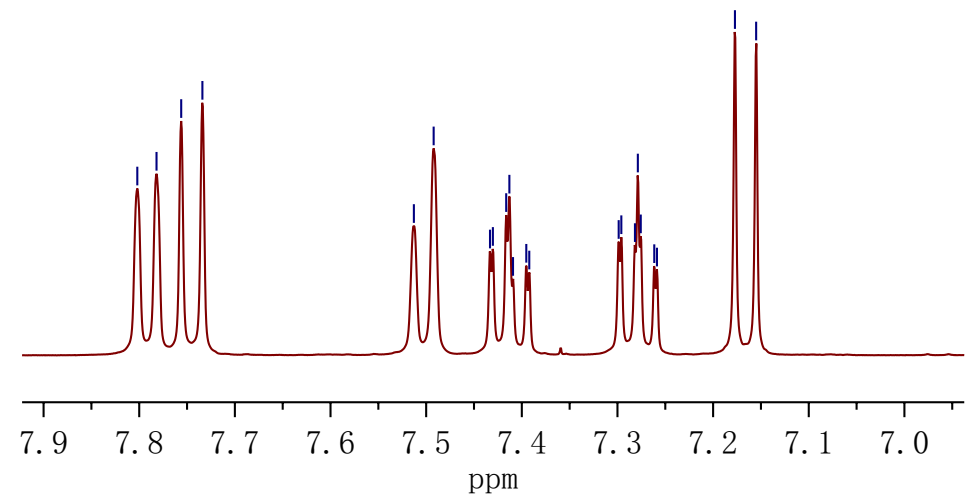

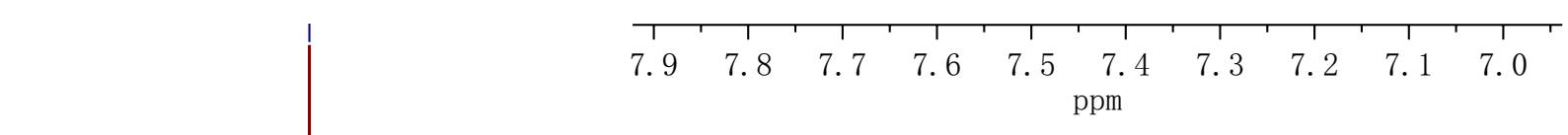
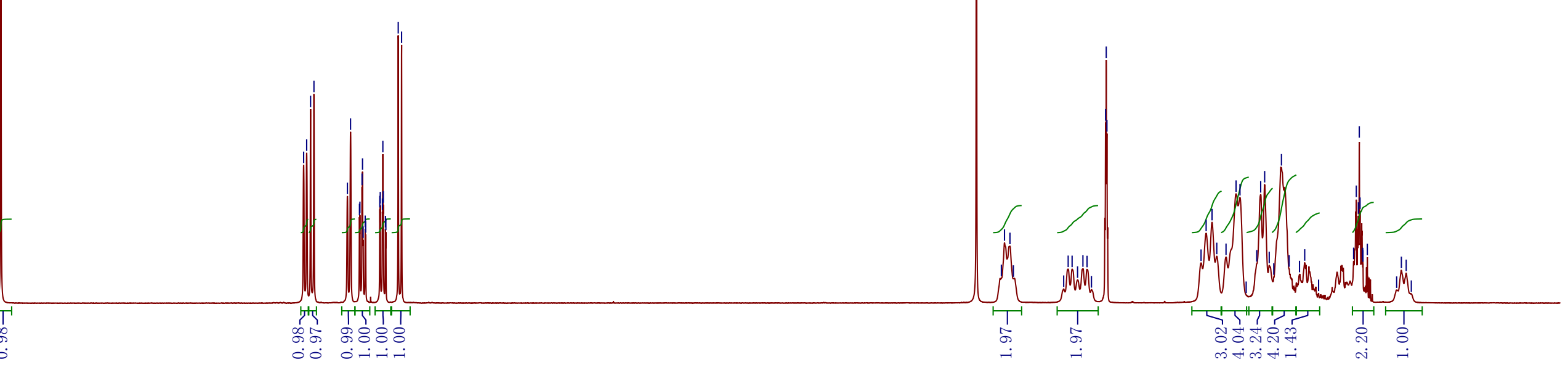

9.5

$9.0 \quad 8.5$

$8.0 \quad 7.5$

$7.0 \quad 6.5$

6. 0

5. 5 S92 5.0

4.5

4. 0

3.5

3. 2. 


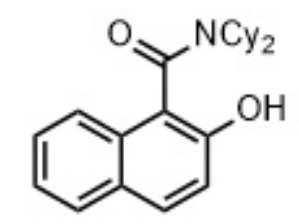

1b

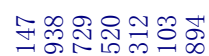

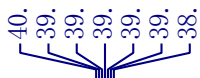

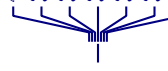

0
0

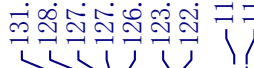

Mil Y

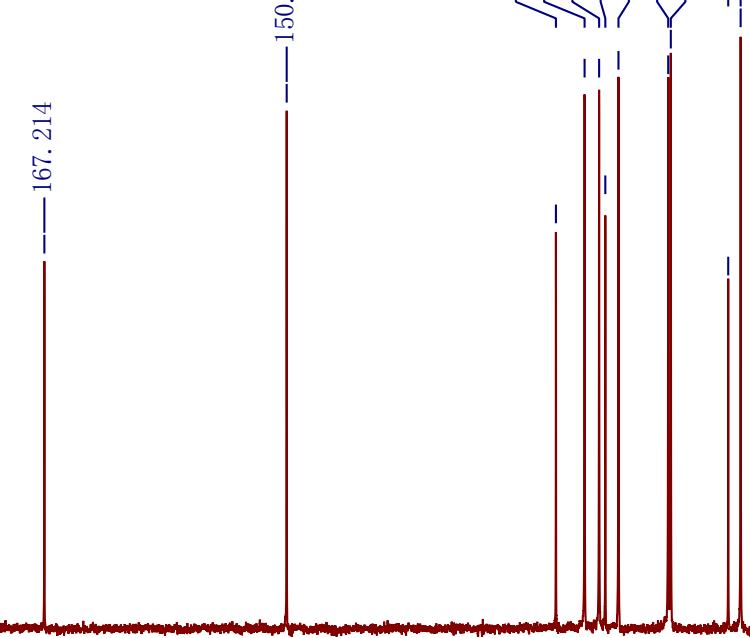

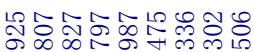

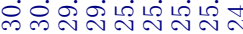

YYY
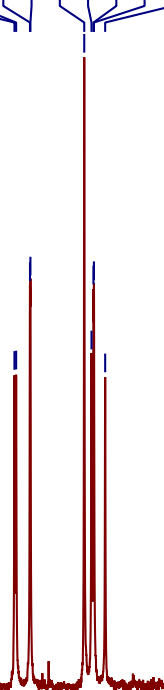
<smiles>O=C(c1ccccc1)c1c(O)ccc2ccccc12</smiles>

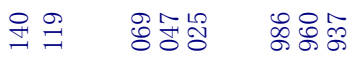

il
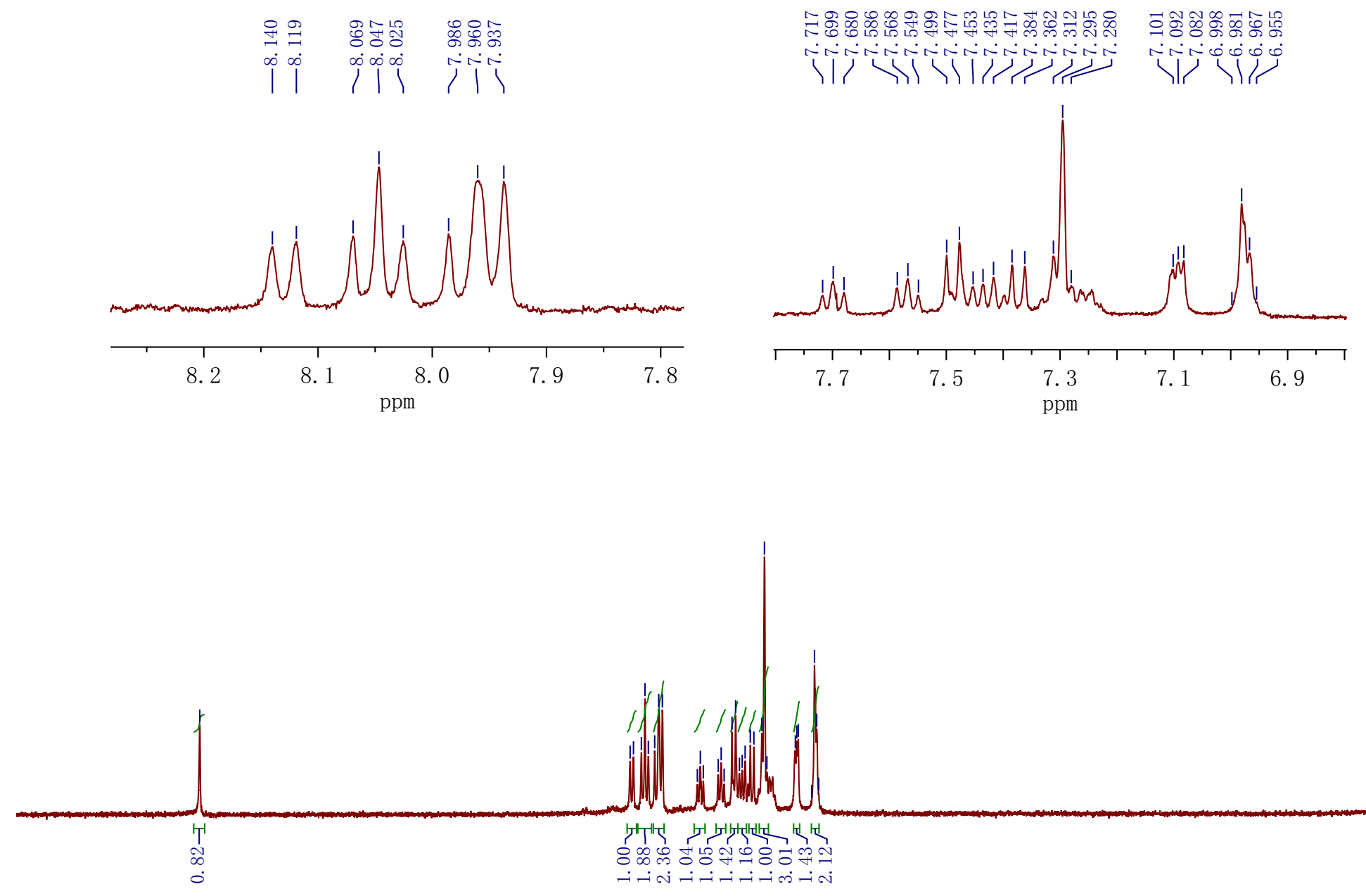

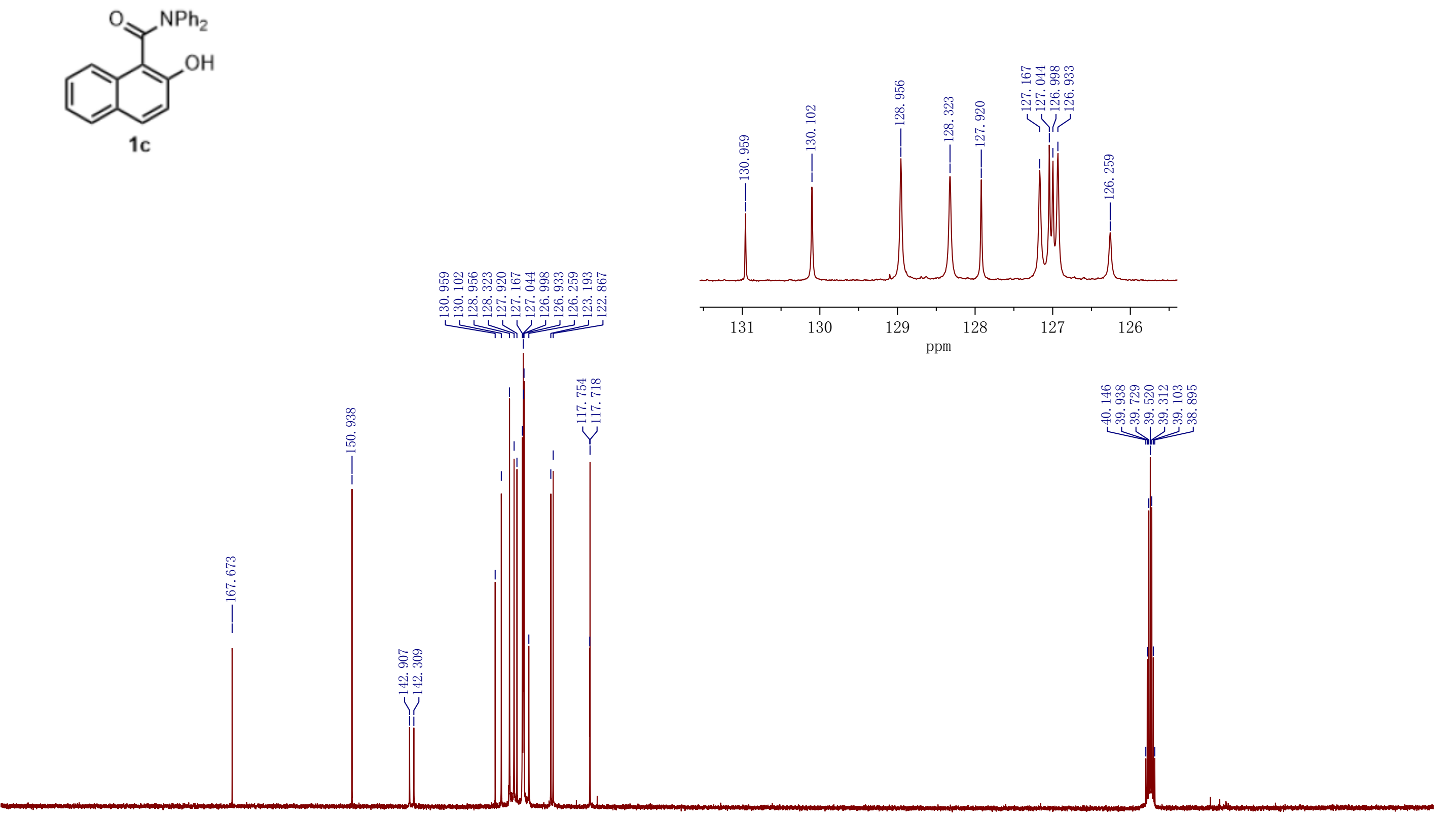

\begin{tabular}{|c|c|c|c|c|c|c|c|c|c|c|c|c|c|c|c|c|c|c|c|}
\hline & $\begin{array}{r}1 \\
180\end{array}$ & 170 & 160 & $\begin{array}{c}1 \\
150\end{array}$ & 140 & 130 & 120 & 110 & $\$ 95$ & 90 & $\begin{array}{l}1 \\
80\end{array}$ & $\begin{array}{c}1 \\
70\end{array}$ & 1 & $\begin{array}{l}1 \\
50\end{array}$ & 40 & 30 & $\begin{array}{l}1 \\
20\end{array}$ & $\begin{array}{l}1 \\
10\end{array}$ \\
\hline
\end{tabular}



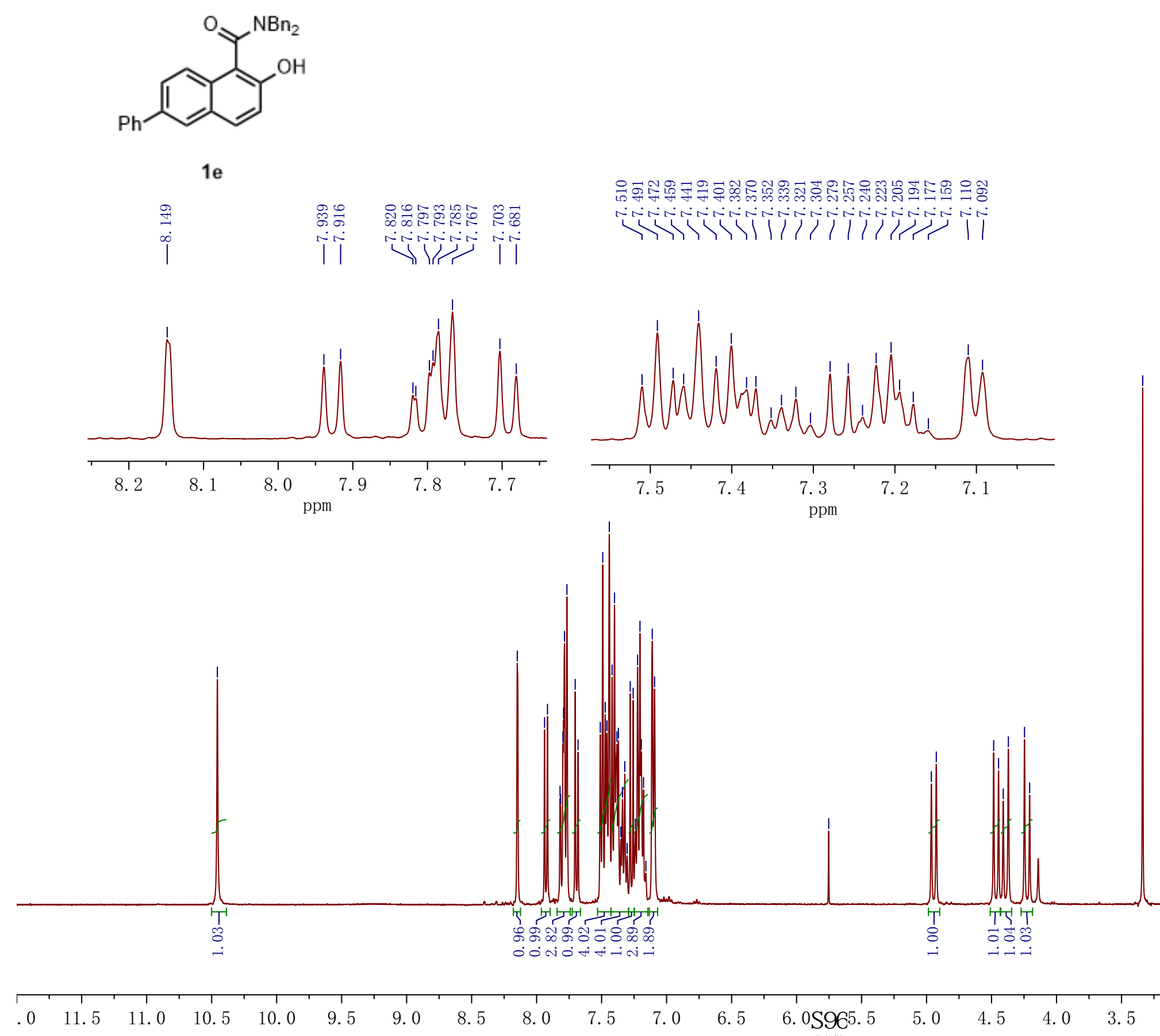

$\underset{\mathrm{ppm}}{0 \mathrm{~S} 96 .}$ 


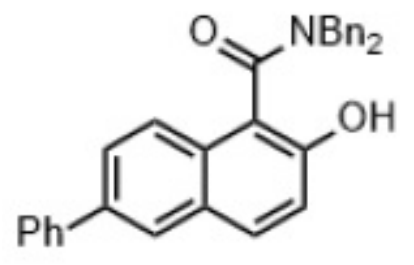

$1 \mathrm{e}$

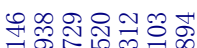

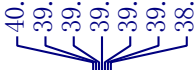

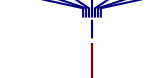

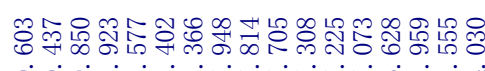

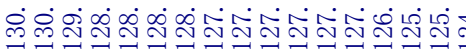

Tाinा।

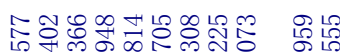

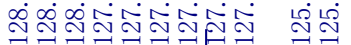

动
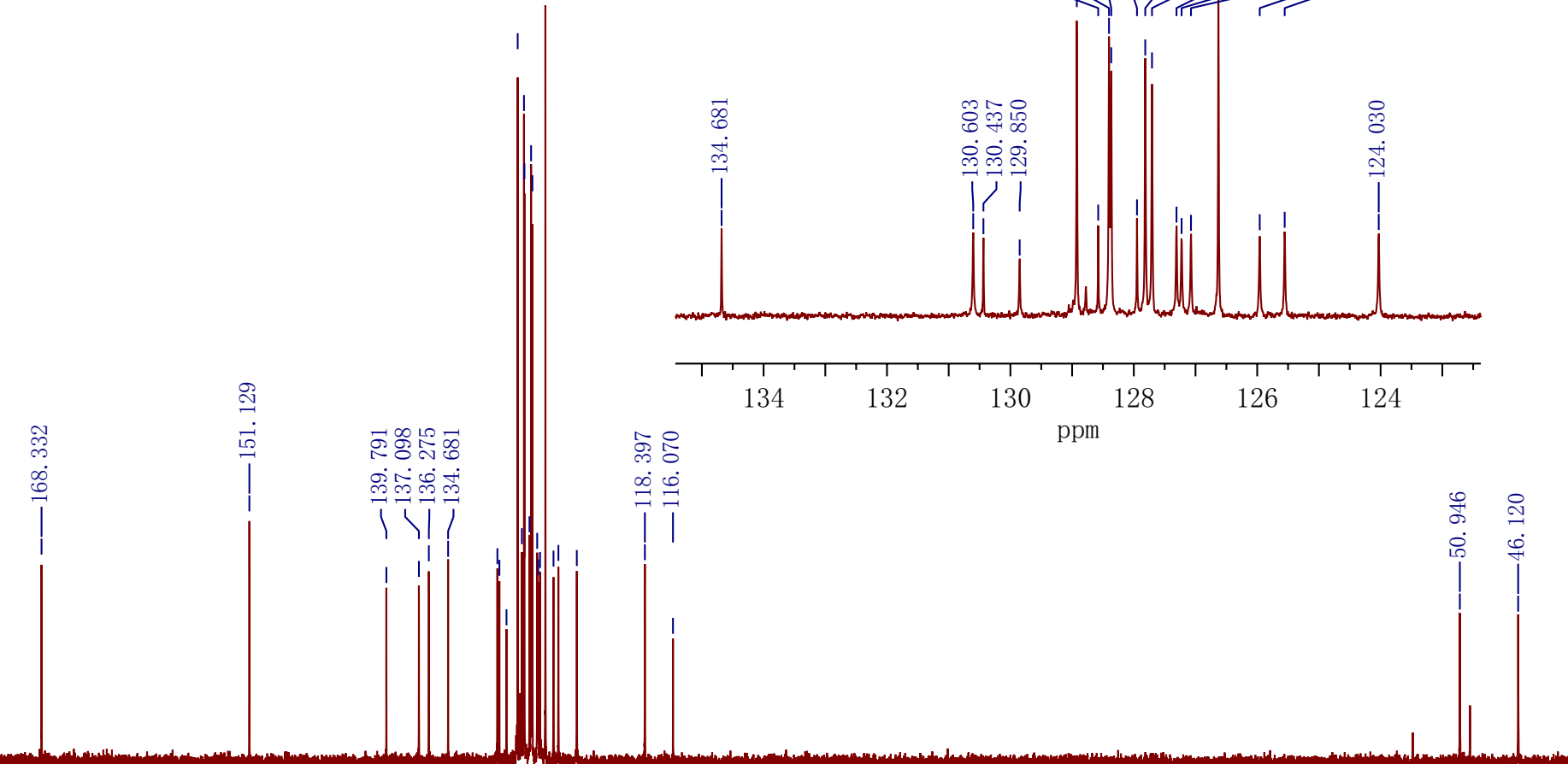

24

$90 \quad 180$

170

160

150

140

130

120

110

$\$ 99$

90

80

70

60

50

40

20 


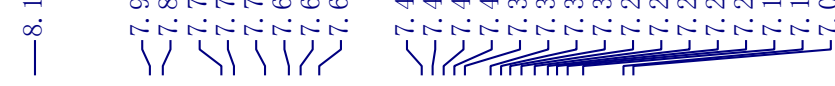
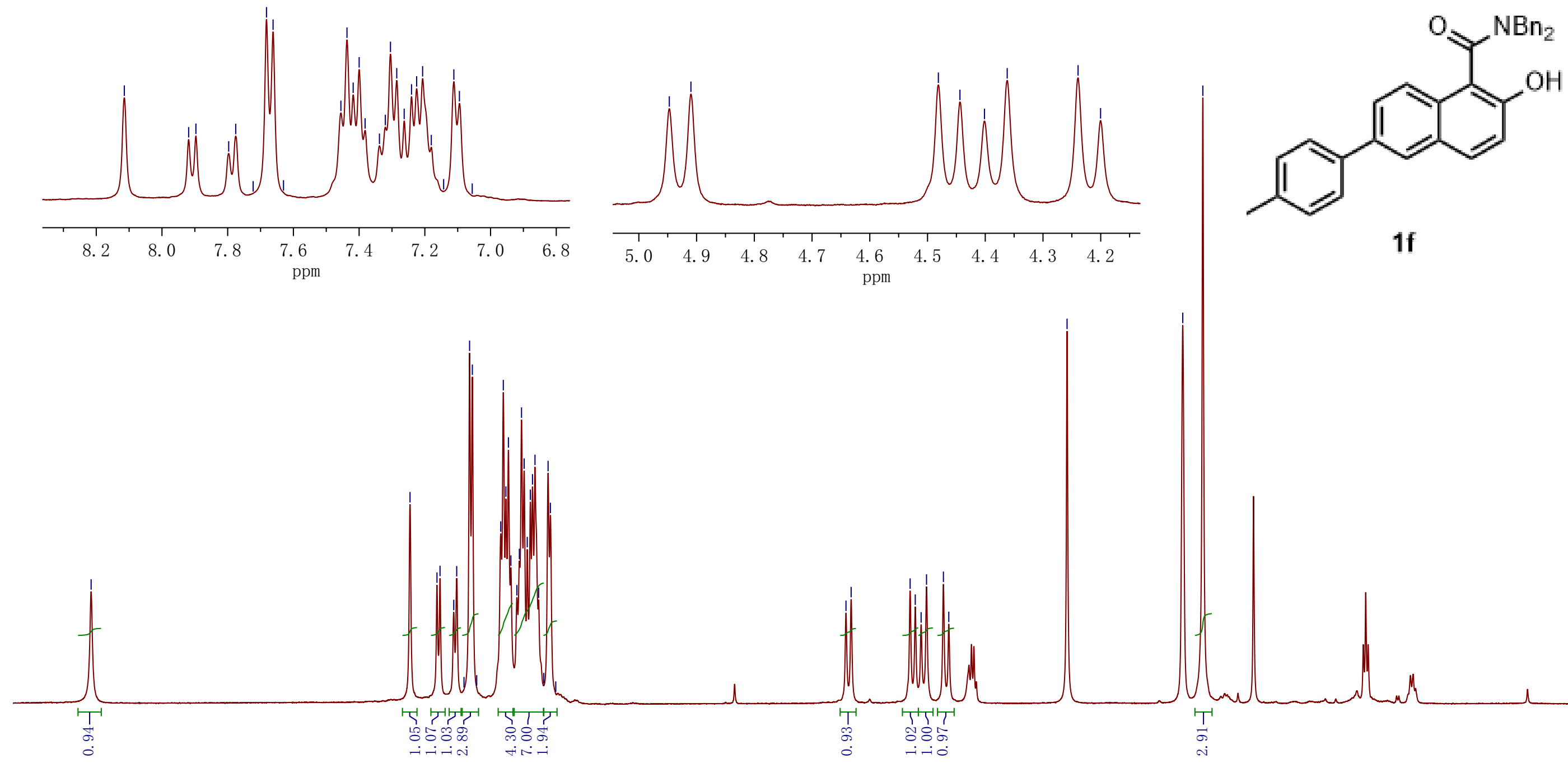

ग 

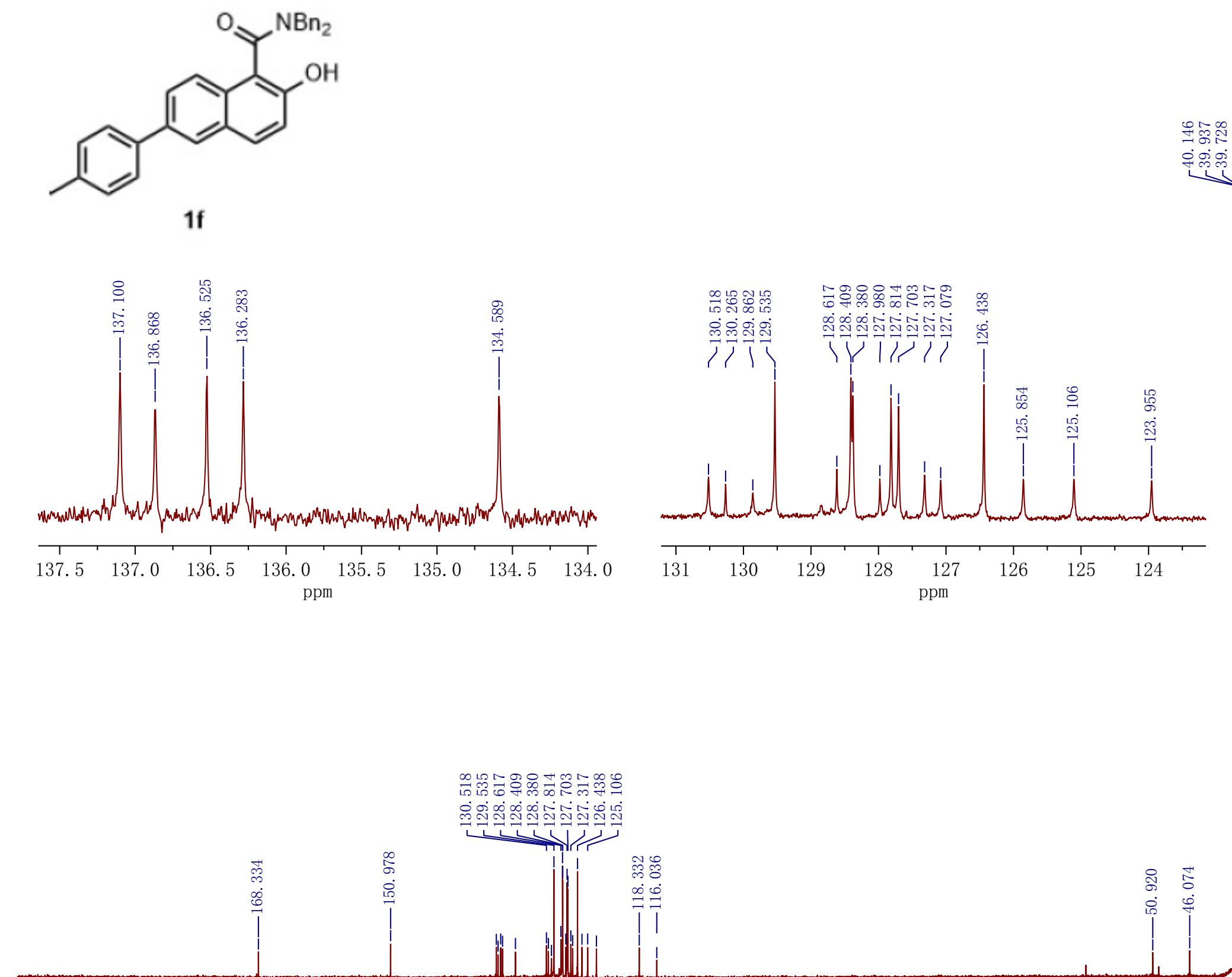

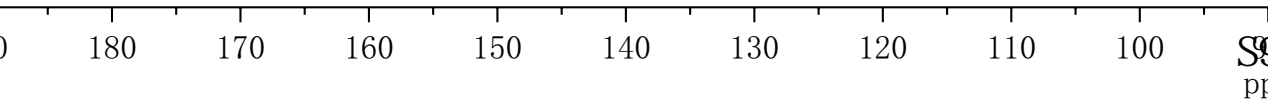




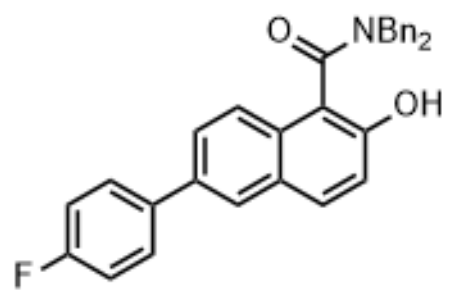

$1 \mathrm{~g}$

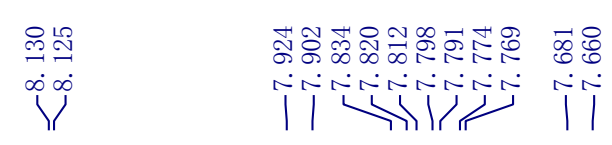

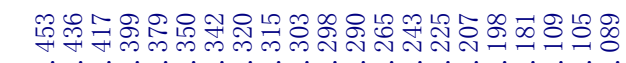

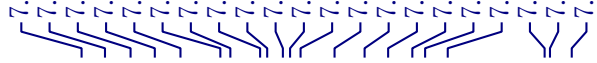
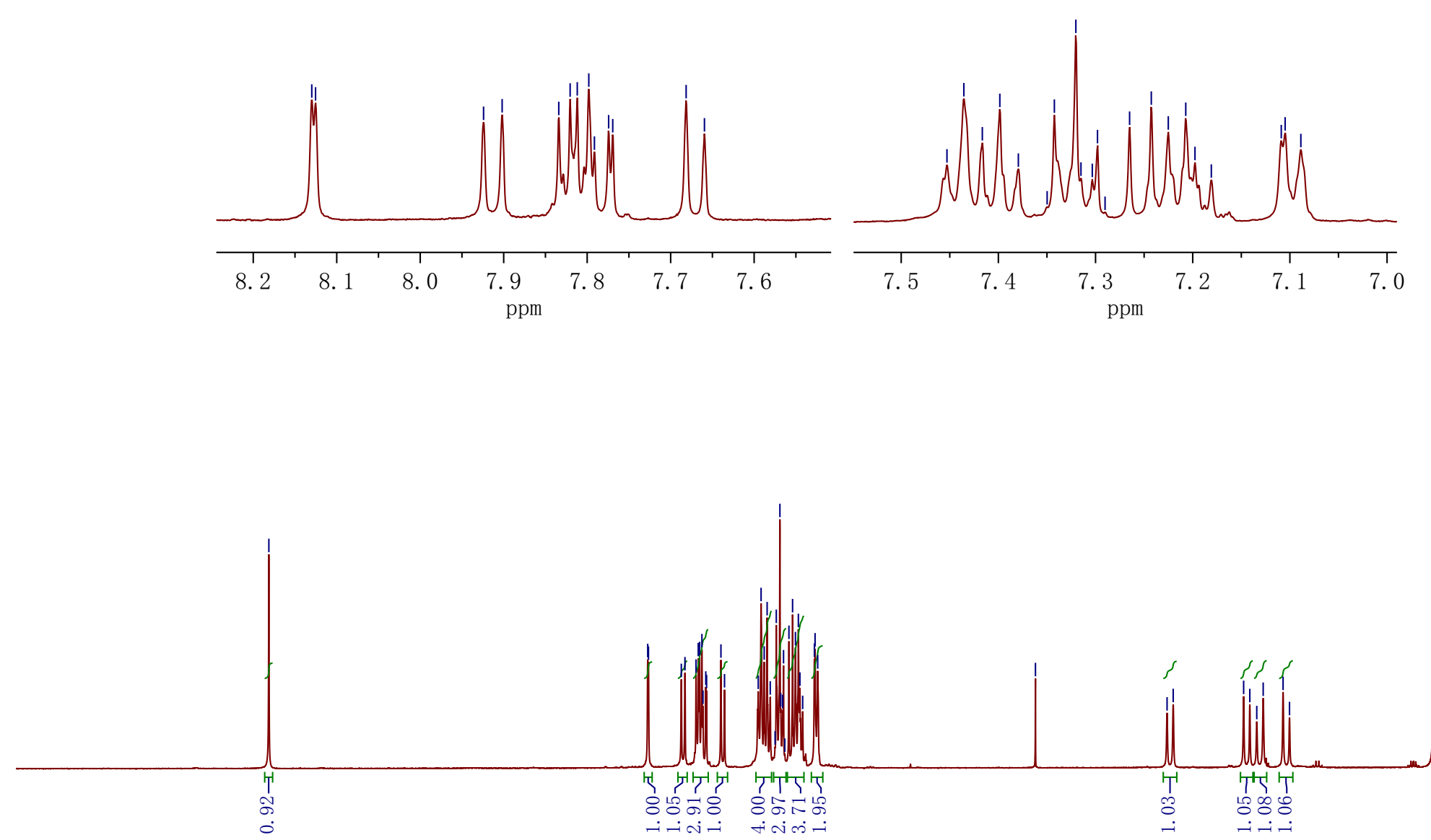

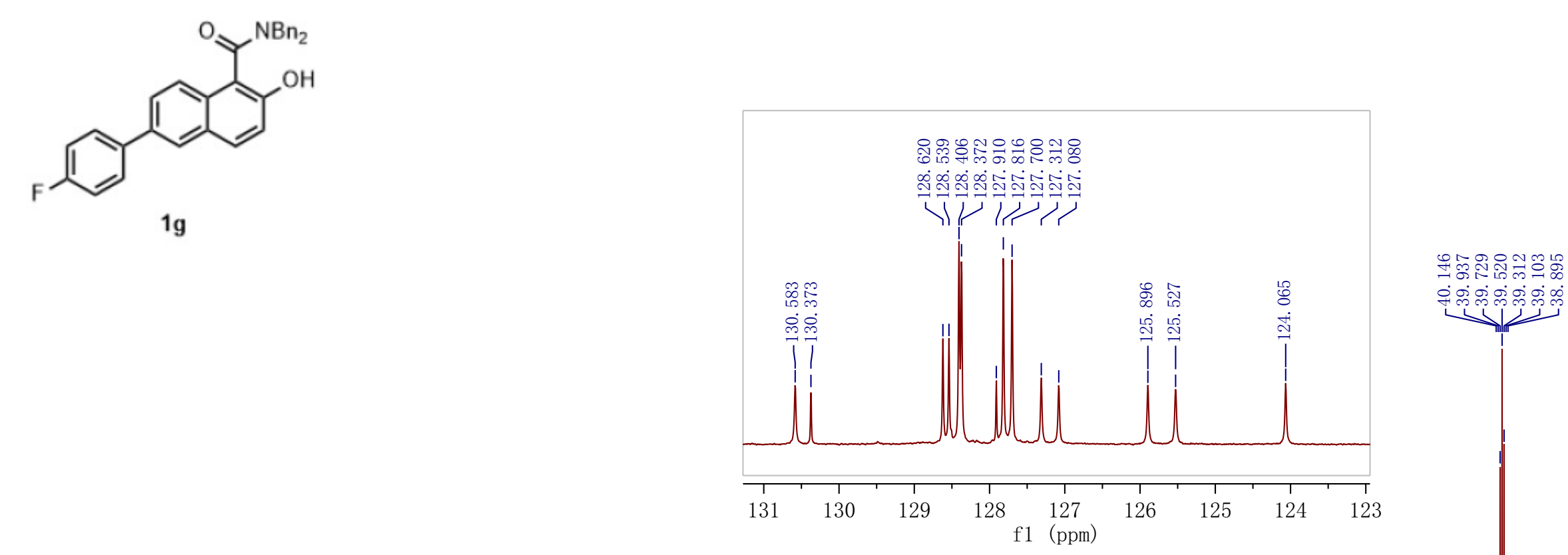

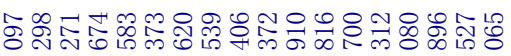

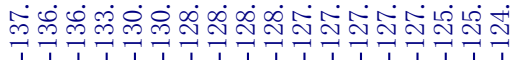
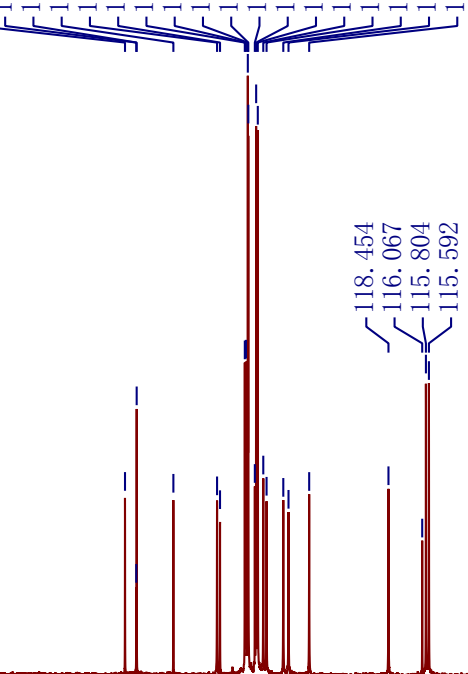


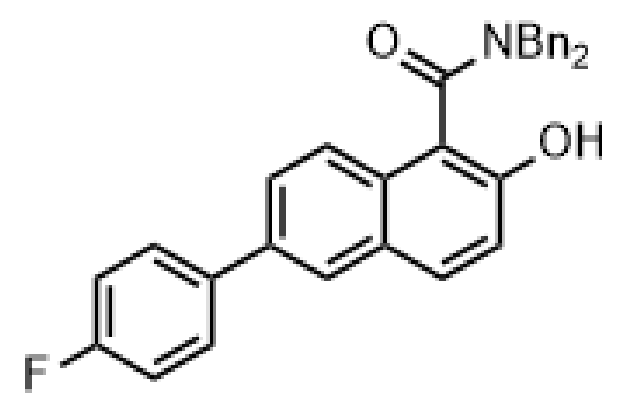

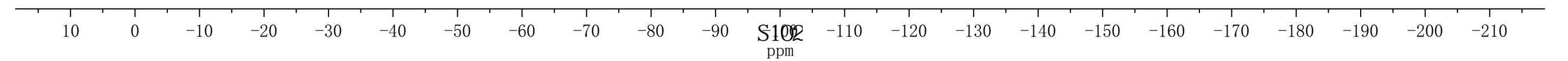



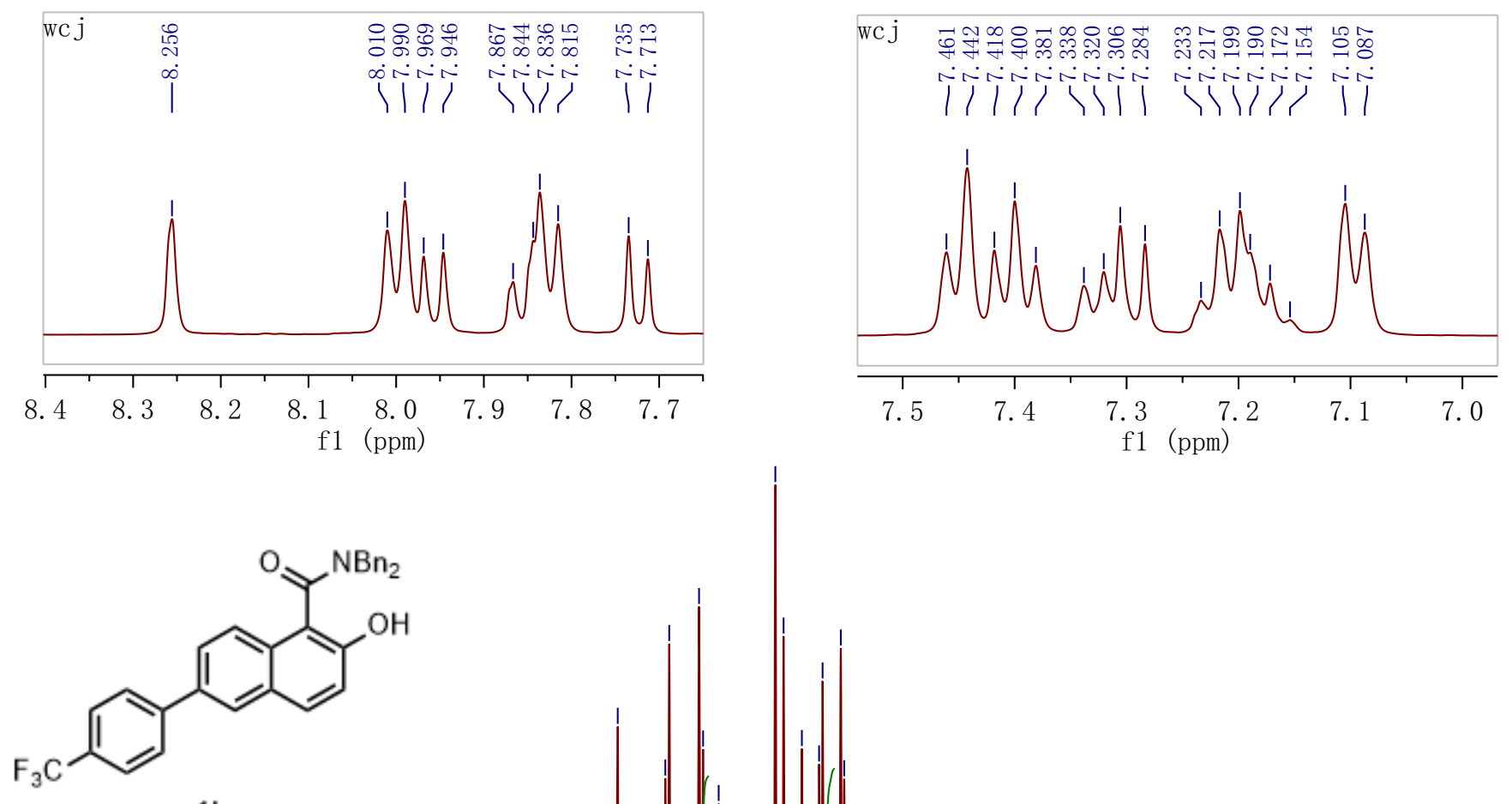

1h
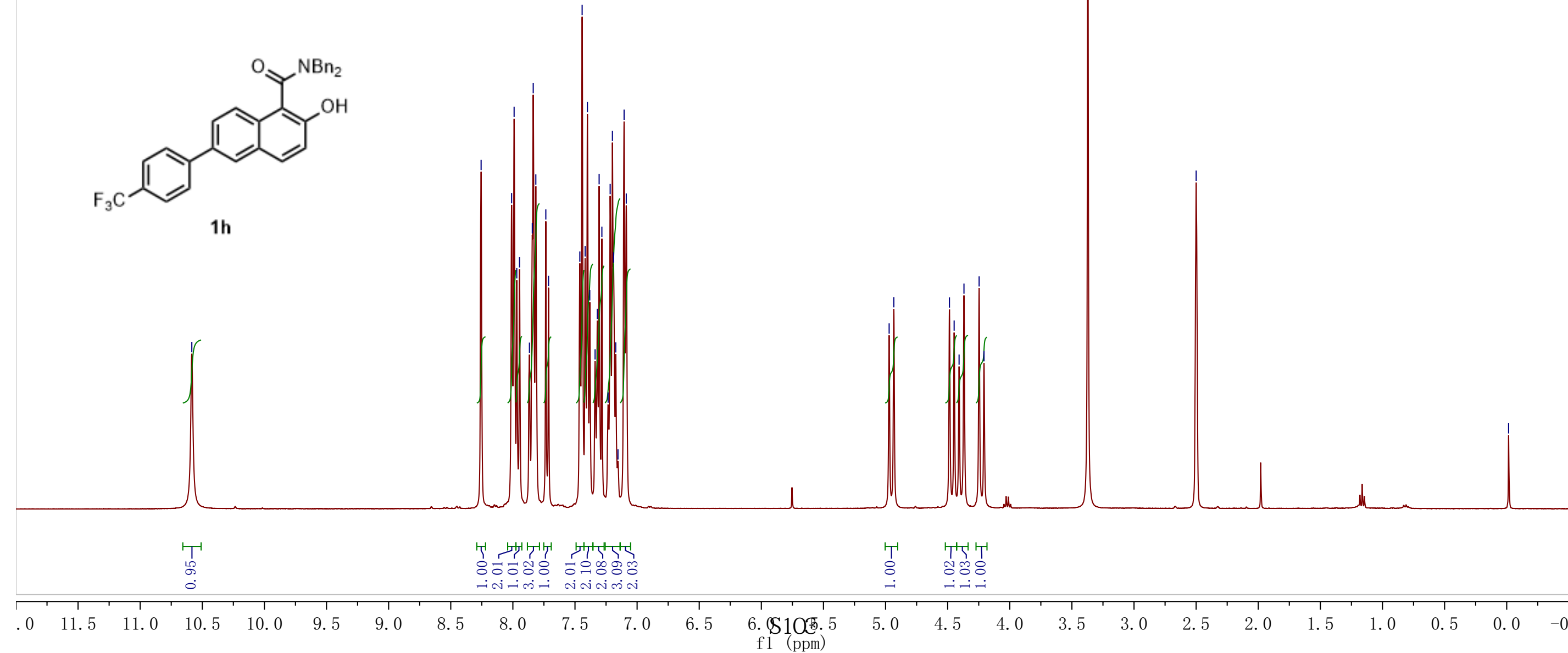


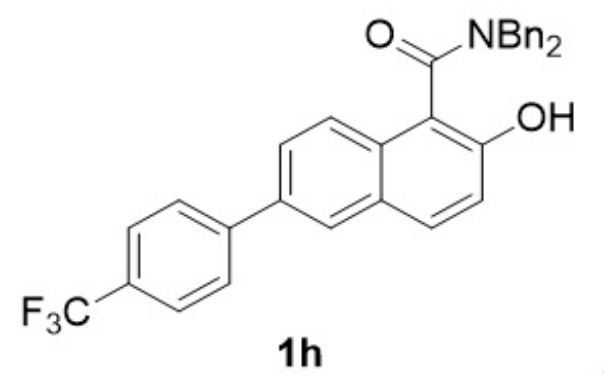

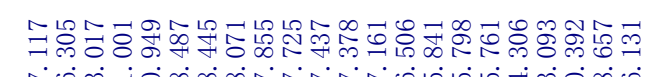

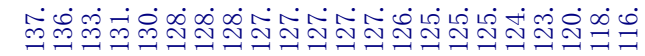

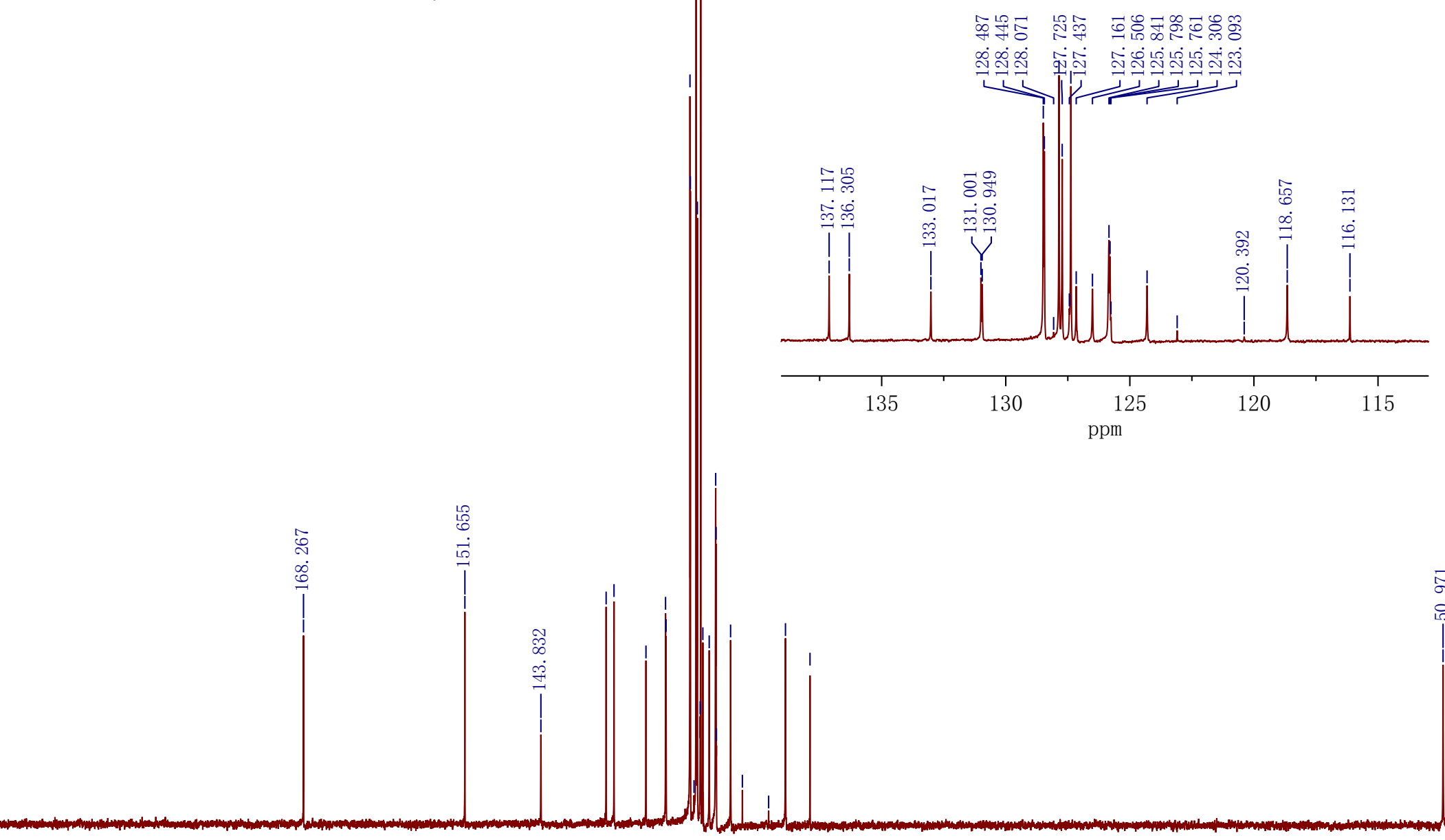

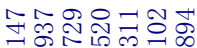

0000000

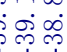

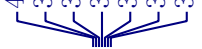




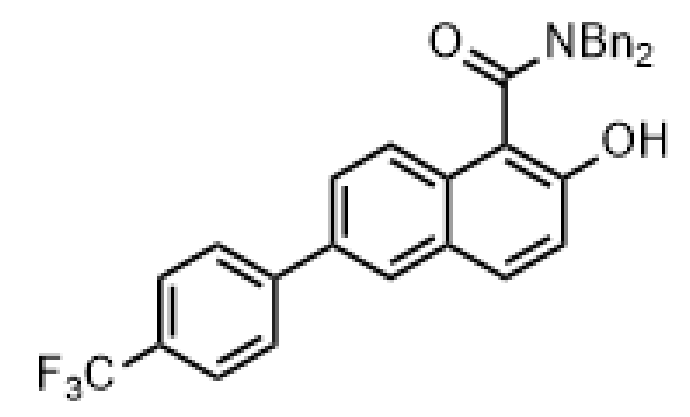

1h
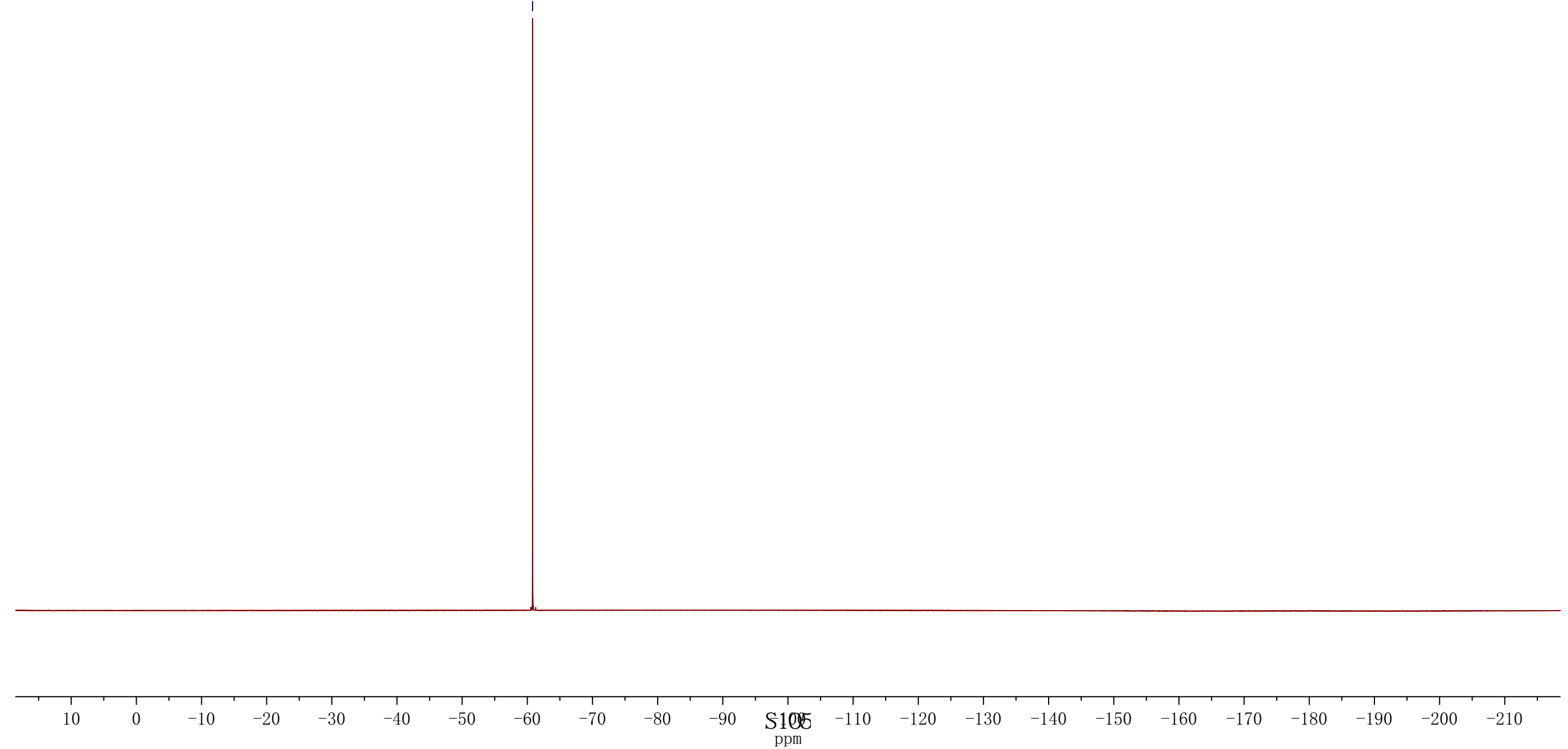
<smiles>CCCCC(=O)c1c(O)ccc2ccc(-c3ccccc3)cc12</smiles>

$1 \mathrm{i}$

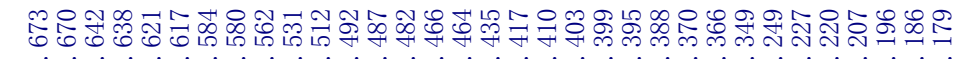

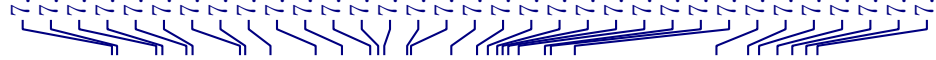

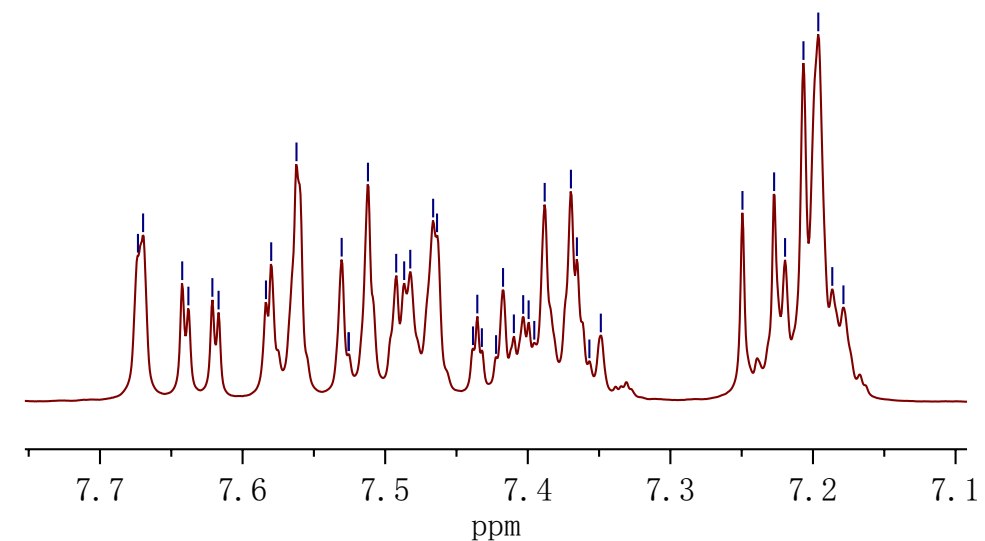

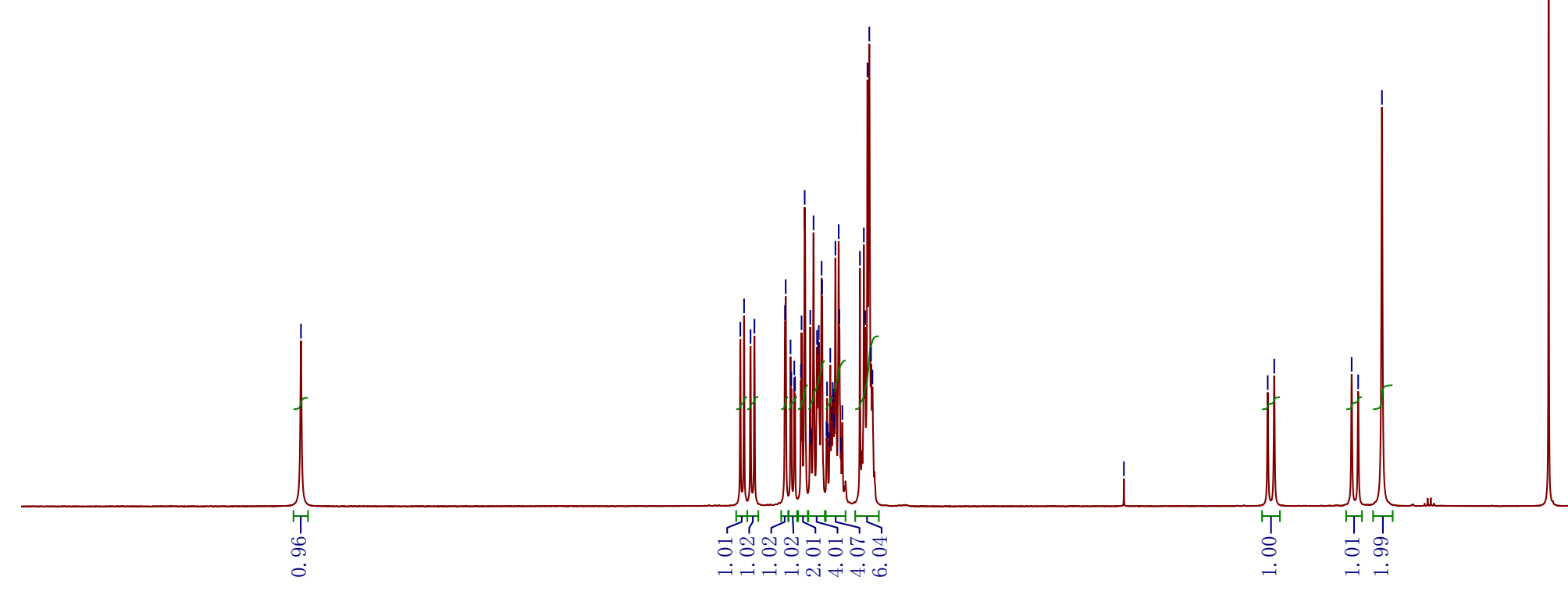




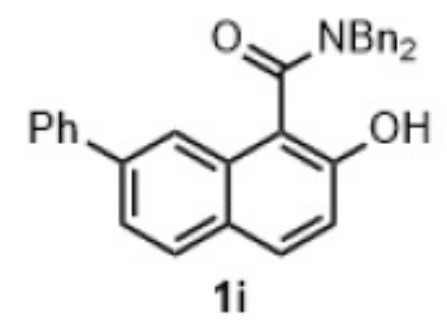

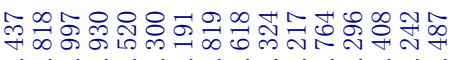

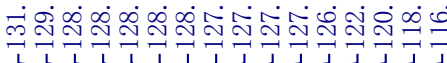
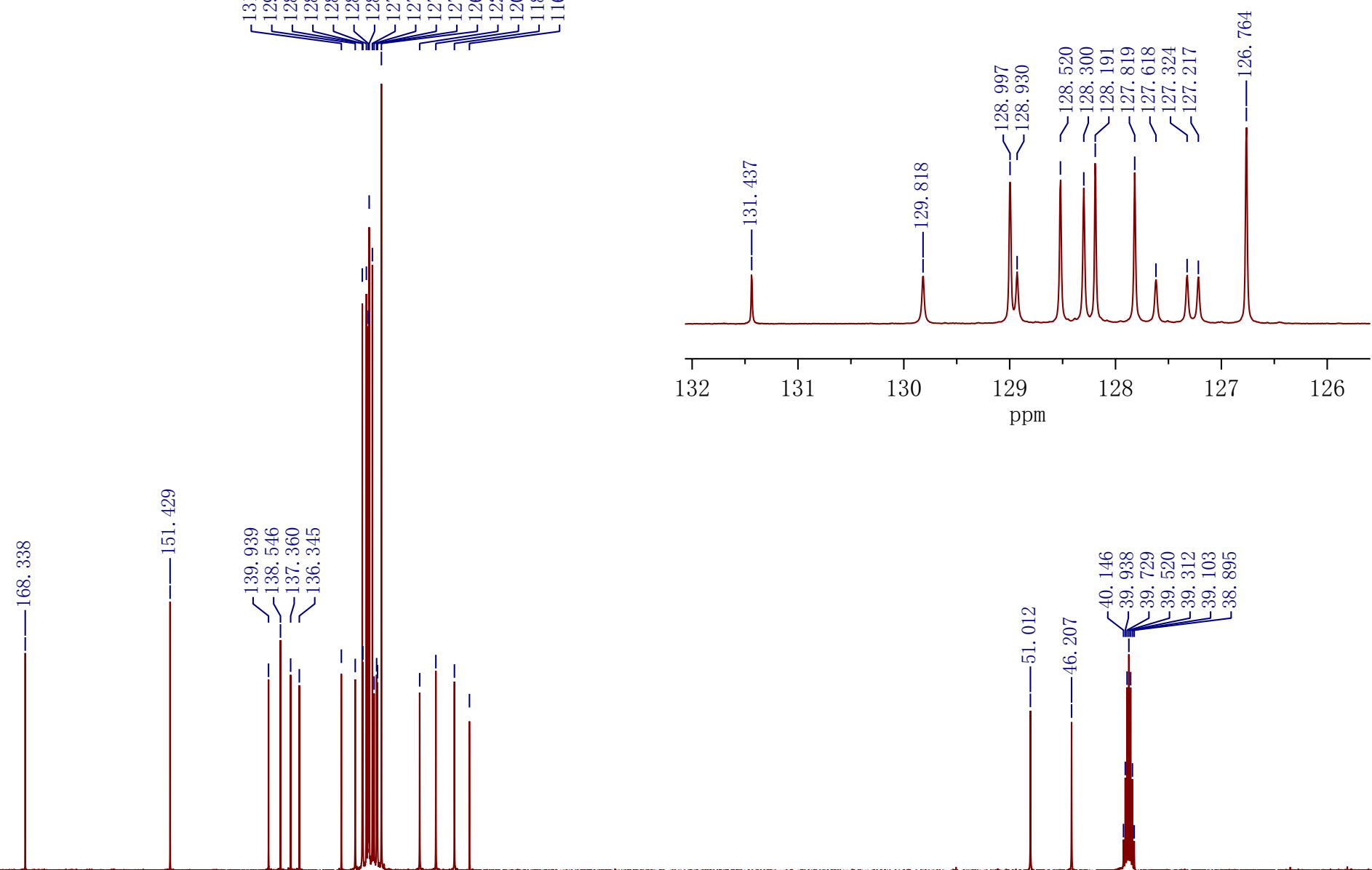

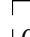


<smiles>CCCCC(=O)c1c(O)ccc2cc(Br)ccc12</smiles>

$1 \mathrm{j}$

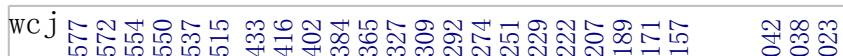

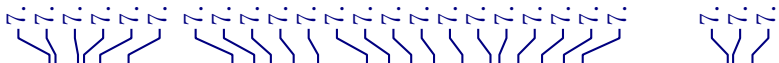

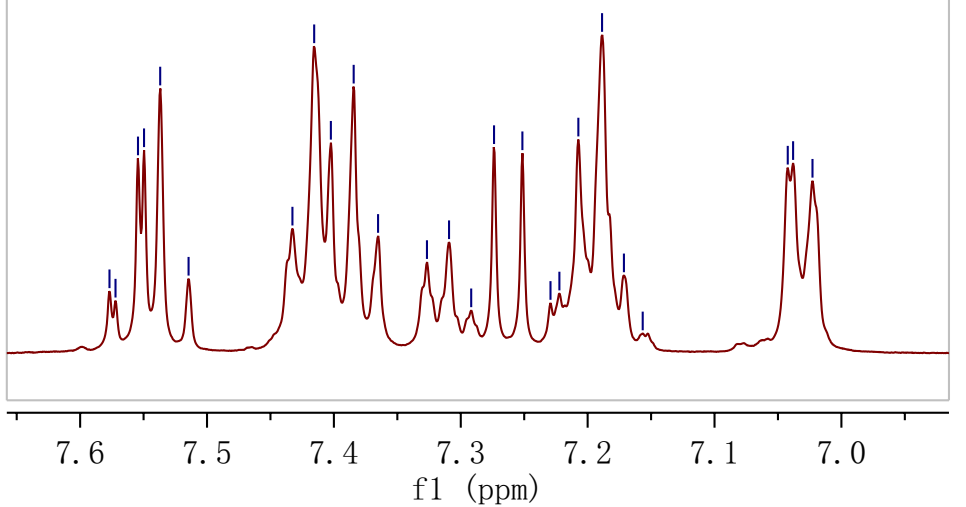

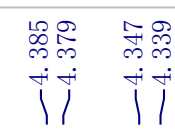

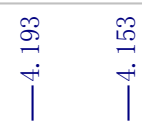

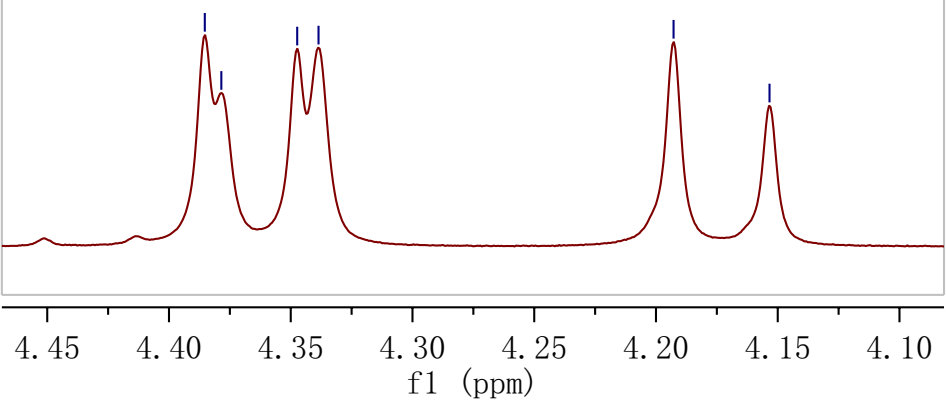

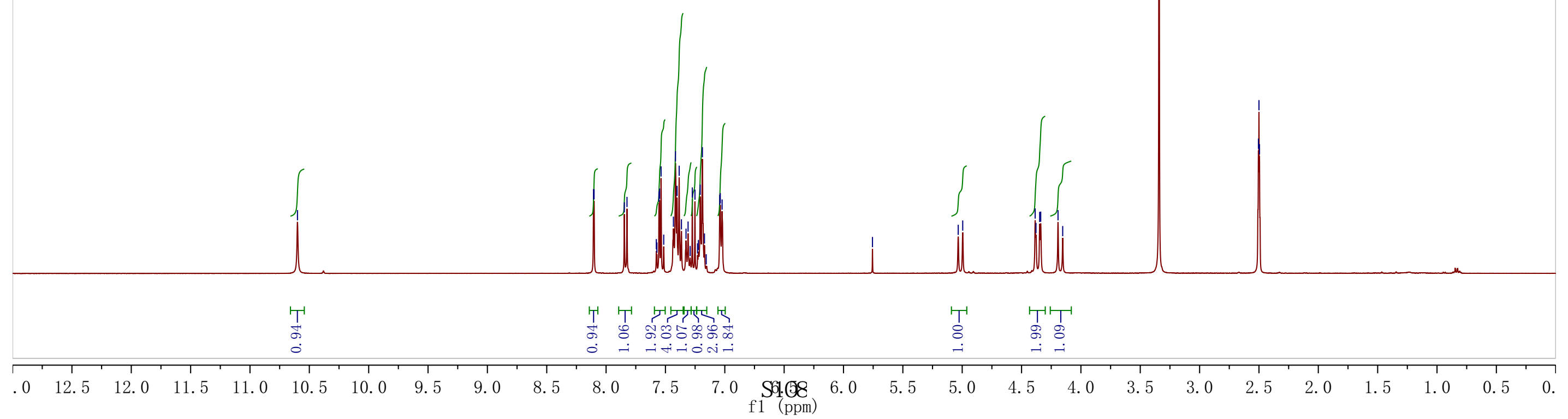



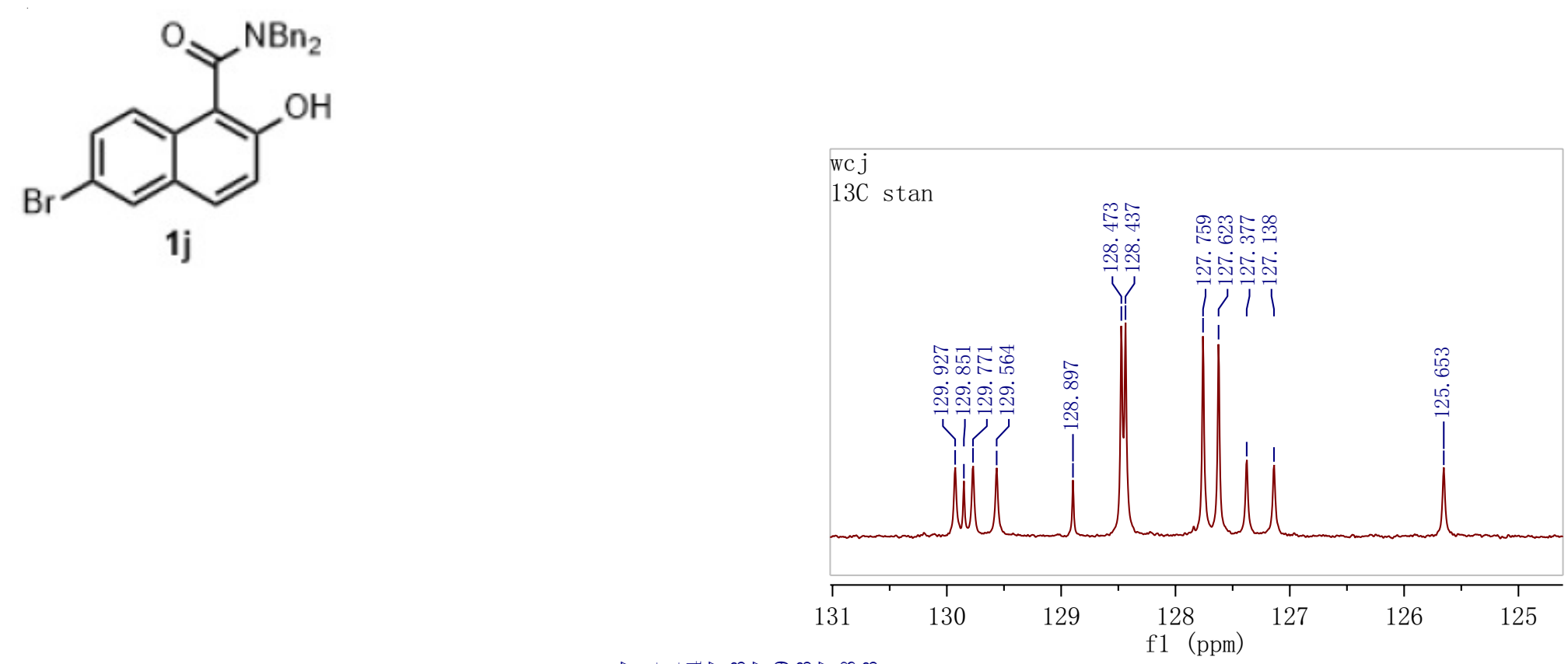

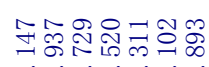

하이요요

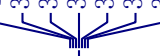

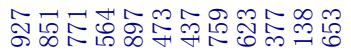

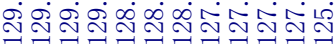

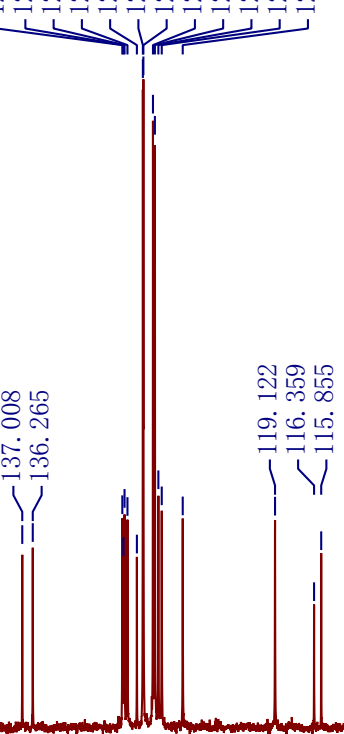




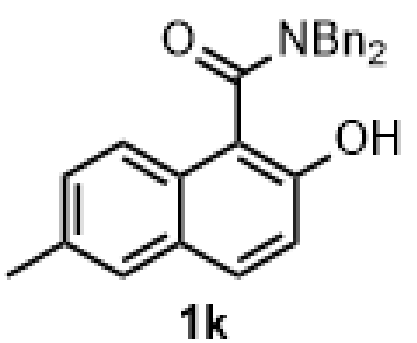

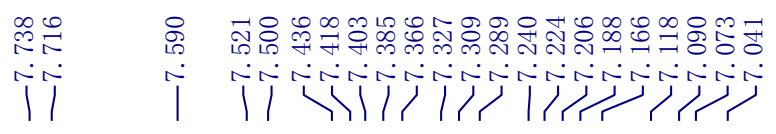

$1 \mathrm{k}$
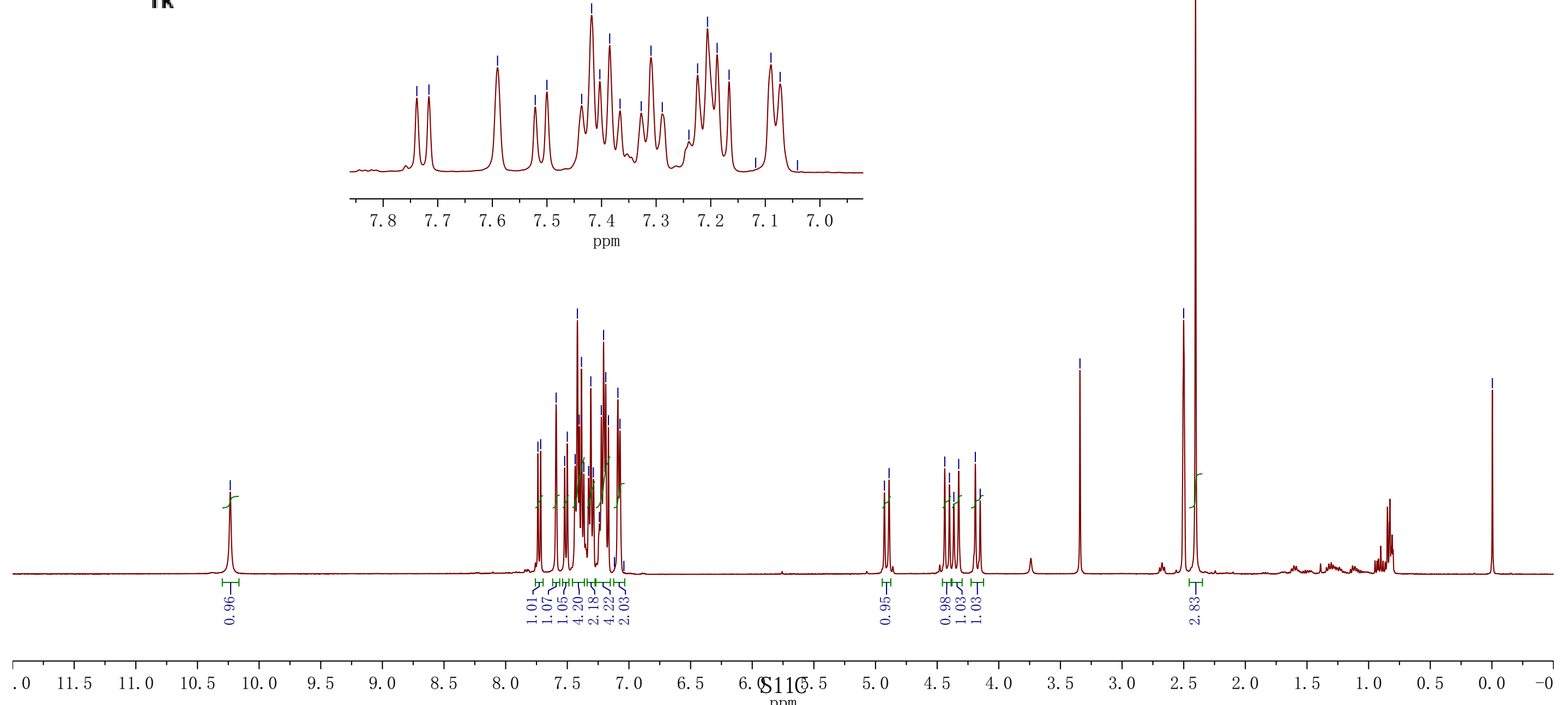


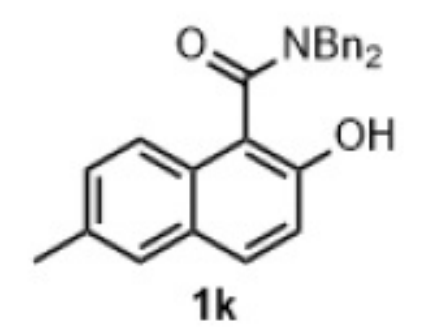

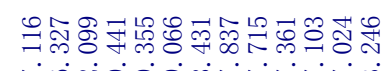

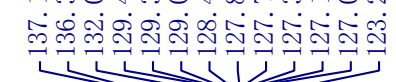

(1)
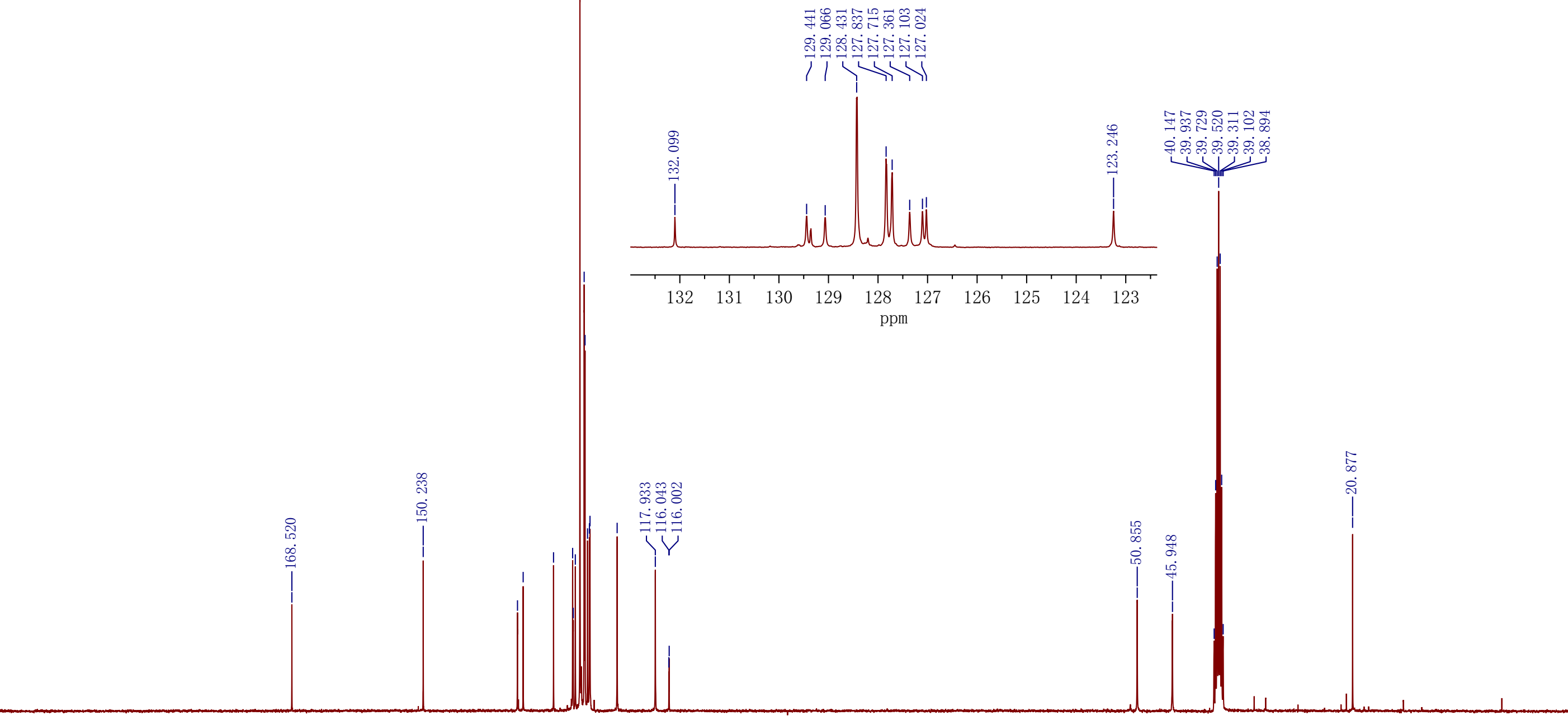
<smiles>CCCCCC(=O)c1c(O)ccc2ccc(OC)cc12</smiles>

11

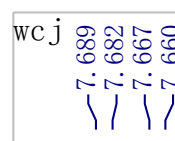

学 杵

ĩ
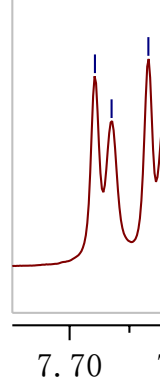

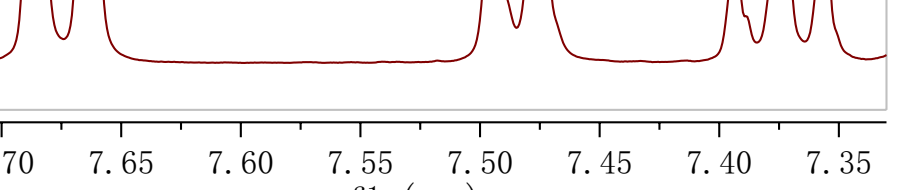

f1 (ppm)

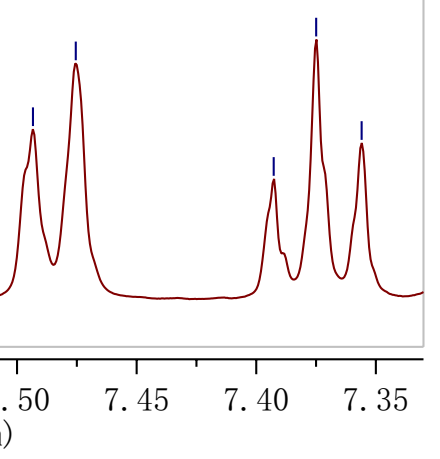

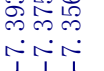

iा
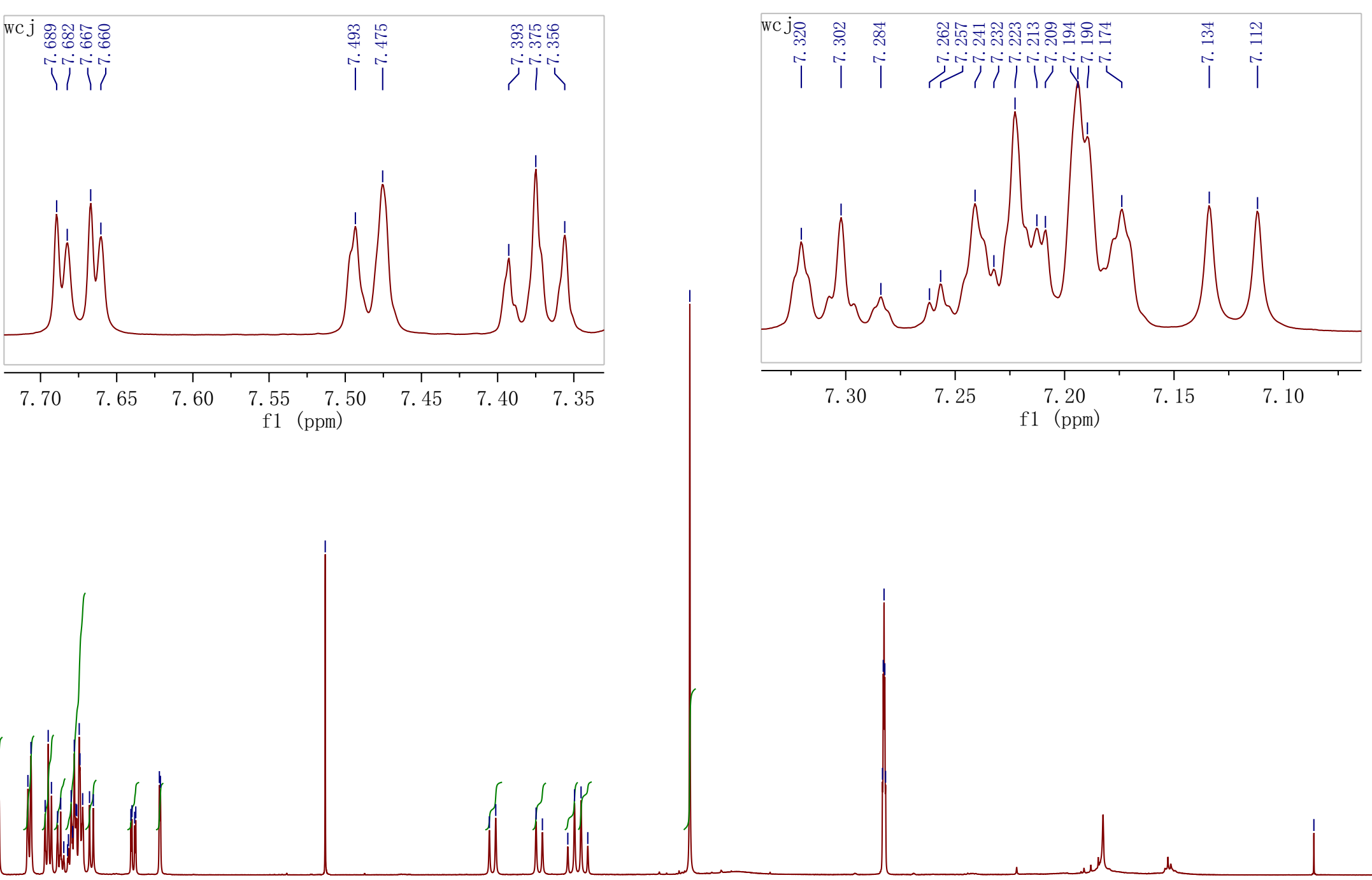

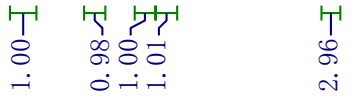

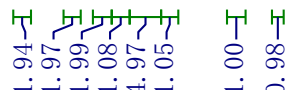

$7.5 \quad 7.0$

6.5
0S1124. 5 f 1 (p1124. 
<smiles>COc1ccc2ccc(O)c(C(=O)CC(C)C)c2c1</smiles>

11

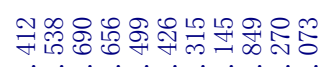

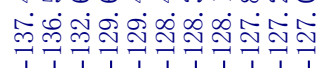

选

$\bar{\infty} \bar{\sigma}$

เं

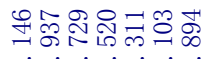

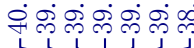

(n) 


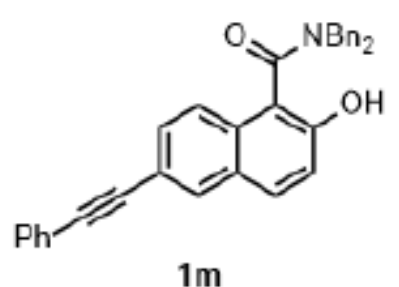

$1 \mathrm{~m}$
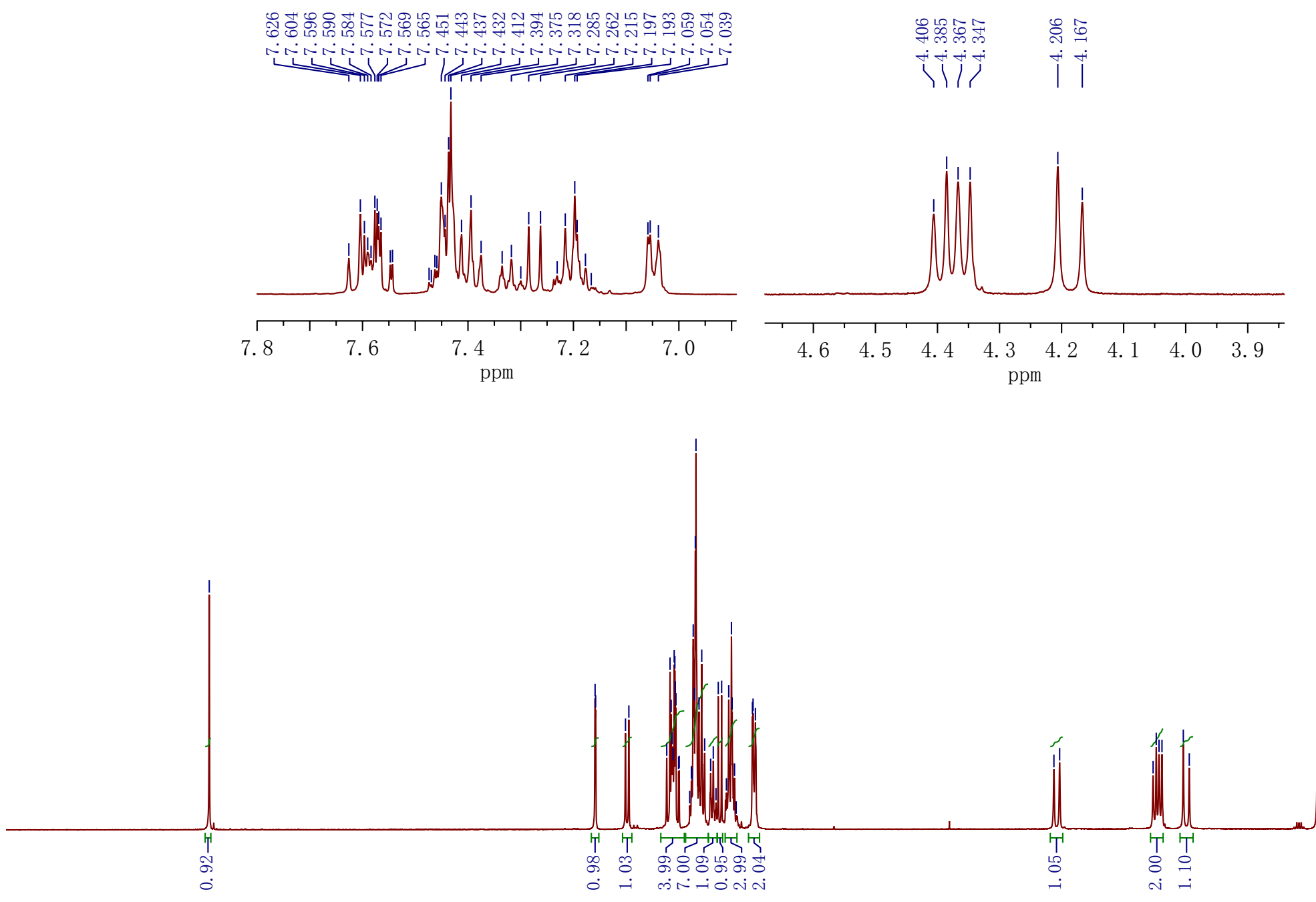


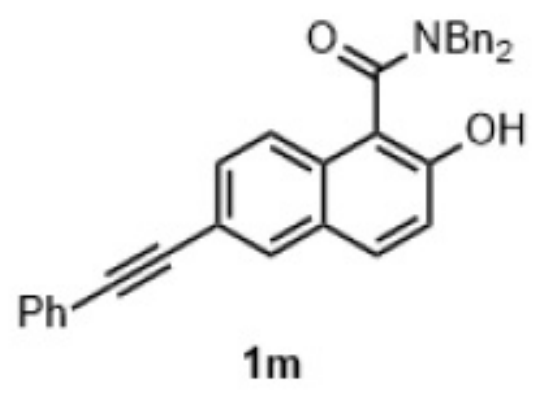

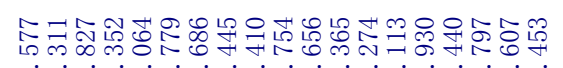

ต.
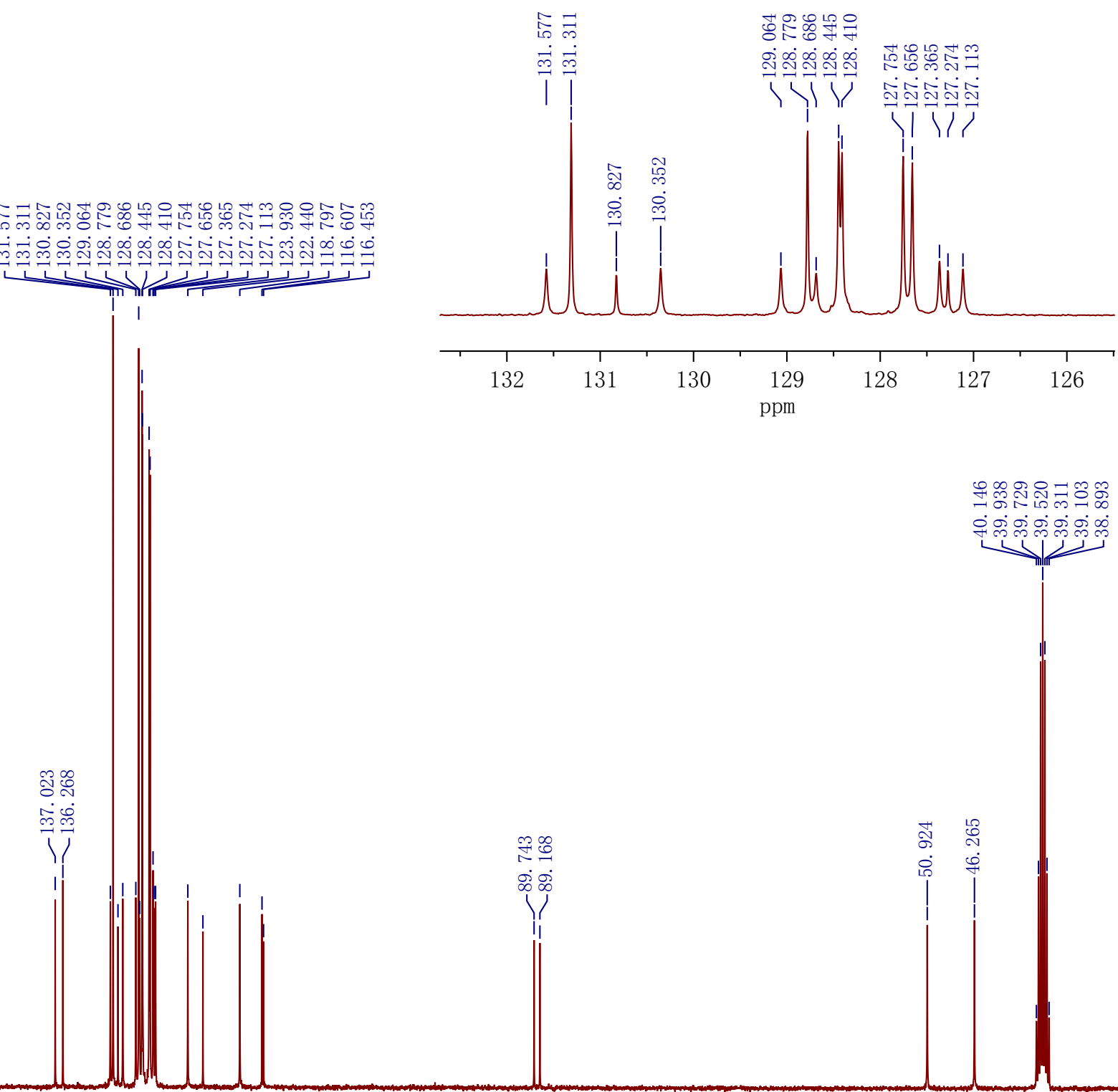

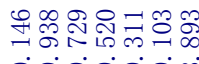

4

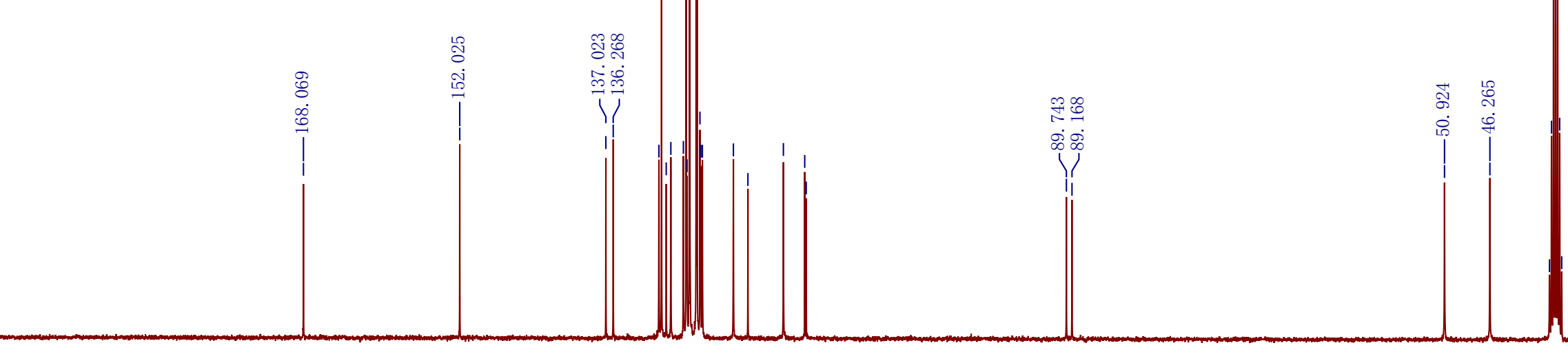



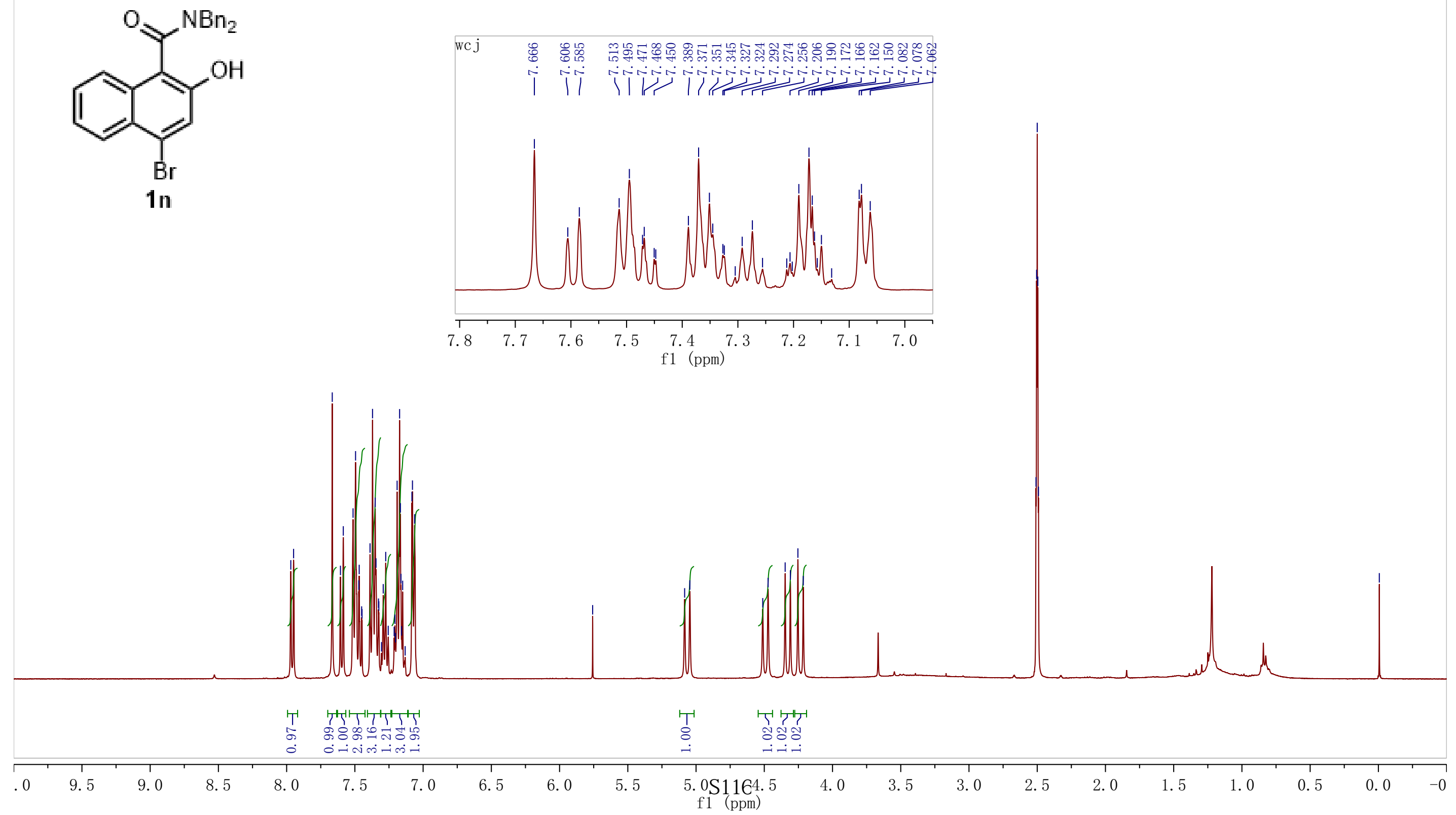

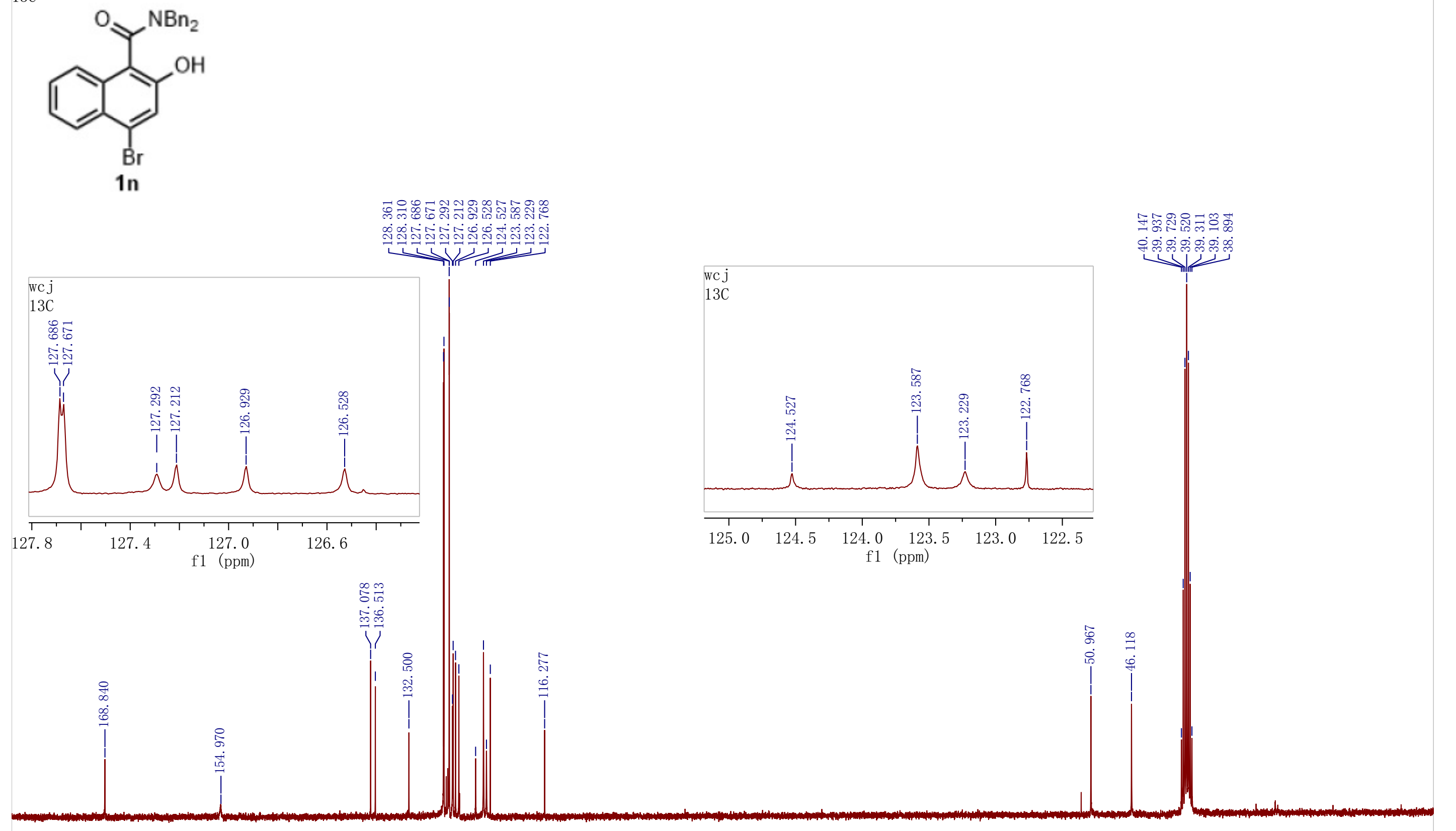

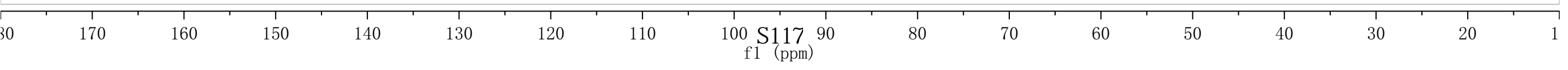



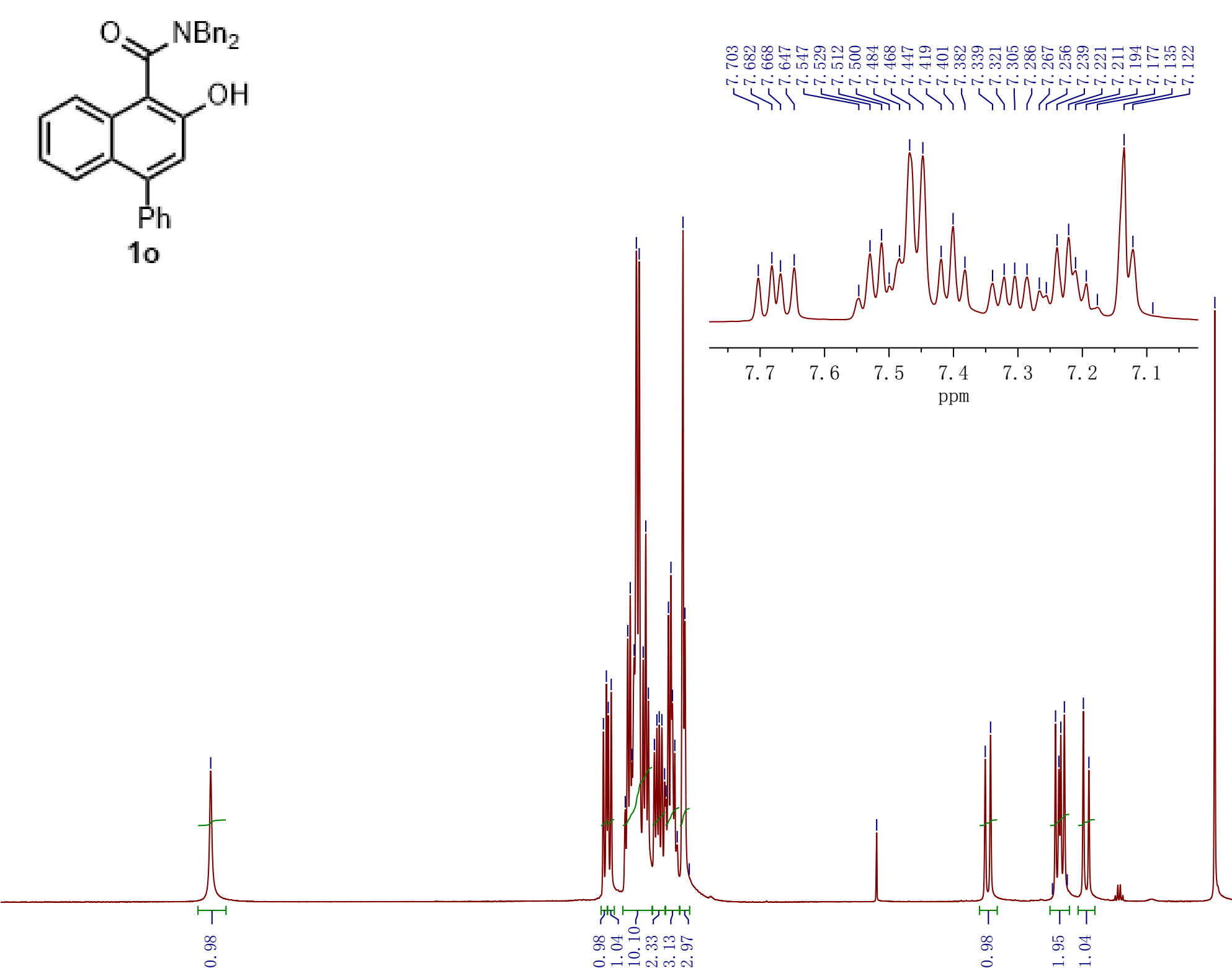

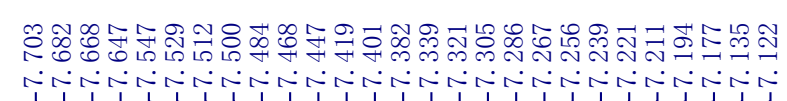

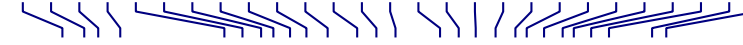

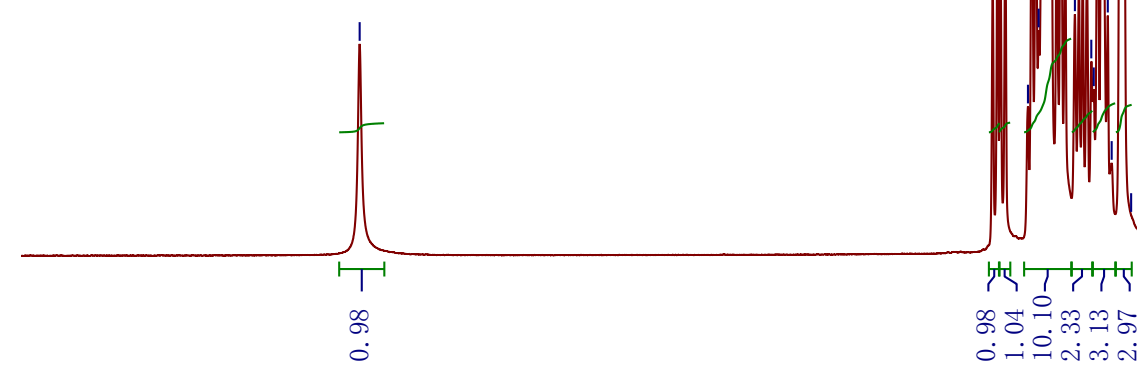



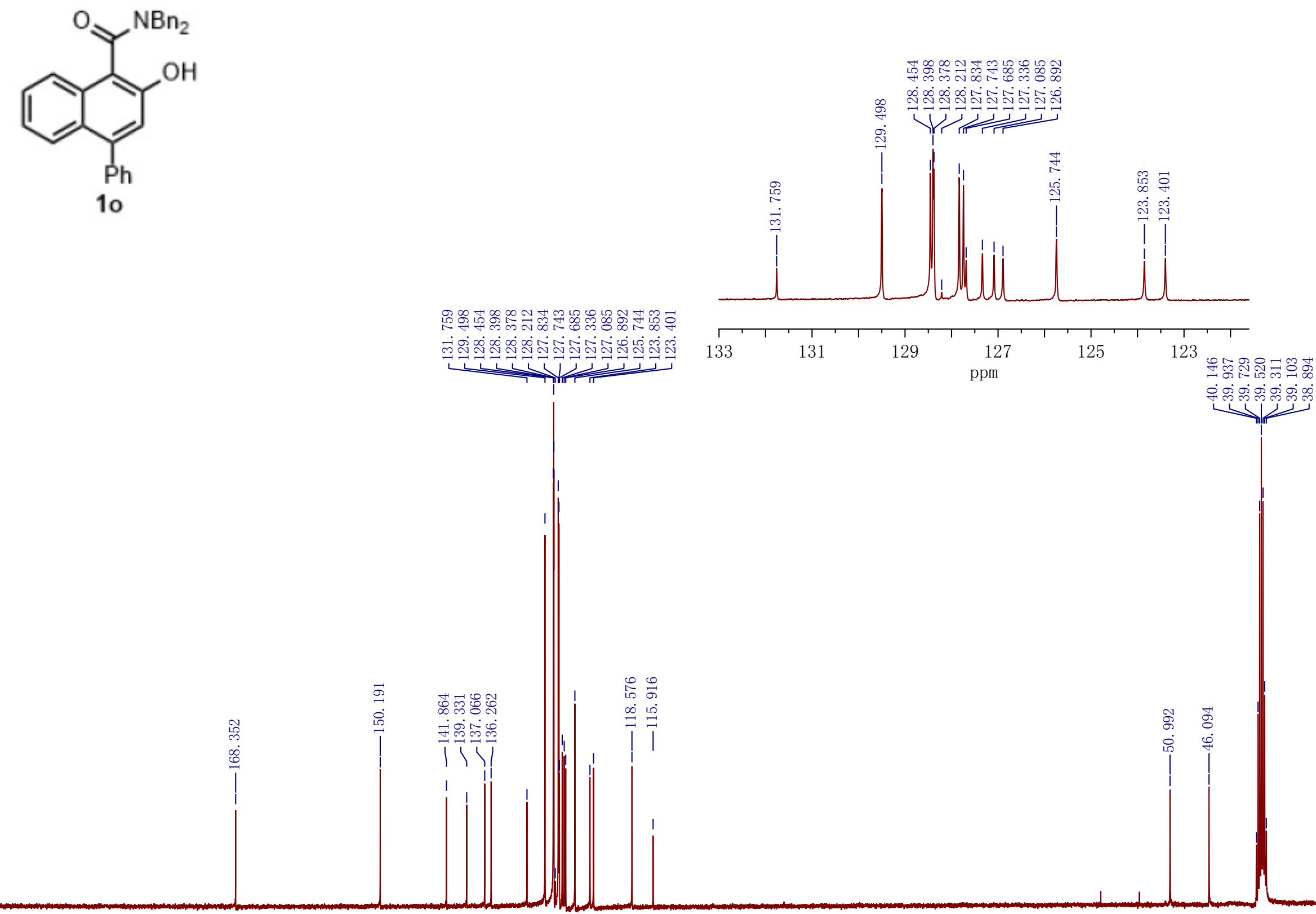

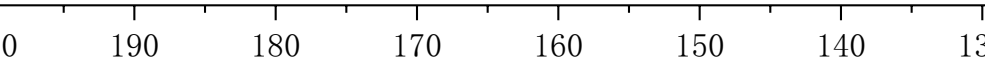

120

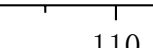

Stopg

$90 \quad 80$

1
$70 \quad 60$

$50+40$

(20)




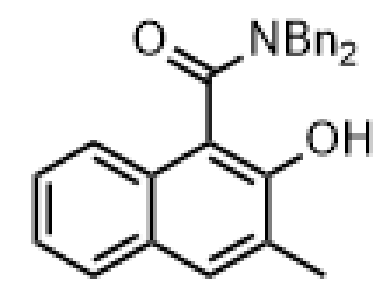

1p

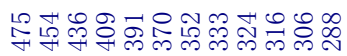

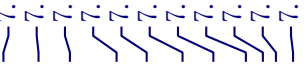 \\ गंग

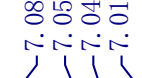

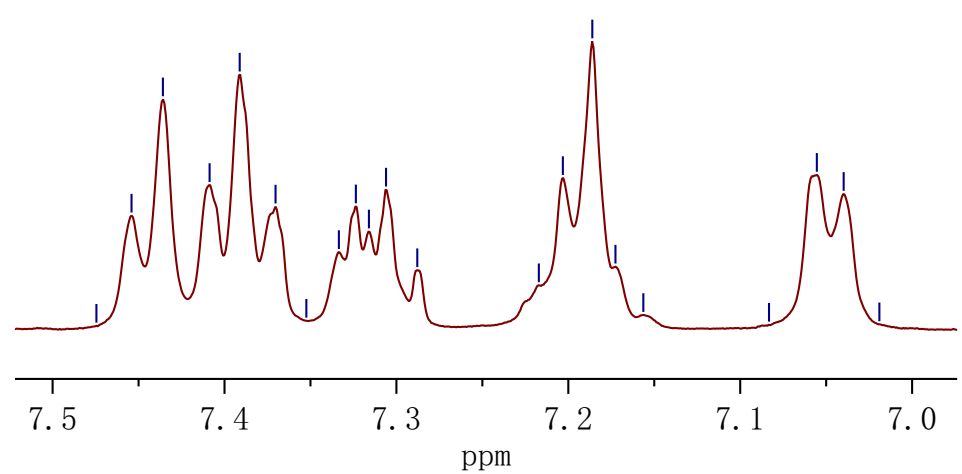

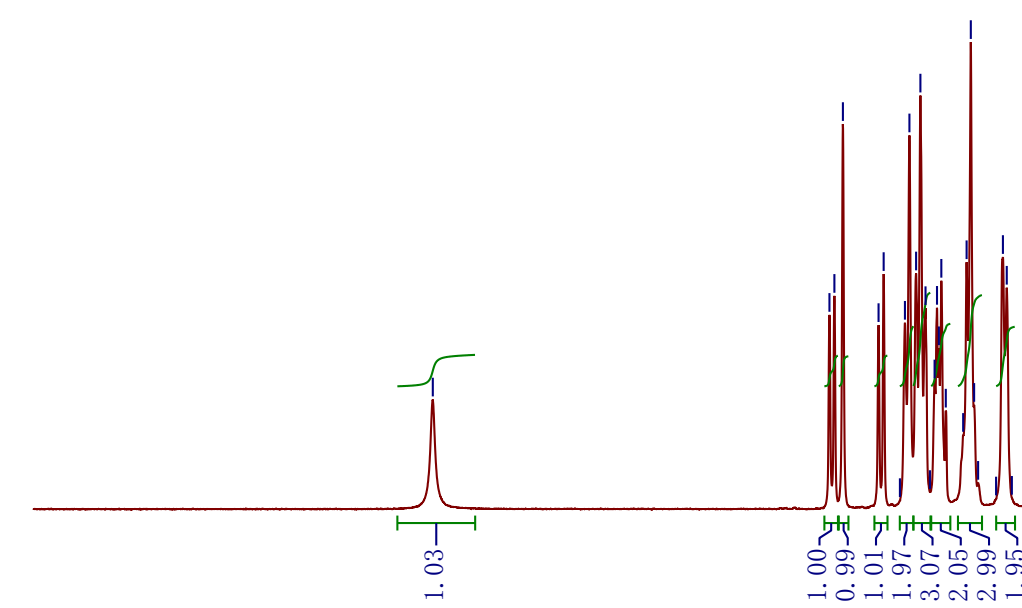
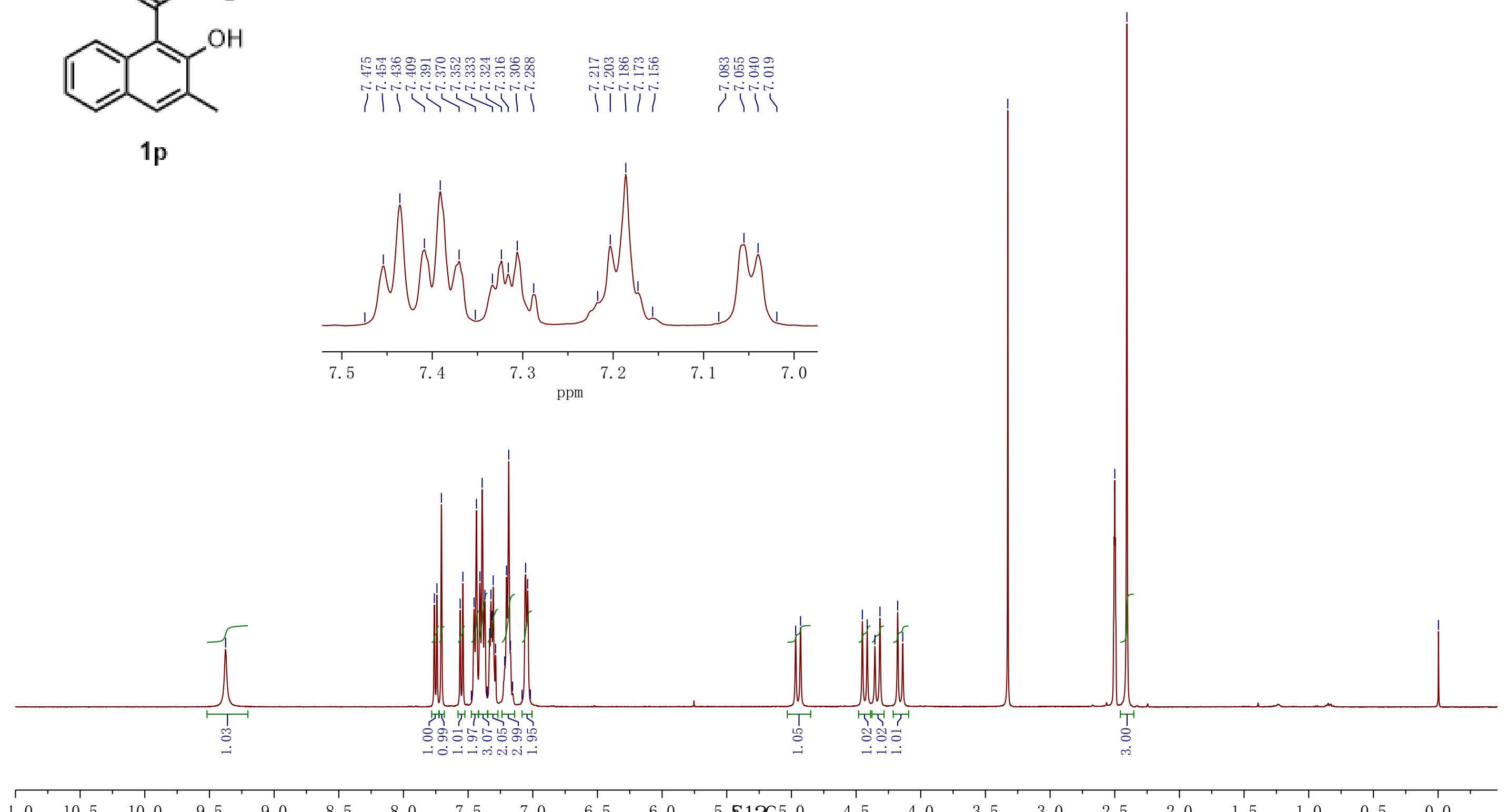

$10.5 \quad 10.0$

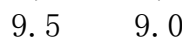

8.5

8. $0 \quad 7.5$

6.5

$\$ 1205$.

4.5

4. 0

3.5

3. 0

2.5

2.0

1.5

1.0

$0.5 \quad 0.0$ 


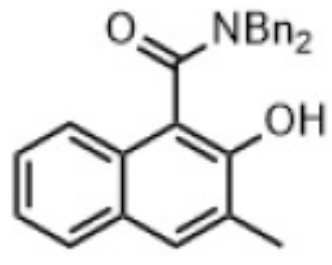

$1 \mathrm{p}$

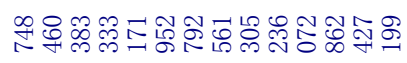

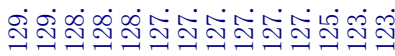
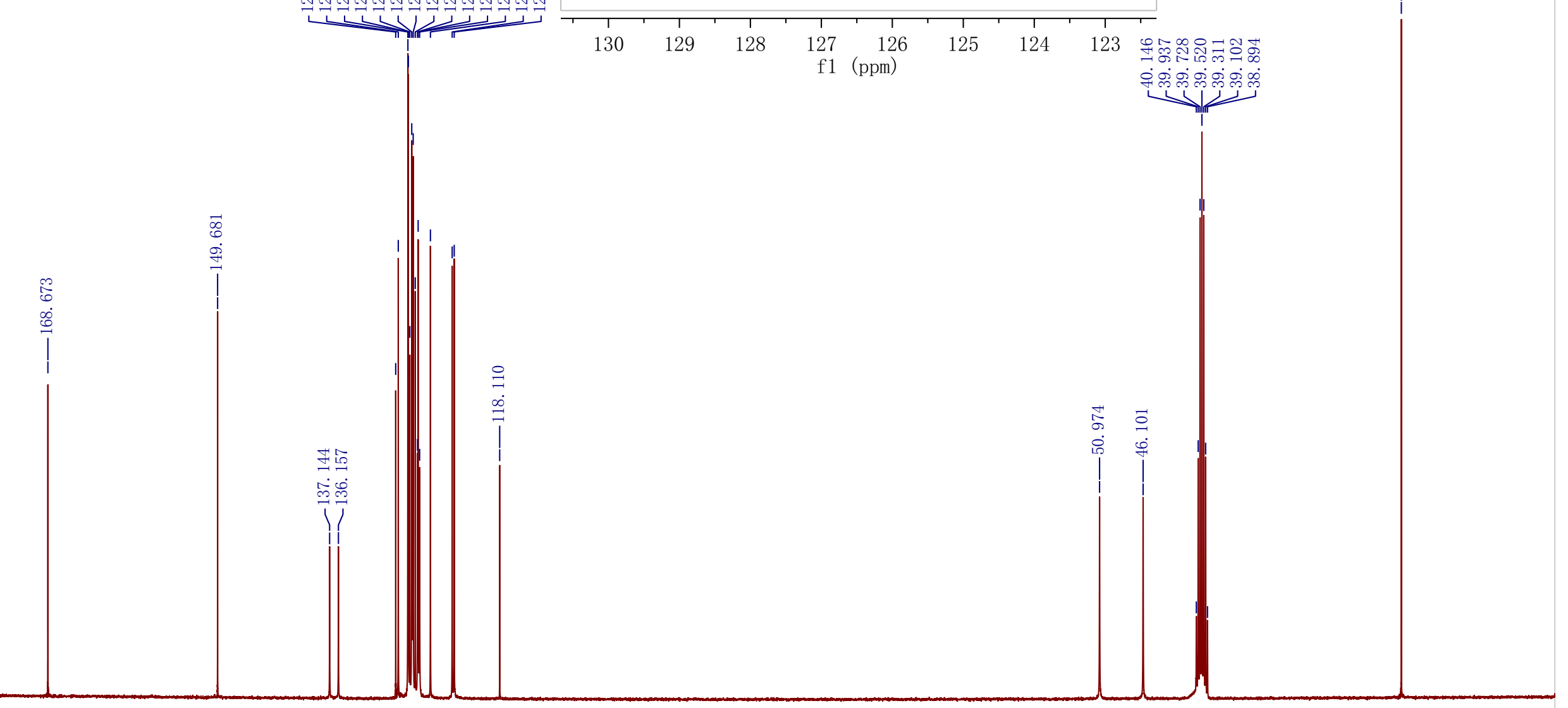

f1 (ppm)

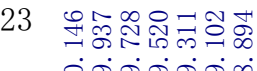

भिल 


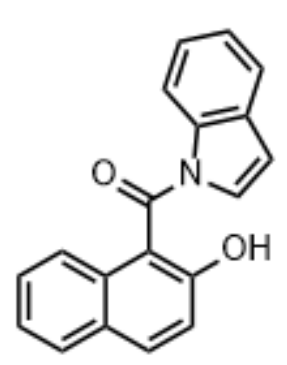
|용용

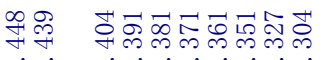
il irivitis

$1 q$
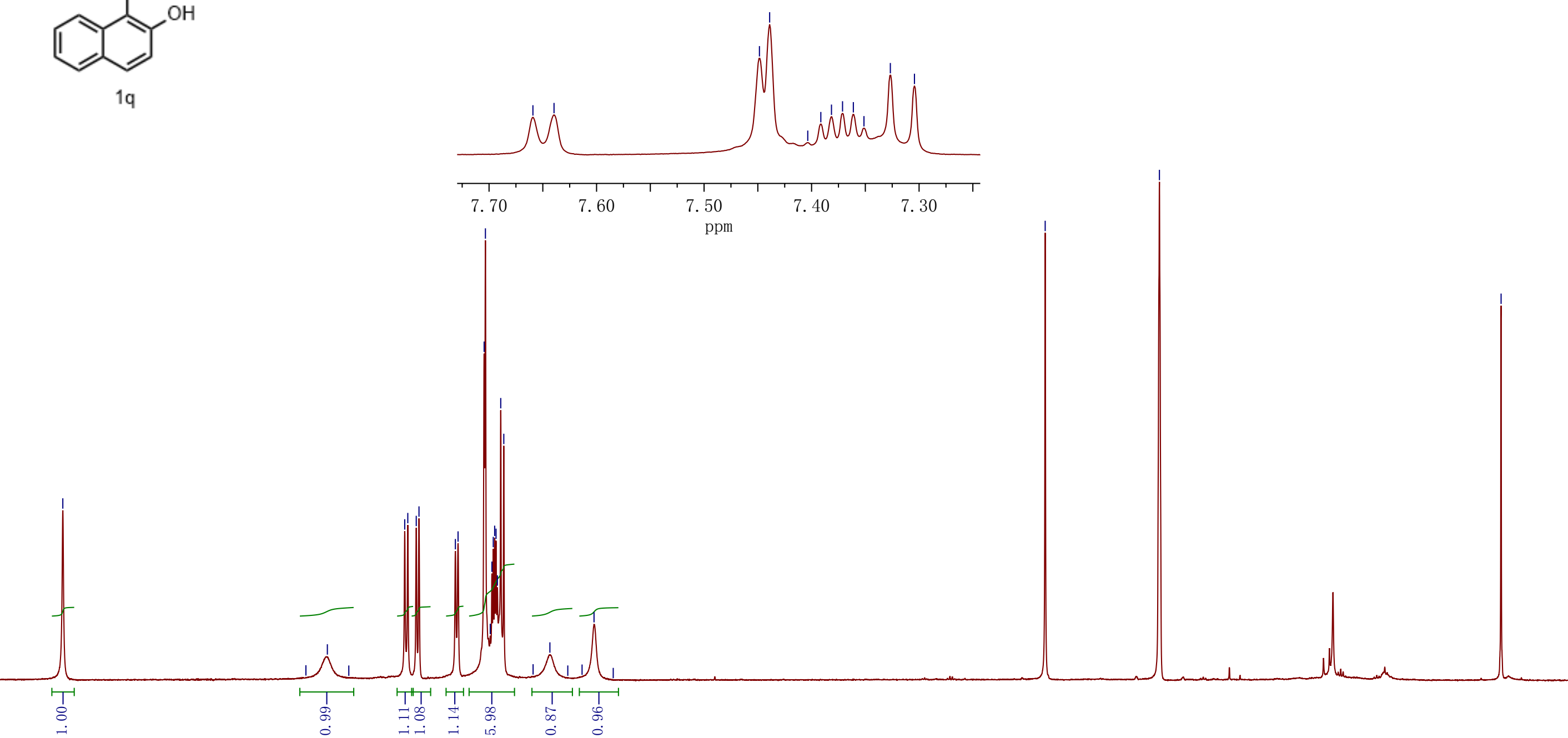

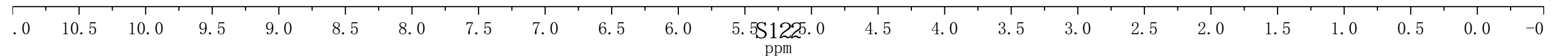




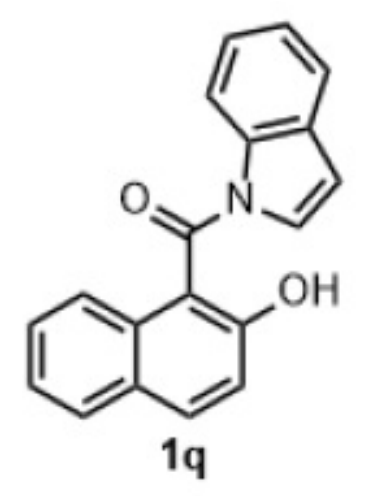

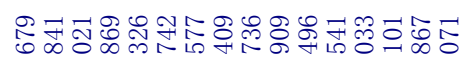

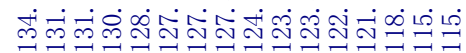
皮

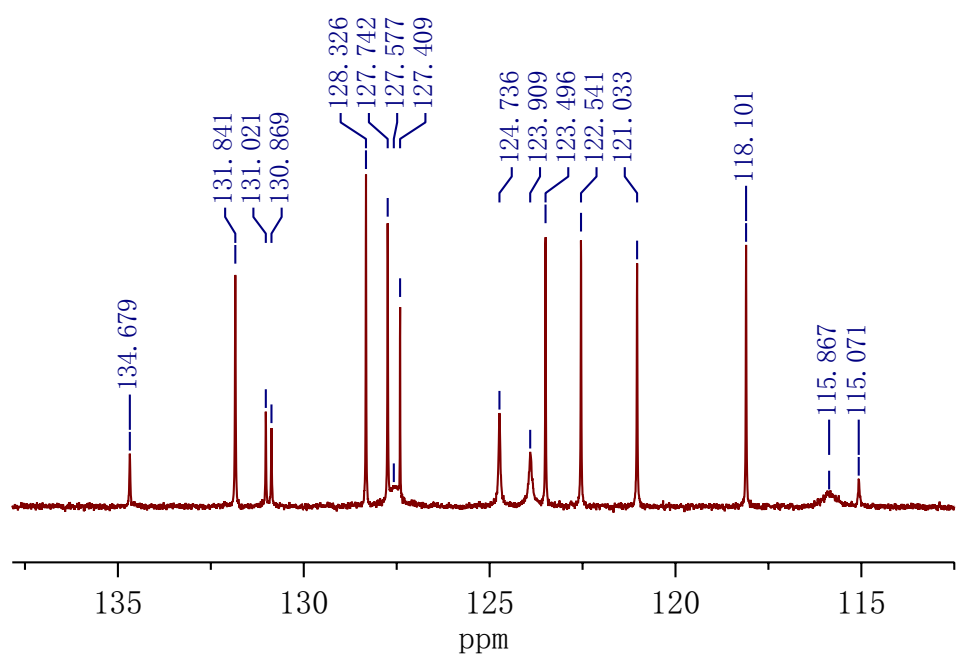




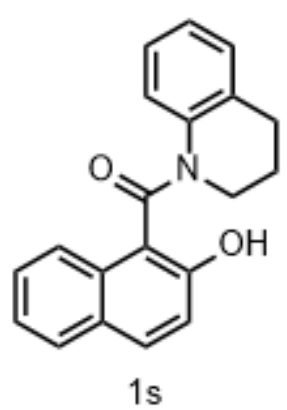

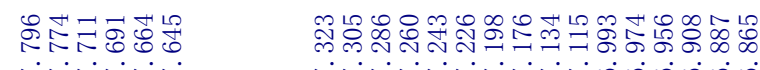

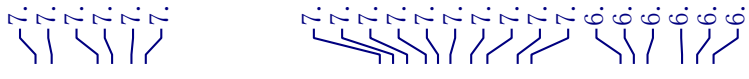
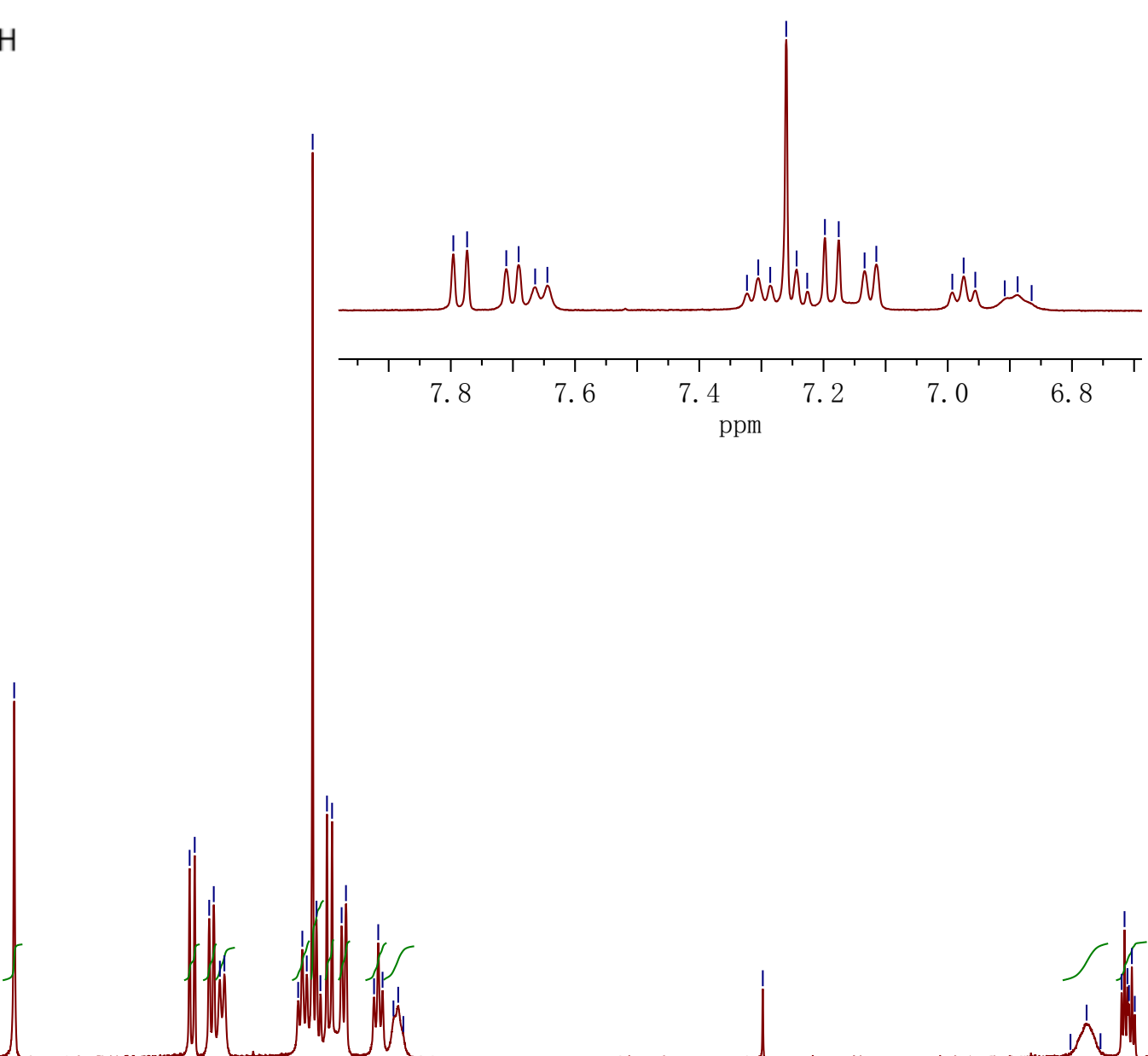

ppm
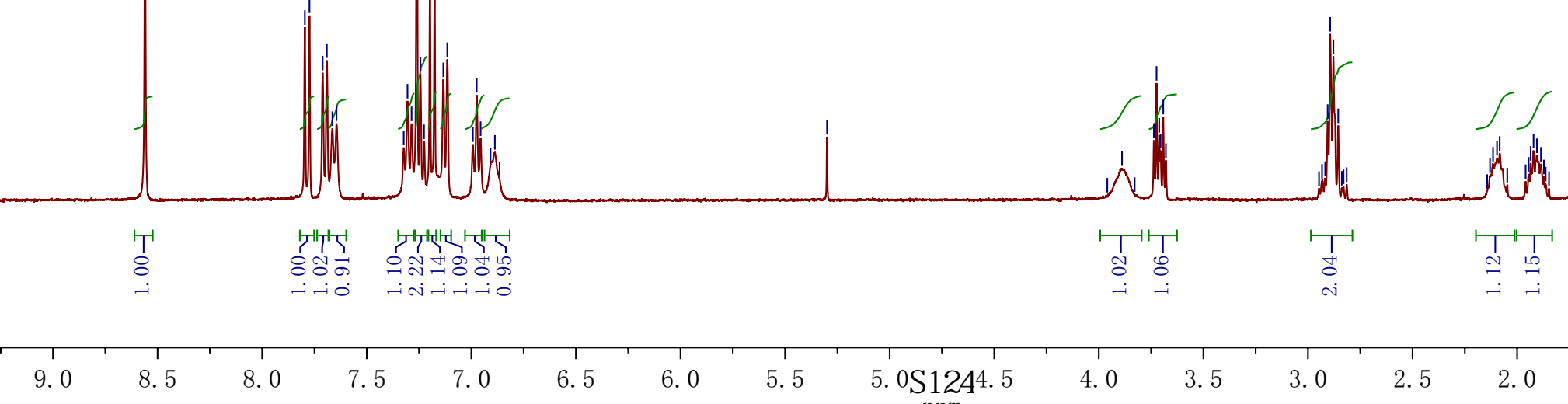

$5 \quad 8.0 \quad 7.5$

7.0

6.5

6. 0

5.5

5. $0 \mathrm{~S} 1244.5$

4. 0

3.5

3.

2.5

2.0

1.5

1. 0

0.5

0. 0 $-0$ 
<smiles>O=C(c1c(O)cc([12F])c2ccccc12)N1CCCc2ccccc21</smiles>
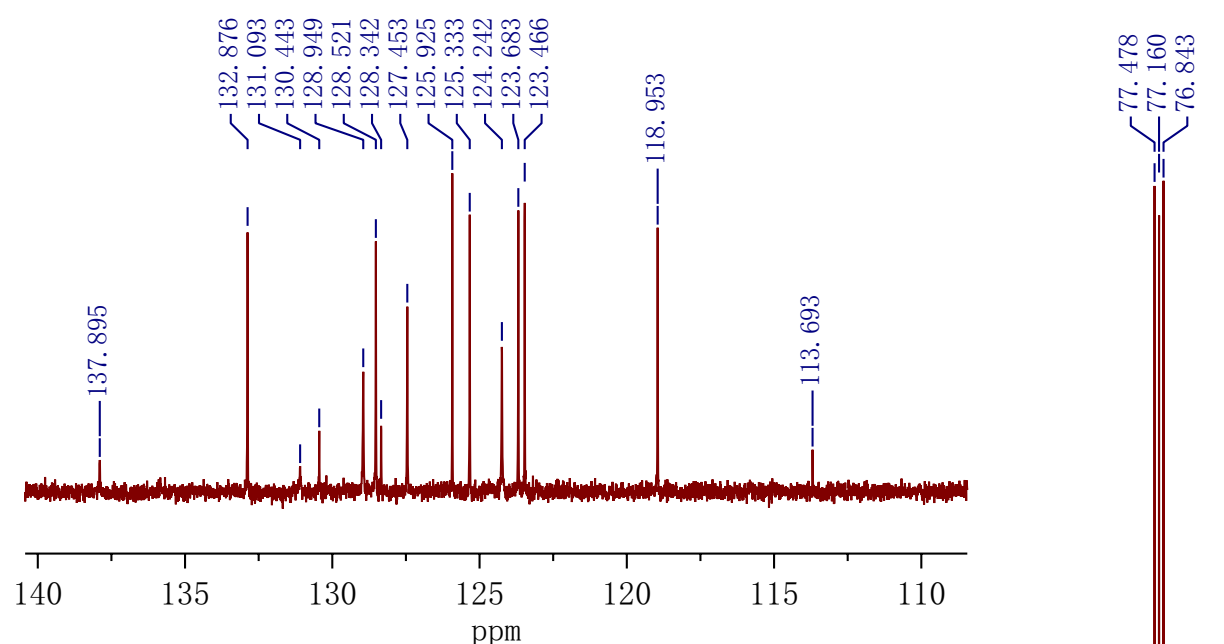

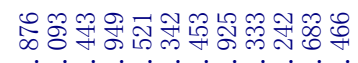

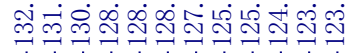

L

$\mid \begin{array}{ll}1 \\ 1 & \substack{: \\ 0} \\ 1 & 0\end{array}$

$\stackrel{0}{1}$

0
0
0
0
0

స

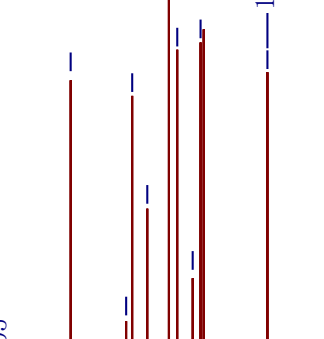

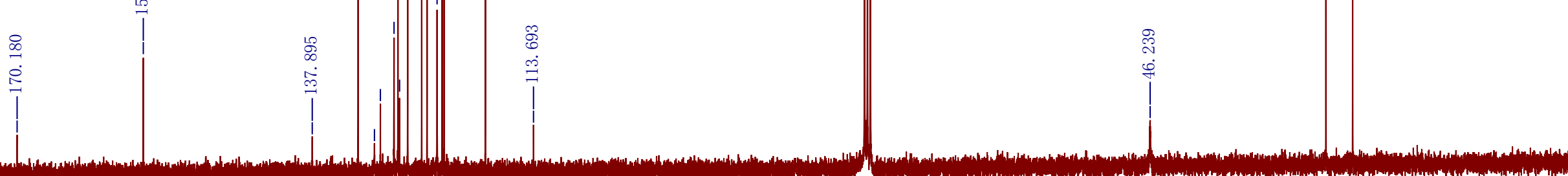




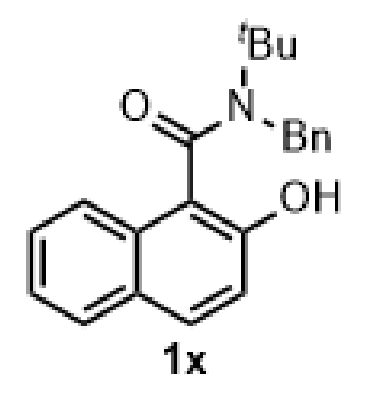

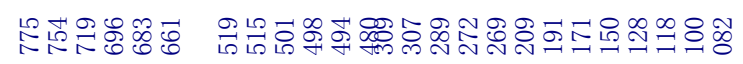

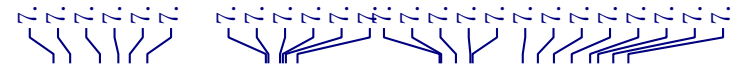
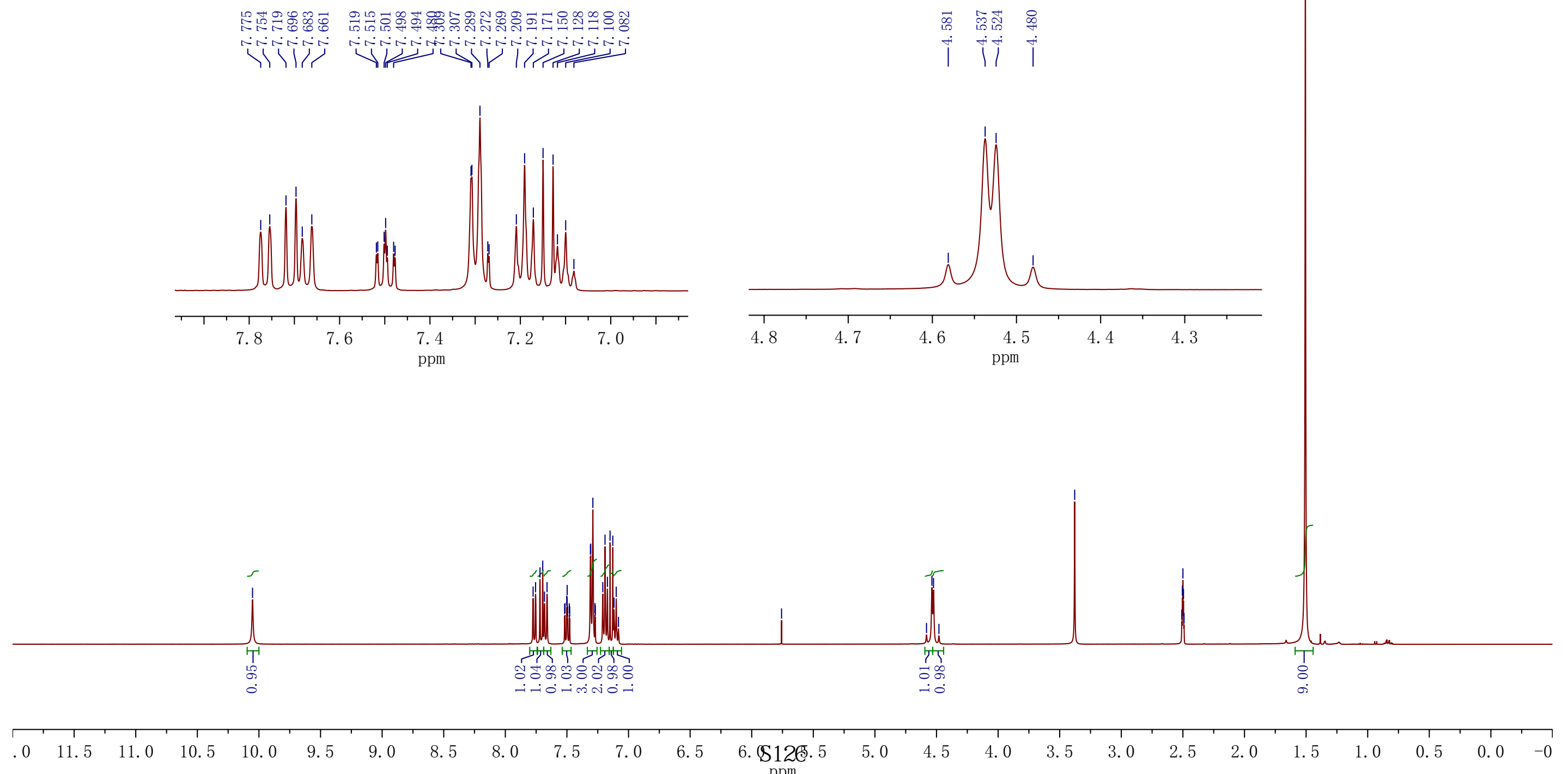


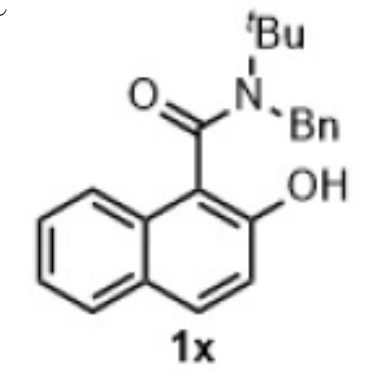

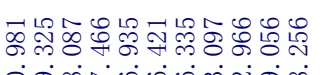

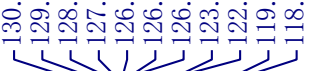

年
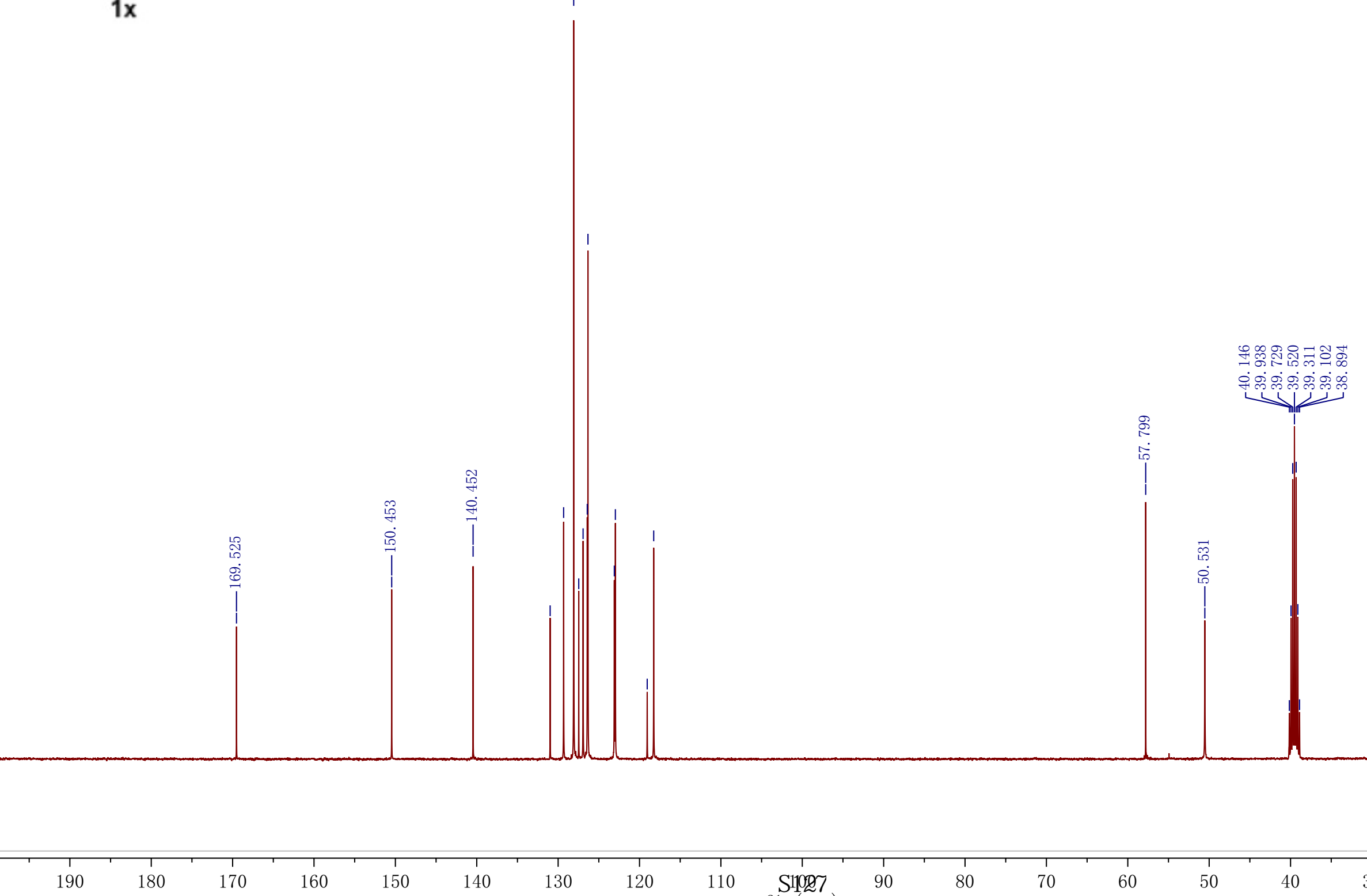

$30 \quad 120$ 

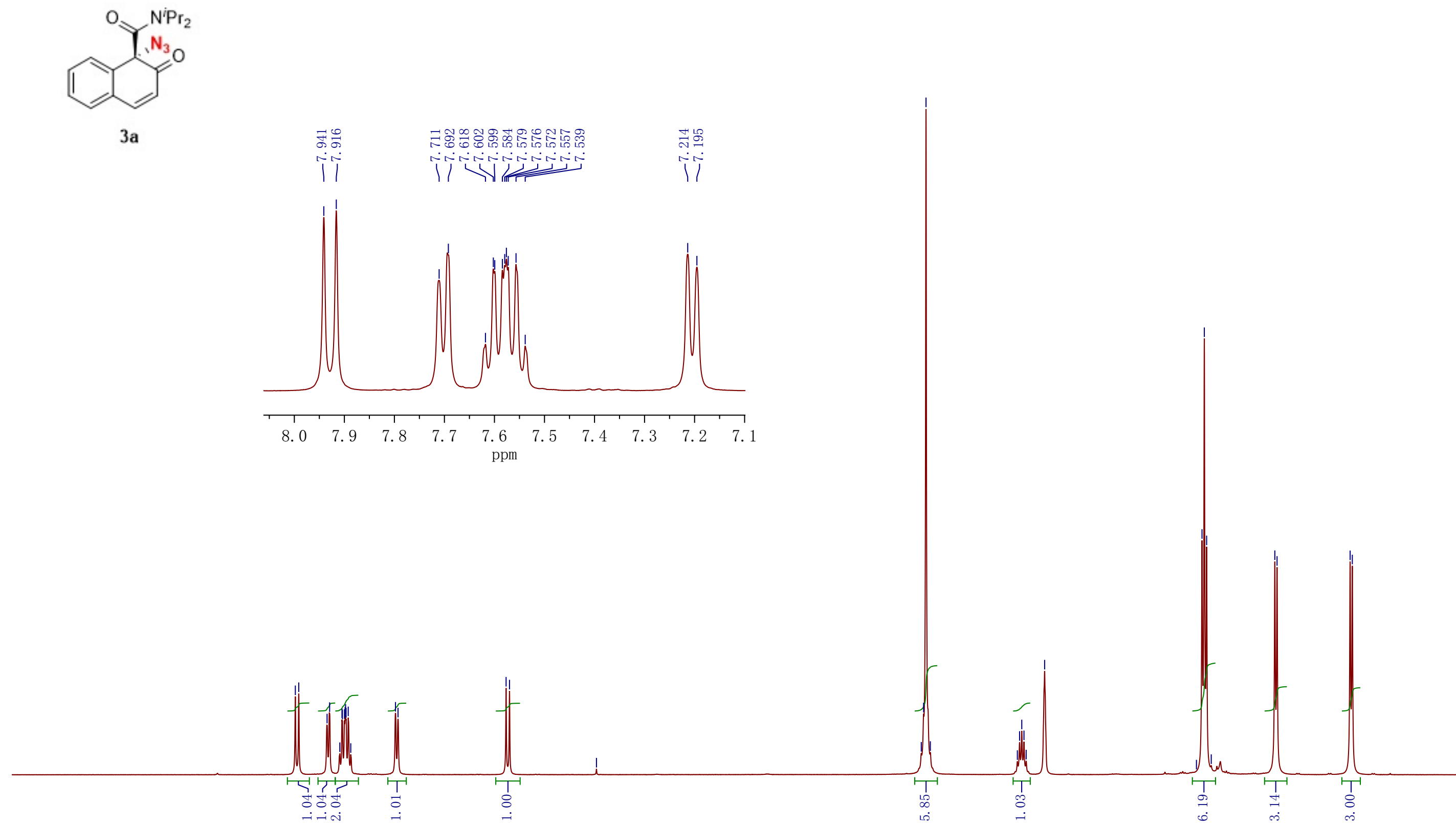

3a 


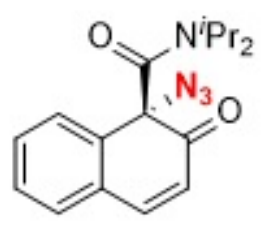

$3 a$
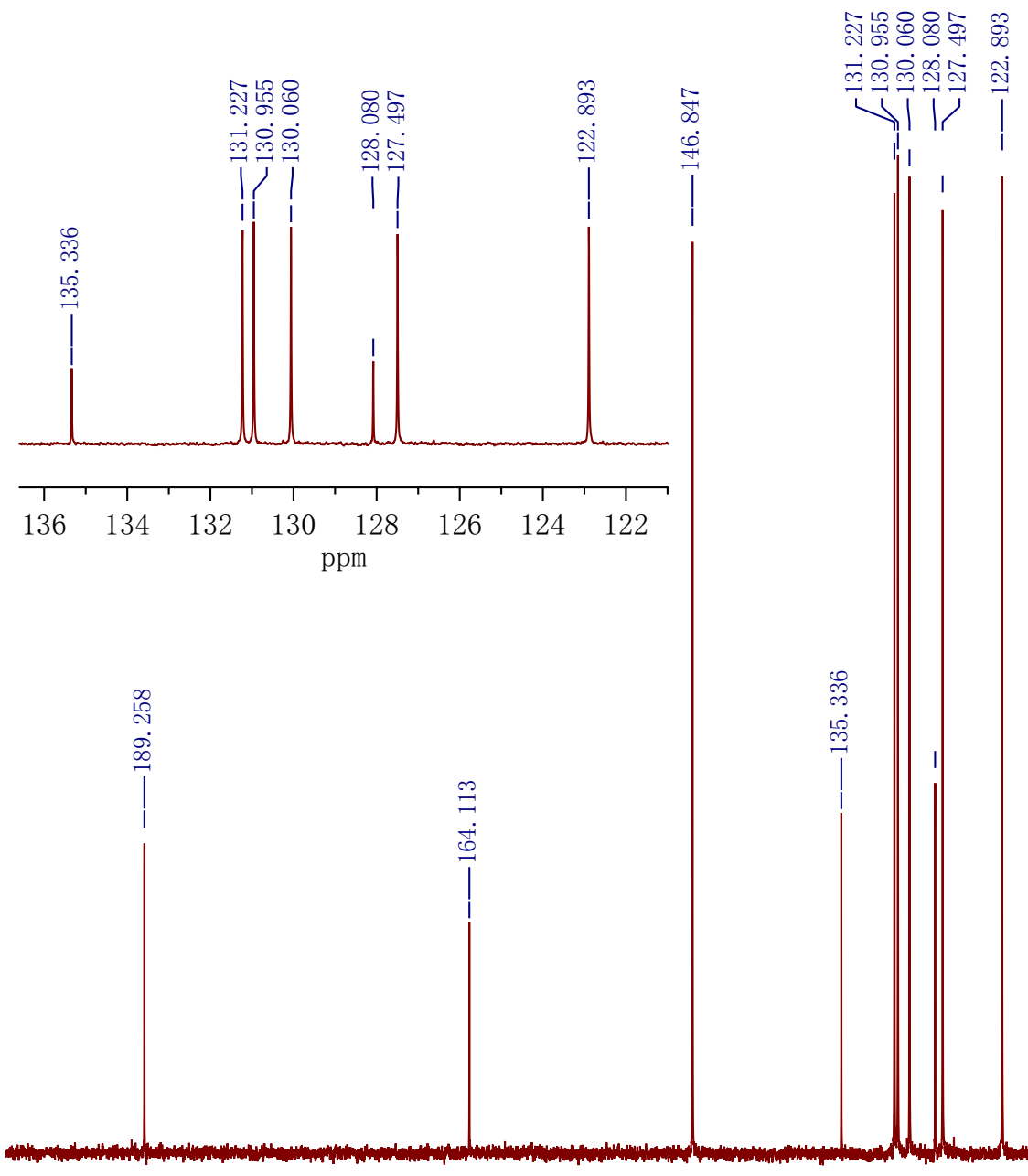
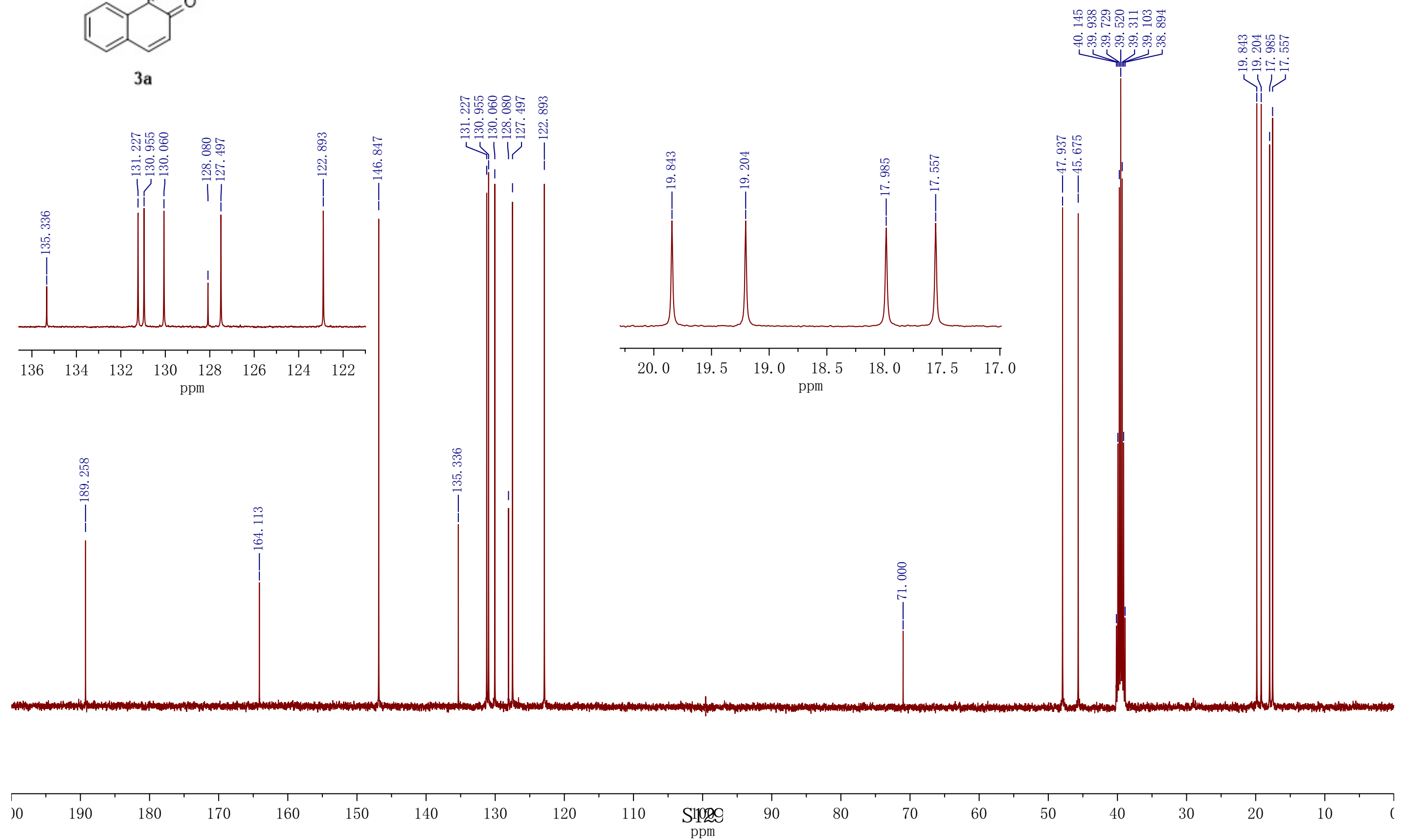

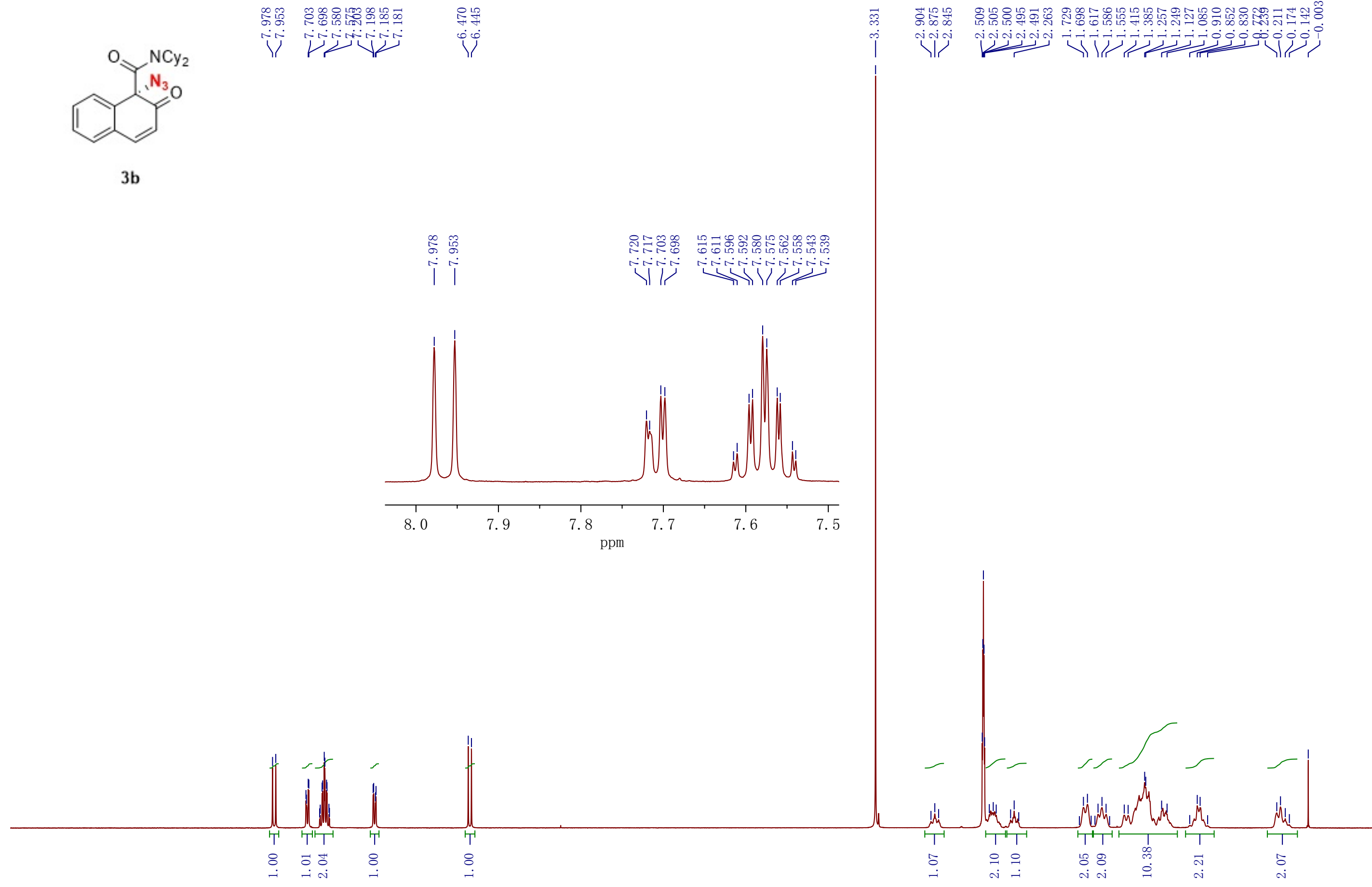

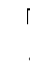

$9.0 \quad 8.5$



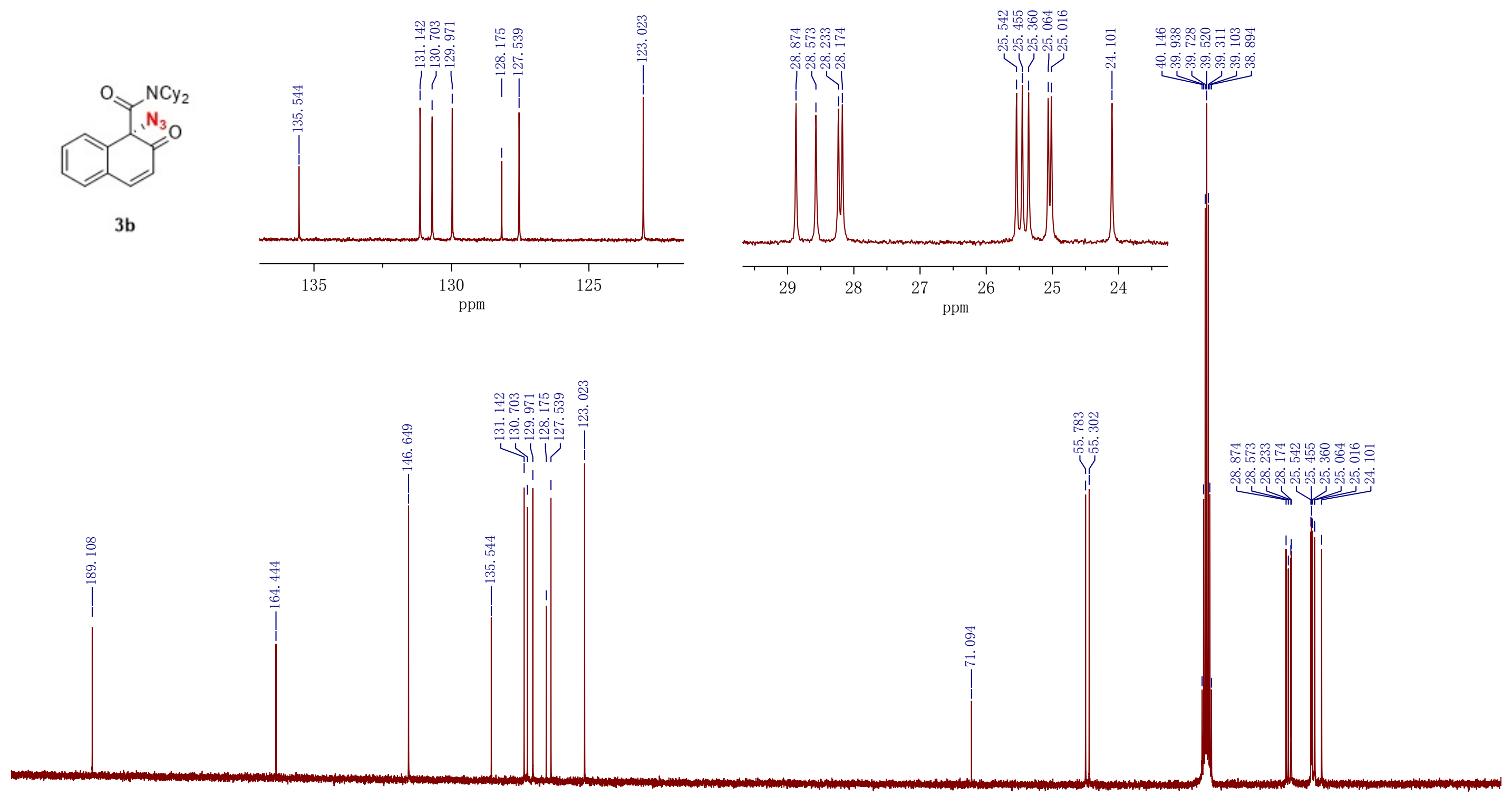

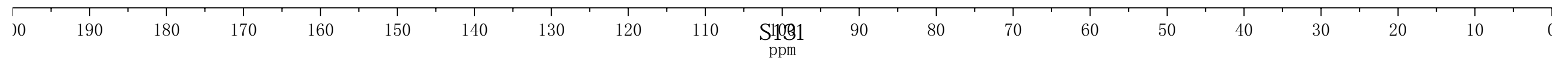




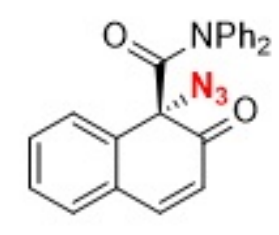

$3 c$

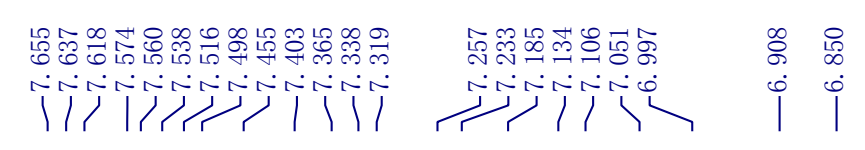

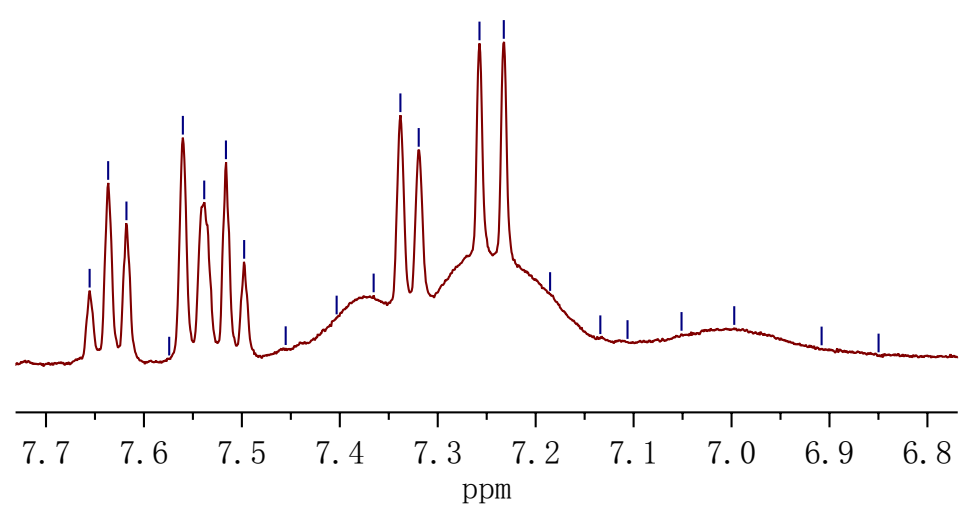

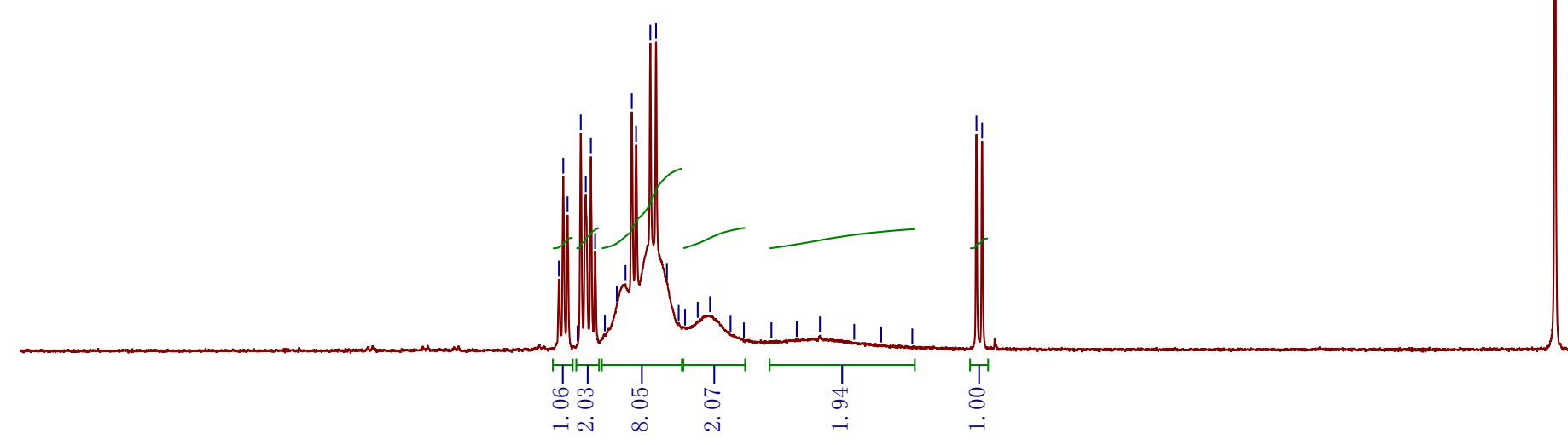

59.

8.5

7.0

6.5

6. 0

5.5

5. $0 \mathrm{~S} 132^{4 .} 5$

4. 0

3. 5

3.

2.5

. 0

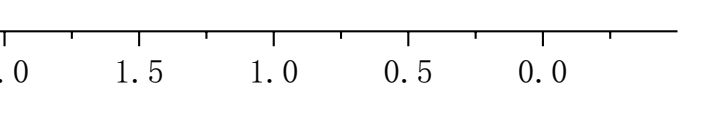




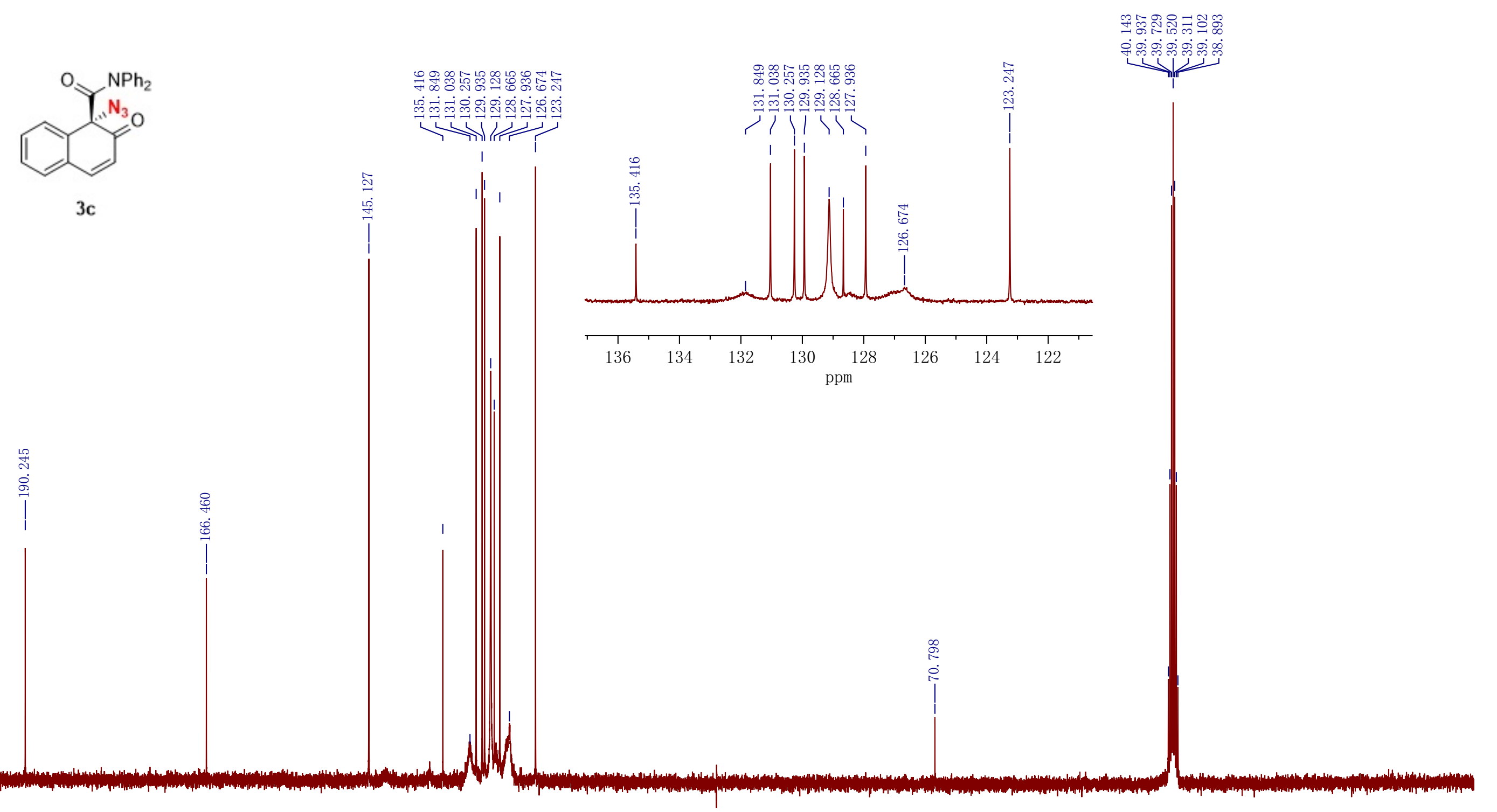

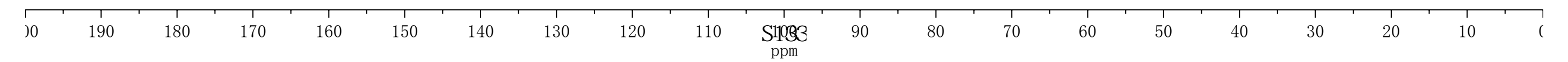



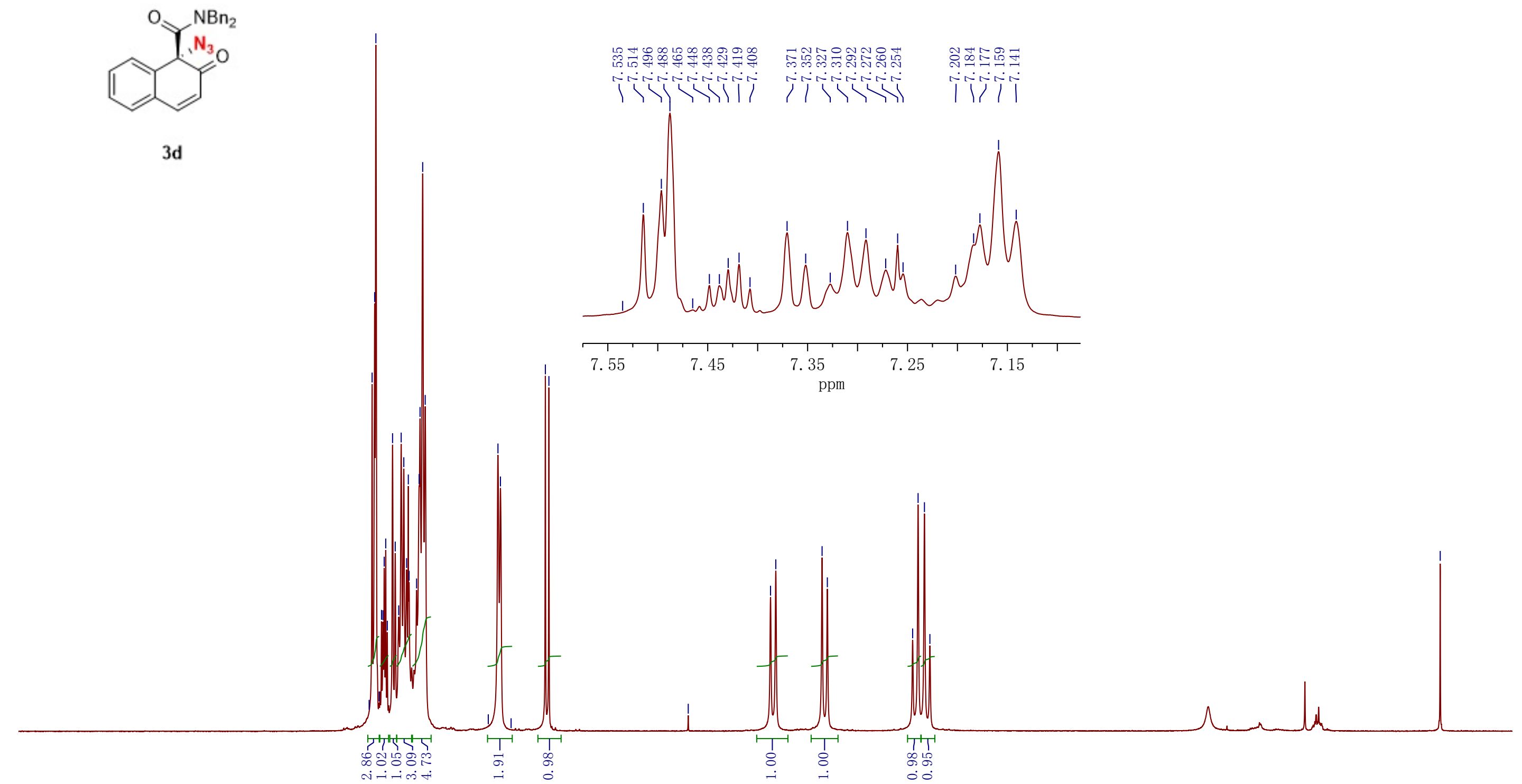

$\begin{array}{lll}9.0 & 8.5 \quad 8.0\end{array}$

7.5 7.0

$6.5 \quad 6.0$

5.5

5. $0 \mathrm{~S} 1344.5$

4. 0

3.5

3. 0

2.5

2.0

1.5

1.0

0.5

$0.0-0$ 


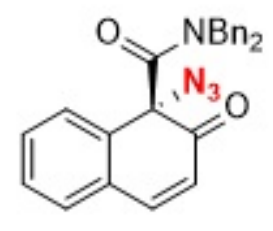

$3 d$

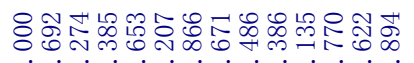

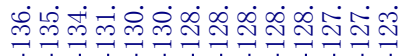

17
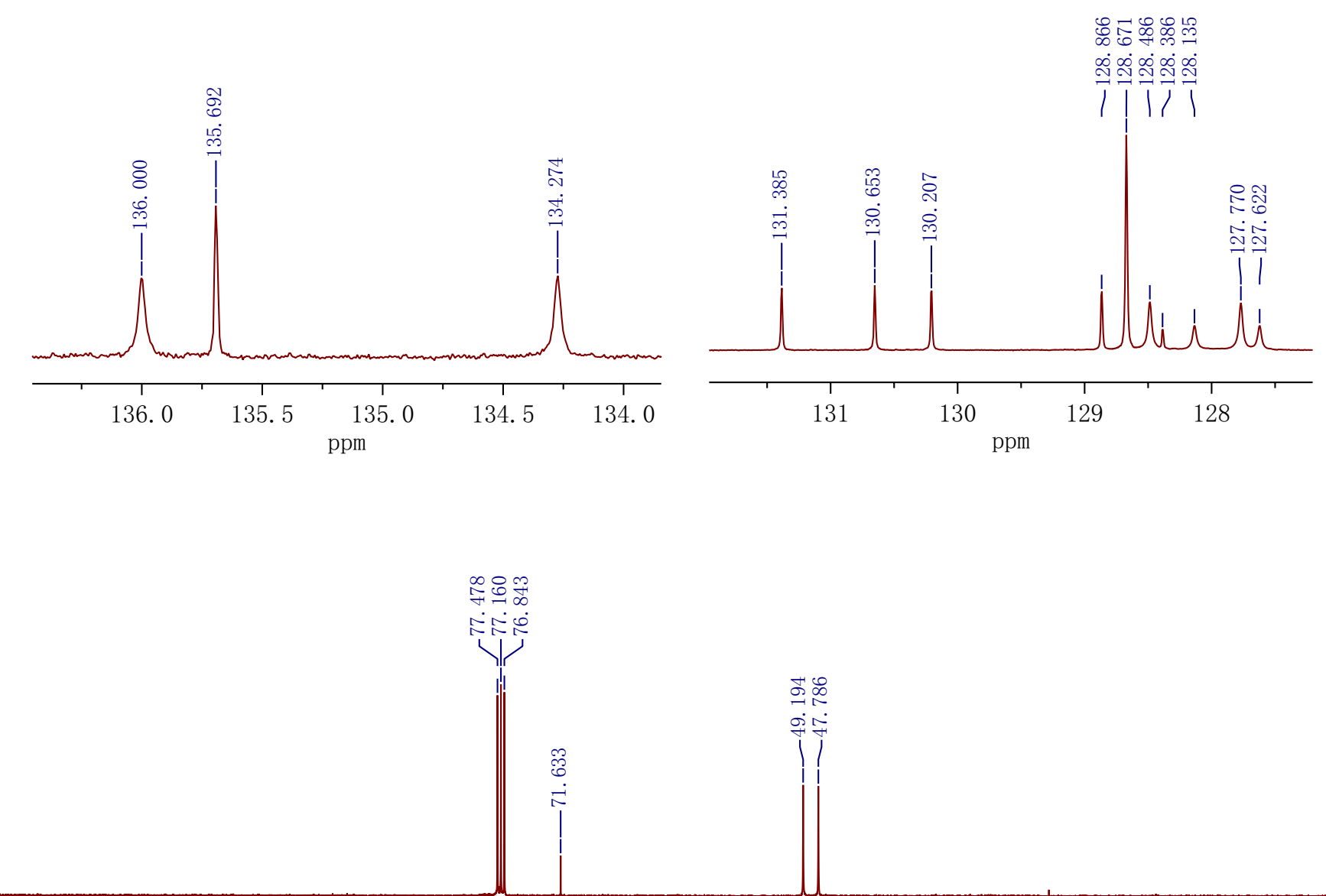

180

170

160

150

140

130

120

110

S11085

90

80

70

60

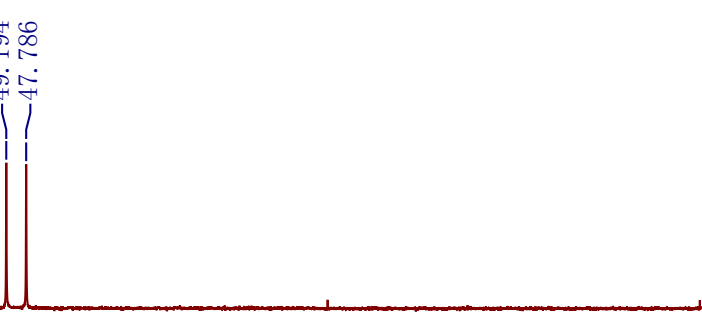


<smiles>O=C1C=Cc2cc(-c3ccccc3)ccc2C12CCCNC2=O</smiles>

3e
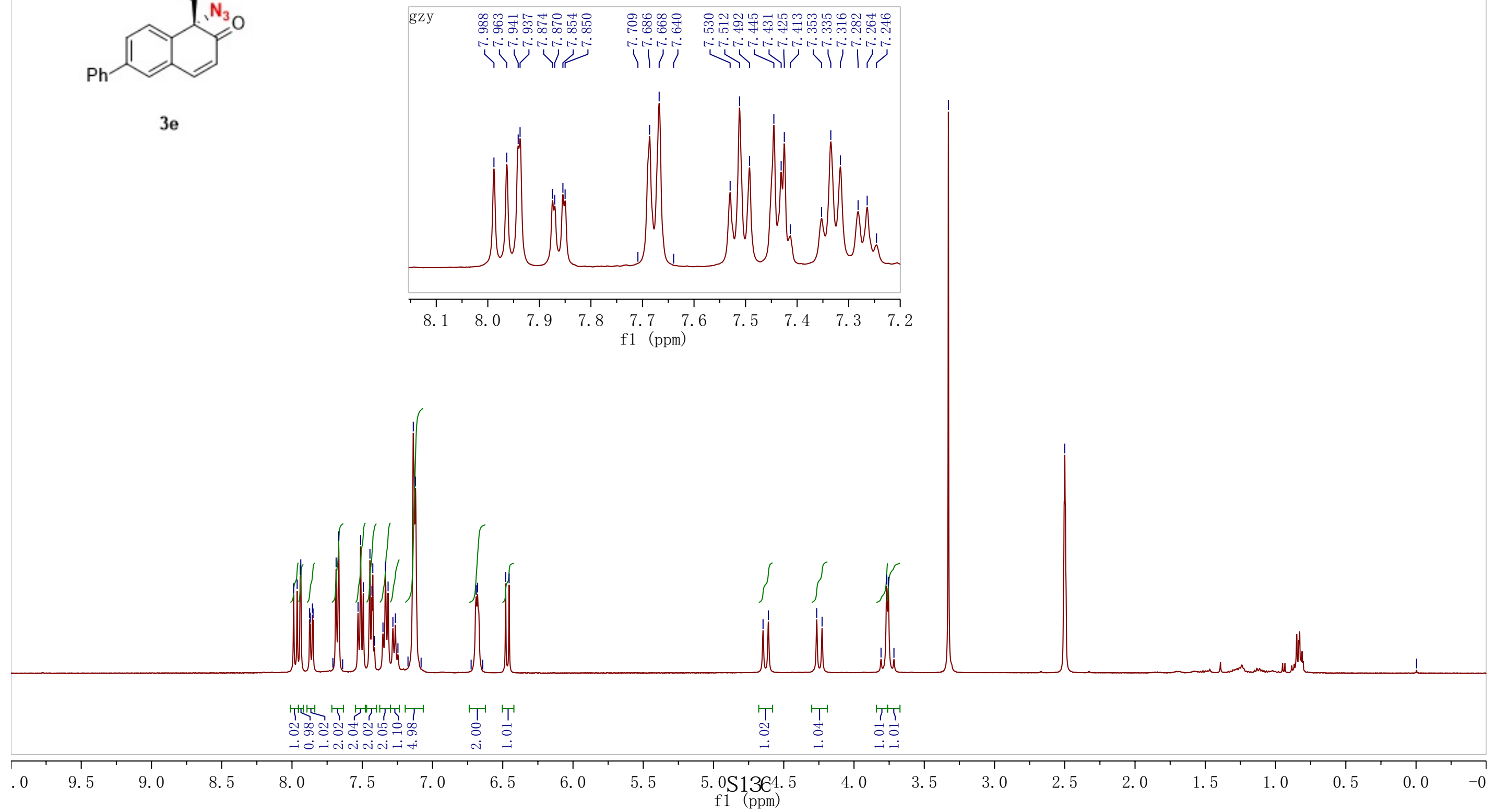


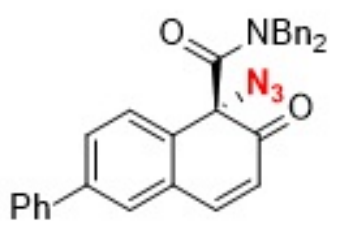

$3 e$

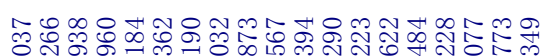

ํ.
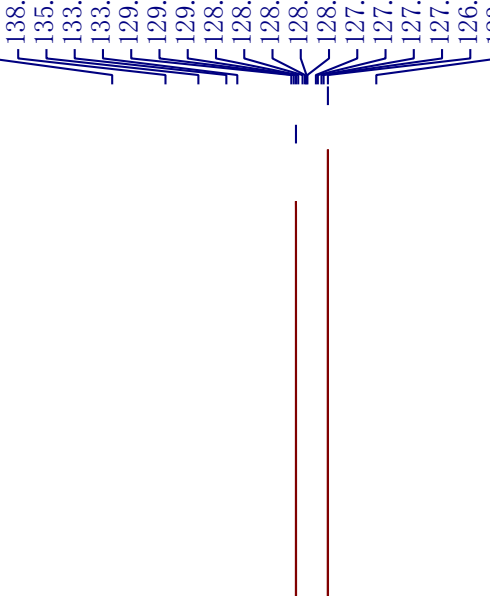

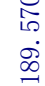
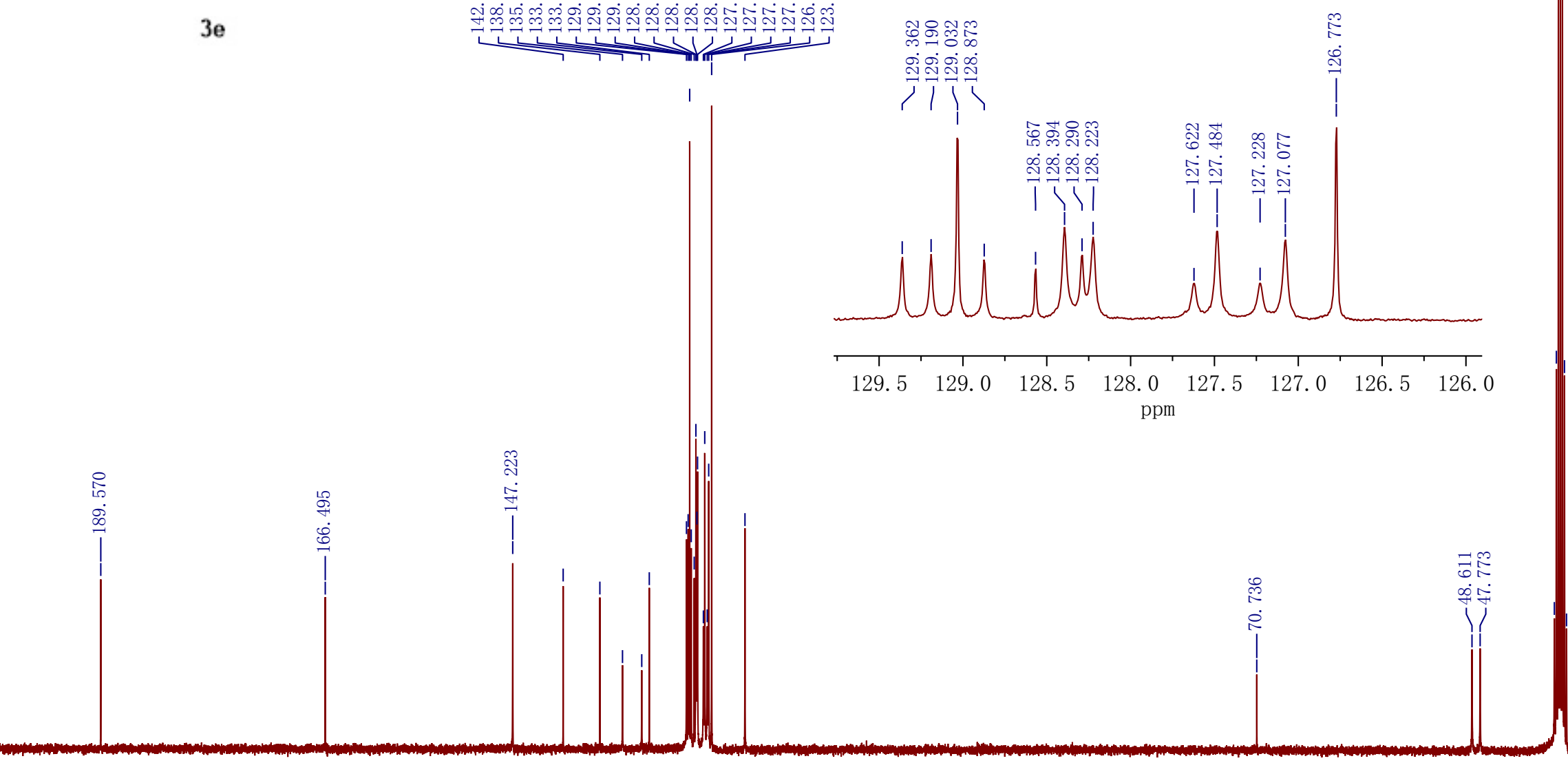

오동엇윰금응

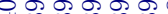

舟

190

180

170

160

150

140

130

120

110

S11087

90

80

70

60

50

40

30

1
20

10 


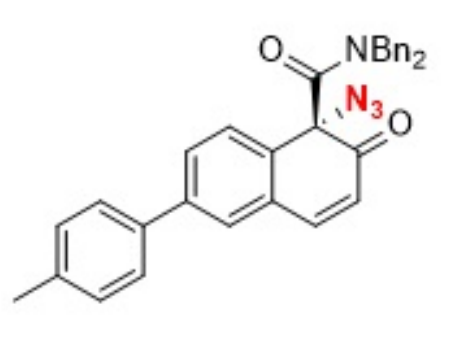

$3 f$
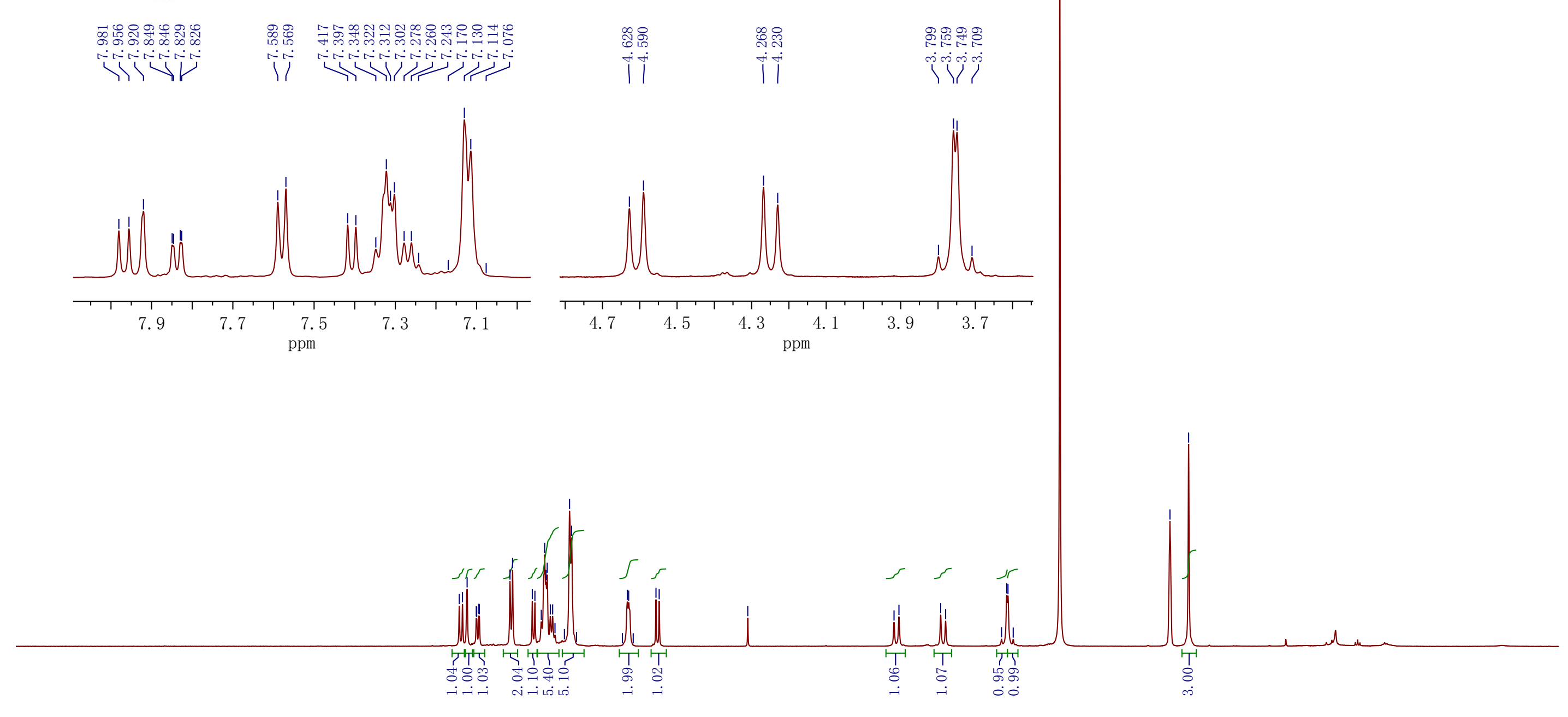

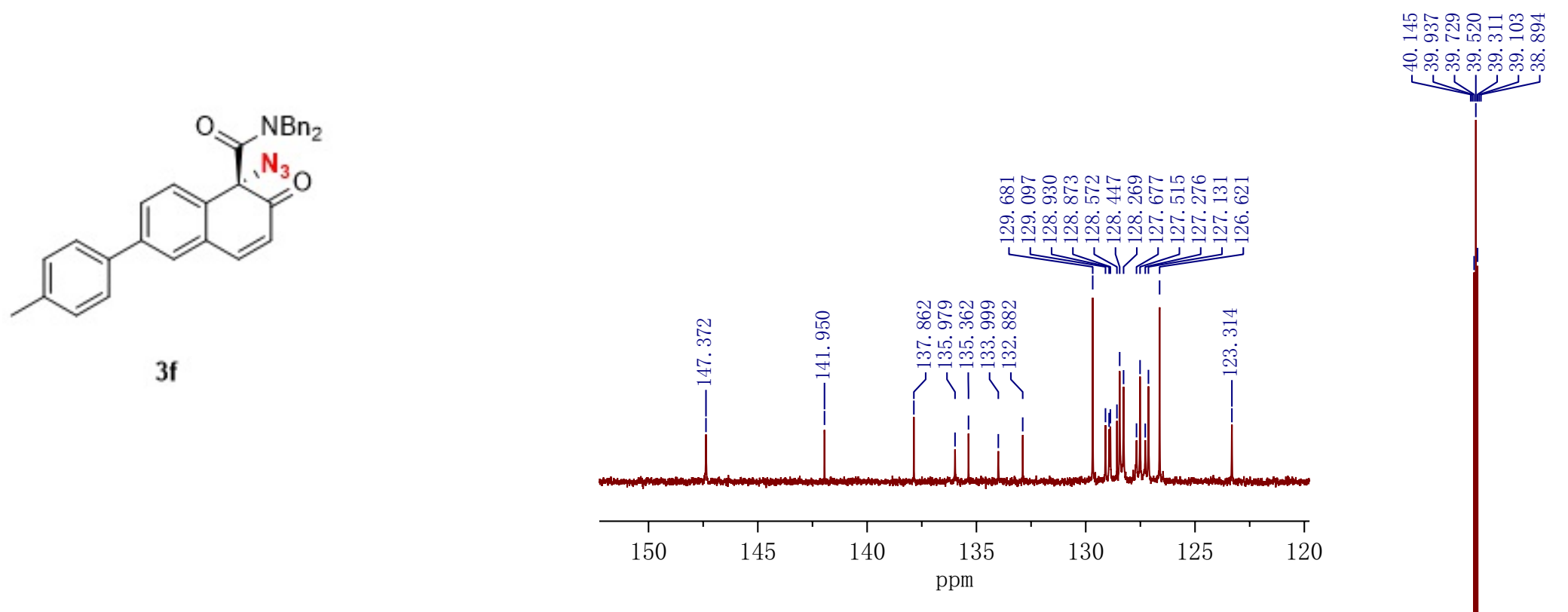

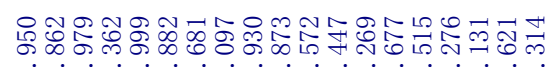

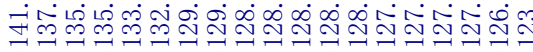
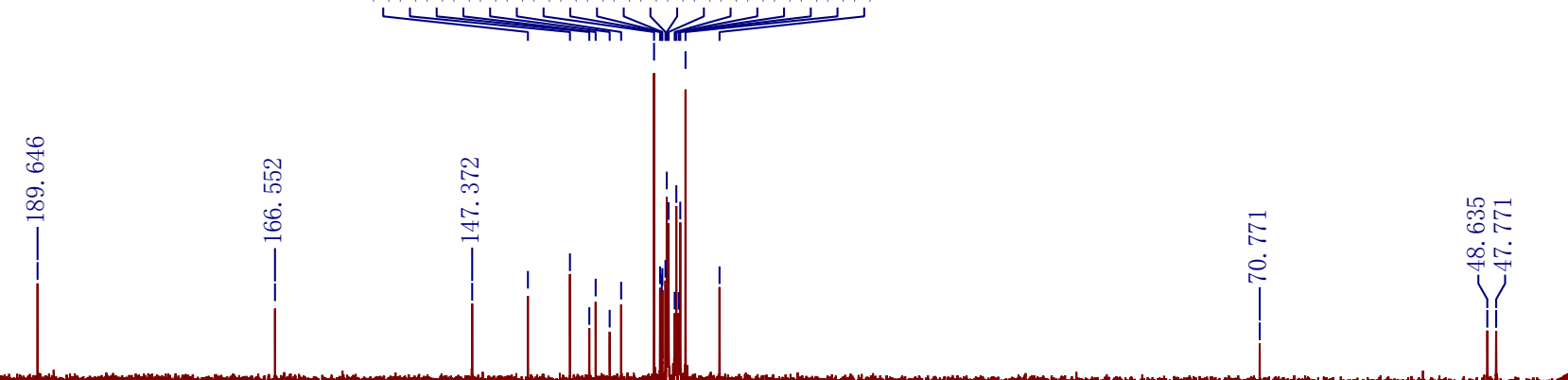


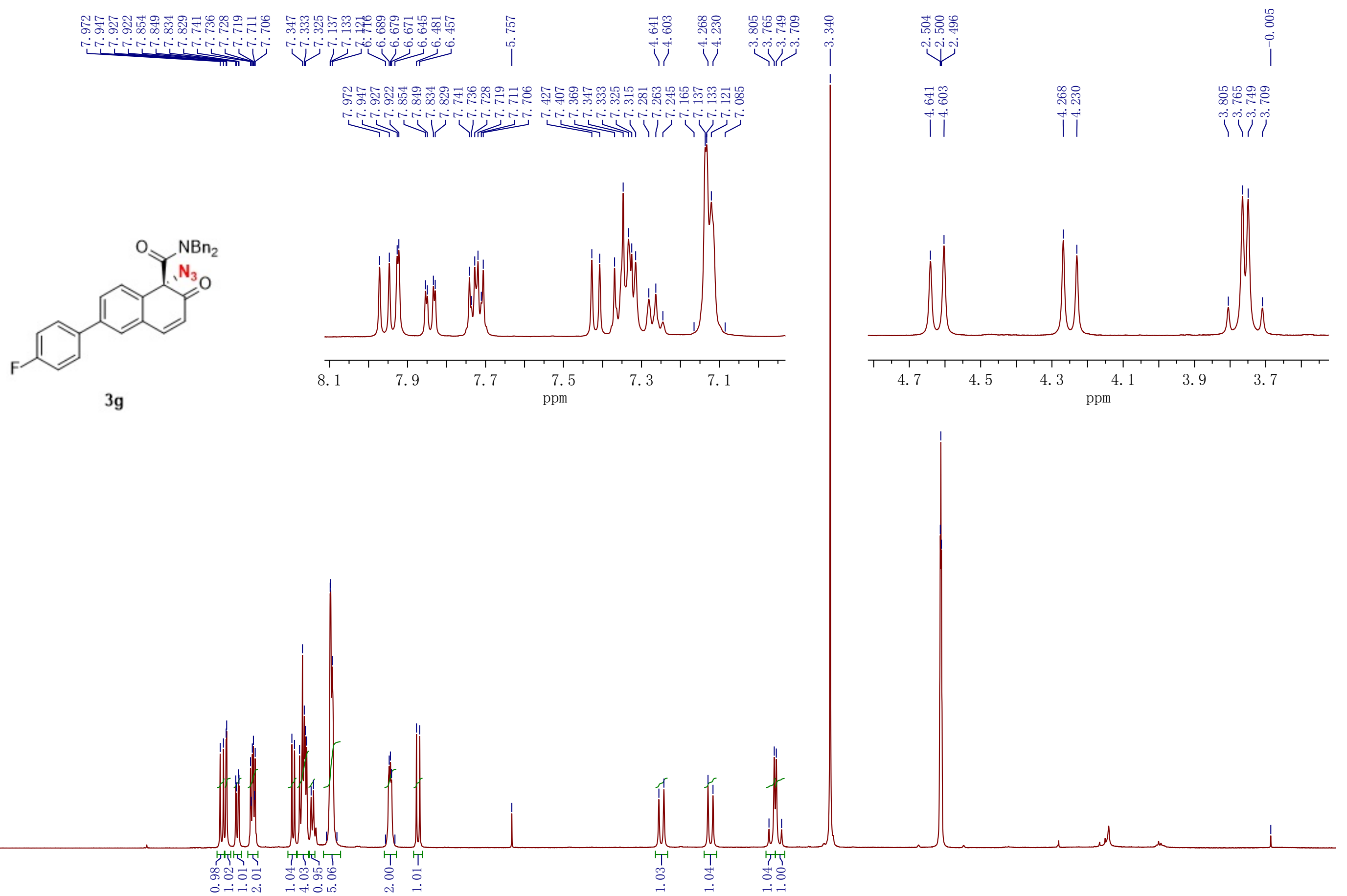

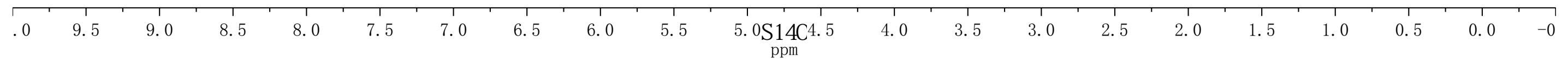




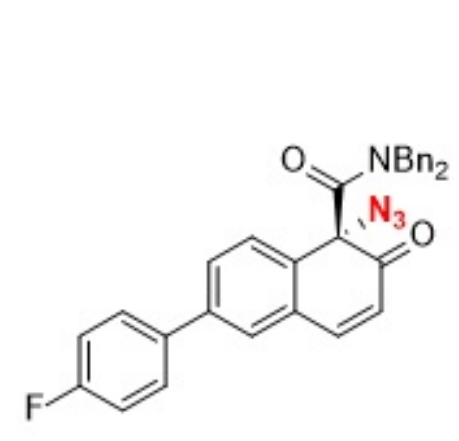

$3 g$

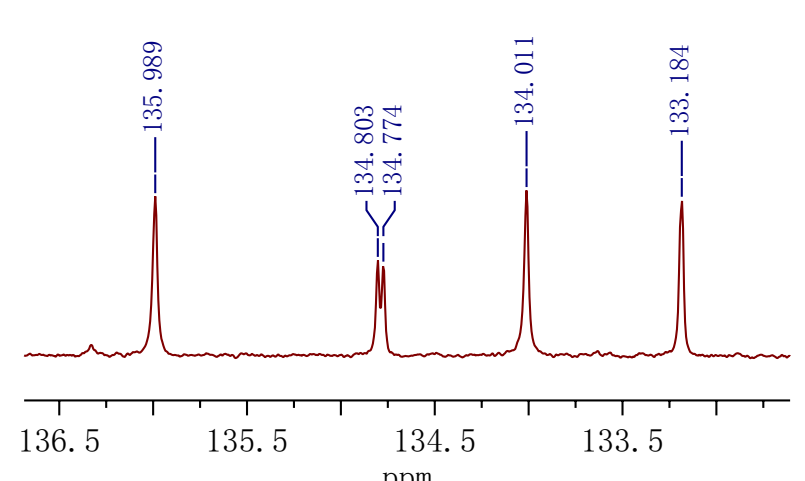

ppm

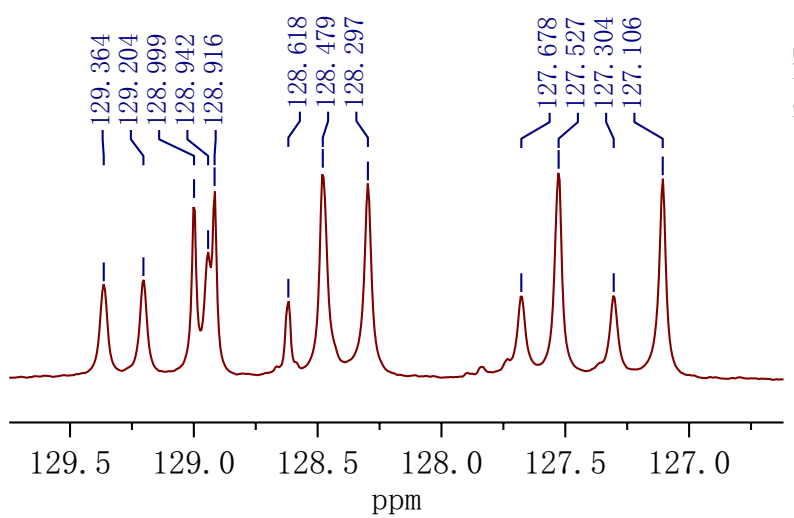

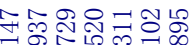

os 0000

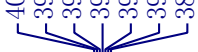

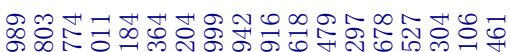

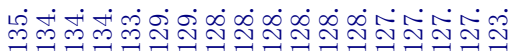

m

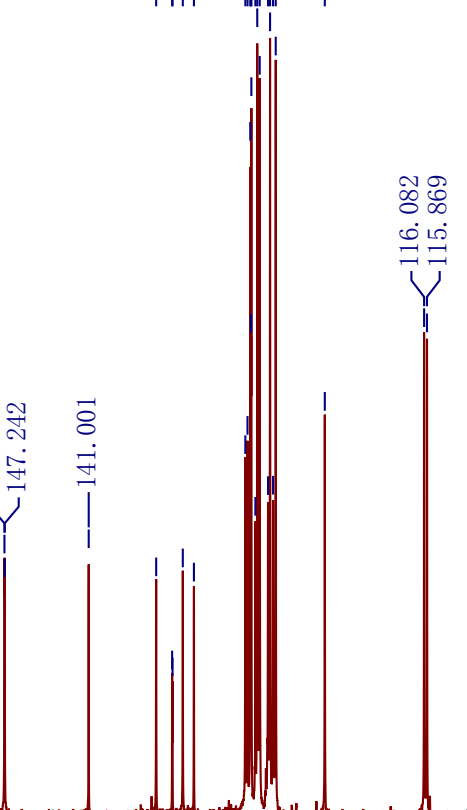

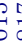




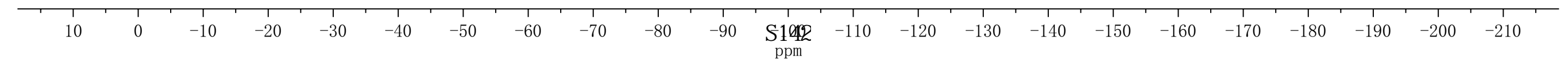



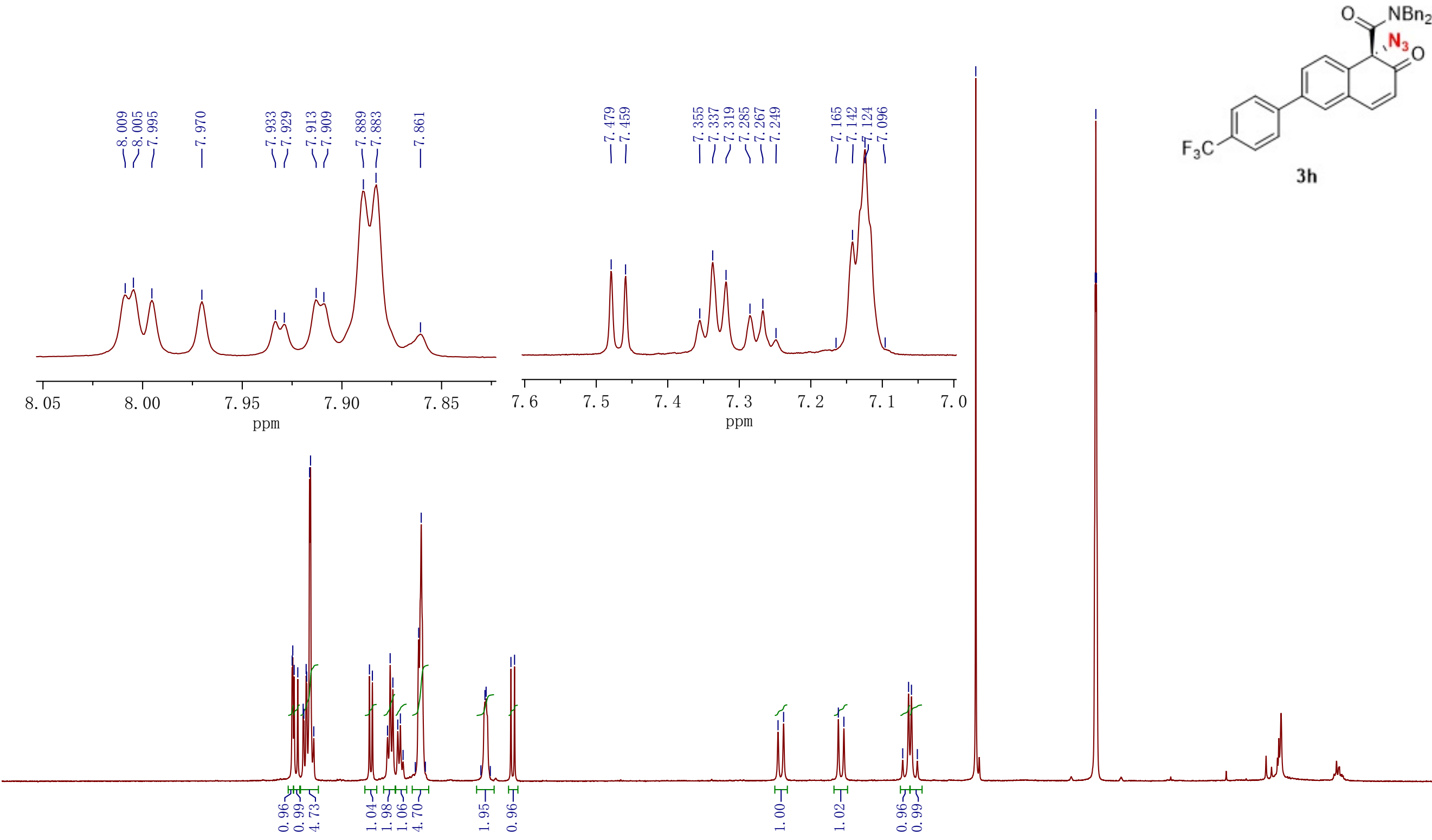

$3 \mathrm{~h}$

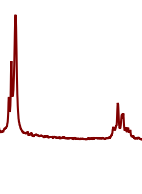

59.

$0 \quad 8.5$

8. $\quad 7.5$

7. 6.5

6. 0

5.5

5. 0 S1434. 5

4. 0

3.5

3. 0

2.5

2.0

1.5

1.0

0.5

$0.0-0$ 

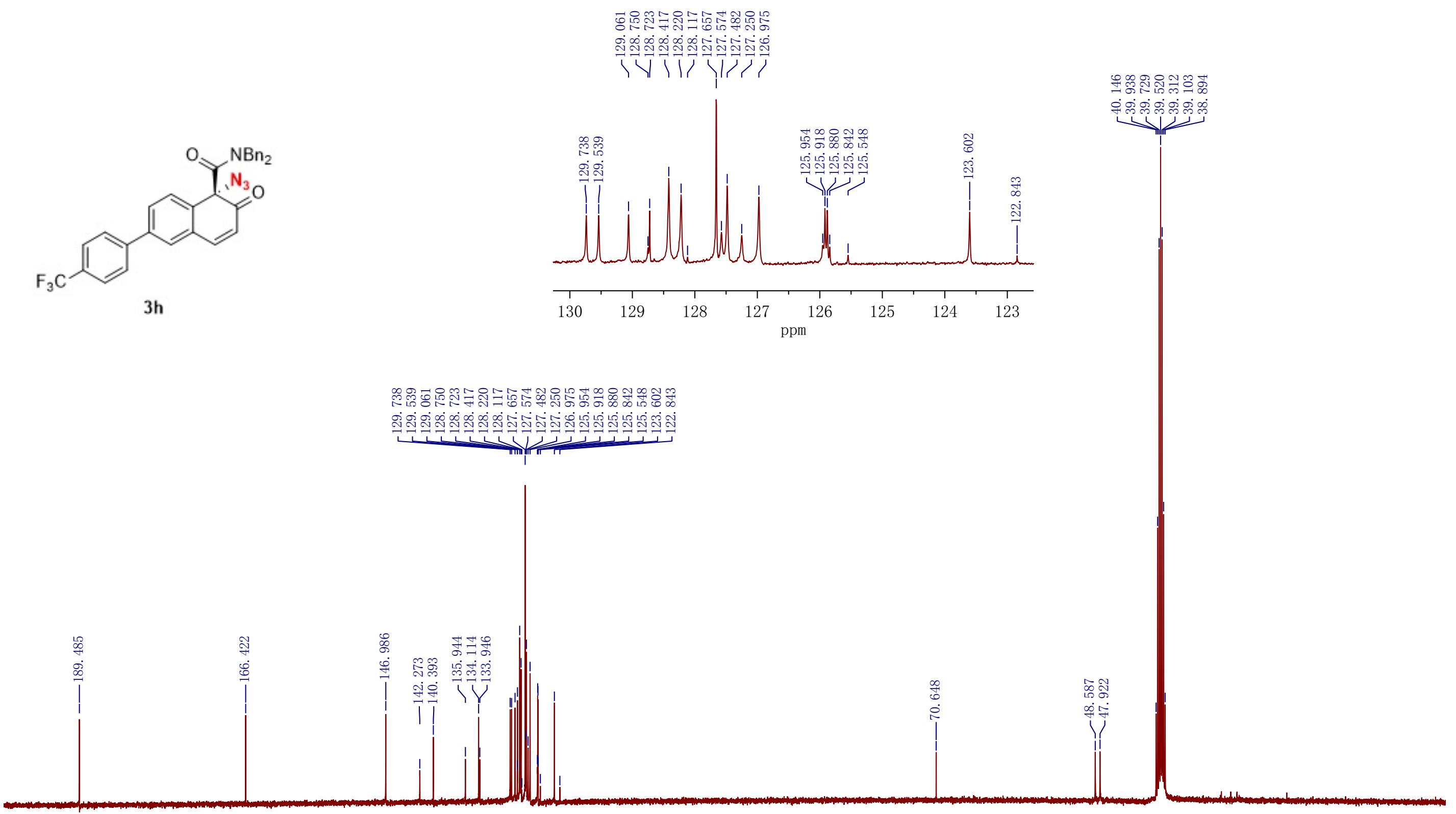

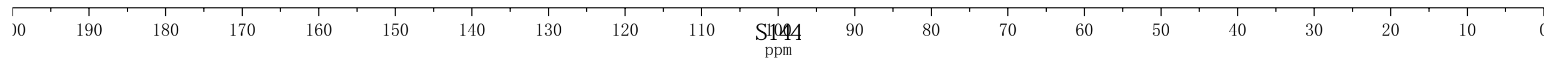




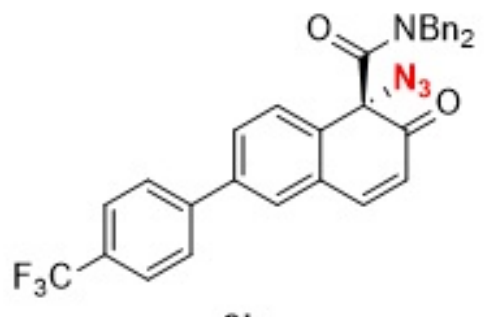

$3 \mathrm{~h}$

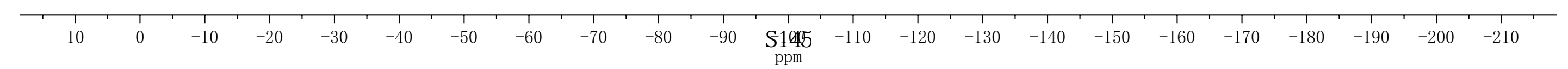




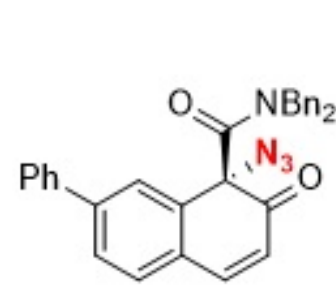

$3 \mathrm{i}$
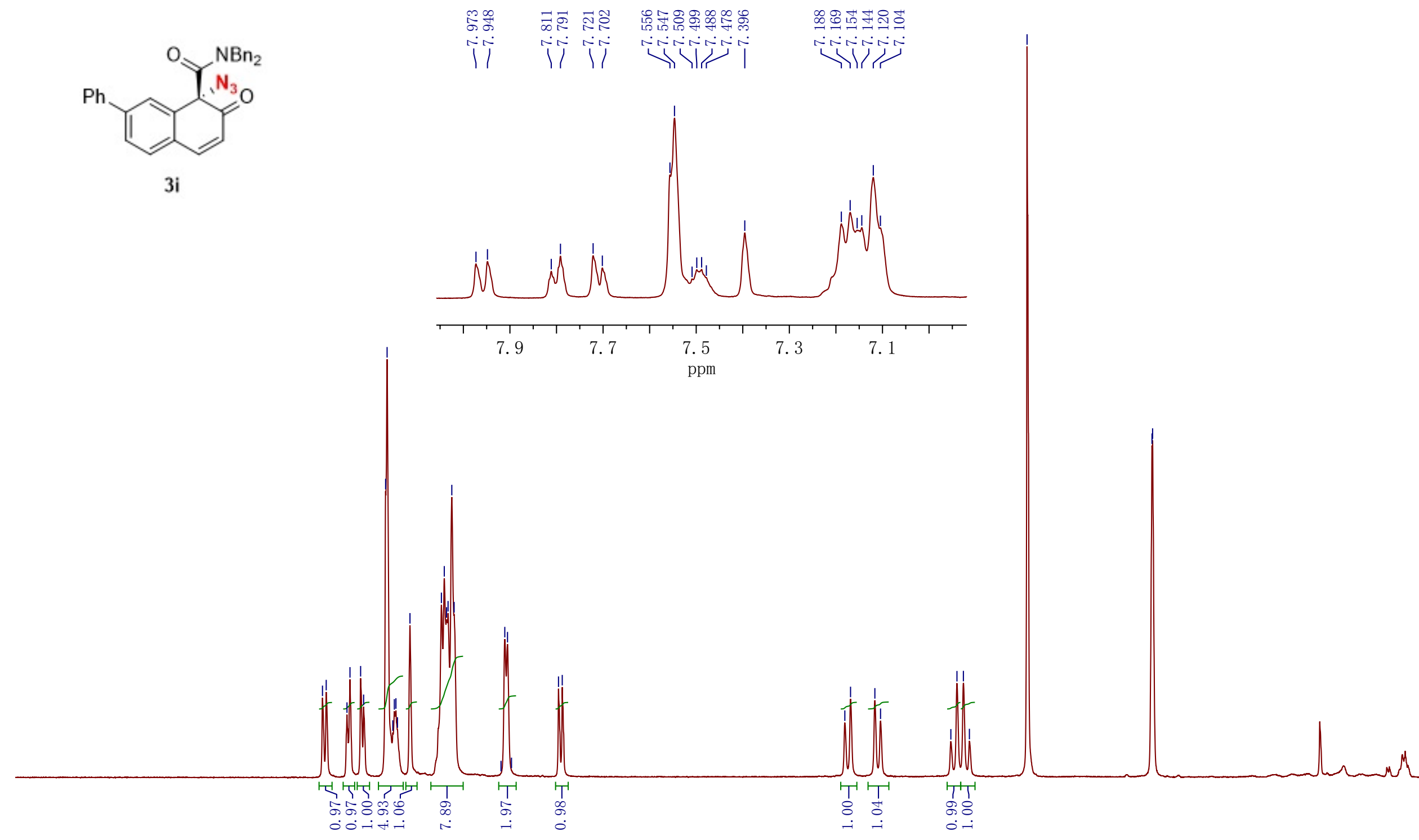

$5 \quad 9.0$

8.5

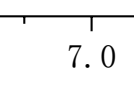

6.5

6. 0

5.5

5. $0 \mathrm{~S} 1464.5$

. 0

3.5

3. 0

2.5

2.0

1.5

1. 0

0.5 

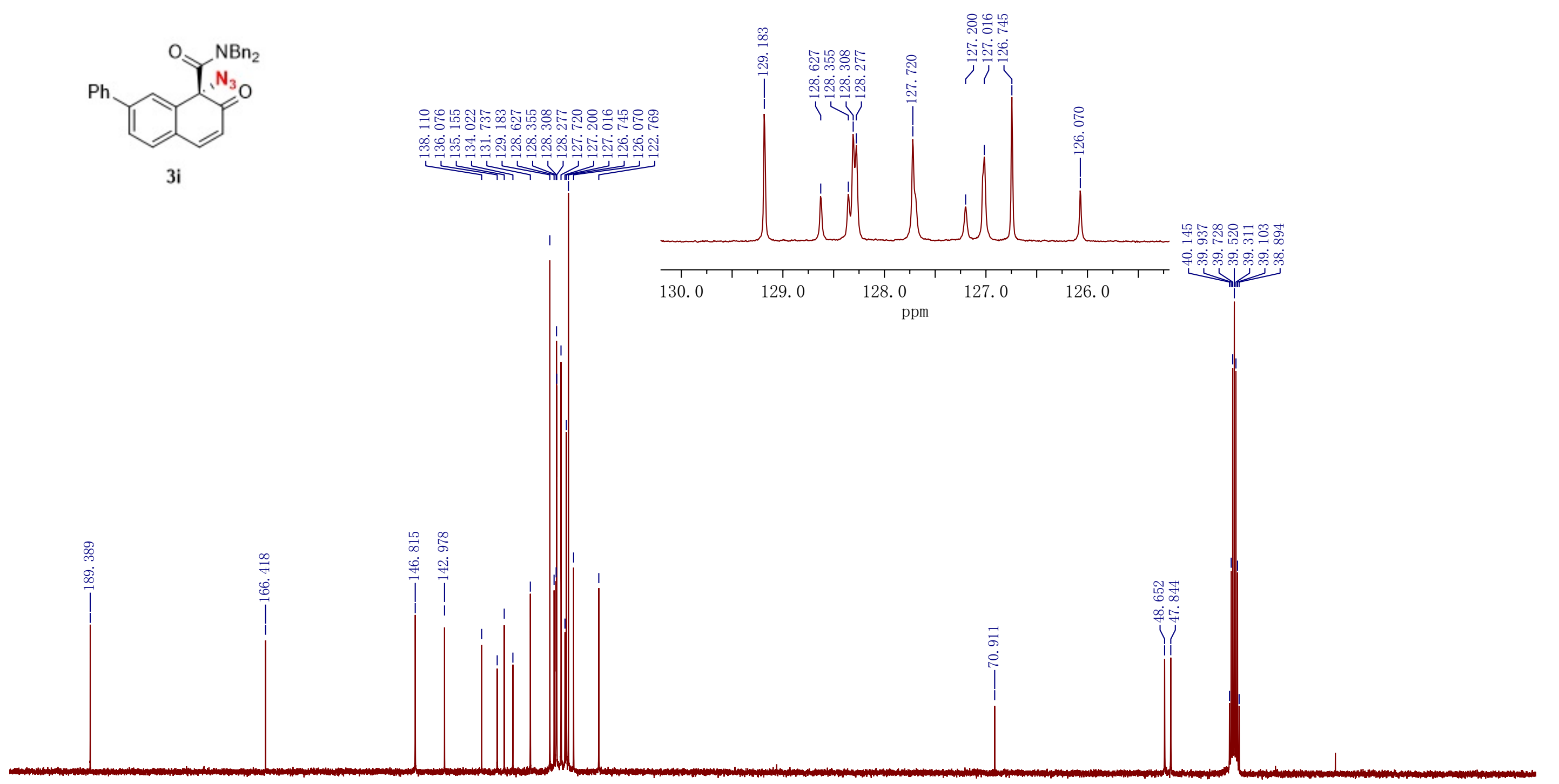

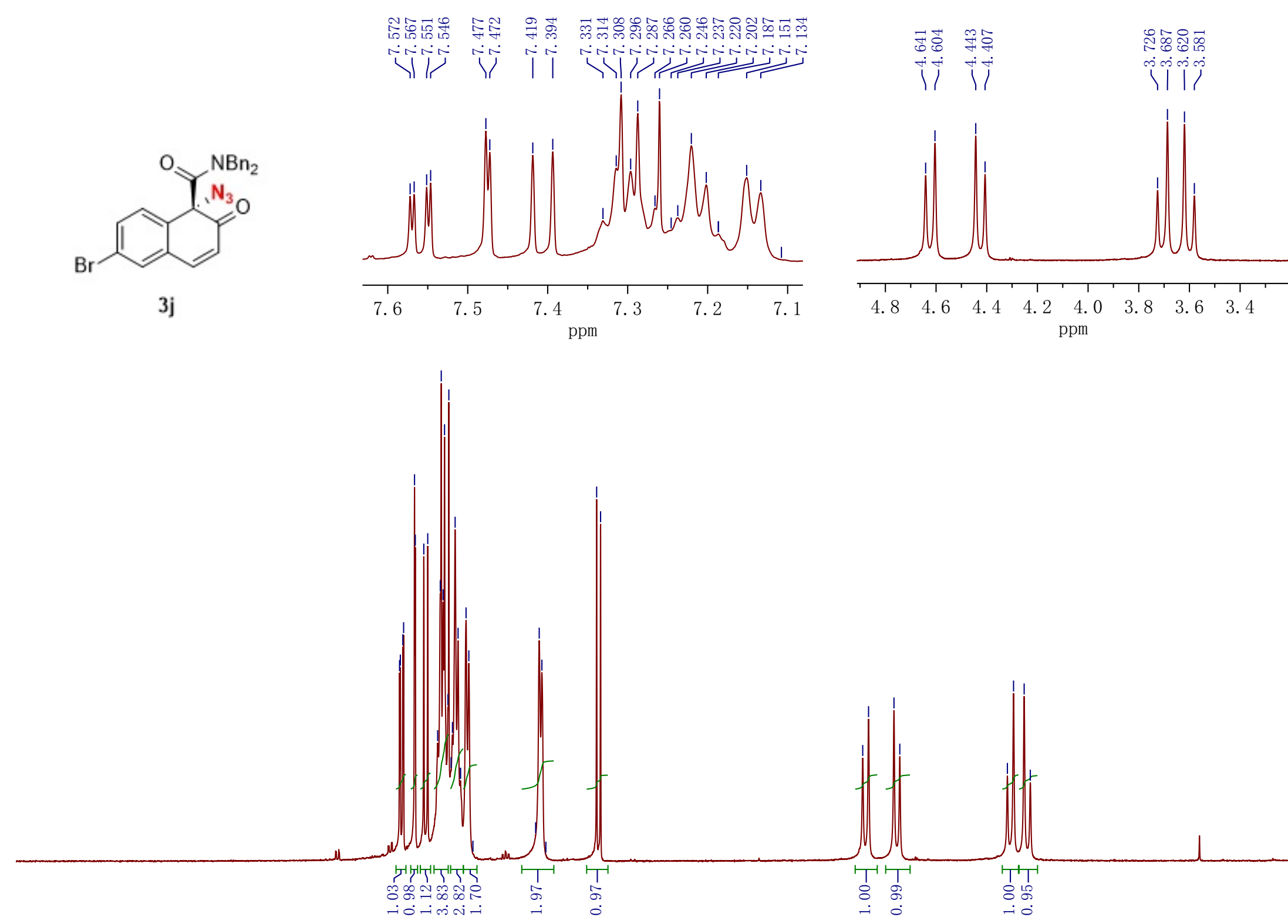

9. $0 \quad 8.5$

8. $0 \quad 7.5$

7. 0

6.5

6. 0

5.5

5. 0 S1484. 5

+ 1.5

3. 0
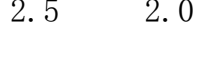


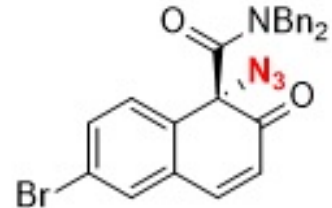

3j

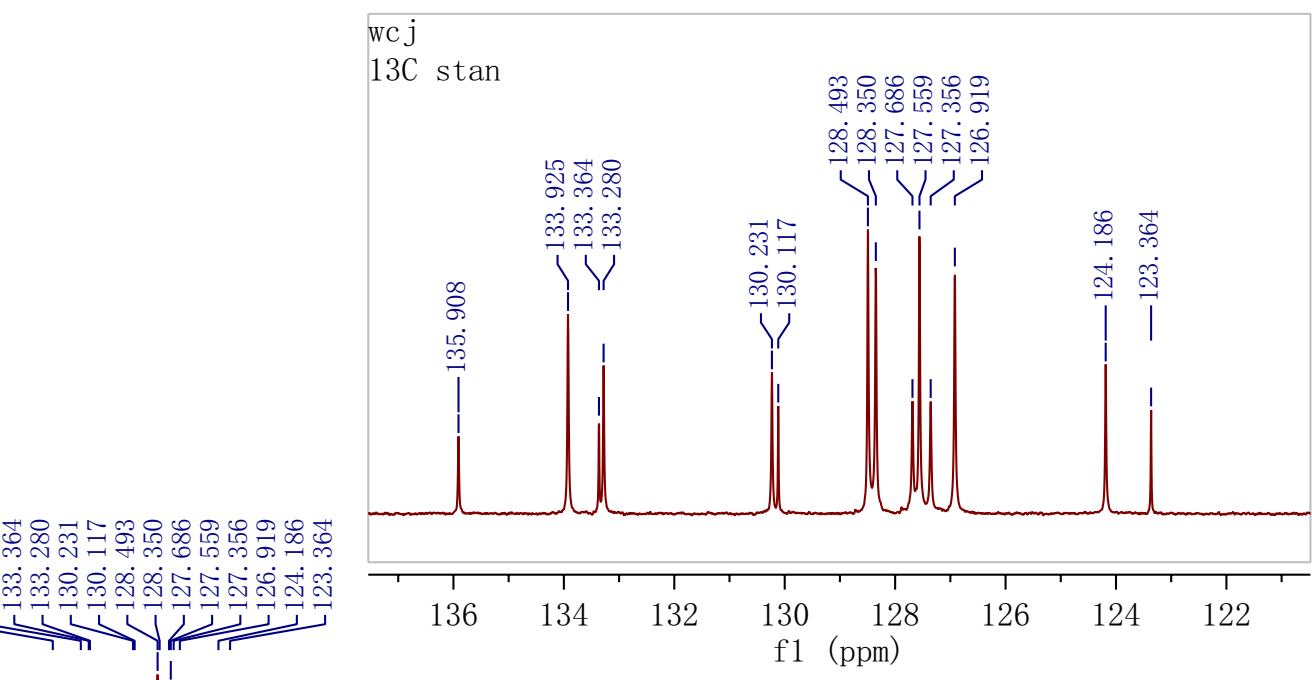

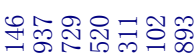
해요요

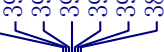

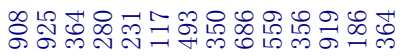
ю의 1

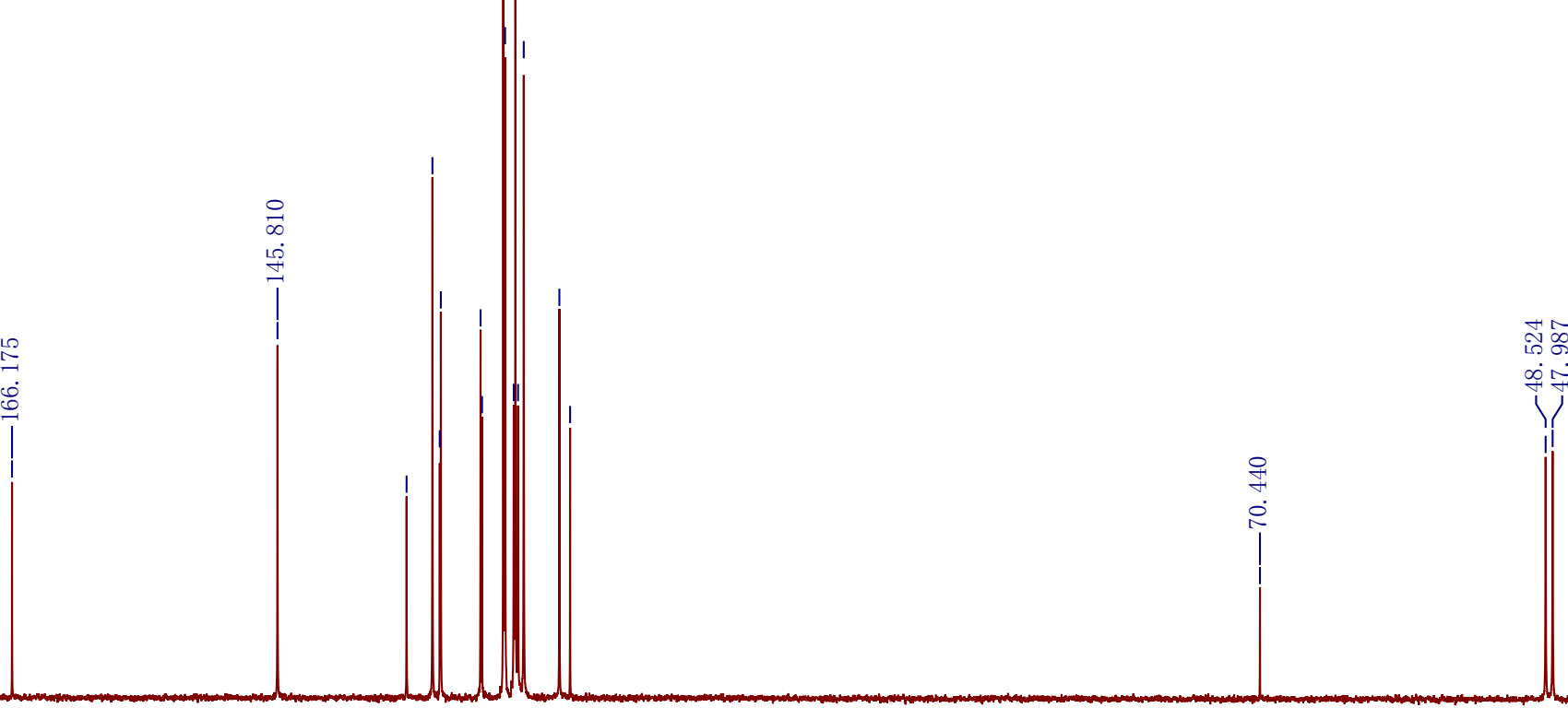

$\stackrel{\vec{D}}{\vec{\infty}}$

$190 \quad 180 \quad 170$

$\begin{array}{rrrr}160 & 150 & 140 & 130\end{array}$

120

$110 \quad$ S1049 (ppm)

$\begin{array}{ll}1 & \\ 90 & 80\end{array}$

$80 \quad 70$

60

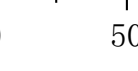

50

40

30 

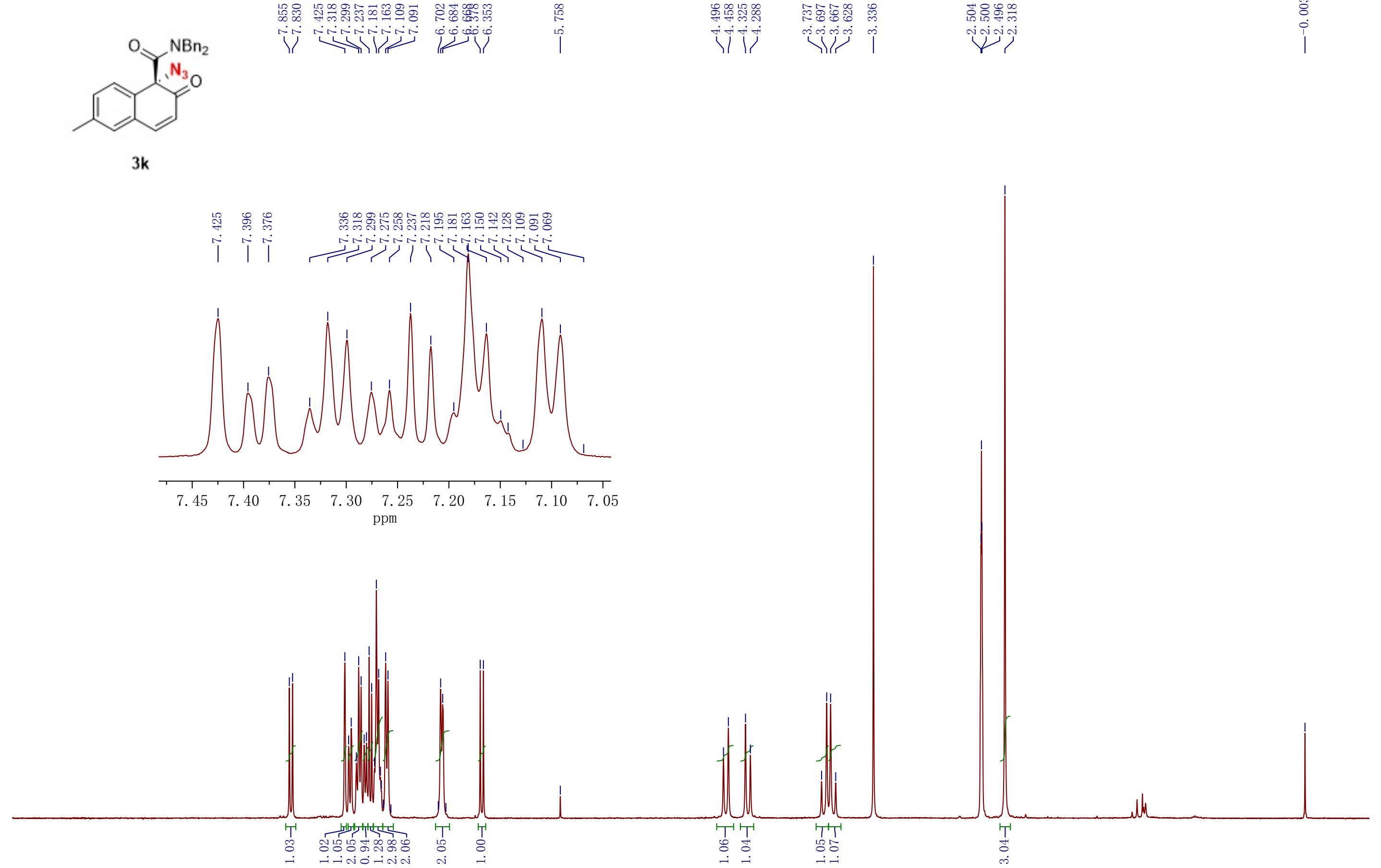

$5 \quad 9.0$

$8.5 \quad 8.0$

$7.5 \quad 7.0$

6.5

6. 0

5.5

5. $0 \mathrm{~S} 1504.5$

3.5

3. 0

2.0

1.5

1. 0

0.5

$0.0-0$ 

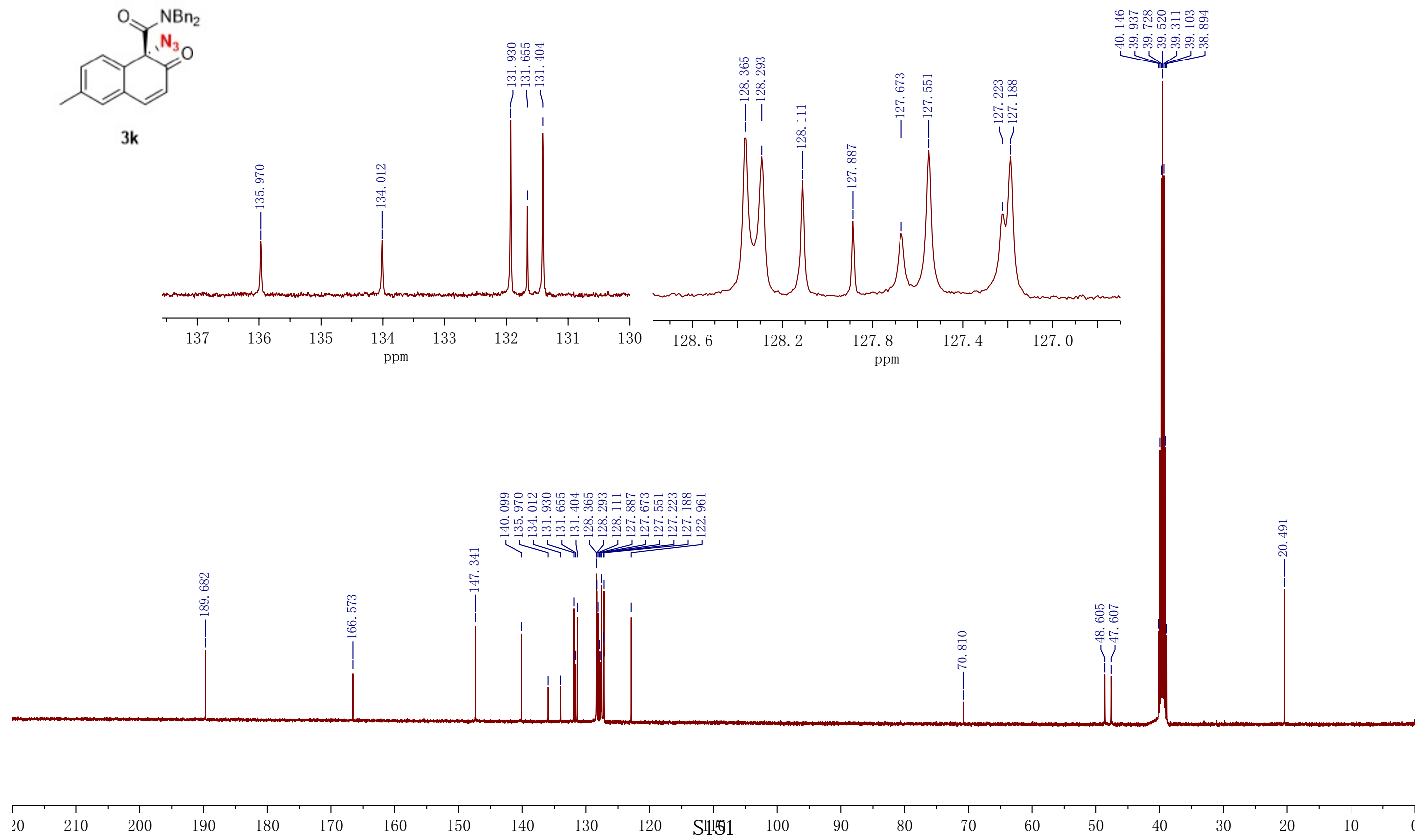

Sn151 

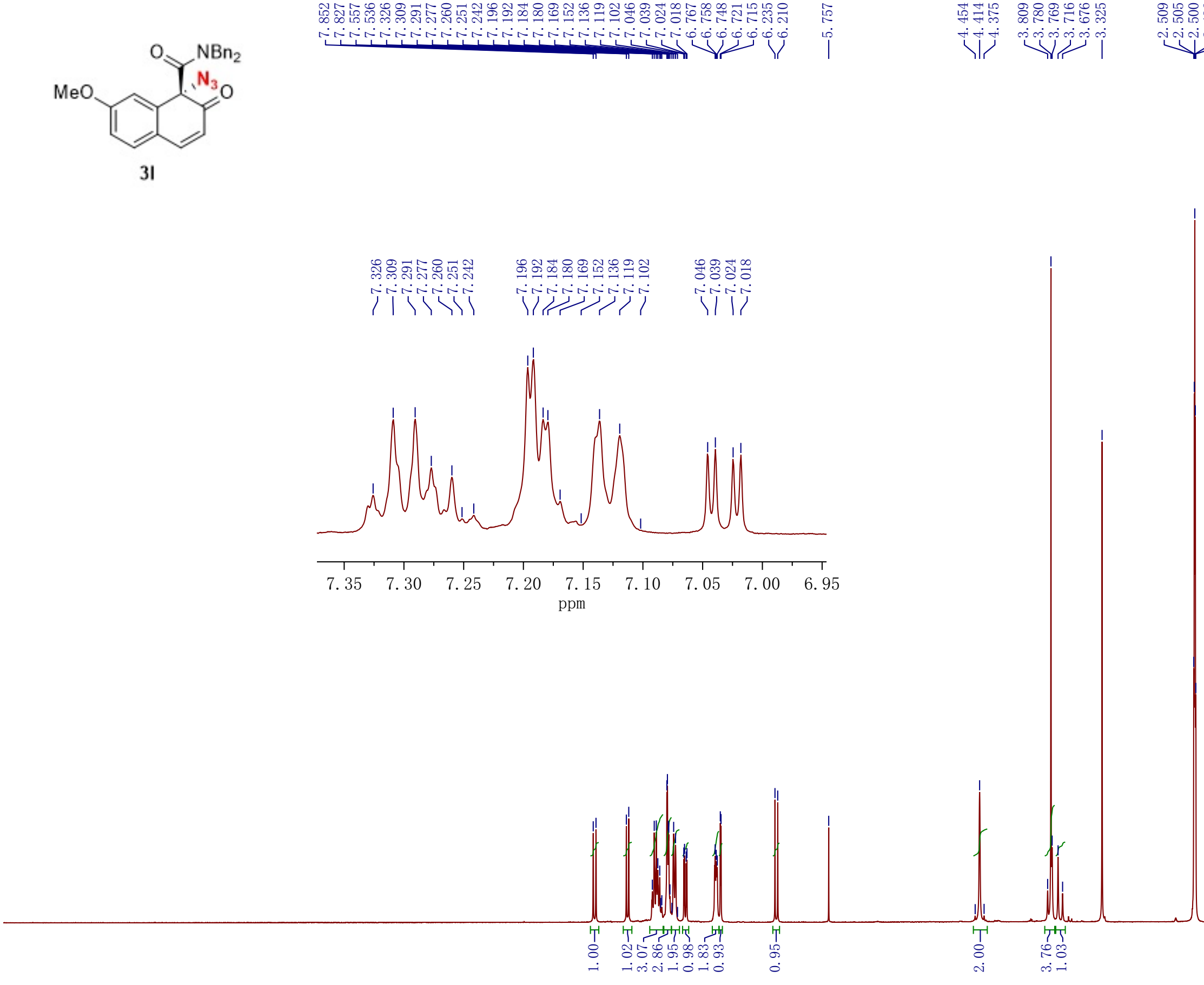


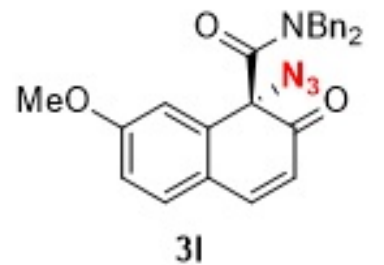

31

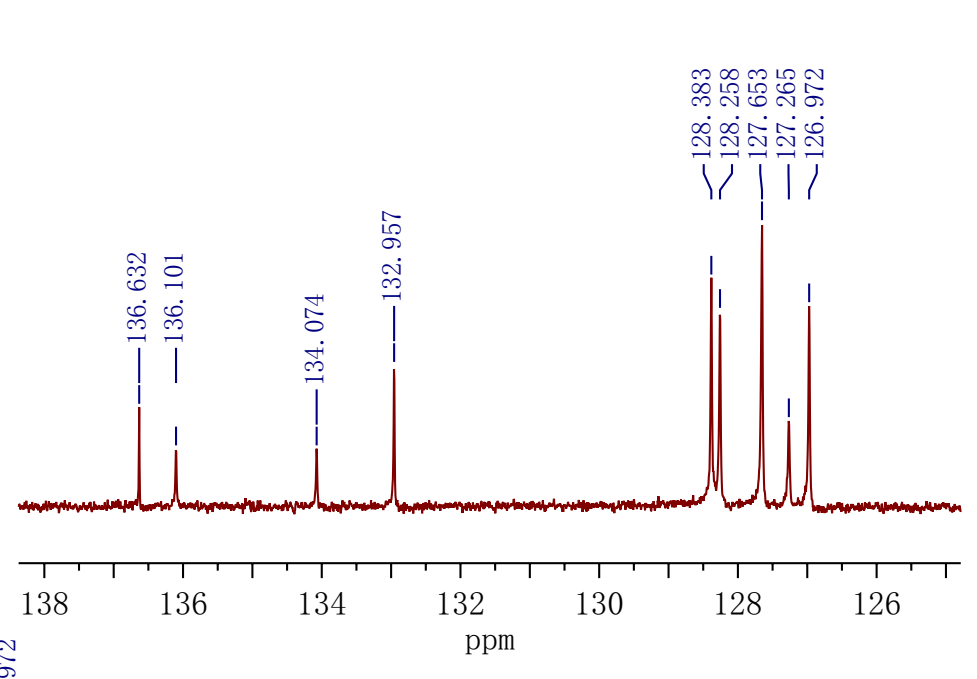

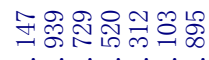

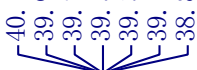

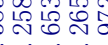

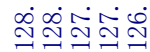

4 


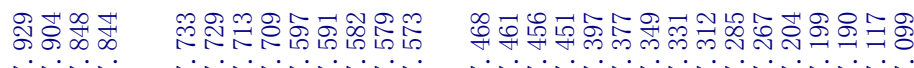

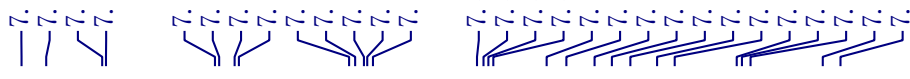
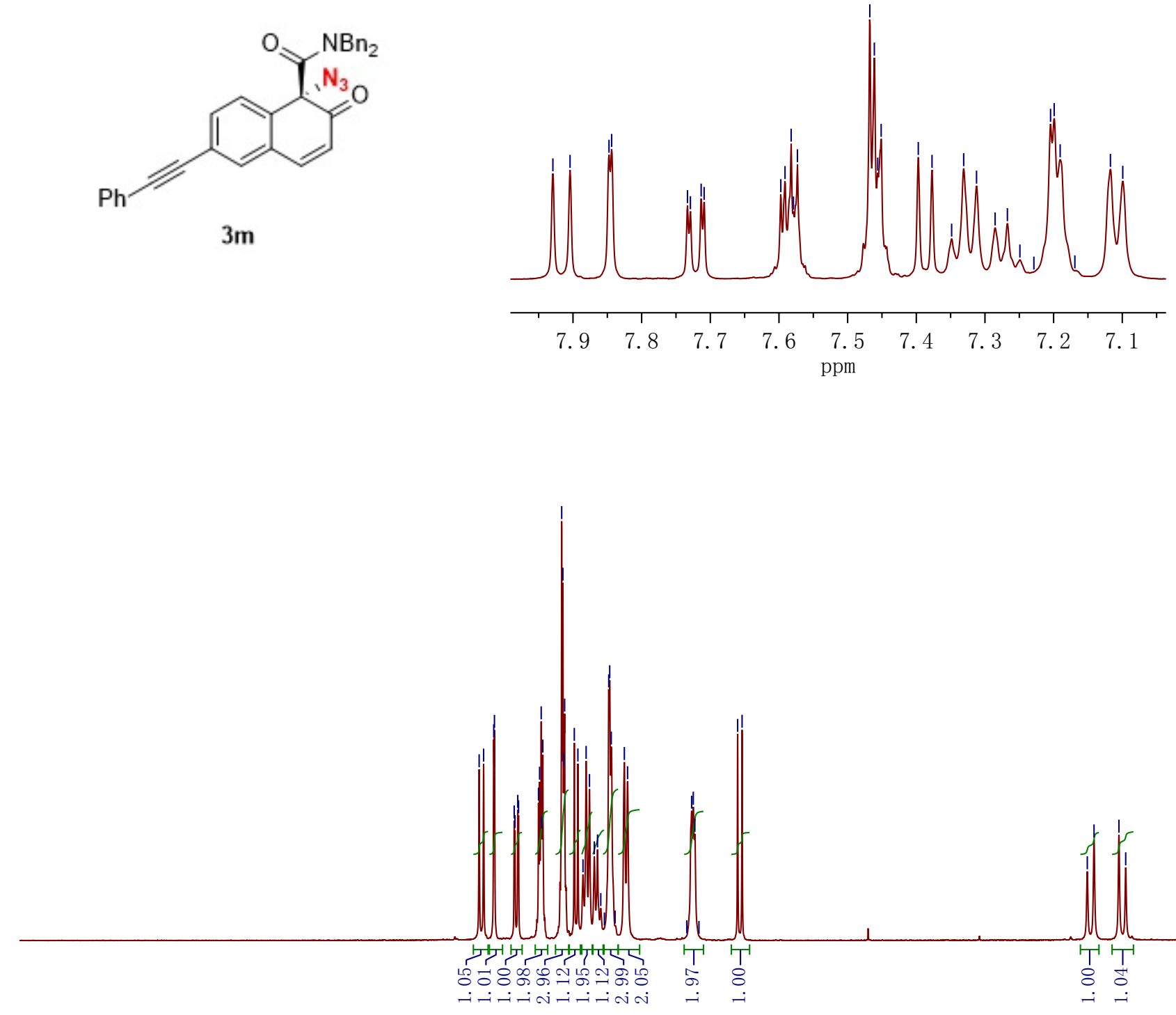

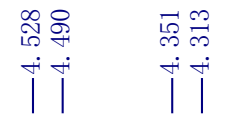

些品获落

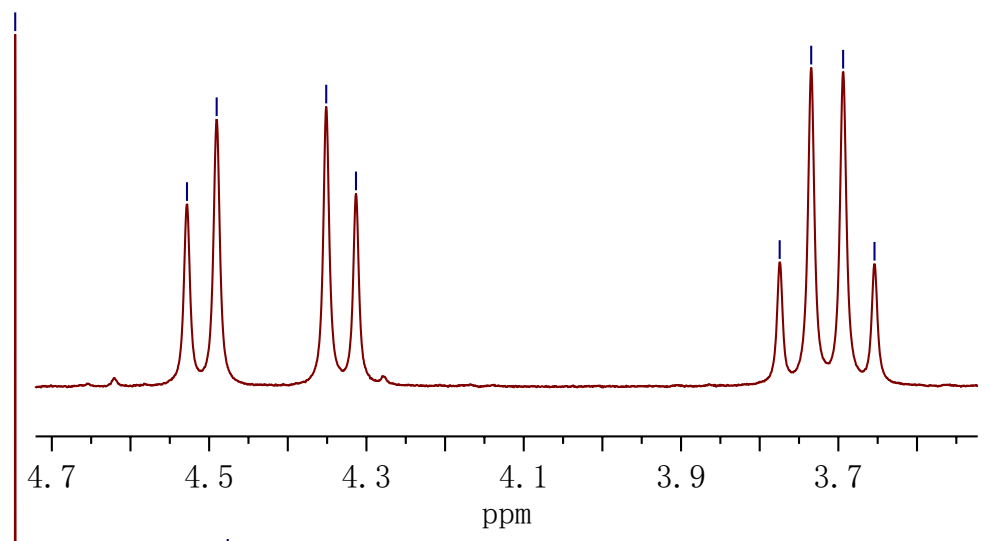




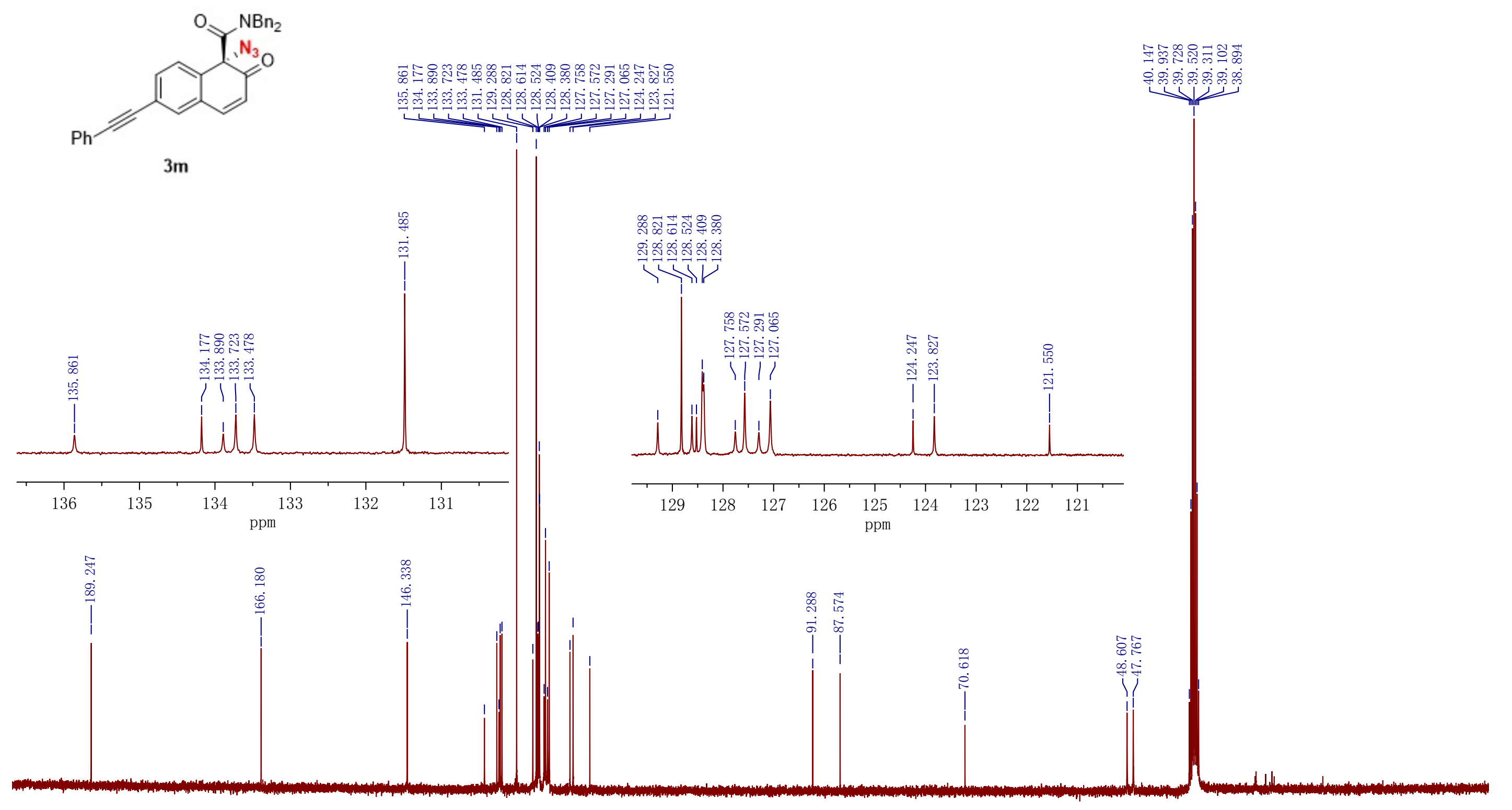

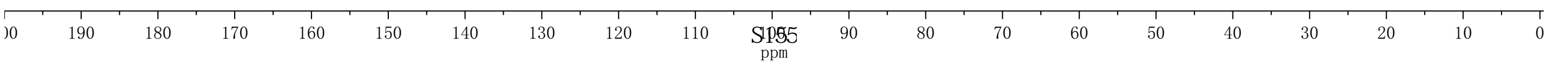




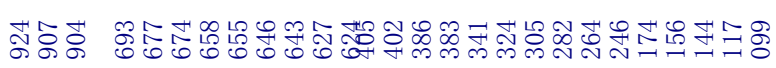

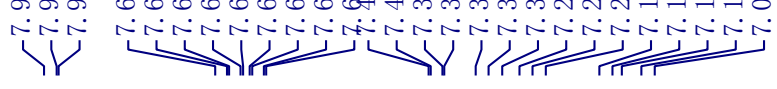
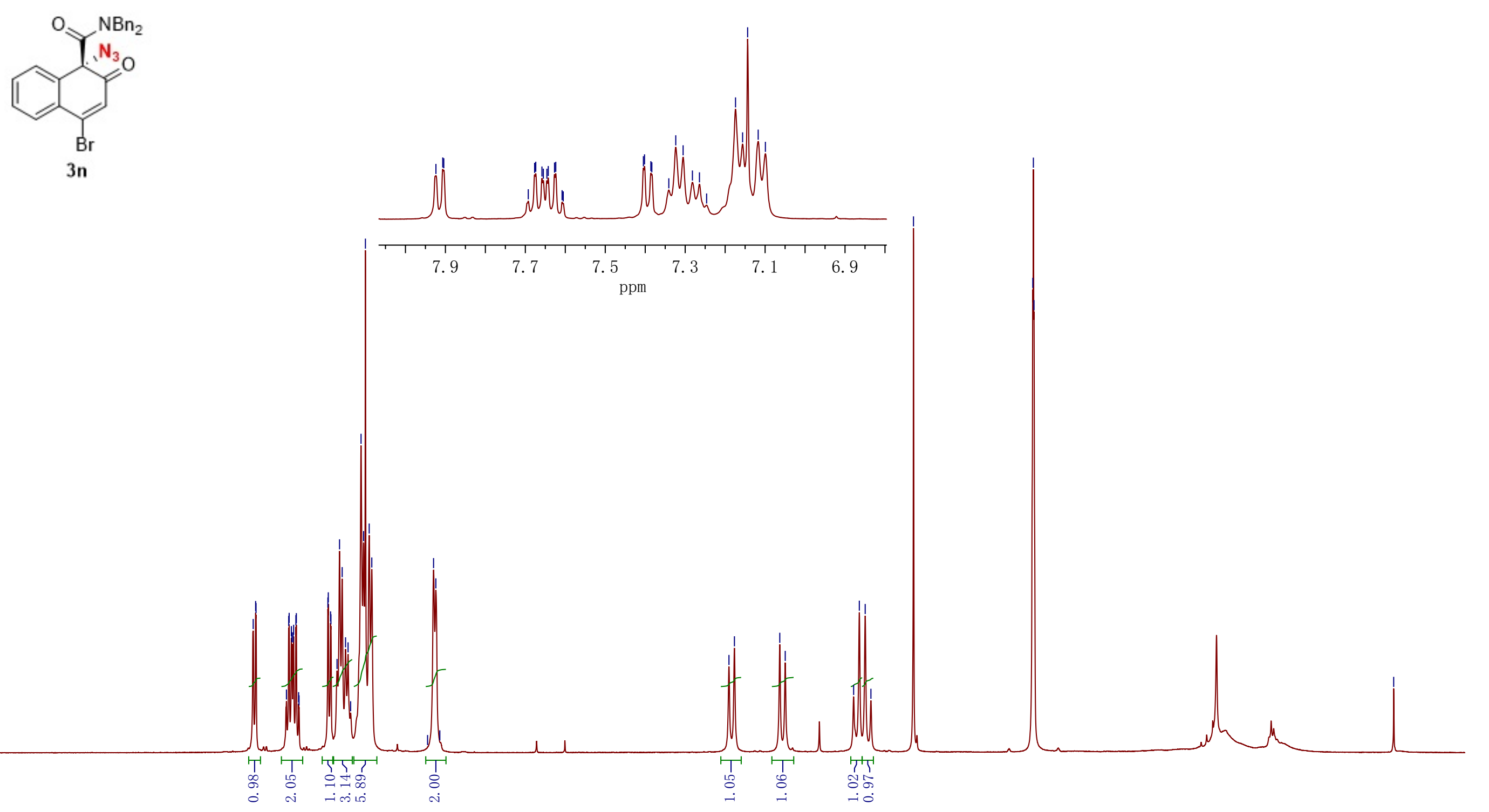


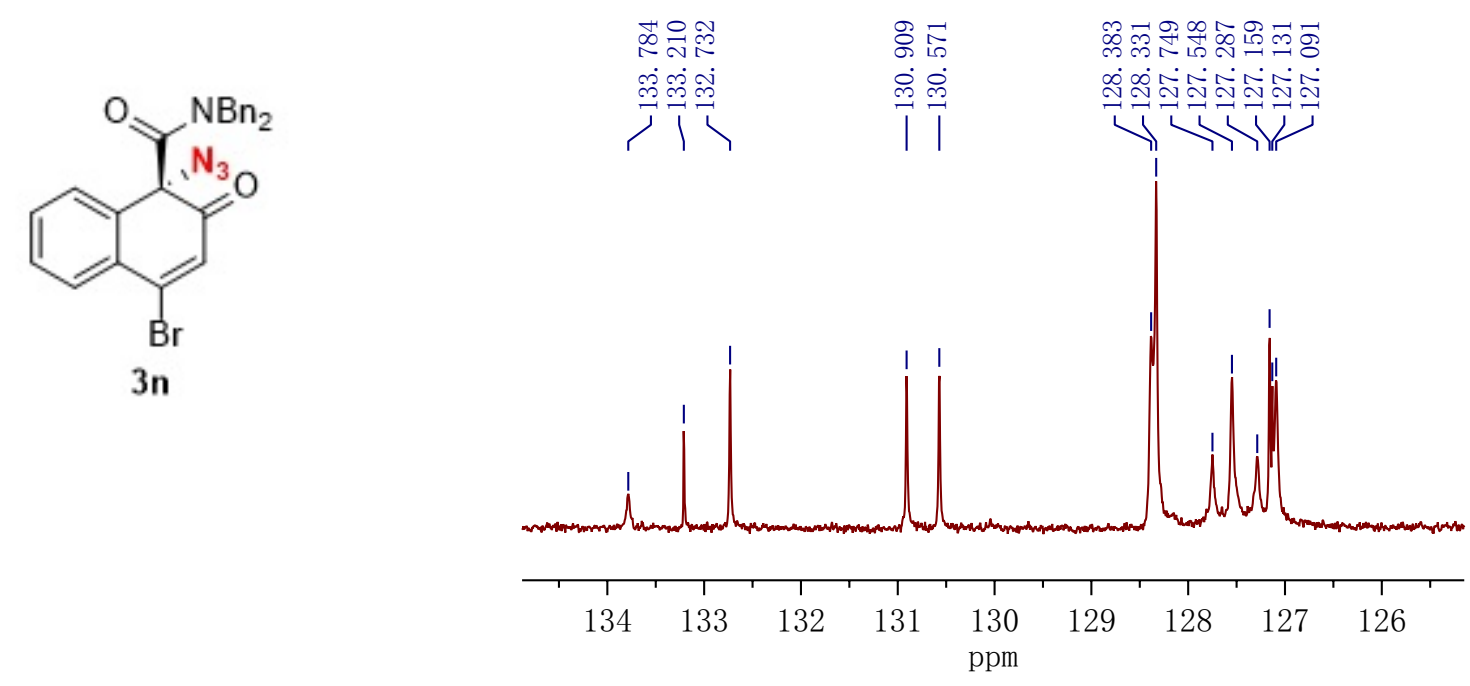

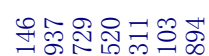

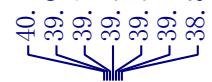

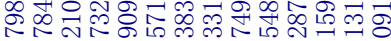

\%

1

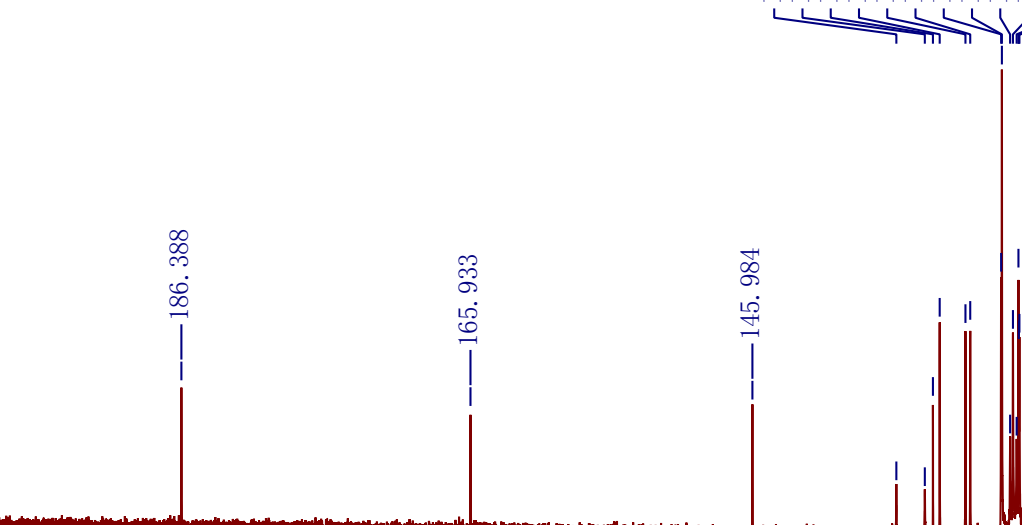

$$
10
$$

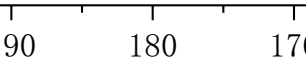

$170+160$

$\begin{array}{rrr}1 & & \\ 150 & 140 & 130\end{array}$

110 S11067

$\quad 70$

40

30 

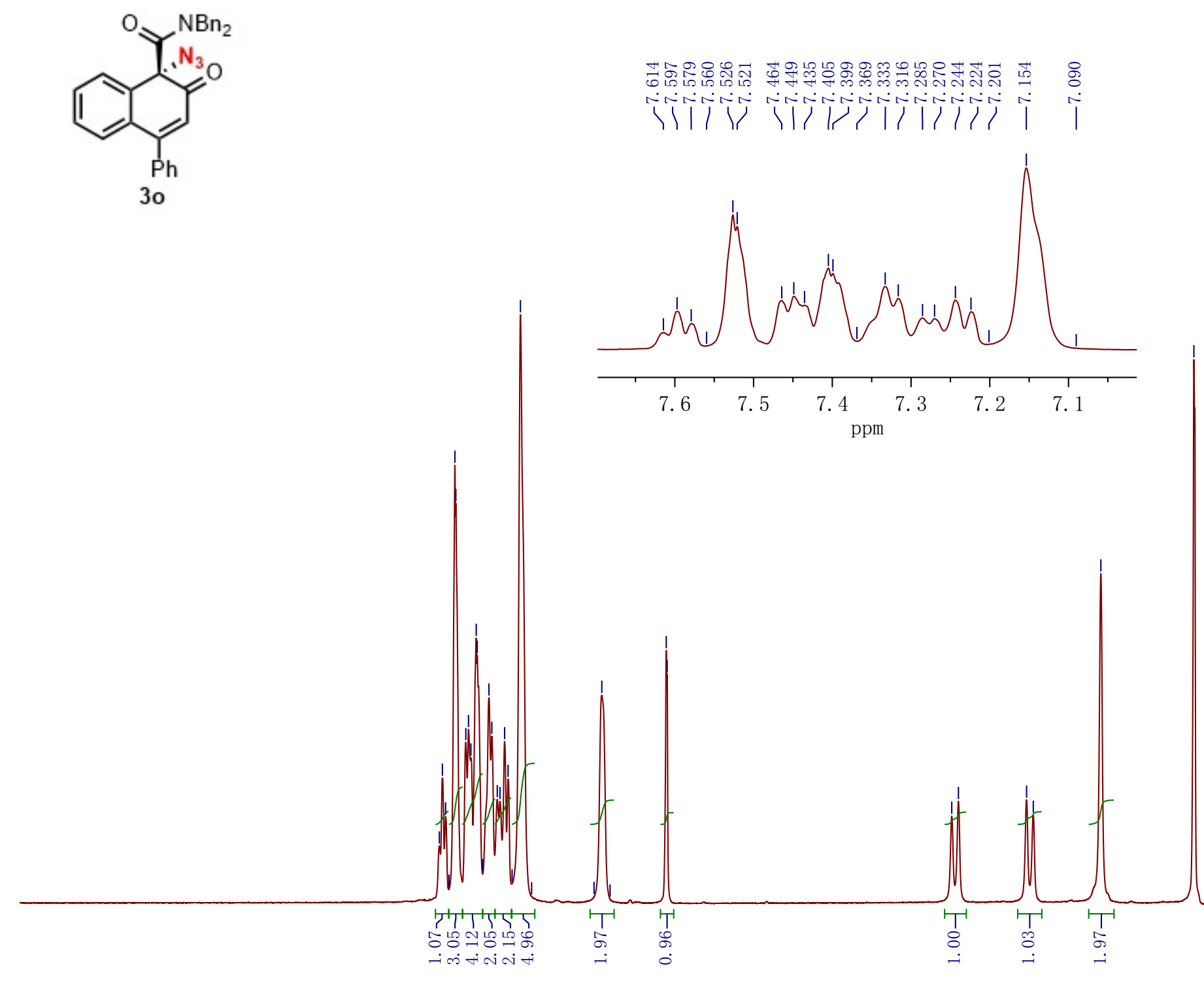

59.

8.58.

7.5 7.0

6.5

5.5

5. $0 \mathrm{~S} 1584.5$

3.5

3. 0

2.5

1.5

1.0

0.5 


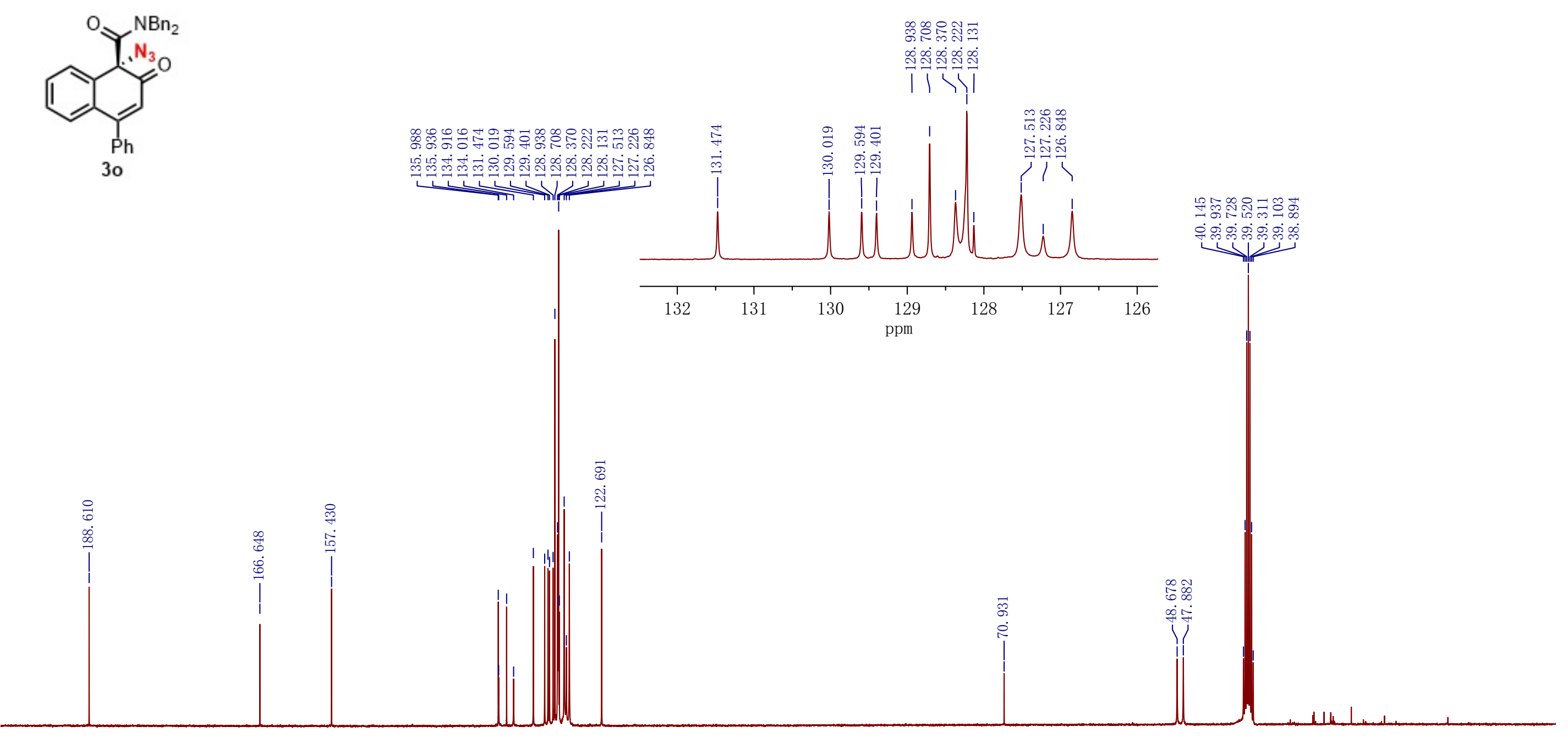

\begin{tabular}{|c|c|c|c|c|c|c|c|c|c|c|c|c|c|c|c|c|c|c|c|}
\hline )0 & $\begin{array}{c}1 \\
190\end{array}$ & 180 & $\begin{array}{c}1 \\
170\end{array}$ & 160 & 150 & 140 & 130 & 120 & 110 & $\begin{array}{c}\text { S10Bg9 } \\
\text { ppm }\end{array}$ & 90 & 80 & 70 & 60 & $\begin{array}{l}1 \\
50\end{array}$ & 40 & 30 & 20 & 10 \\
\hline
\end{tabular}




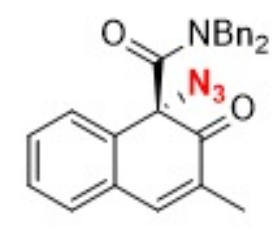

$3 \mathrm{p}$
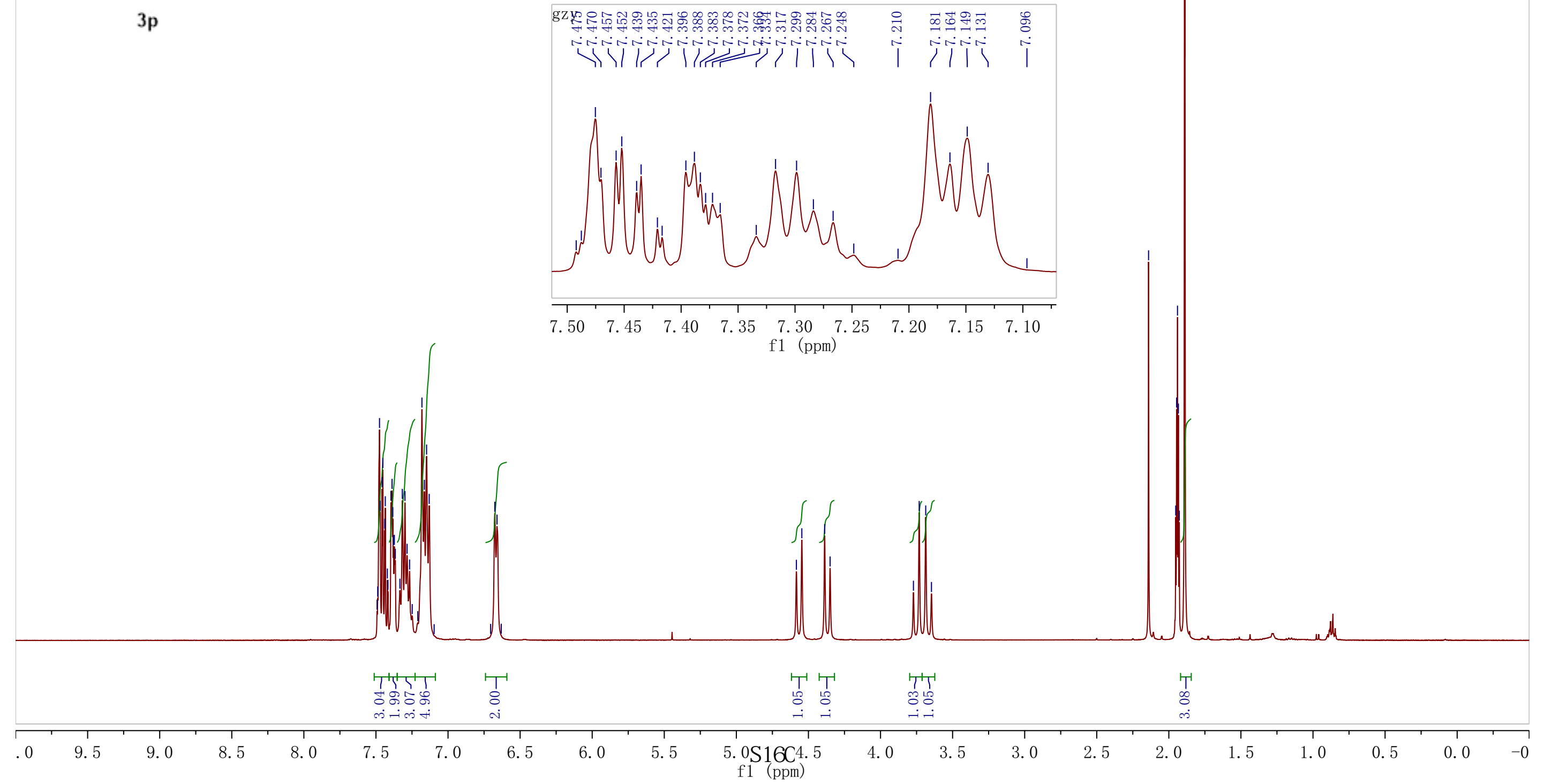

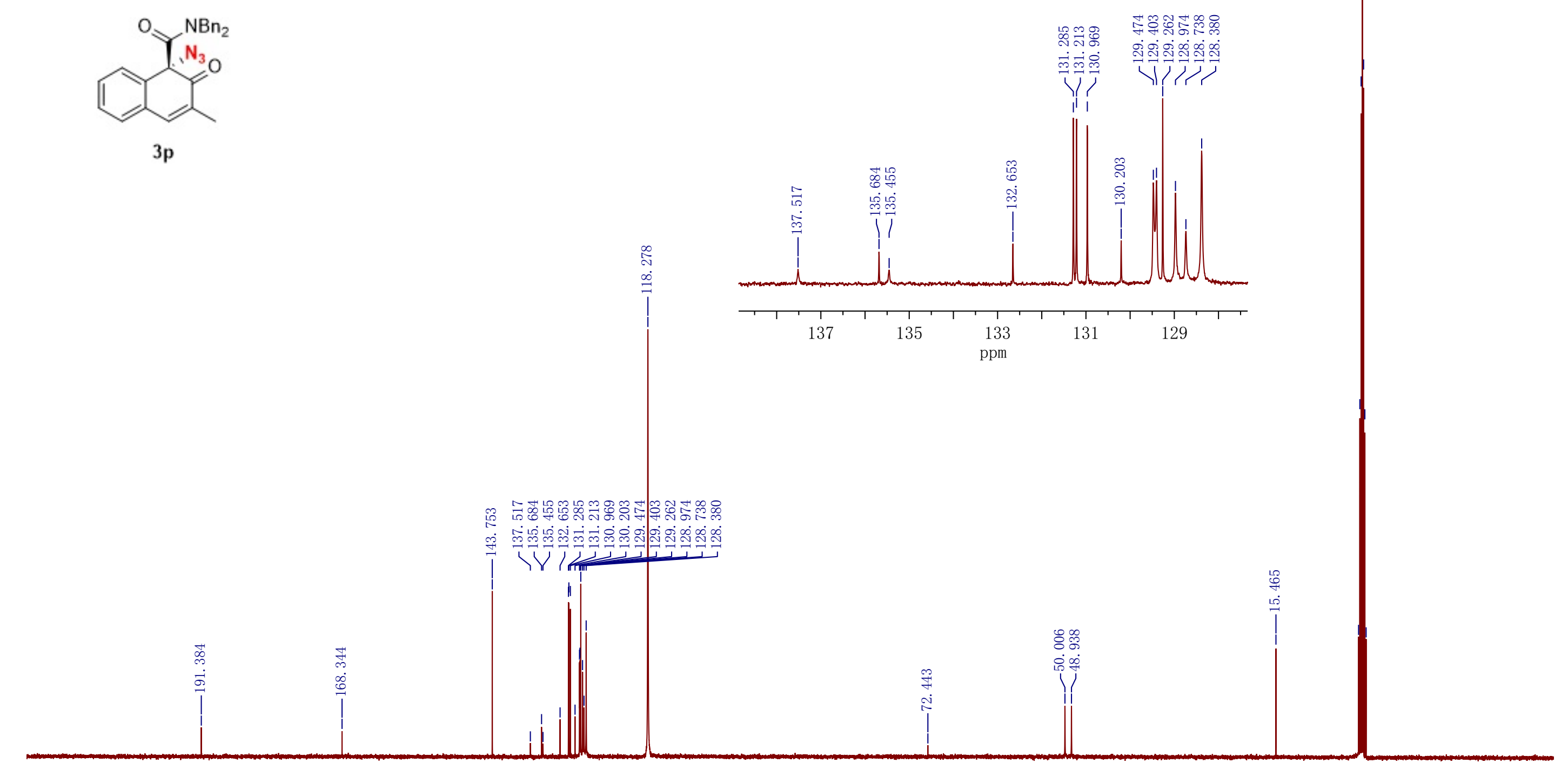

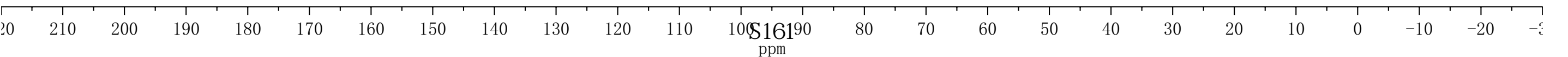




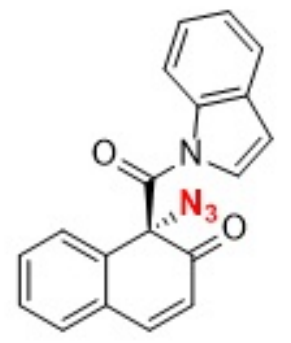

$3 q$

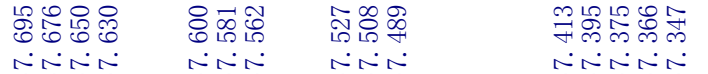
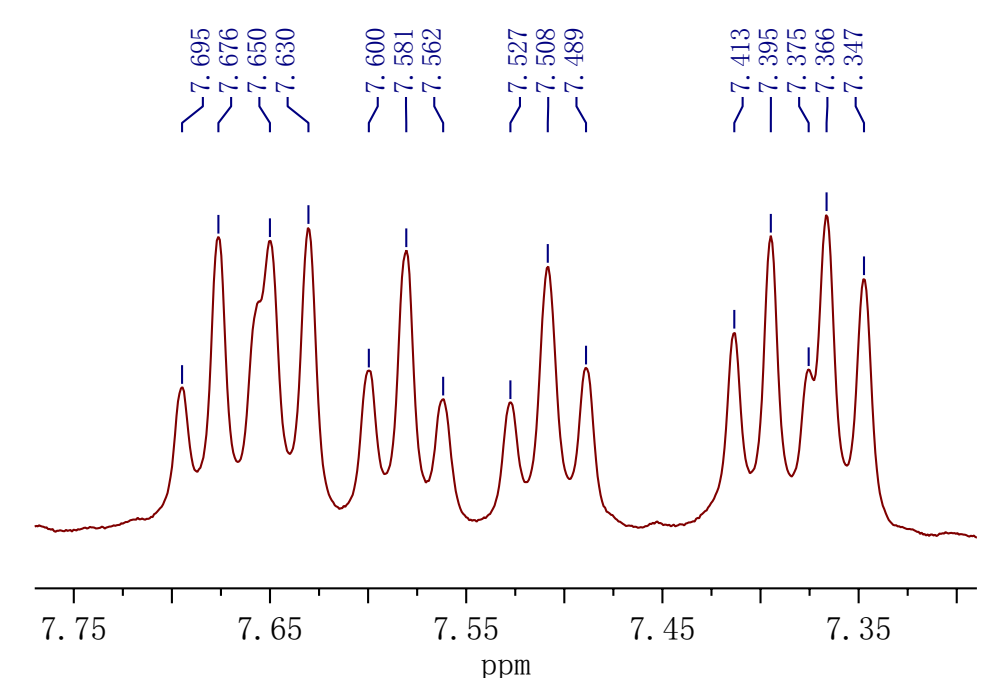

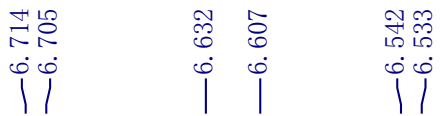

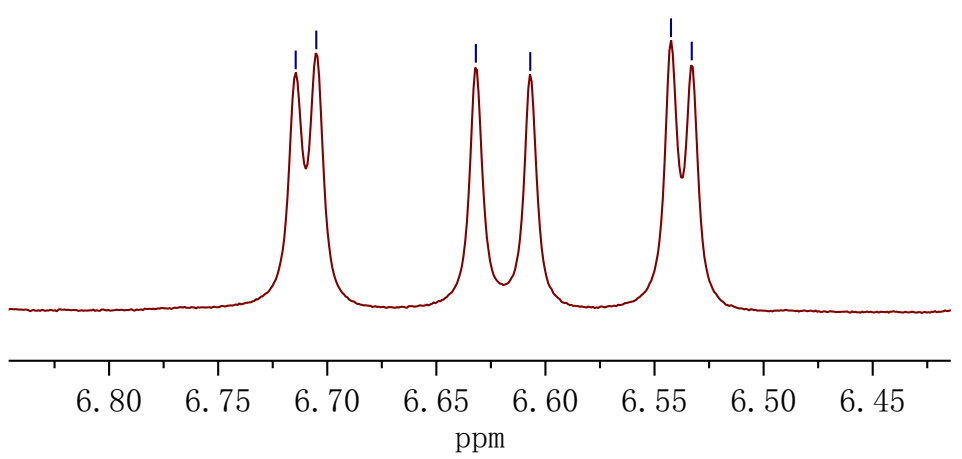

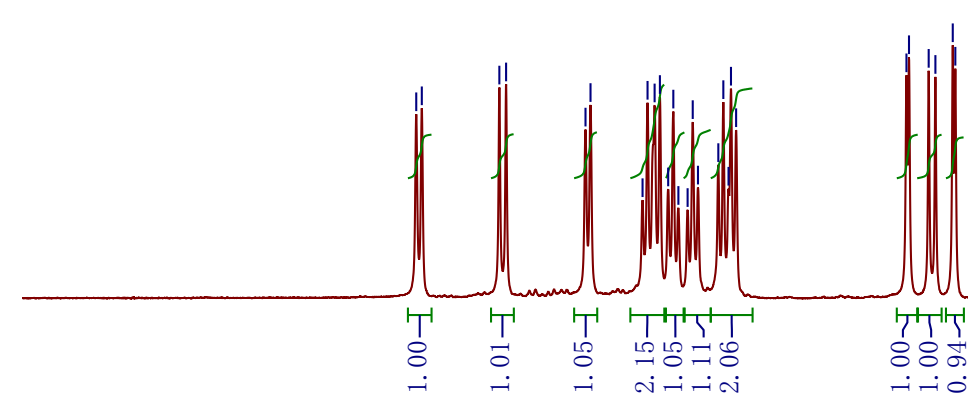

9.5

9.0

8.5

8.0

7.5

7. 0

6.5

6. 0

5.5

5. $0 \mathrm{~S} 162^{4.5}$

4. 0

3.5

3.0

2.5

2.0

1. 5

1.0

$0.5 \quad 0.0 \quad-0$ 


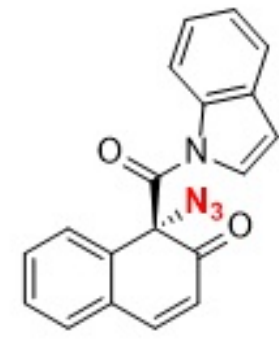

$3 q$
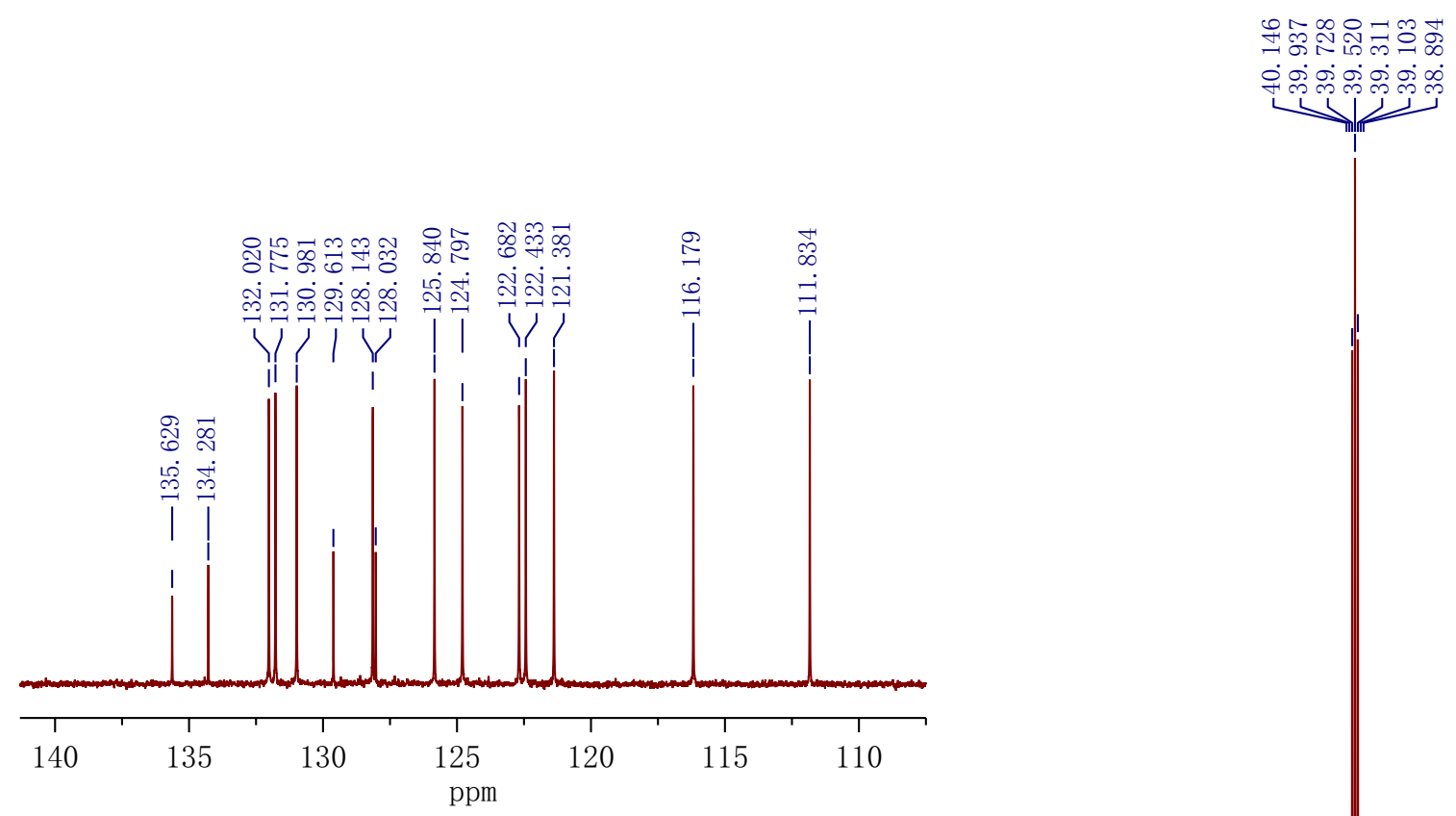

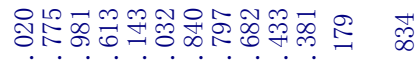

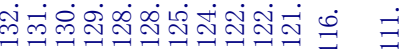

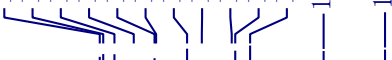
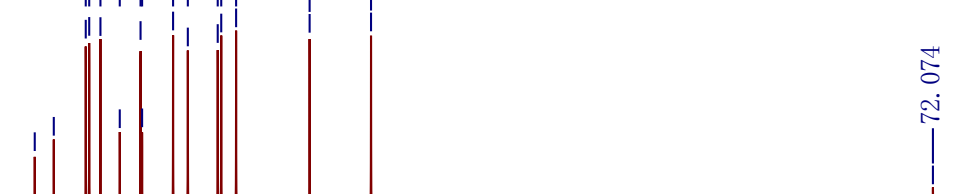

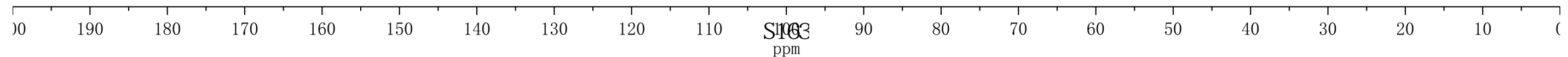




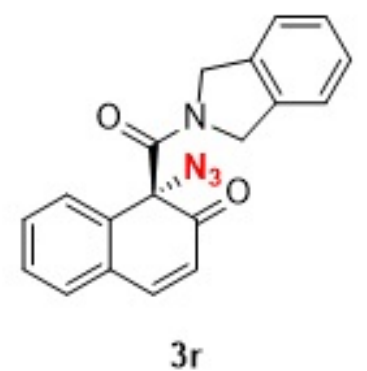

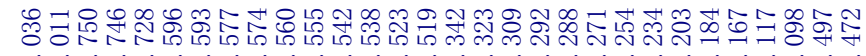

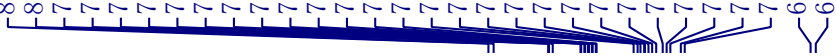

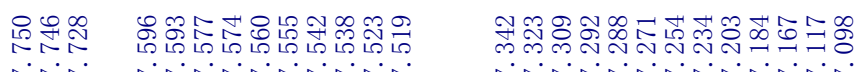

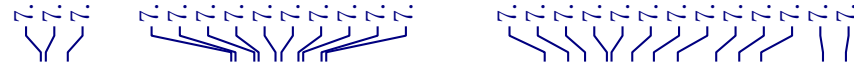
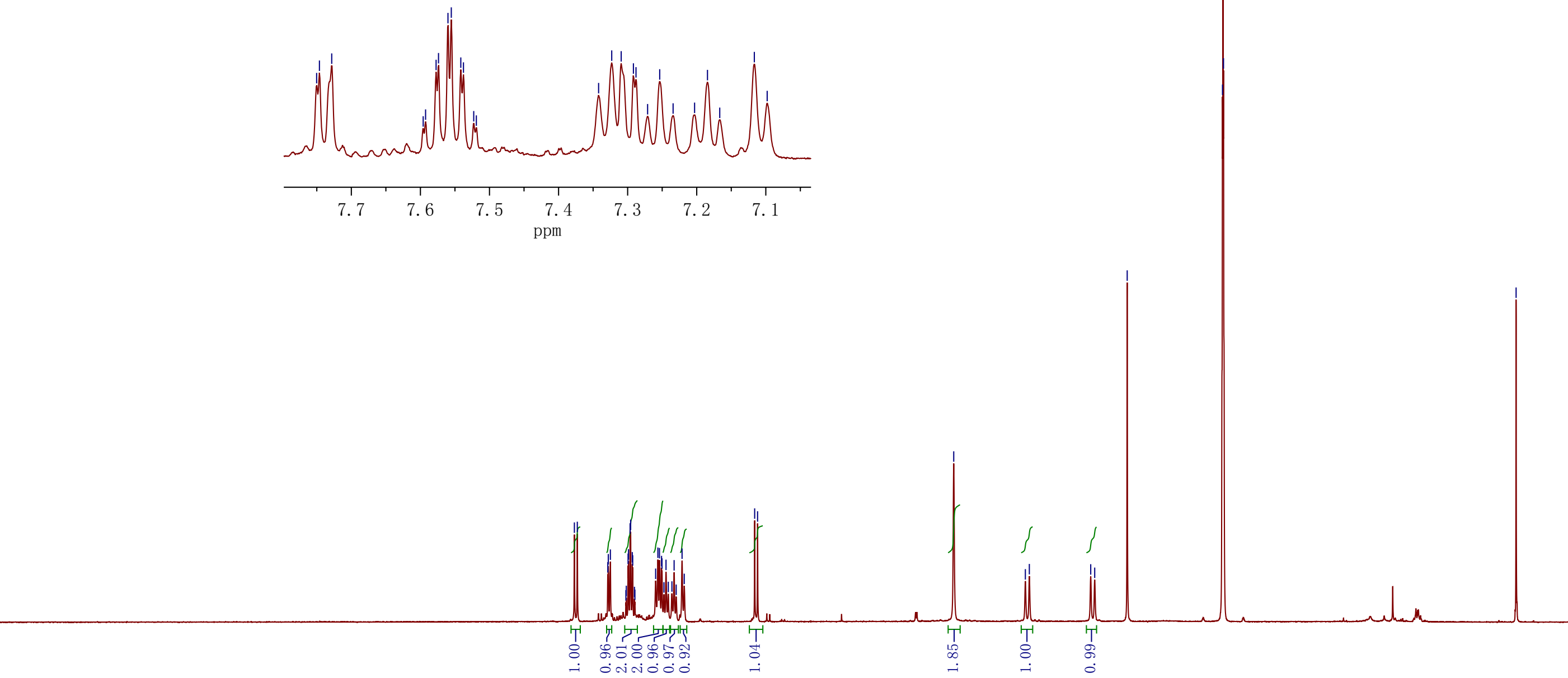

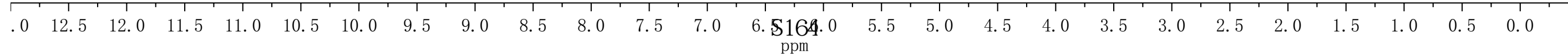



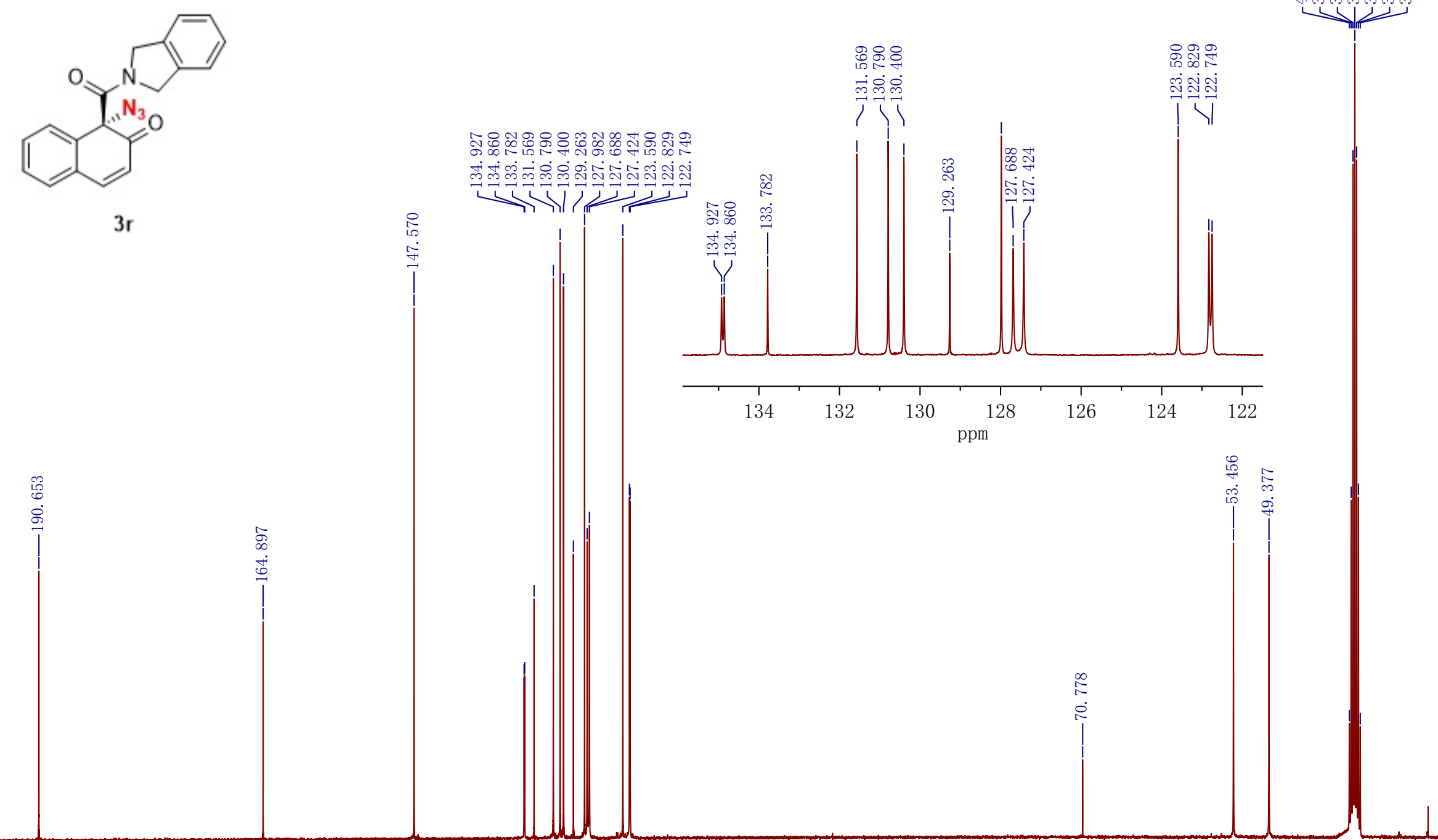

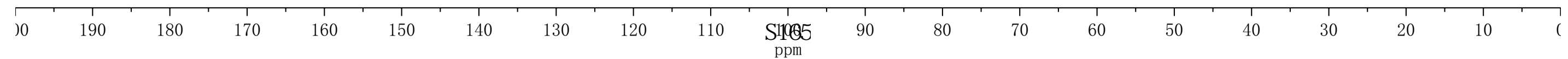




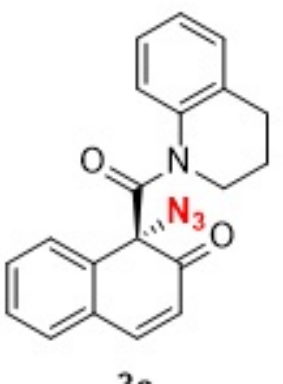

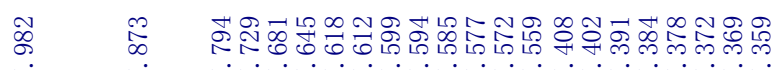

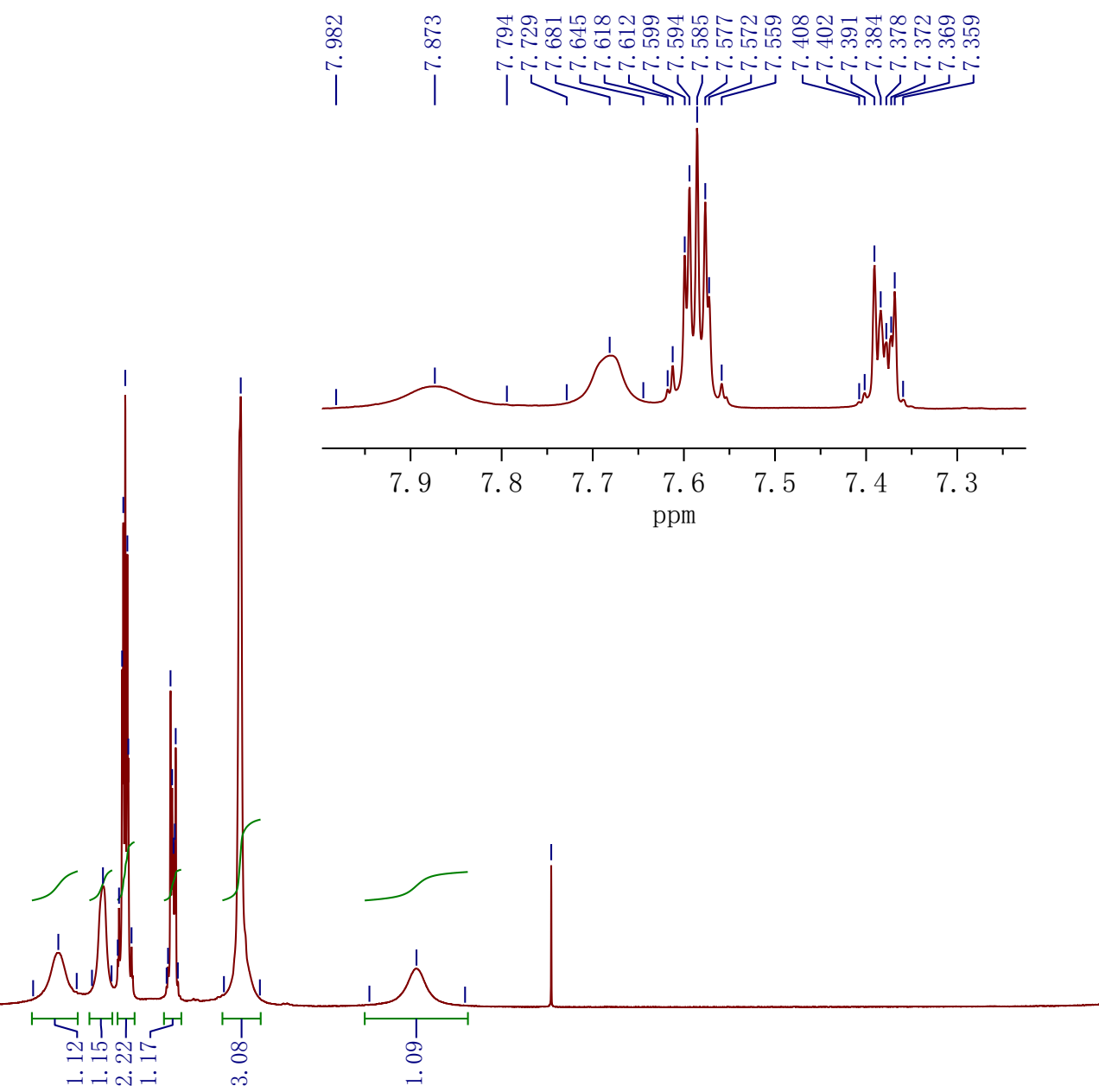

$3 \mathrm{~s}$
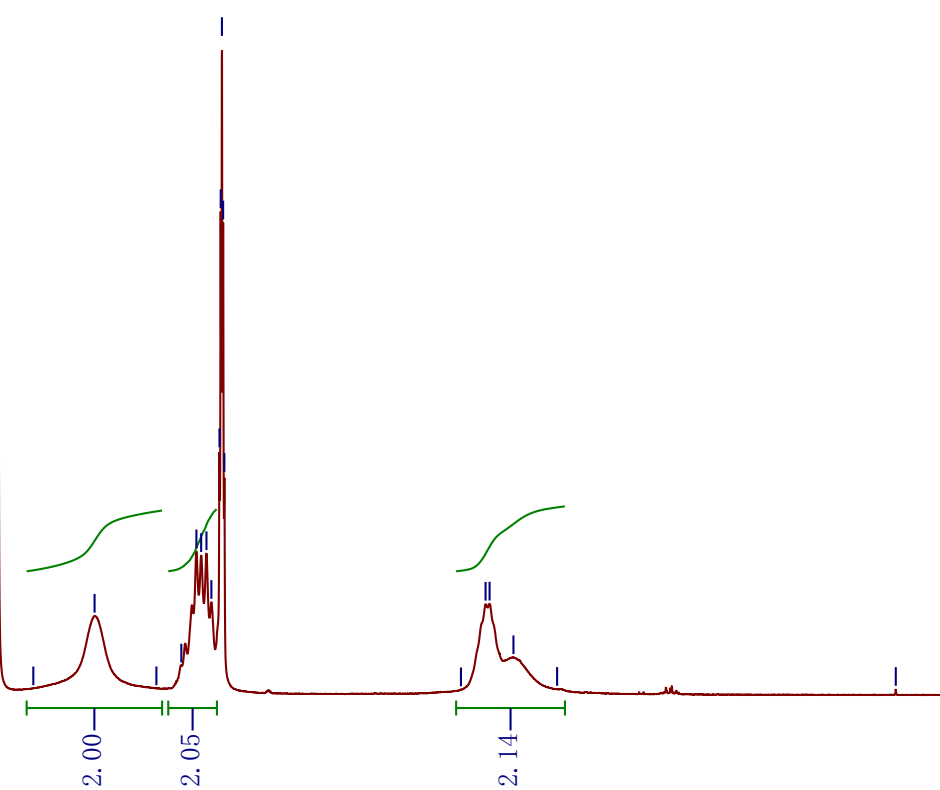

59.

$9.0 \quad 8.5$

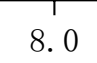

7.5

7. 0

6.5

6. 0

5.5

5. $0 \mathrm{~S} 1664.5$

4. 0

3.5

3.0

2. 5

1. 0

0.5

0.0

$-0$ 

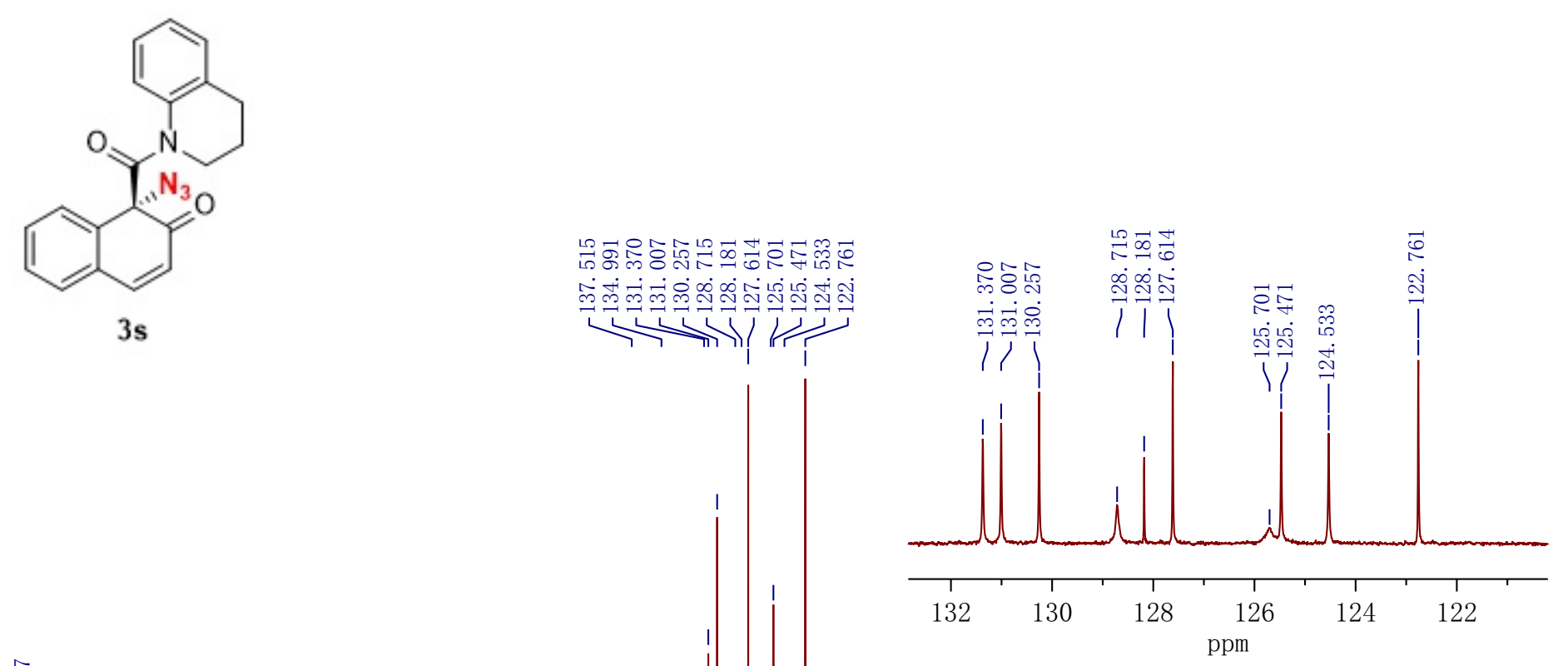

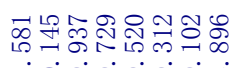

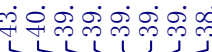

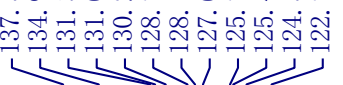

M

도

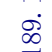

守

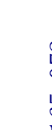

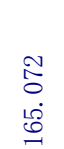

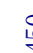

品 


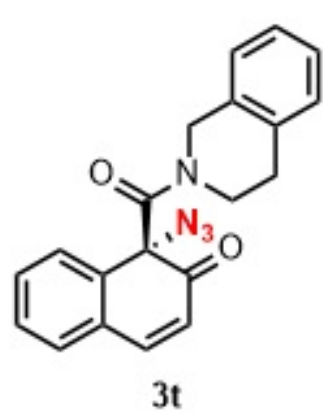

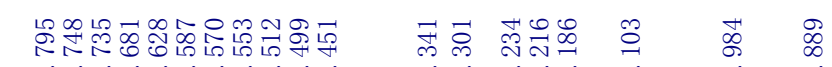

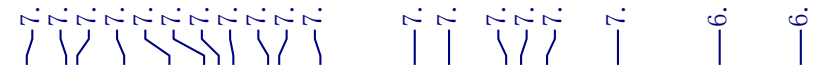
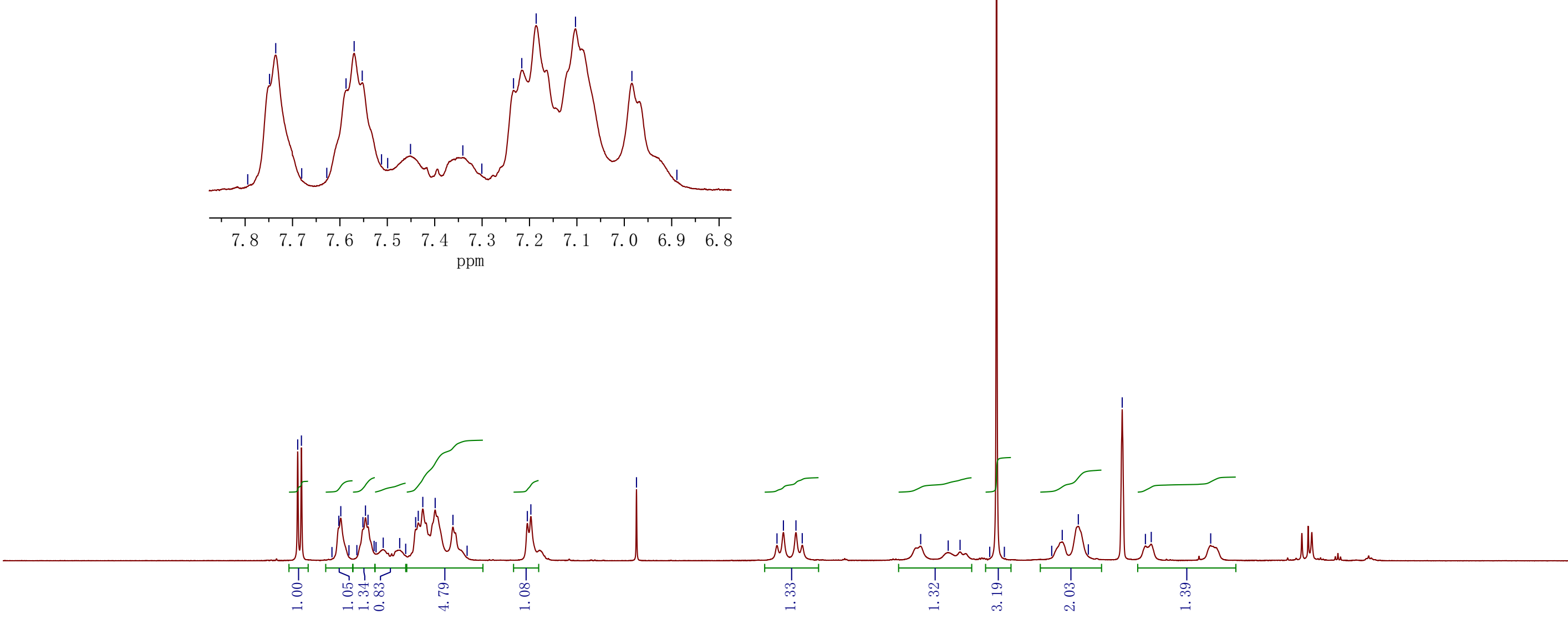

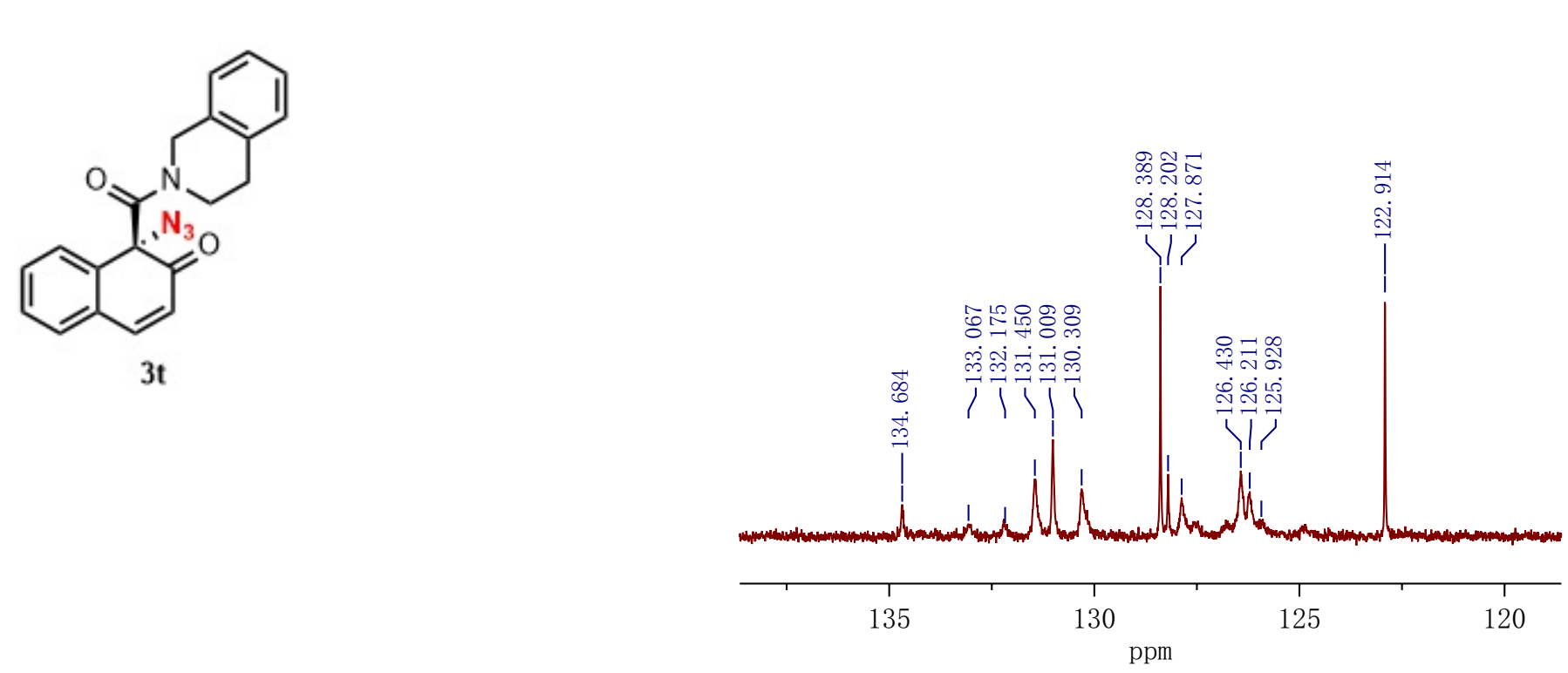

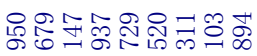

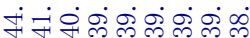



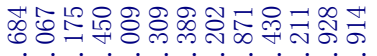

๓⿴囗十心

10-1)

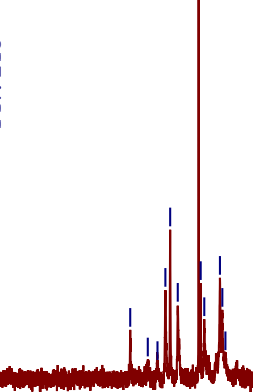




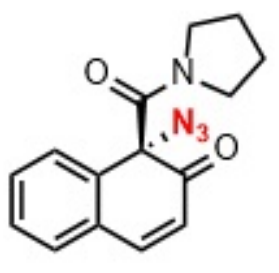

$3 \mathrm{u}$

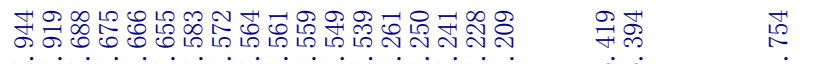

i

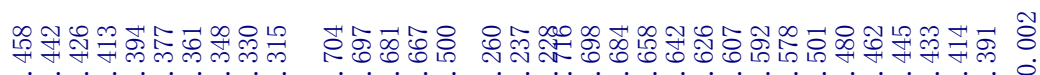

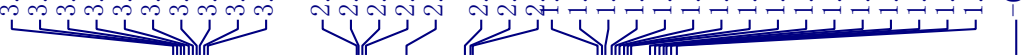
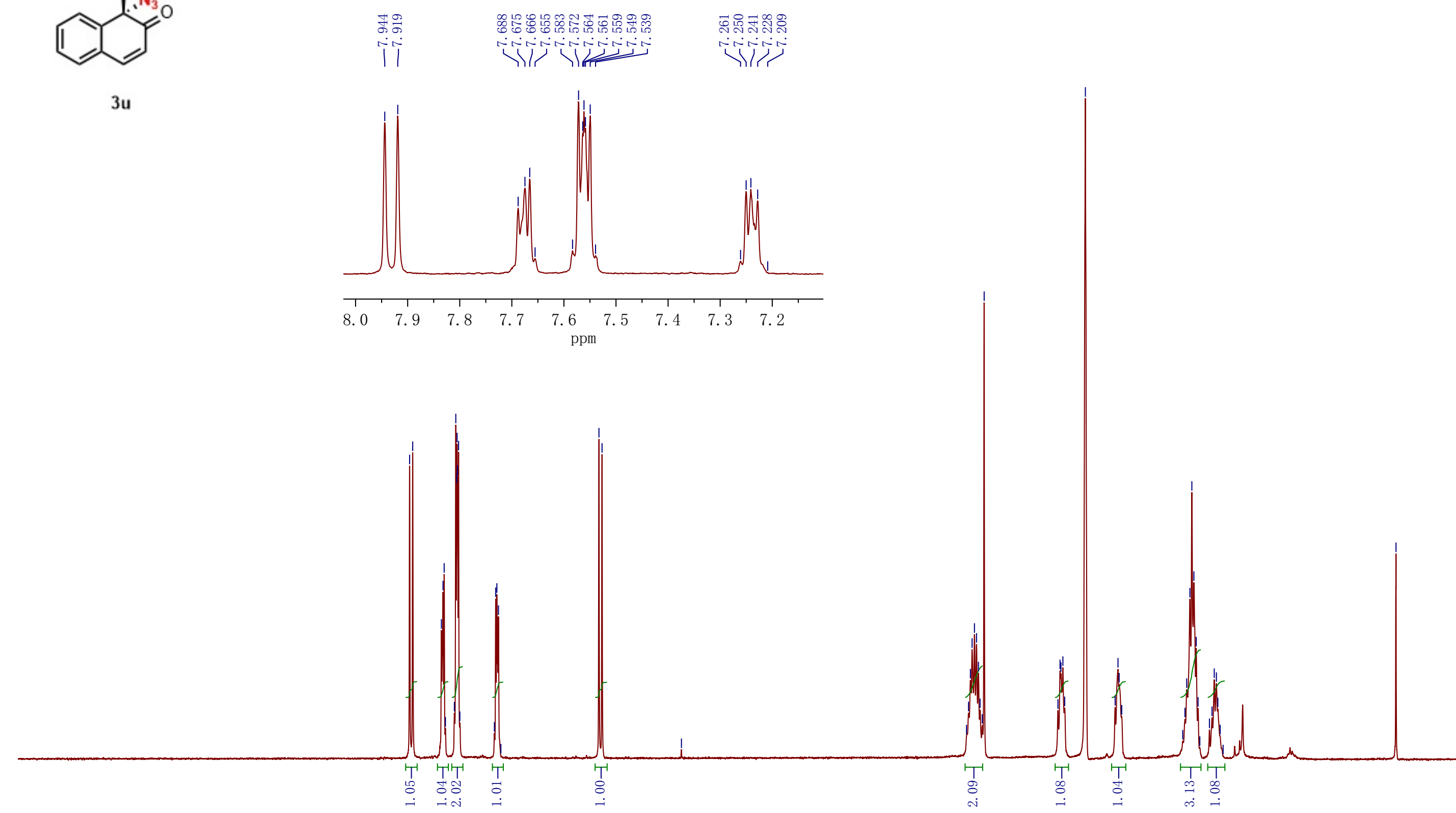

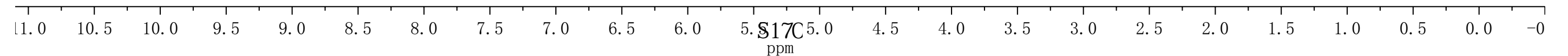



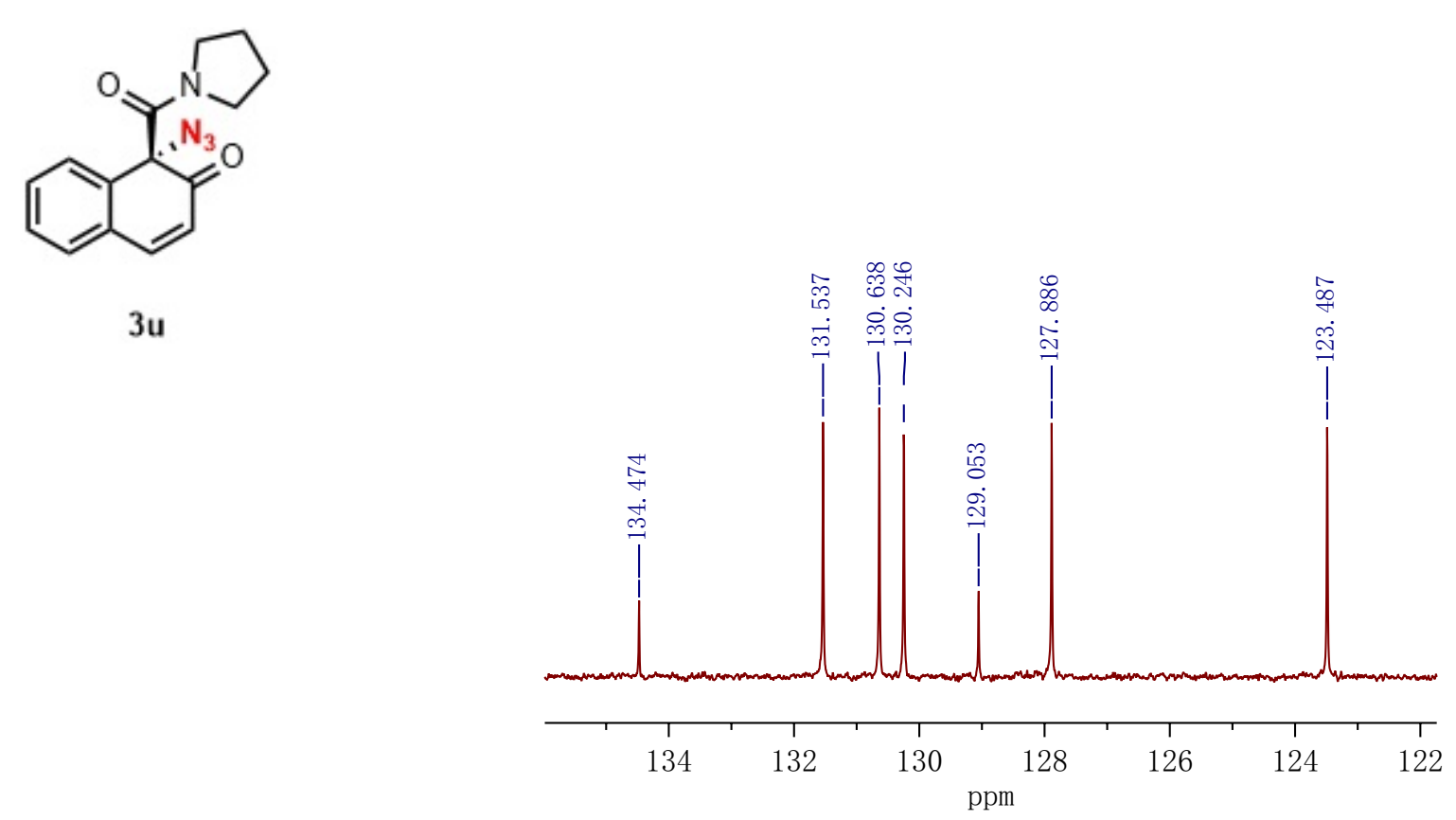

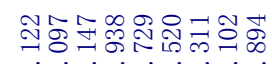

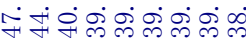

资

3u

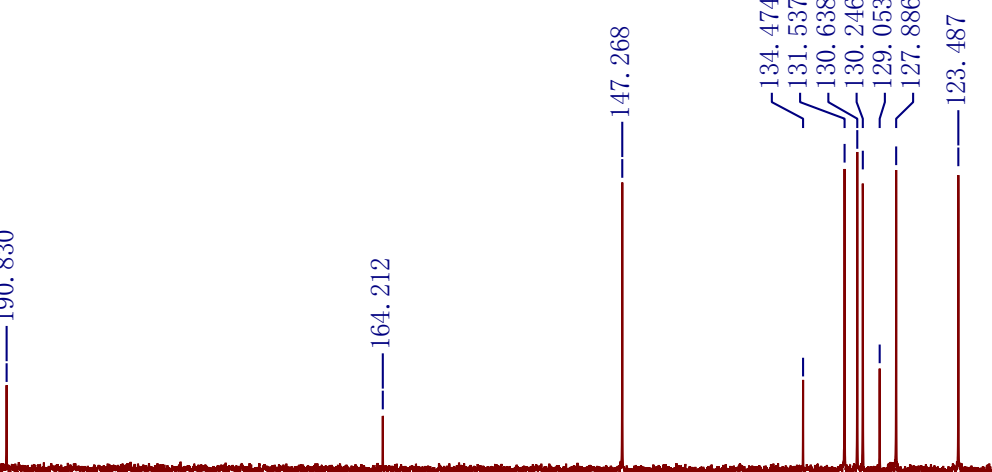

ळ$$
0
$$ 

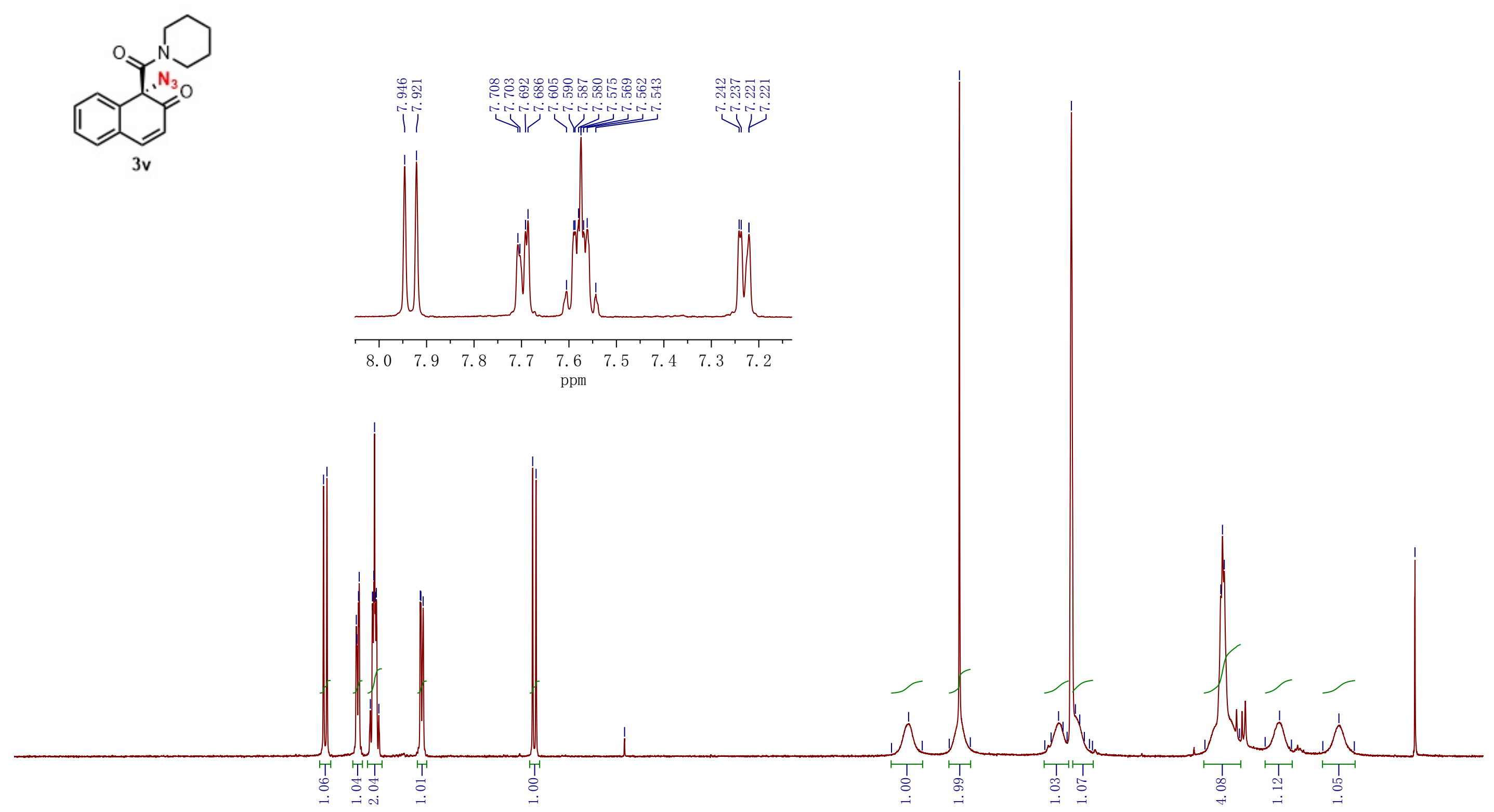

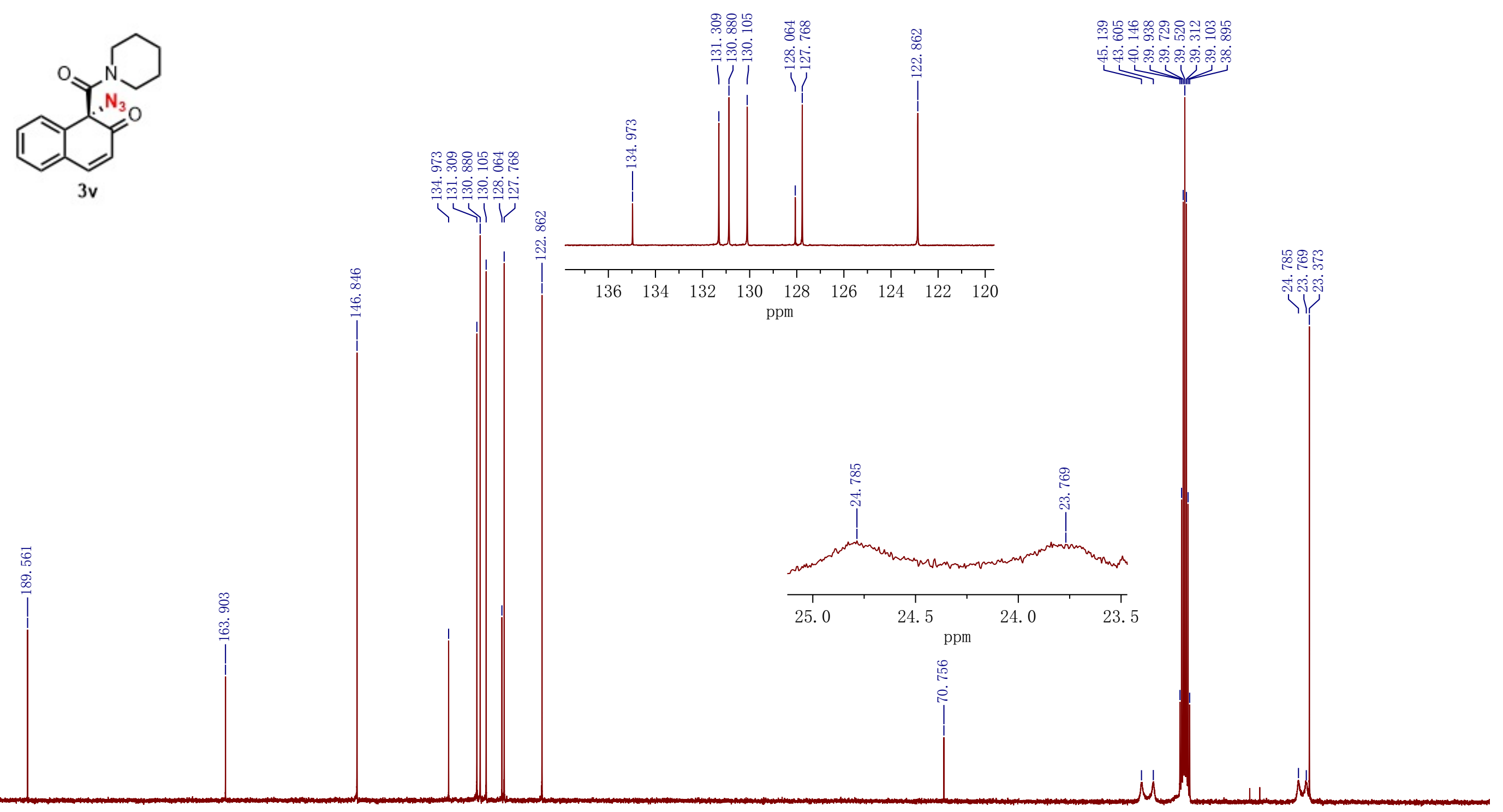

\begin{tabular}{rrrrrr|r}
1 & 1 & 1 & 1 & 1 & 1 & 1 \\
190 & 180 & 170 & 160 & 150 & 140 & 130
\end{tabular}

$\begin{array}{lll}120 & 110 & \substack{\text { SHOP3 } \\ \text { ppm }}\end{array}$



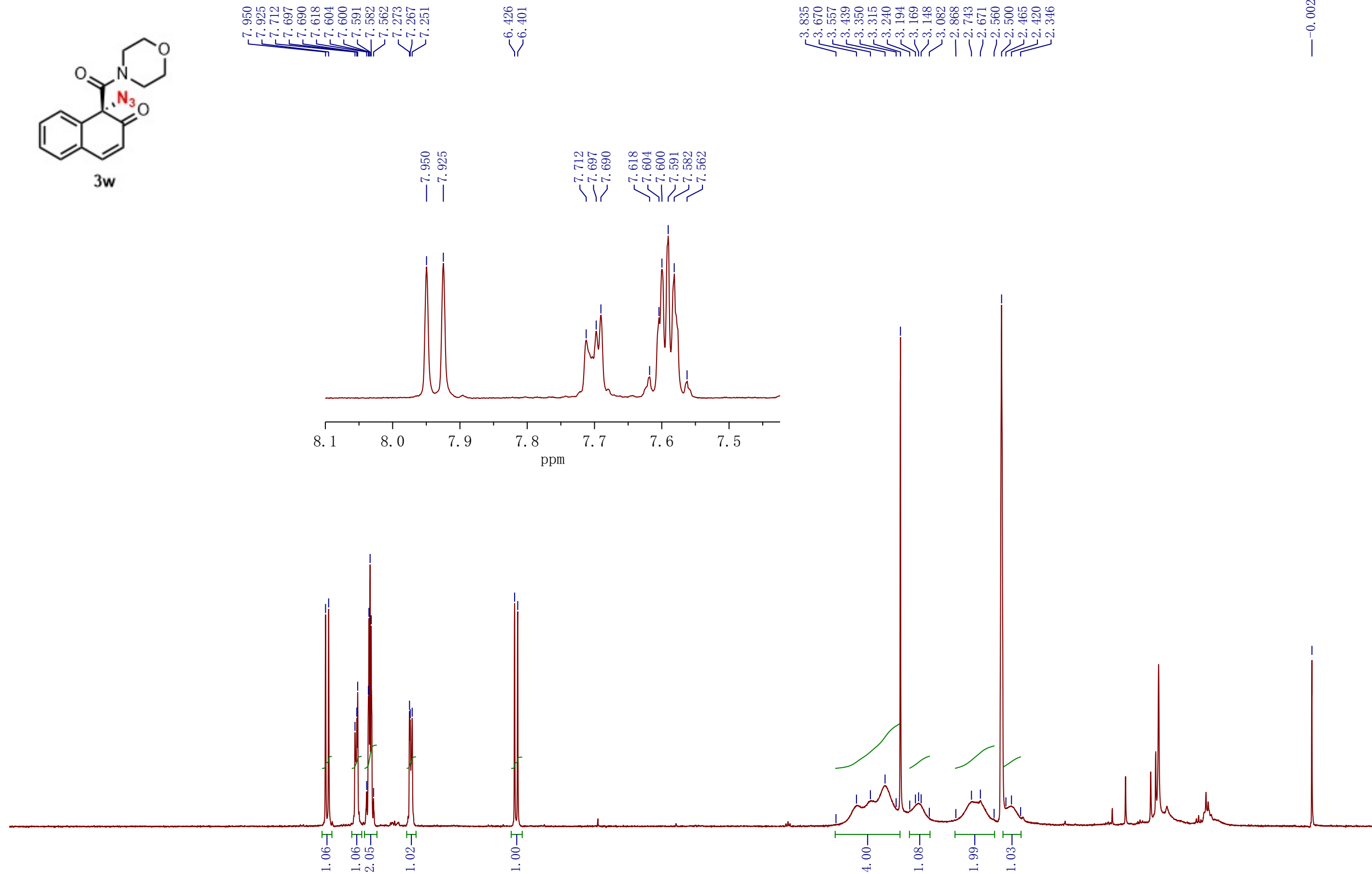

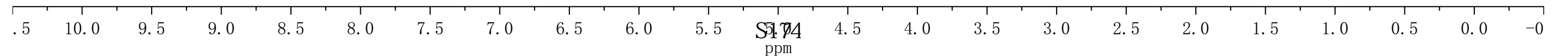



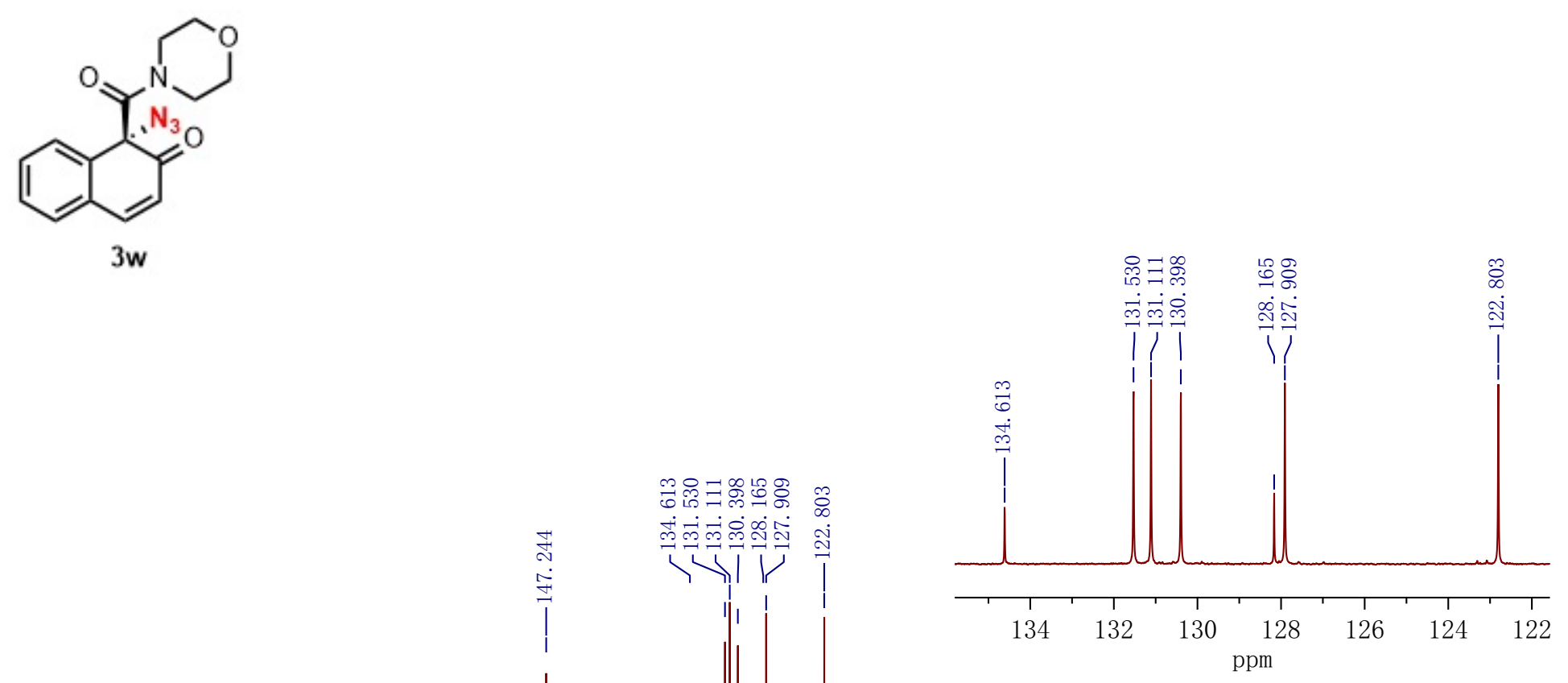

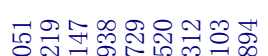

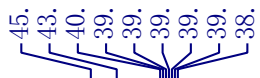

$3 w$

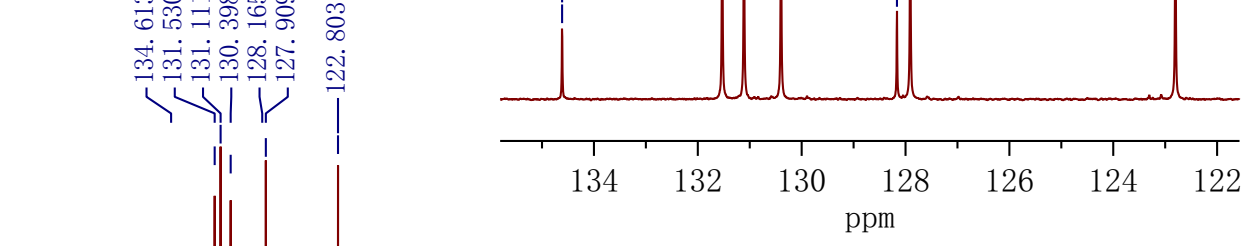

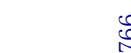

$\stackrel{8}{\circ}$

$\overrightarrow{\stackrel{H}{\circ}}$

ग

궁 공

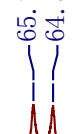

in

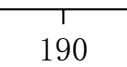

180

170

160

150

140

130

120

110

S10755

$90 \quad 80$

70

60

50

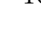



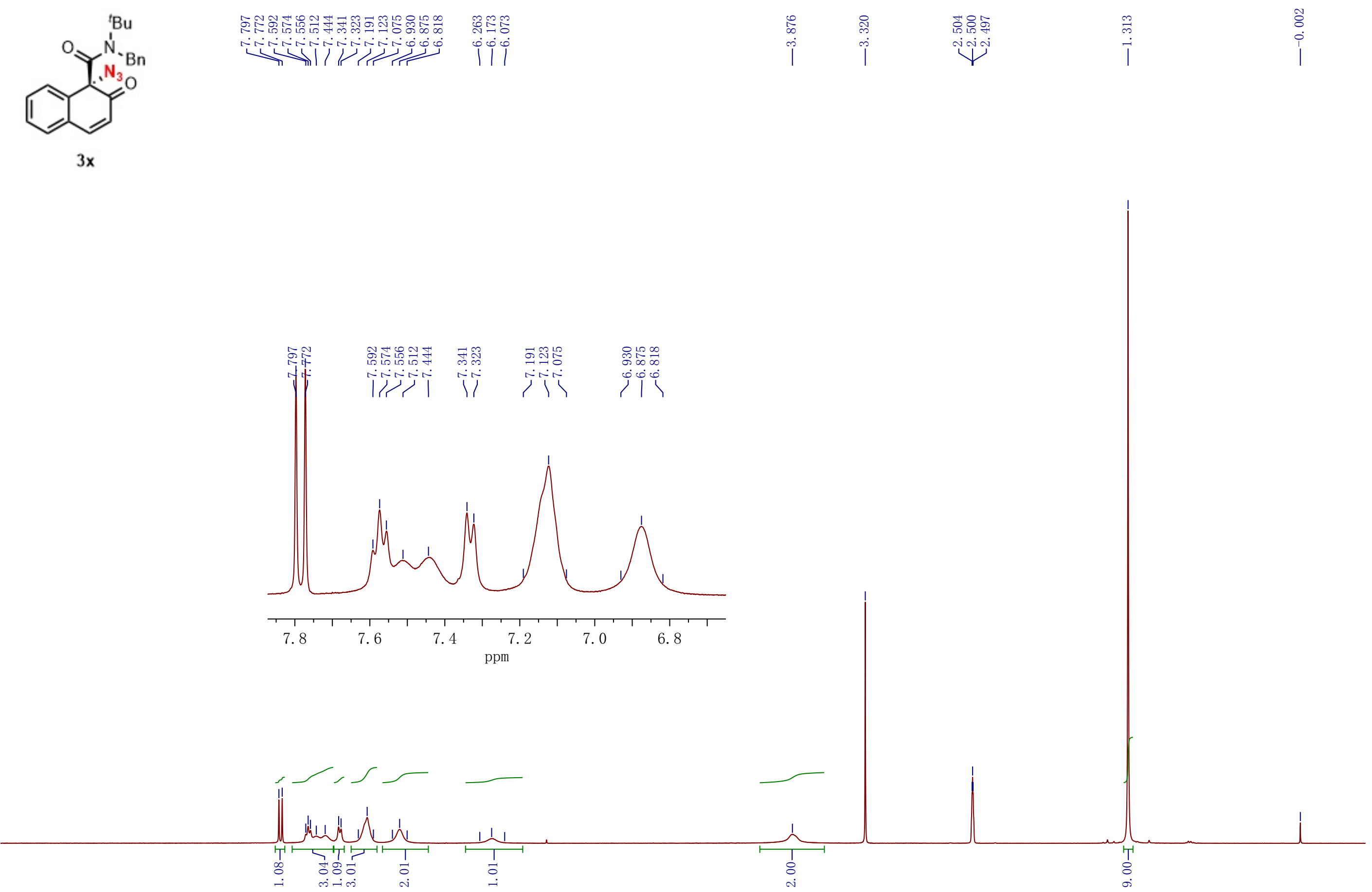

\begin{tabular}{|c|c|c|c|c|c|c|c|c|c|c|c|c|c|c|c|c|c|c|c|}
\hline 9.5 & 9.0 & 8.5 & 8.0 & 7.5 & 7.0 & 6.5 & 6.0 & 5.5 & $5.0 \underset{\mathrm{ppm}}{\mathrm{S} 176} 5$ & 4.0 & 3.5 & 3.0 & 2.5 & 2.0 & 1.5 & 1.0 & 0.5 & 0.0 & -0 \\
\hline
\end{tabular}



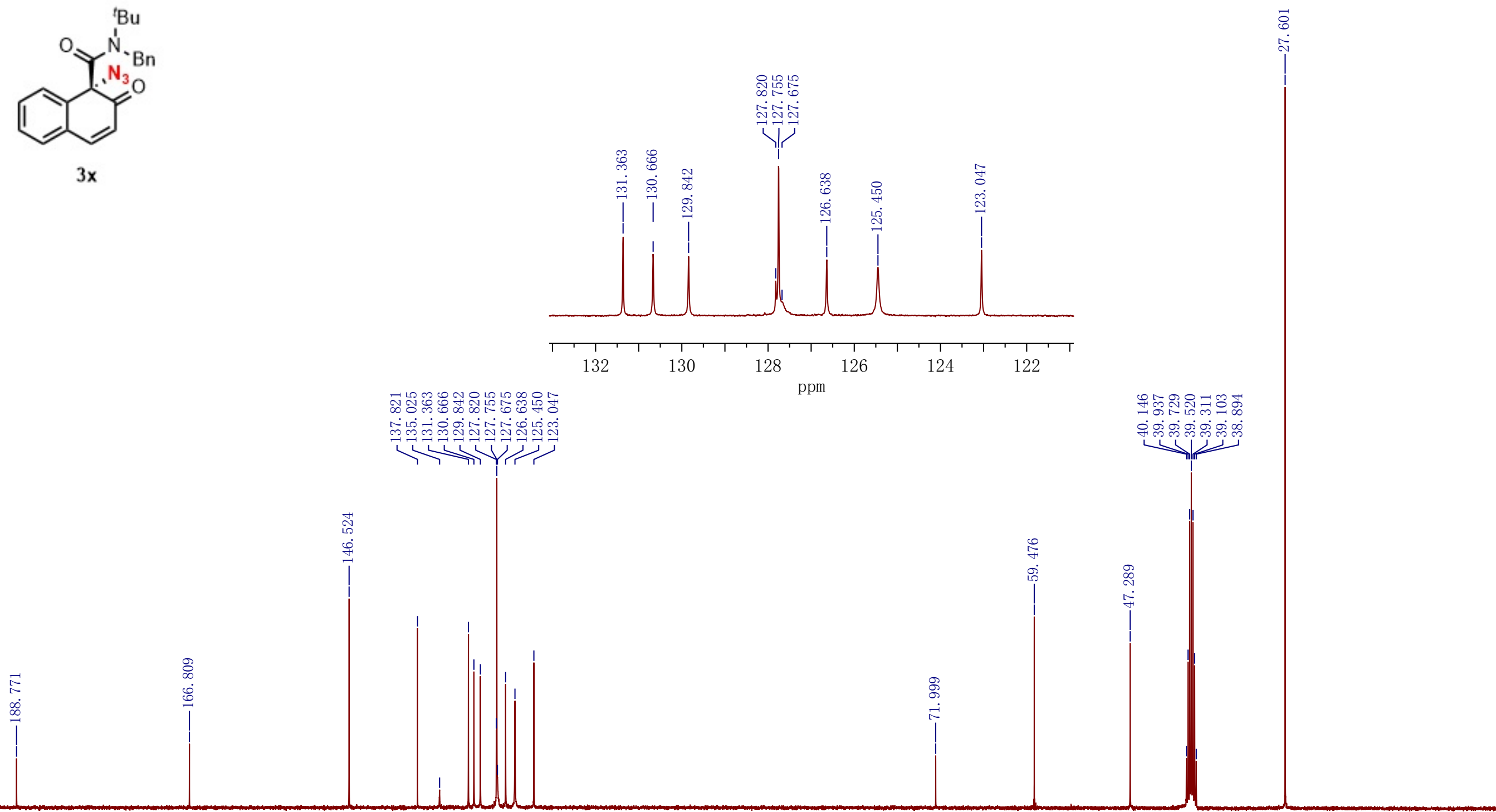

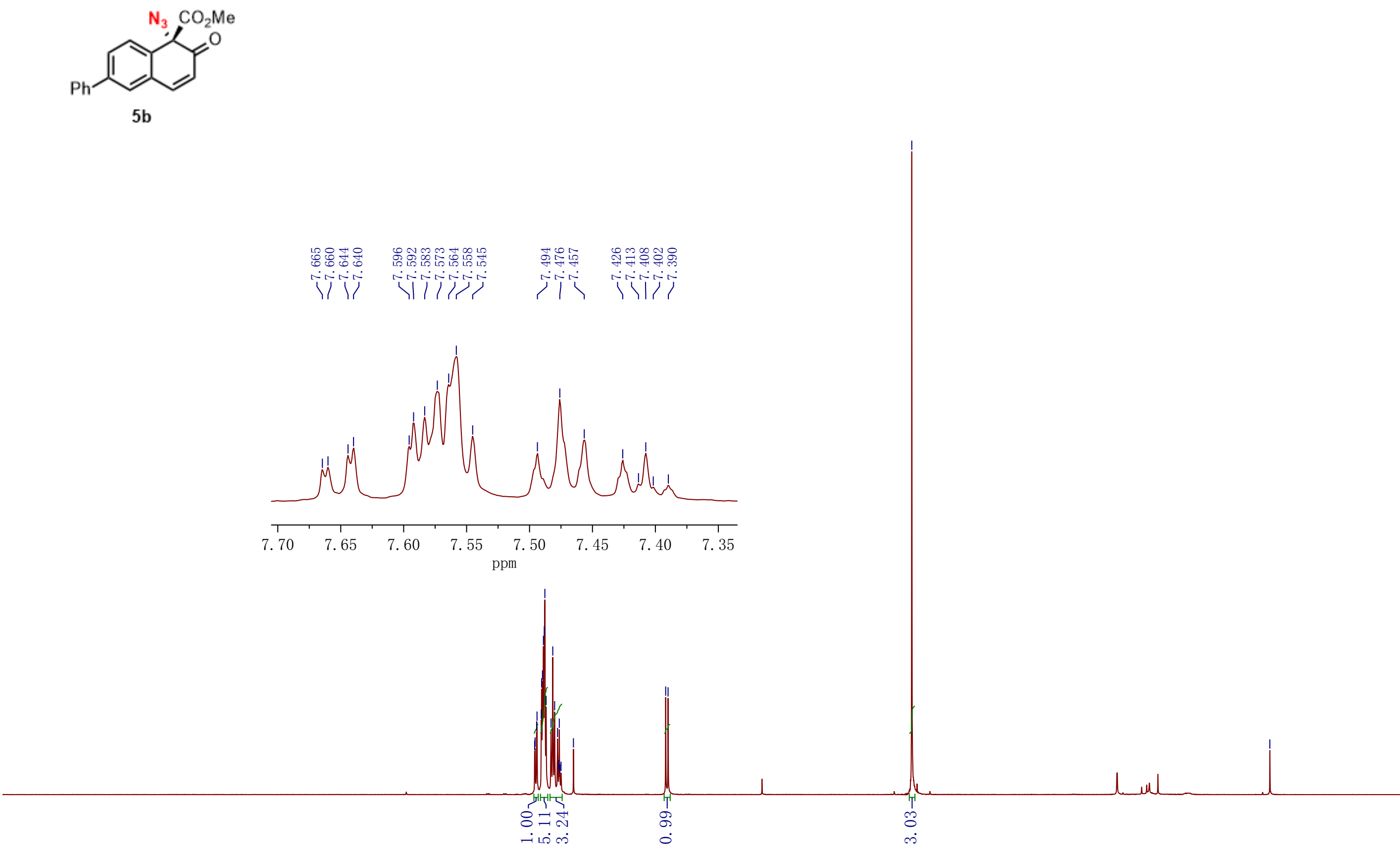


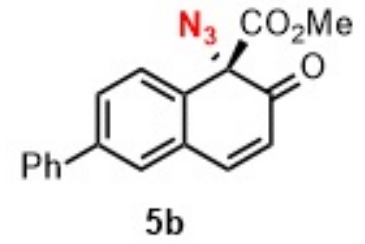

$5 b$

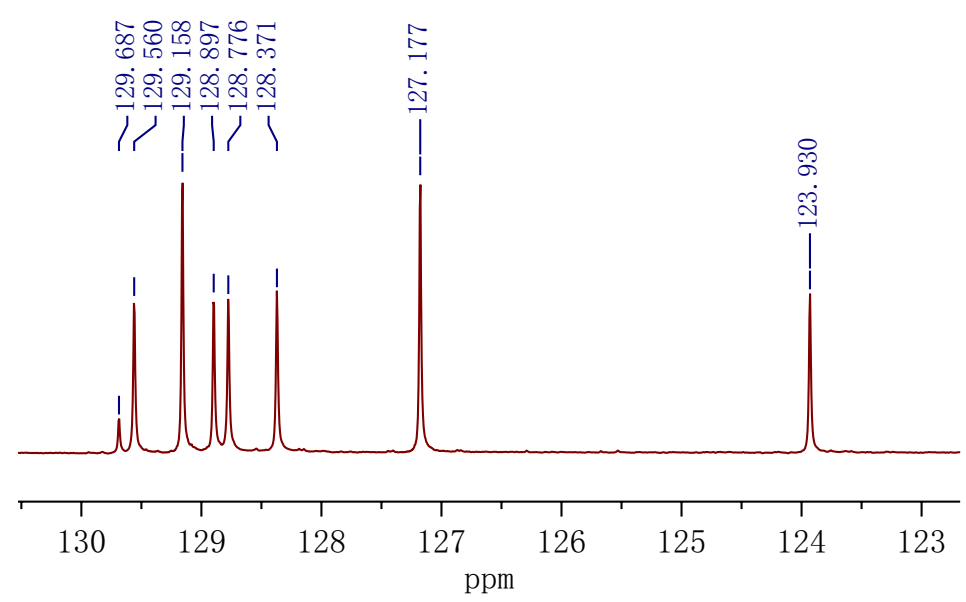

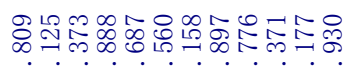

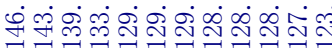

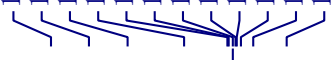

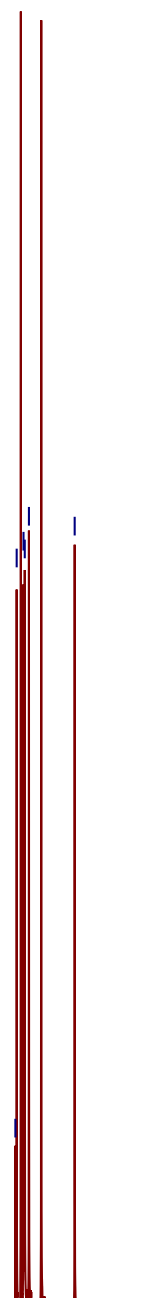

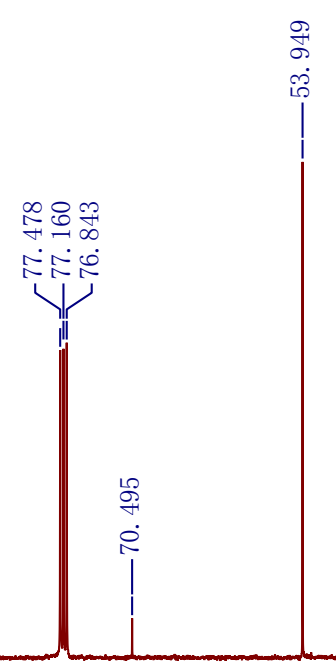




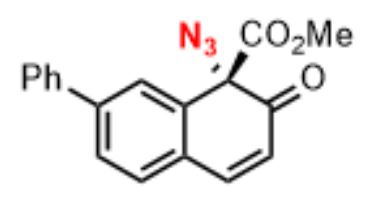

$5 c$

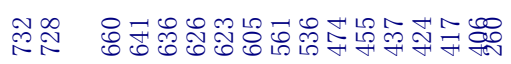

Y I
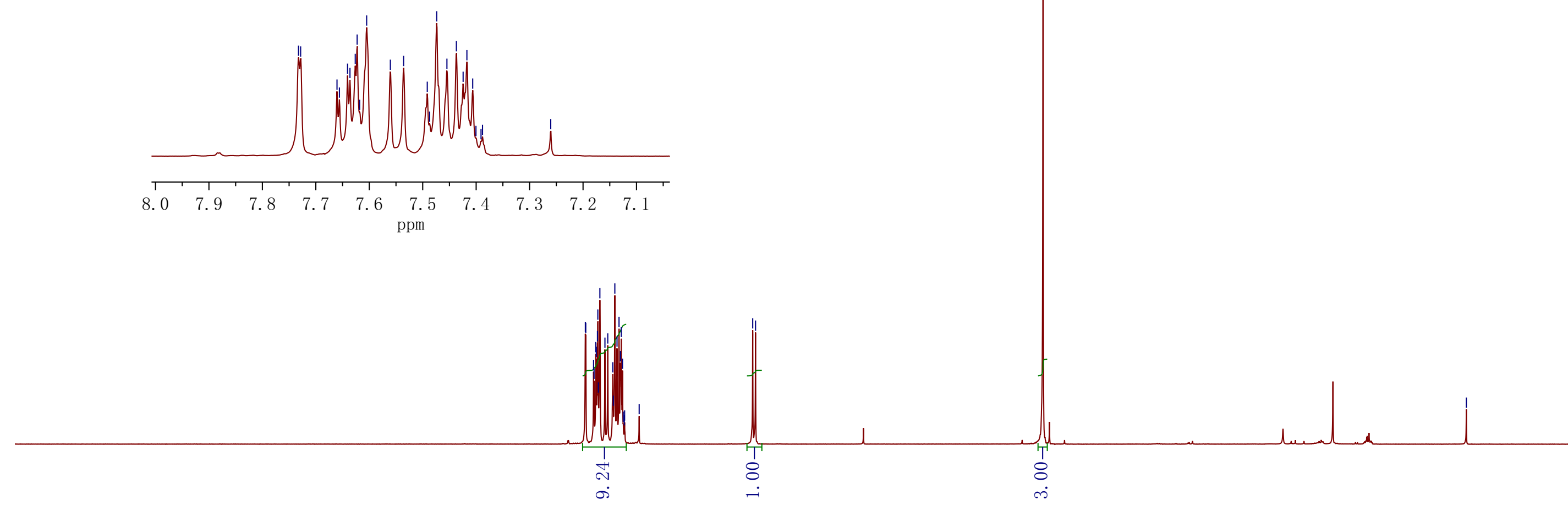


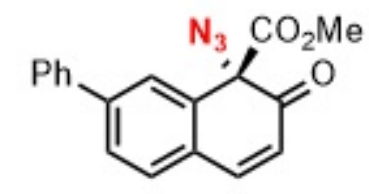

$5 c$

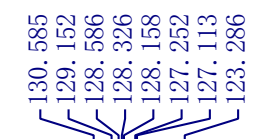
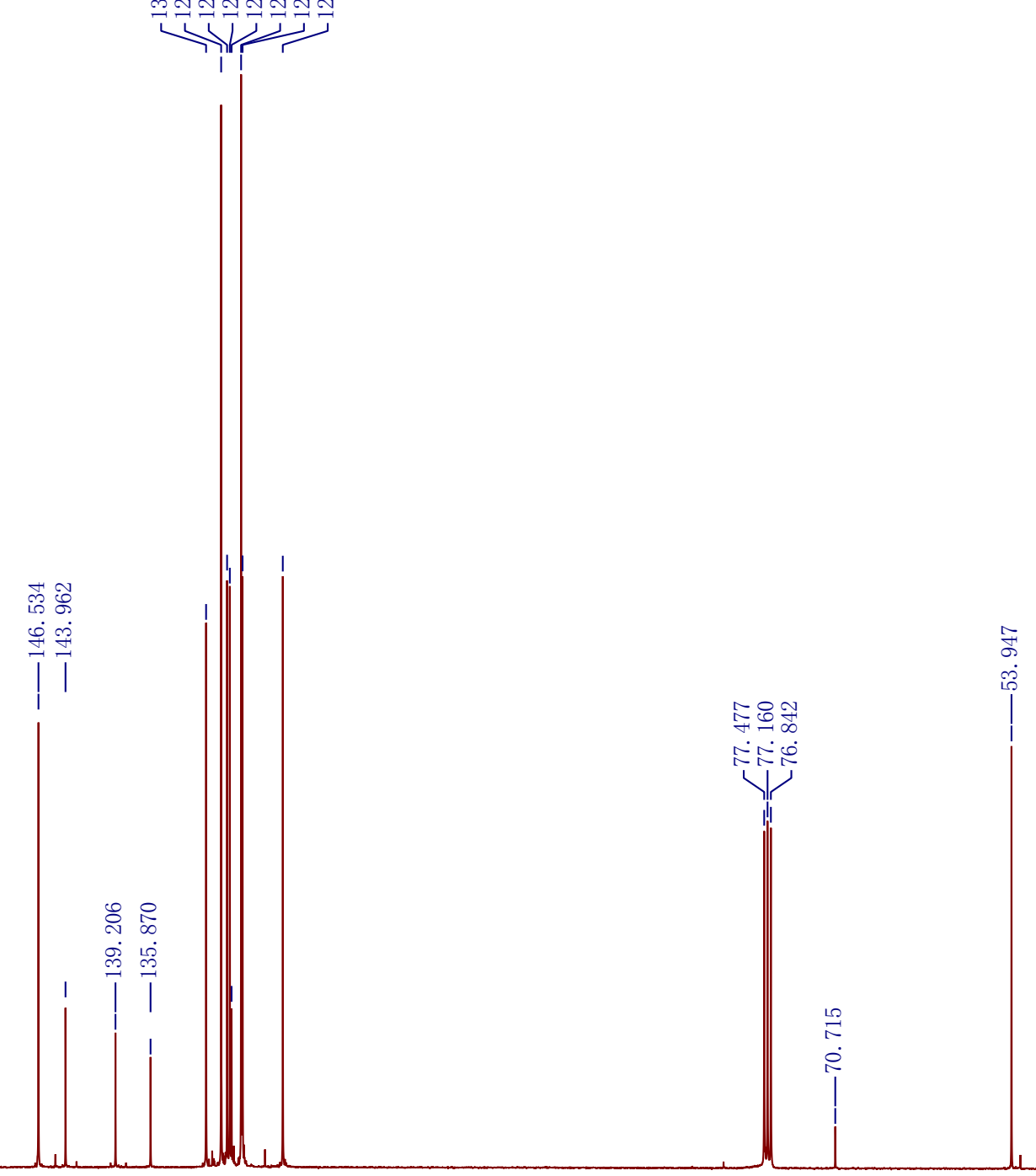$$
210
$$

200

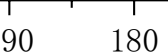

$170 \quad 160$

$150 \quad 140 \quad 130$

120

$1 \$ 181100$ ppm 

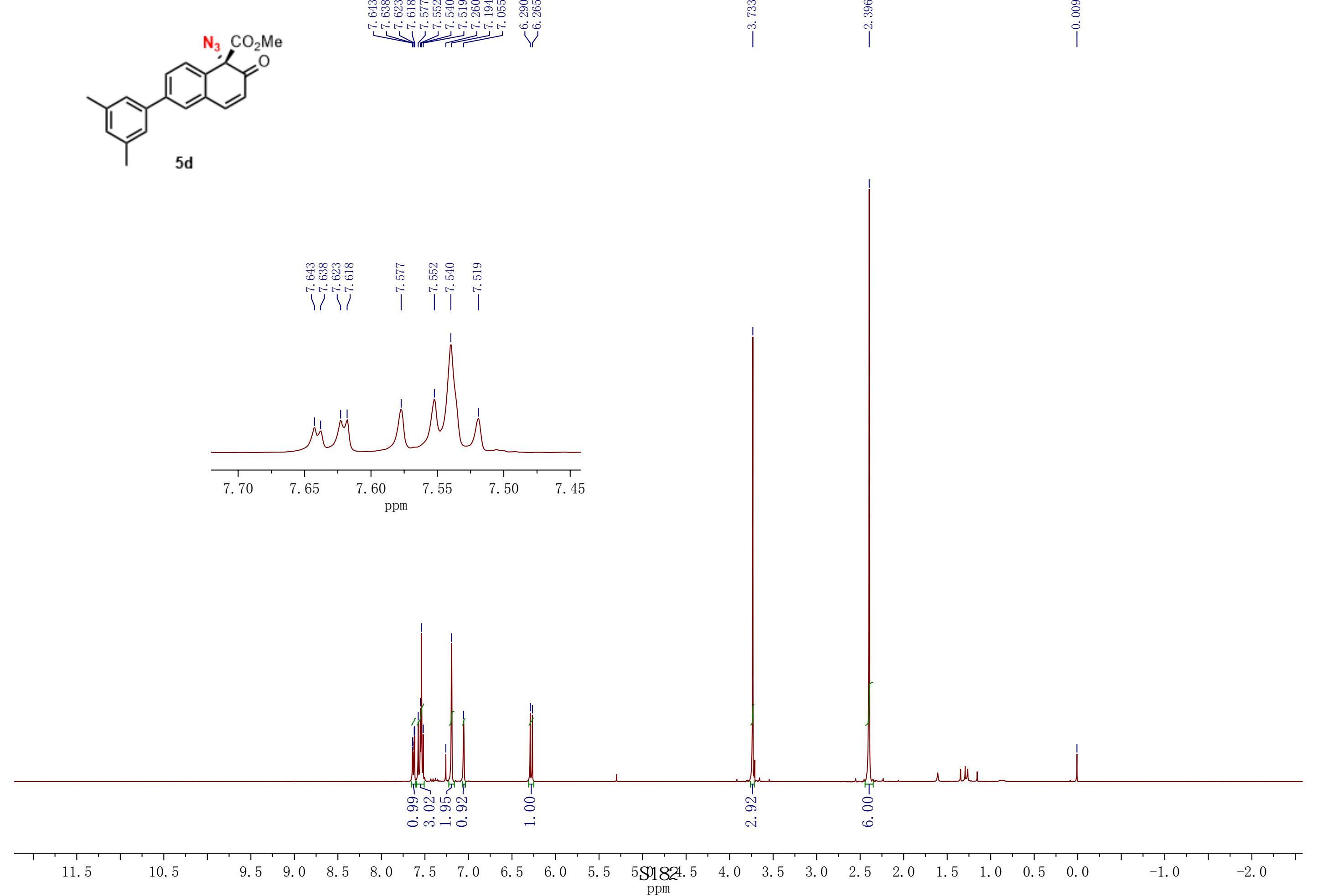


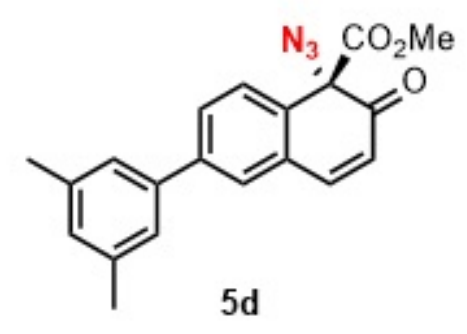

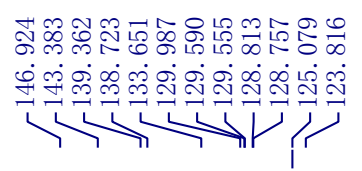

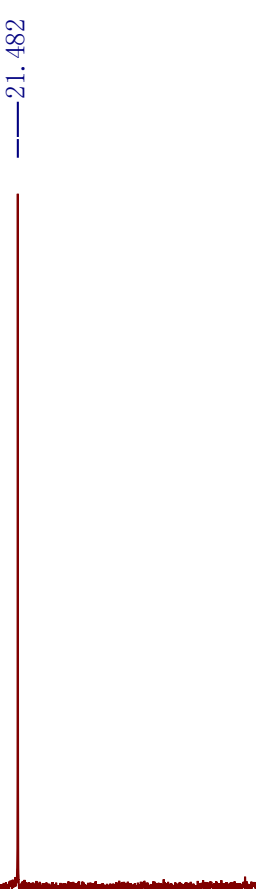




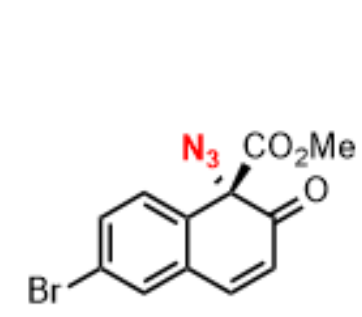

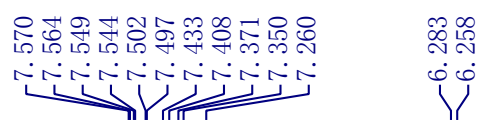

$5 e$
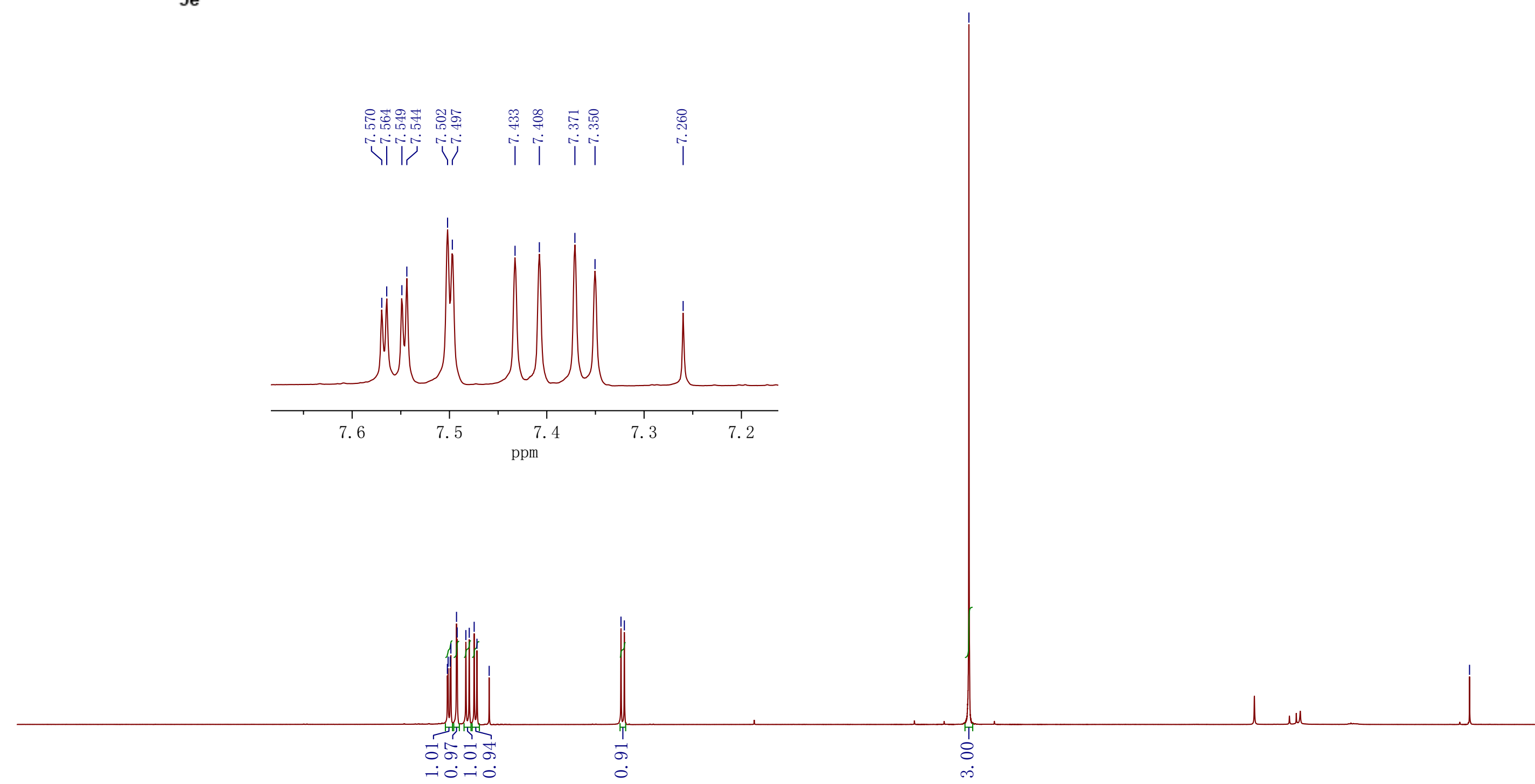

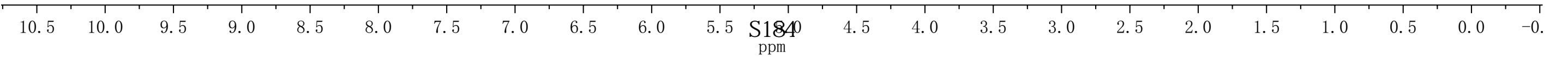




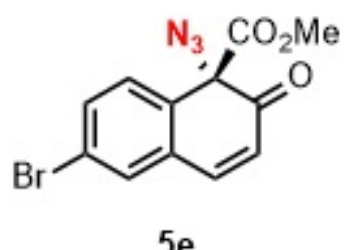

$5 e$

강윤붕으용

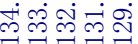

पो人
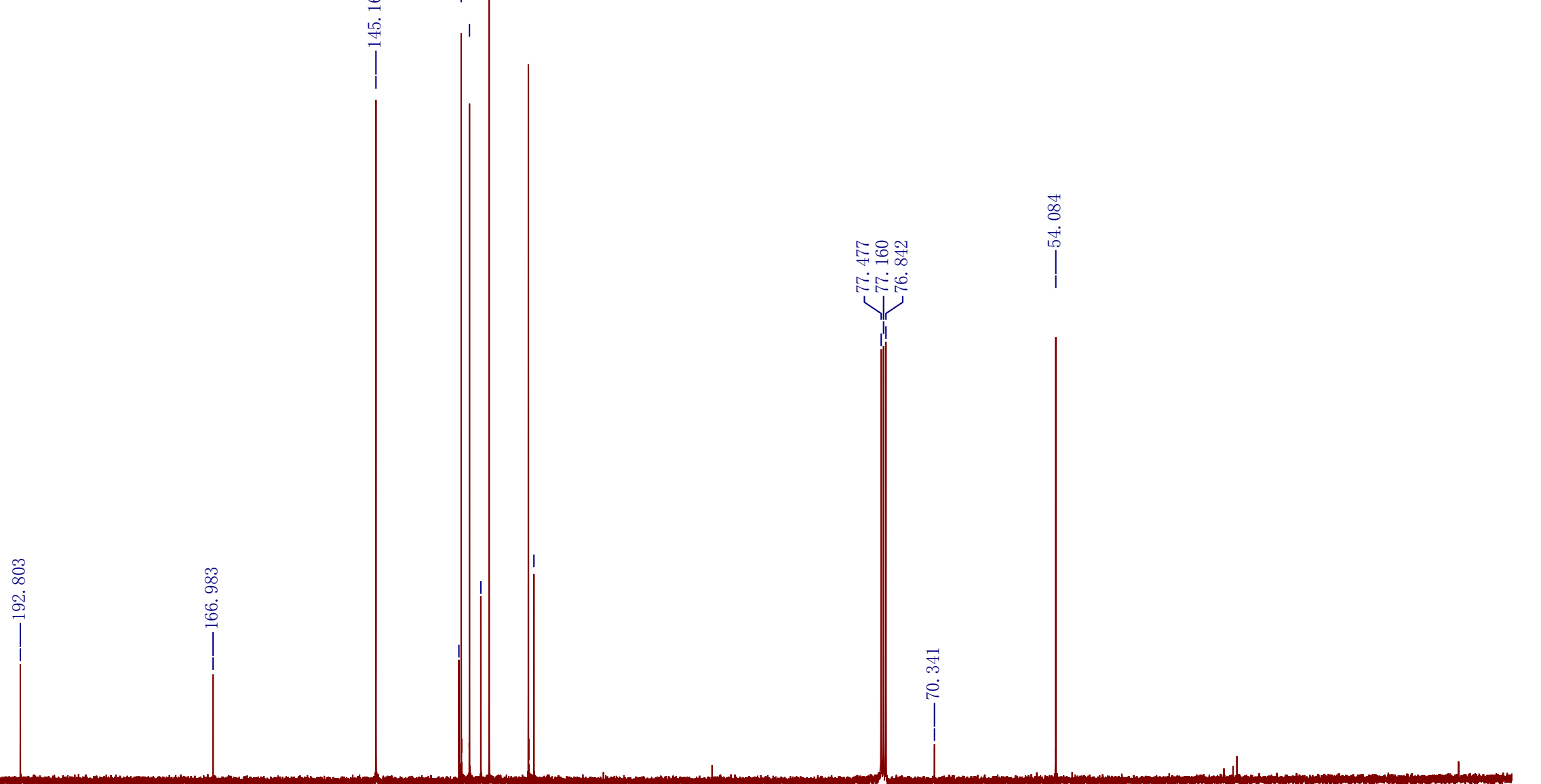

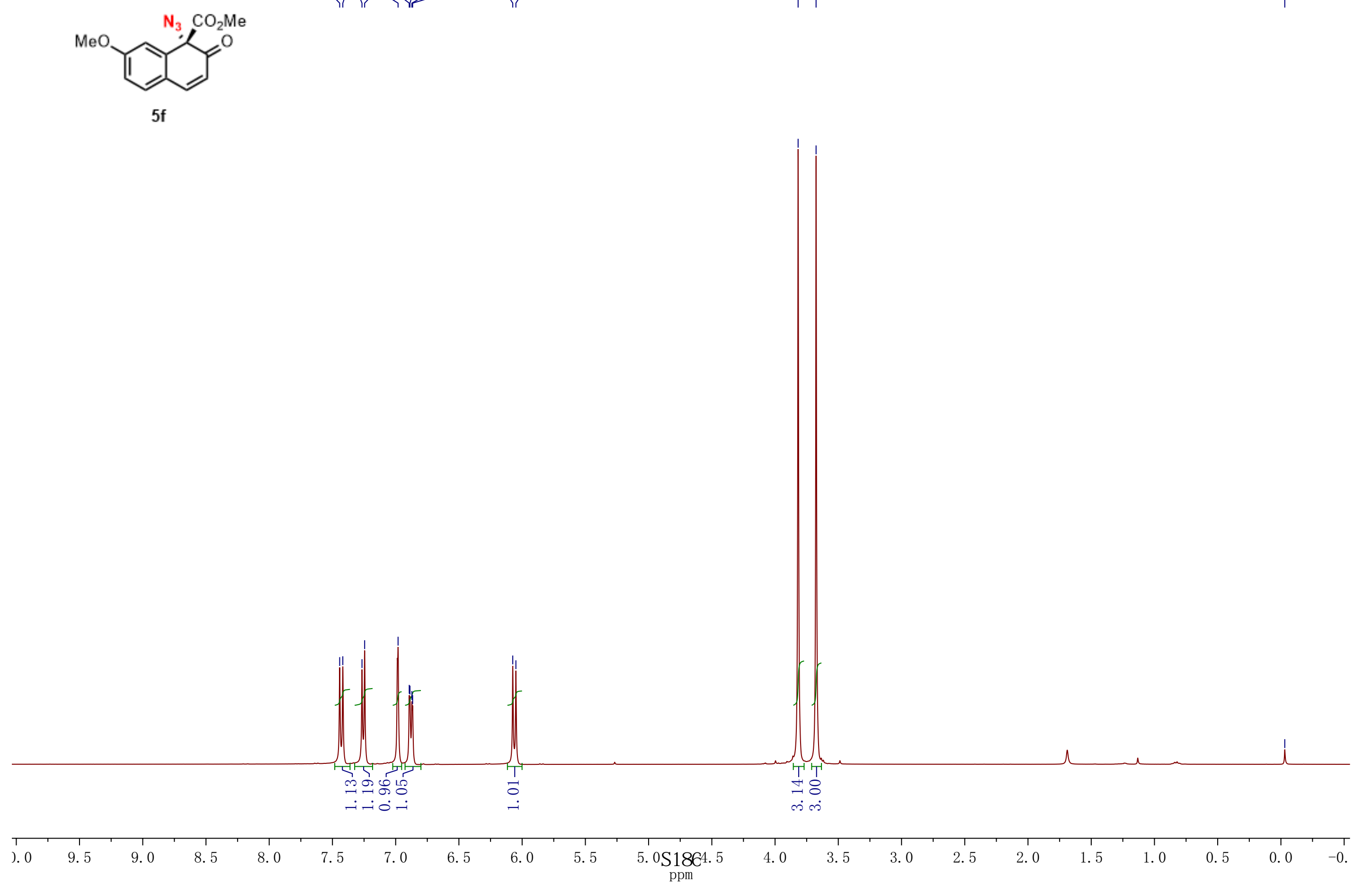

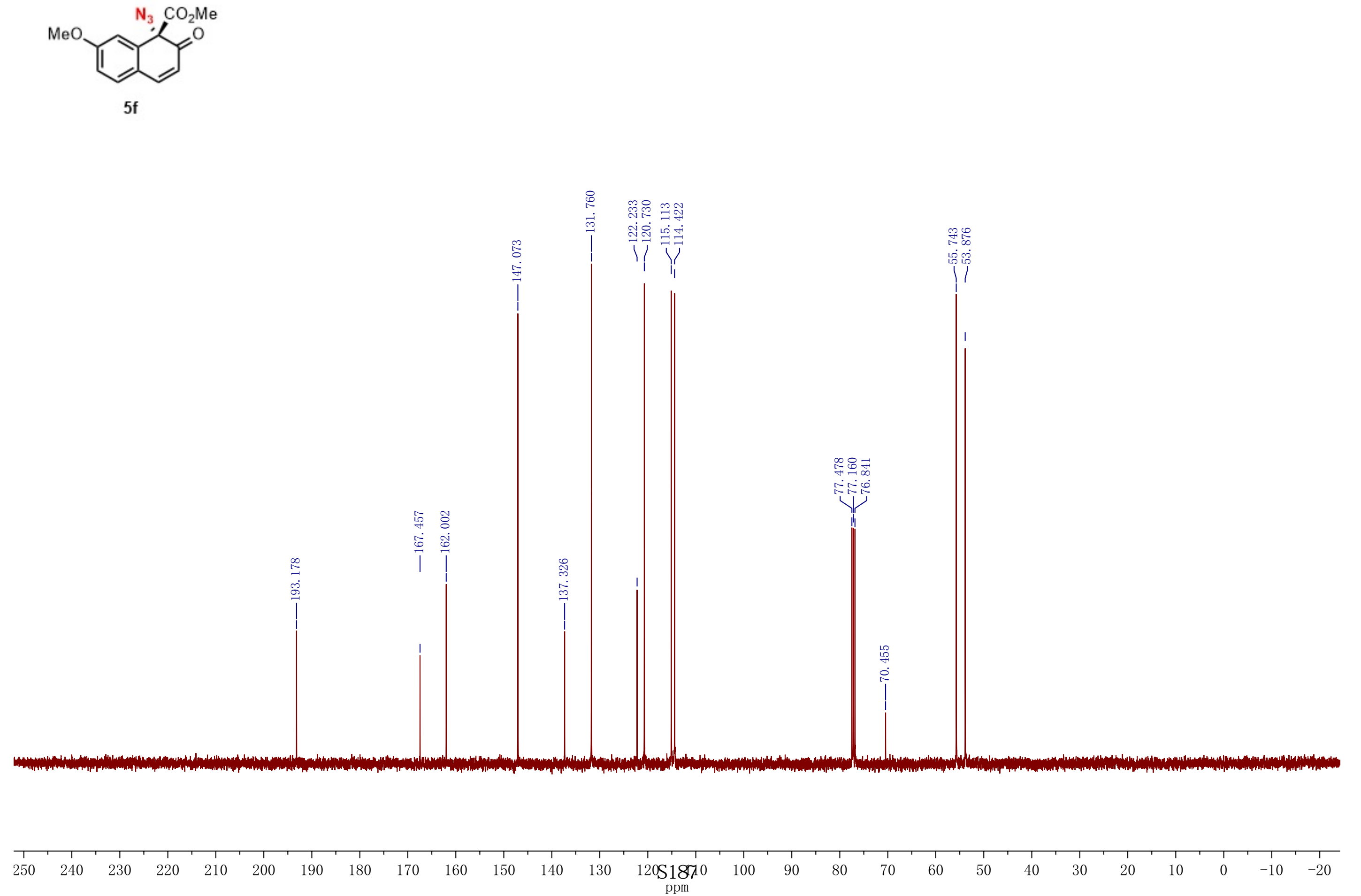

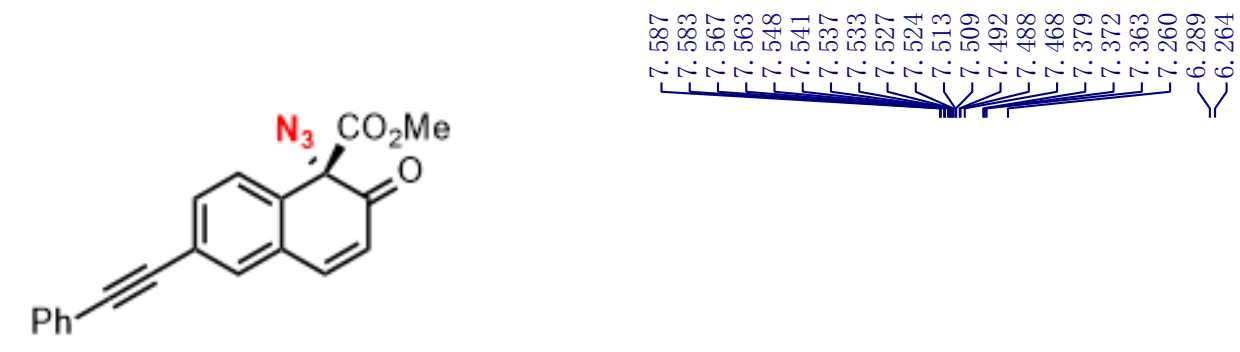

$5 \mathrm{~g}$

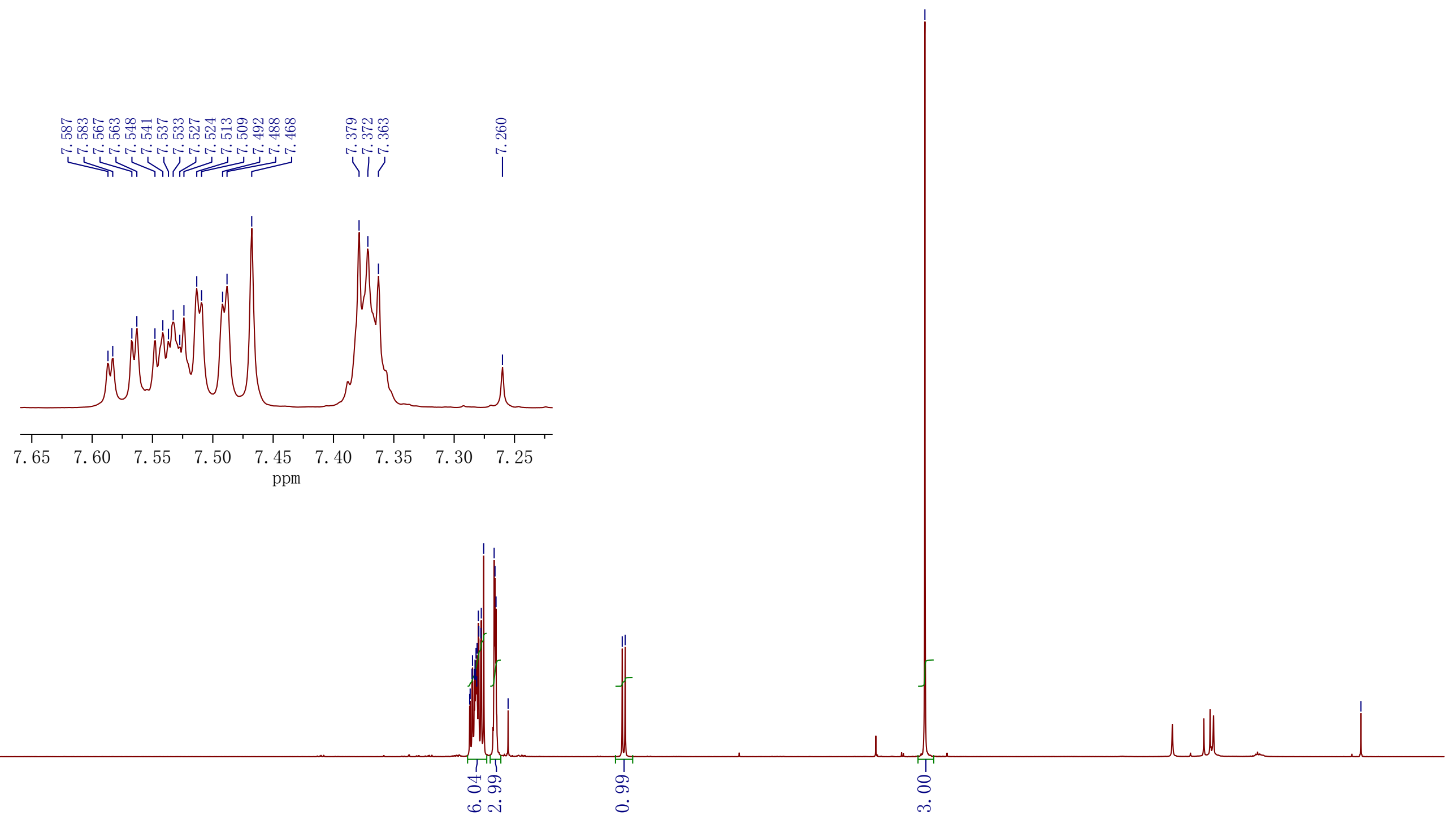




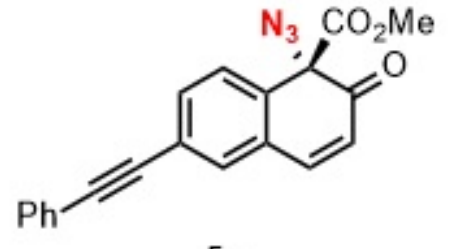

$5 g$

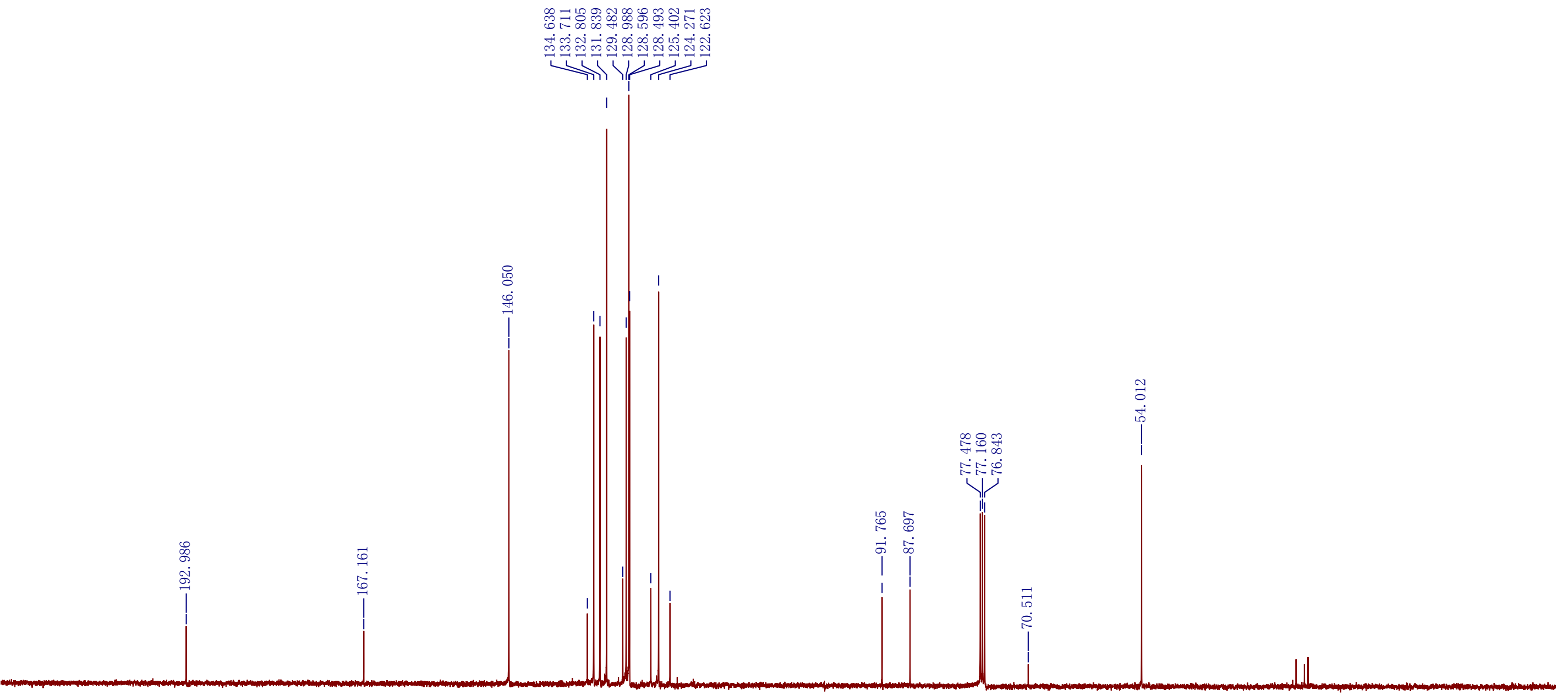

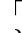

$210 \quad 200$

\begin{tabular}{rrrr|r} 
& 1 & 1 & 1 & \\
& 190 & 180 & 170 & 160
\end{tabular}

$\begin{array}{llll}1 & 1 & 1 \\ 150 & 140 & 130\end{array}$

120



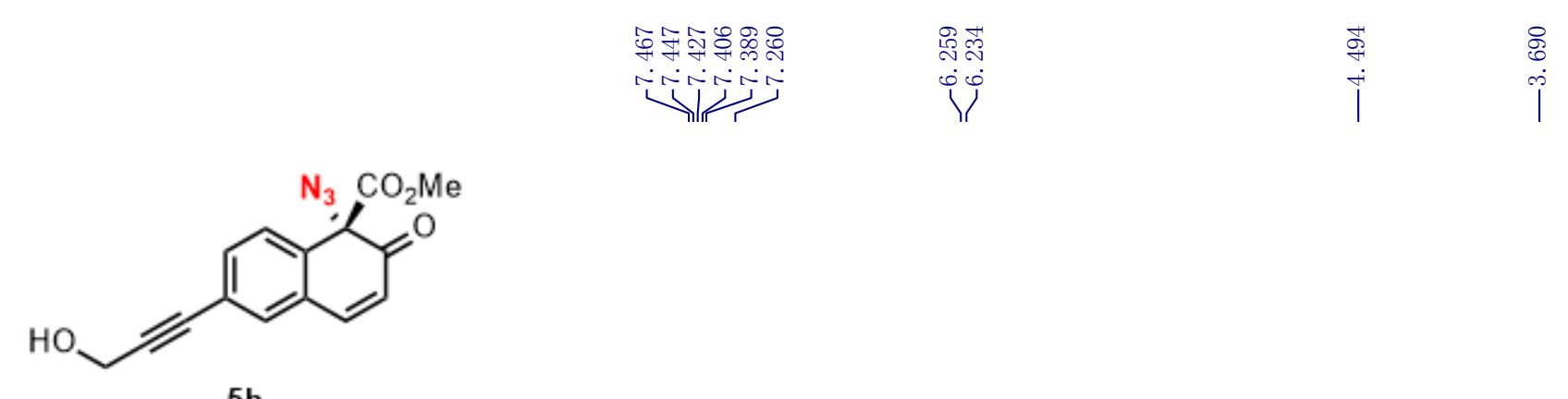

5 h
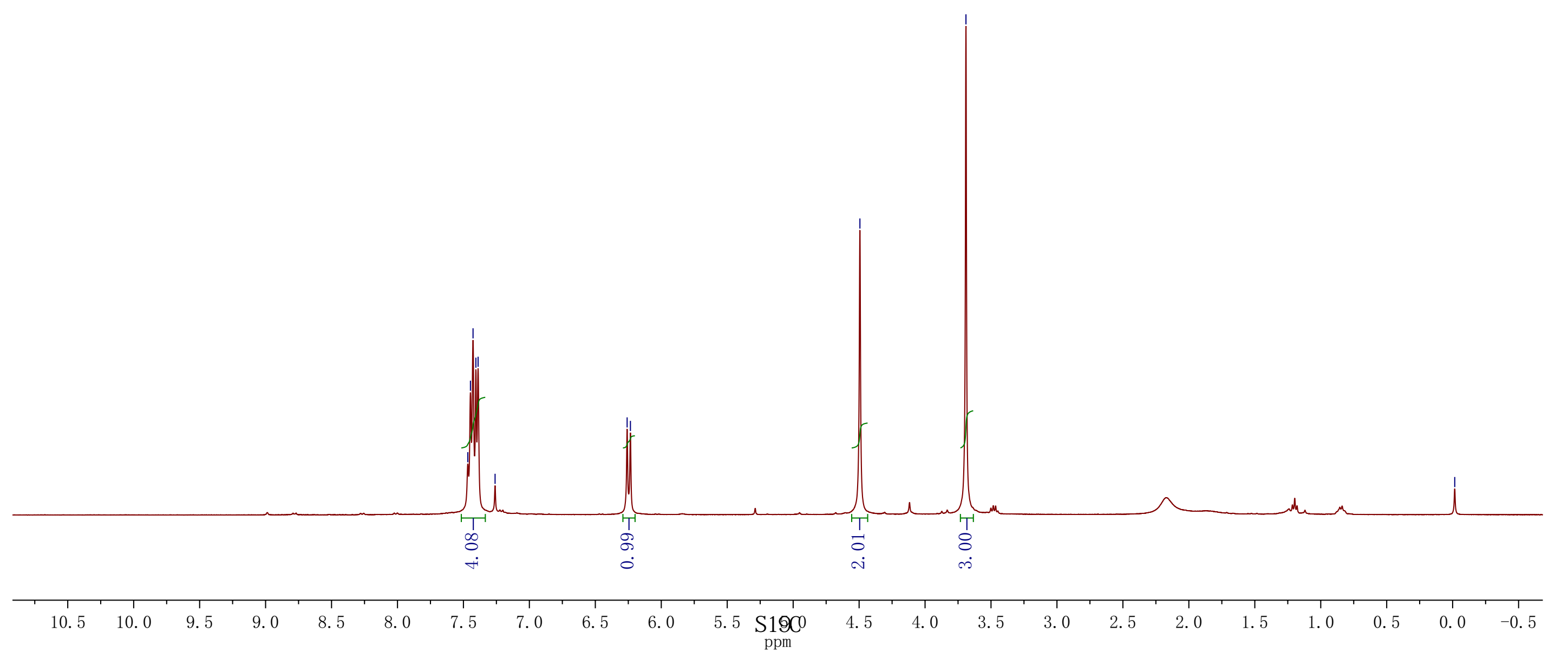


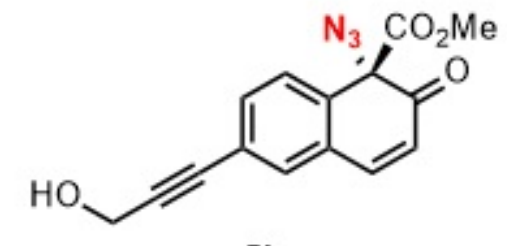

5 h

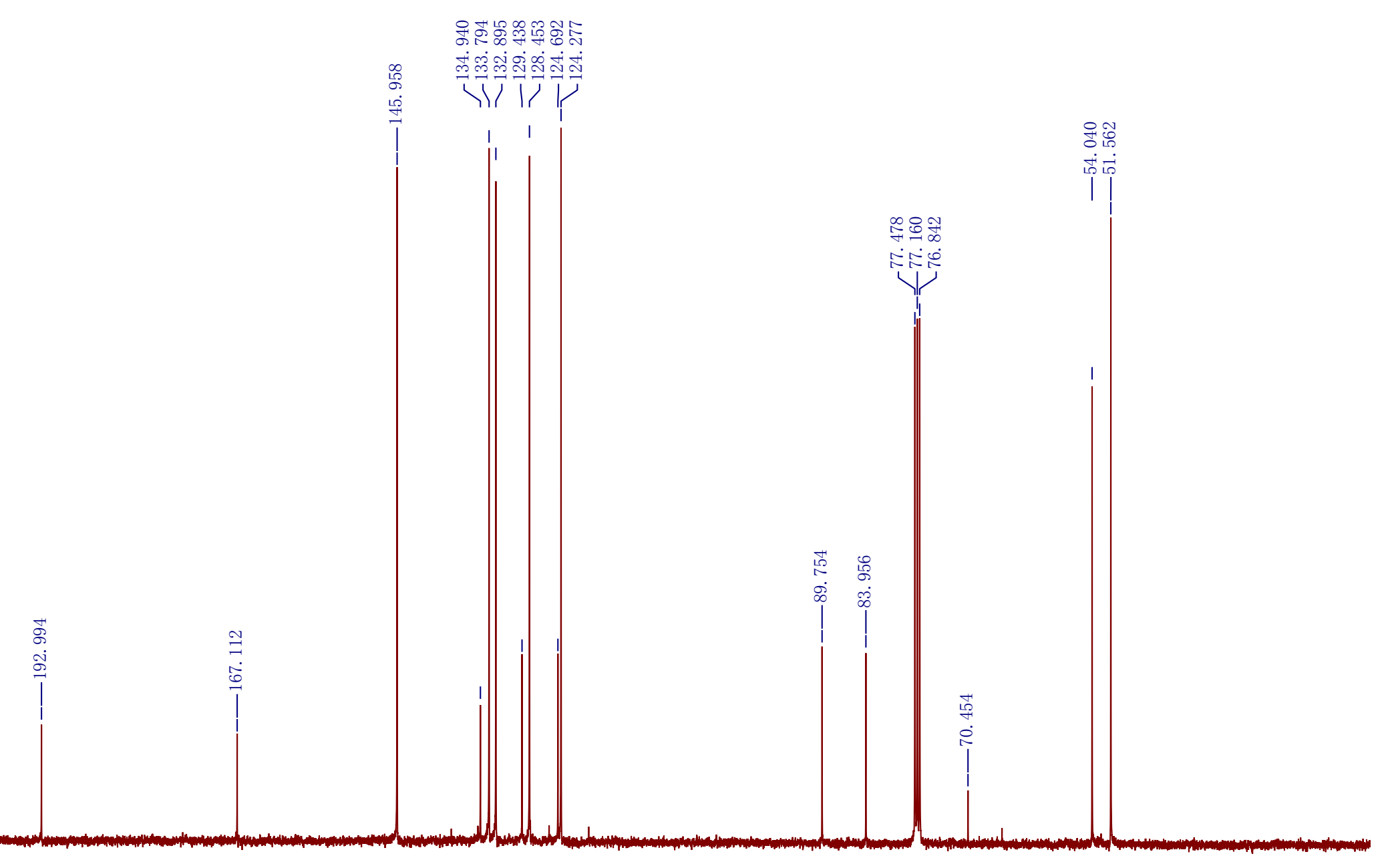

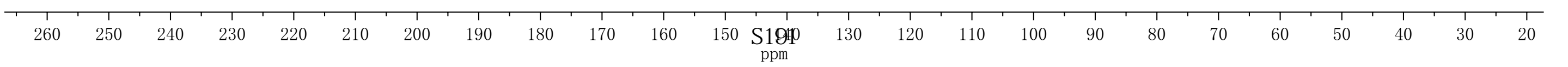




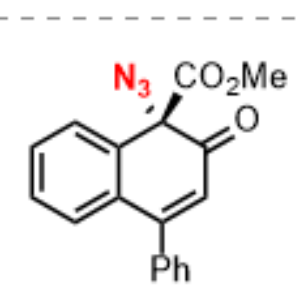

$5 i$

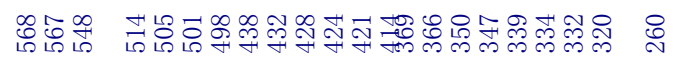

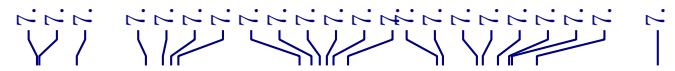

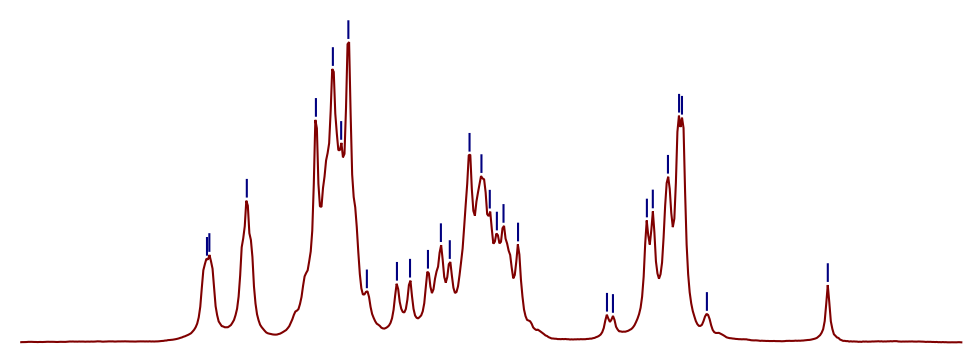

$\begin{array}{llllllllll}7.65 & 7.60 & 7.55 & 7.50 & 7.45 & 7.40 & 7.35 & 7.30 & 7.25 & 7.20\end{array}$ ppm

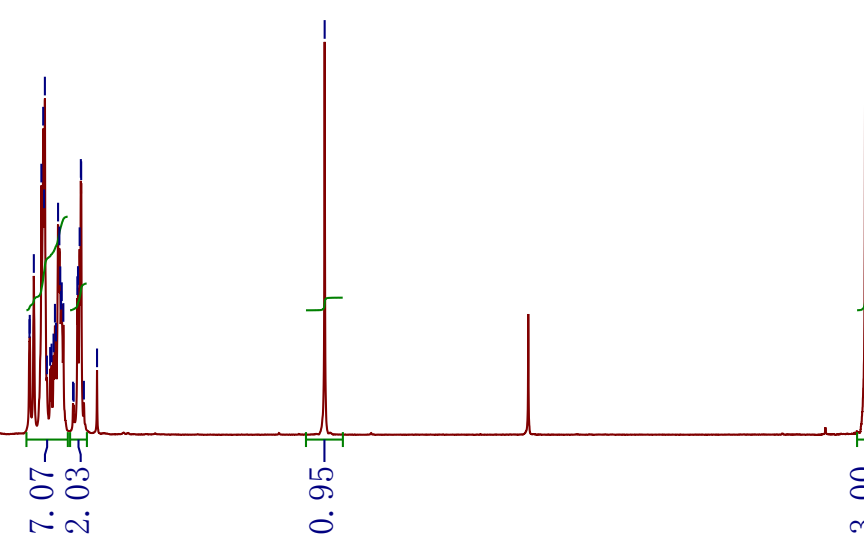

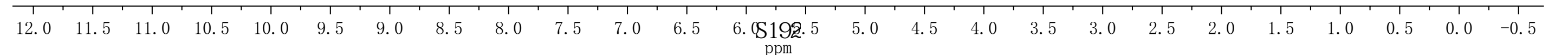




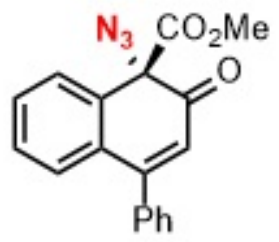

$5 i$

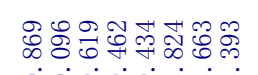

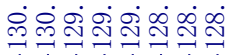

4

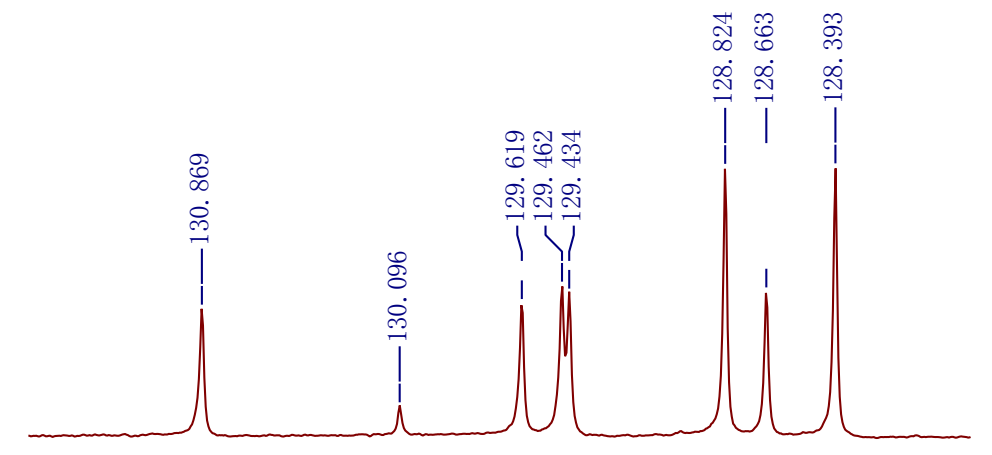

7

131. 5
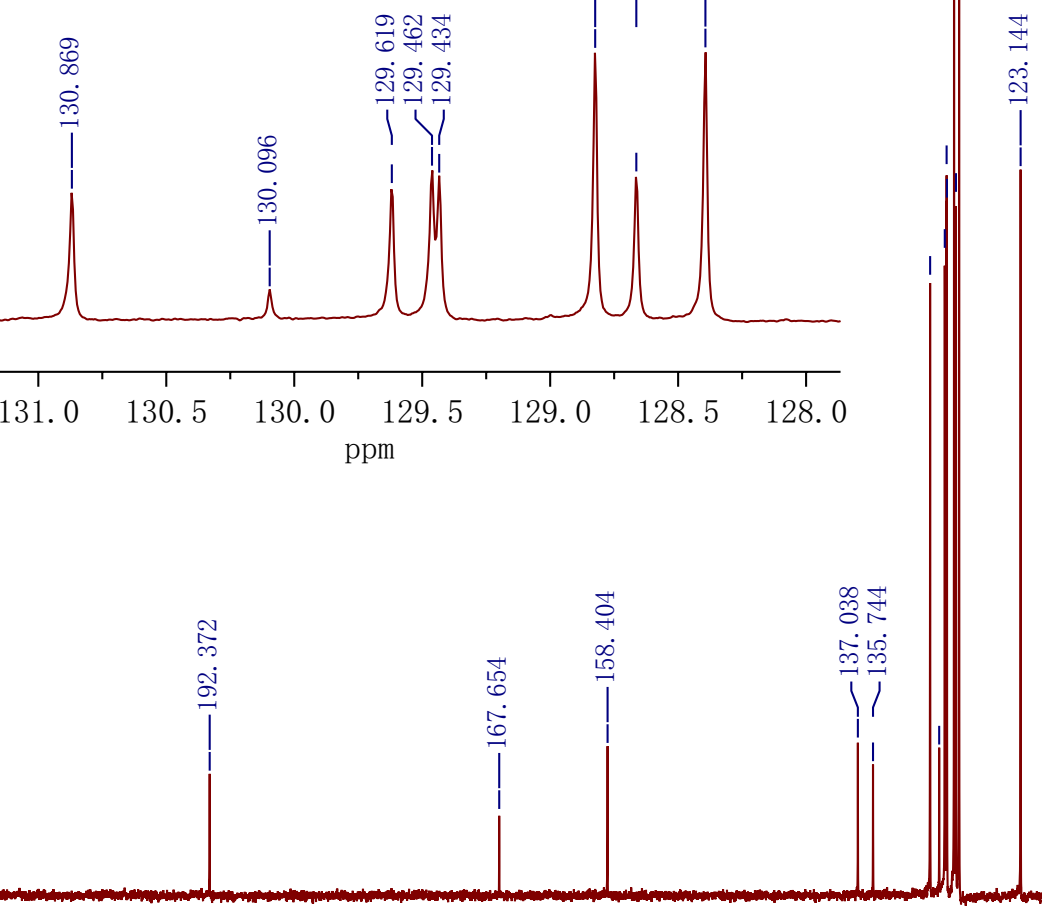

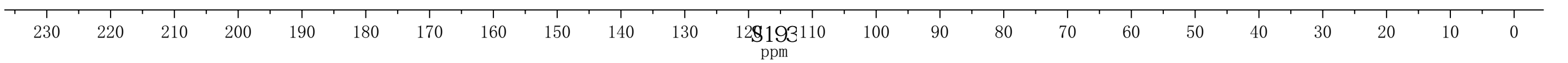



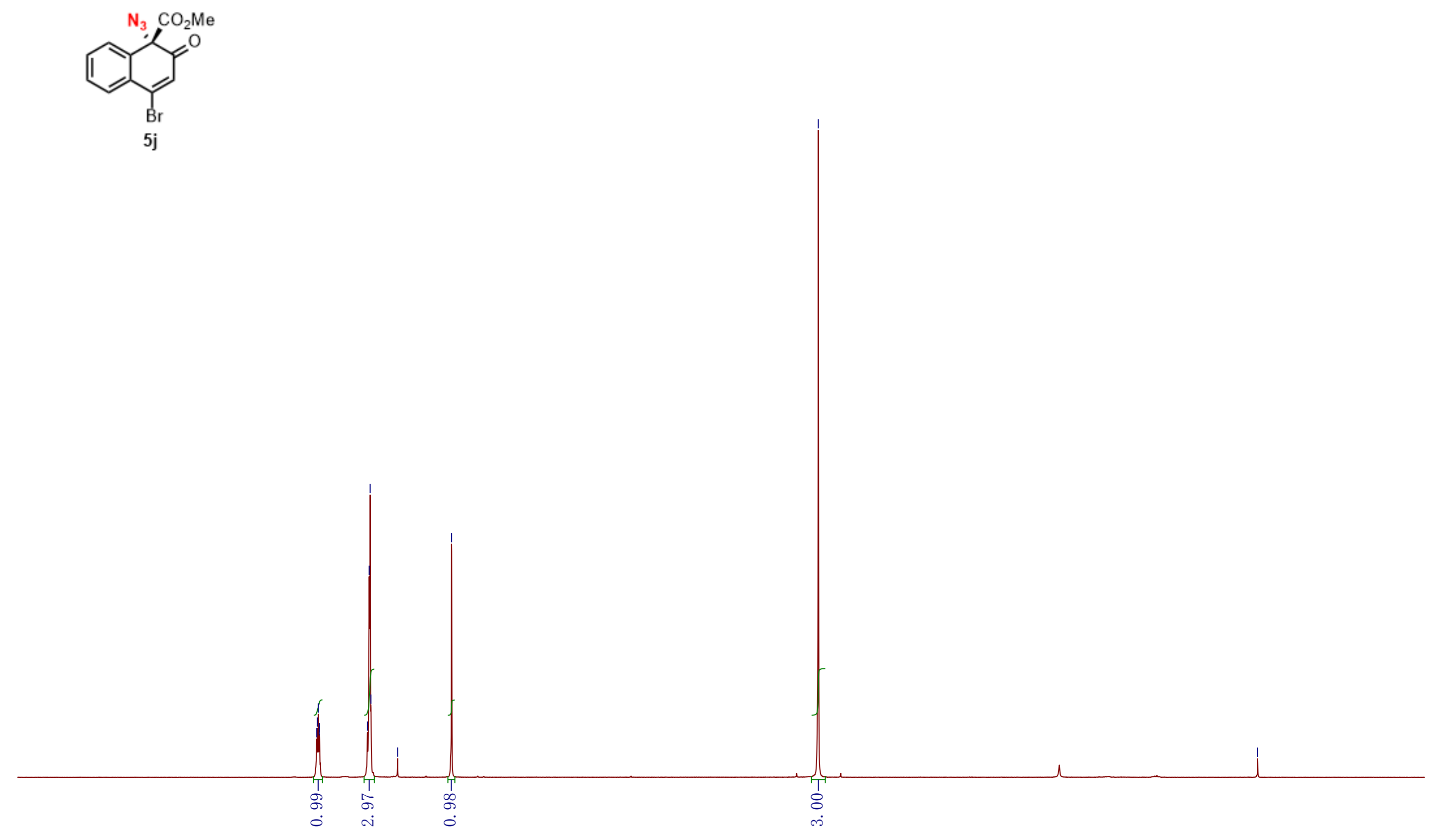

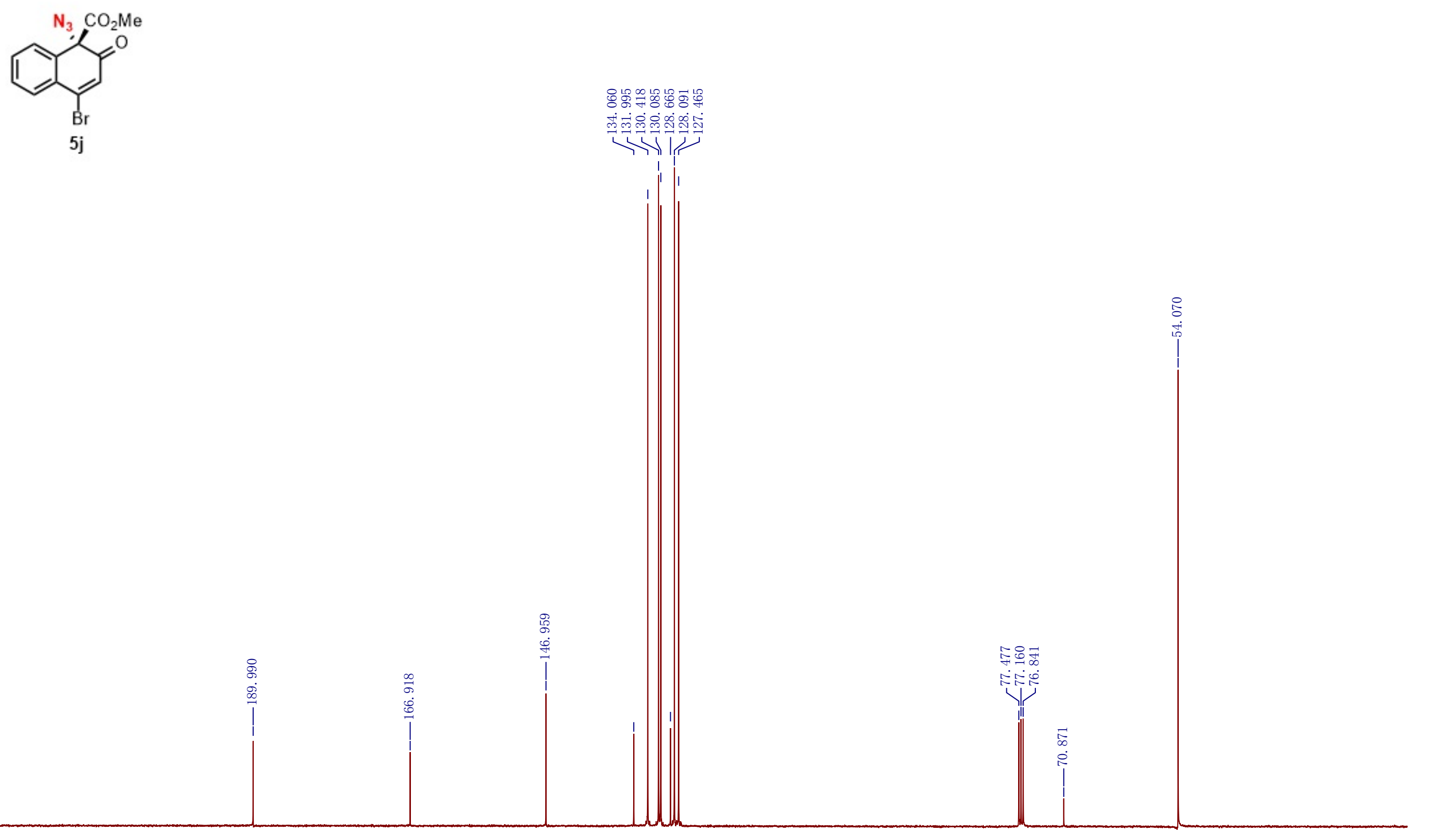

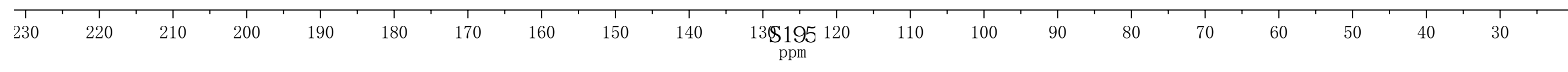



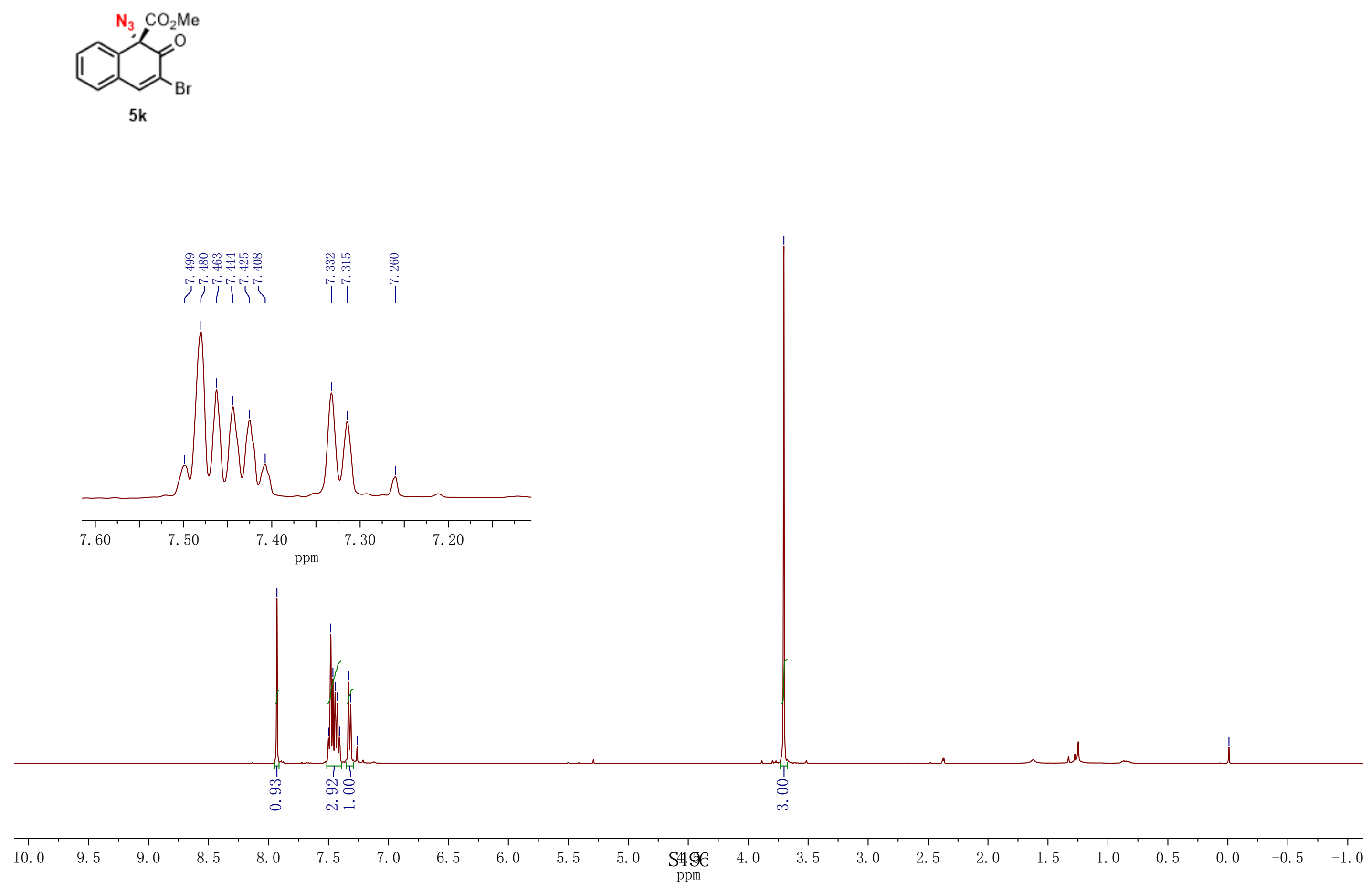


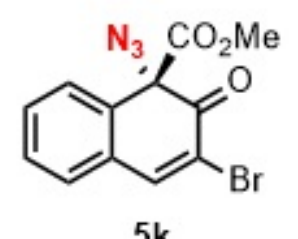

$5 k$
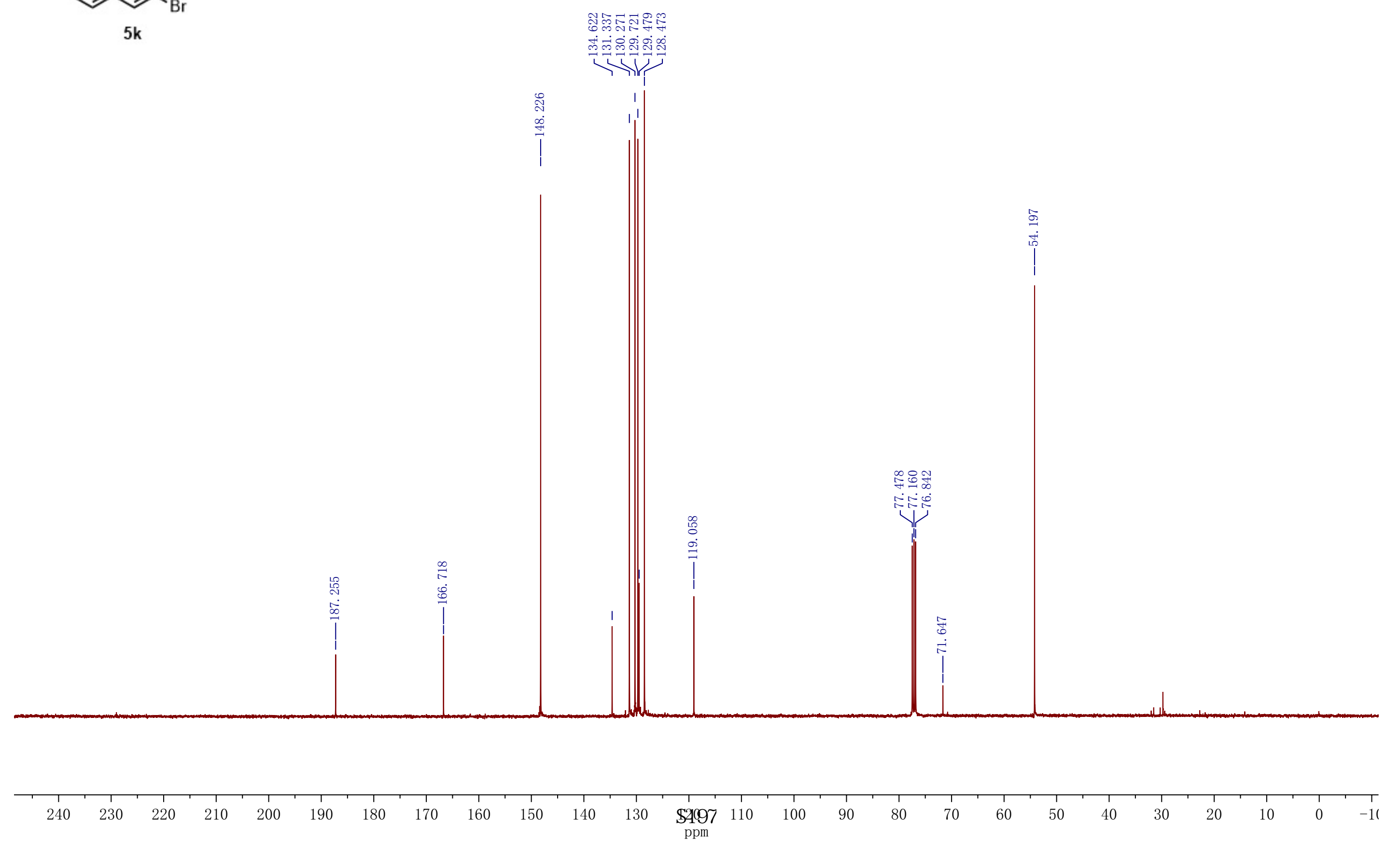


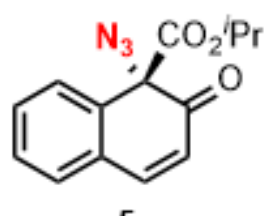

50

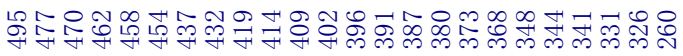

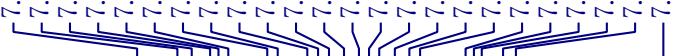

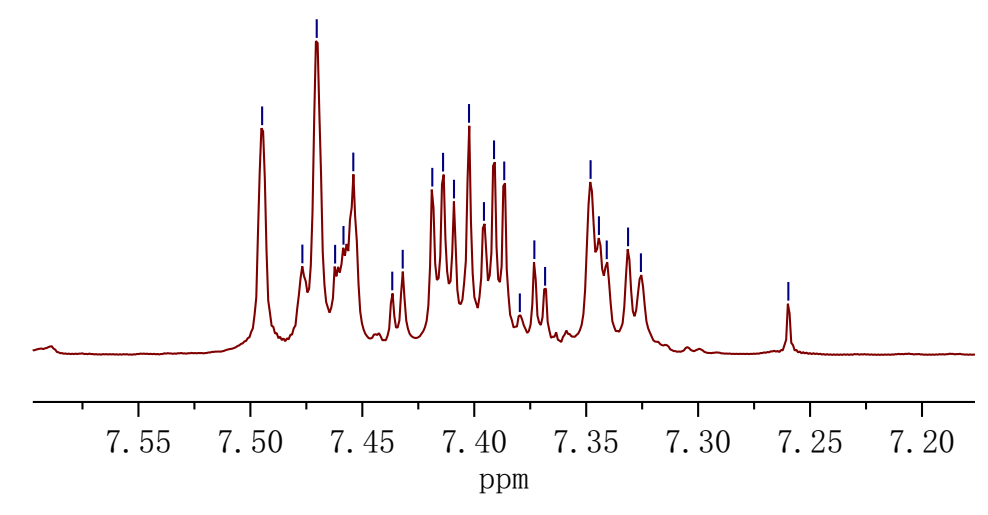

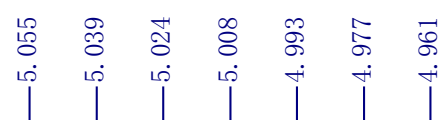

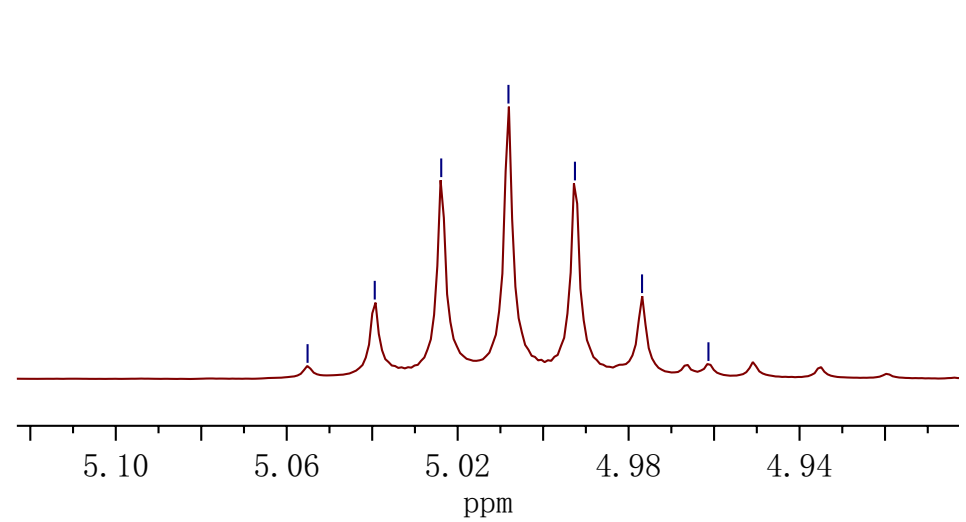

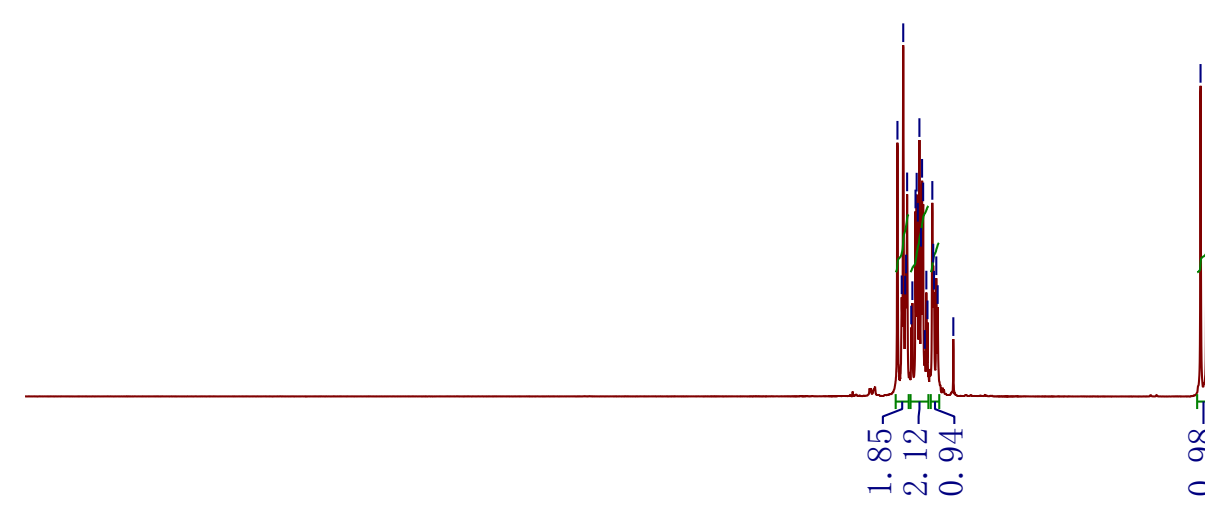
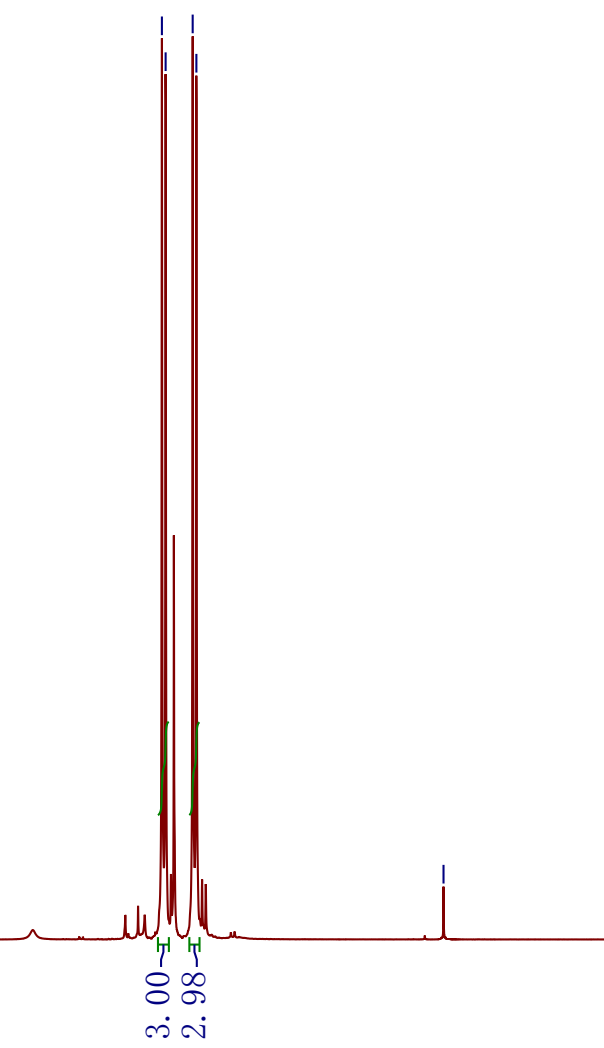

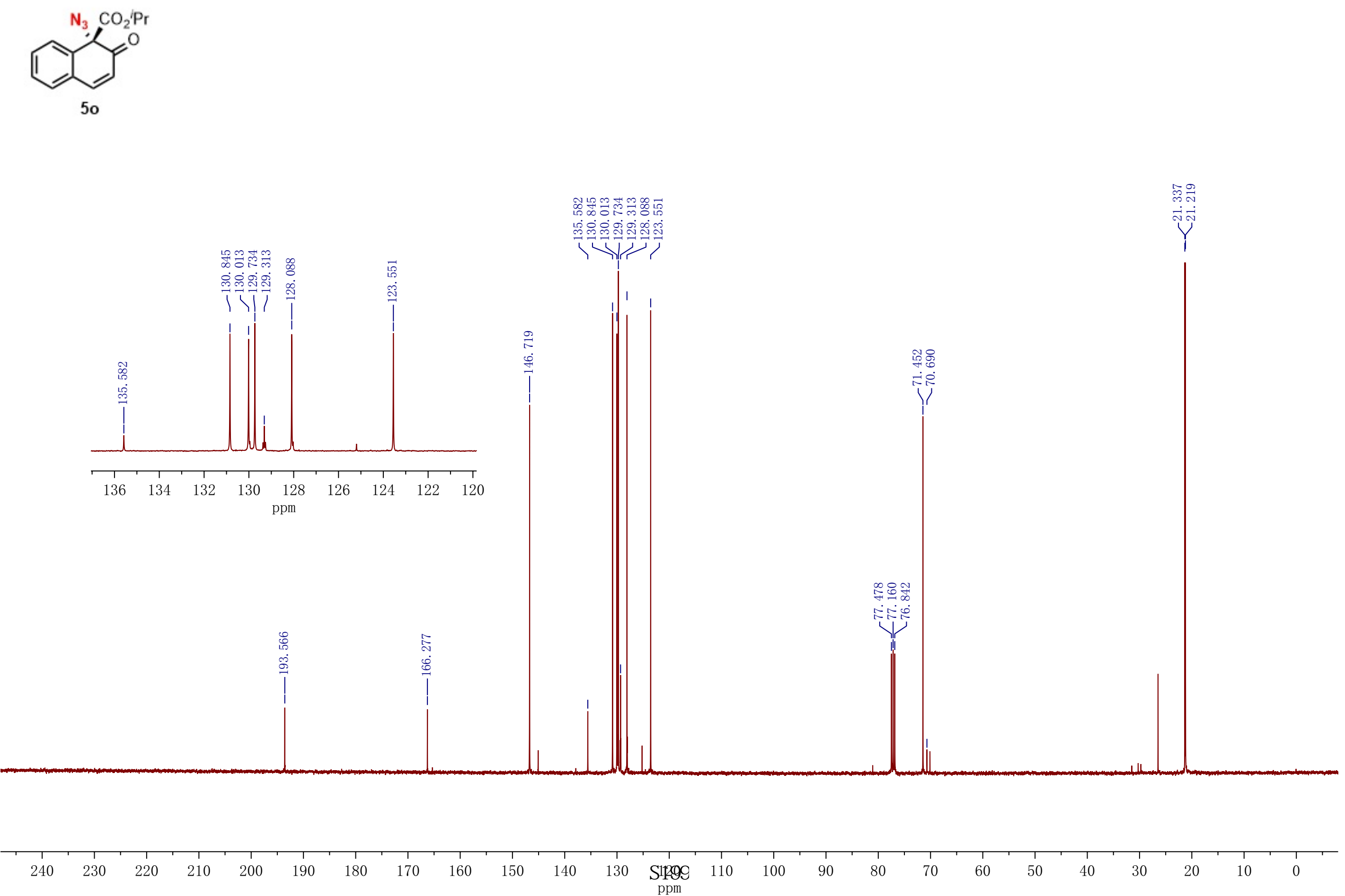


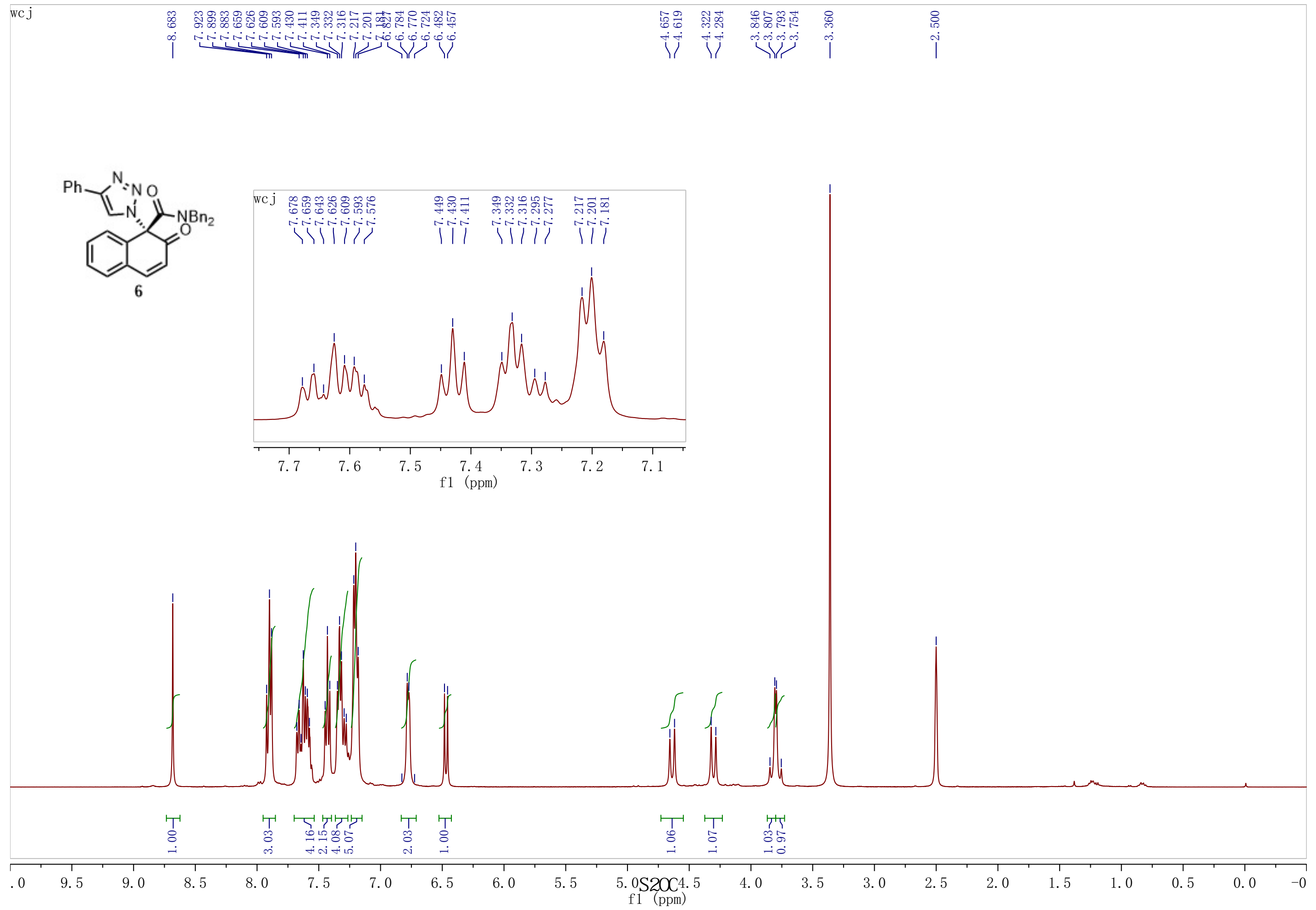




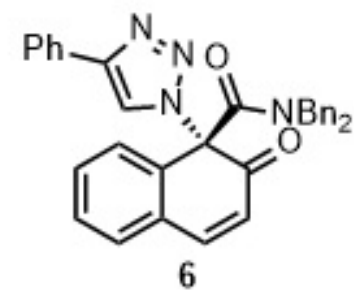

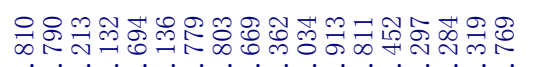

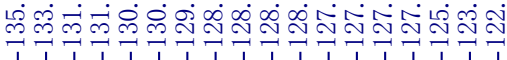

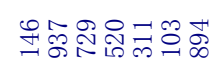

잉 $0^{\circ} \circ \infty$

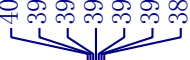
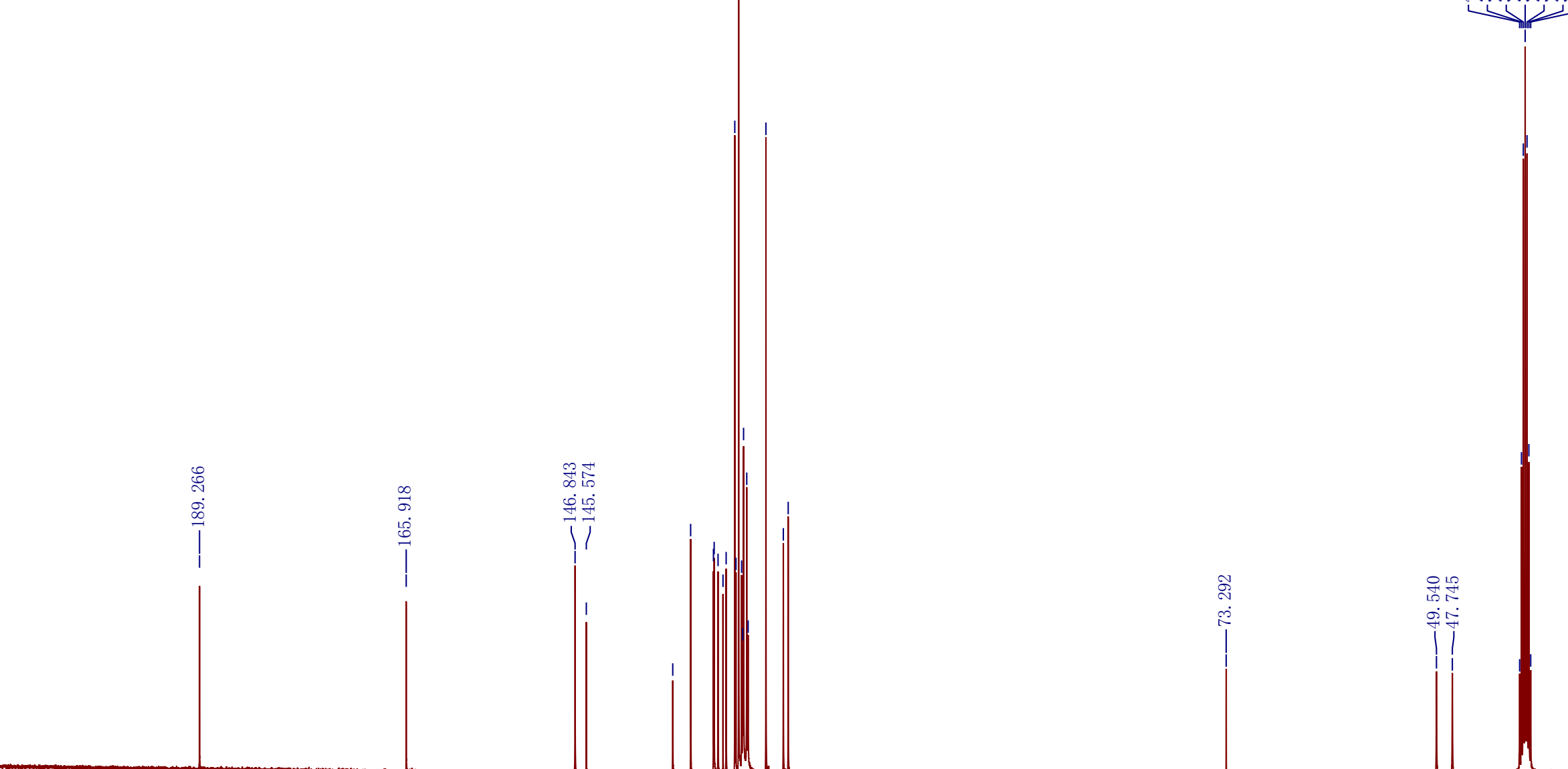

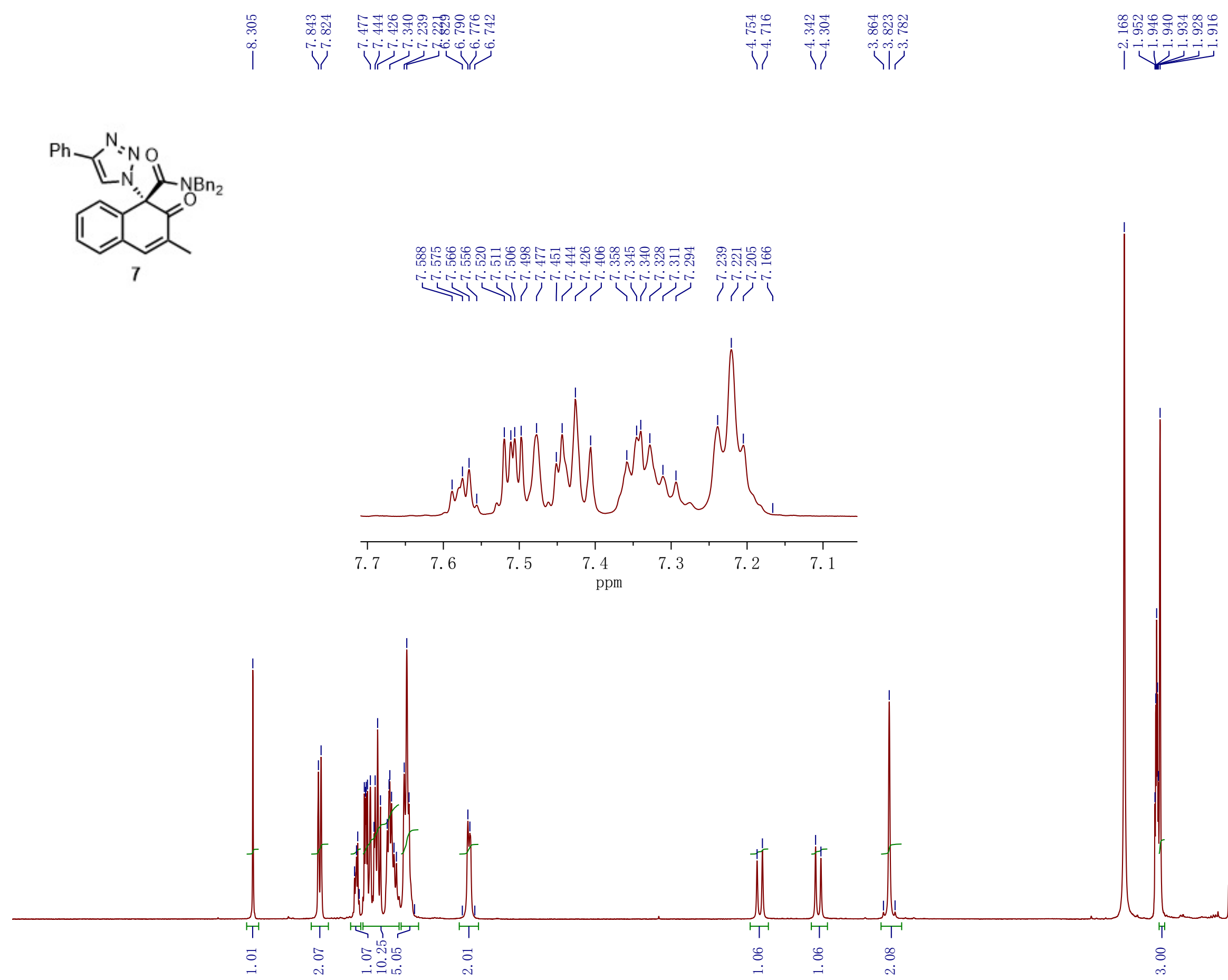

59.

8.58 .0

$7.5 \quad 7.0$

6.5

6.0

5. ${ }_{\mathrm{ppm}}^{\mathrm{S} 2 \mathrm{O} 24}$

3.5 

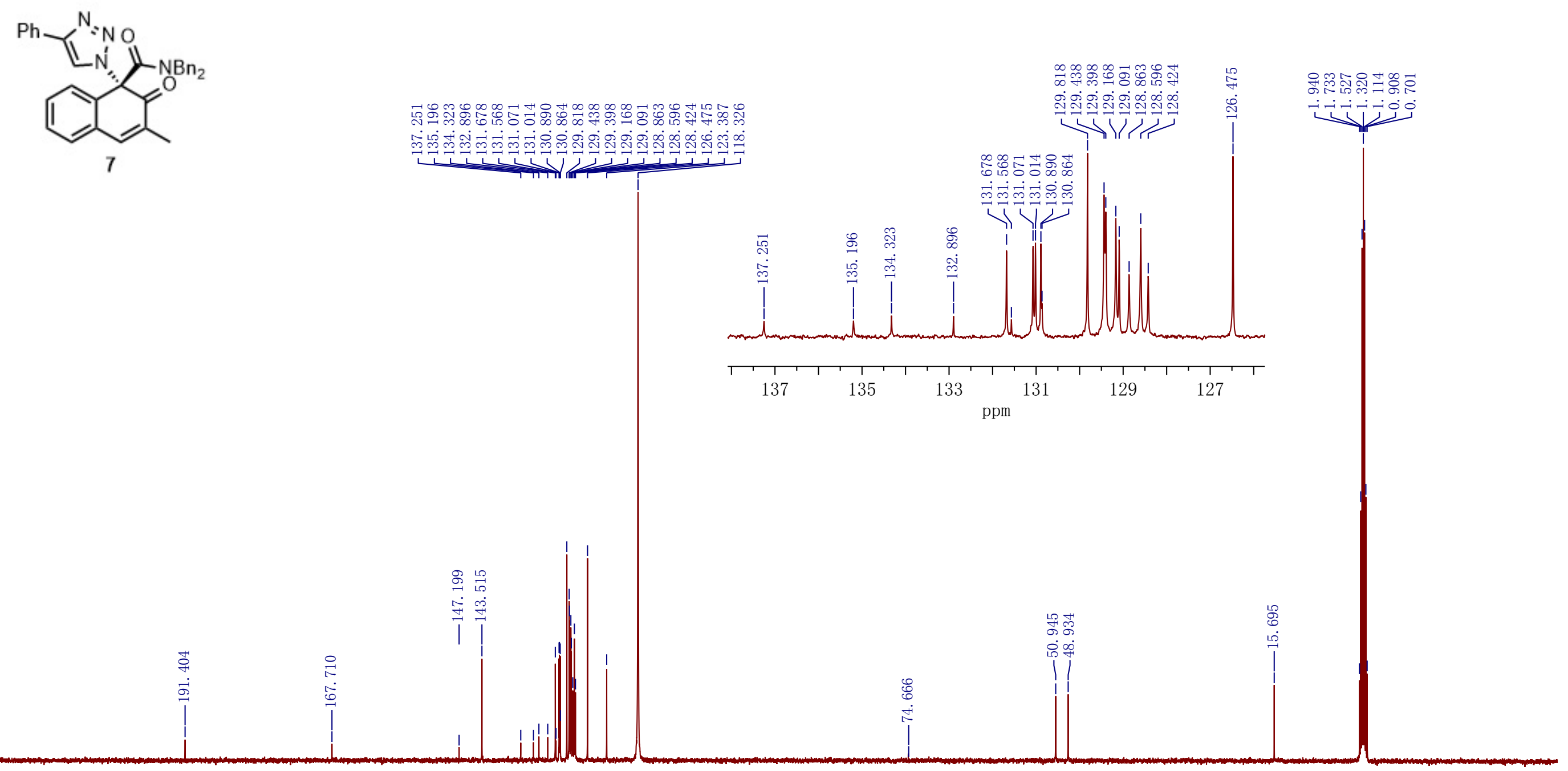


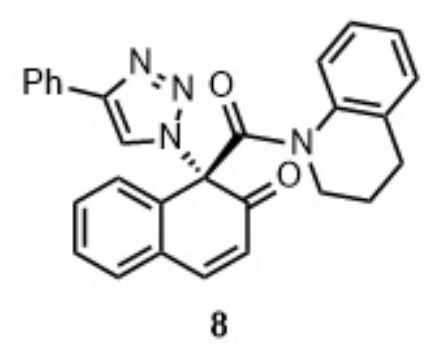

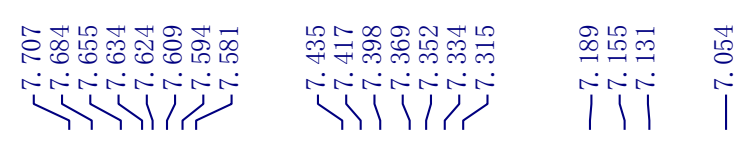
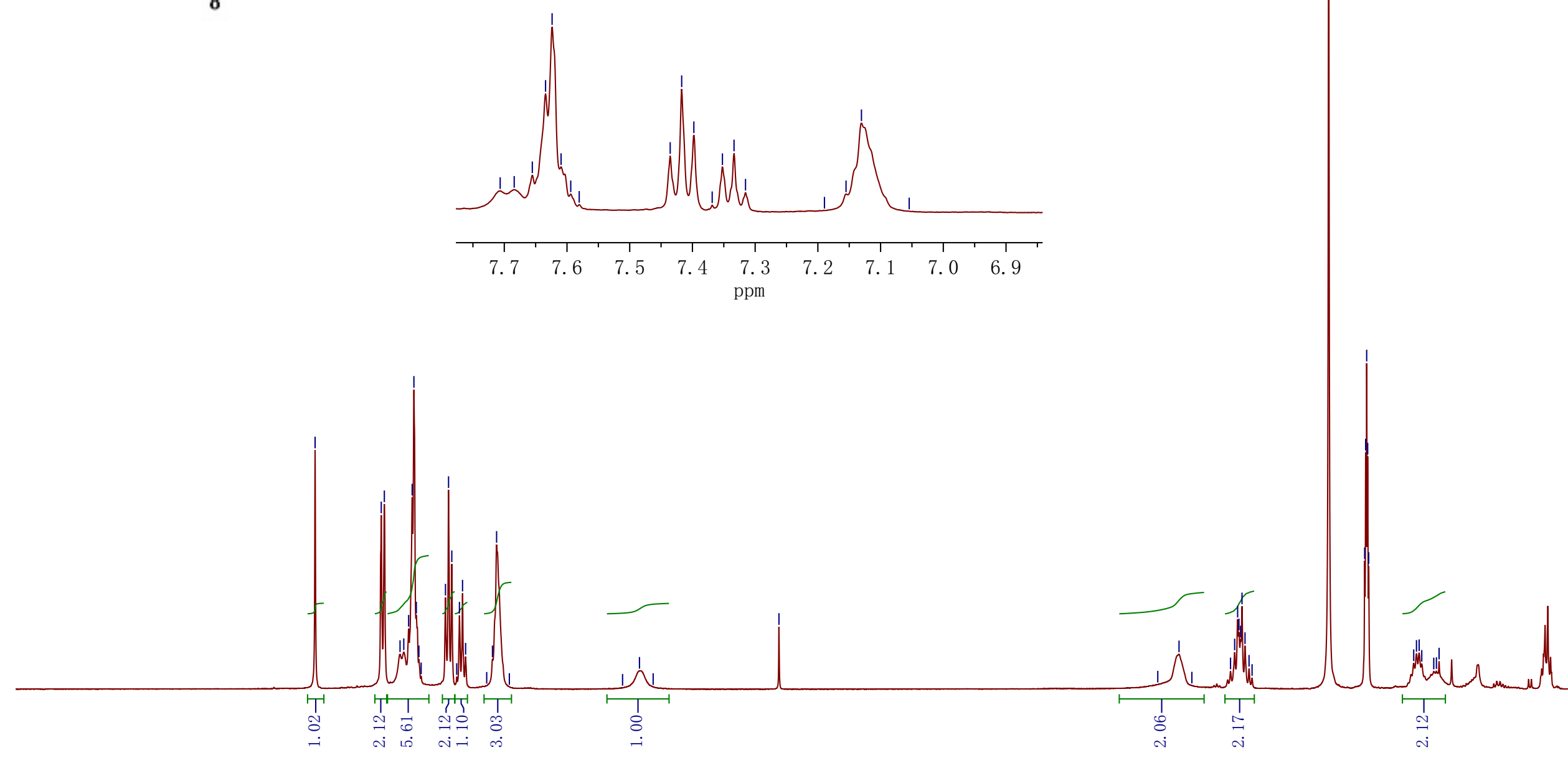

59.

$\begin{array}{llllll}1 & & \\ 0.5 & 8.0 & 7.5 & 7.0\end{array}$
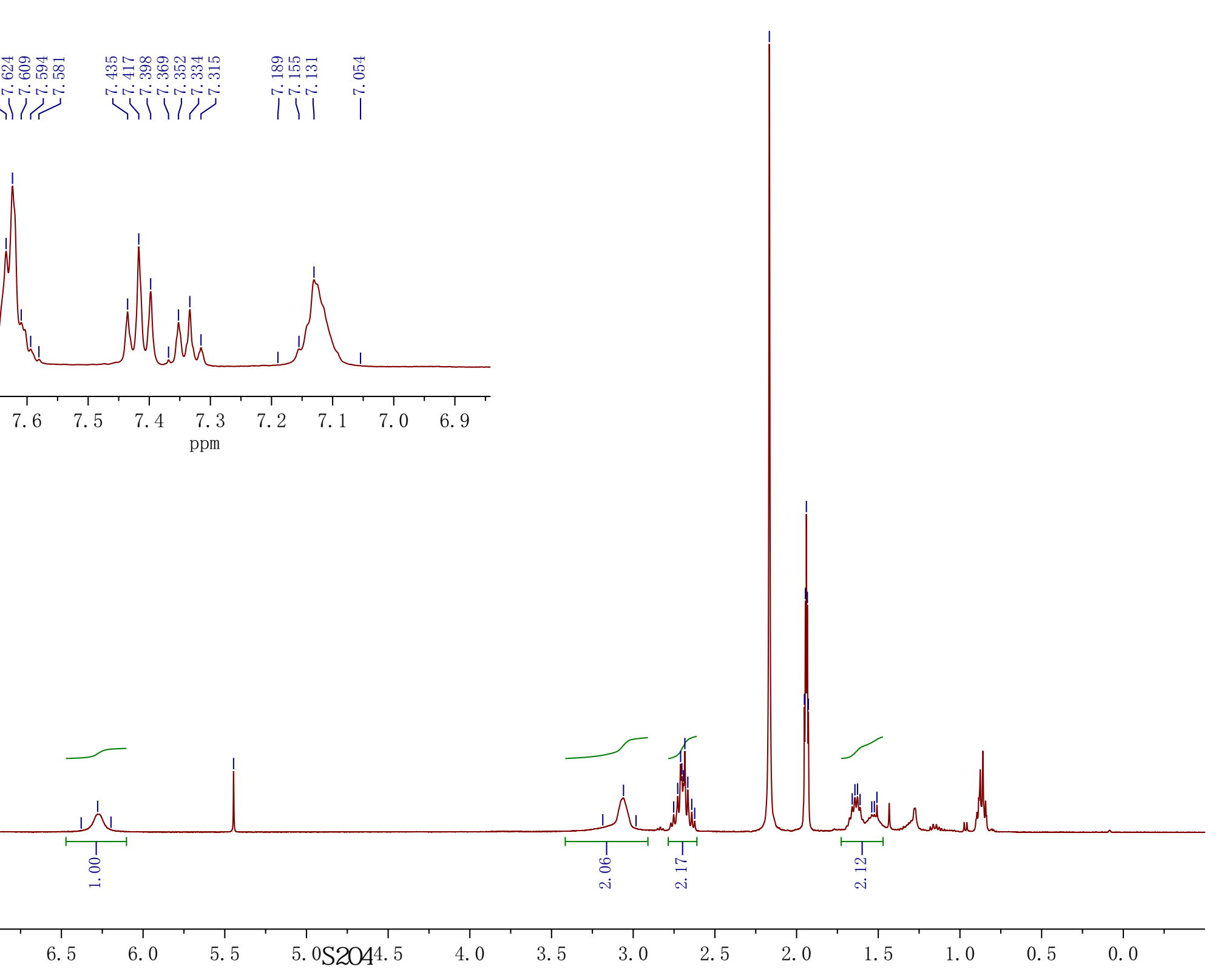


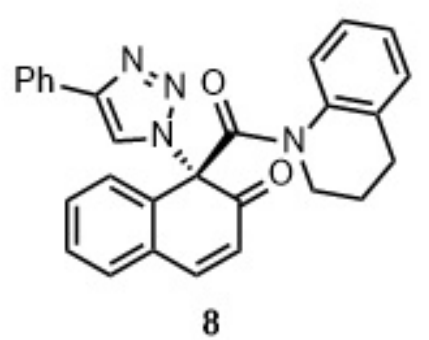

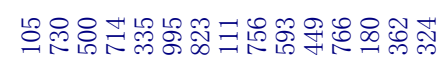

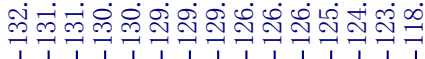
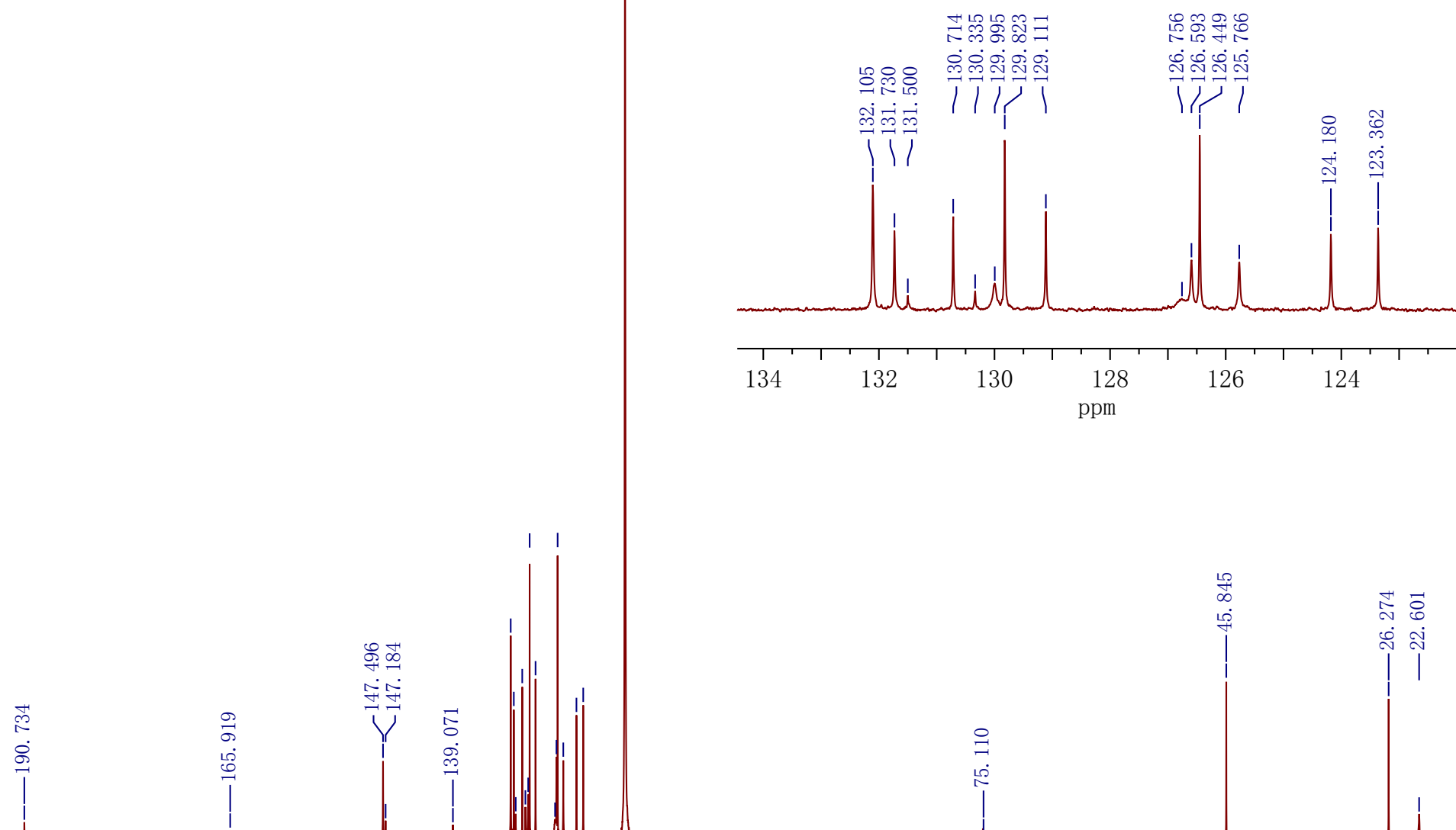


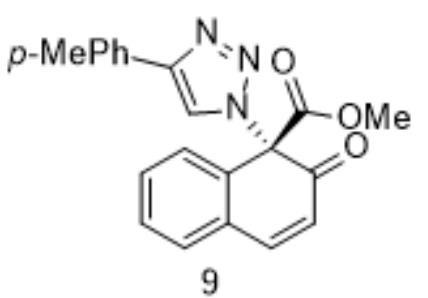

门1
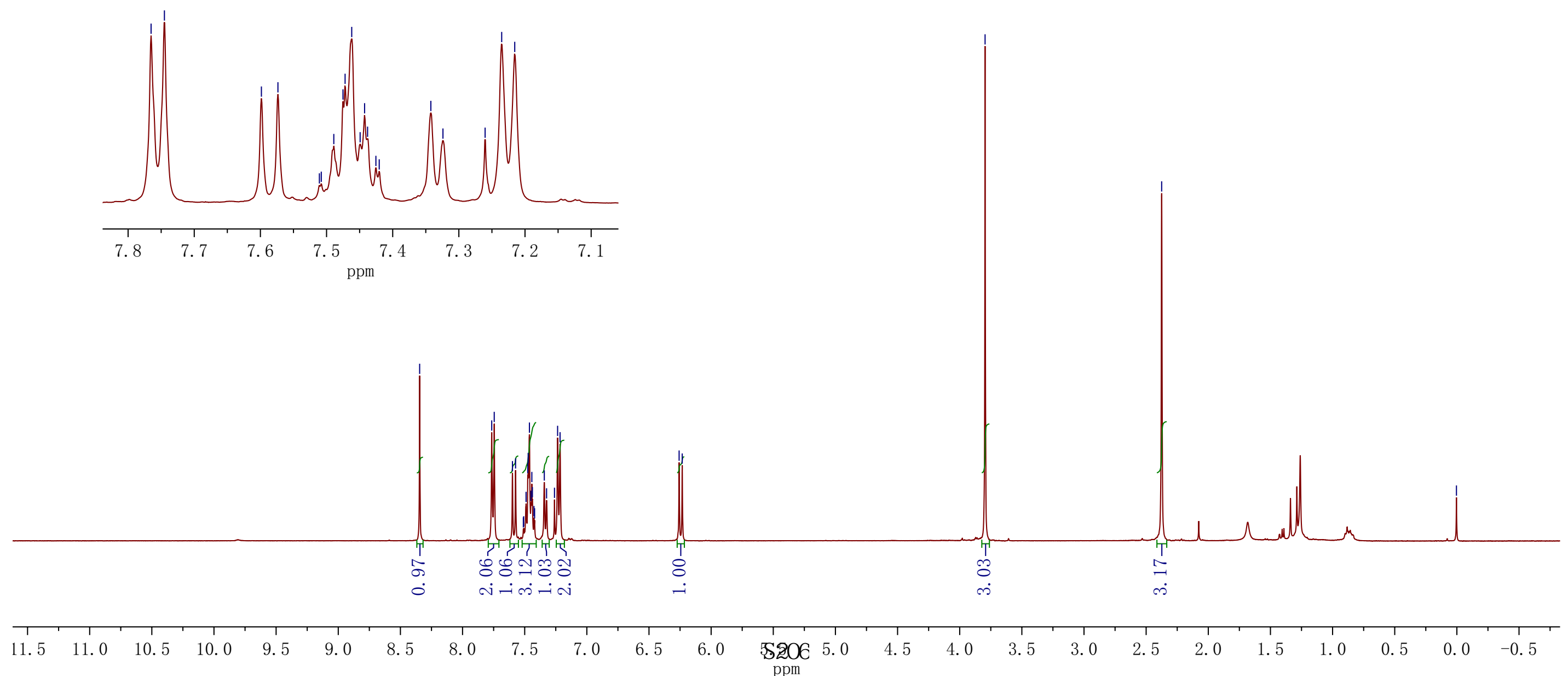

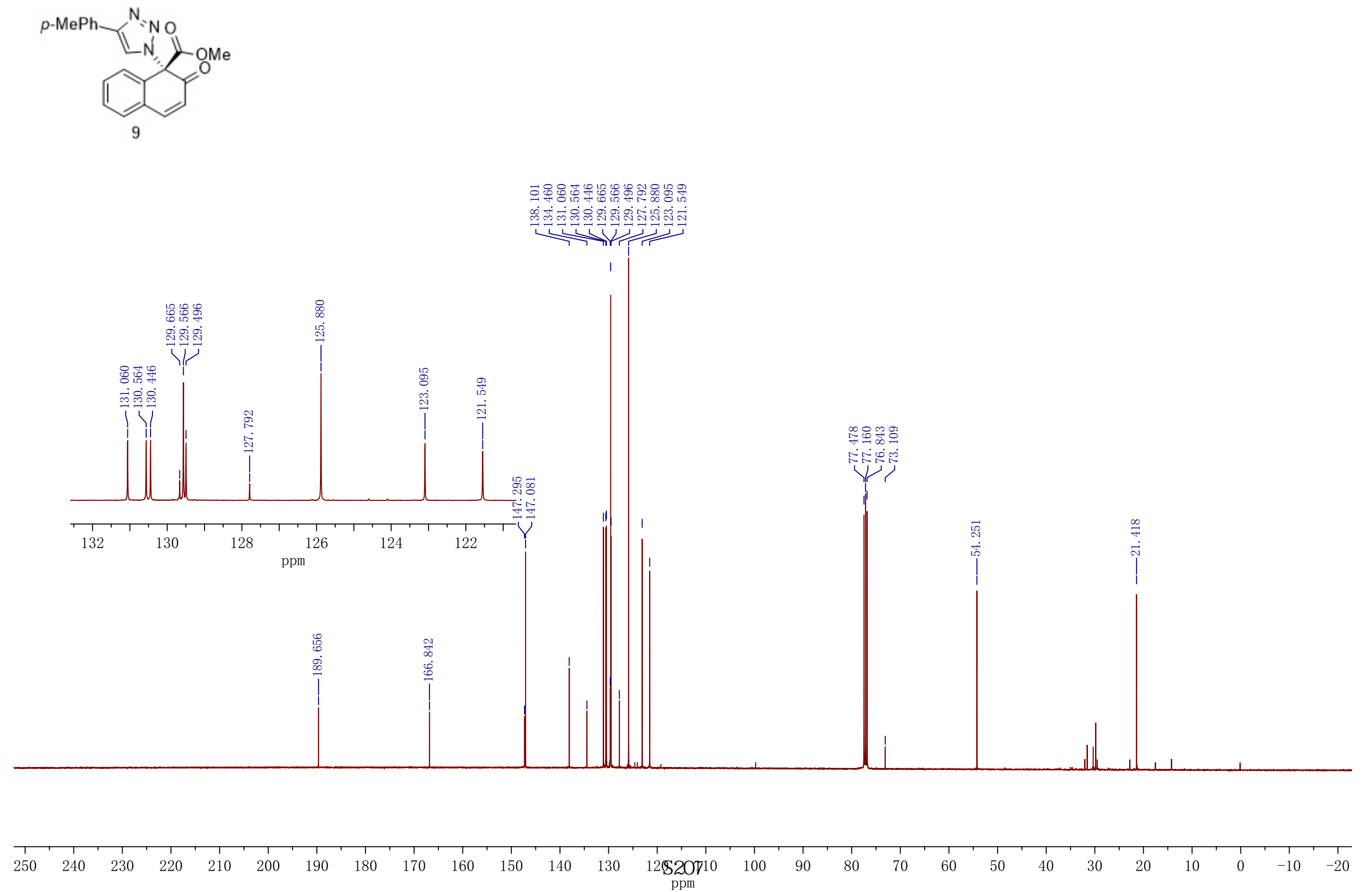